\title{
Photoredox-Catalyzed Intermolecular Hydroalkylative Dearomatization of Electron-Deficient Indole Derivatives
}

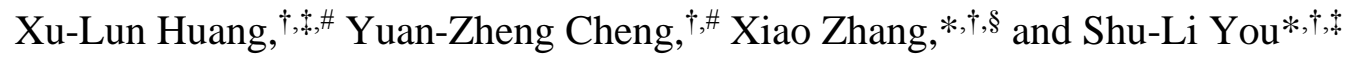

†State Key Laboratory of Organometallic Chemistry, Center for Excellence in Molecular Synthesis, Shanghai Institute of Organic Chemistry, University of Chinese Academy of Sciences, Chinese Academy of Sciences, 345 Lingling Lu, Shanghai 200032, China

${ }^{\ddagger}$ School of Physical Science and Technology, ShanghaiTech University, 100 Haike Road, Shanghai 201210, China

${ }^{\S}$ Fujian Key Laboratory of Polymer Science, Fujian Provincial Key Laboratory of Advanced Materials Oriented Chemical Engineering, College of Chemistry and Materials Science, Fujian Normal University, 32 Shangsan Lu, Fuzhou 350007, China

${ }^{\#}$ These authors contributed equally. 


\section{Table of Contents}

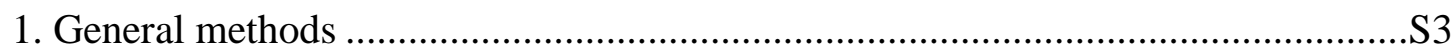

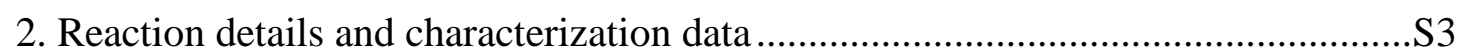

3. Experimental details for mechanistic studies...................................................S42

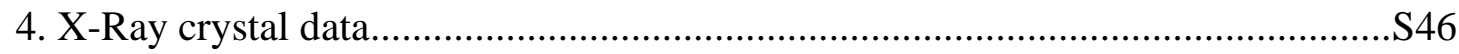

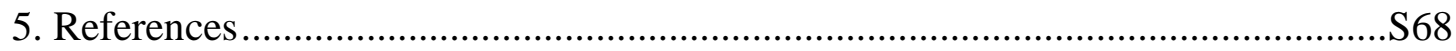

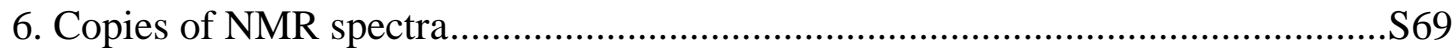




\section{General methods}

Unless stated otherwise, all reactions were carried out in flame-dried glassware under a dry argon atmosphere. All solvents were purified and dried according to standard methods prior to use. Anilinoacetic acid (2a, CAS 103-01-5) was purchased from TCI. Other amino acids (2b-2h) were synthesized according to a previous report. ${ }^{[1]}{ }^{1} \mathrm{H}$ and ${ }^{13} \mathrm{C}$ NMR spectra were recorded on an Agilent instrument $(400 \mathrm{MHz}$ and $100 \mathrm{MHz}$, respectively) or an Agilent instrument (600 MHz and $150 \mathrm{MHz}$, respectively) and internally referenced to tetramethylsilane signal or residual protio solvent signals. ${ }^{19} \mathrm{~F}$ NMR spectra were recorded on an Agilent instrument $(376 \mathrm{MHz})$ and referenced relative to $\mathrm{CFCl}_{3}$. Data for ${ }^{1} \mathrm{H}$ NMR are recorded as follows: chemical shift $(\delta, \mathrm{ppm})$, multiplicity $(\mathrm{s}=$ singlet, $\mathrm{d}=$ doublet, $\mathrm{t}=$ triplet, $\mathrm{m}=$ multiplet or unresolved, $\mathrm{br}=$ broad singlet, coupling constant(s) in $\mathrm{Hz}$, integration). Data for ${ }^{13} \mathrm{C}$ NMR are reported in terms of chemical shift $(\delta, \mathrm{ppm})$. UV/vis absorption spectra were recorded on a Thermo Nanodrop 2000c UV/Vis spectrometer. The mass analyzer type used for the HRMS (High Resolution Mass) measurements is TOF. Irradiation vessel made of borosilicate glass was purchased from synthware glass company. The $24 \mathrm{~W}$ blue LEDs were purchased from Hongchangzhaoming from Chinese Taobao (https://hongchangled.taobao.com), maximum emission wavelength $=450 \mathrm{~nm}$, the distance between blue LEDs and glass tube is $5 \mathrm{~cm}$.

\section{Reaction details and characterization data}

\subsection{General procedure for the preparation of 1a-11:}

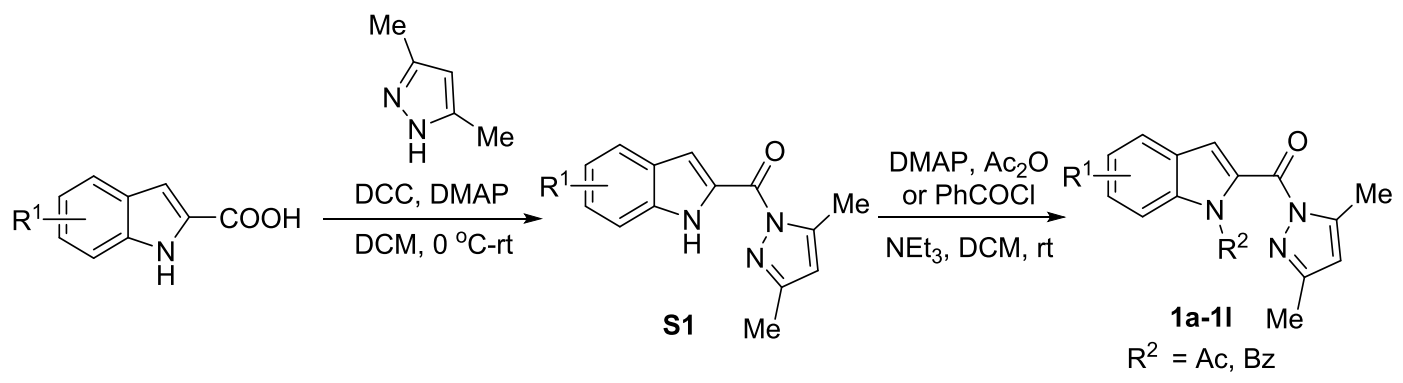


To a solution of substituted $1 H$-indole-2-carboxylic acid (1.0 equiv) and 3,5-dimethyl$1 H$-pyrazole (1.05 equiv) in $\mathrm{CH}_{2} \mathrm{Cl}_{2}(0.25 \mathrm{M})$ were added dicyclohexylcarbodiimide (1.2 equiv) and 4 -dimethylaminopyridine (1.0 equiv) successively at $0{ }^{\circ} \mathrm{C}$. The resulting mixture was stirred at $0{ }^{\circ} \mathrm{C}$ for 30 minutes, then gradually warmed to room temperature and further stirred for 24 hours. The reaction mixture was diluted with $\mathrm{CH}_{2} \mathrm{Cl}_{2}(20 \mathrm{~mL})$ and quenched with water $(20 \mathrm{~mL})$. The layers were separated and the aqueous layer was extracted with $\mathrm{CH}_{2} \mathrm{Cl}_{2}(20 \mathrm{~mL} \times 2)$. The combined organic layers were washed with brine, dried over anhydrous $\mathrm{Na}_{2} \mathrm{SO}_{4}$, filtered and concentrated in vacuo. The residue was purified by column chromatography on silica gel $(\mathrm{PE} / \mathrm{EtOAc}=4 / 1)$ to give $\mathbf{S 1}$.

To a solution of $\mathbf{S 1}$ (1.0 equiv) in $\mathrm{CH}_{2} \mathrm{Cl}_{2}(0.25 \mathrm{M})$, acetic anhydride (or benzoyl chloride) (1.5 equiv), $\mathrm{NEt}_{3}$ (2.5 equiv) and DMAP (10 mol\%) were added. The mixture was stirred at room temperature for $24 \mathrm{~h}$. After completion of the reaction as monitored by TLC, it was washed with a saturated solution of $\mathrm{NH}_{4} \mathrm{Cl}(20 \mathrm{~mL})$ and the aqueous phase was extracted with $\mathrm{CH}_{2} \mathrm{Cl}_{2}(2 \times 20 \mathrm{~mL})$. The combined organic extracts were dried over $\mathrm{Na}_{2} \mathrm{SO}_{4}$ and concentrated under vacuum. Then the residue was purified by column chromatography on silica gel to afford the desired product $\mathbf{1}$.

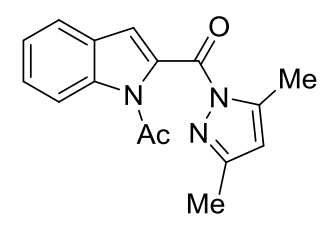

1a, PE/EtOAc $=4 / 1$, white solid, m.p. $=105.3-108.5^{\circ} \mathrm{C} .596 \mathrm{mg}, 42 \%$ yield over 2 steps (5 mmol scale). ${ }^{1} \mathrm{H} \mathrm{NMR}\left(400 \mathrm{MHz}, \mathrm{CDCl}_{3}\right) \delta 7.99(\mathrm{~d}, J=8.8 \mathrm{~Hz}, 1 \mathrm{H}), 7.65(\mathrm{~d}$, $J=7.6,1 \mathrm{H}), 7.44(\mathrm{t}, J=8.0 \mathrm{~Hz}, 1 \mathrm{H}), 7.29$ (t, $J=7.6 \mathrm{~Hz}, 1 \mathrm{H}), 7.26(\mathrm{~s}, 1 \mathrm{H}), 6.05(\mathrm{~s}, 1 \mathrm{H})$, $2.66(\mathrm{~s}, 3 \mathrm{H}), 2.59$ (s, 3H), $2.20(\mathrm{~s}, 3 \mathrm{H}) .{ }^{13} \mathrm{C} \mathrm{NMR}\left(100 \mathrm{MHz}, \mathrm{CDCl}_{3}\right) \delta$ 169.6, 161.9, 153.0, 145.0, 136.8, 132.2, 128.4, 127.3, 123.7, 122.9, 117.6, 114.7, 111.5, 26.3, 14.2, 13.9. IR (thin film): $v_{\max }\left(\mathrm{cm}^{-1}\right)=3106,2930,2853,2307,2114,1918,1755,1692$, 1585, 1561, 1486, 1457, 1431, 1406, 1374, 1351, 1305, 1269, 1232, 1212, 1177, 1150, 1125, 1053, 1027, 991, 949, 881, 845, 824, 800, 749, 686, 664, 633, 599, 579, 540, 498, 448. HRMS (ESI) m/z: [M + Na $]^{+}$Calcd for $\mathrm{C}_{16} \mathrm{H}_{15} \mathrm{~N}_{3} \mathrm{O}_{2} \mathrm{Na} 304.1057$; Found 304.1057. 


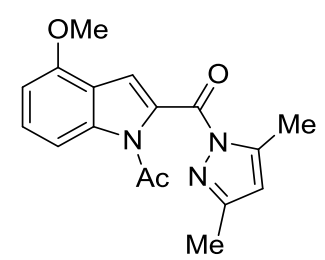

1b, PE/EtOAc $=4 / 1$, yellow solid, m.p. $=161.0-162.5^{\circ} \mathrm{C} .564 \mathrm{mg}, 36 \%$ yield over 2 steps (5 mmol scale). ${ }^{1} \mathrm{H}$ NMR (400 MHz, $\left.\mathrm{CDCl}_{3}\right) \delta 7.57$ (d, $\left.J=8.4 \mathrm{~Hz}, 1 \mathrm{H}\right), 7.43$ (s, 1H), 7.36 (t, $J=8.4 \mathrm{~Hz}, 1 \mathrm{H}), 6.68(\mathrm{~d}, J=8.0 \mathrm{~Hz}, 1 \mathrm{H}), 6.04(\mathrm{~s}, 1 \mathrm{H}), 3.92$ (s, 3H), 2.63 (s, 3H), 2.57 (s, 3H), 2.21 (s, 3H). $\left.{ }^{13} \mathrm{C} \mathrm{NMR} \mathrm{(100} \mathrm{MHz,} \mathrm{CDCl}_{3}\right) \delta$ 170.0, 161.7, 154.4, $152.8,144.9,138.4,130.5,128.9,119.0,116.1,111.4,107.5,103.7,55.6,26.4,14.2$, 13.9. IR (thin film): $v_{\max }\left(\mathrm{cm}^{-1}\right)=2927,1713,1695,1608,1582,1553,1494,1466$, 1436, 1355, 1311, 1289, 1260, 1178, 1147, 1126, 1047, 1007, 982, 959, 906, 854, 837, 822, 769, 733, 652, 607, 577, 510, 439. HRMS (ESI) m/z: $[\mathrm{M}+\mathrm{Na}]^{+}$Calcd for $\mathrm{C}_{17} \mathrm{H}_{17} \mathrm{~N}_{3} \mathrm{O}_{3} \mathrm{Na} 334.1162$; Found 334.1165.

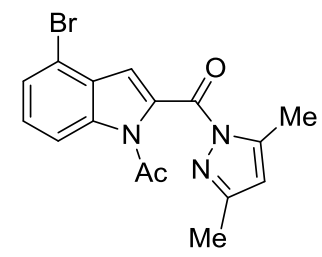

1c, $\mathrm{PE} / \mathrm{EtOAc}=4 / 1$, white solid, m.p. $=114.7-115.9{ }^{\circ} \mathrm{C} .545 \mathrm{mg}, 30 \%$ yield over 2 steps (5 mmol scale). ${ }^{1} \mathrm{H}$ NMR $\left(400 \mathrm{MHz}, \mathrm{CDCl}_{3}\right) \delta 7.96(\mathrm{~d}, J=8.4 \mathrm{~Hz}, 1 \mathrm{H}), 7.46(\mathrm{~d}$, $J=7.6 \mathrm{~Hz}, 1 \mathrm{H}), 7.31-7.26(\mathrm{~m}, 2 \mathrm{H}), 6.07$ (s, 1H), $2.65(\mathrm{~s}, 3 \mathrm{H}), 2.57(\mathrm{~s}, 3 \mathrm{H}), 2.21(\mathrm{~s}, 3 \mathrm{H})$. ${ }^{13} \mathrm{C}$ NMR (100 MHz, $\left.\mathrm{CDCl}_{3}\right) \delta 169.5,161.6,153.5,145.1,137.0,132.5,129.1,128.2$, 126.7, 116.9, 116.7, 113.9, 111.8, 26.3, 14.2, 14.0. IR (thin film): $v_{\max }\left(\mathrm{cm}^{-1}\right)=2929$, 1703, 1574, 1556, 1481, 1435, 1416, 1374, 1345, 1307, 1264, 1177, 1154, 1124, 1056, $1038,985,953,888,833,803,770,748,725,700,649,622,604,579,529,498$. HRMS (ESI) m/z: $[\mathrm{M}+\mathrm{Na}]^{+}$Calcd for $\mathrm{C}_{16} \mathrm{H}_{14} \mathrm{~N}_{3} \mathrm{O}_{2} \mathrm{Na}^{79} \mathrm{Br} 382.0162$; Found 382.0157. 


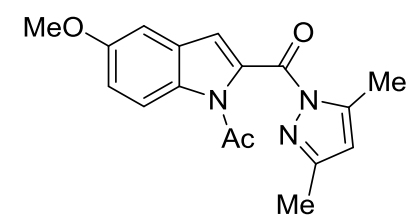

1d, PE/EtOAc $=4 / 1$, white solid, m.p. $=114.7-115.9{ }^{\circ} \mathrm{C} .813 \mathrm{mg}, 52 \%$ yield over 2 steps $(5 \mathrm{mmol} \mathrm{scale}) .{ }^{1} \mathrm{H}$ NMR $\left(400 \mathrm{MHz}, \mathrm{CDCl}_{3}\right) \delta 7.86(\mathrm{~d}, J=8.8 \mathrm{~Hz}, 1 \mathrm{H}), 7.14$ (s, 1H), 7.03-7.01 (m, 2H), $6.03(\mathrm{~s}, 1 \mathrm{H}), 3.81(\mathrm{~s}, 3 \mathrm{H}), 2.62(\mathrm{~s}, 3 \mathrm{H}), 2.53(\mathrm{~s}, 3 \mathrm{H}), 2.17$ (s, $3 \mathrm{H}) .{ }^{13} \mathrm{C} \mathrm{NMR}\left(100 \mathrm{MHz}, \mathrm{CDCl}_{3}\right) \delta 169.3,161.9,156.4,152.9,144.9,132.6,131.7$, 129.2, 117.4, 116.7, 115.7, 111.5, 104.3, 55.6, 26.1, 14.1, 13.9. IR (thin film): $v_{\max }\left(\mathrm{cm}^{-}\right.$ $\left.{ }^{1}\right)=2944,2929,1704,1614,1585,1553,1474,1444,1370,1354,1334,1304,1251$, 1204, 1178, 1160, 1145, 1129, 1056, 1028, 986, 949, 849, 828, 802, 762, 746, 733, 636, 617, 581, 533, 494, 432. HRMS (ESI) m/z: $[\mathrm{M}+\mathrm{Na}]^{+}$Calcd for $\mathrm{C}_{17} \mathrm{H}_{17} \mathrm{~N}_{3} \mathrm{O}_{3} \mathrm{Na}$ 334.1162; Found 334.1155.

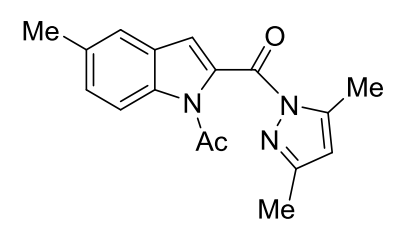

1e, $\mathrm{PE} /$ EtOAc $=4 / 1$, white solid, m.p. $=124.8-126.8{ }^{\circ} \mathrm{C} .580 \mathrm{mg}, 39 \%$ yield over 2 steps $(5 \mathrm{mmol} \mathrm{scale}) .{ }^{1} \mathrm{H} \mathrm{NMR}\left(400 \mathrm{MHz}, \mathrm{CDCl}_{3}\right) \delta 7.84(\mathrm{~d}, J=8.8 \mathrm{~Hz}, 1 \mathrm{H}), 7.41(\mathrm{~s}$, 1H), $7.24(\mathrm{~d}, J=8.8 \mathrm{~Hz}, 1 \mathrm{H}), 7.16(\mathrm{~s}, 1 \mathrm{H}), 6.03(\mathrm{~s}, 1 \mathrm{H}), 2.64(\mathrm{~s}, 3 \mathrm{H}), 2.56(\mathrm{~s}, 3 \mathrm{H}), 2.43$ (s, 3H), 2.18 (s, 3H). $\left.{ }^{13} \mathrm{C} \mathrm{NMR} \mathrm{(100} \mathrm{MHz,} \mathrm{CDCl}_{3}\right) \delta 169.4,162.0,152.9,144.9,135.1$, $133.3,132.2$, 128.8, 128.6, 122.5, 117.5, 114.4, 111.5, 26.2, 21.2, 14.2, 13.9. IR (thin film): $v_{\max }\left(\mathrm{cm}^{-1}\right)=2927,2854,1690,1582,1476,1429,1406,1374,1345,1298,1273$, 1215, 1155, 1135, 1059, 1040, 1024, 990, 953, 891, 802, 775, 741, 648, 621, 577, 533, 495, 443. HRMS (ESI) m/z: $[\mathrm{M}+\mathrm{Na}]^{+}$Calcd for $\mathrm{C}_{17} \mathrm{H}_{17} \mathrm{~N}_{3} \mathrm{O}_{2} \mathrm{Na}$ 318.1213; Found 318.1209 . 


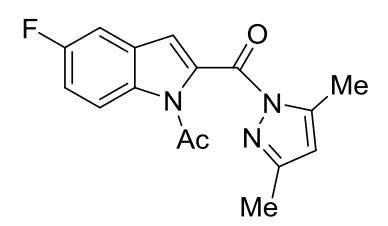

1f, $\mathrm{PE} / \mathrm{EtOAc}=4 / 1$, pale yellow solid, m.p. $=124.5-125.4{ }^{\circ} \mathrm{C} .612 \mathrm{mg}, 41 \%$ yield over 2 steps (5 mmol scale). ${ }^{1} \mathrm{H}$ NMR (400 MHz, $\left.\mathrm{CDCl}_{3}\right) \delta 7.99(\mathrm{dd}, J=9.2,4.4 \mathrm{~Hz}, 1 \mathrm{H})$, $7.28(\mathrm{dd}, J=8.4,2.4 \mathrm{~Hz}, 1 \mathrm{H}), 7.18-7.13(\mathrm{~m}, 2 \mathrm{H}), 6.07(\mathrm{~s}, 1 \mathrm{H}), 2.64(\mathrm{~s}, 3 \mathrm{H}), 2.55$ (s, $3 \mathrm{H}), 2.20(\mathrm{~s}, 3 \mathrm{H}) .{ }^{13} \mathrm{C}$ NMR $\left(100 \mathrm{MHz}, \mathrm{CDCl}_{3}\right) \delta 169.4,161.6,159.5\left(\mathrm{C}-\mathrm{F},{ }^{1} J_{\mathrm{C}-\mathrm{F}}=\right.$ $239.7 \mathrm{~Hz}), 153.4,145.1,133.5,133.3,129.1\left(\mathrm{C}-\mathrm{F},{ }^{3} J_{\mathrm{C}-\mathrm{F}}=10.0 \mathrm{~Hz}\right), 117.0\left(\mathrm{C}-\mathrm{F},{ }^{4} J_{\mathrm{C}-\mathrm{F}}=\right.$ $4.3 \mathrm{~Hz}), 116.2\left(\mathrm{C}-\mathrm{F},{ }^{3} J_{\mathrm{C}-\mathrm{F}}=9.0 \mathrm{~Hz}\right), 115.4\left(\mathrm{C}-\mathrm{F},{ }^{2} J_{\mathrm{C}-\mathrm{F}}=25.1 \mathrm{~Hz}\right), 111.8,107.8(\mathrm{C}-\mathrm{F}$, $\left.{ }^{2} J_{\mathrm{C}-\mathrm{F}}=23.6 \mathrm{~Hz}\right), 26.2,14.2,13.9 .{ }^{19} \mathrm{~F}$ NMR $\left(376 \mathrm{MHz}, \mathrm{CDCl}_{3}\right) \delta-119.17--119.22(\mathrm{~m})$. IR (thin film): $v_{\max }\left(\mathrm{cm}^{-1}\right)=2934,1700,1613,1559,1470,1432,1375,1345,1306$, 1268, 1241, 1210, 1188, 1160, 1132, 1059, 1035, 989, 948, 875, 833, 808, 768, 736, 645, 618, 583, 499, 434. HRMS (ESI) m/z: $[\mathrm{M}+\mathrm{Na}]^{+}$Calcd for $\mathrm{C}_{16} \mathrm{H}_{14} \mathrm{~N}_{3} \mathrm{O}_{2} \mathrm{FNa}$ 322.0962; Found 322.0957.

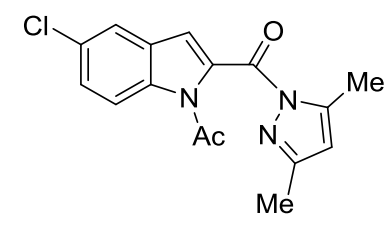

1g, PE/EtOAc $=4 / 1$, white solid, m.p. $=137.4-138.5^{\circ} \mathrm{C} .697 \mathrm{mg}, 44 \%$ yield over 2 steps (5 mmol scale). ${ }^{1} \mathrm{H} \mathrm{NMR}\left(400 \mathrm{MHz}, \mathrm{CDCl}_{3}\right) \delta 7.94(\mathrm{~d}, J=8.8 \mathrm{~Hz}, 1 \mathrm{H}), \delta 7.59$ (s, 1H), $7.37(\mathrm{~d}, J=10.0 \mathrm{~Hz}, 1 \mathrm{H}), 7.16(\mathrm{~s}, 1 \mathrm{H}), 6.06(\mathrm{~s}, 1 \mathrm{H}), 2.64(\mathrm{~s}, 3 \mathrm{H}), 2.54(\mathrm{~s}, 3 \mathrm{H})$, $2.20(\mathrm{~s}, 3 \mathrm{H}) .{ }^{13} \mathrm{C}$ NMR $\left(100 \mathrm{MHz}, \mathrm{CDCl}_{3}\right) \delta 169.4,161.6,153.4,145.1,135.2,133.3$, $129.4,129.3,127.5,122.1,116.5,116.1,111.8,26.3,14.2,13.9$. IR (thin film): $v_{\max }$ $\left(\mathrm{cm}^{-1}\right)=2930,1699,1583,1561,1481,1428,1373,1346,1305,1270,1210,1174$, 1126, 1078, 1059, 1027, 990, 954, 908, 815, 798, 771, 747, 725, 644, 618, 576, 538, 475, 444, 426. HRMS (ESI) m/z: $[\mathrm{M}+\mathrm{Na}]^{+}$Calcd for $\mathrm{C}_{16} \mathrm{H}_{14} \mathrm{~N}_{3} \mathrm{O}_{2} \mathrm{NaCl} 338.0667$; Found 338.0660. 


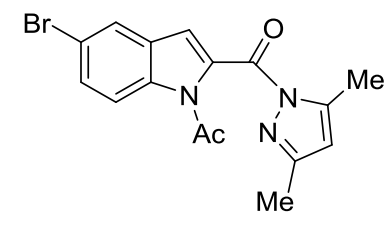

1h, PE/EtOAc $=4 / 1$, white solid, m.p. $=158.9-160.8{ }^{\circ} \mathrm{C} .811 \mathrm{mg}, 45 \%$ yield over 2 steps (5 mmol scale). ${ }^{1} \mathrm{H}$ NMR $\left(400 \mathrm{MHz}, \mathrm{CDCl}_{3}\right) \delta 7.86(\mathrm{~d}, J=9.2 \mathrm{~Hz}, 1 \mathrm{H}), 7.73(\mathrm{~d}$, $J=1.6 \mathrm{~Hz}, 1 \mathrm{H}), 7.48(\mathrm{dd}, J=8.8,2.0 \mathrm{~Hz}, 1 \mathrm{H}), 7.13(\mathrm{~s}, 1 \mathrm{H}), 6.05(\mathrm{~s}, 1 \mathrm{H}), 2.63(\mathrm{~s}, 3 \mathrm{H})$, $2.53(\mathrm{~s}, 3 \mathrm{H}), 2.18(\mathrm{~s}, 3 \mathrm{H}) .{ }^{13} \mathrm{C} \mathrm{NMR}\left(100 \mathrm{MHz}, \mathrm{CDCl}_{3}\right) \delta 169.3,161.5,153.3,145.0$, $135.4,133.0,130.0,129.9,125.1,116.8,116.3,116.2,111.8,26.2,14.2,13.9$. IR (thin film): $v_{\max }\left(\mathrm{cm}^{-1}\right)=2928,1700,1582,1556,1480,1427,1373,1345,1304,1271,1211$, 1174, 1125, 1059, 1027, 989, 954, 896, 799, 771, 745, 710, 642, 615, 574, 536, 512, 443, 419. HRMS (ESI) m/z: $[\mathrm{M}+\mathrm{Na}]^{+}$Calcd for $\mathrm{C}_{16} \mathrm{H}_{14} \mathrm{~N}_{3} \mathrm{O}_{2} \mathrm{Na}^{79} \mathrm{Br} 382.0162$; Found 382.0155 .

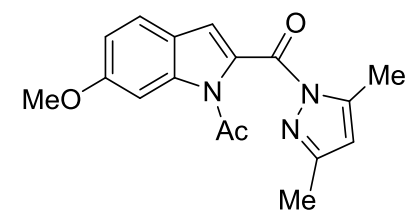

1i, PE/EtOAc $=4 / 1$, white solid, m.p. $=145.5-147.6^{\circ} \mathrm{C} .344 \mathrm{mg}, 22 \%$ yield over 2 steps (5 mmol scale). ${ }^{1} \mathrm{H}$ NMR (400 MHz, $\left.\mathrm{CDCl}_{3}\right) \delta 7.65(\mathrm{~s}, 1 \mathrm{H}), 7.48(\mathrm{~d}, J=8.8 \mathrm{~Hz}, 1 \mathrm{H})$, 7.34 (s, 1H), $6.90(\mathrm{dd}, J=8.4,2.0 \mathrm{~Hz}, 1 \mathrm{H}), 6.05(\mathrm{~s}, 1 \mathrm{H}), 3.87$ (s, 3H), 2.60 (s, 3H), 2.51 (s, 3H), 2.24 (s, 3H). ${ }^{13} \mathrm{C}$ NMR $\left(100 \mathrm{MHz}, \mathrm{CDCl}_{3}\right) \delta 170.7,161.0,160.8,152.8,145.0$, $139.6,130.3,123.6,121.5,121.2,113.9,111.5,98.6,55.7,26.8,14.2,13.9$. IR (thin film): $v_{\max }\left(\mathrm{cm}^{-1}\right)=3354$ 5, 3134, 2991, 2961, 2937, 2838, 2303, 2111, 1995, 1901, 1743, 1701, 1682, 1610, 1585, 1520, 1488, 1440, 1404, 1371, 1357, 1324, 1307, 1276, 1243, 1212, 1189, 1170, 1110, 1062, 1044, 1024, 965, 891, 860, 836, 813, 798, 785, 760, 745, 698, 647, 635, 595, 575, 539, 471, 443, 425. HRMS (ESI) m/z: [M + Na $]^{+}$ Calcd for $\mathrm{C}_{17} \mathrm{H}_{17} \mathrm{~N}_{3} \mathrm{O}_{3} \mathrm{Na} 334.1162$; Found 334.1160. 


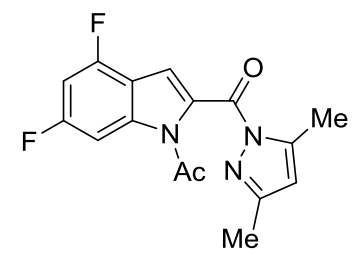

1j, PE/EtOAc $=4 / 1$, yellow solid, m.p. $=161.9-164.4{ }^{\circ} \mathrm{C} .342 \mathrm{mg}, 22 \%$ yield over 2 steps (5 mmol scale). ${ }^{1} \mathrm{H}$ NMR (400 MHz, $\left.\mathrm{CDCl}_{3}\right) \delta 7.67(\mathrm{~d}, J=9.6 \mathrm{~Hz}, 1 \mathrm{H}), 7.37$ (s, $1 \mathrm{H}), 6.80(\mathrm{t}, J=8.8 \mathrm{~Hz}, 1 \mathrm{H}), 6.10(\mathrm{~s}, 1 \mathrm{H}), 2.64(\mathrm{~s}, 3 \mathrm{H}), 2.52(\mathrm{~s}, 3 \mathrm{H}), 2.24(\mathrm{~s}, 3 \mathrm{H}),{ }^{13} \mathrm{C}$ NMR $\left(100 \mathrm{MHz}, \mathrm{CDCl}_{3}\right) \delta 169.8,166.5,162.9\left(\mathrm{C}-\mathrm{F},{ }^{1} J_{\mathrm{C}-\mathrm{F}}=244.7 \mathrm{~Hz} ; \mathrm{C}-\mathrm{F},{ }^{3} J_{\mathrm{C}-\mathrm{F}}=11.5\right.$ $\mathrm{Hz}), 160.8,156.5\left(\mathrm{C}-\mathrm{F},{ }^{1} J_{\mathrm{C}-\mathrm{F}}=252.5 \mathrm{~Hz} ; \mathrm{C}-\mathrm{F},{ }^{3} J_{\mathrm{C}-\mathrm{F}}=11.5 \mathrm{~Hz}\right), 153.6,145.2,138.5(\mathrm{C}-$ $\left.\mathrm{F},{ }^{3} J_{\mathrm{C}-\mathrm{F}}=14.9 \mathrm{~Hz} ; \mathrm{C}-\mathrm{F},{ }^{3} J_{\mathrm{C}-\mathrm{F}}=10.3 \mathrm{~Hz}\right), 132.0\left(\mathrm{C}-\mathrm{F},{ }^{3} J_{\mathrm{C}-\mathrm{F}}=3.7 \mathrm{~Hz}\right), 114.0,113.8$, 112.0, $99.4\left(\mathrm{C}-\mathrm{F},{ }^{2} J_{\mathrm{C}-\mathrm{F}}=28.5 \mathrm{~Hz} ; \mathrm{C}-\mathrm{F},{ }^{2} J_{\mathrm{C}-\mathrm{F}}=22.2 \mathrm{~Hz}\right), 98.8\left(\mathrm{C}-\mathrm{F},{ }^{2} J_{\mathrm{C}-\mathrm{F}}=28.8 \mathrm{~Hz}\right.$; C$\left.\mathrm{F},{ }^{4} J_{\mathrm{C}-\mathrm{F}}=4.6 \mathrm{~Hz}\right), 26.4,14.2,13.9 .{ }^{19} \mathrm{~F} \mathrm{NMR}\left(376 \mathrm{MHz}, \mathrm{CDCl}_{3}\right) \delta-108.81-108.88(\mathrm{~m})$, -115.97--116.01 (m). IR (thin film): $v_{\max }\left(\mathrm{cm}^{-1}\right)=2932,1709,1686,1630,1589,1529$, $1485,1423,1385,1364,1321,1264,1241,1173,1147,1115,1080,1057,984,964$, 891, 851, 835, 809, 775, 748, 726, 677, 621, 598, 546, 525, 483, 425. HRMS (ESI) m/z: $[\mathrm{M}+\mathrm{Na}]^{+}$Calcd for $\mathrm{C}_{16} \mathrm{H}_{13} \mathrm{~N}_{3} \mathrm{O}_{2} \mathrm{~F}_{2} \mathrm{Na}$ 340.0868; Found 340.0860 .

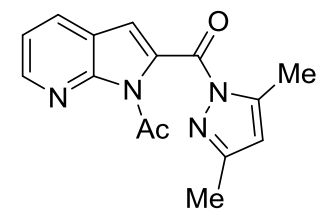

1k, PE/EtOAc $=2 / 1$, white solid, m.p. $=104.4-105.5{ }^{\circ} \mathrm{C} .217 \mathrm{mg}, 15 \%$ yield over 2 steps (5 mmol scale). ${ }^{1} \mathrm{H}$ NMR $\left(400 \mathrm{MHz}, \mathrm{CDCl}_{3}\right) \delta 8.45-8.44(\mathrm{~m}, 1 \mathrm{H}), 7.94(\mathrm{~d}, J=7.6$ $\mathrm{Hz}, 1 \mathrm{H}), 7.23$ (dd, J= 7.8 Hz, 2H), 7.02 (s, 1H), 6.00 (s, 1H), 3.02 (s, 3H), 2.67 (s, 3H), $2.12(\mathrm{~s}, 3 \mathrm{H}) .{ }^{13} \mathrm{C} \mathrm{NMR}\left(100 \mathrm{MHz}, \mathrm{CDCl}_{3}\right) \delta 169.5,162.4,152.9,147.9,145.9,144.7$, 132.8, 130.7, 121.4, 119.2, 111.2, 110.5. IR (thin film): $v_{\max }\left(\mathrm{cm}^{-1}\right)=2931,1720,1700$, 1583, 1554, 1485, 1432, 1403, 1375, 1339, 1323, 1295, 1251, 1230, 1197, 1136, 1058, 1026, 1007, 956, 891, 837, 804, 766, 749, 674, 637, 605, 576, 539, 505. HRMS (ESI) $\mathrm{m} / \mathrm{z}:[\mathrm{M}+\mathrm{Na}]^{+}$Calcd for $\mathrm{C}_{15} \mathrm{H}_{14} \mathrm{~N}_{4} \mathrm{O}_{2} \mathrm{Na}$ 305.1009; Found 305.1010. 


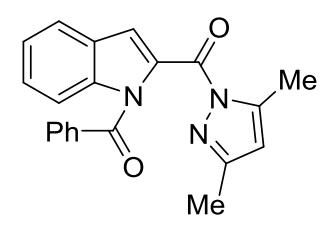

11, PE/EtOAc $=2 / 1$, white solid, m.p. $=131.4-133.7^{\circ} \mathrm{C} .1 .50 \mathrm{~g}, 57 \%$ yield over 2 steps (7.6 mmol scale). ${ }^{1} \mathrm{H}$ NMR (400 MHz, $\left.\mathrm{CDCl}_{3}\right) \delta 7.59(\mathrm{~d}, J=7.6 \mathrm{~Hz}, 1 \mathrm{H}), 7.52-7.48$ (m, 3H), 7.41-7.37 (m, 1H), $7.35(\mathrm{~s}, 1 \mathrm{H}), 7.27-7.20(\mathrm{~m}, 3 \mathrm{H}), 7.15(\mathrm{t}, J=7.6 \mathrm{~Hz}, 1 \mathrm{H})$, $5.70(\mathrm{~s}, 1 \mathrm{H}), 2.15$ (s, 3H), 1.99 (s, 3H). ${ }^{13} \mathrm{C} \mathrm{NMR}\left(100 \mathrm{MHz}, \mathrm{CDCl}_{3}\right) \delta$ 168.7, 161.0, 152.9, 144.6, 137.9, 134.8, 133.0, 132.7, 129.2, 128.5, 127.7, 127.0, 123.4, 122.6, 117.4, 114.5, 111.2, 13.7, 13.6. IR (thin film): $v_{\max }\left(\mathrm{cm}^{-1}\right)=2927,1697,1678,1584,1551$, 1481, 1446, 1356, 1297, 1273, 1248, 1224, 1160, 1119, 1099, 1071, 1021, 961, 929, 887, 863, 811, 786, 766, 741, 723, 701, 655, 604, 537, 473, 435. HRMS (ESI) m/z: [M $+\mathrm{Na}]^{+}$Calcd for $\mathrm{C}_{21} \mathrm{H}_{17} \mathrm{~N}_{3} \mathrm{O}_{2} \mathrm{Na}$ 366.1213; Found 366.1211.

\subsection{General procedure for the preparation of $1 \mathrm{a}^{\prime}-1 \mathrm{f}^{\prime}$ :}

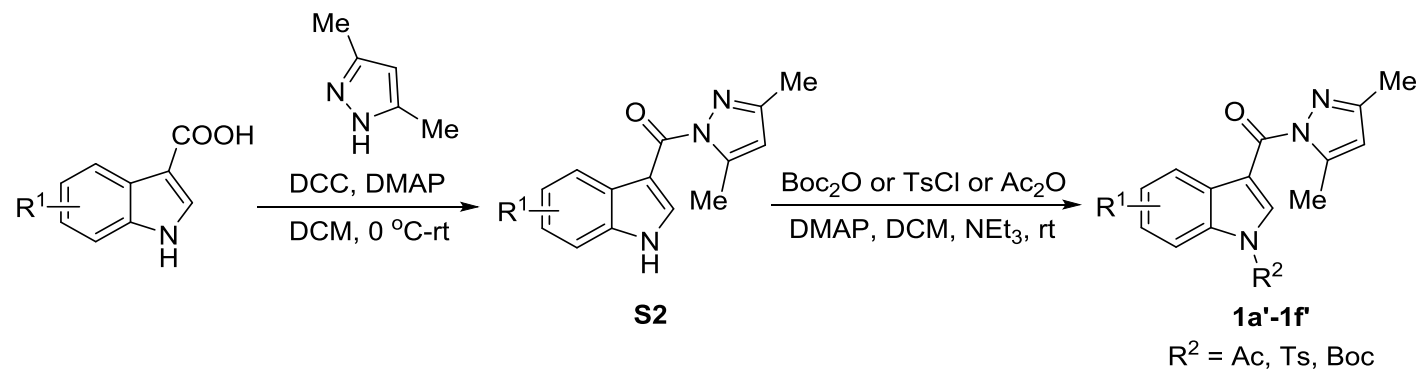

To a solution of $1 H$-indole-3-carboxylic acid (1.0 equiv) and 3,5-dimethyl- $1 H$-pyrazole (1.05 equiv) in $\mathrm{CH}_{2} \mathrm{Cl}_{2}(0.25 \mathrm{M})$ were added dicyclohexylcarbodiimide (1.2 equiv) and 4-dimethylaminopyridine (1.0 equiv) successively at $0{ }^{\circ} \mathrm{C}$. The resulting mixture was stirred at $0{ }^{\circ} \mathrm{C}$ for 30 minutes, then gradually warmed to room temperature and further stirred for 24 hours. The reaction mixture was diluted with $\mathrm{CH}_{2} \mathrm{Cl}_{2}(20 \mathrm{~mL})$ and quenched with water $(20 \mathrm{~mL})$. The layers were separated and the aqueous layer was extracted with $\mathrm{CH}_{2} \mathrm{Cl}_{2}(20 \mathrm{~mL} \times 2)$. The combined organic layers were washed with 
brine, dried over anhydrous $\mathrm{Na}_{2} \mathrm{SO}_{4}$, filtered and concentrated in vacuo. The residue was purified by column chromatography on silica gel to give $\mathbf{S 2}$.

To a solution of $\mathbf{S 2}$ (1.0 equiv) in $\mathrm{CH}_{2} \mathrm{Cl}_{2}(0.25 \mathrm{M})$, acetic anhydride (or $\mathrm{Boc}_{2} \mathrm{O}, \mathrm{TsCl}$ ) (1.5 equiv), $\mathrm{NEt}_{3}$ (2.5 equiv) and DMAP (10 mol\%) were added. The mixture was stirred at room temperature for $24 \mathrm{~h}$. After completion of the reaction as monitored by TLC, it was washed with a saturated solution of $\mathrm{NH}_{4} \mathrm{Cl}(20 \mathrm{~mL})$ and the aqueous layer was extracted with $\mathrm{CH}_{2} \mathrm{Cl}_{2}(2 \times 20 \mathrm{~mL})$. The combined organic extracts were dried over $\mathrm{Na}_{2} \mathrm{SO}_{4}$, filtered, and concentrated under vacuum. Then the residue was purified by column chromatography on silica gel to afford the desired product $\mathbf{1 a}^{\prime} \mathbf{- 1 \mathbf { f } ^ { \prime }}$.

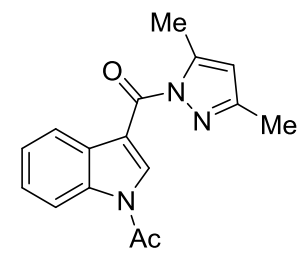

1a', PE/EtOAc $=4 / 1$, white solid, m.p. $=164.0-165.1{ }^{\circ} \mathrm{C} .3 .50 \mathrm{~g}, 50 \%$ yield over 2 steps (25 mmol scale). ${ }^{1} \mathrm{H}$ NMR (400 MHz, $\left.\mathrm{CDCl}_{3}\right) \delta 8.98$ (s, 1H), 8.46-8.43 (m, 1H), 8.39$8.37(\mathrm{~m}, 1 \mathrm{H}), 7.42-7.37(\mathrm{~m}, 2 \mathrm{H}), 6.05(\mathrm{~s}, 1 \mathrm{H}), 2.72(\mathrm{~s}, 3 \mathrm{H}), 2.66(\mathrm{~s}, 3 \mathrm{H}), 2.30(\mathrm{~s}, 3 \mathrm{H})$. ${ }^{13} \mathrm{C} \mathrm{NMR}\left(100 \mathrm{MHz}, \mathrm{CDCl}_{3}\right) \delta 169.2,162.8,152.1,145.4,135.8,135.3,129.3,126.1$, 125.1, 122.2, 116.4, 113.9, 110.9, 24.0, 14.9, 14.1. IR (thin film): $v_{\max }\left(\mathrm{cm}^{-1}\right)=3180$, 2927, 1722, 1667, 1607, 1580, 1532, 1479, 1441, 1387, 1363, 1322, 1305, 1269, 1238, 1206, 1188, 1151, 1113, 1083, 1036, 1014, 969, 938, 858, 821, 810, 771, 750, 730, 651, 607, 583, 561, 512, 455, 425. HRMS (ESI) m/z: $[\mathrm{M}+\mathrm{Na}]^{+}$Calcd for $\mathrm{C}_{16} \mathrm{H}_{15} \mathrm{~N}_{3} \mathrm{O}_{2} \mathrm{Na}$ 304.1057; Found 304.1054.

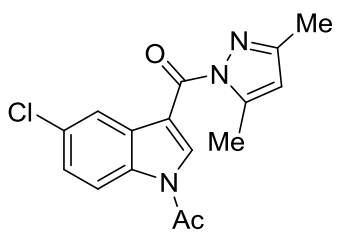


1b', PE/EtOAc $=4 / 1$, white solid, m.p. $=171.1-173.3{ }^{\circ} \mathrm{C} .228 \mathrm{mg}, 14 \%$ yield over 2 steps (5 mmol scale). ${ }^{1} \mathrm{H} \mathrm{NMR}\left(400 \mathrm{MHz}, \mathrm{CDCl}_{3}\right) \delta 9.00(\mathrm{~s}, 1 \mathrm{H}), 8.38(\mathrm{~d}, J=2.4 \mathrm{~Hz}$, $1 \mathrm{H}), 8.34(\mathrm{~d}, J=8.8 \mathrm{~Hz}, 1 \mathrm{H}), 7.32(\mathrm{dd}, J=8.8,2.0 \mathrm{~Hz}, 1 \mathrm{H}), 6.04(\mathrm{~s}, 1 \mathrm{H}), 2.72(\mathrm{~s}, 3 \mathrm{H})$, 2.65 (s, 3H), 2.31 (s, 3H). ${ }^{13} \mathrm{C} \mathrm{NMR}\left(100 \mathrm{MHz}, \mathrm{CDCl}_{3}\right) \delta 169.0,162.3,152.3,145.5$, 136.6, 133.6, 131.0, 130.6, 126.3, 122.0, 117.4, 113.2, 111.1, 23.8, 14.9, 14.1. IR (thin film): $v_{\max }\left(\mathrm{cm}^{-1}\right)=3175,2925,1729,1671,1578,1536,1438,1365,1341,1317,1247$, 1201, 1183, 1117, 1099, 1061, 1045, 1012, 984, 960, 939, 894, 875, 804, 767, 752, 727, 670, 617, 446, 423. HRMS (ESI) m/z: $[\mathrm{M}+\mathrm{Na}]^{+}$Calcd for $\mathrm{C}_{16} \mathrm{H}_{14} \mathrm{~N}_{3} \mathrm{O}_{2} \mathrm{NaCl} 338.0667$; Found 338.0658 .

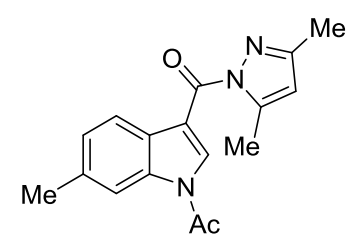

1c', PE/EtOAc $=4 / 1$, white solid, m.p. $=161.4-163.4{ }^{\circ} \mathrm{C} .388 \mathrm{mg}, 26 \%$ yield over 2 steps (5 mmol scale). ${ }^{1} \mathrm{H}$ NMR (400 MHz, $\left.\mathrm{CDCl}_{3}\right) \delta 8.91(\mathrm{~s}, 1 \mathrm{H}), 8.27$ (s, 1H), 8.24 (d, $J=8.4 \mathrm{~Hz}, 1 \mathrm{H}), 7.22(\mathrm{~d}, J=8.4 \mathrm{~Hz}, 1 \mathrm{H}), 6.05(\mathrm{~s}, 1 \mathrm{H}), 2.71(\mathrm{~s}, 3 \mathrm{H}), 2.66(\mathrm{~s}, 3 \mathrm{H}), 2.50$ (s, 3H), 2.30 (s, 3H). $\left.{ }^{13} \mathrm{C} \mathrm{NMR} \mathrm{(100} \mathrm{MHz,} \mathrm{CDCl}_{3}\right) \delta 169.3,162.9,152.0,145.4,136.2$, $135.7,135.3,127.0,126.5,121.7,116.5,113.9,110.9,24.0,22.0,14.9,14.1$. IR (thin film): $v_{\max }\left(\mathrm{cm}^{-1}\right)=3178,2925,1724,1671,1581,1533,1485,1422,1382,1322,1301$, 1270, 1219, 1190, 1150, 1120, 1083, 1017, 966, 950, 898, 818, 784, 757, 728, 701, 651, 620, 586, 465, 429. HRMS (ESI) m/z: $[\mathrm{M}+\mathrm{Na}]^{+}$Calcd for $\mathrm{C}_{17} \mathrm{H}_{17} \mathrm{~N}_{3} \mathrm{O}_{2} \mathrm{Na}$ 318.1213; Found 318.1212 .

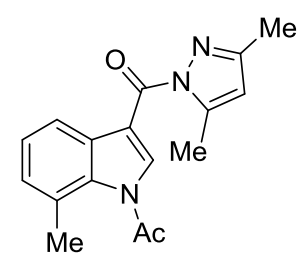

1d', PE/EtOAc = 4/1, white solid, m.p. $=110.3-110.7{ }^{\circ} \mathrm{C} .640 \mathrm{mg}, 43 \%$ yield over 2 steps (5 mmol scale). ${ }^{1} \mathrm{H}$ NMR (400 MHz, $\left.\mathrm{CDCl}_{3}\right) \delta 8.98(\mathrm{~s}, 1 \mathrm{H}), 8.27$ (d, J=7.6 Hz, 1H), 7.33 (t, $J=7.6 \mathrm{~Hz}, 1 \mathrm{H}), 7.21(\mathrm{~d}, J=7.2 \mathrm{~Hz}, 1 \mathrm{H}), 6.06$ (s, 1H), 2.76 (s, 3H), 2.67 
(s, 3H), 2.55 (s, 3H), 2.31 (s, 3H). $\left.{ }^{13} \mathrm{C} \mathrm{NMR} \mathrm{(100} \mathrm{MHz,} \mathrm{CDCl}_{3}\right) \delta$ 168.0, 162.7, 151.8, 145.2, 137.0, 134.7, 130.7, 128.9, 126.1, 125.2, 119.6, 113.2, 110.7, 24.4, 22.5, 14.8, 14.0. IR (thin film): $v_{\max }\left(\mathrm{cm}^{-1}\right)=3188,2928,2112,1731,1581,1540,1484,1438$, 1321, 1307, 1253, 1188, 1166, 1144, 1117, 1080, 1031, 1001, 967, 946, 922, 859, 812, 791, 775, 763, 672, 650, 618, 581, 556, 500, 432. HRMS (ESI) m/z: $[\mathrm{M}+\mathrm{Na}]^{+} \mathrm{Calcd}$ for $\mathrm{C}_{17} \mathrm{H}_{17} \mathrm{~N}_{3} \mathrm{O}_{2} \mathrm{Na} 318.1213$; Found 318.1211.

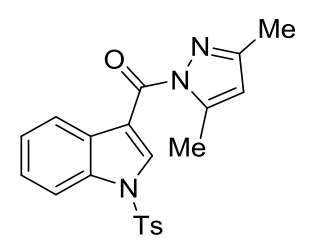

1e', PE/EtOAc $=4 / 1$, white solid, m.p. $=145.4-147.4{ }^{\circ} \mathrm{C} .750 \mathrm{mg}, 54 \%$ yield over 2 steps (3.5 mmol scale). ${ }^{1} \mathrm{H}$ NMR (400 MHz, $\left.\mathrm{CDCl}_{3}\right) \delta 9.00(\mathrm{~s}, 1 \mathrm{H}), 8.27-8.25(\mathrm{~m}, 1 \mathrm{H})$, 7.88-7.85 (m, 1H), 7.74 (d, $J=8.0 \mathrm{~Hz}, 2 \mathrm{H}), 7.27-7.20(\mathrm{~m}, 2 \mathrm{H}), 7.12(\mathrm{~d}, J=8.4 \mathrm{~Hz}, 2 \mathrm{H})$, $5.93(\mathrm{~s}, 1 \mathrm{H}), 2.52$ (s, 3H), 2.23 (s, 3H), 2.19 (s, 3H). ${ }^{13} \mathrm{C} \mathrm{NMR}\left(100 \mathrm{MHz}, \mathrm{CDCl}_{3}\right) \delta$ 162.6, 152.2, 145.8, 145.1, 136.3, 134.7, 134.1, 130.2, 129.7, 127.3, 125.4, 124.6, 122.7, 113.6, 113.2, 111.0, 21.6, 14.8, 14.1. IR (thin film): $v_{\max }\left(\mathrm{cm}^{-1}\right)=3157,1675,1584$, 1531, 1477, 1444, 1370, 1322, 1307, 1217, 1171, 1133, 1111, 1095, 1022, 965, 857, 817, 797, 762, 744, 716, 661, 617, 570, 535, 486, 450, 422. HRMS (ESI) m/z: [M + $\mathrm{Na}]^{+}$Calcd for $\mathrm{C}_{21} \mathrm{H}_{19} \mathrm{~N}_{3} \mathrm{O}_{3} \mathrm{NaS}$ 416.1039; Found 416.1037.

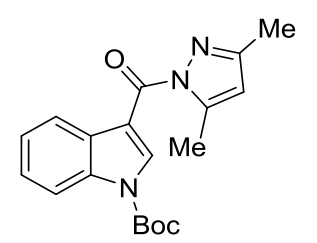

1f', PE/EtOAc $=4 / 1$, white solid, m.p. $=171.1-173.3{ }^{\circ} \mathrm{C} .730 \mathrm{mg}, 61 \%$ yield over 2 steps (3.5 mmol scale). ${ }^{1} \mathrm{H}$ NMR (400 MHz, $\left.\mathrm{CDCl}_{3}\right) \delta 9.07(\mathrm{~s}, 1 \mathrm{H}), 8.42-8.40(\mathrm{~m}, 1 \mathrm{H})$, 8.19-8.17 (m, 1H), 7.41-7.35 (m, 2H), $6.04(\mathrm{~s}, 1 \mathrm{H}), 2.67$ (s, 3H), 2.31 (s, 3H), $1.71(\mathrm{~s}$, 9H). ${ }^{13} \mathrm{C} \mathrm{NMR}\left(100 \mathrm{MHz}, \mathrm{CDCl}_{3}\right) \delta 163.1,151.8,149.2,146.8,145.0,136.6,134.9$, $129.5,125.2,124.2,122.3,115.0,112.5,110.7,85.2,85.1,28.1,27.5,14.8,13.9$. IR (thin film): $v_{\max }\left(\mathrm{cm}^{-1}\right)=3196,2978,2929,1744,1674,1579,1536,1476,1449,1385$, 
1366, 1323, 1306, 1272, 1249, 1220, 1148, 1116, 1084, 1039, 1017, 969, 947, 852, 819, 765, 750, 733, 715, 617, 579, 475, 443, 426. HRMS (ESI) m/z: $[\mathrm{M}+\mathrm{Na}]^{+}$Calcd for $\mathrm{C}_{19} \mathrm{H}_{21} \mathrm{~N}_{3} \mathrm{O}_{3} \mathrm{Na}$ 362.1475; Found 362.1466.

\subsection{General procedure for intermolecular hydroalkylative dearomatization of electron-deficient indole derivatives}

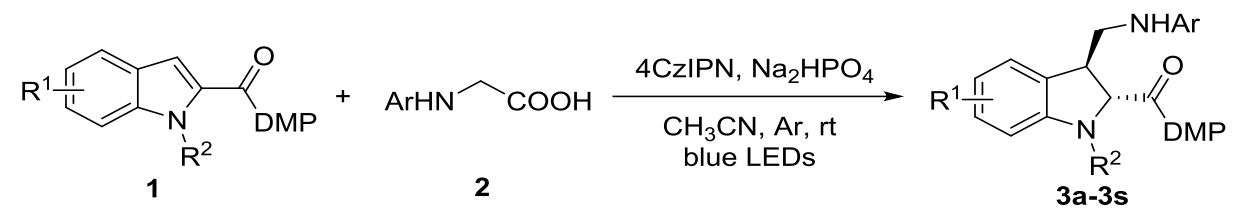

To a sealed tube equipped with a magnetic stir bar were added $\mathbf{1}(0.2 \mathrm{mmol}, 1.0$ equiv), 2 (0.4 mmol, 2.0 equiv), 4CzIPN (3.2 mg, $0.004 \mathrm{mmol}, 2.0 \mathrm{~mol} \%$ ), $\mathrm{Na}_{2} \mathrm{HPO}_{4}$ (56.8 mg, 0.4 mmol, 2.0 equiv) and $\mathrm{CH}_{3} \mathrm{CN}(2 \mathrm{~mL})$. The reaction mixture was degassed via freeze-pump-thaw for 3 cycles. After the mixture was thoroughly degassed, the tube was sealed and positioned approximately $5 \mathrm{~cm}$ from $24 \mathrm{~W}$ blue LEDs (maximum emission wavelength $=450 \mathrm{~nm}$ ). Then the reaction mixture was stirred at room temperature irradiated by $24 \mathrm{~W}$ blue LEDs. After 1 was consumed completely (monitored by TLC), the solution was quenched with water and extracted with EtOAc $(10 \mathrm{~mL} \times 3)$. The combined organic layers were washed with brine, dried over anhydrous $\mathrm{Na}_{2} \mathrm{SO}_{4}$ and concentrated in vacuo. The residue was purified by column chromatograph on silica gel to afford 3a-3s.

Two rotamers were observed by NMR for all compounds except $\mathbf{3 k}$ at room temperature. In $\mathrm{CDCl}_{3}$, two sets of NMR signals have equal altitude (3a, 3j, 3q, 3r). In DMSO- $d_{6}$, the peaks of the minor one are marked with asterisk $(\mathbf{3 b}, \mathbf{3 c}, \mathbf{3 d}, \mathbf{3 e}, \mathbf{3 f}, \mathbf{3 g}$, 3h, 3i, 3j, 3m, 3n, 3o, 3p, 3q, 3s). The analytical data of the products 3a-3s are summarized below.

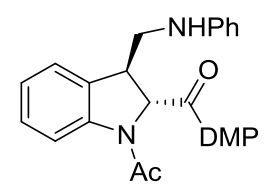

Reaction time: 5 hours. 
3a, $\mathrm{PE} / \mathrm{EtOAc}=2 / 1$, pale yellow solid, m.p. $=144.0-145.0{ }^{\circ} \mathrm{C} .76 .9 \mathrm{mg}, 99 \%$ yield $(0.2$ mmol scale). ${ }^{1} \mathrm{H}$ NMR (600 MHz, DMSO- $\left.d_{6}, 110{ }^{\circ} \mathrm{C}\right) \delta 7.90(\mathrm{br}, 1 \mathrm{H}), 7.28-7.25(\mathrm{~m}$, 2H), $7.08(\mathrm{t}, J=8.4 \mathrm{~Hz}, 2 \mathrm{H}), 7.02(\mathrm{t}, J=7.8 \mathrm{~Hz}, 1 \mathrm{H}), 6.69(\mathrm{~d}, J=7.8 \mathrm{~Hz}, 2 \mathrm{H}), 6.58(\mathrm{~d}$, $J=7.2 \mathrm{~Hz}, 1 \mathrm{H}), 6.27$ (s, 1H), $6.00(\mathrm{~s}, 1 \mathrm{H}), 5.51$ (br, 1H), 3.69-3.60 (m, 2H), 3.47-3.38 (m, 1H), 2.48 (s, 3H), 2.34 (br, 3H). $\left.{ }^{13} \mathrm{C} \mathrm{NMR} \mathrm{(100} \mathrm{MHz,} \mathrm{CDCl}_{3}\right) \delta$ 169.5, 169.1, 169.0, $168.5,153.7,153.0,147.9,147.8,145.5,145.2$, 143.1, 141.5, 132.9, 130.3, 129.4, 129.3, 129.0, 128.8, 125.9, 124.3, 124.0, 123.5, 117.5, 117.4, 117.3, 113.9, 112.5, 112.4, 111.9, 111.5, 66.1, 65.1, 49.2, 48.9, 46.0, 43.4, 24.5, 23.7, 14.2, 14.1. IR (thin film): $v_{\max }\left(\mathrm{cm}^{-}\right.$ $\left.{ }^{1}\right)=3356,2836,1740,1648,1595,1518,1481,1462,1435,1377,1313,1252,1177$, 1127, 1069, 1025, 968, 913, 887, 851, 832, 769, 743, 710, 688, 661, 632, 603, 508, 480, 426. HRMS (ESI) m/z: $[\mathrm{M}+\mathrm{Na}]^{+}$Calcd for $\mathrm{C}_{23} \mathrm{H}_{24} \mathrm{~N}_{4} \mathrm{O}_{2} \mathrm{Na} 411.1792$; Found 411.1789.

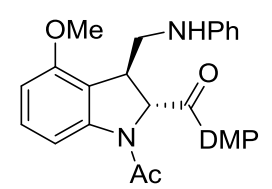

Reaction time: 10 hours.

3b, PE/EtOAc $=2 / 1$, white solid, m.p. $=176.0-178.2^{\circ} \mathrm{C} .77 .0 \mathrm{mg}, 92 \%$ yield $(0.2 \mathrm{mmol}$ scale). ${ }^{1} \mathrm{H}$ NMR (600 MHz, DMSO- $\left.d_{6}, 110{ }^{\circ} \mathrm{C}\right) \delta 7.52(\mathrm{br}, 1 \mathrm{H}), 7.25(\mathrm{t}, J=7.8 \mathrm{~Hz}, 1 \mathrm{H})$, $7.08(\mathrm{t}, J=8.4 \mathrm{~Hz}, 2 \mathrm{H}), 6.73(\mathrm{~d}, J=8.4 \mathrm{~Hz}, 1 \mathrm{H}), 6.66(\mathrm{~d}, J=7.8 \mathrm{~Hz}, 2 \mathrm{H}), 6.56(\mathrm{t}, J=$ $7.2 \mathrm{~Hz}, 1 \mathrm{H}), 6.23$ (s, 1H), 6.08 (s, 1H), 5.43 (br, 1H), 3.81 (s, 3H), 3.69 (br, 1H), 3.58 (dd, $J=13.2,4.8 \mathrm{~Hz}, 1 \mathrm{H}), 3.41(\mathrm{dd}, J=13.2,7.8 \mathrm{~Hz}, 1 \mathrm{H}), 2.43$ (s, 3H), 2.27 (s, 3H), 2.12 (br, 3H). ${ }^{13} \mathrm{C}$ NMR (100 MHz, DMSO-d $) \delta 169.9,169.1^{*}, 168.8,168.0^{*}, 156.3^{*}$, 155.6, 153.2, 152.7*, 148.5, 144.7, 144.4, 144.3*, 142.9*, 130.2*, 129.7, 128.9, 118.9*, $117.4,115.9^{*}, 115.8,112.0,111.9,111.7^{*}, 109.2,106.9^{*}, 106.6,106.2,66.1,64.9$, $55.4^{*}, 55.3,45.9^{*}, 45.6,43.8,41.2,24.2^{*}, 23.6,13.8,13.6,13.5$. IR (thin film): $v_{\max }$ $\left(\mathrm{cm}^{-1}\right)=3342,2929,1731,1657,1601,1514,1465,1438,1377,1353,1295,1259$, 1177, 1095, 1025, 991, 964, 925, 865, 819, 749, 692, 607, 510. HRMS (ESI) m/z: [M $+\mathrm{Na}]^{+}$Calcd for $\mathrm{C}_{24} \mathrm{H}_{26} \mathrm{~N}_{4} \mathrm{O}_{3} \mathrm{Na}$ 441.1897; Found 441.1892.

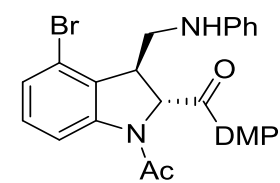

Reaction time: 5 hours. 
3c, $\mathrm{PE} / \mathrm{EtOAc}=2 / 1$, colorless oil. $86.9 \mathrm{mg}, 93 \%$ yield $(0.2 \mathrm{mmol} \mathrm{scale}) .{ }^{1} \mathrm{H}$ NMR $(600$ $\left.\mathrm{MHz}, \mathrm{DMSO}-d_{6}, 110{ }^{\circ} \mathrm{C}\right) \delta 7.96(\mathrm{br}, 1 \mathrm{H}), 7.22-7.20(\mathrm{~m}, 2 \mathrm{H}), 7.08(\mathrm{t}, J=7.8 \mathrm{~Hz}, 2 \mathrm{H})$, $6.71(\mathrm{~d}, J=7.8 \mathrm{~Hz}, 1 \mathrm{H}), 6.57(\mathrm{t}, J=7.2 \mathrm{~Hz}, 1 \mathrm{H}), 6.23(\mathrm{~s}, 1 \mathrm{H}), 6.18(\mathrm{~s}, 1 \mathrm{H}), 5.64(\mathrm{br}$, 1H), 3.74-3.69 (m, 2H), 3.44 (dd, $J=7.2,5.4 \mathrm{~Hz}, 1 \mathrm{H}), 2.46$ (s, 3H), 2.24 (s, 3H), 2.13 (br, 3H). ${ }^{13} \mathrm{C}$ NMR (100 MHz, DMSO- $\left.d_{6}\right) \delta$ 167.0, 169.7, 169.3*, 168.7*, 153.8, 153.3*, $148.9,145.2,145.1,144.8^{*}, 143.7^{*}, 132.5^{*}, 131.1,130.9,129.4,127.0,126.6^{*}, 119.9^{*}$, $118.8,116.4^{*}, 116.3,115.7,113.7^{*}, 112.43^{*}, 112.35^{*}, 112.3,65.7,64.5^{*}, 47.5,45.6^{*}$, $45.4,45.0^{*}, 24.6^{*}, 23.9,14.2,13.9^{*}, 13.8$. IR (thin film): $v_{\max }\left(\mathrm{cm}^{-1}\right)=3346,2927$, 1728, 1668, 1601, 1499, 1447, 1378, 1351, 1319, 1254, 1179, 1135, 1051, 1025, 1006, 964, 881 820, 752, 693 670, 606, 567, 508. HRMS (ESI) m/z: $[\mathrm{M}+\mathrm{Na}]^{+}$Calcd for $\mathrm{C}_{23} \mathrm{H}_{23} \mathrm{~N}_{4} \mathrm{O}_{2} \mathrm{Na}^{79} \mathrm{Br} 489.0897$; Found 489.0898.

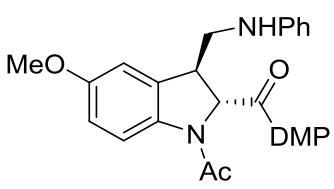

Reaction time: 5 hours.

3d, $\mathrm{PE} / \mathrm{EtOAc}=2 / 1$, yellow oil. $81.4 \mathrm{mg}, 97 \%$ yield $(0.2 \mathrm{mmol} \mathrm{scale}) .{ }^{1} \mathrm{H}$ NMR $(600$ MHz, DMSO- $\left.d_{6}, 110{ }^{\circ} \mathrm{C}\right) \delta 7.91(\mathrm{br}, 1 \mathrm{H}), 7.08(\mathrm{t}, J=8.4 \mathrm{~Hz}, 2 \mathrm{H}), 6.84-6.81(\mathrm{~m}, 2 \mathrm{H})$, $6.69(\mathrm{~d}, J=7.8 \mathrm{~Hz}, 2 \mathrm{H}), 6.57(\mathrm{t}, J=7.2 \mathrm{~Hz}, 1 \mathrm{H}), 6.26(\mathrm{~s}, 1 \mathrm{H}), 5.96(\mathrm{~s}, 1 \mathrm{H}), 5.56(\mathrm{~s}$, $1 \mathrm{H}), 3.73(\mathrm{~s}, 3 \mathrm{H}), 3.67-3.65(\mathrm{~m}, 1 \mathrm{H}), 3.60-3.59(\mathrm{~m}, 1 \mathrm{H}), 3.44-3.40(\mathrm{~m}, 1 \mathrm{H}), 2.47(\mathrm{~s}$, $3 \mathrm{H}), 2.34$ (s, 3H), 2.12 (br, 3H). ${ }^{13} \mathrm{C}$ NMR (100 MHz, DMSO-d $) \delta 169.9,169.1^{*}, 168.2$, $167.4^{*}, 155.5,155.3^{*}, 153.3,152.7^{*}, 148.12^{*}, 148.09,144.8,144.3^{*}, 136.6,134.9^{*}$, $134.7^{*}, 132.9,129.1,116.5,116.0^{*}, 115.8,114.3,113.0^{*}, 112.6,112.3^{*}, 111.89$, 111.86, 111.82, 111.7*, 111.3* $65.9,64.7^{*}, 55.3^{*}, 55.2,47.4^{*}, 47.3^{*}, 44.7,42.4^{*}$, $24.0^{*}, 23.3,13.8,13.6$. IR (thin film): $v_{\max }\left(\mathrm{cm}^{-1}\right)=3349,2929,1730,1651,1601,1488$, 1436, 1379, 1355, 1321, 1260, 1181, 1137, 1025, 989, 963, 927, 852, 816, 750, 694, 616, 589, 511, 446, 415. HRMS (ESI) $\mathrm{m} / \mathrm{z}:[\mathrm{M}+\mathrm{Na}]^{+}$Calcd for $\mathrm{C}_{24} \mathrm{H}_{26} \mathrm{~N}_{4} \mathrm{O}_{3} \mathrm{Na}$ 441.1897; Found 441.1898.

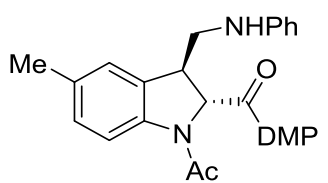

Reaction time: 5 hours. 
3e, $\mathrm{PE} / \mathrm{EtOAc}=2 / 1$, pale yellow solid, m.p. $=108.4-110.0^{\circ} \mathrm{C} .70 .8 \mathrm{mg}, 88 \%$ yield $(0.2$ mmol scale). ${ }^{1} \mathrm{H}$ NMR (600 MHz, DMSO- $\left.d_{6}, 110{ }^{\circ} \mathrm{C}\right) \delta 7.86(\mathrm{br}, 1 \mathrm{H}), 7.10-7.06(\mathrm{~m}$, 4H), $6.69(\mathrm{~d}, J=7.8 \mathrm{~Hz}, 2 \mathrm{H}), 6.58(\mathrm{t}, J=7.2 \mathrm{~Hz}, 1 \mathrm{H}), 6.27(\mathrm{~s}, 1 \mathrm{H}), 5.98(\mathrm{~d}, J=1.8 \mathrm{~Hz}$, $1 \mathrm{H}), 5.56(\mathrm{~s}, 1 \mathrm{H}), 3.64-3.58(\mathrm{~m}, 2 \mathrm{H}), 3.43-3.39(\mathrm{~m}, 1 \mathrm{H}), 2.47$ (s, 3H), $2.34(\mathrm{~s}, 3 \mathrm{H}), 2.27$ (s, 3H), 2.15 (br, 3H). ${ }^{13} \mathrm{C}$ NMR (100 MHz, DMSO-d6) $\delta$ 167.0, 169.2*, 168.6, 167.7*, $153.2,152.7^{*}, 148.2,144.8,144.3^{*}, 140.8,139.2,133.2^{*}, 132.3,132.0^{*}, 131.5,129.0$, 128.6* $128.3,126.8^{*}, 125.8,116.0^{*}, 115.8,115.7,113.5^{*}, 111.91,111.85,111.7^{*}$, $65.8,64.6^{*}, 47.7^{*}, 47.6,44.8,42.4^{*}, 24.2^{*}, 23.5,20.7,20.4^{*}, 13.8,13.6$. IR (thin film): $v_{\max }\left(\mathrm{cm}^{-1}\right)=3348,2926,1730,1654,1601,1489,1433,1378,1352,1309,1258,1179$, 1142, 1026, 988, 963, 928, 880, 852, 818, 749, 693, 638, 612, 502. HRMS (ESI) m/z: $[\mathrm{M}+\mathrm{Na}]^{+}$Calcd for $\mathrm{C}_{24} \mathrm{H}_{26} \mathrm{~N}_{4} \mathrm{O}_{2} \mathrm{Na}$ 425.1948; Found 425.1945.

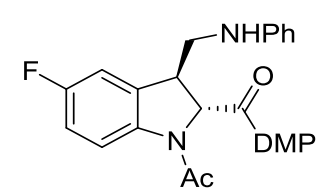

Reaction time: 12 hours.

3f, $\mathrm{PE} /$ EtOAc $=2 / 1$, pale yellow solid, m.p. $=162.3-163.9^{\circ} \mathrm{C} .74 .0 \mathrm{mg}, 91 \%$ yield $(0.2$ mmol scale). ${ }^{1} \mathrm{H}$ NMR (600 MHz, DMSO- $\left.d_{6}, 110{ }^{\circ} \mathrm{C}\right) \delta 7.95(\mathrm{br}, 1 \mathrm{H}), 7.10-7.07(\mathrm{~m}$, 2H), 7.04-7.01 (m, 2H), $6.70(\mathrm{~d}, J=7.8 \mathrm{~Hz}, 2 \mathrm{H}), 6.58(\mathrm{t}, J=7.2 \mathrm{~Hz}, 1 \mathrm{H}), 6.27(\mathrm{~s}, 1 \mathrm{H})$, $6.01(\mathrm{~d}, J=2.4 \mathrm{~Hz}, 1 \mathrm{H}), 5.59(\mathrm{br}, 1 \mathrm{H}), 3.70-3.64(\mathrm{~m}, 2 \mathrm{H}), 3.47-3.44(\mathrm{~m}, 1 \mathrm{H}), 2.48(\mathrm{~s}$, $3 \mathrm{H}), 2.34$ (s, 3H), 2.13 (br, 3H). ${ }^{13} \mathrm{C}$ NMR (100 MHz, DMSO-d $) \delta$ 169.7, 169.0*, 168.8, $167.7^{*}, 158.3\left(\mathrm{C}-\mathrm{F},{ }^{1} J_{\mathrm{C}-\mathrm{F}}=238.0 \mathrm{~Hz}\right), 158.2^{*}\left(\mathrm{C}-\mathrm{F},{ }^{1} J_{\mathrm{C}-\mathrm{F}}=237.6 \mathrm{~Hz}\right), 153.3,152.8^{*}$, $148.1,144.8,144.4^{*}, 139.4,137.9^{*}, 135.5^{*}\left(\mathrm{C}-\mathrm{F},{ }^{3} J_{\mathrm{C}-\mathrm{F}}=8.4 \mathrm{~Hz}\right), 133.8\left(\mathrm{C}-\mathrm{F},{ }^{3} J_{\mathrm{C}-\mathrm{F}}=\right.$ $8.4 \mathrm{~Hz}), 129.1,116.7\left(\mathrm{C}-\mathrm{F},{ }^{3} J_{\mathrm{C}-\mathrm{F}}=8.0 \mathrm{~Hz}\right), 116.0^{*}, 115.9^{*}, 114.5^{*}\left(\mathrm{C}-\mathrm{F},{ }^{2} J_{\mathrm{C}-\mathrm{F}}=23.7\right.$ $\mathrm{Hz}), 114.0\left(\mathrm{C}-\mathrm{F},{ }^{2} J_{\mathrm{C}-\mathrm{F}}=22.4 \mathrm{~Hz}\right), 113.6 *\left(\mathrm{C}-\mathrm{F},{ }^{2} J_{\mathrm{C}-\mathrm{F}}=24.3 \mathrm{~Hz}\right), 112.6\left(\mathrm{C}-\mathrm{F},{ }^{2} J_{\mathrm{C}-\mathrm{F}}=\right.$ $24.1 \mathrm{~Hz}), 111.94,111.91,111.85,111.71,66.0,64.9^{*}, 47.3^{*}, 47.2,44.7,42.4^{*}, 24.1^{*}$, 23.4, 13.7, 13.6. ${ }^{19}$ F NMR (376 MHz, DMSO-d6) $\delta-114.96--115.02,-116.09--116.15$. IR (thin film): $v_{\max }\left(\mathrm{cm}^{-1}\right)=3435,2924,1724,1661,1597,1507,1482,1397,1378$, 1353, 1306, 1252, 1231, 1212, 1177, 1128, 1073, 1024, 987, 965, 929, 890, 871, 849, 822, 753, 697, 635, 608, 499, 447, 411. HRMS (ESI) m/z: $[\mathrm{M}+\mathrm{Na}]^{+}$Calcd for $\mathrm{C}_{23} \mathrm{H}_{23} \mathrm{~N}_{4} \mathrm{O}_{2} \mathrm{Na}$ 429.1697; Found 429.1692. 


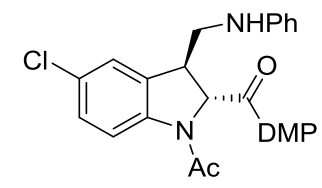

Reaction time: 8 hours.

3g, PE/EtOAc = 2/1, white foam. $79.7 \mathrm{mg}, 94 \%$ yield (0.2 mmol scale). ${ }^{1} \mathrm{H}$ NMR (600 $\left.\mathrm{MHz}, \mathrm{DMSO}-d_{6}, 110^{\circ} \mathrm{C}\right) \delta 7.92(\mathrm{br}, 1 \mathrm{H}), 7.28-7.25(\mathrm{~m}, 2 \mathrm{H}), 7.08(\mathrm{t}, J=7.8 \mathrm{~Hz}, 2 \mathrm{H})$, $6.69(\mathrm{~d}, J=8.4 \mathrm{~Hz}, 2 \mathrm{H}), 6.57(\mathrm{t}, J=7.2 \mathrm{~Hz}, 1 \mathrm{H}), 6.27$ (s, 1H), $6.00(\mathrm{~d}, J=1.2 \mathrm{~Hz}, 1 \mathrm{H})$, $5.61(\mathrm{~s}, 1 \mathrm{H}), 3.68-3.65(\mathrm{~m}, 2 \mathrm{H}), 3.65-3.44(\mathrm{~m}, 1 \mathrm{H}), 2.48(\mathrm{~s}, 3 \mathrm{H}), 2.33$ (s, 3H), 2.14 (br, 3H). ${ }^{13} \mathrm{C}$ NMR (100 MHz, DMSO-d $) \delta 169.6,169.1,168.9^{*}, 167.9^{*}, 153.3,152.8^{*}$, $148.1,144.8,144.3^{*}, 142.0,140.4^{*}, 135.5^{*}, 133.9,129.1,127.9^{*} 127.7,126.8,126.7^{*}$, $126.1^{*}, 125.3,117.0,115.92,115.0,111.9,111.8,65.9,64.7^{*}, 47.2,44.6,42.3^{*}, 24.2^{*}$, 23.4, 13.7, 13.5. IR (thin film): $v_{\max }\left(\mathrm{cm}^{-1}\right)=3342,2927,1729,1664,1601,1474,1436$, 1378, 1352, 1309, 1256, 1171, 1109, 1052, 1025, 1007, 990, 963, 924, 819, 749, 693, 623, 511, 480, 423. HRMS (ESI) m/z: $[\mathrm{M}+\mathrm{Na}]^{+} \mathrm{Calcd}$ for $\mathrm{C}_{23} \mathrm{H}_{23} \mathrm{~N}_{4} \mathrm{O}_{2} \mathrm{NaCl}$ 445.1402; Found 445.1401.

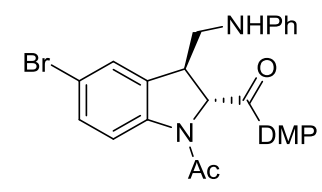

Reaction time: 5 hours.

3h, PE/EtOAc $=2 / 1$, white foam. $89.7 \mathrm{mg}, 96 \%$ yield $(0.2 \mathrm{mmol} \mathrm{scale}) .{ }^{1} \mathrm{H}$ NMR (600 $\left.\mathrm{MHz}, \mathrm{DMSO}-d_{6}, 110{ }^{\circ} \mathrm{C}\right) \delta 7.90(\mathrm{br}, 1 \mathrm{H}), 7.41(\mathrm{~d}, J=9.0 \mathrm{~Hz}, 1 \mathrm{H}), 7.38(\mathrm{~s}, 1 \mathrm{H}), 7.08$ $(\mathrm{t}, J=7.2 \mathrm{~Hz}, 2 \mathrm{H}), 6.70(\mathrm{~d}, J=8.4 \mathrm{~Hz}, 2 \mathrm{H}), 6.58(\mathrm{t}, J=7.2 \mathrm{~Hz}, 1 \mathrm{H}), 6.27(\mathrm{~s}, 1 \mathrm{H}), 6.00$ (s, 1H), $5.63(\mathrm{br}, 1 \mathrm{H}), 3.68-3.66(\mathrm{~m}, 2 \mathrm{H}), 3.47-3.33(\mathrm{~m}, 1 \mathrm{H}), 2.48(\mathrm{~s}, 3 \mathrm{H}), 2.33(\mathrm{~s}, 3 \mathrm{H})$, 2.14 (br, 3H). ${ }^{13} \mathrm{C}$ NMR (100 MHz, DMSO-d $) \delta 169.6,169.2,168.8^{*}, 168.0^{*}, 153.3$, $152.8^{*}, 148.2^{*}, 148.1,144.8,144.3^{*}, 142.4,140.9^{*}, 135.9^{*}, 134.3,130.8^{*}, 130.6$, 129.1, 128.1, 117.5, 116.0*, 115.9, 115.5*, 114.8, 114.7*, 111.9, 111.8, 111.7*, 65.8, 64.7*, 47.2, 44.6, 42.3*, 24.2*, 23.5, 13.7, 13.6. IR (thin film): $v_{\max }\left(\mathrm{cm}^{-1}\right)=3343,2927$, 1729, 1662, 1601, 1512, 1471, 1436, 1377, 1350, 1308, 1256, 1169, 1109, 1026, 989, 962, 922, 874, 818, 748, 693, 620, 510, 474, 422. HRMS (ESI) m/z: $[\mathrm{M}+\mathrm{Na}]^{+}$Calcd for $\mathrm{C}_{23} \mathrm{H}_{23} \mathrm{~N}_{4} \mathrm{O}_{2} \mathrm{Na}^{79} \mathrm{Br} 489.0897$; Found 489.0899. 


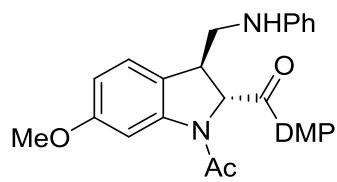

Reaction time: 8 hours.

3i, PE/EtOAc $=2 / 1$, red solid, m.p. $=158.4-159.6{ }^{\circ} \mathrm{C} .80 .3 \mathrm{mg}, 96 \%$ yield $(0.2 \mathrm{mmol}$ scale). ${ }^{1} \mathrm{H}$ NMR (600 MHz, DMSO- $\left.d_{6}, 110{ }^{\circ} \mathrm{C}\right) \delta 7.64(\mathrm{br}, 1 \mathrm{H}), 7.12(\mathrm{~d}, J=7.8 \mathrm{~Hz}$, 1H), $7.07(\mathrm{t}, J=7.8 \mathrm{~Hz}, 2 \mathrm{H}), 6.67(\mathrm{~d}, J=7.8 \mathrm{~Hz}, 2 \mathrm{H}), 6.60(\mathrm{~d}, J=7.8 \mathrm{~Hz}, 1 \mathrm{H}), 6.56(\mathrm{t}$, $J=7.2 \mathrm{~Hz}, 1 \mathrm{H}), 6.27$ (s, 1H), $5.97(\mathrm{~s}, 1 \mathrm{H}), 5.53(\mathrm{br}, 1 \mathrm{H}), 3.79$ (s, 3H), 3.62-3.58 (m, $1 \mathrm{H}), 3.55-3.54(\mathrm{~m}, 1 \mathrm{H}), 3.38-3.34(\mathrm{~m}, 1 \mathrm{H}), 2.47$ (s, 3H), $2.33(\mathrm{~s}, 3 \mathrm{H}), 2.15$ (br, 3H). ${ }^{13} \mathrm{C}$ NMR (100 MHz, DMSO-d $\left.d_{6}\right) \delta 167.0,169.1,168.0^{*}, 159.8^{*}, 159.4,153.3,152.7^{*}$, $151.9^{*}, 148.2,144.8,144.3^{*}, 144.2,142.5^{*}, 129.0,126.5^{*}, 125.5^{*}, 125.1^{*}, 123.4$, $115.9^{*}, 115.8,111.9,111.8,111.7^{*}, 108.4,107.7^{*}, 102.8,101.2^{*}, 66.4,65.2^{*}, 55.5^{*}$, 55.3, 47.8*, 47.7, 44.1, 41.7* $24.3^{*}, 23.7,13.8,13.6$. IR (thin film) $: v_{\max }\left(\mathrm{cm}^{-1}\right)=3354$, 2927, 2836, 1729, 1660, 1600, 1493, 1448, 1378, 1355, 1309, 1257, 1218, 1167, 1112, 1029, 990, 963, 853, 809, 748, 693, 657, 630, 591, 509. HRMS (ESI) m/z: $[\mathrm{M}+\mathrm{Na}]^{+}$ Calcd for $\mathrm{C}_{24} \mathrm{H}_{26} \mathrm{~N}_{4} \mathrm{O}_{3} \mathrm{Na} 441.1897$; Found 441.1897.

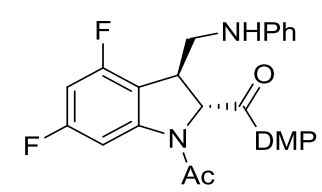

Reaction time: 9 hours.

3j, PE/EtOAc $=2 / 1$, yellow solid, m.p. $=161.0-162.5{ }^{\circ} \mathrm{C} .81 .5 \mathrm{mg}, 96 \%$ yield $(0.2$ mmol scale). ${ }^{1} \mathrm{H}$ NMR (600 MHz, DMSO- $\left.d_{6}, 110{ }^{\circ} \mathrm{C}\right) \delta 7.61(\mathrm{br}, 1 \mathrm{H}), 7.07$ (t, $J=7.8$ $\mathrm{Hz}, 1 \mathrm{H}), 6.74(\mathrm{td}, J=9.4,1.8 \mathrm{~Hz}, 1 \mathrm{H}), 6.68(\mathrm{~d}, J=8.4 \mathrm{~Hz}, 2 \mathrm{H}), 6.56(\mathrm{t}, J=7.2 \mathrm{~Hz}$, $1 \mathrm{H}), 6.26(\mathrm{~s}, 1 \mathrm{H}), 6.13(\mathrm{~s}, 1 \mathrm{H}), 5.54(\mathrm{~s}, 1 \mathrm{H}), 3.84(\mathrm{~s}, 1 \mathrm{H}), 3.63-3.59(\mathrm{~m}, 2 \mathrm{H}), 2.48(\mathrm{~s}$, 3H), 2.31 (s, 3H), 2.13 (br, 3H). $\left.{ }^{13} \mathrm{C} \mathrm{NMR} \mathrm{(100} \mathrm{MHz,} \mathrm{CDCl}_{3}\right) \delta$ 169.4, 169.1, 168.5*, $168.4^{*}, 163.9\left(\mathrm{C}-\mathrm{F},{ }^{1} J_{\mathrm{C}-\mathrm{F}}=244.7 \mathrm{~Hz} ; \mathrm{C}-\mathrm{F},{ }^{3} J_{\mathrm{C}-\mathrm{F}}=12.2 \mathrm{~Hz}\right), 158.5\left(\mathrm{C}-\mathrm{F},{ }^{1} J_{\mathrm{C}-\mathrm{F}}=245.5\right.$ $\left.\mathrm{Hz} ; \mathrm{C}-\mathrm{F},{ }^{3} J_{\mathrm{C}-\mathrm{F}}=14.7 \mathrm{~Hz}\right), 154.3,153.5^{*}, 148.0^{*}, 147.9,145.9,145.8,145.6,145.4$, $129.5,129.4^{*}, 117.8,117.5^{*}, 112.6,112.5^{*}, 112.3,112.1^{*}, 112.06,112.03,111.9$, $102.1\left(\mathrm{C}-\mathrm{F},{ }^{2} J_{\mathrm{C}-\mathrm{F}}=29.1 \mathrm{~Hz} ; \mathrm{C}-\mathrm{F},{ }^{4} J_{\mathrm{C}-\mathrm{F}}=3.8 \mathrm{~Hz}\right), 99.4\left(\mathrm{C}-\mathrm{F},{ }^{2} J_{\mathrm{C}-\mathrm{F}}=27.0 \mathrm{~Hz} ; \mathrm{C}-\mathrm{F},{ }^{2} J_{\mathrm{C}-\mathrm{F}}\right.$ $=27.1 \mathrm{~Hz}), 98.9,98.6,66.9,66.1^{*}, 60.5,47.5^{*}, 47.2,43.6,40.7,24.5^{*}, 23.7,14.3,14.2$. ${ }^{19}$ F NMR (376 MHz, DMSO-d $d_{6}$-104.39--104.44 (m), -105.19--105.27 (m), -109.11-109.16 (m), -109.79--109.83 (m). IR (thin film): $v_{\max }\left(\mathrm{cm}^{-1}\right)=3345,2845,1627,1602$, 
1511, 1492 1445, 1379, 1357, 1257, 1221, 1198, 1140, 1105, 1084, 1029, 994, 979, 963, 930, 869, 852, 830, 807, 642, 620, 588, 510, 455, 418. HRMS (ESI) m/z: [M + $\mathrm{Na}]^{+}$Calcd for $\mathrm{C}_{23} \mathrm{H}_{22} \mathrm{~N}_{4} \mathrm{O}_{2} \mathrm{~F}_{2} \mathrm{Na}$ 447.1603; Found 447.1596.

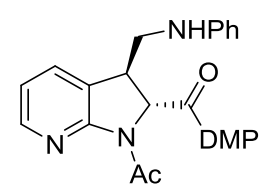

Reaction time: 9 hours.

3k, PE/EtOAc $=1 / 1$, white solid, m.p. $=179.5-182.3{ }^{\circ} \mathrm{C} .50 .1 \mathrm{mg}, 64 \%$ yield $(0.2 \mathrm{mmol}$ scale). ${ }^{1} \mathrm{H}$ NMR $\left(600 \mathrm{MHz}, \mathrm{DMSO}-d_{6}, 110{ }^{\circ} \mathrm{C}\right) \delta 8.21(\mathrm{~d}, J=4.8 \mathrm{~Hz}, 1 \mathrm{H}), 7.62(\mathrm{~d}, J=$ $7.8 \mathrm{~Hz}, 1 \mathrm{H}), 7.07$ (t, $J=7.8 \mathrm{~Hz}, 2 \mathrm{H}), 7.00(\mathrm{dd}, J=7.2,5.4 \mathrm{~Hz}, 1 \mathrm{H}), 6.67$ (d, $J=8.4 \mathrm{~Hz}$, 2H), $6.57(\mathrm{td}, J=7.2,1.2 \mathrm{~Hz}, 1 \mathrm{H}), 6.27(\mathrm{~s}, 1 \mathrm{H}), 5.95(\mathrm{~d}, J=2.4 \mathrm{~Hz}, 1 \mathrm{H}), 5.60(\mathrm{~s}, 1 \mathrm{H})$, 3.71-3.67 (m, 1H), 3.55-3.52 (m, 1H), 3.42-3.38 (m, 1H), $2.68(\mathrm{~s}, 3 \mathrm{H}), 2.46(\mathrm{~s}, 3 \mathrm{H})$, $2.33(\mathrm{~s}, 3 \mathrm{H}) .{ }^{13} \mathrm{C} \mathrm{NMR}\left(100 \mathrm{MHz}, \mathrm{CDCl}_{3}\right) \delta 170.3,168.9,155.7,153.2,147.64,147.60$, 145.3, 133.7, 129.5, 125.6, 118.4, 117.7, 112.6, 111.7, 62.8, 48.9, 41.1, 25.0, 14.3, 14.1. IR (thin film): $v_{\max }\left(\mathrm{cm}^{-1}\right)=3323,1739,1663,1592,1522,1469,1421,1380,1354$, 1319, 1254, 1235, 1127, 1095, 989, 884, 813, 796, 770, 749, 692, 611, 557, 515, 481. HRMS (ESI) m/z: $[\mathrm{M}+\mathrm{Na}]^{+}$Calcd for $\mathrm{C}_{22} \mathrm{H}_{23} \mathrm{~N}_{5} \mathrm{O}_{2} \mathrm{Na} 412.1744$; Found 412.1742.

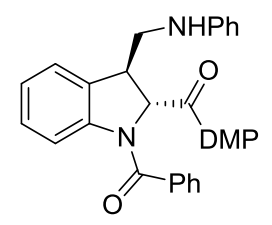

Reaction time: 17 hours.

31, PE/EtOAc $=1 / 1$, white solid, m.p. $=131.4-133.7^{\circ} \mathrm{C} .81 .1 \mathrm{mg}, 90 \%$ yield $(0.2 \mathrm{mmol}$ scale). ${ }^{1} \mathrm{H}$ NMR $\left(600 \mathrm{MHz}, \mathrm{DMSO}-d_{6}, 110{ }^{\circ} \mathrm{C}\right) \delta 7.49-7.41(\mathrm{~m}, 5 \mathrm{H}), 7.29(\mathrm{~d}, J=7.2$ $\mathrm{Hz}, 1 \mathrm{H}), 7.12-7.08(\mathrm{~m}, 3 \mathrm{H}), 7.02-7.00(\mathrm{~m}, 1 \mathrm{H}), 6.72(\mathrm{~d}, J=7.8 \mathrm{~Hz}, 2 \mathrm{H}), 6.59$ (t, $J=$ $7.2 \mathrm{~Hz}, 1 \mathrm{H}), 6.20(\mathrm{~s}, 1 \mathrm{H}), 5.49$ (s, 1H), 3.72-3.62 (m, 2H), 3.59-3.55 (m, 1H), 2.43 (s, $3 \mathrm{H}), 2.28(\mathrm{~s}, 3 \mathrm{H}) .{ }^{13} \mathrm{C} \mathrm{NMR}\left(150 \mathrm{MHz}, \mathrm{DMSO}-d_{6}, 110{ }^{\circ} \mathrm{C}\right) \delta 169.0,167.7,152.1,147.9$, 143.7, 142.2, 135.7, 131.9, 129.7, 128.3, 128.0, 127.1, 126.3, 125.0, 122.9, 115.8, 114.4, 111.8, 1110, 65.5, 47.3, 44.3, 12.73, 12.69. IR (thin film): $v_{\max }\left(\mathrm{cm}^{-1}\right)=3349,2923$, 1730, 1643, 1600, 1479, 1376, 1357, 1314, 1256, 1175, 1025, 987, 963, 816, 787, 747, 694, 652, 620, 510. HRMS (ESI) m/z: $[\mathrm{M}+\mathrm{Na}]^{+}$Calcd for $\mathrm{C}_{28} \mathrm{H}_{26} \mathrm{~N}_{4} \mathrm{O}_{2} \mathrm{Na} 473.1948$; Found 473.1941. 


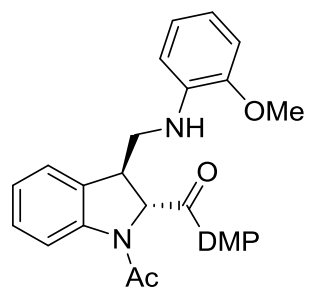

Reaction time: 41 hours.

3m, PE/EtOAc $=1 / 1$, white solid, m.p. $=134.8-135.5^{\circ} \mathrm{C} .59 .3 \mathrm{mg}, 71 \%$ yield $(0.2$ mmol scale). ${ }^{1} \mathrm{H}$ NMR (600 MHz, DMSO- $\left.d_{6}, 110{ }^{\circ} \mathrm{C}\right) \delta 7.96(\mathrm{br}, 1 \mathrm{H}), 7.27(\mathrm{t}, J=7.2$ $\mathrm{Hz}, 1 \mathrm{H}), 7.20$ (d, $J=7.2 \mathrm{~Hz}, 1 \mathrm{H}), 7.03$ (t, $J=7.2,1 \mathrm{H}), 6.84$ (d, $J=7.8 \mathrm{~Hz}, 1 \mathrm{H}), 6.76$ (t, $J=7.8 \mathrm{~Hz}, 1 \mathrm{H}), 6.69(\mathrm{t}, J=7.8 \mathrm{~Hz}, 1 \mathrm{H}), 6.58(\mathrm{t}, J=7.8 \mathrm{~Hz}, 1 \mathrm{H}), 6.27(\mathrm{~s}, 1 \mathrm{H}), 5.98$ (s, 1H), 5.02 (br, 1H), 3.79 (s, 3H), 3.67-3.62 (m, 2H), 3.49-3.46 (m, 1H), $2.46(\mathrm{~s}, 3 \mathrm{H})$, 2.32 (s, 3H), 2.15 (br, 3H). ${ }^{13} \mathrm{C}$ NMR (100 MHz, DMSO-d $) \delta$ 169.9, 169.0, 168.0*, $153.3,152.9^{*}, 146.42,146.40,144.7,144.3^{*}, 143.0,141.3^{*}, 137.5,133.0 *, 131.3$, 128.4*, 128.0, 126.0*, 125.0, 123.3, 123.0*, 121.1, 116.0, 115.9, 113.9*, 111.8, 111.7*, 110.1, 110.0*, 109.2* 109.0, 65.7, 64.4*, 55.4, 47.8*, 47.7, 44.8, 42.4*, 24.2*, 23.6, 13.8, 13.4. IR (thin film): $v_{\max }\left(\mathrm{cm}^{-1}\right)=2925,1728,1668,1597,1513,1481,1460,1433$, 1378, 1357, 1308, 1253, 1222, 1172, 1131, 1022, 990, 962, 920, 841, 819, 755, 738, 661, 620, 604, 582, 513, 467, 423. HRMS (ESI) m/z: $\left[\mathrm{M}+\mathrm{Na}^{+}\right.$Calcd for $\mathrm{C}_{24} \mathrm{H}_{26} \mathrm{~N}_{4} \mathrm{O}_{3} \mathrm{Na}$ 441.1897; Found 441.1891.

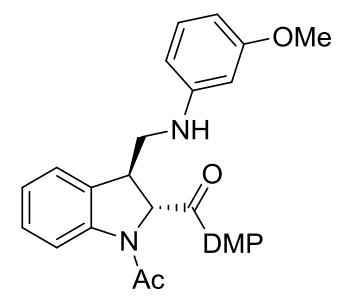

Reaction time: 19 hours.

3n, $\mathrm{PE} / \mathrm{EtOAc}=1 / 1$, white foam. $74.0 \mathrm{mg}, 88 \%$ yield $(0.2 \mathrm{mmol}$ scale $) .{ }^{1} \mathrm{H}$ NMR $(600$ $\left.\mathrm{MHz}, \mathrm{DMSO}-d_{6}, 110^{\circ} \mathrm{C}\right) \delta 7.93(\mathrm{br}, 1 \mathrm{H}), 7.27-7.23(\mathrm{~m}, 2 \mathrm{H}), 7.02(\mathrm{t}, J=7.8 \mathrm{~Hz}, 1 \mathrm{H})$, 6.99-6.96 (m, 1H), $6.29(\mathrm{~d}, J=8.4 \mathrm{~Hz}, 1 \mathrm{H}), 6.27(\mathrm{~s}, 1 \mathrm{H}), 6.24(\mathrm{~s}, 1 \mathrm{H}), 6.18-6.17$ (m, 1H), 5.98 (s, 1H), $5.58(\mathrm{br}, 1 \mathrm{H}), 3.68(\mathrm{~s}, 3 \mathrm{H}), 3.65-3.62(\mathrm{~m}, 2 \mathrm{H}), 3.42-3.38(\mathrm{~m}, 1 \mathrm{H})$, 2.47 (s, 3H), 2.34 (s, 3H), 2.17 (br, 3H). ${ }^{13} \mathrm{C}$ NMR (100 MHz, DMSO-d $d_{6} \delta$ 169.9, $169.1^{*}, 168.9,167.9^{*}, 160.52,160.49^{*}, 153.4,152.8^{*}, 149.5,144.8,144.3^{*}, 143.1$, $141.4^{*}, 133.0^{*}, 131.4,129.8,129.7^{*}, 128.3^{*}, 127.9,126.3 *, 125.4,123.3,122.9 *$, 
$115.9,113.7^{*}, 111.9,111.7^{*}, 105.0,101.5^{*}, 101.2,97.7,65.6,64.4^{*}, 54.6,47.5^{*}, 47.4$, 44.7, 42.2*, 24.3*, 23.6, 13.8, 13.6. IR (thin film): $v_{\max }\left(\mathrm{cm}^{-1}\right)=3344,2931,1730$, 1657, 1593, 1520, 1480, 1463, 1379, 1356, 1312, 1257, 1211, 1163, 1025, 990, 963, 923, 819, 755, 690, 604, 564, 514, 461. HRMS (ESI) m/z: $[\mathrm{M}+\mathrm{Na}]^{+}$Calcd for $\mathrm{C}_{24} \mathrm{H}_{26} \mathrm{~N}_{4} \mathrm{O}_{3} \mathrm{Na} 441.1897$; Found 441.1891 .

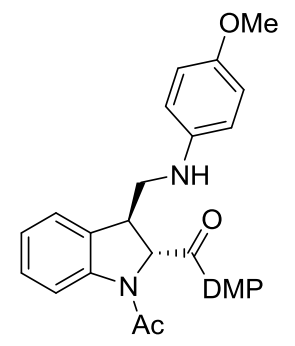

Reaction time: 33 hours.

3o, $\mathrm{PE} /$ EtOAc $=1 / 1$, pink solid, m.p. $=176.4-178.7^{\circ} \mathrm{C} .59 .7 \mathrm{mg}, 72 \%$ yield $(0.2 \mathrm{mmol}$ scale). ${ }^{1} \mathrm{H}$ NMR (600 MHz, DMSO- $\left.d_{6}, 110{ }^{\circ} \mathrm{C}\right) \delta 7.88(\mathrm{br}, 1 \mathrm{H}), 7.27-7.24(\mathrm{~m}, 2 \mathrm{H}), 7.02$ $(\mathrm{t}, J=7.2 \mathrm{~Hz}, 1 \mathrm{H}), 6.73-6.71(\mathrm{~m}, 2 \mathrm{H}), 6.64-6.61(\mathrm{~m}, 2 \mathrm{H}), 6.27(\mathrm{~s}, 1 \mathrm{H}), 5.98(\mathrm{~s}, 1 \mathrm{H})$, 5.12 (br, 1H), 3.67 (s, 3H), 3.60-3.57 (m, 2H), 3.38 (dd, $J=15.0,9.0 \mathrm{~Hz}, 1 \mathrm{H}), 2.47$ (s, $3 \mathrm{H}), 2.34$ (s, 3H), 2.17 (br, 3H). ${ }^{13} \mathrm{C}$ NMR (100 MHz, DMSO-d $) \delta 170.0,169.2^{*}, 168.9$, $168.0^{*}, 153.3,152.7^{*}, 150.9^{*}, 150.8,144.8,144.3^{*}, 143.0,142.3^{*}, 141.4^{*}, 133.1^{*}$, $131.5,128.3^{*}, 127.9,126.2^{*}, 125.3,123.3,122.9^{*}, 115.9,114.8,113.7^{*}, 112.9^{*}, 112.8$, $111.9,111.7^{*}, 65.7,64.5^{*}, 55.3,48.5^{*}, 48.3,44.9,42.4^{*}, 24.3^{*}, 23.6,13.8,13.6$. IR (thin film): $v_{\max }\left(\mathrm{cm}^{-1}\right)=3326,2925,2827,1730,1652,1587,1510,1482,1438,1380$, 1332, 1299, 1249, 1230, 1176, 1143, 1122, 1100, 1036, 965, 883, 821, 790, 755, 655, 627, 601, 543, 523. HRMS (ESI) m/z: $[\mathrm{M}+\mathrm{Na}]^{+}$Calcd for $\mathrm{C}_{24} \mathrm{H}_{26} \mathrm{~N}_{4} \mathrm{O}_{3} \mathrm{Na} 441.1897$; Found 441.1891.

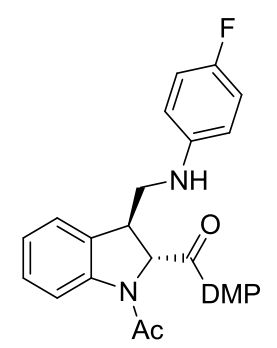

Reaction time: 11 hours.

3p, PE/EtOAc = 2/1, white foam. $77.3 \mathrm{mg}, 95 \%$ yield $(0.2 \mathrm{mmol}$ scale $) .{ }^{1} \mathrm{H}$ NMR (600 $\left.\mathrm{MHz}, \mathrm{DMSO}-d_{6}, 110^{\circ} \mathrm{C}\right) \delta 7.91(\mathrm{br}, 1 \mathrm{H}), 7.27-7.24(\mathrm{~m}, 2 \mathrm{H}), 7.24(\mathrm{t}, J=7.8 \mathrm{~Hz}, 1 \mathrm{H})$, 
$7.02(\mathrm{t}, J=7.2 \mathrm{~Hz}, 1 \mathrm{H}), 6.87(\mathrm{~d}, J=9.0 \mathrm{~Hz}, 2 \mathrm{H}), 6.66(\mathrm{dd}, J=8.6,4.4 \mathrm{~Hz}, 1 \mathrm{H}), 6.27$ (s, 1H), $5.98(\mathrm{~s}, 1 \mathrm{H}), 5.54(\mathrm{br}, 1 \mathrm{H}), 3.62-3.58(\mathrm{~m}, 2 \mathrm{H}), 3.42-3.37(\mathrm{~m}, 1 \mathrm{H}), 2.47,(\mathrm{~s}, 3 \mathrm{H})$, 2.32 (s, 3H), 2.18 (br, 3H). ${ }^{13} \mathrm{C}$ NMR (100 MHz, DMSO-d 6 ) $\delta$ 169.9, 169.2*, 169.0, $168.0^{*}, 155.5^{*}\left(\mathrm{C}-\mathrm{F},{ }^{1} J_{\mathrm{C}-\mathrm{F}}=229.7 \mathrm{~Hz}\right), 154.3\left(\mathrm{C}-\mathrm{F},{ }^{1} J_{\mathrm{C}-\mathrm{F}}=229.7 \mathrm{~Hz}\right), 153.3,152.8$, $145.0^{*}, 144.9,144.8,144.3^{*}, 143.1,141.4^{*}, 133.1^{*}, 131.4,128.3^{*}, 127.9,126.2^{*}$, $125.3,123.3,122.9^{*}, 116.0,115.4\left(\mathrm{C}-\mathrm{F},{ }^{2} J_{\mathrm{C}-\mathrm{F}}=21.6 \mathrm{~Hz}\right), 113.7^{*}, 112.6,112.53,112.50$, $112.42,111.9,111.7^{*} 65.7,64.5^{*}, 48.1^{*}, 48.0,44.8,42.4^{*}, 24.3^{*}, 23.6,13.8,13.6 .{ }^{19} \mathrm{~F}$ NMR (376 MHz, DMSO-d $)^{\text {) } \delta ~-124.77--124.83 ~(m), ~-124.91--124.99 ~(m) . ~ I R ~(t h i n ~}$ film): $v_{\max }\left(\mathrm{cm}^{-1}\right)=3345,2926,1723,1658,1609,1591,1518,1484,1428,1378,1354$, 1324, 1298, 1251, 1204, 1175, 1155, 1120, 1094, 1022, 966, 890, 821, 807, 765, 750, 657, 629, 602, 585, 513, 467. HRMS (ESI) m/z: $[\mathrm{M}+\mathrm{Na}]^{+}$Calcd for $\mathrm{C}_{23} \mathrm{H}_{23} \mathrm{~N}_{4} \mathrm{O}_{2} \mathrm{FNa}$ 429.1697; Found 429.1691.

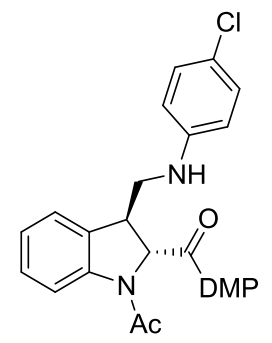

Reaction time: 8 hours.

3q, PE/EtOAc $=2 / 1$, white solid, m.p. $=205.8-207.3{ }^{\circ} \mathrm{C} .76 .4 \mathrm{mg}, 90 \%$ yield $(0.2 \mathrm{mmol}$ scale). ${ }^{1} \mathrm{H}$ NMR (600 MHz, DMSO- $\left.d_{6}, 110{ }^{\circ} \mathrm{C}\right) \delta 7.87(\mathrm{br}, 1 \mathrm{H}), 7.27-7.23(\mathrm{~m}, 2 \mathrm{H})$, 7.07-7.04 (m, 2H), $7.01(\mathrm{t}, J=7.2 \mathrm{~Hz}, 1 \mathrm{H}), 6.68-6.65(\mathrm{~m}, 2 \mathrm{H}), 6.27(\mathrm{~s}, 1 \mathrm{H}), 5.96$ (s, $1 \mathrm{H}), 5.77(\mathrm{br}, 1 \mathrm{H}), 3.63-3.60(\mathrm{~m}, 2 \mathrm{H}), 3.43-3.38(\mathrm{~m}, 1 \mathrm{H}), 2.47(\mathrm{~s}, 3 \mathrm{H}), 2.31(\mathrm{~s}, 3 \mathrm{H})$, 2.18 (br, 3H). ${ }^{13} \mathrm{C}$ NMR (100 MHz, $\left.\mathrm{CDCl}_{3}\right) \delta 169.5,169.2,169.0,168.7,153.9,153.1$, 146.6, 146.5, 145.8, 145.5, 143.2, 141.6, 132.7, 130.1, 129.4, 129.3, 129.2, 129.0, 125.9, $124.4,124.2$, 123.7, 122.1, 121.9, 117.6, 114.0, 113.6, 113.5, 112.0, 111.6, 66.2, 65.2, 49.4, 49.1, 46.0, 43.4, 24.7, 23.9, 14.35, 14.33, 14.2. IR (thin film): $v_{\max }\left(\mathrm{cm}^{-1}\right)=3339$, 2922, 2851, 1726, 1658, 1595, 1513, 1484, 1427, 1377, 1324, 1298, 1251, 1174, 1127 , 1091, 1022, 966, 887, 818, 753, 708, 653, 627, 600, 509. HRMS (ESI) m/z: $[\mathrm{M}+\mathrm{Na}]^{+}$ Calcd for $\mathrm{C}_{23} \mathrm{H}_{23} \mathrm{~N}_{4} \mathrm{O}_{2} \mathrm{NaCl}$ 445.1402; Found 445.1394. 


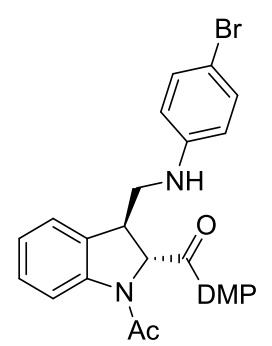

Reaction time: 8 hours.

3r, $\mathrm{PE} / \mathrm{EtOAc}=2 / 1$, white solid, m.p. $=203.4-204.9^{\circ} \mathrm{C} .92 .3 \mathrm{mg}, 99 \%$ yield $(0.2 \mathrm{mmol}$ scale). ${ }^{1} \mathrm{H}$ NMR (600 MHz, DMSO- $\left.d_{6}, 110{ }^{\circ} \mathrm{C}\right) \delta 7.88(\mathrm{br}, 1 \mathrm{H}), 7.27-7.23(\mathrm{~m}, 2 \mathrm{H})$, 7.19-7.17 (m, 2H), $7.01(\mathrm{td}, J=7.8,0.6 \mathrm{~Hz}, 1 \mathrm{H}), 6.65-6.62(\mathrm{~m}, 2 \mathrm{H}), 6.26(\mathrm{~s}, 1 \mathrm{H}), 5.97$ $(\mathrm{d}, J=2.4 \mathrm{~Hz}, 1 \mathrm{H}), 5.80(\mathrm{br}, 1 \mathrm{H}), 3.64-3.60(\mathrm{~m}, 2 \mathrm{H}), 3.44-3.39(\mathrm{~m}, 1 \mathrm{H}), 2.47(\mathrm{~s}, 3 \mathrm{H})$, 2.32 (s, 3H), 2.19 (br, 3H). ${ }^{13} \mathrm{C}$ NMR (100 MHz, $\left.\mathrm{CDCl}_{3}\right) \delta 169.5,169.2,169.0,168.7$, 153.9, 153.2, 147.0, 146.9, 145.8, 145.5, 143.2, 141.7, 132.7, 132.2, 132.1, 130.1, 129.3, 129.0, 126.0, 124.4, 124.2, 123.7, 117.6, 114.14, 114.05, 112.0, 111.7,109.2, 109.0, $66.2,65.2,49.3,49.0,45.9,43.3,24.7,23.9,14.4,14.3,14.2$. IR (thin film): $v_{\max }\left(\mathrm{cm}^{-}\right.$ $\left.{ }^{1}\right)=3332,2920,2850,1728,1656,1591,1511,1482,1432,1378,1355,1316,1253$, 1175, 1126, 1070, 1023, 964, 923, 811, 751, 625, 599, 565, 506. HRMS (ESI) m/z: [M $+\mathrm{Na}]^{+}$Calcd for $\mathrm{C}_{23} \mathrm{H}_{23} \mathrm{~N}_{4} \mathrm{O}_{2} \mathrm{Na}^{79} \mathrm{Br} 489.0897$; Found 489.0885 .

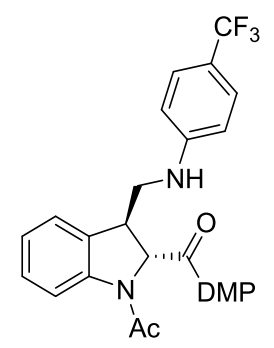

Reaction time: 5 hours.

3s, PE/EtOAc $=2 / 1$, white solid, m.p. $=205.7-207.0^{\circ} \mathrm{C} .75 .7 \mathrm{mg}, 83 \%$ yield $(0.2 \mathrm{mmol}$ scale). ${ }^{1} \mathrm{H}$ NMR (600 MHz, DMSO- $\left.d_{6}, 110{ }^{\circ} \mathrm{C}\right) \delta 7.88(\mathrm{br}, 1 \mathrm{H}), 7.35(\mathrm{~d}, J=8.4 \mathrm{~Hz}$, 2H), 7.27-7.24 (m, 2H), $7.02(\mathrm{t}, J=7.2,1 \mathrm{H}), 6.78(\mathrm{~d}, J=9.0 \mathrm{~Hz}, 2 \mathrm{H}), 6.34(\mathrm{~s}, 1 \mathrm{H})$, $6.26(\mathrm{~s}, 1 \mathrm{H}), 5.89(\mathrm{~d}, J=1.8 \mathrm{~Hz}, 1 \mathrm{H}), 3.71-3.65(\mathrm{~m}, 2 \mathrm{H}), 3.51-3.47(\mathrm{~m}, 1 \mathrm{H}), 2.47$ (s, $3 \mathrm{H}), 2.30$ (s, 3H), 2.19 (br, 3H). ${ }^{13} \mathrm{C}$ NMR (100 MHz, DMSO-d $) \delta 169.8,169.0,168.0^{*}$, $153.3,152.8^{*}, 151.3,144.8,144.3^{*}, 143.1,141.5^{*}, 132.8^{*}, 131.1,128.4^{*}, 128.0$, $126.7^{*}, 126.3,125.3,124.0^{*}, 123.3,122.9^{*}, 116.0,115.7^{*}, 115.4^{*}, 113.8,111.9$, $111.7^{*}, 111.3^{*}, 111.2,65.6,64.4^{*}, 46.9,44.6,42.2^{*}, 24.3^{*}, 23.6,13.7,13.5 .{ }^{19} \mathrm{~F}$ NMR $\left(376 \mathrm{MHz}, \mathrm{DMSO}-d_{6}\right) \delta-54.27--54.28(\mathrm{~m})$. IR (thin film): $v_{\max }\left(\mathrm{cm}^{-1}\right)=3318,1742$, 
$1651,1613,1592,1540,1481,1380,1357,1317,1253,1181,1155,1124,1101,1061$, 1033, 969, 940, 825, 809, 758, 639, 588, 513. HRMS (ESI) m/z: $[\mathrm{M}+\mathrm{Na}]^{+}$Calcd for $\mathrm{C}_{24} \mathrm{H}_{23} \mathrm{~N}_{4} \mathrm{O}_{2} \mathrm{~F}_{3} \mathrm{Na} 479.1665$; Found 479.1664.

\subsection{General procedure for formal [3+2] cycloadditive dearomatization of C2-} substituted indoles

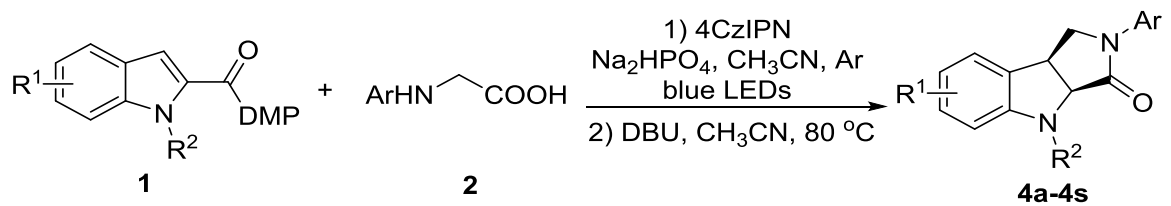

To a sealed tube equipped with a magnetic stir bar were added 1 ( $0.2 \mathrm{mmol}, 1.0$ equiv), 2 (0.4 mmol, 2.0 equiv), 4CzIPN (3.2 mg, $0.004 \mathrm{mmol}, 2.0 \mathrm{~mol} \%$ ), $\mathrm{Na}_{2} \mathrm{HPO}_{4}$ (56.8 mg, 0.4 mmol, 2.0 equiv) and $\mathrm{CH}_{3} \mathrm{CN}(2 \mathrm{~mL})$. The reaction mixture was degassed via freeze-pump-thaw for 3 cycles. After the mixture was thoroughly degassed, the tube was sealed and positioned approximately $5 \mathrm{~cm}$ from $24 \mathrm{~W}$ blue LEDs (maximum emission wavelength $=450 \mathrm{~nm}$ ). Then the reaction mixture was stirred at room temperature irradiated by $24 \mathrm{~W}$ blue LEDs. After 1 was consumed completely (monitored by TLC), the solution was quenched with water and extracted with EtOAc $(10 \mathrm{~mL} \times 3)$. The combined organic layers were washed with brine, dried over anhydrous $\mathrm{Na}_{2} \mathrm{SO}_{4}$ and concentrated in vacuo. The residue was purified by column chromatograph on silica gel to afford the desired product 3.

To a $25 \mathrm{~mL}$ flask equipped with a magnetic stir bar were added $3, \mathrm{CH}_{3} \mathrm{CN}(2 \mathrm{~mL})$, and DBU (152 mg, $1.0 \mathrm{mmol}, 5.0$ equiv). The reaction mixture was stirred at $80{ }^{\circ} \mathrm{C}$ for 1.5 h. After 3 was consumed completely (monitored by TLC), the reaction mixture was diluted with $\mathrm{CH}_{2} \mathrm{Cl}_{2}(20 \mathrm{~mL})$ and quenched with water $(20 \mathrm{~mL})$. The layers were separated and the aqueous layer was extracted with $\mathrm{CH}_{2} \mathrm{Cl}_{2}(20 \mathrm{~mL} \times 2)$. The combined organic layers were washed with brine, dried over anhydrous $\mathrm{Na}_{2} \mathrm{SO}_{4}$, and concentrated in vacuo. The residue was purified by column chromatography on silica gel to afford the desired product $\mathbf{4 a - 4 s}$. The analytical data of the products $\mathbf{4 a - 4 s}$ are summarized below. 


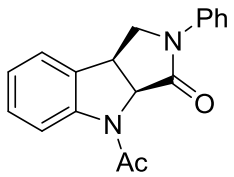

4a, $\mathrm{CH}_{2} \mathrm{Cl}_{2} / \mathrm{MeOH}=120: 1$, white solid, m.p. $=225.5-228.1{ }^{\circ} \mathrm{C} .47 .9 \mathrm{mg}, 81 \%$ yield over two steps (0.2 mmol scale). ${ }^{1} \mathrm{H}$ NMR $\left(400 \mathrm{MHz} \mathrm{CDCl}_{3}\right) \delta 8.24(\mathrm{~d}, J=8.0 \mathrm{~Hz}$, $1 \mathrm{H}), 7.60(\mathrm{~d}, J=8.0 \mathrm{~Hz}, 2 \mathrm{H}), 7.36(\mathrm{t}, J=8.0 \mathrm{~Hz}, 2 \mathrm{H}), 7.31-7.25(\mathrm{~m}, 2 \mathrm{H}), 7.18(\mathrm{t}, J=$ $7.6 \mathrm{~Hz}, 1 \mathrm{H}), 7.10(\mathrm{t}, J=7.2 \mathrm{~Hz}, 1 \mathrm{H}), 5.13(\mathrm{~d}, J=9.2 \mathrm{~Hz}, 1 \mathrm{H}), 4.43(\mathrm{dd}, J=10.0,7.2$ $\mathrm{Hz}, 1 \mathrm{H}), 4.26(\mathrm{t}, J=8 \mathrm{~Hz}, 1 \mathrm{H}), 4.05(\mathrm{~d}, J=10 \mathrm{~Hz}, 1 \mathrm{H}), 2.58(\mathrm{~s}, 3 \mathrm{H}) .{ }^{13} \mathrm{C}$ NMR $(100$ $\left.\mathrm{MHz}, \mathrm{CDCl}_{3}\right) \delta 169.93,169.85,142.6,138.5,131.2,129.4,129.1,125.7,124.6,123.8$, 120.3, 118.1, 64.7, 52.2, 37.5, 24.5. IR (thin film): $v_{\max }\left(\mathrm{cm}^{-1}\right)=2918,1700,1656,1597$, 1493, 1478, 1460, 1433, 1410, 1389, 1355, 1303, 1257, 1194, 1163, 1142, 1093, 1057, 1032, 935, 912, 876, 852, 792, 757, 708, 691, 658, 631, 565, 542, 508, 496, 434, 414. HRMS (ESI) m/z: [M + Na] ${ }^{+}$Calcd for $\mathrm{C}_{18} \mathrm{H}_{16} \mathrm{~N}_{2} \mathrm{O}_{2} \mathrm{Na} 315.1104$; Found 315.1101.

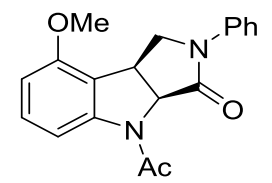

4b, $\mathrm{CH}_{2} \mathrm{Cl}_{2} / \mathrm{MeOH}=120: 1$, white solid, m.p. $=203.9-206.0{ }^{\circ} \mathrm{C} .41 .9 \mathrm{mg}, 65 \%$ yield over two steps $(0.2 \mathrm{mmol} \mathrm{scale}) .{ }^{1} \mathrm{H}$ NMR $\left(400 \mathrm{MHz}, \mathrm{CDCl}_{3}\right) \delta 7.85(\mathrm{~d}, J=8.4 \mathrm{~Hz}$, $1 \mathrm{H}), 7.62(\mathrm{~d}, J=8.0 \mathrm{~Hz}, 2 \mathrm{H}), 7.35(\mathrm{t}, J=7.6 \mathrm{~Hz}, 2 \mathrm{H}), 7.31-7.25(\mathrm{~m}, 2 \mathrm{H}), 7.24(\mathrm{t}, J=$ $8.0 \mathrm{~Hz}, 1 \mathrm{H}), 7.17(\mathrm{~d}, J=7.6 \mathrm{~Hz}, 1 \mathrm{H}), 6.61(\mathrm{~d}, J=8.0 \mathrm{~Hz}, 1 \mathrm{H}), 5.12(\mathrm{~d}, J=8.4 \mathrm{~Hz}, 1 \mathrm{H})$, 4.33-4.28 (m, 3H), $3.84(\mathrm{~s}, 3 \mathrm{H}), 2.57,(\mathrm{~s}, 3 \mathrm{H}) .{ }^{13} \mathrm{C} \mathrm{NMR}\left(100 \mathrm{MHz}, \mathrm{CDCl}_{3}\right) \delta 167.0$, 156.2, 143.8, 138.7, 130.7, 129.0, 125.5, 120.2, 117.6, 110.9, 106.6, 64.9, 55.4, 50.4, 36.0, 24.5. IR (thin film): $v_{\max }\left(\mathrm{cm}^{-1}\right)=2920,2845,1685,1658,1597,1486,1459,1389$, 1304, 1278, 1254, 1218, 1199, 1141, 1119, 1094, 1034, 999, 961, 891, 785, 756, 734, 706, 688, 654, 633, 589, 549, 506, 491, 448. HRMS (ESI) m/z: [M + Na] ${ }^{+}$Calcd for $\mathrm{C}_{19} \mathrm{H}_{19} \mathrm{~N}_{2} \mathrm{O}_{3} \mathrm{Na} 323.1390$; Found 323.1384.

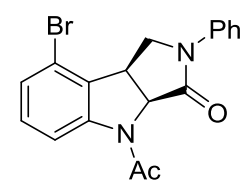


4c, $\mathrm{CH}_{2} \mathrm{Cl}_{2} / \mathrm{MeOH}=120: 1$, yellow solid, m.p. $=198.2-200.1^{\circ} \mathrm{C} .45 .4 \mathrm{mg}, 61 \%$ yield over two steps (0.2 mmol scale). ${ }^{1} \mathrm{H}$ NMR $\left(400 \mathrm{MHz}, \mathrm{CDCl}_{3}\right) \delta 8.21(\mathrm{~d}, J=8 \mathrm{~Hz}, 1 \mathrm{H})$, $7.61(\mathrm{~d}, J=8.0 \mathrm{~Hz}, 2 \mathrm{H}), 7.37$ (t, $J=7.6 \mathrm{~Hz}, 2 \mathrm{H}), 7.23-7.14(\mathrm{~m}, 3 \mathrm{H}), 5.18$ (d, $J=9.2$ $\mathrm{Hz}, 1 \mathrm{H}), 4.39-4.28$ (m, 3H), 2.60 (s, 3H). ${ }^{13} \mathrm{C} \mathrm{NMR}\left(100 \mathrm{MHz}, \mathrm{CDCl}_{3}\right) \delta 170.3,169.4$, 144.1, 138.2, 131.0, 130.5, 129.1, 127.7, 125.9, 120.5, 119.1, 116.9, 64.1, 51.0, 38.6, 24.5. IR (thin film): $v_{\max }\left(\mathrm{cm}^{-1}\right)=2910,1693,1672,1595,1495,1465,1443,1414$, 1378, 1343, 1306, 1272, 1254, 1212, 1159, 1140, 1113, 1070, 1033, 983, 945, 895, 854, 828, 781, 753 726, 688, 673, 634, 592, 552, 533, 487, 464, 434. HRMS (ESI) m/z: [M $+\mathrm{Na}]^{+}$Calcd for $\mathrm{C}_{18} \mathrm{H}_{15} \mathrm{~N}_{2} \mathrm{O}_{2} \mathrm{Na}^{79} \mathrm{Br}$ 393.0209; Found 393.0211.

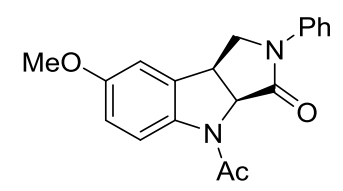

4d, $\mathrm{CH}_{2} \mathrm{Cl}_{2} / \mathrm{MeOH}=120: 1$, white solid, m.p. $=173.4-176.2{ }^{\circ} \mathrm{C} .43 .4 \mathrm{mg}, 67 \%$ yield over two steps (0.2 mmol scale). ${ }^{1} \mathrm{H}$ NMR $\left(400 \mathrm{MHz}, \mathrm{CDCl}_{3}\right) \delta 8.15-8.12(\mathrm{~m}, 1 \mathrm{H}), 7.57$ $(\mathrm{d}, J=8.0 \mathrm{~Hz}, 2 \mathrm{H}), 7.34(\mathrm{t}, J=7.2 \mathrm{~Hz}, 2 \mathrm{H}), 7.16(\mathrm{t}, J=7.6 \mathrm{~Hz}, 1 \mathrm{H}), 6.81-6.78(\mathrm{~m}, 2 \mathrm{H})$, 5.11-5.07 (m, 1H), 4.41-4.35 (m, 1H), 4.22-4.17 (m, 1H), 4.02-3.99 (m, 1H), $3.76(\mathrm{~s}$, 3H), $2.53(\mathrm{~s}, 3 \mathrm{H}) .{ }^{13} \mathrm{C} \mathrm{NMR}\left(100 \mathrm{MHz}, \mathrm{CDCl}_{3}\right) \delta 167.0,169.2,157.0,138.5,136.2$, $132.7,129.1,125.7,120.2$, 118.7, 113.6, 110.1, 64.9, 55.8, 52.0, 37.5, 24.1. IR (thin film): $v_{\max }\left(\mathrm{cm}^{-1}\right)=2921,1691,1659,1594,1547,1487,1389,1327,1303,1287,1263$, 1203, 1184, 1145, 1121, 1060, 1028, 996, 939, 907, 869, 821, 753, 688, 624, 594, 506, 451. HRMS (ESI) m/z: [M+ Na] $]^{+}$Calcd for $\mathrm{C}_{19} \mathrm{H}_{19} \mathrm{~N}_{2} \mathrm{O}_{3} \mathrm{Na} 323.1390$; Found 323.1387.

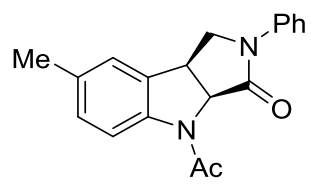

4e, $\mathrm{CH}_{2} \mathrm{Cl}_{2} / \mathrm{MeOH}=120: 1$, white solid, m.p. = 223.0-225.2 ${ }^{\circ} \mathrm{C} .36 .9 \mathrm{mg}, 60 \%$ yield over two steps (0.2 mmol scale). ${ }^{1} \mathrm{H}$ NMR (400 MHz, $\left.\mathrm{CDCl}_{3}\right) \delta 8.09(\mathrm{~d}, J=8.4 \mathrm{~Hz}$, $1 \mathrm{H}), 7.59(\mathrm{~d}, J=8.4 \mathrm{~Hz}, 2 \mathrm{H}), 7.36-7.32(\mathrm{~m}, 2 \mathrm{H}), 7.16(\mathrm{t}, J=7.2 \mathrm{~Hz}, 1 \mathrm{H}), 7.08-7.05(\mathrm{~m}$, 2H), $5.08(\mathrm{~d}, J=8.8 \mathrm{~Hz}, 1 \mathrm{H}), 4.38(\mathrm{dd}, J=9.6,6.8 \mathrm{~Hz}, 1 \mathrm{H}), 4.19(\mathrm{t}, J=8.0 \mathrm{~Hz}, 1 \mathrm{H})$, $4.01(\mathrm{~d}, J=10.0 \mathrm{~Hz}, 1 \mathrm{H}), 2.53(\mathrm{~s}, 3 \mathrm{H}), 2.30(\mathrm{~s}, 3 \mathrm{H}) .{ }^{13} \mathrm{C} \mathrm{NMR}\left(100 \mathrm{MHz}, \mathrm{CDCl}_{3}\right) \delta$ 
170.0, 169.5, 140.3, 138.5, 134.3, 131.3, 129.8, 129.0, 125.6, 124.4, 120.2, 117.6, 64.8, 52.1, 37.4, 24.3, 21.1. IR (thin film): $v_{\max }\left(\mathrm{cm}^{-1}\right)=2914,1708,1651,1596,1485,1460$, 1433, 1389, 1353, 1305, 1288, 1255, 1204, 1144, 1121, 1060, 1034, 977, 940, 916, 815, 785, 755, 708, 686, 625, 583, 510, 483 429. HRMS (ESI) m/z: $[\mathrm{M}+\mathrm{Na}]^{+}$Calcd for $\mathrm{C}_{19} \mathrm{H}_{18} \mathrm{~N}_{2} \mathrm{O}_{2} \mathrm{Na}$ 329.1261; Found 329.1260.

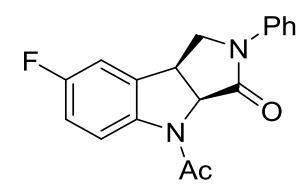

4f, $\mathrm{CH}_{2} \mathrm{Cl}_{2} / \mathrm{MeOH}=120: 1$, yellow solid, m.p. $=225.6-226.1{ }^{\circ} \mathrm{C} .44 .5 \mathrm{mg}, 72 \%$ yield over two steps (0.2 mmol scale). ${ }^{1} \mathrm{H}$ NMR (400 $\left.\mathrm{MHz}, \mathrm{CDCl}_{3}\right) \delta 8.20(\mathrm{dd}, J=8.8,4.8$ $\mathrm{Hz}, 1 \mathrm{H}), 7.60-7.57$ (m, 2H), 7.39-7.35 (m, 2H), 7.19 (t, J=7.2 Hz, 1H), 7.01-6.95 (m, $2 \mathrm{H}), 5.15(\mathrm{~d}, J=8.8 \mathrm{~Hz}, 1 \mathrm{H}), 4.42(\mathrm{dd}, J=10.0,7.2 \mathrm{~Hz}, 1 \mathrm{H}), 4.25(\mathrm{t}, J=8.0 \mathrm{~Hz}, 1 \mathrm{H})$, $4.01(\mathrm{~d}, J=10.0 \mathrm{~Hz}, 1 \mathrm{H}), 2.57$ (s, 3H). ${ }^{13} \mathrm{C} \mathrm{NMR}\left(100 \mathrm{MHz}, \mathrm{CDCl}_{3}\right) \delta 169.62,169.59$, $159.8\left(\mathrm{C}-\mathrm{F},{ }^{1} J_{\mathrm{C}-\mathrm{F}}=242.4 \mathrm{~Hz}\right), 138.8,138.3,133.0\left(\mathrm{C}-\mathrm{F},{ }^{3} J_{\mathrm{C}-\mathrm{F}}=8.0 \mathrm{~Hz}\right), 129.2,125.9$, $120.3,119.1\left(\mathrm{C}-\mathrm{F},{ }^{3} J_{\mathrm{C}-\mathrm{F}}=8.1 \mathrm{~Hz}\right), 115.9\left(\mathrm{C}-\mathrm{F},{ }^{2} J_{\mathrm{C}-\mathrm{F}}=22.5 \mathrm{~Hz}\right), 111.1\left(\mathrm{C}-\mathrm{F},{ }^{2} J_{\mathrm{C}-\mathrm{F}}=24.1\right.$ $\mathrm{Hz}), 65.0,52.0,37.5\left(\mathrm{C}-\mathrm{F},{ }^{4} J_{\mathrm{C}-\mathrm{F}}=2.0 \mathrm{~Hz}\right), 24.2 .{ }^{19} \mathrm{~F}$ NMR $\left(376 \mathrm{MHz}, \mathrm{CDCl}_{3}\right)-117.7--$ 117.8 (m). IR (thin film): $v_{\max }\left(\mathrm{cm}^{-1}\right)=3064,2920,2851,1675,1598,1477,1383,1346$, 1302, 1250, 1217, 1199, 1177, 1142, 1119, 1061, 1031, 998, 949, 919, 895, 838, 785, 754, 707, 683, 624, 586, 514, 484, 448, 433. HRMS (ESI) m/z: $[\mathrm{M}+\mathrm{Na}]^{+}$Calcd for $\mathrm{C}_{18} \mathrm{H}_{15} \mathrm{~N}_{2} \mathrm{O}_{2} \mathrm{FNa} 333.1010$; Found 333.1001.

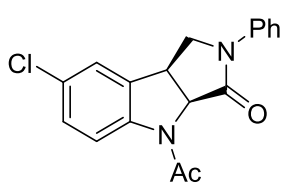

4g, $\mathrm{CH}_{2} \mathrm{Cl}_{2} / \mathrm{MeOH}=120: 1$, white foam, $48.4 \mathrm{mg}$, $74 \%$ yield over two steps $(0.2 \mathrm{mmol}$ scale). ${ }^{1} \mathrm{H}$ NMR $\left(400 \mathrm{MHz}, \mathrm{CDCl}_{3}\right) \delta 8.17(\mathrm{~d}, J=8.4 \mathrm{~Hz}, 1 \mathrm{H}), 7.58(\mathrm{~d}, J=8.0 \mathrm{~Hz}, 2 \mathrm{H})$, $7.37(\mathrm{t}, J=7.6 \mathrm{~Hz}, 2 \mathrm{H}), 7.26-7.18(\mathrm{~m}, 3 \mathrm{H}), 5.15(\mathrm{~d}, J=9.2 \mathrm{~Hz}, 1 \mathrm{H}), 4.42(\mathrm{dd}, J=10.0$, $7.2 \mathrm{~Hz}, 1 \mathrm{H}), 4.25(\mathrm{t}, J=8.0 \mathrm{~Hz}, 1 \mathrm{H}), 4.02(\mathrm{~d}, J=10.4 \mathrm{~Hz}, 1 \mathrm{H}), 2.58(\mathrm{~s}, 3 \mathrm{H}) .{ }^{13} \mathrm{C} \mathrm{NMR}$ $\left(100 \mathrm{MHz}, \mathrm{CDCl}_{3}\right) \delta 169.8,169.5,141.3,138.3,133.1,129.5,129.4,129.2,125.9$, 124.1, 120.3, 119.0, 64.9, 52.0, 37.4, 24.3. IR (thin film): $v_{\max }\left(\mathrm{cm}^{-1}\right)=2987,2919$, 
$1692,1648,1595,1486,1469,1387,1309,1288,1250,1205,1164,1105,1073,1059$, 1032, 981, 939, 916, 889, 821, 785, 756, 690, 666, 641, 611, 576, 504, 452, 429, 415. HRMS (ESI) m/z: [M + Na] ${ }^{+}$Calcd for $\mathrm{C}_{18} \mathrm{H}_{15} \mathrm{~N}_{2} \mathrm{O}_{2} \mathrm{NaCl}$ 349.0714; Found 349.0709.

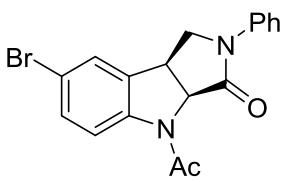

4h, $\mathrm{CH}_{2} \mathrm{Cl}_{2} / \mathrm{MeOH}=120: 1$, white solid, m.p. $=259.7-260.2{ }^{\circ} \mathrm{C} .46 .2 \mathrm{mg}, 62 \%$ yield over two steps (0.2 mmol scale). ${ }^{1} \mathrm{H}$ NMR (400 MHz, $\left.\mathrm{CDCl}_{3}\right) \delta 8.13(\mathrm{~d}, J=8.8 \mathrm{~Hz}$, 1H), 7.60-7.57 (m, 2H), 7.42-7.35 (m, 2H), $7.20(\mathrm{t}, J=7.6 \mathrm{~Hz}, 1 \mathrm{H}), 5.14$ (d, $J=8.8 \mathrm{~Hz}$, $1 \mathrm{H}), 4.42(\mathrm{dd}, J=10.0,7.2 \mathrm{~Hz}, 1 \mathrm{H}), 4.26(\mathrm{t}, J=8.4 \mathrm{~Hz}, 1 \mathrm{H}), 4.02(\mathrm{dd}, J=10.0,0.8 \mathrm{~Hz}$, 1H), 2.58 (s, 3H). ${ }^{13} \mathrm{C}$ NMR (100 MHz, $\left.\mathrm{CDCl}_{3}\right) \delta 169.9,169.5,141.8,138.3,133.4$, $132.4,129.2,127.0,126.0,120.4,119.5,116.9,64.8,52.0,37.4,24.4$. IR (thin film): $v_{\max }\left(\mathrm{cm}^{-1}\right)=2919,1691,1648,1594,1485,1468,1386,1308,1288,1252,1202,1163$, 1119, 1059, 1032, 1001, 981, 938, 916, 888, 819, 784, 756, 690, 664, 636, 600, 575, 542, 500, 424. HRMS (ESI) m/z: $[\mathrm{M}+\mathrm{Na}]^{+}$Calcd for $\mathrm{C}_{18} \mathrm{H}_{15} \mathrm{~N}_{2} \mathrm{O}_{2} \mathrm{Na}^{79} \mathrm{Br}$ 393.0209; Found 393.0205.

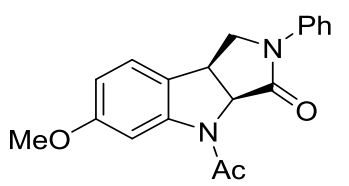

$4 \mathbf{i}, \mathrm{CH}_{2} \mathrm{Cl}_{2} / \mathrm{MeOH}=120: 1$, white solid, m.p. $=221.8-223.0{ }^{\circ} \mathrm{C} .36 .1 \mathrm{mg}, 56 \%$ yield over two steps (0.2 mmol scale). ${ }^{1} \mathrm{H}$ NMR (400 MHz, $\left.\mathrm{CDCl}_{3}\right) \delta 7.90(\mathrm{~d}, J=2.4 \mathrm{~Hz}$, 1H), 7.60-7.58 (m, 2H), 7.37-7.33 (m, 2H), 7.19 (t, $J=7.2 \mathrm{~Hz}, 1 \mathrm{H}), 7.12(\mathrm{~d}, J=8.4 \mathrm{~Hz}$, $1 \mathrm{H}), 6.64(\mathrm{dd}, J=8.0,2.4 \mathrm{~Hz}, 1 \mathrm{H}), 5.13(\mathrm{~d}, J=8.8 \mathrm{~Hz}, 1 \mathrm{H}), 4.38(\mathrm{dd}, J=10.0,6.8 \mathrm{~Hz}$, $1 \mathrm{H}), 4.19(\mathrm{t}, J=8.0 \mathrm{~Hz}, 1 \mathrm{H}), 3.99(\mathrm{~d}, J=10.0 \mathrm{~Hz}, 1 \mathrm{H}), 3.80(\mathrm{~s}, 3 \mathrm{H}), 2.58(\mathrm{~s}, 3 \mathrm{H}) .{ }^{13} \mathrm{C}$ NMR (100 MHz, $\left.\mathrm{CDCl}_{3}\right) \delta 170.00,169.97,160.8,143.9,138.6,129.1,125.7,124.1$, 123.0, 120.3, 111.3, 103.5, 65.5, 55.8, 52.3, 36.9, 24.5. IR (thin film): $v_{\max }\left(\mathrm{cm}^{-1}\right)=$ 2920, 1701, 1653, 1598, 1497, 1446, 1396, 1353, 1309, 1283, 1259, 1220, 1200, 1184, 1116, 1096, 1057, 1032, 996, 950, 899, 851, 808, 761, 741, 704, 686, 659, 631, 534, 
498, 460, 440, 413. HRMS (ESI) m/z: $[\mathrm{M}+\mathrm{Na}]^{+}$Calcd for $\mathrm{C}_{19} \mathrm{H}_{18} \mathrm{~N}_{2} \mathrm{O}_{3} \mathrm{Na}$ 345.1210; Found 345.1211.

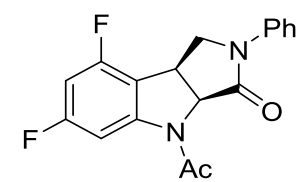

4j, $\mathrm{CH}_{2} \mathrm{Cl}_{2} / \mathrm{MeOH}=120: 1$, white solid, m.p. $=190.4-191.2{ }^{\circ} \mathrm{C} .47 .4 \mathrm{mg}, 72 \%$ yield over two steps $(0.2 \mathrm{mmol} \mathrm{scale}) .{ }^{1} \mathrm{H} \mathrm{NMR}\left(400 \mathrm{MHz}, \mathrm{CDCl}_{3}\right) \delta 7.82(\mathrm{~d}, J=9.6 \mathrm{~Hz}$, 1H), 7.61-7.58 (m, 2H), 7.40-7.35 (m, 2H), 7.20 (t, J=7.2 Hz, 1H), $7.12(\mathrm{t}, J=7.2 \mathrm{~Hz}$, $1 \mathrm{H}), 6.53(\mathrm{td}, J=9.2,2.4 \mathrm{~Hz}, 1 \mathrm{H}), 5.19-5.17(\mathrm{~m}, 1 \mathrm{H}), 4.39-4.33(\mathrm{~m}, 2 \mathrm{H}), 4.20-4.15(\mathrm{~m}$, 1H), 3.99 (d, $J=10.0 \mathrm{~Hz}, 1 \mathrm{H}), 2.57(\mathrm{~s}, 3 \mathrm{H}) .{ }^{13} \mathrm{C} \mathrm{NMR}\left(100 \mathrm{MHz}, \mathrm{CDCl}_{3}\right) \delta 170.1$, $169.1,165.5\left(\mathrm{C}-\mathrm{F},{ }^{1} J_{\mathrm{C}-\mathrm{F}}=245.4 \mathrm{~Hz} ; \mathrm{C}-\mathrm{F},{ }^{3} J_{\mathrm{C}-\mathrm{F}}=12.4 \mathrm{~Hz}\right), 159.0\left(\mathrm{C}-\mathrm{F},{ }^{1} J_{\mathrm{C}-\mathrm{F}}=245.1\right.$ $\left.\mathrm{Hz} ; \mathrm{C}-\mathrm{F},{ }^{3} J_{\mathrm{C}-\mathrm{F}}=15.0 \mathrm{~Hz}\right), 145.0\left(\mathrm{C}-\mathrm{F},{ }^{3} J_{\mathrm{C}-\mathrm{F}}=15.0 \mathrm{~Hz} ; \mathrm{C}-\mathrm{F},{ }^{3} J_{\mathrm{C}-\mathrm{F}}=10.0 \mathrm{~Hz}\right), 138.2$, $129.2,126.0,112.8\left(\mathrm{C}-\mathrm{F},{ }^{2} J_{\mathrm{C}-\mathrm{F}}=19.7 ; \mathrm{C}-\mathrm{F},{ }^{4} J_{\mathrm{C}-\mathrm{F}}=3.2 \mathrm{~Hz}\right), 102.4\left(\mathrm{C}-\mathrm{F},{ }^{2} J_{\mathrm{C}-\mathrm{F}}=28.7 \mathrm{~Hz}\right.$; $\left.\mathrm{C}-\mathrm{F},{ }^{4} J_{\mathrm{C}-\mathrm{F}}=2.9 \mathrm{~Hz}\right), 99.7\left(\mathrm{C}-\mathrm{F},{ }^{2} J_{\mathrm{C}-\mathrm{F}}=27.0 \mathrm{~Hz} ; \mathrm{C}-\mathrm{F},{ }^{2} J_{\mathrm{C}-\mathrm{F}}=23.6 \mathrm{~Hz}\right), 65.4,50.6,35.2$, 24.4. ${ }^{19}$ F NMR (376 MHz, $\mathrm{CDCl}_{3}$ ) -107.16--107.23 (m), -117.2--117.32 (m). IR (thin film): $v_{\max }\left(\mathrm{cm}^{-1}\right)=2920,2851,1698,1675,1627,1599,1487,1442,1390,1361,1336$, 1304, 1263, 1223, 1195, 1144, 1104, 1079, 1055, 978, 898, 850, 823, 755, 706, 687, 672, 629, 571, 550, 514, 475, 463. HRMS (ESI) m/z: $[\mathrm{M}+\mathrm{Na}]^{+}$Calcd for $\mathrm{C}_{18} \mathrm{H}_{14} \mathrm{~N}_{2} \mathrm{O}_{2}-$ $\mathrm{F}_{2} \mathrm{Na}$ 351.0916; Found 351.0911.

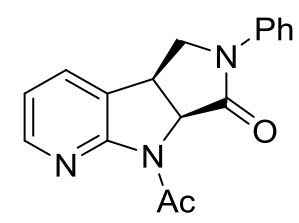

$4 \mathbf{k}, \mathrm{CH}_{2} \mathrm{Cl}_{2} / \mathrm{MeOH}=80: 1$, white foam, $26.3 \mathrm{mg}, 45 \%$ yield over two steps $(0.2 \mathrm{mmol}$ scale). ${ }^{1} \mathrm{H}$ NMR (400 MHz, $\left.\mathrm{CDCl}_{3}\right) \delta 8.23(\mathrm{~d}, J=7.6 \mathrm{~Hz}, 1 \mathrm{H}), 7.62-7.60(\mathrm{~m}, 3 \mathrm{H}), 7.32$ (t, $J=7.6 \mathrm{~Hz}, 2 \mathrm{H}), 7.13(\mathrm{t}, J=7.2 \mathrm{~Hz}, 1 \mathrm{H}), 7.12(\mathrm{t}, J=7.2 \mathrm{~Hz}, 1 \mathrm{H}), 6.98(\mathrm{dd}, J=7.6$, $5.2 \mathrm{~Hz}, 1 \mathrm{H}), 5.72(\mathrm{~d}, J=8.8 \mathrm{~Hz}, 1 \mathrm{H}), 4.40(\mathrm{dd}, J=10.0,7.6 \mathrm{~Hz}, 1 \mathrm{H}), 4.10$ (t, $J=8.0$ $\mathrm{Hz}, 1 \mathrm{H}), 4.00(\mathrm{~d}, J=10.0 \mathrm{~Hz}, 1 \mathrm{H}), 2.73(\mathrm{~s}, 3 \mathrm{H}) .{ }^{13} \mathrm{C} \mathrm{NMR}\left(100 \mathrm{MHz}, \mathrm{CDCl}_{3}\right) \delta 169.8$, $169.6,155.5,148.2$, 138.7, 133.3, 129.0, 126.3, 125.3, 119.9, 119.1, 61.3, 51.4, 34.3, 
24.7. IR (thin film): $v_{\max }\left(\mathrm{cm}^{-1}\right)=2920,2851,1695,1660,1586,1501,1470,1414$, 1374, 1357, 1305, 1267, 1233, 1207, 1188, 1158, 1117, 1091, 1035, 976, 902, 864, 798, 783, 755, 721, 692, 632, 590, 572, 547, 513, 494. HRMS (ESI) m/z: $[\mathrm{M}+\mathrm{Na}]^{+}$Calcd for $\mathrm{C}_{17} \mathrm{H}_{16} \mathrm{~N}_{3} \mathrm{O}_{2} \mathrm{Na}$ 294.1237; Found 294.1225.

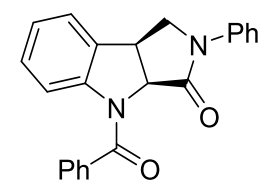

4l, $\mathrm{CH}_{2} \mathrm{Cl}_{2} / \mathrm{MeOH}=80: 1$, white solid, m.p. $=203.0-204.1{ }^{\circ} \mathrm{C} .49 .8 \mathrm{mg}, 70 \%$ yield over two steps (0.2 mmol scale). ${ }^{1} \mathrm{H}$ NMR $\left(400 \mathrm{MHz}, \mathrm{CDCl}_{3}\right) \delta$ 7.98-7.96 (m, 3H), $7.57(\mathrm{~d}$, $J=7.6 \mathrm{~Hz}, 2 \mathrm{H}), 7.49-7.42(\mathrm{~m}, 4 \mathrm{H}), 7.35-7.29$ (m, 4H), 7.15 (t, $J=7.6 \mathrm{~Hz}, 2 \mathrm{H}), 5.18$ (br, 1H), 4.30 (dd, $J=10.0,6.4 \mathrm{~Hz}, 1 \mathrm{H}), 4.19$ (t, $J=7.6 \mathrm{~Hz}, 1 \mathrm{H}), 4.03(\mathrm{~d}, J=10.0 \mathrm{~Hz}$, 1H). ${ }^{13} \mathrm{C}$ NMR $\left(100 \mathrm{MHz}, \mathrm{CDCl}_{3}\right) \delta 170.1,170.0,142.6,138.6,136.3,132.1,130.6$, $129.1,129.0,128.5,128.2,125.5,125.3,123.9,120.1,119.1,65.8,51.1,38.2$. IR (thin film): $v_{\max }\left(\mathrm{cm}^{-1}\right)=3066,2921,1696,1642,1595,1497,1474,1381,1307,1257,1224$, 1201, 1175, 1118, 1096, 1048, 1028, 998, 939, 913, 883, 840, 801, 784, 755, 711, 692, 667, 641, 607, 570, 520, 497. HRMS (ESI) m/z: $[\mathrm{M}+\mathrm{Na}]^{+}$Calcd for $\mathrm{C}_{23} \mathrm{H}_{18} \mathrm{~N}_{2} \mathrm{O}_{2} \mathrm{Na}$ 377.1261; Found 377.1255.

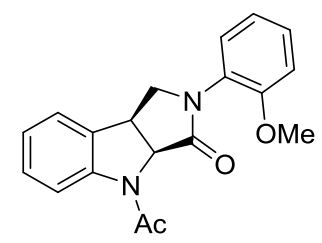

4m, $\mathrm{CH}_{2} \mathrm{Cl}_{2} / \mathrm{MeOH}=80: 1$, white solid, m.p. $=172.0-173.4{ }^{\circ} \mathrm{C} .36 .7 \mathrm{mg}$, 57\% yield over two steps (0.2 mmol scale). ${ }^{1} \mathrm{H}$ NMR (400 $\left.\mathrm{MHz} \mathrm{CDCl}_{3}\right) \delta 8.23(\mathrm{~d}, J=8.0 \mathrm{~Hz}$, 1H), 7.30-7.23 (m, 3H), 7.12-7.07 (m, 2H), 6.92-6.88 (m, 2H), $5.11(\mathrm{~d}, J=8.8 \mathrm{~Hz}, 1 \mathrm{H})$, $4.36(\mathrm{dd}, J=10.0,7.2 \mathrm{~Hz}, 1 \mathrm{H}), 4.21(\mathrm{t}, J=8.0 \mathrm{~Hz}, 1 \mathrm{H}), 3.85$ (d, $J=10.0 \mathrm{~Hz}, 1 \mathrm{H}), 3.67$ $(\mathrm{s}, 3 \mathrm{H}), 2.55$ (s, 3H). ${ }^{13} \mathrm{C}$ NMR $\left(100 \mathrm{MHz}, \mathrm{CDCl}_{3}\right) \delta 170.6,170.0,154.7,142.5,131.9$, 129.4, 129.0, 128.6, 126.1, 124.3, 123.8, 120.9, 117.9, 112.2, 63.6, 55.7, 53.3, 38.7, 24.4. IR (thin film): $v_{\max }\left(\mathrm{cm}^{-1}\right)=2921,1693,1658,1596,1503,1478,1460,1391$, 
1355, 1296, 1256, 1162, 1124, 1022, 938, 916, 855, 798, 751, 707, 658, 632, 596, 557, 483. HRMS (ESI) m/z: [M+ Na $]^{+}$Calcd for $\mathrm{C}_{19} \mathrm{H}_{18} \mathrm{~N}_{2} \mathrm{O}_{3} \mathrm{Na} 345.1210$; Found 345.1209.

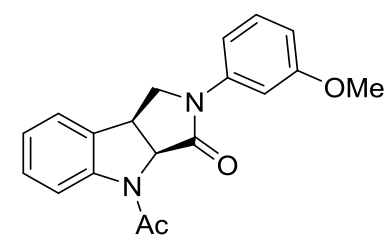

4n, $\mathrm{CH}_{2} \mathrm{Cl}_{2} / \mathrm{MeOH}=80: 1$, white solid, m.p. $=199.7-201.4^{\circ} \mathrm{C} .40 .3 \mathrm{mg}, 63 \%$ yield over two steps (0.2 mmol scale). ${ }^{1} \mathrm{H}$ NMR (400 MHz, $\left.\mathrm{CDCl}_{3}\right) \delta 8.23(\mathrm{~d}, J=8.0 \mathrm{~Hz}, 1 \mathrm{H})$, 7.35-7.22 (m, 4H), 7.11-7.06 (m, 2H), $6.72(\mathrm{dd}, J=8.4,1.6 \mathrm{~Hz}, 1 \mathrm{H}), 5.11(\mathrm{~d}, J=9.2$ $\mathrm{Hz}, 1 \mathrm{H}), 4.41-4.37$ (m, 1H), 4.23 (t, $J=7.6 \mathrm{~Hz}, 1 \mathrm{H}), 4.03$ (d, J=10.0 Hz, 1H), 3.78 (s, 3H), $2.57(\mathrm{~s}, 3 \mathrm{H}) .{ }^{13} \mathrm{C} \mathrm{NMR}\left(100 \mathrm{MHz}, \mathrm{CDCl}_{3}\right) \delta 170.0,169.8,160.1,142.6,139.7$, 131.2, 129.8, 129.7, 129.3, 124.6, 123.9, 118.1, 112.0, 111.5, 106.2, 64.8, 55.5, 52.3, 37.4, 24.4. Found: 345.1204. IR (thin film): $v_{\max }\left(\mathrm{cm}^{-1}\right)=2919,1707,1651,1602,1478$, 1460, 1389, 1355, 1302, 1256, 1221, 1202, 1175, 1137, 1097, 1052, 980, 927, 878, 845, 772, 754, 707, 684, 657, 631, 567, 533, 494, 457, 413. HRMS (ESI) m/z: $[\mathrm{M}+\mathrm{Na}]^{+}$ Calcd for $\mathrm{C}_{19} \mathrm{H}_{18} \mathrm{~N}_{2} \mathrm{O}_{3} \mathrm{Na} 345.1210$; Found 345.1204.

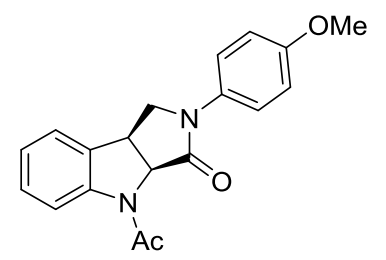

4o, $\mathrm{CH}_{2} \mathrm{Cl}_{2} / \mathrm{MeOH}=80: 1$, white solid, m.p. $=231.2-233.5^{\circ} \mathrm{C} .37 .5 \mathrm{mg}$, 58\% yield over two steps (0.2 mmol scale). ${ }^{1} \mathrm{H}$ NMR (400 MHz, $\left.\mathrm{CDCl}_{3}\right) \delta 8.23(\mathrm{~d}, J=8.4 \mathrm{~Hz}, 1 \mathrm{H})$, 7.49-7.45 (m, 4H), 7.31-7.25 (m, 2H), 7.09 (t, $J=7.6 \mathrm{~Hz}, 1 \mathrm{H}), 6.69-6.85$ (m, 2H), 5.12 $(\mathrm{d}, J=8.8 \mathrm{~Hz}, 1 \mathrm{H}), 4.39(\mathrm{dd}, J=9.6,7.2 \mathrm{~Hz}, 1 \mathrm{H}), 4.24(\mathrm{t}, J=8.4 \mathrm{~Hz}, 1 \mathrm{H}), 3.98(\mathrm{~d}, J=$ $9.6 \mathrm{~Hz}, 1 \mathrm{H}), 3.78(\mathrm{~s}, 3 \mathrm{H}), 2.58(\mathrm{~s}, 3 \mathrm{H}) .{ }^{13} \mathrm{C} \mathrm{NMR}\left(100 \mathrm{MHz}, \mathrm{CDCl}_{3}\right) \delta$ 169.9, 169.5, 157.4, 142.6, 131.6, 131.4, 129.3, 124.6, 123.8, 122.1, 118.1, 114.3, 64.6, 55.6, 52.7, 37.5, 24.5. IR (thin film): $v_{\max }\left(\mathrm{cm}^{-1}\right)=3361,2894,1690,1660,1512,1476,1459,1394$, 1359, 1313, 1291, 1251, 1208, 1186, 1140, 1107, 1055, 1028, 994, 936, 829, 753, 710, 
648, 602, 567, 542, 521, 460. HRMS (ESI) m/z: $[\mathrm{M}+\mathrm{Na}]^{+}$Calcd for $\mathrm{C}_{19} \mathrm{H}_{18} \mathrm{~N}_{2} \mathrm{O}_{3} \mathrm{Na}$ 345.1210; Found 345.1200.

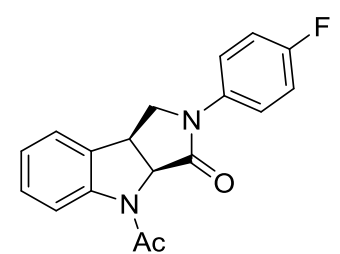

4p, $\mathrm{CH}_{2} \mathrm{Cl}_{2} / \mathrm{MeOH}=120: 1$, white solid, m.p. = 255.4-256.8 ${ }^{\circ} \mathrm{C} .42 .2 \mathrm{mg}, 68 \%$ yield over two steps (0.2 mmol scale). ${ }^{1} \mathrm{H}$ NMR (400 MHz, $\left.\mathrm{CDCl}_{3}\right) \delta 8.24(\mathrm{~d}, J=8.4 \mathrm{~Hz}$, 1H), 7.57-7.54 (m, 2H), 7.32-7.26 (m, 2H), 7.11 (t, J=7.6 Hz, 1H), 7.07-7.02 (m, 2H), $5.13(\mathrm{~d}, J=8.8 \mathrm{~Hz}, 1 \mathrm{H}), 4.41(\mathrm{dd}, J=9.6,7.2 \mathrm{~Hz}, 1 \mathrm{H}), 4.27(\mathrm{t}, J=8.4 \mathrm{~Hz}, 1 \mathrm{H}), 4.01$ $(\mathrm{d}, J=10.0 \mathrm{~Hz}, 1 \mathrm{H}), 2.58(\mathrm{~s}, 3 \mathrm{H}) .{ }^{13} \mathrm{C} \mathrm{NMR}\left(100 \mathrm{MHz}, \mathrm{CDCl}_{3}\right) \delta 169.9,160.2(\mathrm{C}-\mathrm{F}$, $\left.{ }^{1} J_{\mathrm{C}-\mathrm{F}}=244.5 \mathrm{~Hz}\right), 142.6,134.6,131.1,129.5,124.6,123.8,122.2\left(\mathrm{C}-\mathrm{F},{ }^{3} J_{\mathrm{C}-\mathrm{F}}=8.0 \mathrm{~Hz}\right)$, $118.1,115.9\left(\mathrm{C}-\mathrm{F},{ }^{2} J_{\mathrm{C}-\mathrm{F}}=22.4 \mathrm{~Hz}\right), 64.5,52.5,37.5,24.4 .{ }^{19} \mathrm{~F} \mathrm{NMR}\left(376 \mathrm{MHz}, \mathrm{CDCl}_{3}\right)$ -115.87--115.94 (m). IR (thin film): $v_{\max }\left(\mathrm{cm}^{-1}\right)=3071,2893,1697,1660,1597,1508$, 1474, 1432, 1392, 1309, 1288, 1260, 1224, 1207, 1171, 1142, 1120, 1055, 1036, 994, 936, 915, 860, 842, 816, 756, 711, 648, 597, 567, 521, 488, 429. HRMS (ESI) m/z: [M $+\mathrm{Na}]^{+}$Calcd for $\mathrm{C}_{18} \mathrm{H}_{15} \mathrm{~N}_{2} \mathrm{O}_{2} \mathrm{FNa}$ 333.1010; Found 333.0999.

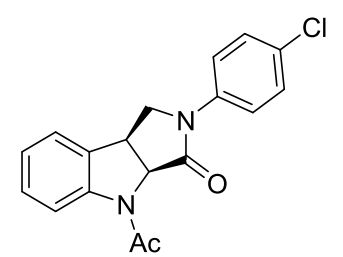

4q, $\mathrm{CH}_{2} \mathrm{Cl}_{2} / \mathrm{MeOH}=120: 1$, white solid, m.p. $=172.2-174.4^{\circ} \mathrm{C} .44 .0 \mathrm{mg}, 67 \%$ yield over two steps $(0.2 \mathrm{mmol} \mathrm{scale}) .{ }^{1} \mathrm{H}$ NMR $\left(400 \mathrm{MHz}, \mathrm{CDCl}_{3}\right) \delta 8.23(\mathrm{~d}, J=8.4 \mathrm{~Hz}$, $1 \mathrm{H}), 7.58-7.55(\mathrm{~m}, 2 \mathrm{H}), 7.33-7.30(\mathrm{~m}, 3 \mathrm{H}), 7.28-7.26(\mathrm{~m}, 1 \mathrm{H}), 7.11(\mathrm{t}, J=7.2 \mathrm{~Hz}, 1 \mathrm{H})$, $5.13(\mathrm{~d}, J=8.8 \mathrm{~Hz}, 1 \mathrm{H}), 4.40(\mathrm{dd}, J=10.0,7.2 \mathrm{~Hz}, 1 \mathrm{H}), 4.27(\mathrm{t}, J=8.4 \mathrm{~Hz}, 1 \mathrm{H}), 4.02$ $(\mathrm{d}, J=10.0 \mathrm{~Hz}, 1 \mathrm{H}), 2.58(\mathrm{~s}, 3 \mathrm{H}) .{ }^{13} \mathrm{C} \mathrm{NMR}\left(100 \mathrm{MHz}, \mathrm{CDCl}_{3}\right) \delta 170.0,169.8,142.6$, 137.1, 131.0, 130.9, 129.5, 129.2, 124.7, 123.8, 121.3, 118.1, 64.6, 52.2, 37.4, 24.4. IR (thin film): $v_{\max }\left(\mathrm{cm}^{-1}\right)=2918,1697,1655,1597,1480,1460,1426,1390,1308,1277$, 1256, 1203, 1144, 1125, 1092, 1057, 1005, 980, 937, 915, 853, 835, 808, 792, 750, 710, 
642, 569, 507, 456. HRMS (ESI) m/z: $[\mathrm{M}+\mathrm{Na}]^{+}$Calcd for $\mathrm{C}_{18} \mathrm{H}_{15} \mathrm{~N}_{2} \mathrm{O}_{2} \mathrm{NaCl} 349.0714$; Found 349.0711.

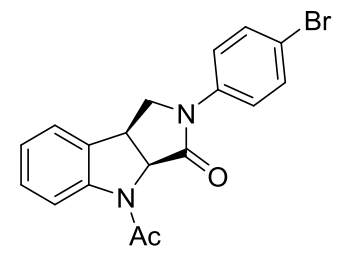

4r, $\mathrm{CH}_{2} \mathrm{Cl}_{2} / \mathrm{MeOH}=120: 1$, white solid, m.p. = 192.1-193.9 ${ }^{\circ} \mathrm{C} .51 .7 \mathrm{mg}, 70 \%$ yield over two steps (0.2 mmol scale). ${ }^{1} \mathrm{H}$ NMR $\left(400 \mathrm{MHz}, \mathrm{CDCl}_{3}\right) \delta 8.23(\mathrm{~d}, J=8.0 \mathrm{~Hz}$, 1H), 7.52-7.47 (m, 4H), $7.30(\mathrm{t}, J=8.0 \mathrm{~Hz}, 1 \mathrm{H}), 7.10(\mathrm{t}, J=7.6 \mathrm{~Hz}, 1 \mathrm{H}), 5.13(\mathrm{~d}, J=$ $8.8 \mathrm{~Hz}, 1 \mathrm{H}), 4.40(\mathrm{dd}, J=10.0,7.6 \mathrm{~Hz}, 1 \mathrm{H}), 4.27$ (t, $J=8.0 \mathrm{~Hz}, 1 \mathrm{H}), 4.01$ (d, $J=10.0$ $\mathrm{Hz}, 1 \mathrm{H}), 2.57$ (s, 3H). ${ }^{13} \mathrm{C} \mathrm{NMR}\left(100 \mathrm{MHz}, \mathrm{CDCl}_{3}\right) \delta 170.0,169.8,142.5,137.6,132.1$, 131.0, 129.5, 124.7, 123.8, 121.6, 118.7, 118.1, 64.6, 52.1, 37.4, 24.4. IR (thin film): $v_{\max }\left(\mathrm{cm}^{-1}\right)=1697,1656,1598,1480,1461,1424,1389,1309,1257,1204,1144,1076$, 1057, 1003, 833, 809, 751, 709, 641, 502. HRMS (ESI) m/z: $[\mathrm{M}+\mathrm{Na}]^{+}$Calcd for $\mathrm{C}_{18} \mathrm{H}_{15} \mathrm{~N}_{2} \mathrm{O}_{2} \mathrm{Na}^{79} \mathrm{Br} 393.0209$; Found 393.0206.

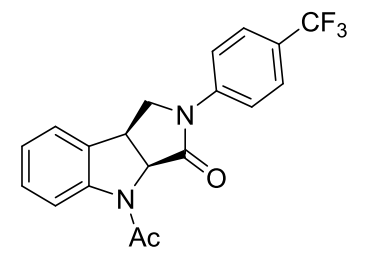

4s, $\mathrm{CH}_{2} \mathrm{Cl}_{2} / \mathrm{MeOH}=120: 1$, white solid, m.p. = 247.2-249.3 ${ }^{\circ} \mathrm{C} .51 .8 \mathrm{mg}, 72 \%$ yield over two steps (0.2 mmol scale). ${ }^{1} \mathrm{H}$ NMR $\left(400 \mathrm{MHz}, \mathrm{CDCl}_{3}\right) \delta 8.23(\mathrm{~d}, J=8.4 \mathrm{~Hz}$, 1H), $7.77(\mathrm{~d}, J=8.4 \mathrm{~Hz}, 2 \mathrm{H}), 7.60(\mathrm{~d}, J=8.8 \mathrm{~Hz}, 2 \mathrm{H}), 7.29$ (q, $J=7.6 \mathrm{~Hz}, 1 \mathrm{H}), 7.11$ $(\mathrm{t}, J=7.6 \mathrm{~Hz}, 1 \mathrm{H}), 5.16(\mathrm{~d}, J=8.8 \mathrm{~Hz}, 1 \mathrm{H}), 4.45(\mathrm{dd}, J=9.6,7.2 \mathrm{~Hz}, 1 \mathrm{H}), 4.30(\mathrm{t}, J=$ $8.0 \mathrm{~Hz}, 1 \mathrm{H}), 4.08(\mathrm{~d}, J=9.2 \mathrm{~Hz}, 1 \mathrm{H}), 2.58(\mathrm{~s}, 3 \mathrm{H}) .{ }^{13} \mathrm{C} \mathrm{NMR}\left(100 \mathrm{MHz}, \mathrm{CDCl}_{3}\right) \delta$ 170.6, 169.7, 142.5, 141.4, 130.8, 129.5, 126.3 (q, $J=3.2 \mathrm{~Hz}), 124.7,123.9,119.7$, 118.1, 64.6, 51.9, 37.4, 24.4. ${ }^{19} \mathrm{~F}$ NMR $\left(376 \mathrm{MHz}, \mathrm{CDCl}_{3}\right)-62.36$ (s). IR (thin film): $v_{\max }\left(\mathrm{cm}^{-1}\right)=2917,1707,1656,1615,1519,1479,1461,1433,1393,1359,1328,1311$, 1258, 1226, 1203, 1166, 1110, 1073, 1057, 1013, 979, 939, 915, 844, 817, 752, 736, 707, 672, 641, 592, 570, 535, 504, 438. HRMS (ESI) m/z: $[\mathrm{M}+\mathrm{Na}]^{+}$Calcd for $\mathrm{C}_{19} \mathrm{H}_{15} \mathrm{~N}_{2} \mathrm{O}_{2} \mathrm{~F}_{3} \mathrm{Na}$ 383.0978; Found 383.0979. 


\subsection{General procedure for formal [3+2] cycloadditive dearomatization of C3-}

\section{substituted indoles}

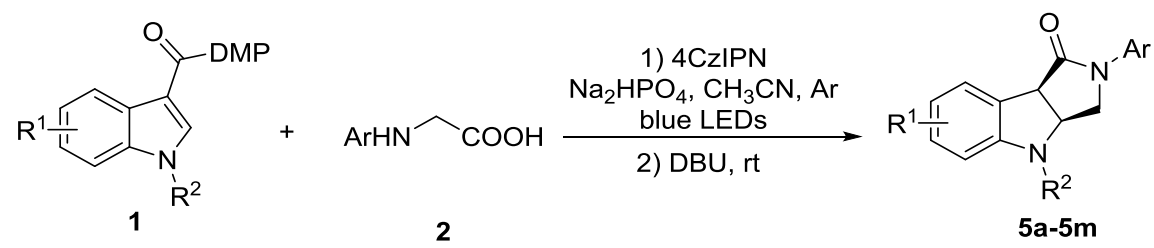

To a sealed tube equipped with a magnetic stir bar were added 1 ( $0.2 \mathrm{mmol}, 1.0$ equiv), 2 (0.4 mmol, 2.0 equiv), 4CzIPN (3.2 mg, $0.004 \mathrm{mmol}, 2.0 \mathrm{~mol} \%), \mathrm{Na}_{2} \mathrm{HPO}_{4}(56.8 \mathrm{mg}$, 0.4 mmol, 2.0 equiv) and $\mathrm{CH}_{3} \mathrm{CN}(2 \mathrm{~mL})$. The reaction mixture was degassed via freeze-pump-thaw for 3 cycles. After the mixture was thoroughly degassed, the tube was sealed and positioned approximately $5 \mathrm{~cm}$ from $24 \mathrm{~W}$ blue LEDs (maximum emission wavelength $=450 \mathrm{~nm}$ ). Then the reaction mixture was stirred at room temperature irradiated by $24 \mathrm{~W}$ blue LEDs. After 1 was consumed completely (monitored by TLC), DBU (152 mg, $1.0 \mathrm{mmol}, 5.0$ equiv) was added and the mixture was stirred at room temperature. When the reaction was complete ( monitored by TLC), the reaction mixture was diluted with $\mathrm{CH}_{2} \mathrm{Cl}_{2}(20 \mathrm{~mL})$ and quenched with water $(20$ $\mathrm{mL}$ ). The layers were separated and the aqueous layer was extracted with $\mathrm{CH}_{2} \mathrm{Cl}_{2}(20$ $\mathrm{mL} \times 2$ ). The combined organic layers were washed with brine, dried over anhydrous $\mathrm{Na}_{2} \mathrm{SO}_{4}$, and concentrated in vacuo. The residue was purified by column chromatography on silica gel to afford the desired products $\mathbf{5 a - 5 m}$. Two rotamers were observed by NMR for all compounds except 5e at room temperature. The analytical data of the products $\mathbf{5 a - 5 m}$ are summarized below.<smiles></smiles>

Reaction time: 11 hours.

5a, $\mathrm{CH}_{2} \mathrm{Cl}_{2} / \mathrm{MeOH}=120: 1$, white solid, m.p. $=172.3-174.3{ }^{\circ} \mathrm{C}, 54.9 \mathrm{mg}$, $94 \%$ yield (0.2 mmol scale). ${ }^{1} \mathrm{H}$ NMR (600 MHz, DMSO- $\left.d_{6}, 110{ }^{\circ} \mathrm{C}\right) \delta 7.93(\mathrm{br}, 1 \mathrm{H}), 7.65(\mathrm{~d}, J=$ 
$8.4 \mathrm{~Hz}, 2 \mathrm{H}), 7.50(\mathrm{~d}, J=7.8 \mathrm{~Hz}, 1 \mathrm{H}), 7.37-7.35(\mathrm{~m}, 2 \mathrm{H}), 7.28(\mathrm{t}, J=7.8 \mathrm{~Hz}, 1 \mathrm{H}), 7.16$ (t, $J=7.2 \mathrm{~Hz}, 1 \mathrm{H}), 7.08(\mathrm{t}, J=7.2 \mathrm{~Hz}, 1 \mathrm{H}), 5.37-5.34(\mathrm{~m}, 1 \mathrm{H}), 4.53(\mathrm{dd}, J=10.8,8.4$ $\mathrm{Hz}, 1 \mathrm{H}), 4.44$ (d, $J=10.2 \mathrm{~Hz}, 1 \mathrm{H}), 3.98(\mathrm{dd}, J=10.8,2.4 \mathrm{~Hz}, 1 \mathrm{H}), 2.33(\mathrm{~s}, 3 \mathrm{H}) .{ }^{13} \mathrm{C}$ NMR (100 MHz, DMSO- $\left.d_{6}\right) \delta 170.6,168.9,142.4,138.6,129.0,128.8,128.6,128.0$, 124.7, 124.5, 123.7, 120.0, 116.3, 55.5, 49.8, 23.9. IR (thin film): $v_{\max }\left(\mathrm{cm}^{-1}\right)=1697$, 1661, 1594, 1477, 1463, 1407, 1390, 1354, 1333, 1290, 1270, 1248, 1227, 1200, 1162 , 1141, 1124, 1093, 1078, 1025, 996, 968, 938, 910, 877, 844, 797, 764, 728, 693, 660, 642, 590, 561, 512, 447, 419. HRMS (ESI) m/z: $[\mathrm{M}+\mathrm{Na}]^{+}$Calcd for $\mathrm{C}_{18} \mathrm{H}_{16} \mathrm{~N}_{2} \mathrm{O}_{2} \mathrm{Na}$ 315.1104; Found 315.1100.

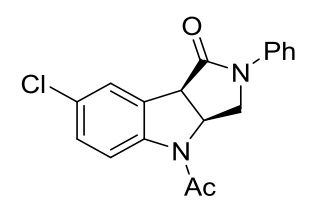

Reaction time: 15 hours.

5b, $\mathrm{CH}_{2} \mathrm{Cl}_{2} / \mathrm{MeOH}=120: 1$, white solid, m.p. = 191.2-192.8 ${ }^{\circ} \mathrm{C}, 59.5 \mathrm{mg}, 91 \%$ yield (0.2 mmol scale). ${ }^{1} \mathrm{H}$ NMR (600 MHz, DMSO- $\left.d_{6}, 110{ }^{\circ} \mathrm{C}\right) \delta 7.94(\mathrm{br}, 1 \mathrm{H}), 7.64(\mathrm{~d}, J=$ $7.2 \mathrm{~Hz}, 2 \mathrm{H}), 7.44(\mathrm{~m}, 1 \mathrm{H}), 7.38-7.35(\mathrm{t}, J=8.4 \mathrm{~Hz}, 2 \mathrm{H}), 7.30(\mathrm{dd}, J=8.4,2.4 \mathrm{~Hz}, 1 \mathrm{H})$, $7.17(\mathrm{t}, J=7.2 \mathrm{~Hz}, 1 \mathrm{H}), 5.38(\mathrm{ddd}, J=11.4,7.8,3.6 \mathrm{~Hz}, 1 \mathrm{H}), 4.54(\mathrm{dd}, J=10.8,8.4$ $\mathrm{Hz}, 1 \mathrm{H}), 4.47$ (d, $J=10.2 \mathrm{~Hz}, 1 \mathrm{H}), 4.03(\mathrm{dd}, J=10.8,3.0 \mathrm{~Hz}, 1 \mathrm{H}), 2.32(\mathrm{~s}, 3 \mathrm{H}) .{ }^{13} \mathrm{C}$ NMR (100 MHz, DMSO- $\left.d_{6}\right) \delta 170.5,169.5,141.8,138.9,130.8,129.2,128.8,127.4$, 125.2, 124.7, 120.5, 117.7, 56.2, 55.8, 50.0, 24.2. IR (thin film): $v_{\max }\left(\mathrm{cm}^{-1}\right)=3430$, 2921, 1684, 1650, 1593, 1491, 1475, 1415, 1385, 1348, 1334, 1293, 1260, 1228, 1198, 1169, 1126, 1108, 1054, 1026, 1005, 947, 904, 888, 864, 829, 768, 730, 690, 660, 651, 614, 594, 535, 514, 482, 444. HRMS (ESI) m/z: $[\mathrm{M}+\mathrm{H}]^{+}$Calcd for $\mathrm{C}_{18} \mathrm{H}_{16} \mathrm{~N}_{2} \mathrm{O}_{2} \mathrm{Cl}$ 327.0895; Found 327.0895.

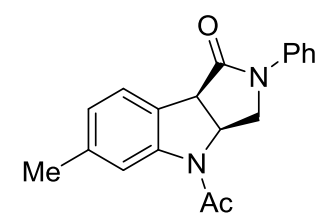

Reaction time: 15 hours.

5c, $\mathrm{CH}_{2} \mathrm{Cl}_{2} / \mathrm{MeOH}=120: 1$, white solid, m.p. = 86.4-89.4 ${ }^{\circ} \mathrm{C} .56 .4 \mathrm{mg}, 92 \%$ yield $(0.2$ mmol scale). ${ }^{1} \mathrm{H}$ NMR (600 MHz, DMSO- $\left.d_{6}, 110{ }^{\circ} \mathrm{C}\right) \delta 7.77$ (br, $\left.1 \mathrm{H}\right), 7.64(\mathrm{~d}, J=7.8$, 
2H), 7.46-7.43 (m, 1H), 7.37-7.34 (m, 3H), $7.15(\mathrm{t}, J=7.8 \mathrm{~Hz}, 1 \mathrm{H}), 6.90(\mathrm{~d}, J=7.2 \mathrm{~Hz}$, 1H), 5.34 (ddd, $J=12.0,8.4,3.6 \mathrm{~Hz}, 1 \mathrm{H}), 4.52$ (dd, $J=10.2,7.8 \mathrm{~Hz}, 1 \mathrm{H}), 3.94$ (dd, $J$ $=10.8,3.6 \mathrm{~Hz}, 1 \mathrm{H}), 4.02(\mathrm{dd}, J=10.8,2.4 \mathrm{~Hz}, 1 \mathrm{H}), 2.33-2.32(\mathrm{~s}, 6 \mathrm{H}) .{ }^{13} \mathrm{C} \mathrm{NMR}(100$ MHz, DMSO-d6) $\delta 170.7,168.7,142.5,138.6,138.1,128.7,125.2,124.6,124.3,124.0$, 119.9, 116.9, 55.7, 55.4, 49.5, 23.9, 21.4. IR (thin film): $v_{\max }\left(\mathrm{cm}^{-1}\right)=2920,1693,1659$, 1595, 1493, 1424, 1402, 1384, 1360, 1335, 1290, 1265, 1226, 1159, 1124, 1028, 1005 , 949, 885, 834, 806, 759, 691, 638, 613, 578, 520, 480, 446. HRMS (ESI) m/z: [M + $\mathrm{Na}]^{+}$Calcd for $\mathrm{C}_{19} \mathrm{H}_{19} \mathrm{~N}_{2} \mathrm{O}_{2} \mathrm{Na}$ 307.1441; Found 307.1436.

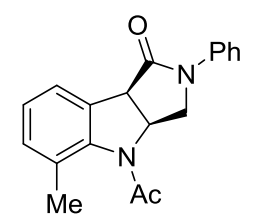

Reaction time: 15 hours.

5d, $\mathrm{CH}_{2} \mathrm{Cl}_{2} / \mathrm{MeOH}=120: 1$, white solid, m.p. $=212.3-215.8{ }^{\circ} \mathrm{C} .60 .3 \mathrm{mg}, 98 \%$ yield (0.2 mmol scale). ${ }^{1} \mathrm{H}$ NMR (600 MHz, DMSO- $\left.d_{6}, 110{ }^{\circ} \mathrm{C}\right) \delta 7.60(\mathrm{~d}, J=9.0 \mathrm{~Hz}, 2 \mathrm{H})$, $7.35(\mathrm{t}, J=7.2 \mathrm{~Hz}, 2 \mathrm{H}), 7.31(\mathrm{~d}, J=7.2 \mathrm{~Hz}, 1 \mathrm{H}), 7.15-7.13(\mathrm{~m}, 2 \mathrm{H}), 7.09$ (t, $J=7.8 \mathrm{~Hz}$, $1 \mathrm{H}), 5.39(\mathrm{td}, J=9.0,5.4 \mathrm{~Hz}, 1 \mathrm{H}), 4.53(\mathrm{dd}, J=10.8,9.0 \mathrm{~Hz}, 1 \mathrm{H}), 4.43(\mathrm{~d}, J=8.4 \mathrm{~Hz}$, $1 \mathrm{H}), 3.73(\mathrm{dd}, J=10.2,5.4 \mathrm{~Hz}, 1 \mathrm{H}), 2.30$ (s, 3H), 2.24 (s, 3H). ${ }^{13} \mathrm{C} \mathrm{NMR}(100 \mathrm{MHz}$, DMSO- $\left.d_{6}\right) \delta 170.7,168.6,140.3,138.6,131.0,120.0,128.9,128.8,125.3,124.5,121.2$, 119.7, 56.2, 54.2, 50.3, 23.6, 20.8. IR (thin film): $v_{\max }\left(\mathrm{cm}^{-1}\right)=2920,2849,1678,1595$, 1499, 1456, 1405, 1376, 1352, 1329, 1286, 1244, 1226, 1127, 1078, 1031, 999, 962, 907, 773, 753, 692, 670, 649, 627, 587, 506, 434. HRMS (ESI) m/z: $[\mathrm{M}+\mathrm{H}]^{+} \mathrm{Calcd}$ for $\mathrm{C}_{19} \mathrm{H}_{19} \mathrm{~N}_{2} \mathrm{O}_{2}$ 307.1441; Found 307.1437.

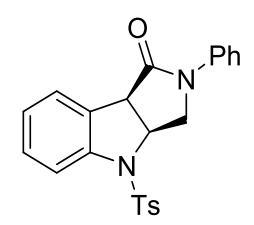

Reaction time: 6 hours.

5e, $\mathrm{CH}_{2} \mathrm{Cl}_{2} / \mathrm{MeOH}=120: 1$, white solid, m.p. = 217.1-219.6 ${ }^{\circ} \mathrm{C} .72 .8 \mathrm{mg}, 90 \%$ yield (0.2 mmol scale). ${ }^{1} \mathrm{H}$ NMR (600 MHz, DMSO- $\left.d_{6}, 110{ }^{\circ} \mathrm{C}\right) \delta 7.74(\mathrm{~d}, J=8.4 \mathrm{~Hz}, 2 \mathrm{H})$, $7.61(\mathrm{~d}, J=9.0 \mathrm{~Hz}, 2 \mathrm{H}), 7.51(\mathrm{~d}, J=8.4 \mathrm{~Hz}, 1 \mathrm{H}), 7.41-7.36(\mathrm{~m}, 5 \mathrm{H}), 7.31(\mathrm{t}, J=7.8$ $\mathrm{Hz}, 1 \mathrm{H}), 7.17(\mathrm{t}, J=7.2 \mathrm{~Hz}, 1 \mathrm{H}), 7.09$ (t, $J=7.2 \mathrm{~Hz}, 1 \mathrm{H}), 5.14-5.11(\mathrm{~m}, 1 \mathrm{H}), 4.51(\mathrm{dd}$, 
$J=11.4,7.2 \mathrm{~Hz}, 1 \mathrm{H}), 4.27(\mathrm{~d}, J=9.6 \mathrm{~Hz}, 1 \mathrm{H}), 4.17(\mathrm{dd}, J=11.4,1.8 \mathrm{~Hz}, 1 \mathrm{H}), 2.37$ (s, $3 \mathrm{H}) .{ }^{13} \mathrm{C}$ NMR (100 MHz, DMSO- $\left.d_{6}\right) \delta 170.0,145.0,141.0,138.5,132.8,130.1,129.1$, $128.9,128.1,127.4,125.7,124.8,124.4,119.9,114.0,58.7,55.4,49.4,21.0$. IR (thin film): $v_{\max }\left(\mathrm{cm}^{-1}\right)=2920,2849,1678,1595,1499,1456,1405,1376,1352,1330,1286$, 1244, 1226, 1127, 1078, 1031, 999, 962, 907, 773, 753, 692, 670, 649, 627, 587, 506, 434. HRMS (ESI) m/z: $[\mathrm{M}+\mathrm{Na}]^{+}$Calcd for $\mathrm{C}_{23} \mathrm{H}_{21} \mathrm{~N}_{2} \mathrm{O}_{3} \mathrm{~S} 405.1267$; Found 405.1263.

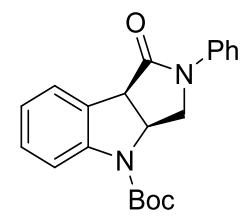

Reaction time: 8 hours.

5f, $\mathrm{CH}_{2} \mathrm{Cl}_{2} / \mathrm{MeOH}=120: 1$, pale yellow solid, m.p. $=133.1-137.5^{\circ} \mathrm{C} .61 .4 \mathrm{mg}, 88 \%$ yield $(0.2 \mathrm{mmol} \mathrm{scale}) .{ }^{1} \mathrm{H} \mathrm{NMR}\left(600 \mathrm{MHz}, \mathrm{DMSO}-d_{6}, 110^{\circ} \mathrm{C}\right) \delta 7.68(\mathrm{~d}, J=7.8 \mathrm{~Hz}$, 1H), $7.63(\mathrm{~d}, J=7.8 \mathrm{~Hz}, 2 \mathrm{H}), 7.47(\mathrm{~d}, J=7.8 \mathrm{~Hz}, 1 \mathrm{H}), 7.36(\mathrm{t}, J=7.8 \mathrm{~Hz}, 2 \mathrm{H}), 7.26$ (t, $J=8.4 \mathrm{~Hz}, 1 \mathrm{H}), 7.15(\mathrm{t}, J=7.8 \mathrm{~Hz}, 1 \mathrm{H}), 7.02(\mathrm{t}, J=7.8 \mathrm{~Hz}, 1 \mathrm{H}), 5.15(\mathrm{ddd}, J=10.2$, 7.8, 3.0 Hz, 1H), 4.42 (dd, $J=10.8,7.2 \mathrm{~Hz}, 1 \mathrm{H}), 4.38$ (d, $J=10.2 \mathrm{~Hz}, 1 \mathrm{H}), 3.97$ (dd, $J$ $=11.4,3.0 \mathrm{~Hz}, 1 \mathrm{H}), 1.59(\mathrm{~s}, 9 \mathrm{H}) .{ }^{13} \mathrm{C} \mathrm{NMR}\left(150 \mathrm{MHz}, \mathrm{DMSO}-d_{6}, 110{ }^{\circ} \mathrm{C}\right) \delta 170.2$, 150.9, 141.3, 138.4, 128.0, 128.0, 127.9, 127.1, 124.3, 124.0, 122.0, 119.8, 113.6, 80.9, 55.1, 54.9, 48.4, 27.6. IR (thin film): $v_{\max }\left(\mathrm{cm}^{-1}\right)=2971,2923,1695,1597,1482,1462$, 1406, 1382, 1335, 1302, 1287, 1267, 1225, 1144, 1047, 996, 943, 903, 848, 827, 756, 710, 692, 656, 602, 507, 452. HRMS (ESI) m/z: $[\mathrm{M}+\mathrm{Na}]^{+}$Calcd for $\mathrm{C}_{21} \mathrm{H}_{23} \mathrm{~N}_{2} \mathrm{O}_{3}$ 351.1703; Found 351.1699.

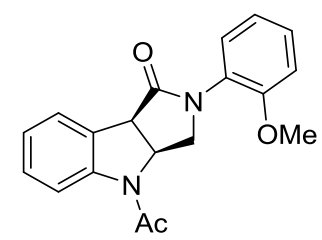

Reaction time: 12 hours.

5g, $\mathrm{CH}_{2} \mathrm{Cl}_{2} / \mathrm{MeOH}=80: 1$, white solid, m.p. $=159.6-160.9^{\circ} \mathrm{C} .55 .2 \mathrm{mg}, 86 \%$ yield $(0.2$ mmol scale). ${ }^{1} \mathrm{H}$ NMR (600 MHz, DMSO- $\left.d_{6}, 110{ }^{\circ} \mathrm{C}\right) \delta 7.95$ (br, $\left.1 \mathrm{H}\right), 7.47$ (d, $J=7.2$ $\mathrm{Hz}, 1 \mathrm{H}), 7.31-7.27$ (m, 2H), $7.18(\mathrm{dd}, J=7.8,1.8 \mathrm{~Hz}, 1 \mathrm{H}), 7.10-7.07(\mathrm{~m}, 2 \mathrm{H}), 6.95$ (td, $J=7.8,1.8 \mathrm{~Hz}, 1 \mathrm{H}), 5.39-5.36(\mathrm{~m}, 1 \mathrm{H}), 4.35-4.32(\mathrm{~m}, 2 \mathrm{H}), 3.77$ (dd, $J=10.2,3.6 \mathrm{~Hz}$, 1H), 3.70 (s, 3H), 2.27 (s, 3H). ${ }^{13} \mathrm{C}$ NMR (100 MHz, DMSO-d6) $\delta$ 170.9, 168.9, 154.7, 
$142.4,129.1,128.7,128.5,128.2,126.2,124.6,123.8,120.6,116.3,112.5,56.7,56.4$, 55.7, 48.3, 23.8. IR (thin film): $v_{\max }\left(\mathrm{cm}^{-1}\right)=2918,2847,1696,1661,1593,1503,1475$, 1458, 1413, 1390, 1357, 1337, 1298, 1275, 1247, 1184, 1132, 1114, 1045, 1023, 940, 818, 755, 707, 637, 595, 562, 515, 470, 441. HRMS (ESI) m/z: $[\mathrm{M}+\mathrm{Na}]^{+}$Calcd for $\mathrm{C}_{19} \mathrm{H}_{18} \mathrm{~N}_{2} \mathrm{O}_{3} \mathrm{Na}$ 345.1210; Found 345.1207.

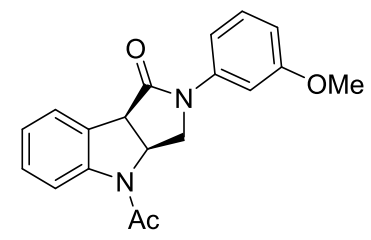

Reaction time: 12 hours.

5h, $\mathrm{CH}_{2} \mathrm{Cl}_{2} / \mathrm{MeOH}=80: 1$, white solid, m.p. $=160.5-164.7^{\circ} \mathrm{C} .57 .4 \mathrm{mg}, 89 \%$ yield $(0.2$ mmol scale). ${ }^{1} \mathrm{H}$ NMR (600 MHz, DMSO- $\left.d_{6}, 110{ }^{\circ} \mathrm{C}\right) \delta 7.93$ (br, $\left.1 \mathrm{H}\right), 7.45$ (d, $J=7.2$ $\mathrm{Hz}, 1 \mathrm{H}), 7.35-7.32(\mathrm{~m}, 1 \mathrm{H}), 7.29-7.25(\mathrm{~m}, 1 \mathrm{H}), 7.20(\mathrm{~d}, J=7.2 \mathrm{~Hz}, 1 \mathrm{H}), 7.08$ (t, $J=$ $7.2 \mathrm{~Hz}, 1 \mathrm{H}), 6.76-6.74(\mathrm{~m}, 1 \mathrm{H}), 5.34$ (ddd, $J=11.4,8.4,3.6 \mathrm{~Hz}, 1 \mathrm{H}), 4.52(\mathrm{dd}, J=10.8$, $8.4 \mathrm{~Hz}, 1 \mathrm{H}), 4.40$ (d, $J=9.6 \mathrm{~Hz}, 1 \mathrm{H}), 3.98(\mathrm{dd}, J=10.8,3.0 \mathrm{~Hz}, 1 \mathrm{H}), 3.77$ (s, 3H), 2.33 $(\mathrm{s}, 3 \mathrm{H}) .{ }^{13} \mathrm{C}$ NMR $\left(100 \mathrm{MHz}, \mathrm{DMSO}-d_{6}\right) \delta 170.7,168.9,159.5,159.4,142.3,139.7$, 129.5, 129.4, 128.6, 128.0, 124.5, 123.7, 116.2, 112.1, 110.1, 105.9, 55.6, 55.4, 55.2, 50.0, 23.9. IR (thin film): $v_{\max }\left(\mathrm{cm}^{-1}\right)=2922,2850,1695,1667,1595,1478,1456,1401$, 1381, 1359, 1334, 1286, 1255, 1139, 1120, 1035, 999, 944, 818, 795, 759, 686, 639, 591, 562, 521, 446. HRMS (ESI) m/z: $[\mathrm{M}+\mathrm{H}]^{+}$Calcd for $\mathrm{C}_{19} \mathrm{H}_{19} \mathrm{~N}_{2} \mathrm{O}_{3} 323.1390$; Found 323.1383 .

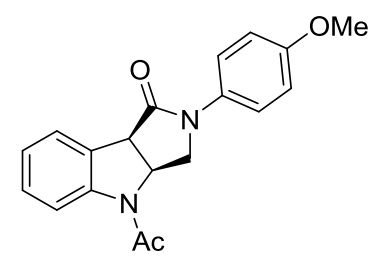

Reaction time: 18 hours.

5i, $\mathrm{CH}_{2} \mathrm{Cl}_{2} / \mathrm{MeOH}=80: 1$, brown solid, m.p. $=184.3-186.7^{\circ} \mathrm{C} .51 .3 \mathrm{mg}, 80 \%$ yield $(0.2$ mmol scale). ${ }^{1} \mathrm{H}$ NMR (600 MHz, DMSO- $\left.d_{6}, 110{ }^{\circ} \mathrm{C}\right) \delta 7.92(\mathrm{br}, 1 \mathrm{H}), 7.53$ (d, $J=9.0$ $\mathrm{Hz}, 2 \mathrm{H}), 7.49$ (d, $J=7.2 \mathrm{~Hz}, 1 \mathrm{H}), 7.28$ (t, $J=7.2 \mathrm{~Hz}, 1 \mathrm{H}), 7.08$ (t, $J=7.2 \mathrm{~Hz}, 1 \mathrm{H}), 6.92$ $(\mathrm{d}, J=9.0 \mathrm{~Hz}, 2 \mathrm{H}), 5.35-5.32(\mathrm{~m}, 1 \mathrm{H}), 4.48(\mathrm{dd}, J=10.2,7.8 \mathrm{~Hz}, 1 \mathrm{H}), 4.40(\mathrm{~d}, J=9.6$ $\mathrm{Hz}, 1 \mathrm{H}), 3.93(\mathrm{dd}, J=10.8,3.6 \mathrm{~Hz}, 1 \mathrm{H}), 3.76(\mathrm{~s}, 3 \mathrm{H}), 2.32(\mathrm{~s}, 3 \mathrm{H}) .{ }^{13} \mathrm{C} \mathrm{NMR}(100$ 
MHz, DMSO- $\left.d_{6}, 110^{\circ} \mathrm{C}\right) \delta 169.5,167.8,156.1,141.5,131.5,127.8,124.1,122.8,121.6$, 115.2, 113.6, 55.3, 55.1, 54.9, 48.6, 23.1. IR (thin film): $v_{\max }\left(\mathrm{cm}^{-1}\right)=2920,2841,1662$, 1598, 1512, 1481, 1462, 1438, 1411, 1388, 1356, 1329, 1289, 1272, 1245, 1179, 1122 , 1069, 1028, 989, 932, 835, 779, 747, 707, 635, 610, 586, 566, 527, 445, 430. HRMS (ESI) m/z: $[\mathrm{M}+\mathrm{H}]^{+}$Calcd for $\mathrm{C}_{19} \mathrm{H}_{19} \mathrm{~N}_{2} \mathrm{O}_{3}$ 323.1390; Found 323.1384.

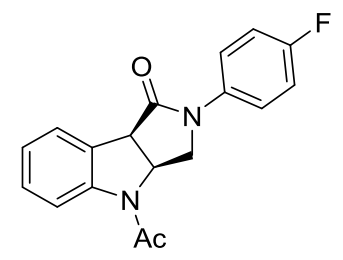

Reaction time: 18 hours.

5j, $\mathrm{CH}_{2} \mathrm{Cl}_{2} / \mathrm{MeOH}=120: 1$, yellow solid, m.p. $=125.7-126.3{ }^{\circ} \mathrm{C} .60 .7 \mathrm{mg}$, 98\% yield (0.2 mmol scale). ${ }^{1} \mathrm{H}$ NMR (600 MHz, DMSO- $\left.d_{6}, 110{ }^{\circ} \mathrm{C}\right) \delta 7.92(\mathrm{br}, 1 \mathrm{H}), 7.69-7.65$ (m, 2H), 7.49 (d, $J=7.8 \mathrm{~Hz}, 1 \mathrm{H}), 7.28$ (t, $J=8.4 \mathrm{~Hz}, 1 \mathrm{H}), 7.17-7.13(\mathrm{~m}, 2 \mathrm{H}), 7.08$ (t, $J=7.2 \mathrm{~Hz}, 1 \mathrm{H}), 5.35(\mathrm{ddd}, J=11.4,7.8,3.6 \mathrm{~Hz}, 1 \mathrm{H}), 4.52(\mathrm{dd}, J=10.8,8.4 \mathrm{~Hz}, 1 \mathrm{H})$, $4.43(\mathrm{~d}, J=9.6 \mathrm{~Hz}, 1 \mathrm{H}), 3.97(\mathrm{dd}, J=10.8,3.6 \mathrm{~Hz}, 1 \mathrm{H}), 2.33$ (s, 3H). ${ }^{13} \mathrm{C} \mathrm{NMR}(100$ MHz, DMSO- $\left.d_{6}\right) \delta 170.6,168.9,158.8\left(\mathrm{C}-\mathrm{F},{ }^{1} J_{\mathrm{C}-\mathrm{F}}=240.4 \mathrm{~Hz}\right), 142.4,135.0,128.6$, $127.9,124.5,123.7,122.1\left(\mathrm{C}-\mathrm{F},{ }^{3} J_{\mathrm{C}-\mathrm{F}}=8.1 \mathrm{~Hz}\right), 116.2,115.4\left(\mathrm{C}-\mathrm{F},{ }^{2} J_{\mathrm{C}-\mathrm{F}}=22.1 \mathrm{~Hz}\right)$, 55.7, 55.4, 49.7, 23.9. ${ }^{19} \mathrm{~F}$ NMR (376 MHz, DMSO- $\left.d_{6}\right) \delta-112.72--112.89(\mathrm{~m})$. IR (thin film): $v_{\max }\left(\mathrm{cm}^{-1}\right)=3056,1680,1652,1592,1513,1480,1461,1439,1414,1391,1358$, 1323, 1309, 1289, 1272, 1249, 1215, 1170, 1122, 1092, 1053, 1026, 994, 937, 852, 826, 742, 730, 635, 605, 578, 546, 528, 513, 446, 432. HRMS (ESI) m/z: $[\mathrm{M}+\mathrm{H}]^{+}$Calcd for $\mathrm{C}_{18} \mathrm{H}_{16} \mathrm{~N}_{2} \mathrm{O}_{2} \mathrm{~F} 311.1190$; Found 311.1189.

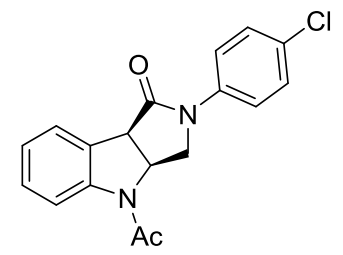

Reaction time: 16 hours.

5k, $\mathrm{CH}_{2} \mathrm{Cl}_{2} / \mathrm{MeOH}=120: 1$, white solid, m.p. = 197.3-199.5 ${ }^{\circ} \mathrm{C} .62 .1 \mathrm{mg}$, 95\% yield (0.2 mmol scale). ${ }^{1} \mathrm{H}$ NMR (600 MHz, DMSO- $\left.d_{6}, 110{ }^{\circ} \mathrm{C}\right) \delta 7.91(\mathrm{br}, 1 \mathrm{H}), 7.70-7.67$ (m, 2H), 7.49 (d, J=7.2 Hz, 1H), 7.39-7.37 (m, 2H), 7.28 (t, $J=7.8 \mathrm{~Hz}, 1 \mathrm{H}), 5.35$ (ddd, $J=11.4,8.4,3.6 \mathrm{~Hz}, 1 \mathrm{H}), 4.52(\mathrm{dd}, J=10.2,7.8 \mathrm{~Hz}, 1 \mathrm{H}), 4.45(\mathrm{~d}, J=10.2 \mathrm{~Hz}, 1 \mathrm{H})$, 
$3.98(\mathrm{dd}, J=10.8,3.6 \mathrm{~Hz}, 1 \mathrm{H}), 2.33(\mathrm{~s}, 3 \mathrm{H}) .{ }^{13} \mathrm{C}$ NMR (100 MHz, DMSO-d $\left.d_{6}\right) \delta 170.9$, 168.8, 142.4, 137.5, 128.6, 128.4, 127.8, 124.5, 123.7, 121.4, 116.2, 55.4, 55.3, 49.8, 23.9. IR (thin film): $v_{\max }\left(\mathrm{cm}^{-1}\right)=2919,2849,1682,1654,1592,1500,1477,1461$, 1432, 1407, 1388, 1357, 1322, 1308, 1278, 1246, 1122, 1091, 1015, 986, 935, 843, 812, 740, 693, 632, 597, 568, 526, 501, 443. HRMS (ESI) m/z: $[\mathrm{M}+\mathrm{H}]^{+}$Calcd for $\mathrm{C}_{18} \mathrm{H}_{16} \mathrm{~N}_{2} \mathrm{O}_{2} \mathrm{Cl} 327.0895$; Found 327.0891 .

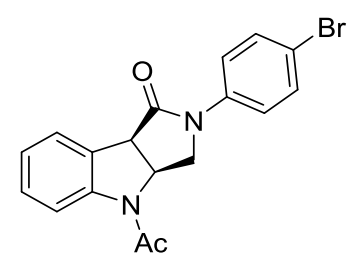

Reaction time: 16 hours.

5l, $\mathrm{CH}_{2} \mathrm{Cl}_{2} / \mathrm{MeOH}=120: 1$, white solid, m.p. $=185.1-186.7^{\circ} \mathrm{C} .71 .3 \mathrm{mg}, 96 \%$ yield $(0.2$ mmol scale). ${ }^{1} \mathrm{H}$ NMR (600 MHz, DMSO- $\left.d_{6}, 110{ }^{\circ} \mathrm{C}\right) \delta 7.91(\mathrm{br}, 1 \mathrm{H}), 7.65-7.63(\mathrm{~m}$, 2H), 7.52-7.48 (m, 3H), $7.28(\mathrm{t}, J=8.4 \mathrm{~Hz}, 1 \mathrm{H}), 7.08(\mathrm{t}, J=7.2 \mathrm{~Hz}, 1 \mathrm{H}), 5.34$ (ddd, $J$ $=11.4,7.8,3.0 \mathrm{~Hz}, 1 \mathrm{H}), 4.52(\mathrm{dd}, J=10.8,8.4 \mathrm{~Hz}, 1 \mathrm{H}), 4.45(\mathrm{~d}, J=9.6 \mathrm{~Hz}, 1 \mathrm{H}), 3.98$ $(\mathrm{dd}, J=10.8,3.0 \mathrm{~Hz}, 1 \mathrm{H}), 2.33$ (s, 3H). ${ }^{13} \mathrm{C}$ NMR (100 MHz, DMSO- $\left.d_{6}\right) \delta 170.8,168.8$, 142.3, 137.9, 131.5, 128.6, 127.8, 124.5, 123.7, 121.7, 116.5, 116.2, 55.3, 49.8, 23.9. IR (thin film): $v_{\max }\left(\mathrm{cm}^{-1}\right)=2920,1683,1656,1589,1476,1429,1386,1357,1307$, 1279, 1122, 1074, 1009, 984, 935, 838, 810, 742, 631, 596, 568, 522, 443. HRMS (ESI) $\mathrm{m} / \mathrm{z}:[\mathrm{M}+\mathrm{H}]^{+}$Calcd for $\mathrm{C}_{18} \mathrm{H}_{16} \mathrm{~N}_{2} \mathrm{O}_{2}{ }^{79} \mathrm{Br} 371.0390$; Found 371.0382.

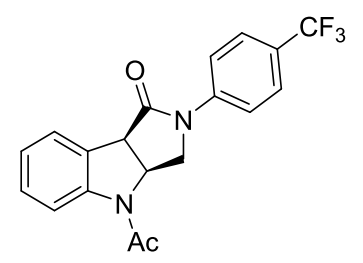

Reaction time: 13 hours.

5m, $\mathrm{CH}_{2} \mathrm{Cl}_{2} / \mathrm{MeOH}=120: 1$, white solid, m.p. $=185.1-186.7{ }^{\circ} \mathrm{C} .64 .9 \mathrm{mg}$, $90 \%$ yield (0.2 mmol scale). ${ }^{1} \mathrm{H}$ NMR (600 MHz, DMSO- $\left.d_{6}, 110{ }^{\circ} \mathrm{C}\right) \delta 7.90(\mathrm{~d}, J=8.4 \mathrm{~Hz}, 3 \mathrm{H})$, $7.69(\mathrm{~d}, J=9.0 \mathrm{~Hz}, 2 \mathrm{H}), 7.50(\mathrm{~d}, J=7.2 \mathrm{~Hz}, 1 \mathrm{H}), 7.28$ (t, $J=7.2 \mathrm{~Hz}, 1 \mathrm{H}), 7.09$ (t, $J=$ $7.2 \mathrm{~Hz}, 1 \mathrm{H}), 5.40-5.36(\mathrm{~m}, 1 \mathrm{H}), 4.59$ (t, $J=10.2 \mathrm{~Hz}, 1 \mathrm{H}), 4.50(\mathrm{~d}, J=9.6 \mathrm{~Hz}, 1 \mathrm{H}), 4.06$ $(\mathrm{dd}, J=10.8,3.0 \mathrm{~Hz}, 1 \mathrm{H}), 2.35$ (s, 3H). ${ }^{13} \mathrm{C}$ NMR (100 MHz, DMSO-d $) \delta 171.5,168.9$, $142.4,142.0,128.7,127.7^{*}, 125.9$ (q, $\left.J=3.0 \mathrm{~Hz}\right), 125.6^{*}, 124.5,124.3^{*}, 123.7,122.9^{*}$, 
119.7, 116.3, 55.3, 50.0, 23.9. ${ }^{19} \mathrm{~F}$ NMR (376 MHz, DMSO- $\left.d_{6}\right) \delta-55.86$ (s). IR (thin film): $v_{\max }\left(\mathrm{cm}^{-1}\right)=2921,1695,1653,1613,1520,1485,1468,1432,1401,1381,1318$, 1295, 1266, 1221, 1199, 1159, 1126, 1100, 1068, 1011, 993, 952, 844, 753, 720, 667, 618, 591, 531, 511, 439, 423. HRMS (ESI) m/z: $[\mathrm{M}+\mathrm{H}]^{+}$Calcd for $\mathrm{C}_{19} \mathrm{H}_{16} \mathrm{~N}_{2} \mathrm{O}_{2} \mathrm{~F}_{3}$ 361.1158; Found 361.1154.

\section{Experimental details for mechanistic studies}

\subsection{Stern-Volmer Quenching Experiments}

Stern-Volmer quenching experiments were conducted on a Hitachi F4600 Fluorescence Spectrophotometer. Stern-Volmer luminescence quenching experiments were run with freshly prepared solutions of $1.0 \times 10^{-5} \mathrm{M} 4 \mathrm{CzIPN}$ and the appropriate amount of quencher in $\mathrm{CH}_{3} \mathrm{CN}$ (for Tables $\mathrm{S} 1$ and $\mathrm{S} 2$ ) at room temperature. After degassing with argon for $5 \mathrm{~min}$, the emission spectra of the samples were collected. The solutions were irradiated at $340 \mathrm{~nm}$ and luminescence was measured at $548 \mathrm{~nm}\left(\mathrm{CH}_{3} \mathrm{CN}\right.$ as the solvent). The data summarized in the tables are the phosphorescence intensity measured three times for each sample. The data illustrated in the graphs are the average of three experiments.

Table S1: Luminescence quenching data for $4 \mathrm{CzIPN}$ and $\mathbf{1 a}$ in $\mathrm{CH}_{3} \mathrm{CN}$.

\begin{tabular}{ccccccc}
\hline Vial & $\mathbf{1}$ & $\mathbf{2}$ & $\mathbf{3}$ & Average & $\mathbf{I} \mathbf{l} / \mathbf{I}$ & {$[\mathbf{1 a}]$} \\
\hline $\mathbf{0}$ & 876.3 & 842.2 & 797.9 & 848.8 & 1.00 & 0 \\
$\mathbf{1}$ & 786.3 & 757.2 & 720.8 & 753.9 & 1.13 & 0.0002 \\
$\mathbf{2}$ & 631.0 & 613.3 & 587.0 & 610.4 & 1.39 & 0.0005 \\
$\mathbf{3}$ & 528.2 & 509.0 & 481.9 & 506.4 & 1.68 & 0.0008 \\
$\mathbf{4}$ & 475.7 & 430.8 & 413.5 & 440 & 1.93 & 0.002 \\
$\mathbf{5}$ & 409.7 & 387.0 & 369.1 & 388.6 & 2.18 & 0.005 \\
$\mathbf{6}$ & 313.2 & 303.4 & 280.0 & 298.9 & 2.84 & 0.008
\end{tabular}


Table S2: Luminescence quenching data for $4 \mathrm{CzIPN}$ and $\mathbf{2 a}$ in $\mathrm{CH}_{3} \mathrm{CN}$.

\begin{tabular}{ccccccc}
\hline Vial & $\mathbf{1}$ & $\mathbf{2}$ & $\mathbf{3}$ & Average & I0/I & [2a] \\
\hline $\mathbf{0}$ & 710.1 & 724.0 & 730.5 & 721.5 & 1.00 & 0 \\
$\mathbf{1}$ & 518.2 & 494.4 & 479.6 & 497.4 & 1.45 & 0.002 \\
$\mathbf{2}$ & 359.4 & 356.3 & 354.1 & 356.6 & 2.02 & 0.004 \\
$\mathbf{3}$ & 306.0 & 297.4 & 301.6 & 301.7 & 2.39 & 0.008 \\
$\mathbf{4}$ & 267.9 & 261.3 & 269.5 & 268.2 & 2.69 & 0.01 \\
$\mathbf{5}$ & 247.8 & 241.2 & 239.9 & 243.0 & 2.97 & 0.012 \\
$\mathbf{6}$ & 206.7 & 205.8 & 206.1 & 206.2 & 3.50 & 0.014 \\
$\mathbf{7}$ & 181.0 & 180.7 & 179.6 & 180.4 & 4.02 & 0.016 \\
\hline
\end{tabular}

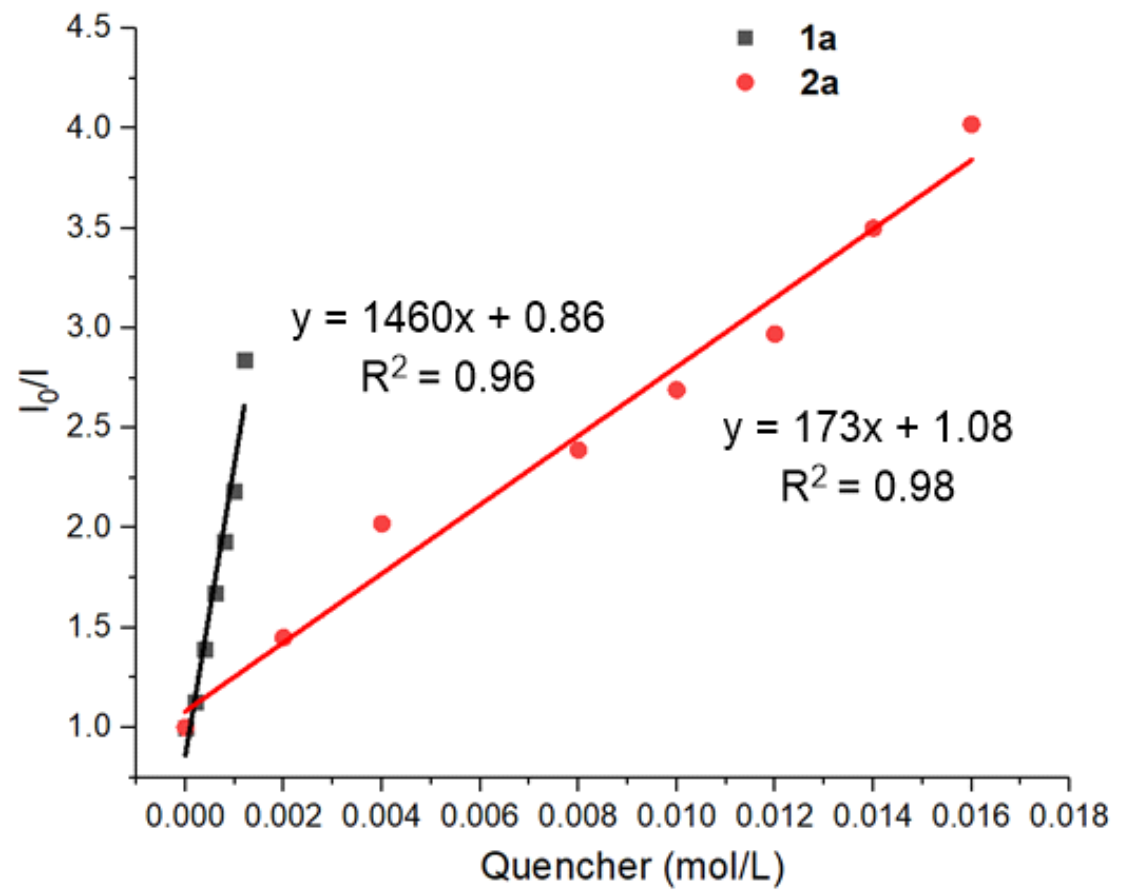

Figure S1: Luminescence quenching of $4 \mathrm{CzIPN}$ with varying concentrations of $\mathbf{1 a}$ (black) and $2 \mathbf{a}$ (red) in $\mathrm{CH}_{3} \mathrm{CN}$

\subsection{Radical trapping experiments}




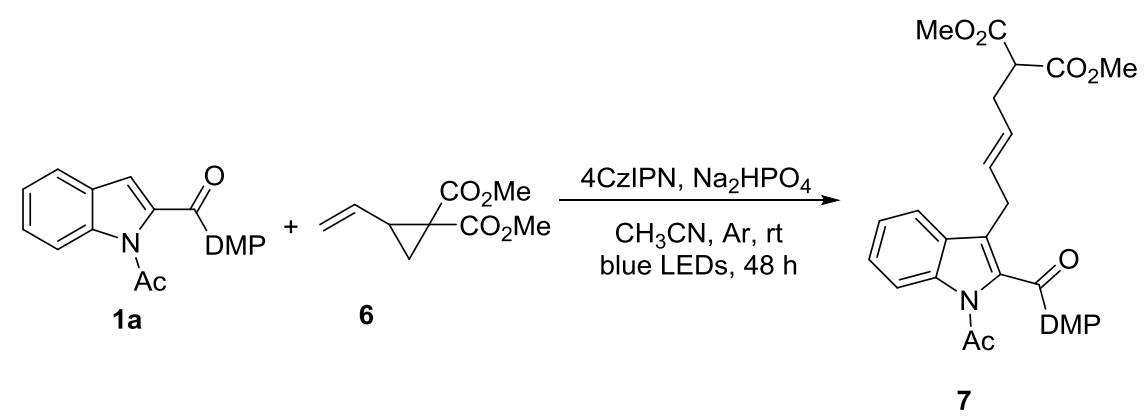

To a sealed tube equipped with a magnetic stir bar were added $1 \mathbf{a}(112.5 \mathrm{mg}, 0.4 \mathrm{mmol}$, 1.0 equiv), 6 (147.4 mg, $0.8 \mathrm{mmol}, 2.0$ equiv), 4CzIPN (6.4 mg, $0.008 \mathrm{mmol}, 2.0 \mathrm{~mol} \%$ ), $\mathrm{Na}_{2} \mathrm{HPO}_{4}\left(113.6 \mathrm{mg}, 0.8 \mathrm{mmol}, 2.0\right.$ equiv) and $\mathrm{CH}_{3} \mathrm{CN}(4 \mathrm{~mL})$. The reaction mixture was degassed via freeze-pump-thaw for 3 cycles. After the mixture was thoroughly degassed, the tube was sealed and positioned approximately $5 \mathrm{~cm}$ from $24 \mathrm{~W}$ blue LEDs (maximum emission wavelength $=450 \mathrm{~nm}$ ). Then the reaction mixture was stirred at room temperature irradiated by $24 \mathrm{~W}$ blue LEDs. After $48 \mathrm{~h}$, the solution was quenched with water and extracted with $\mathrm{CH}_{2} \mathrm{Cl}_{2}(10 \mathrm{~mL} \times 3)$. The combined organic layers were washed with brine, dried over anhydrous $\mathrm{Na}_{2} \mathrm{SO}_{4}$ and concentrated in vacuo. The residue was purified by column chromatograph on silica gel $(\mathrm{PE} / \mathrm{EtOAc}=4 / 1)$ to afford compound 7.

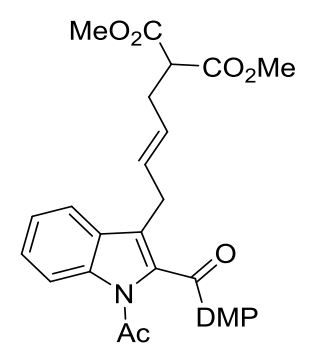

Reaction time: 48 hours.

7, PE/EtOAc $=4: 1$, yellow oil. $39.1 \mathrm{mg}, 21 \%$ yield. ${ }^{1} \mathrm{H} \mathrm{NMR}\left(400 \mathrm{MHz}, \mathrm{CDCl}_{3}\right) \delta$ $7.83(\mathrm{~d}, J=8.4 \mathrm{~Hz}, 1 \mathrm{H}), 7.59$ (d, $J=7.6 \mathrm{~Hz}, 1 \mathrm{H}), 7.42(\mathrm{t}, J=7.2 \mathrm{~Hz}, 1 \mathrm{H}), 7.29$ (t, $J=$ $7.2 \mathrm{~Hz}, 1 \mathrm{H}), 6.01(\mathrm{~s}, 1 \mathrm{H}), 5.69-5.62(\mathrm{~m}, 1 \mathrm{H}), 5.52-5.45(\mathrm{~m}, 1 \mathrm{H}), 3.64(\mathrm{~s}, 6 \mathrm{H}), 3.40(\mathrm{~d}$, $J=6.8 \mathrm{~Hz}, 2 \mathrm{H}), 3.33(\mathrm{t}, J=7.6 \mathrm{~Hz}, 1 \mathrm{H}), 2.66(\mathrm{~s}, 3 \mathrm{H}), 2.59(\mathrm{~s}, 3 \mathrm{H}), 2.55-2.51(\mathrm{~m}, 2 \mathrm{H})$, $2.13(\mathrm{~s}, 3 \mathrm{H}) .{ }^{13} \mathrm{C} \mathrm{NMR}\left(100 \mathrm{MHz}, \mathrm{CDCl}_{3}\right) \delta 169.4,168.6,162.8,153.0,144.6,135.4$, $130.3,129.8,129.3,127.5,126.7,124.9,123.4,121.3,114.5,111.4,52.6,51.7,31.8$, 27.6, 25.8, 14.2, 13.9. IR (thin film): $v_{\max }\left(\mathrm{cm}^{-1}\right)=2953,2929,1733,1701,1584,1480$, 
1435, 1390, 1373, 1339, 1308, 1271, 1224, 1153, 1109, 1027, 962, 911, 806, 764, 730, 648, 619, 604, 575, 433. HRMS (ESI) m/z: $[\mathrm{M}+\mathrm{Na}]^{+}$Calcd for $\mathrm{C}_{25} \mathrm{H}_{27} \mathrm{~N}_{3} \mathrm{O}_{6} \mathrm{Na}$ 488.1792. Found 488.1798. 


\section{X-Ray crystal data}

Single crystals of $\mathrm{C}_{23} \mathrm{H}_{24} \mathrm{~N}_{4} \mathrm{O}_{2}$ [mj20370_0m] were obtained by slow evaporation of the solution of 3a in DCM and PE. A suitable crystal was selected on a 'Bruker D8 Venture' diffractometer. The crystal was kept at $173.0 \mathrm{~K}$ during data collection. Using Olex $2^{[2]}$, the structure was solved with the ShelXT ${ }^{[3]}$ structure solution program using Intrinsic Phasing and refined with the ShelXL ${ }^{[4]}$ refinement package using Least Squares minimisation.

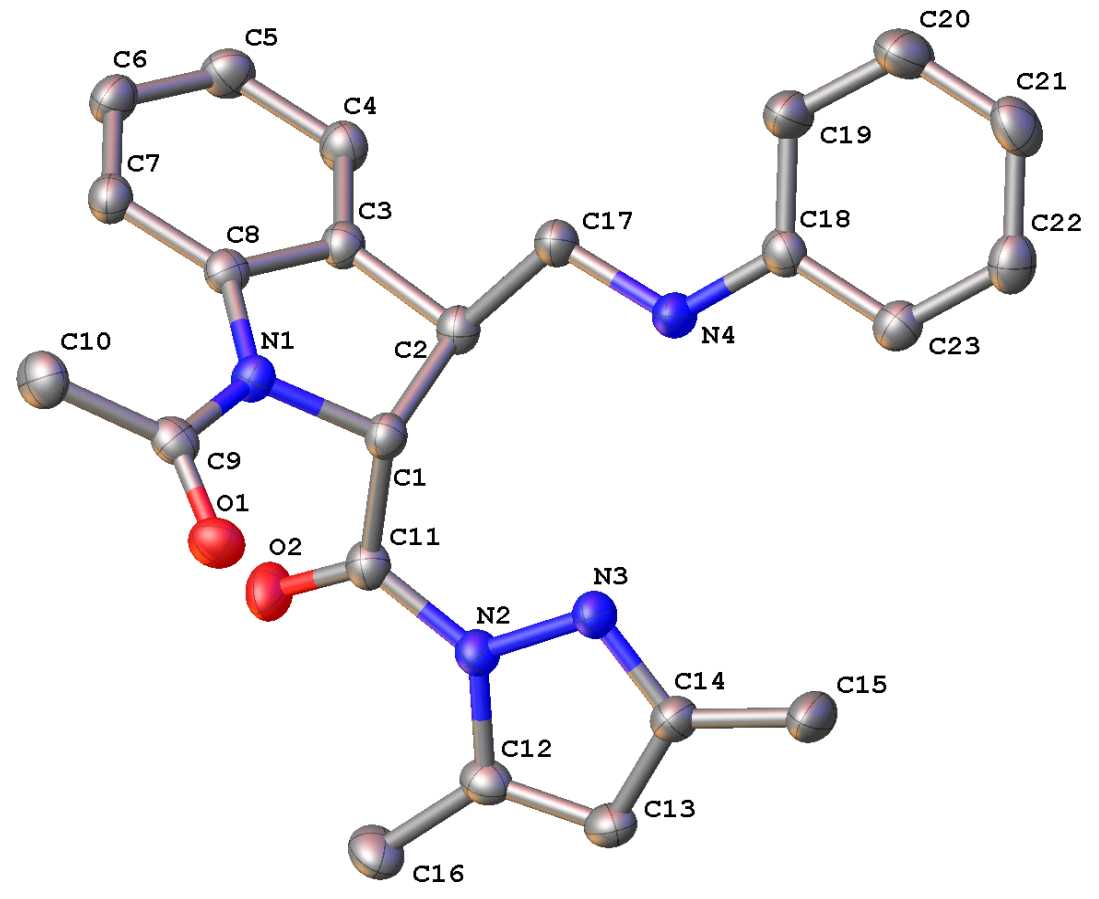

Figure S2: ORTEP drawing of 3a with 30\% probablitity ellipsoids. (CCDC 2036627)

Table 1. Crystal data and structure refinement for mj20370_0m.

Identification code

Empirical formula

Formula weight

Temperature

Wavelength

Crystal system

Space group

Unit cell dimensions

Volume mj20370_0m

$\mathrm{C} 23 \mathrm{H} 24 \mathrm{~N} 4 \mathrm{O} 2$

388.46

$173.0 \mathrm{~K}$

$1.34139 \AA$

Triclinic

P-1

$\mathrm{a}=9.1221(8) \AA$

$\alpha=83.623(3)^{\circ}$.

$\mathrm{b}=9.8731(8) \AA$

$\beta=75.411(3)^{\circ}$.

$\mathrm{c}=11.5340(9) \AA$

$\gamma=83.642(3)^{\circ}$. 
Z

Density (calculated)

Absorption coefficient

$\mathrm{F}(000)$

Crystal size

Theta range for data collection

Index ranges

Reflections collected

Independent reflections

Completeness to theta $=53.594^{\circ}$

Absorption correction

Max. and min. transmission

Refinement method

Data / restraints / parameters

Goodness-of-fit on $\mathrm{F}^{2}$

Final $\mathrm{R}$ indices [I $>2 \operatorname{sigma}(\mathrm{I})]$

$\mathrm{R}$ indices (all data)

Extinction coefficient

Largest diff. peak and hole
2

$1.296 \mathrm{Mg} / \mathrm{m}^{3}$

$0.437 \mathrm{~mm}^{-1}$

412

$0.08 \times 0.06 \times 0.06 \mathrm{~mm}^{3}$

6.135 to $55.022^{\circ}$.

$-11<=\mathrm{h}<=11,-12<=\mathrm{k}<=12,-14<=\mathrm{l}<=14$

27150

$3705[\mathrm{R}(\mathrm{int})=0.0503]$

$97.7 \%$

Semi-empirical from equivalents

0.7508 and 0.5607

Full-matrix least-squares on $\mathrm{F}^{2}$

3705 / 0 / 265

1.068

$\mathrm{R} 1=0.0414, \mathrm{wR} 2=0.1024$

$\mathrm{R} 1=0.0429, \mathrm{wR} 2=0.1036$

$\mathrm{n} / \mathrm{a}$

0.222 and -0.203 e. $\AA^{-3}$ 
Table 2. Atomic coordinates ( $\left.\times 10^{4}\right)$ and equivalent isotropic displacement parameters $\left(\AA^{2} \times 10^{3}\right)$ for mj20370_0m. U(eq) is defined as one third of the trace of the orthogonalized $\mathrm{U}^{\mathrm{ij}}$ tensor.

\begin{tabular}{|c|c|c|c|c|}
\hline & $\mathrm{x}$ & $\mathrm{y}$ & $\mathrm{z}$ & $\mathrm{U}(\mathrm{eq})$ \\
\hline $\mathrm{O}(1)$ & $6168(1)$ & 6122(1) & 4181(1) & $31(1)$ \\
\hline $\mathrm{O}(2)$ & 7672(1) & $7780(1)$ & 5806(1) & $34(1)$ \\
\hline $\mathrm{N}(1)$ & 4807(1) & $7310(1)$ & $5698(1)$ & $23(1)$ \\
\hline $\mathrm{N}(2)$ & $8172(1)$ & $5666(1)$ & 6691(1) & $25(1)$ \\
\hline $\mathrm{N}(3)$ & 7581(1) & $4504(1)$ & $7345(1)$ & $27(1)$ \\
\hline $\mathrm{N}(4)$ & 4164(1) & $4461(1)$ & $8788(1)$ & $30(1)$ \\
\hline $\mathrm{C}(1)$ & $5546(1)$ & $6400(1)$ & $6522(1)$ & $22(1)$ \\
\hline $\mathrm{C}(2)$ & $4650(1)$ & $6768(1)$ & 7797(1) & $24(1)$ \\
\hline $\mathrm{C}(3)$ & $3842(1)$ & $8145(1)$ & $7538(1)$ & $23(1)$ \\
\hline $\mathrm{C}(4)$ & $3055(2)$ & 9069(1) & $8341(1)$ & $28(1)$ \\
\hline$C(5)$ & 2299(2) & 10249(1) & 7924(1) & $30(1)$ \\
\hline$C(6)$ & $2338(2)$ & 10481(1) & 6711(1) & $29(1)$ \\
\hline $\mathrm{C}(7)$ & $3137(1)$ & $9569(1)$ & $5886(1)$ & $26(1)$ \\
\hline $\mathrm{C}(8)$ & $3896(1)$ & $8396(1)$ & 6314(1) & $22(1)$ \\
\hline $\mathrm{C}(9)$ & $5195(1)$ & $7056(1)$ & $4507(1)$ & $24(1)$ \\
\hline$C(10)$ & 4389(2) & $7896(2)$ & $3654(1)$ & $31(1)$ \\
\hline $\mathrm{C}(11)$ & 7203(1) & $6709(1)$ & $6286(1)$ & $25(1)$ \\
\hline $\mathrm{C}(12)$ & 9744(1) & $5589(1)$ & $6505(1)$ & $27(1)$ \\
\hline $\mathrm{C}(13)$ & 10141(2) & $4361(1)$ & $7045(1)$ & $29(1)$ \\
\hline$C(14)$ & $8775(2)$ & $3729(1)$ & 7556(1) & $28(1)$ \\
\hline$C(15)$ & $8594(2)$ & 2361(2) & $8239(2)$ & $41(1)$ \\
\hline$C(16)$ & $10718(2)$ & $6665(2)$ & $5823(1)$ & $36(1)$ \\
\hline $\mathrm{C}(17)$ & $3472(2)$ & $5769(1)$ & $8429(1)$ & $27(1)$ \\
\hline $\mathrm{C}(18)$ & $3325(2)$ & $3480(1)$ & $9519(1)$ & $25(1)$ \\
\hline C(19) & $1750(2)$ & $3696(2)$ & $9968(1)$ & $37(1)$ \\
\hline $\mathrm{C}(20)$ & $950(2)$ & 2713(2) & $10756(2)$ & $43(1)$ \\
\hline $\mathrm{C}(21)$ & $1672(2)$ & $1495(2)$ & 11111(1) & $37(1)$ \\
\hline$C(22)$ & $3225(2)$ & $1258(1)$ & $10652(1)$ & $33(1)$ \\
\hline $\mathrm{C}(23)$ & 4037(2) & 2224(1) & 9861(1) & $27(1)$ \\
\hline
\end{tabular}


Table 3. Bond lengths $[\AA \AA]$ and angles $\left[{ }^{\circ}\right]$ for mj20370_0m.

\begin{tabular}{|c|c|}
\hline $\mathrm{O}(1)-\mathrm{C}(9)$ & $1.2276(16)$ \\
\hline $\mathrm{O}(2)-\mathrm{C}(11)$ & $1.2071(16)$ \\
\hline $\mathrm{N}(1)-\mathrm{C}(1)$ & $1.4704(15)$ \\
\hline $\mathrm{N}(1)-\mathrm{C}(8)$ & $1.4209(15)$ \\
\hline $\mathrm{N}(1)-\mathrm{C}(9)$ & $1.3741(16)$ \\
\hline $\mathrm{N}(2)-\mathrm{N}(3)$ & $1.3831(15)$ \\
\hline $\mathrm{N}(2)-\mathrm{C}(11)$ & $1.4002(16)$ \\
\hline $\mathrm{N}(2)-\mathrm{C}(12)$ & $1.3901(16)$ \\
\hline $\mathrm{N}(3)-\mathrm{C}(14)$ & $1.3186(17)$ \\
\hline $\mathrm{N}(4)-\mathrm{H}(4)$ & 0.8800 \\
\hline $\mathrm{N}(4)-\mathrm{C}(17)$ & $1.4387(16)$ \\
\hline $\mathrm{N}(4)-\mathrm{C}(18)$ & $1.3755(17)$ \\
\hline $\mathrm{C}(1)-\mathrm{H}(1)$ & 1.0000 \\
\hline$C(1)-C(2)$ & $1.5523(17)$ \\
\hline $\mathrm{C}(1)-\mathrm{C}(11)$ & $1.5263(17)$ \\
\hline $\mathrm{C}(2)-\mathrm{H}(2)$ & 1.0000 \\
\hline $\mathrm{C}(2)-\mathrm{C}(3)$ & $1.5094(17)$ \\
\hline $\mathrm{C}(2)-\mathrm{C}(17)$ & $1.5293(17)$ \\
\hline$C(3)-C(4)$ & $1.3834(18)$ \\
\hline $\mathrm{C}(3)-\mathrm{C}(8)$ & $1.3956(17)$ \\
\hline $\mathrm{C}(4)-\mathrm{H}(4 \mathrm{~A})$ & 0.9500 \\
\hline$C(4)-C(5)$ & $1.3892(19)$ \\
\hline $\mathrm{C}(5)-\mathrm{H}(5)$ & 0.9500 \\
\hline$C(5)-C(6)$ & $1.385(2)$ \\
\hline $\mathrm{C}(6)-\mathrm{H}(6)$ & 0.9500 \\
\hline$C(6)-C(7)$ & $1.3934(18)$ \\
\hline $\mathrm{C}(7)-\mathrm{H}(7)$ & 0.9500 \\
\hline$C(7)-C(8)$ & $1.3907(17)$ \\
\hline $\mathrm{C}(9)-\mathrm{C}(10)$ & $1.4979(18)$ \\
\hline $\mathrm{C}(10)-\mathrm{H}(10 \mathrm{~A})$ & 0.9800 \\
\hline $\mathrm{C}(10)-\mathrm{H}(10 \mathrm{~B})$ & 0.9800 \\
\hline $\mathrm{C}(10)-\mathrm{H}(10 \mathrm{C})$ & 0.9800 \\
\hline $\mathrm{C}(12)-\mathrm{C}(13)$ & $1.3556(19)$ \\
\hline$C(12)-C(16)$ & $1.4865(18)$ \\
\hline $\mathrm{C}(13)-\mathrm{H}(13)$ & 0.9500 \\
\hline
\end{tabular}




\begin{tabular}{|c|c|}
\hline $\mathrm{C}(13)-\mathrm{C}(14)$ & $1.4181(19)$ \\
\hline $\mathrm{C}(14)-\mathrm{C}(15)$ & $1.4900(19)$ \\
\hline $\mathrm{C}(15)-\mathrm{H}(15 \mathrm{~A})$ & 0.9800 \\
\hline $\mathrm{C}(15)-\mathrm{H}(15 \mathrm{~B})$ & 0.9800 \\
\hline $\mathrm{C}(15)-\mathrm{H}(15 \mathrm{C})$ & 0.9800 \\
\hline $\mathrm{C}(16)-\mathrm{H}(16 \mathrm{~A})$ & 0.9800 \\
\hline $\mathrm{C}(16)-\mathrm{H}(16 \mathrm{~B})$ & 0.9800 \\
\hline $\mathrm{C}(16)-\mathrm{H}(16 \mathrm{C})$ & 0.9800 \\
\hline $\mathrm{C}(17)-\mathrm{H}(17 \mathrm{~A})$ & 0.9900 \\
\hline $\mathrm{C}(17)-\mathrm{H}(17 \mathrm{~B})$ & 0.9900 \\
\hline $\mathrm{C}(18)-\mathrm{C}(19)$ & $1.3998(19)$ \\
\hline $\mathrm{C}(18)-\mathrm{C}(23)$ & $1.3988(18)$ \\
\hline $\mathrm{C}(19)-\mathrm{H}(19)$ & 0.9500 \\
\hline$C(19)-C(20)$ & $1.389(2)$ \\
\hline $\mathrm{C}(20)-\mathrm{H}(20)$ & 0.9500 \\
\hline$C(20)-C(21)$ & $1.378(2)$ \\
\hline $\mathrm{C}(21)-\mathrm{H}(21)$ & 0.9500 \\
\hline$C(21)-C(22)$ & $1.385(2)$ \\
\hline $\mathrm{C}(22)-\mathrm{H}(22)$ & 0.9500 \\
\hline$C(22)-C(23)$ & $1.3842(19)$ \\
\hline $\mathrm{C}(23)-\mathrm{H}(23)$ & 0.9500 \\
\hline $\mathrm{C}(8)-\mathrm{N}(1)-\mathrm{C}(1)$ & $110.21(10)$ \\
\hline $\mathrm{C}(9)-\mathrm{N}(1)-\mathrm{C}(1)$ & $117.50(10)$ \\
\hline $\mathrm{C}(9)-\mathrm{N}(1)-\mathrm{C}(8)$ & $132.00(11)$ \\
\hline $\mathrm{N}(3)-\mathrm{N}(2)-\mathrm{C}(11)$ & $119.92(10)$ \\
\hline $\mathrm{N}(3)-\mathrm{N}(2)-\mathrm{C}(12)$ & $111.42(10)$ \\
\hline $\mathrm{C}(12)-\mathrm{N}(2)-\mathrm{C}(11)$ & $128.64(11)$ \\
\hline $\mathrm{C}(14)-\mathrm{N}(3)-\mathrm{N}(2)$ & $104.65(10)$ \\
\hline $\mathrm{C}(17)-\mathrm{N}(4)-\mathrm{H}(4)$ & 119.0 \\
\hline $\mathrm{C}(18)-\mathrm{N}(4)-\mathrm{H}(4)$ & 119.0 \\
\hline $\mathrm{C}(18)-\mathrm{N}(4)-\mathrm{C}(17)$ & $121.98(11)$ \\
\hline $\mathrm{N}(1)-\mathrm{C}(1)-\mathrm{H}(1)$ & 110.8 \\
\hline $\mathrm{N}(1)-\mathrm{C}(1)-\mathrm{C}(2)$ & $104.56(9)$ \\
\hline $\mathrm{N}(1)-\mathrm{C}(1)-\mathrm{C}(11)$ & $109.08(10)$ \\
\hline $\mathrm{C}(2)-\mathrm{C}(1)-\mathrm{H}(1)$ & 110.8 \\
\hline $\mathrm{C}(11)-\mathrm{C}(1)-\mathrm{H}(1)$ & 110.8 \\
\hline
\end{tabular}




\begin{tabular}{|c|c|}
\hline $\mathrm{C}(11)-\mathrm{C}(1)-\mathrm{C}(2)$ & $110.71(10)$ \\
\hline $\mathrm{C}(1)-\mathrm{C}(2)-\mathrm{H}(2)$ & 110.4 \\
\hline$C(3)-C(2)-C(1)$ & $102.40(10)$ \\
\hline $\mathrm{C}(3)-\mathrm{C}(2)-\mathrm{H}(2)$ & 110.4 \\
\hline $\mathrm{C}(3)-\mathrm{C}(2)-\mathrm{C}(17)$ & $109.21(10)$ \\
\hline $\mathrm{C}(17)-\mathrm{C}(2)-\mathrm{C}(1)$ & $113.83(10)$ \\
\hline $\mathrm{C}(17)-\mathrm{C}(2)-\mathrm{H}(2)$ & 110.4 \\
\hline$C(4)-C(3)-C(2)$ & $128.59(11)$ \\
\hline$C(4)-C(3)-C(8)$ & $120.59(11)$ \\
\hline$C(8)-C(3)-C(2)$ & $110.73(11)$ \\
\hline $\mathrm{C}(3)-\mathrm{C}(4)-\mathrm{H}(4 \mathrm{~A})$ & 120.2 \\
\hline$C(3)-C(4)-C(5)$ & $119.53(12)$ \\
\hline $\mathrm{C}(5)-\mathrm{C}(4)-\mathrm{H}(4 \mathrm{~A})$ & 120.2 \\
\hline $\mathrm{C}(4)-\mathrm{C}(5)-\mathrm{H}(5)$ & 120.2 \\
\hline$C(6)-C(5)-C(4)$ & $119.54(12)$ \\
\hline $\mathrm{C}(6)-\mathrm{C}(5)-\mathrm{H}(5)$ & 120.2 \\
\hline $\mathrm{C}(5)-\mathrm{C}(6)-\mathrm{H}(6)$ & 119.1 \\
\hline$C(5)-C(6)-C(7)$ & $121.82(12)$ \\
\hline $\mathrm{C}(7)-\mathrm{C}(6)-\mathrm{H}(6)$ & 119.1 \\
\hline $\mathrm{C}(6)-\mathrm{C}(7)-\mathrm{H}(7)$ & 121.0 \\
\hline $\mathrm{C}(8)-\mathrm{C}(7)-\mathrm{C}(6)$ & $118.07(12)$ \\
\hline $\mathrm{C}(8)-\mathrm{C}(7)-\mathrm{H}(7)$ & 121.0 \\
\hline $\mathrm{C}(3)-\mathrm{C}(8)-\mathrm{N}(1)$ & $108.81(10)$ \\
\hline $\mathrm{C}(7)-\mathrm{C}(8)-\mathrm{N}(1)$ & $130.75(11)$ \\
\hline$C(7)-C(8)-C(3)$ & $120.44(11)$ \\
\hline $\mathrm{O}(1)-\mathrm{C}(9)-\mathrm{N}(1)$ & $118.75(12)$ \\
\hline $\mathrm{O}(1)-\mathrm{C}(9)-\mathrm{C}(10)$ & $122.05(12)$ \\
\hline $\mathrm{N}(1)-\mathrm{C}(9)-\mathrm{C}(10)$ & $119.17(11)$ \\
\hline $\mathrm{C}(9)-\mathrm{C}(10)-\mathrm{H}(10 \mathrm{~A})$ & 109.5 \\
\hline $\mathrm{C}(9)-\mathrm{C}(10)-\mathrm{H}(10 \mathrm{~B})$ & 109.5 \\
\hline $\mathrm{C}(9)-\mathrm{C}(10)-\mathrm{H}(10 \mathrm{C})$ & 109.5 \\
\hline $\mathrm{H}(10 \mathrm{~A})-\mathrm{C}(10)-\mathrm{H}(10 \mathrm{~B})$ & 109.5 \\
\hline $\mathrm{H}(10 \mathrm{~A})-\mathrm{C}(10)-\mathrm{H}(10 \mathrm{C})$ & 109.5 \\
\hline $\mathrm{H}(10 \mathrm{~B})-\mathrm{C}(10)-\mathrm{H}(10 \mathrm{C})$ & 109.5 \\
\hline $\mathrm{O}(2)-\mathrm{C}(11)-\mathrm{N}(2)$ & $120.98(12)$ \\
\hline $\mathrm{O}(2)-\mathrm{C}(11)-\mathrm{C}(1)$ & $123.63(11)$ \\
\hline $\mathrm{N}(2)-\mathrm{C}(11)-\mathrm{C}(1)$ & $115.37(10)$ \\
\hline
\end{tabular}




\begin{tabular}{|c|c|}
\hline $\mathrm{N}(2)-\mathrm{C}(12)-\mathrm{C}(16)$ & $124.60(12)$ \\
\hline $\mathrm{C}(13)-\mathrm{C}(12)-\mathrm{N}(2)$ & $105.76(11)$ \\
\hline$C(13)-C(12)-C(16)$ & $129.63(12)$ \\
\hline $\mathrm{C}(12)-\mathrm{C}(13)-\mathrm{H}(13)$ & 126.7 \\
\hline$C(12)-C(13)-C(14)$ & $106.61(11)$ \\
\hline $\mathrm{C}(14)-\mathrm{C}(13)-\mathrm{H}(13)$ & 126.7 \\
\hline $\mathrm{N}(3)-\mathrm{C}(14)-\mathrm{C}(13)$ & $111.56(12)$ \\
\hline $\mathrm{N}(3)-\mathrm{C}(14)-\mathrm{C}(15)$ & $120.60(12)$ \\
\hline$C(13)-C(14)-C(15)$ & $127.83(12)$ \\
\hline $\mathrm{C}(14)-\mathrm{C}(15)-\mathrm{H}(15 \mathrm{~A})$ & 109.5 \\
\hline $\mathrm{C}(14)-\mathrm{C}(15)-\mathrm{H}(15 \mathrm{~B})$ & 109.5 \\
\hline $\mathrm{C}(14)-\mathrm{C}(15)-\mathrm{H}(15 \mathrm{C})$ & 109.5 \\
\hline $\mathrm{H}(15 \mathrm{~A})-\mathrm{C}(15)-\mathrm{H}(15 \mathrm{~B})$ & 109.5 \\
\hline $\mathrm{H}(15 \mathrm{~A})-\mathrm{C}(15)-\mathrm{H}(15 \mathrm{C})$ & 109.5 \\
\hline $\mathrm{H}(15 \mathrm{~B})-\mathrm{C}(15)-\mathrm{H}(15 \mathrm{C})$ & 109.5 \\
\hline $\mathrm{C}(12)-\mathrm{C}(16)-\mathrm{H}(16 \mathrm{~A})$ & 109.5 \\
\hline $\mathrm{C}(12)-\mathrm{C}(16)-\mathrm{H}(16 \mathrm{~B})$ & 109.5 \\
\hline $\mathrm{C}(12)-\mathrm{C}(16)-\mathrm{H}(16 \mathrm{C})$ & 109.5 \\
\hline $\mathrm{H}(16 \mathrm{~A})-\mathrm{C}(16)-\mathrm{H}(16 \mathrm{~B})$ & 109.5 \\
\hline $\mathrm{H}(16 \mathrm{~A})-\mathrm{C}(16)-\mathrm{H}(16 \mathrm{C})$ & 109.5 \\
\hline $\mathrm{H}(16 \mathrm{~B})-\mathrm{C}(16)-\mathrm{H}(16 \mathrm{C})$ & 109.5 \\
\hline $\mathrm{N}(4)-\mathrm{C}(17)-\mathrm{C}(2)$ & $112.33(10)$ \\
\hline N(4)-C(17)-H(17A) & 109.1 \\
\hline $\mathrm{N}(4)-\mathrm{C}(17)-\mathrm{H}(17 \mathrm{~B})$ & 109.1 \\
\hline $\mathrm{C}(2)-\mathrm{C}(17)-\mathrm{H}(17 \mathrm{~A})$ & 109.1 \\
\hline $\mathrm{C}(2)-\mathrm{C}(17)-\mathrm{H}(17 \mathrm{~B})$ & 109.1 \\
\hline $\mathrm{H}(17 \mathrm{~A})-\mathrm{C}(17)-\mathrm{H}(17 \mathrm{~B})$ & 107.9 \\
\hline $\mathrm{N}(4)-\mathrm{C}(18)-\mathrm{C}(19)$ & $122.13(12)$ \\
\hline $\mathrm{N}(4)-\mathrm{C}(18)-\mathrm{C}(23)$ & $120.46(12)$ \\
\hline $\mathrm{C}(23)-\mathrm{C}(18)-\mathrm{C}(19)$ & $117.40(12)$ \\
\hline C(18)-C(19)-H(19) & 119.7 \\
\hline$C(20)-C(19)-C(18)$ & $120.65(13)$ \\
\hline $\mathrm{C}(20)-\mathrm{C}(19)-\mathrm{H}(19)$ & 119.7 \\
\hline $\mathrm{C}(19)-\mathrm{C}(20)-\mathrm{H}(20)$ & 119.3 \\
\hline$C(21)-C(20)-C(19)$ & $121.34(14)$ \\
\hline $\mathrm{C}(21)-\mathrm{C}(20)-\mathrm{H}(20)$ & 119.3 \\
\hline $\mathrm{C}(20)-\mathrm{C}(21)-\mathrm{H}(21)$ & 120.7 \\
\hline
\end{tabular}




\begin{tabular}{ll}
$\mathrm{C}(20)-\mathrm{C}(21)-\mathrm{C}(22)$ & $118.51(13)$ \\
$\mathrm{C}(22)-\mathrm{C}(21)-\mathrm{H}(21)$ & 120.7 \\
$\mathrm{C}(21)-\mathrm{C}(22)-\mathrm{H}(22)$ & 119.6 \\
$\mathrm{C}(23)-\mathrm{C}(22)-\mathrm{C}(21)$ & $120.84(13)$ \\
$\mathrm{C}(23)-\mathrm{C}(22)-\mathrm{H}(22)$ & 119.6 \\
$\mathrm{C}(18)-\mathrm{C}(23)-\mathrm{H}(23)$ & 119.4 \\
$\mathrm{C}(22)-\mathrm{C}(23)-\mathrm{C}(18)$ & $121.21(13)$ \\
$\mathrm{C}(22)-\mathrm{C}(23)-\mathrm{H}(23)$ & 119.4 \\
\hline
\end{tabular}

Symmetry transformations used to generate equivalent atoms: 
Table 4. Anisotropic displacement parameters $\left(\AA^{2} \times 10^{3}\right)$ for mj20370_0m. The anisotropic displacement factor exponent takes the form: $-2 \pi^{2}\left[h^{2} a^{* 2} U^{11}+\ldots+2 h k a^{*} b^{*} U^{12}\right]$

\begin{tabular}{|c|c|c|c|c|c|c|}
\hline & $\mathrm{U}^{11}$ & $\mathrm{U}^{22}$ & $\mathrm{U}^{33}$ & $\mathrm{U}^{23}$ & $\mathrm{U}^{13}$ & $\mathrm{U}^{12}$ \\
\hline $\mathrm{O}(1)$ & $30(1)$ & $30(1)$ & $30(1)$ & $-7(1)$ & $-3(1)$ & $-2(1)$ \\
\hline $\mathrm{O}(2)$ & $32(1)$ & $28(1)$ & $42(1)$ & 11(1) & $-12(1)$ & $-10(1)$ \\
\hline $\mathrm{N}(1)$ & $24(1)$ & 21(1) & $23(1)$ & $-2(1)$ & $-7(1)$ & $-1(1)$ \\
\hline $\mathrm{N}(2)$ & $22(1)$ & $23(1)$ & $29(1)$ & $3(1)$ & $-6(1)$ & $-5(1)$ \\
\hline $\mathrm{N}(3)$ & 24(1) & 24(1) & $31(1)$ & $5(1)$ & $-4(1)$ & $-3(1)$ \\
\hline $\mathrm{N}(4)$ & $24(1)$ & $26(1)$ & $34(1)$ & $8(1)$ & $-1(1)$ & $1(1)$ \\
\hline $\mathrm{C}(1)$ & $24(1)$ & $19(1)$ & 24(1) & $0(1)$ & $-7(1)$ & $-2(1)$ \\
\hline $\mathrm{C}(2)$ & $26(1)$ & $22(1)$ & $23(1)$ & $-1(1)$ & $-7(1)$ & $-2(1)$ \\
\hline $\mathrm{C}(3)$ & 24(1) & 21(1) & $25(1)$ & $0(1)$ & $-7(1)$ & $-3(1)$ \\
\hline $\mathrm{C}(4)$ & $32(1)$ & $26(1)$ & $25(1)$ & $-2(1)$ & $-6(1)$ & $-1(1)$ \\
\hline $\mathrm{C}(5)$ & $31(1)$ & $26(1)$ & $32(1)$ & $-6(1)$ & $-4(1)$ & 1(1) \\
\hline $\mathrm{C}(6)$ & $28(1)$ & $22(1)$ & $34(1)$ & 2(1) & $-7(1)$ & $1(1)$ \\
\hline $\mathrm{C}(7)$ & $27(1)$ & $24(1)$ & $26(1)$ & $2(1)$ & $-7(1)$ & $-3(1)$ \\
\hline $\mathrm{C}(8)$ & $22(1)$ & 19(1) & $25(1)$ & $-1(1)$ & $-5(1)$ & $-5(1)$ \\
\hline $\mathrm{C}(9)$ & $24(1)$ & $25(1)$ & $25(1)$ & $-2(1)$ & $-5(1)$ & $-9(1)$ \\
\hline $\mathrm{C}(10)$ & $36(1)$ & $35(1)$ & $24(1)$ & $-3(1)$ & $-10(1)$ & $-4(1)$ \\
\hline $\mathrm{C}(11)$ & $26(1)$ & $23(1)$ & $24(1)$ & $0(1)$ & $-6(1)$ & $-4(1)$ \\
\hline $\mathrm{C}(12)$ & $22(1)$ & $32(1)$ & $27(1)$ & $-2(1)$ & $-5(1)$ & $-6(1)$ \\
\hline $\mathrm{C}(13)$ & 21(1) & $34(1)$ & $32(1)$ & $-1(1)$ & $-6(1)$ & $0(1)$ \\
\hline $\mathrm{C}(14)$ & 24(1) & $26(1)$ & $31(1)$ & $2(1)$ & $-5(1)$ & $1(1)$ \\
\hline $\mathrm{C}(15)$ & $29(1)$ & $34(1)$ & $52(1)$ & $14(1)$ & $-5(1)$ & 2(1) \\
\hline $\mathrm{C}(16)$ & $27(1)$ & $42(1)$ & $40(1)$ & $6(1)$ & $-7(1)$ & $-12(1)$ \\
\hline $\mathrm{C}(17)$ & $26(1)$ & $24(1)$ & $27(1)$ & $3(1)$ & $-3(1)$ & $0(1)$ \\
\hline$C(18)$ & $27(1)$ & $24(1)$ & $22(1)$ & $-1(1)$ & $-4(1)$ & $-2(1)$ \\
\hline $\mathrm{C}(19)$ & $29(1)$ & $32(1)$ & $43(1)$ & $9(1)$ & $0(1)$ & 2(1) \\
\hline $\mathrm{C}(20)$ & $30(1)$ & $40(1)$ & $48(1)$ & $5(1)$ & $5(1)$ & $-2(1)$ \\
\hline $\mathrm{C}(21)$ & $42(1)$ & $30(1)$ & $35(1)$ & $5(1)$ & $0(1)$ & $-10(1)$ \\
\hline $\mathrm{C}(22)$ & $42(1)$ & $22(1)$ & $34(1)$ & $1(1)$ & $-11(1)$ & $-3(1)$ \\
\hline $\mathrm{C}(23)$ & $28(1)$ & $25(1)$ & $29(1)$ & $-2(1)$ & $-8(1)$ & $-1(1)$ \\
\hline
\end{tabular}


Table 5. Hydrogen coordinates ( $\left.\times 10^{4}\right)$ and isotropic displacement parameters $\left(\AA^{2} \times 10^{3}\right)$ for mj20370_0m.

\begin{tabular}{|c|c|c|c|c|}
\hline & $\mathrm{x}$ & $\mathrm{y}$ & $\mathrm{z}$ & $\mathrm{U}(\mathrm{eq})$ \\
\hline $\mathrm{H}(4)$ & 5153 & 4284 & 8533 & 36 \\
\hline $\mathrm{H}(1)$ & 5475 & 5419 & 6419 & 27 \\
\hline $\mathrm{H}(2)$ & 5366 & 6852 & 8309 & 28 \\
\hline $\mathrm{H}(4 \mathrm{~A})$ & 3031 & 8898 & 9173 & 33 \\
\hline $\mathrm{H}(5)$ & 1758 & 10892 & 8467 & 36 \\
\hline $\mathrm{H}(6)$ & 1807 & 11283 & 6433 & 35 \\
\hline $\mathrm{H}(7)$ & 3162 & 9745 & 5054 & 31 \\
\hline $\mathrm{H}(10 \mathrm{~A})$ & 4698 & 8831 & 3534 & 47 \\
\hline $\mathrm{H}(10 \mathrm{~B})$ & 3288 & 7915 & 3990 & 47 \\
\hline $\mathrm{H}(10 \mathrm{C})$ & 4654 & 7493 & 2882 & 47 \\
\hline $\mathrm{H}(13)$ & 11140 & 3995 & 7075 & 35 \\
\hline $\mathrm{H}(15 \mathrm{~A})$ & 7511 & 2219 & 8518 & 62 \\
\hline $\mathrm{H}(15 \mathrm{~B})$ & 9041 & 2315 & 8932 & 62 \\
\hline $\mathrm{H}(15 \mathrm{C})$ & 9112 & 1648 & 7715 & 62 \\
\hline $\mathrm{H}(16 \mathrm{~A})$ & 10518 & 6876 & 5024 & 55 \\
\hline $\mathrm{H}(16 \mathrm{~B})$ & 11791 & 6337 & 5739 & 55 \\
\hline $\mathrm{H}(16 \mathrm{C})$ & 10489 & 7492 & 6256 & 55 \\
\hline $\mathrm{H}(17 \mathrm{~A})$ & 2803 & 6168 & 9148 & 33 \\
\hline $\mathrm{H}(17 \mathrm{~B})$ & 2831 & 5638 & 7880 & 33 \\
\hline $\mathrm{H}(19)$ & 1224 & 4524 & 9731 & 45 \\
\hline $\mathrm{H}(20)$ & -117 & 2884 & 11057 & 51 \\
\hline $\mathrm{H}(21)$ & 1117 & 833 & 11658 & 45 \\
\hline $\mathrm{H}(22)$ & 3739 & 420 & 10884 & 39 \\
\hline $\mathrm{H}(23)$ & 5098 & 2032 & 9544 & 33 \\
\hline
\end{tabular}


Single crystals of $\mathrm{C}_{23} \mathrm{H}_{24} \mathrm{~N}_{4} \mathrm{O}_{2}$ [d8v191006] were obtained by slow evaporation of the solution of 4a in DCM and PE. A suitable crystal was selected and on a 'Bruker D8 Venture' diffractometer. The crystal was kept at $193.0 \mathrm{~K}$ during data collection. Using Olex $2^{[2]}$, the structure was solved with the ShelXT ${ }^{[3]}$ structure solution program using Intrinsic Phasing and refined with the ShelXL ${ }^{[4]}$ refinement package using Least Squares minimisation.
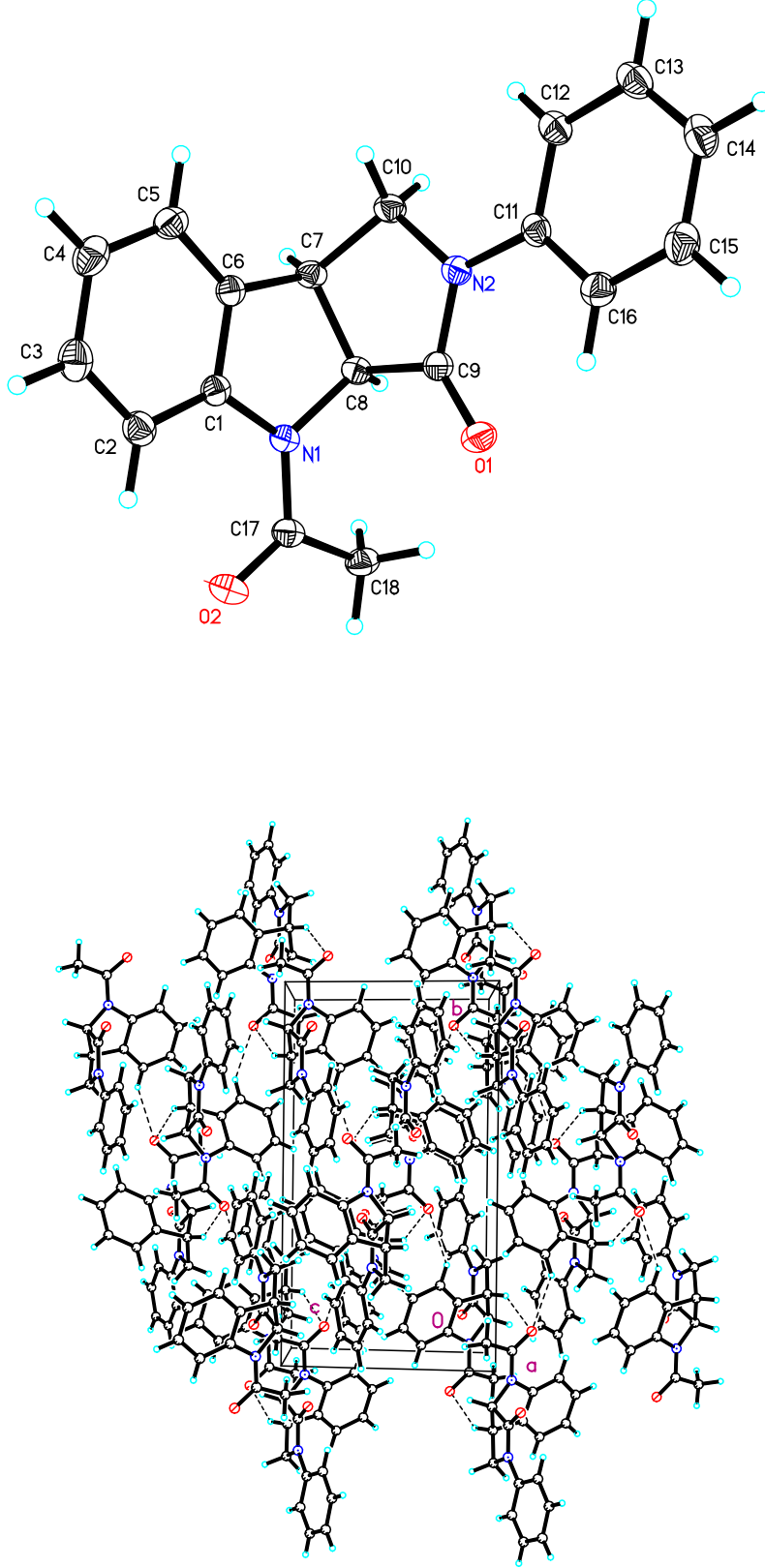

Figure S3: ORTEP drawing of $\mathbf{4 a}$ with $30 \%$ probablitity ellipsoids. (CCDC 2036626) 
Table 1. Crystal data and structure refinement for d8v191006.

Identification code

Empirical formula

Formula weight

Temperature

Wavelength

Crystal system

Space group

Unit cell dimensions

Volume

$\mathrm{Z}$

Density (calculated)

Absorption coefficient

$\mathrm{F}(000)$

Crystal size

Theta range for data collection

Index ranges

Reflections collected

Independent reflections

Completeness to theta $=25.242^{\circ}$

Absorption correction

Max. and min. transmission

Refinement method

Data / restraints / parameters

Goodness-of-fit on $\mathrm{F}^{2}$

Final R indices [I $>2 \operatorname{sigma}(\mathrm{I})]$

$\mathrm{R}$ indices (all data)

Extinction coefficient

Largest diff. peak and hole d8v191006

C18 H16 N2 O2

292.33

193(2) K

$0.71073 \AA$

Monoclinic

P 21/c

$$
\begin{array}{ll}
\mathrm{a}=10.0715(8) \AA & \alpha=90^{\circ} . \\
\mathrm{b}=16.0131(14) \AA & \beta=100.686(3)^{\circ} . \\
\mathrm{c}=9.0150(8) \AA & \gamma=90^{\circ} .
\end{array}
$$

1428.7(2) $\AA^{3}$

4

$1.359 \mathrm{Mg} / \mathrm{m}^{3}$

$0.090 \mathrm{~mm}^{-1}$

616

$0.180 \times 0.140 \times 0.100 \mathrm{~mm}^{3}$

2.544 to $25.997^{\circ}$.

$-11<=\mathrm{h}<=12,-16<=\mathrm{k}<=19,-11<=\mathrm{l}<=11$

13607

$2797[\mathrm{R}(\mathrm{int})=0.0376]$

$99.7 \%$

Semi-empirical from equivalents

0.7456 and 0.6784

Full-matrix least-squares on $\mathrm{F}^{2}$

$2797 / 0 / 212$

1.017

$\mathrm{R} 1=0.0396, \mathrm{wR} 2=0.0893$

$\mathrm{R} 1=0.0543, \mathrm{wR} 2=0.0992$

$0.021(3)$

0.185 and -0.177 e. $\AA^{-3}$ 
Table 2. Atomic coordinates ( $\left.\times 10^{4}\right)$ and equivalent isotropic displacement parameters $\left(\AA^{2} \times 10^{3}\right)$ for $\mathrm{d} 8 \mathrm{v} 191006$. $U(\mathrm{eq})$ is defined as one third of the trace of the orthogonalized $\mathrm{U}^{\mathrm{ij}}$ tensor.

\begin{tabular}{|c|c|c|c|c|}
\hline & $\mathrm{x}$ & $\mathrm{y}$ & z & $\mathrm{U}(\mathrm{eq})$ \\
\hline $\mathrm{O}(1)$ & $1160(1)$ & $3890(1)$ & $6242(1)$ & $42(1)$ \\
\hline $\mathrm{O}(2)$ & $3809(1)$ & 5893(1) & 6902(1) & $43(1)$ \\
\hline $\mathrm{N}(1)$ & $3767(1)$ & $4583(1)$ & $5975(1)$ & $30(1)$ \\
\hline $\mathrm{N}(2)$ & 2189(1) & $2650(1)$ & $5747(1)$ & $30(1)$ \\
\hline $\mathrm{C}(1)$ & $4828(2)$ & $4232(1)$ & $7052(2)$ & $30(1)$ \\
\hline$C(2)$ & $5544(2)$ & $4578(1)$ & $8370(2)$ & $37(1)$ \\
\hline $\mathrm{C}(3)$ & $6528(2)$ & $4085(1)$ & $9246(2)$ & $43(1)$ \\
\hline $\mathrm{C}(4)$ & $6796(2)$ & $3282(1)$ & 8818(2) & $44(1)$ \\
\hline$C(5)$ & $6077(2)$ & 2942(1) & $7490(2)$ & $37(1)$ \\
\hline$C(6)$ & 5091(1) & $3420(1)$ & $6608(2)$ & $30(1)$ \\
\hline $\mathrm{C}(7)$ & $4184(2)$ & $3207(1)$ & $5125(2)$ & $31(1)$ \\
\hline $\mathrm{C}(8)$ & $3123(2)$ & $3898(1)$ & $5021(2)$ & $30(1)$ \\
\hline C(9) & 2015(2) & $3499(1)$ & $5739(2)$ & $31(1)$ \\
\hline$C(10)$ & $3383(2)$ & $2399(1)$ & $5129(2)$ & $34(1)$ \\
\hline $\mathrm{C}(11)$ & $1329(2)$ & 2044(1) & $6221(2)$ & $31(1)$ \\
\hline$C(12)$ & $1521(2)$ & 1204(1) & $5915(2)$ & $36(1)$ \\
\hline$C(13)$ & $735(2)$ & $595(1)$ & $6417(2)$ & $40(1)$ \\
\hline$C(14)$ & $-267(2)$ & $810(1)$ & $7200(2)$ & $43(1)$ \\
\hline$C(15)$ & $-474(2)$ & $1643(1)$ & $7478(2)$ & $44(1)$ \\
\hline$C(16)$ & $314(2)$ & $2262(1)$ & 7013(2) & $38(1)$ \\
\hline$C(17)$ & $3298(2)$ & $5386(1)$ & $5944(2)$ & $33(1)$ \\
\hline $\mathrm{C}(18)$ & 2174(2) & 5614(1) & $4676(2)$ & $38(1)$ \\
\hline
\end{tabular}


Table 3. Bond lengths $[\AA ̊]$ and angles $\left[{ }^{\circ}\right]$ for d8v191006.

\begin{tabular}{|c|c|}
\hline $\mathrm{O}(1)-\mathrm{C}(9)$ & $1.2173(18)$ \\
\hline $\mathrm{O}(2)-\mathrm{C}(17)$ & $1.2269(18)$ \\
\hline N(1)-C(17) & $1.3681(19)$ \\
\hline $\mathrm{N}(1)-\mathrm{C}(1)$ & $1.4197(19)$ \\
\hline $\mathrm{N}(1)-\mathrm{C}(8)$ & $1.4690(18)$ \\
\hline $\mathrm{N}(2)-\mathrm{C}(9)$ & $1.3706(19)$ \\
\hline $\mathrm{N}(2)-\mathrm{C}(11)$ & $1.4185(19)$ \\
\hline $\mathrm{N}(2)-\mathrm{C}(10)$ & $1.4722(19)$ \\
\hline$C(1)-C(2)$ & $1.386(2)$ \\
\hline$C(1)-C(6)$ & $1.399(2)$ \\
\hline $\mathrm{C}(2)-\mathrm{C}(3)$ & $1.392(2)$ \\
\hline $\mathrm{C}(2)-\mathrm{H}(2)$ & 0.9500 \\
\hline$C(3)-C(4)$ & $1.383(2)$ \\
\hline $\mathrm{C}(3)-\mathrm{H}(3)$ & 0.9500 \\
\hline$C(4)-C(5)$ & $1.391(2)$ \\
\hline $\mathrm{C}(4)-\mathrm{H}(4)$ & 0.9500 \\
\hline$C(5)-C(6)$ & $1.383(2)$ \\
\hline $\mathrm{C}(5)-\mathrm{H}(5)$ & 0.9500 \\
\hline$C(6)-C(7)$ & $1.512(2)$ \\
\hline $\mathrm{C}(7)-\mathrm{C}(10)$ & $1.525(2)$ \\
\hline$C(7)-C(8)$ & $1.528(2)$ \\
\hline $\mathrm{C}(7)-\mathrm{H}(7)$ & 1.0000 \\
\hline $\mathrm{C}(8)-\mathrm{C}(9)$ & $1.529(2)$ \\
\hline $\mathrm{C}(8)-\mathrm{H}(8)$ & 1.0000 \\
\hline $\mathrm{C}(10)-\mathrm{H}(10 \mathrm{~A})$ & 0.9900 \\
\hline $\mathrm{C}(10)-\mathrm{H}(10 \mathrm{~B})$ & 0.9900 \\
\hline $\mathrm{C}(11)-\mathrm{C}(12)$ & $1.394(2)$ \\
\hline$C(11)-C(16)$ & $1.395(2)$ \\
\hline $\mathrm{C}(12)-\mathrm{C}(13)$ & $1.384(2)$ \\
\hline $\mathrm{C}(12)-\mathrm{H}(12)$ & 0.9500 \\
\hline $\mathrm{C}(13)-\mathrm{C}(14)$ & $1.378(2)$ \\
\hline $\mathrm{C}(13)-\mathrm{H}(13)$ & 0.9500 \\
\hline C(14)-C(15) & $1.380(2)$ \\
\hline C(14)-H(14) & 0.9500 \\
\hline$C(15)-C(16)$ & $1.383(2)$ \\
\hline
\end{tabular}




\begin{tabular}{|c|c|}
\hline $\mathrm{C}(15)-\mathrm{H}(15)$ & 0.9500 \\
\hline $\mathrm{C}(16)-\mathrm{H}(16)$ & 0.9500 \\
\hline $\mathrm{C}(17)-\mathrm{C}(18)$ & $1.498(2)$ \\
\hline $\mathrm{C}(18)-\mathrm{H}(18 \mathrm{~A})$ & $0.99(2)$ \\
\hline $\mathrm{C}(18)-\mathrm{H}(18 \mathrm{~B})$ & $0.98(2)$ \\
\hline C(18)-H(18C) & $0.999(19)$ \\
\hline $\mathrm{C}(17)-\mathrm{N}(1)-\mathrm{C}(1)$ & $126.83(13)$ \\
\hline $\mathrm{C}(17)-\mathrm{N}(1)-\mathrm{C}(8)$ & $125.24(12)$ \\
\hline $\mathrm{C}(1)-\mathrm{N}(1)-\mathrm{C}(8)$ & $107.38(11)$ \\
\hline $\mathrm{C}(9)-\mathrm{N}(2)-\mathrm{C}(11)$ & $126.55(13)$ \\
\hline$C(9)-N(2)-C(10)$ & $112.43(12)$ \\
\hline $\mathrm{C}(11)-\mathrm{N}(2)-\mathrm{C}(10)$ & $120.97(12)$ \\
\hline$C(2)-C(1)-C(6)$ & 121.34(14) \\
\hline $\mathrm{C}(2)-\mathrm{C}(1)-\mathrm{N}(1)$ & $129.32(14)$ \\
\hline $\mathrm{C}(6)-\mathrm{C}(1)-\mathrm{N}(1)$ & 109.34(13) \\
\hline $\mathrm{C}(1)-\mathrm{C}(2)-\mathrm{C}(3)$ & $117.53(15)$ \\
\hline $\mathrm{C}(1)-\mathrm{C}(2)-\mathrm{H}(2)$ & 121.2 \\
\hline $\mathrm{C}(3)-\mathrm{C}(2)-\mathrm{H}(2)$ & 121.2 \\
\hline $\mathrm{C}(4)-\mathrm{C}(3)-\mathrm{C}(2)$ & $121.59(16)$ \\
\hline $\mathrm{C}(4)-\mathrm{C}(3)-\mathrm{H}(3)$ & 119.2 \\
\hline $\mathrm{C}(2)-\mathrm{C}(3)-\mathrm{H}(3)$ & 119.2 \\
\hline$C(3)-C(4)-C(5)$ & $120.48(16)$ \\
\hline $\mathrm{C}(3)-\mathrm{C}(4)-\mathrm{H}(4)$ & 119.8 \\
\hline $\mathrm{C}(5)-\mathrm{C}(4)-\mathrm{H}(4)$ & 119.8 \\
\hline$C(6)-C(5)-C(4)$ & $118.77(15)$ \\
\hline $\mathrm{C}(6)-\mathrm{C}(5)-\mathrm{H}(5)$ & 120.6 \\
\hline $\mathrm{C}(4)-\mathrm{C}(5)-\mathrm{H}(5)$ & 120.6 \\
\hline$C(5)-C(6)-C(1)$ & $120.29(14)$ \\
\hline$C(5)-C(6)-C(7)$ & $129.41(14)$ \\
\hline$C(1)-C(6)-C(7)$ & $110.30(12)$ \\
\hline$C(6)-C(7)-C(10)$ & 115.01(12) \\
\hline$C(6)-C(7)-C(8)$ & 101.07(11) \\
\hline$C(10)-C(7)-C(8)$ & $104.47(12)$ \\
\hline $\mathrm{C}(6)-\mathrm{C}(7)-\mathrm{H}(7)$ & 111.8 \\
\hline $\mathrm{C}(10)-\mathrm{C}(7)-\mathrm{H}(7)$ & 111.8 \\
\hline $\mathrm{C}(8)-\mathrm{C}(7)-\mathrm{H}(7)$ & 111.8 \\
\hline
\end{tabular}




\begin{tabular}{|c|c|}
\hline $\mathrm{N}(1)-\mathrm{C}(8)-\mathrm{C}(7)$ & $106.07(12)$ \\
\hline $\mathrm{N}(1)-\mathrm{C}(8)-\mathrm{C}(9)$ & $109.93(12)$ \\
\hline $\mathrm{C}(7)-\mathrm{C}(8)-\mathrm{C}(9)$ & $103.24(12)$ \\
\hline $\mathrm{N}(1)-\mathrm{C}(8)-\mathrm{H}(8)$ & 112.3 \\
\hline $\mathrm{C}(7)-\mathrm{C}(8)-\mathrm{H}(8)$ & 112.3 \\
\hline $\mathrm{C}(9)-\mathrm{C}(8)-\mathrm{H}(8)$ & 112.3 \\
\hline $\mathrm{O}(1)-\mathrm{C}(9)-\mathrm{N}(2)$ & $127.29(14)$ \\
\hline $\mathrm{O}(1)-\mathrm{C}(9)-\mathrm{C}(8)$ & $124.44(14)$ \\
\hline $\mathrm{N}(2)-\mathrm{C}(9)-\mathrm{C}(8)$ & $108.25(12)$ \\
\hline $\mathrm{N}(2)-\mathrm{C}(10)-\mathrm{C}(7)$ & $103.80(11)$ \\
\hline $\mathrm{N}(2)-\mathrm{C}(10)-\mathrm{H}(10 \mathrm{~A})$ & 111.0 \\
\hline $\mathrm{C}(7)-\mathrm{C}(10)-\mathrm{H}(10 \mathrm{~A})$ & 111.0 \\
\hline $\mathrm{N}(2)-\mathrm{C}(10)-\mathrm{H}(10 \mathrm{~B})$ & 111.0 \\
\hline $\mathrm{C}(7)-\mathrm{C}(10)-\mathrm{H}(10 \mathrm{~B})$ & 111.0 \\
\hline $\mathrm{H}(10 \mathrm{~A})-\mathrm{C}(10)-\mathrm{H}(10 \mathrm{~B})$ & 109.0 \\
\hline$C(12)-C(11)-C(16)$ & $118.95(14)$ \\
\hline $\mathrm{C}(12)-\mathrm{C}(11)-\mathrm{N}(2)$ & $119.06(14)$ \\
\hline $\mathrm{C}(16)-\mathrm{C}(11)-\mathrm{N}(2)$ & $121.97(14)$ \\
\hline$C(13)-C(12)-C(11)$ & $120.40(16)$ \\
\hline $\mathrm{C}(13)-\mathrm{C}(12)-\mathrm{H}(12)$ & 119.8 \\
\hline $\mathrm{C}(11)-\mathrm{C}(12)-\mathrm{H}(12)$ & 119.8 \\
\hline $\mathrm{C}(14)-\mathrm{C}(13)-\mathrm{C}(12)$ & $120.68(16)$ \\
\hline $\mathrm{C}(14)-\mathrm{C}(13)-\mathrm{H}(13)$ & 119.7 \\
\hline $\mathrm{C}(12)-\mathrm{C}(13)-\mathrm{H}(13)$ & 119.7 \\
\hline$C(13)-C(14)-C(15)$ & $118.90(16)$ \\
\hline $\mathrm{C}(13)-\mathrm{C}(14)-\mathrm{H}(14)$ & 120.6 \\
\hline $\mathrm{C}(15)-\mathrm{C}(14)-\mathrm{H}(14)$ & 120.6 \\
\hline $\mathrm{C}(14)-\mathrm{C}(15)-\mathrm{C}(16)$ & $121.56(16)$ \\
\hline $\mathrm{C}(14)-\mathrm{C}(15)-\mathrm{H}(15)$ & 119.2 \\
\hline $\mathrm{C}(16)-\mathrm{C}(15)-\mathrm{H}(15)$ & 119.2 \\
\hline$C(15)-C(16)-C(11)$ & $119.48(15)$ \\
\hline $\mathrm{C}(15)-\mathrm{C}(16)-\mathrm{H}(16)$ & 120.3 \\
\hline $\mathrm{C}(11)-\mathrm{C}(16)-\mathrm{H}(16)$ & 120.3 \\
\hline $\mathrm{O}(2)-\mathrm{C}(17)-\mathrm{N}(1)$ & $120.66(14)$ \\
\hline $\mathrm{O}(2)-\mathrm{C}(17)-\mathrm{C}(18)$ & $122.33(14)$ \\
\hline $\mathrm{N}(1)-\mathrm{C}(17)-\mathrm{C}(18)$ & $116.99(14)$ \\
\hline $\mathrm{C}(17)-\mathrm{C}(18)-\mathrm{H}(18 \mathrm{~A})$ & $113.6(11)$ \\
\hline
\end{tabular}




$\begin{array}{ll}\mathrm{C}(17)-\mathrm{C}(18)-\mathrm{H}(18 \mathrm{~B}) & 109.2(12) \\ \mathrm{H}(18 \mathrm{~A})-\mathrm{C}(18)-\mathrm{H}(18 \mathrm{~B}) & 110.4(16) \\ \mathrm{C}(17)-\mathrm{C}(18)-\mathrm{H}(18 \mathrm{C}) & 112.4(10) \\ \mathrm{H}(18 \mathrm{~A})-\mathrm{C}(18)-\mathrm{H}(18 \mathrm{C}) & 103.6(16) \\ \mathrm{H}(18 \mathrm{~B})-\mathrm{C}(18)-\mathrm{H}(18 \mathrm{C}) & 107.6(15)\end{array}$

Symmetry transformations used to generate equivalent atoms: 
Table 4. Anisotropic displacement parameters $\left(\AA^{2} \times 10^{3}\right)$ for $\mathrm{d} 8 \mathrm{v} 191006$. The anisotropic displacement factor exponent takes the form: $-2 \pi^{2}\left[h^{2} a^{* 2} U^{11}+\ldots+2 h k a^{*} b^{*} U^{12}\right]$

\begin{tabular}{|c|c|c|c|c|c|c|}
\hline & $\mathrm{U}^{11}$ & $\mathrm{U}^{22}$ & $\mathrm{U}^{33}$ & $\mathrm{U}^{23}$ & $\mathrm{U}^{13}$ & $\mathrm{U}^{12}$ \\
\hline $\mathrm{O}(1)$ & $37(1)$ & $34(1)$ & $60(1)$ & $-1(1)$ & $19(1)$ & $4(1)$ \\
\hline $\mathrm{O}(2)$ & $55(1)$ & $29(1)$ & $46(1)$ & $-6(1)$ & $11(1)$ & $0(1)$ \\
\hline $\mathrm{N}(1)$ & $32(1)$ & $26(1)$ & $32(1)$ & $-1(1)$ & $5(1)$ & $0(1)$ \\
\hline $\mathrm{N}(2)$ & $30(1)$ & $28(1)$ & $33(1)$ & $-2(1)$ & $8(1)$ & $-1(1)$ \\
\hline$C(1)$ & $29(1)$ & $31(1)$ & $32(1)$ & $2(1)$ & $9(1)$ & $-1(1)$ \\
\hline$C(2)$ & $36(1)$ & $38(1)$ & $36(1)$ & $-5(1)$ & $8(1)$ & $-3(1)$ \\
\hline$C(3)$ & $39(1)$ & $54(1)$ & $34(1)$ & $-2(1)$ & $3(1)$ & $-4(1)$ \\
\hline $\mathrm{C}(4)$ & $37(1)$ & $50(1)$ & $44(1)$ & $12(1)$ & $4(1)$ & $5(1)$ \\
\hline $\mathrm{C}(5)$ & $34(1)$ & $34(1)$ & $44(1)$ & $6(1)$ & $12(1)$ & $2(1)$ \\
\hline$C(6)$ & $28(1)$ & $30(1)$ & $34(1)$ & $2(1)$ & 11(1) & $-2(1)$ \\
\hline$C(7)$ & $34(1)$ & $28(1)$ & $33(1)$ & $-2(1)$ & $12(1)$ & 1(1) \\
\hline C(8) & $34(1)$ & $27(1)$ & $28(1)$ & $-1(1)$ & $6(1)$ & $0(1)$ \\
\hline $\mathrm{C}(9)$ & $31(1)$ & $29(1)$ & $31(1)$ & $-2(1)$ & $3(1)$ & 2(1) \\
\hline$C(10)$ & $36(1)$ & $29(1)$ & $37(1)$ & $-5(1)$ & 11(1) & $1(1)$ \\
\hline $\mathrm{C}(11)$ & $29(1)$ & $32(1)$ & $28(1)$ & $1(1)$ & $0(1)$ & $-3(1)$ \\
\hline $\mathrm{C}(12)$ & $38(1)$ & $34(1)$ & $36(1)$ & $-3(1)$ & $4(1)$ & $-4(1)$ \\
\hline $\mathrm{C}(13)$ & $44(1)$ & $34(1)$ & $38(1)$ & $-2(1)$ & $-2(1)$ & $-7(1)$ \\
\hline$C(14)$ & $39(1)$ & $44(1)$ & $44(1)$ & $9(1)$ & $2(1)$ & $-11(1)$ \\
\hline $\mathrm{C}(15)$ & $36(1)$ & $50(1)$ & $48(1)$ & $9(1)$ & $11(1)$ & $-2(1)$ \\
\hline$C(16)$ & $36(1)$ & $36(1)$ & $42(1)$ & $3(1)$ & $9(1)$ & $1(1)$ \\
\hline $\mathrm{C}(17)$ & $38(1)$ & $27(1)$ & $36(1)$ & $1(1)$ & $16(1)$ & $-2(1)$ \\
\hline $\mathrm{C}(18)$ & $41(1)$ & $31(1)$ & $44(1)$ & $4(1)$ & $10(1)$ & $6(1)$ \\
\hline
\end{tabular}


Table 5. Hydrogen coordinates $\left(\mathrm{x} 10^{4}\right)$ and isotropic displacement parameters $\left(\AA^{2} \mathrm{x} 10^{3}\right)$ for d8v191006.

\begin{tabular}{|c|c|c|c|c|}
\hline & $\mathrm{x}$ & $\mathrm{y}$ & $\mathrm{z}$ & $\mathrm{U}(\mathrm{eq})$ \\
\hline $\mathrm{H}(2)$ & 5371 & 5131 & 8664 & 44 \\
\hline $\mathrm{H}(3)$ & 7027 & 4305 & 10160 & 52 \\
\hline $\mathrm{H}(4)$ & 7476 & 2961 & 9436 & 53 \\
\hline $\mathrm{H}(5)$ & 6260 & 2391 & 7193 & 44 \\
\hline $\mathrm{H}(7)$ & 4681 & 3229 & 4263 & 37 \\
\hline $\mathrm{H}(8)$ & 2783 & 4079 & 3957 & 36 \\
\hline $\mathrm{H}(10 \mathrm{~A})$ & 3117 & 2174 & 4094 & 40 \\
\hline $\mathrm{H}(10 \mathrm{~B})$ & 3917 & 1972 & 5775 & 40 \\
\hline $\mathrm{H}(12)$ & 2197 & 1048 & 5359 & 43 \\
\hline $\mathrm{H}(13)$ & 887 & 24 & 6219 & 48 \\
\hline $\mathrm{H}(14)$ & -806 & 391 & 7543 & 52 \\
\hline $\mathrm{H}(15)$ & -1174 & 1794 & 8002 & 53 \\
\hline $\mathrm{H}(16)$ & 166 & 2831 & 7231 & 45 \\
\hline $\mathrm{H}(18 \mathrm{~A})$ & $1340(20)$ & $5282(12)$ & $4660(20)$ & $63(6)$ \\
\hline $\mathrm{H}(18 \mathrm{~B})$ & 1982(19) & $6210(13)$ & $4730(20)$ & $61(6)$ \\
\hline $\mathrm{H}(18 \mathrm{C})$ & $2408(18)$ & $5503(11)$ & $3660(20)$ & $52(5)$ \\
\hline
\end{tabular}


Table 6. Torsion angles $\left[{ }^{\circ}\right]$ for d8v191006.

\begin{tabular}{|c|c|}
\hline $\mathrm{C}(17)-\mathrm{N}(1)-\mathrm{C}(1)-\mathrm{C}(2)$ & $6.5(2)$ \\
\hline $\mathrm{C}(8)-\mathrm{N}(1)-\mathrm{C}(1)-\mathrm{C}(2)$ & $-165.37(15)$ \\
\hline$C(17)-N(1)-C(1)-C(6)$ & $-174.15(13)$ \\
\hline $\mathrm{C}(8)-\mathrm{N}(1)-\mathrm{C}(1)-\mathrm{C}(6)$ & $14.00(16)$ \\
\hline $\mathrm{C}(6)-\mathrm{C}(1)-\mathrm{C}(2)-\mathrm{C}(3)$ & $-0.6(2)$ \\
\hline $\mathrm{N}(1)-\mathrm{C}(1)-\mathrm{C}(2)-\mathrm{C}(3)$ & $178.68(15)$ \\
\hline$C(1)-C(2)-C(3)-C(4)$ & $0.6(2)$ \\
\hline$C(2)-C(3)-C(4)-C(5)$ & $-0.3(3)$ \\
\hline$C(3)-C(4)-C(5)-C(6)$ & $0.0(2)$ \\
\hline$C(4)-C(5)-C(6)-C(1)$ & $0.1(2)$ \\
\hline$C(4)-C(5)-C(6)-C(7)$ & $179.35(15)$ \\
\hline $\mathrm{C}(2)-\mathrm{C}(1)-\mathrm{C}(6)-\mathrm{C}(5)$ & $0.3(2)$ \\
\hline$N(1)-C(1)-C(6)-C(5)$ & $-179.15(13)$ \\
\hline$C(2)-C(1)-C(6)-C(7)$ & $-179.14(13)$ \\
\hline $\mathrm{N}(1)-\mathrm{C}(1)-\mathrm{C}(6)-\mathrm{C}(7)$ & $1.43(16)$ \\
\hline$C(5)-C(6)-C(7)-C(10)$ & $53.6(2)$ \\
\hline$C(1)-C(6)-C(7)-C(10)$ & $-127.09(13)$ \\
\hline$C(5)-C(6)-C(7)-C(8)$ & $165.41(15)$ \\
\hline$C(1)-C(6)-C(7)-C(8)$ & $-15.24(15)$ \\
\hline $\mathrm{C}(17)-\mathrm{N}(1)-\mathrm{C}(8)-\mathrm{C}(7)$ & $164.42(13)$ \\
\hline $\mathrm{C}(1)-\mathrm{N}(1)-\mathrm{C}(8)-\mathrm{C}(7)$ & $-23.56(15)$ \\
\hline $\mathrm{C}(17)-\mathrm{N}(1)-\mathrm{C}(8)-\mathrm{C}(9)$ & $-84.60(17)$ \\
\hline $\mathrm{C}(1)-\mathrm{N}(1)-\mathrm{C}(8)-\mathrm{C}(9)$ & $87.42(14)$ \\
\hline $\mathrm{C}(6)-\mathrm{C}(7)-\mathrm{C}(8)-\mathrm{N}(1)$ & $22.95(14)$ \\
\hline $\mathrm{C}(10)-\mathrm{C}(7)-\mathrm{C}(8)-\mathrm{N}(1)$ & $142.64(12)$ \\
\hline$C(6)-C(7)-C(8)-C(9)$ & $-92.66(13)$ \\
\hline $\mathrm{C}(10)-\mathrm{C}(7)-\mathrm{C}(8)-\mathrm{C}(9)$ & $27.03(14)$ \\
\hline $\mathrm{C}(11)-\mathrm{N}(2)-\mathrm{C}(9)-\mathrm{O}(1)$ & $6.3(2)$ \\
\hline $\mathrm{C}(10)-\mathrm{N}(2)-\mathrm{C}(9)-\mathrm{O}(1)$ & $-176.19(15)$ \\
\hline $\mathrm{C}(11)-\mathrm{N}(2)-\mathrm{C}(9)-\mathrm{C}(8)$ & $-175.52(13)$ \\
\hline $\mathrm{C}(10)-\mathrm{N}(2)-\mathrm{C}(9)-\mathrm{C}(8)$ & $2.02(16)$ \\
\hline $\mathrm{N}(1)-\mathrm{C}(8)-\mathrm{C}(9)-\mathrm{O}(1)$ & $47.0(2)$ \\
\hline $\mathrm{C}(7)-\mathrm{C}(8)-\mathrm{C}(9)-\mathrm{O}(1)$ & $159.80(15)$ \\
\hline $\mathrm{N}(1)-\mathrm{C}(8)-\mathrm{C}(9)-\mathrm{N}(2)$ & $-131.30(12)$ \\
\hline $\mathrm{C}(7)-\mathrm{C}(8)-\mathrm{C}(9)-\mathrm{N}(2)$ & $-18.48(15)$ \\
\hline
\end{tabular}




$\begin{array}{lc}\mathrm{C}(9)-\mathrm{N}(2)-\mathrm{C}(10)-\mathrm{C}(7) & 15.38(16) \\ \mathrm{C}(11)-\mathrm{N}(2)-\mathrm{C}(10)-\mathrm{C}(7) & -166.93(12) \\ \mathrm{C}(6)-\mathrm{C}(7)-\mathrm{C}(10)-\mathrm{N}(2) & 83.79(15) \\ \mathrm{C}(8)-\mathrm{C}(7)-\mathrm{C}(10)-\mathrm{N}(2) & -26.04(15) \\ \mathrm{C}(9)-\mathrm{N}(2)-\mathrm{C}(11)-\mathrm{C}(12) & 169.04(14) \\ \mathrm{C}(10)-\mathrm{N}(2)-\mathrm{C}(11)-\mathrm{C}(12) & -8.3(2) \\ \mathrm{C}(9)-\mathrm{N}(2)-\mathrm{C}(11)-\mathrm{C}(16) & -12.4(2) \\ \mathrm{C}(10)-\mathrm{N}(2)-\mathrm{C}(11)-\mathrm{C}(16) & 170.25(14) \\ \mathrm{C}(16)-\mathrm{C}(11)-\mathrm{C}(12)-\mathrm{C}(13) & -1.3(2) \\ \mathrm{N}(2)-\mathrm{C}(11)-\mathrm{C}(12)-\mathrm{C}(13) & 177.35(14) \\ \mathrm{C}(11)-\mathrm{C}(12)-\mathrm{C}(13)-\mathrm{C}(14) & 1.2(2) \\ \mathrm{C}(12)-\mathrm{C}(13)-\mathrm{C}(14)-\mathrm{C}(15) & -0.1(2) \\ \mathrm{C}(13)-\mathrm{C}(14)-\mathrm{C}(15)-\mathrm{C}(16) & -1.1(3) \\ \mathrm{C}(14)-\mathrm{C}(15)-\mathrm{C}(16)-\mathrm{C}(11) & 1.1(2) \\ \mathrm{C}(12)-\mathrm{C}(11)-\mathrm{C}(16)-\mathrm{C}(15) & 0.1(2) \\ \mathrm{N}(2)-\mathrm{C}(11)-\mathrm{C}(16)-\mathrm{C}(15) & -178.45(14) \\ \mathrm{C}(1)-\mathrm{N}(1)-\mathrm{C}(17)-\mathrm{O}(2) & 0.9(2) \\ \mathrm{C}(8)-\mathrm{N}(1)-\mathrm{C}(17)-\mathrm{O}(2) & 171.34(14) \\ \mathrm{C}(1)-\mathrm{N}(1)-\mathrm{C}(17)-\mathrm{C}(18) & 179.22(14) \\ \mathrm{C}(8)-\mathrm{N}(1)-\mathrm{C}(17)-\mathrm{C}(18) & -10.3(2) \\ & \\ & \end{array}$

Symmetry transformations used to generate equivalent atoms: 
Table 7. Hydrogen bonds for d8v191006 [^ and $\left.{ }^{\circ}\right]$.

\begin{tabular}{lcccc}
\hline D-H...A & d(D-H) & $d(H \ldots A)$ & $d(D \ldots A)$ & $<($ DHA $)$ \\
\hline $\mathrm{C}(16)-\mathrm{H}(16) \ldots \mathrm{O}(1)$ & 0.95 & 2.24 & $2.867(2)$ & 123.0 \\
$\mathrm{C}(7)-\mathrm{H}(7) \ldots \mathrm{O}(2) \# 1$ & 1.00 & 2.45 & $3.2966(19)$ & 142.6 \\
$\mathrm{C}(2)-\mathrm{H}(2) \ldots \mathrm{O}(2)$ & 0.95 & 2.36 & $2.896(2)$ & 115.4 \\
\hline
\end{tabular}

Symmetry transformations used to generate equivalent atoms:

\#1 -x+1,-y+1,-z+1 


\section{References}

[1] Yao, Z.; Wu, X.; Zhang, X.; Xiong, Q.; Jiang, S.; Yu, Z. Org. Biomol. Chem. 2019, 17,6777 .

[2] Dolomanov, O. V.; Bourhis, L. J.; Gildea, R. J.; Howard, J. A. K.; Puschmann, H.;

J. Appl. Cryst. 2009, 42, 339.

[3] Sheldrick, G. M. Acta Cryst A. 2015, 71, 3.

[4] Sheldrick, G. M. Acta Cryst C. 2015, 71, 3. 


\section{Copies of NMR spectra}

${ }^{1} \mathrm{H}$ NMR Spectrum of $\mathbf{1 a}$

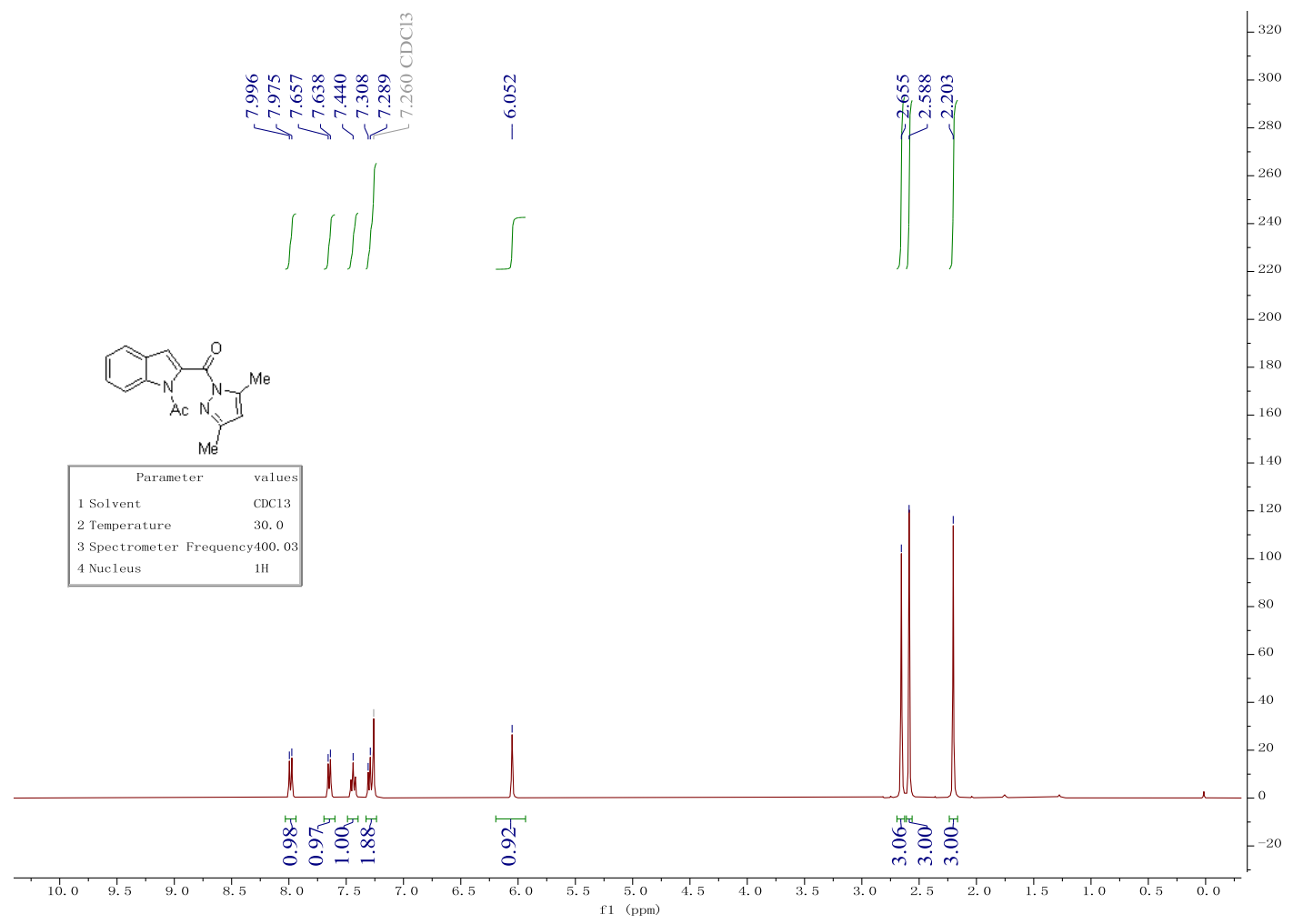

${ }^{13} \mathrm{C}$ NMR Spectrum of $\mathbf{1 a}$

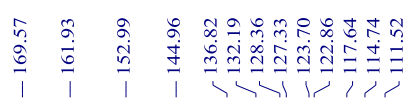
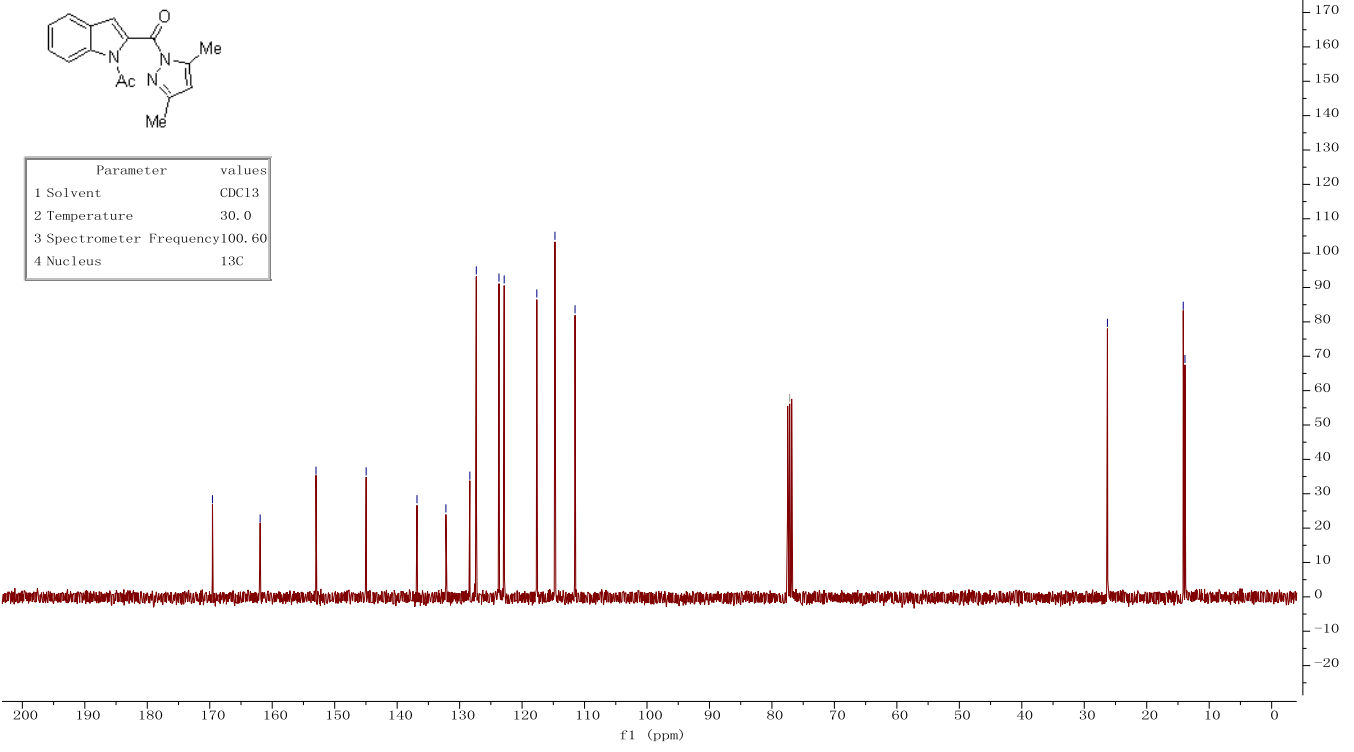
${ }^{1} \mathrm{H}$ NMR Spectrum of $\mathbf{1 b}$

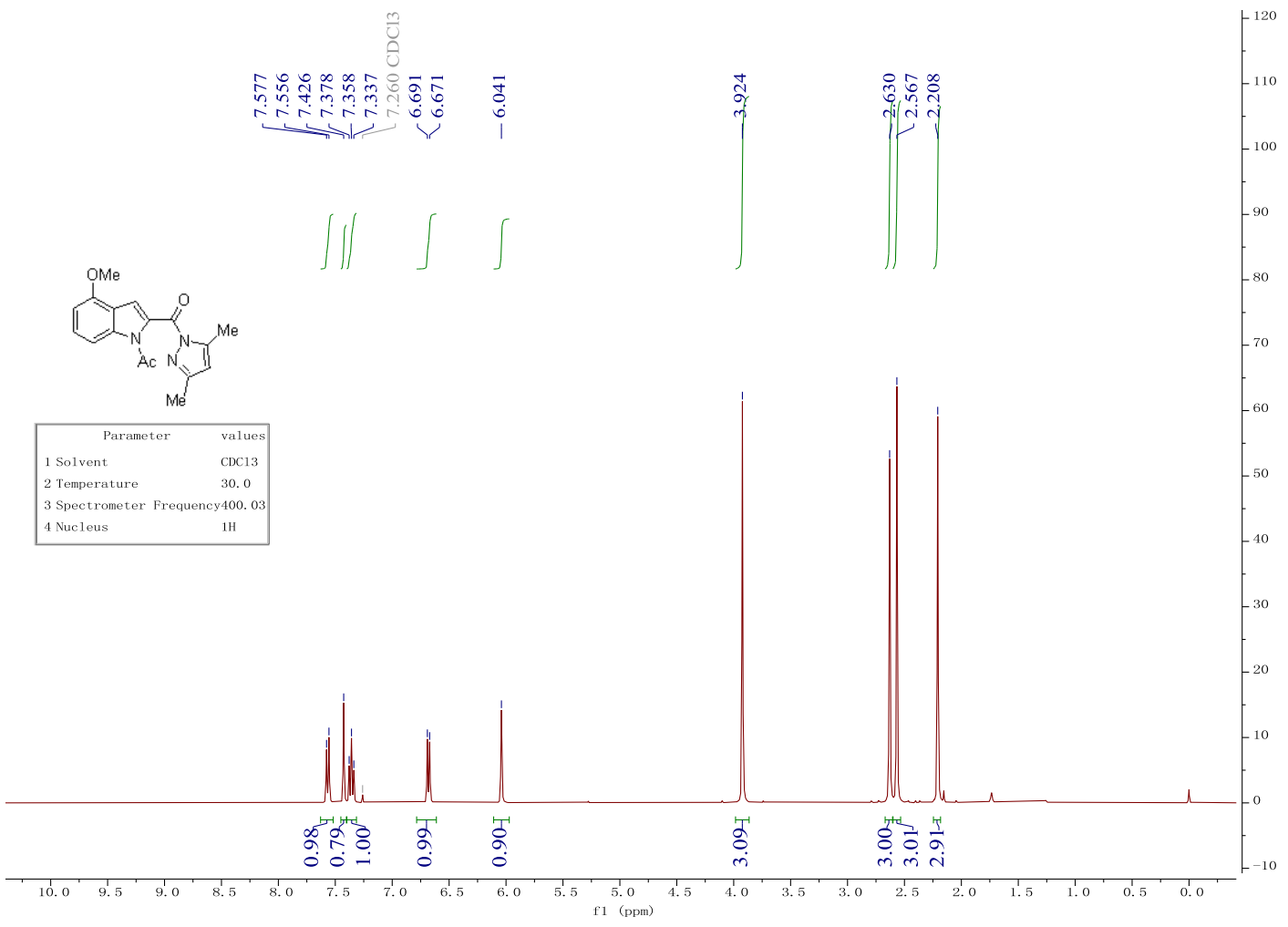

${ }^{13} \mathrm{C}$ NMR Spectrum of $\mathbf{1 b}$

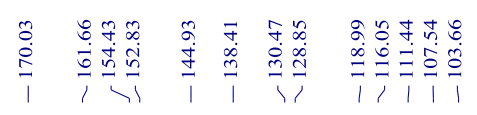

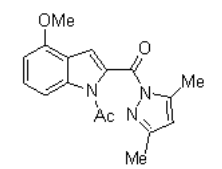

\begin{tabular}{|lc|}
\hline \multicolumn{1}{|c|}{ Parameter } & values \\
1 Solvent & CDC13 \\
2 Temperature & 30.0 \\
3 Spectrometer & Frequency 100. 60 \\
4 Nucl leus & $13 \mathrm{C}$ \\
\hline
\end{tabular}

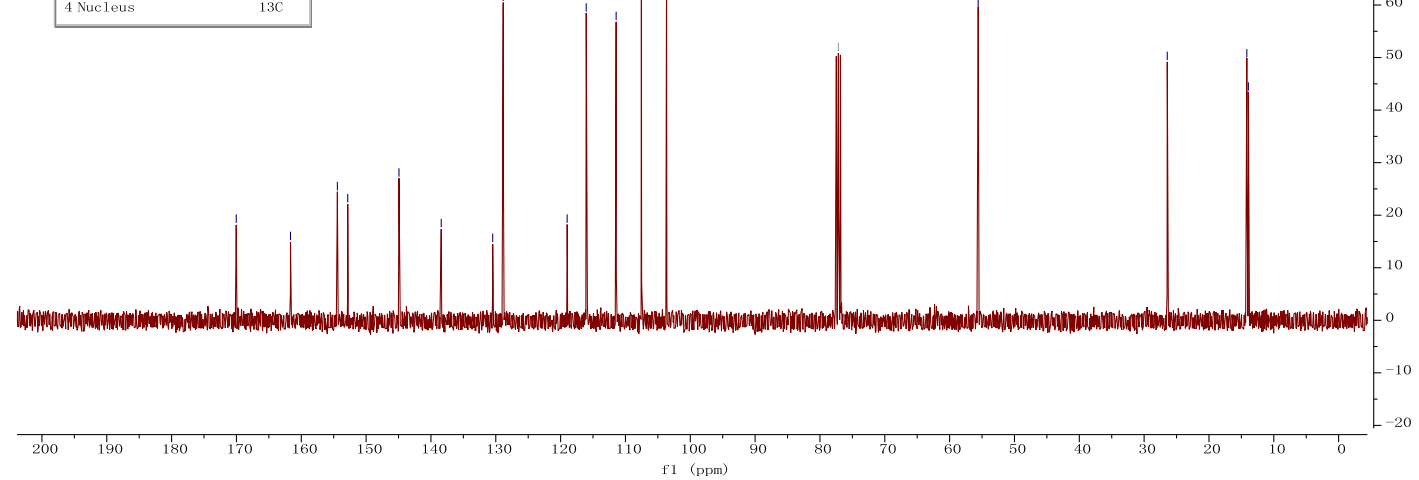


${ }^{1} \mathrm{H}$ NMR Spectrum of $\mathbf{1 c}$

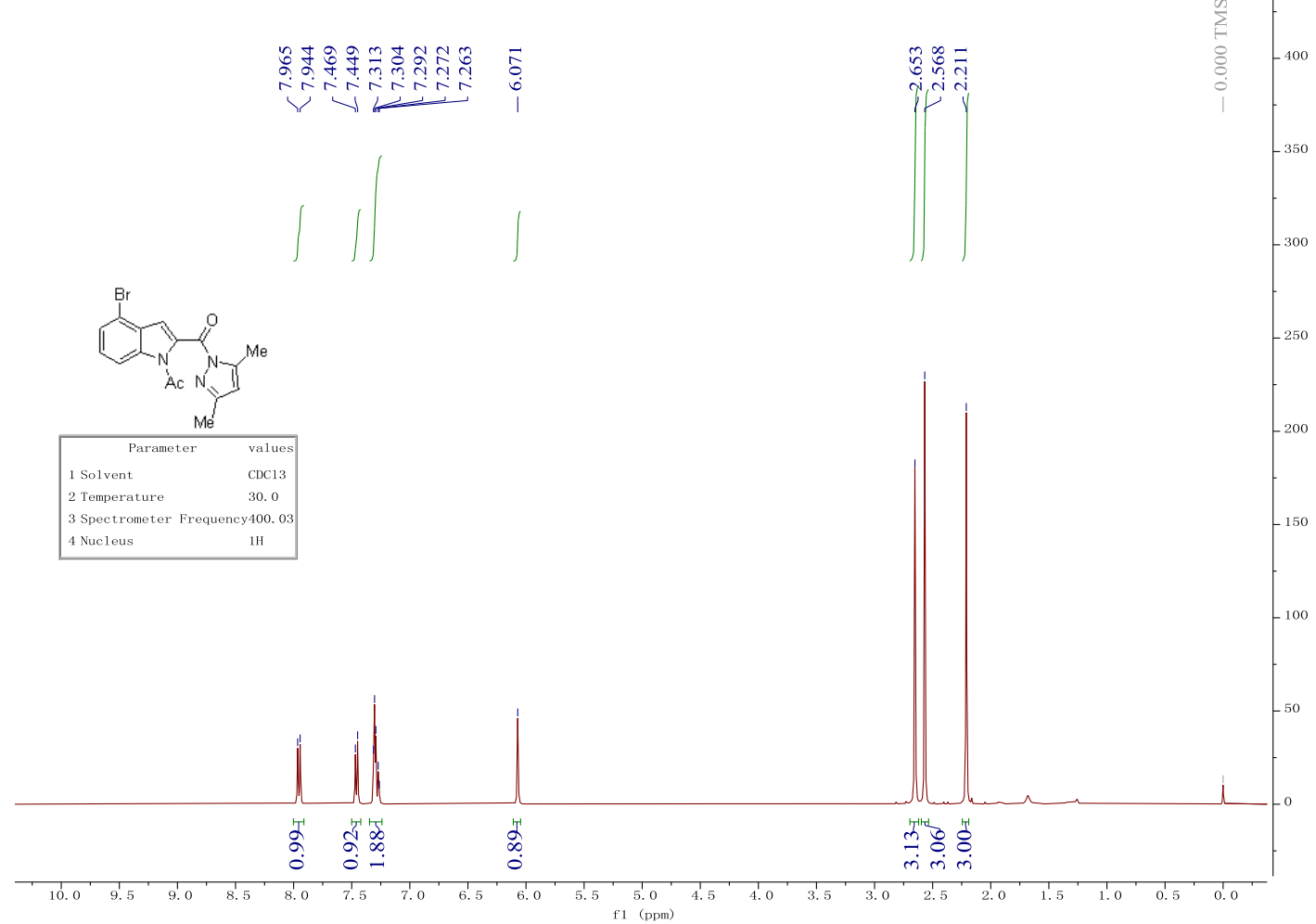

${ }^{13} \mathrm{C}$ NMR Spectrum of $\mathbf{1 c}$

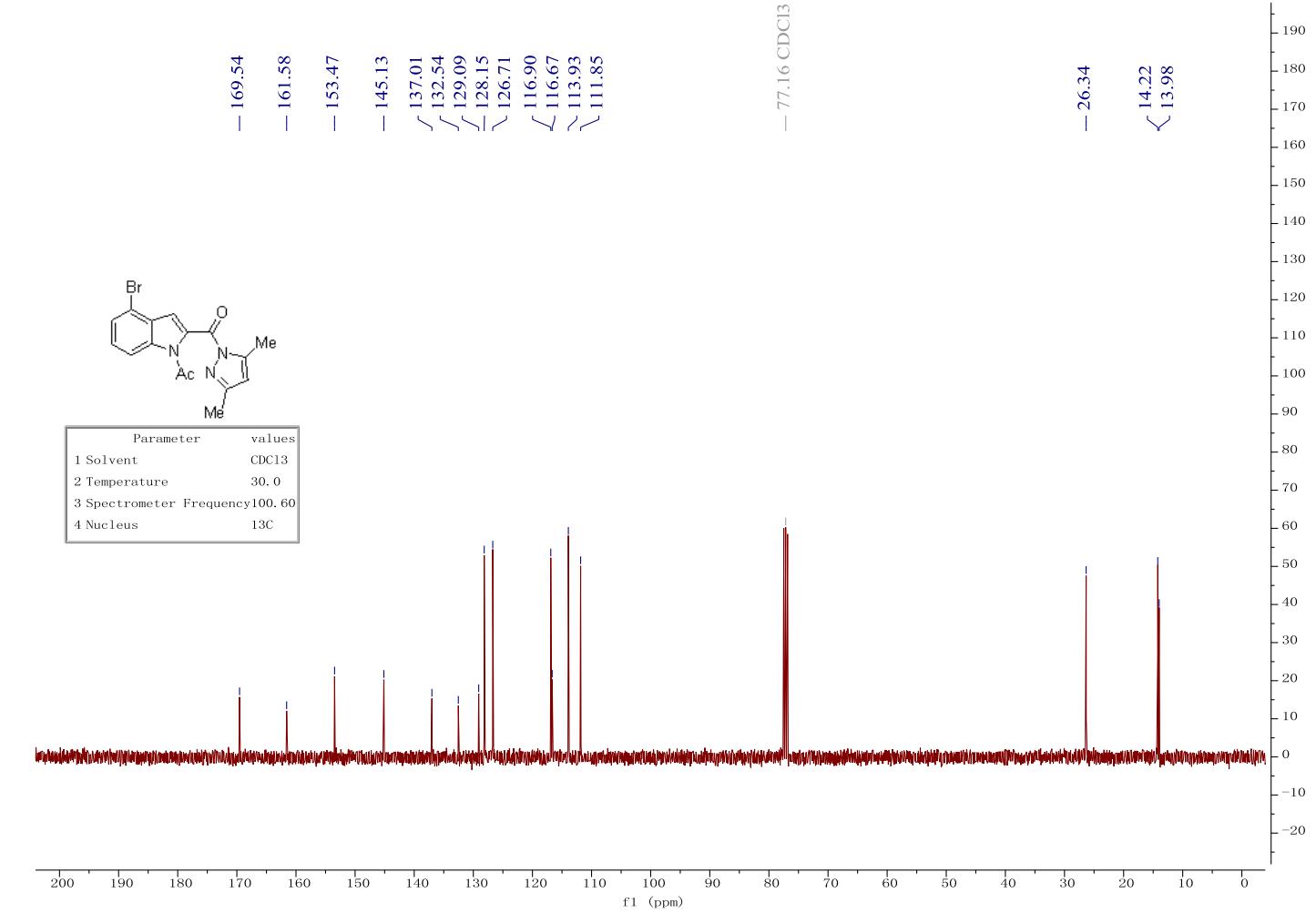




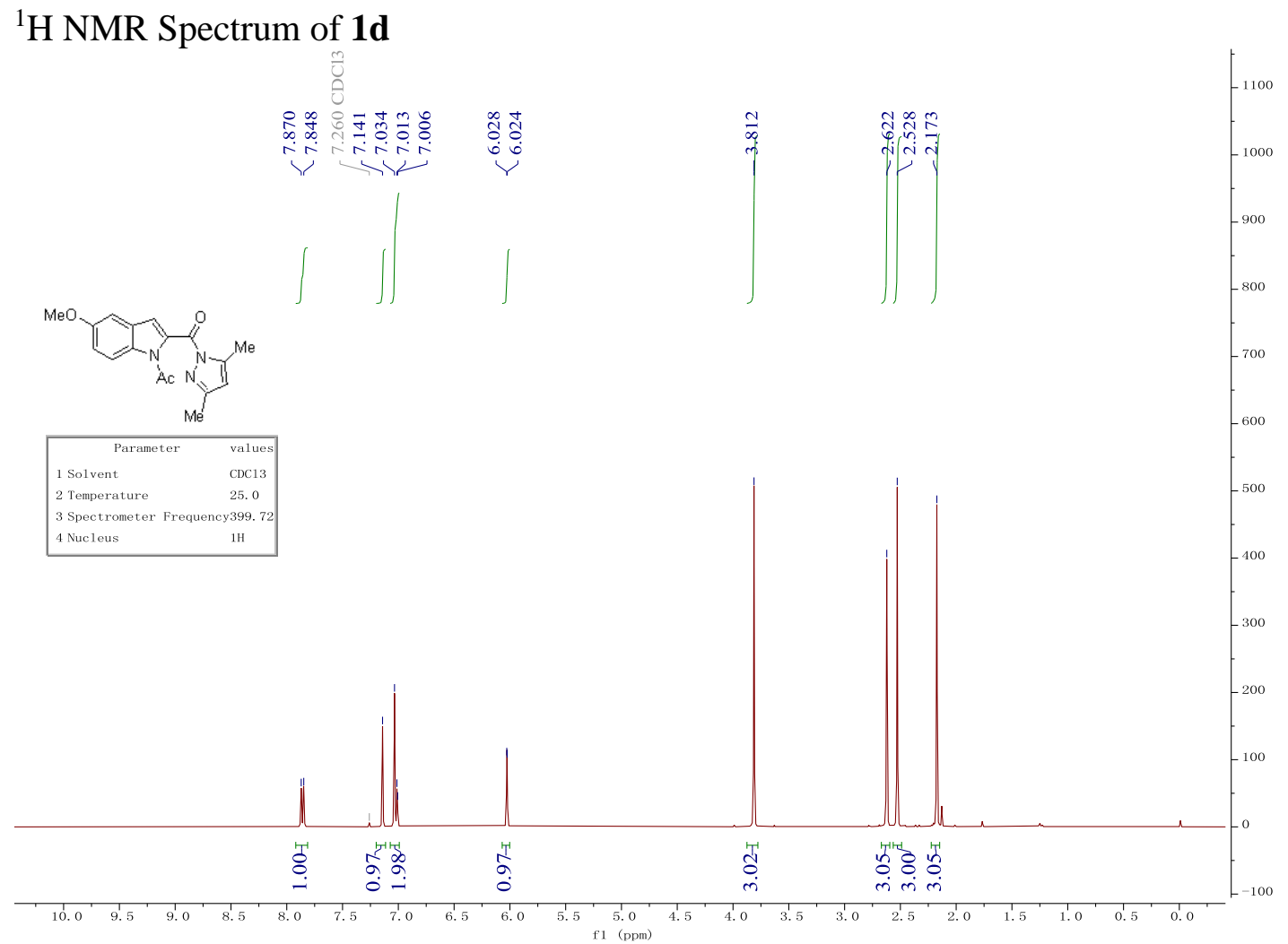

${ }^{13} \mathrm{C}$ NMR Spectrum of $\mathbf{1 d}$

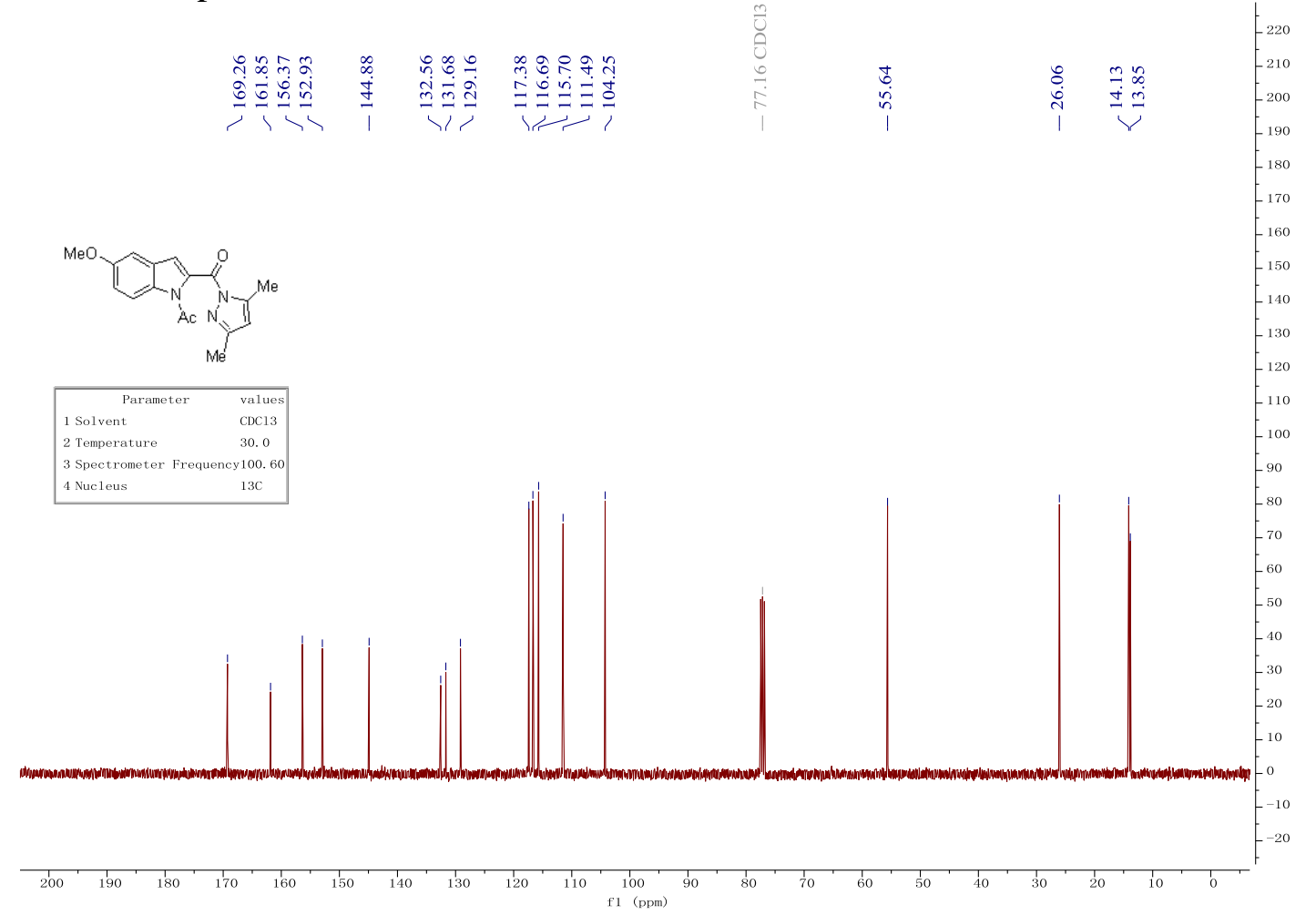


${ }^{1} \mathrm{H}$ NMR Spectrum of $\mathbf{1 e}$

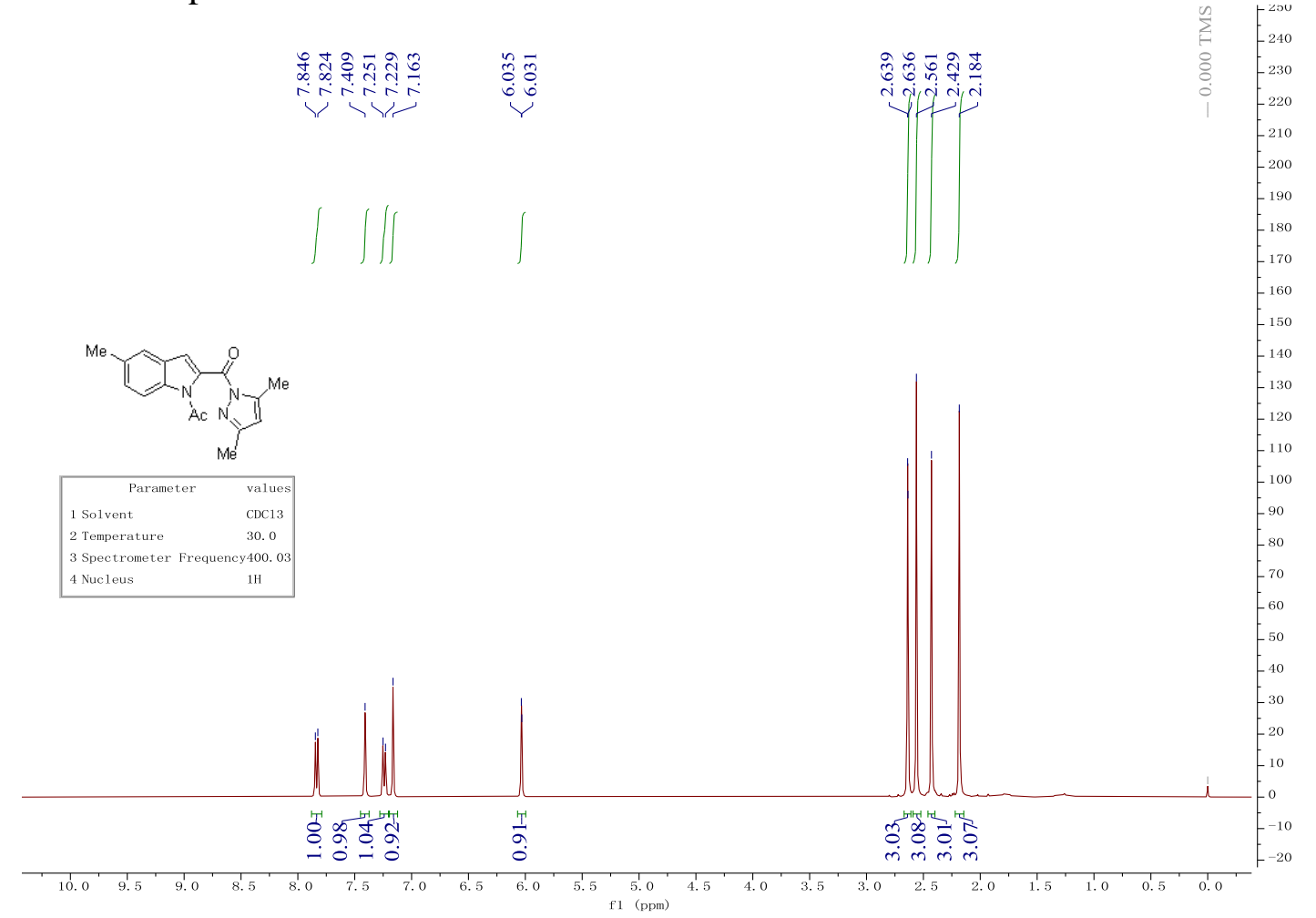

${ }^{13} \mathrm{C}$ NMR Spectrum of $\mathbf{1 e}$

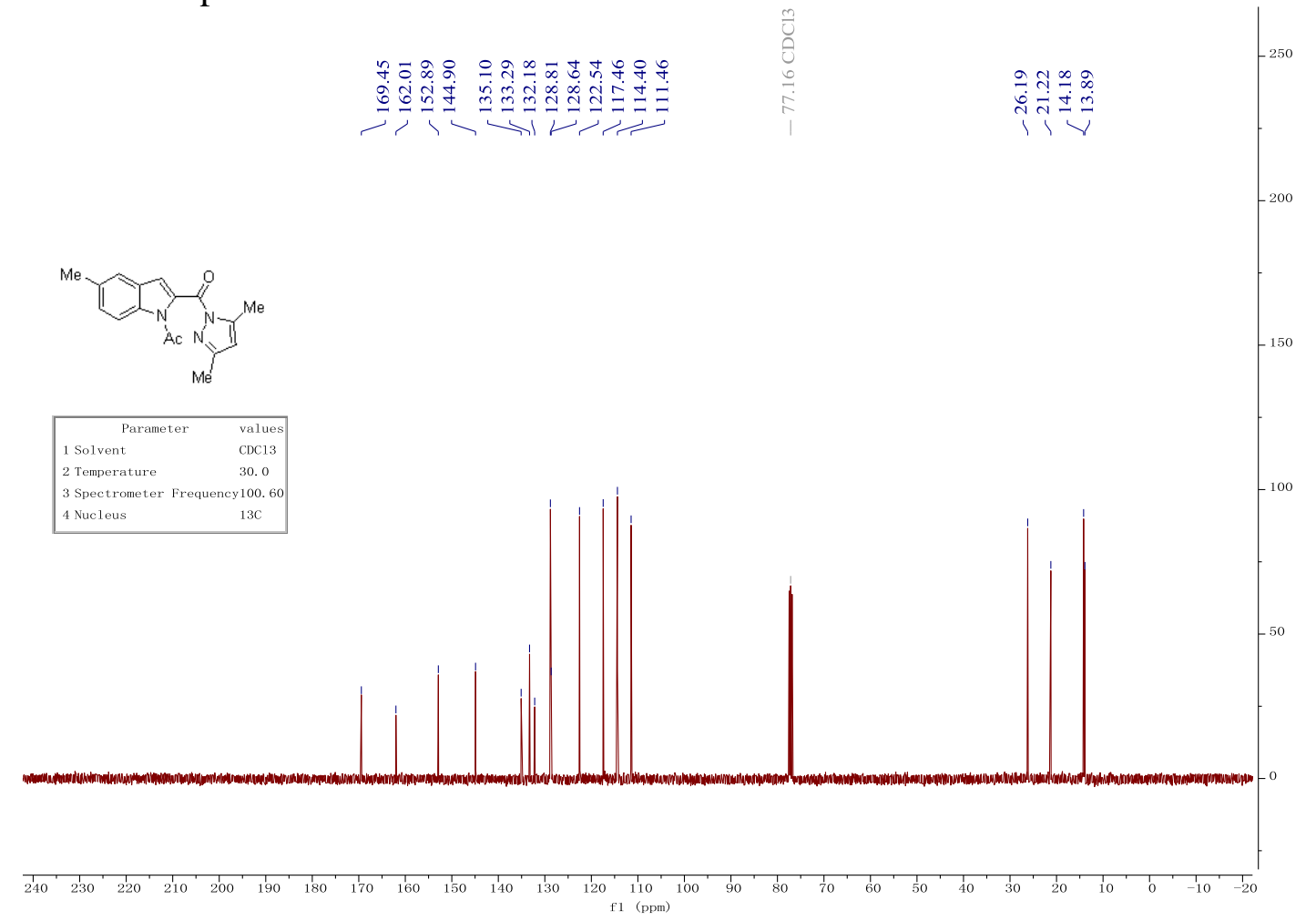


${ }^{1} \mathrm{H}$ NMR Spectrum of $\mathbf{1 f}$

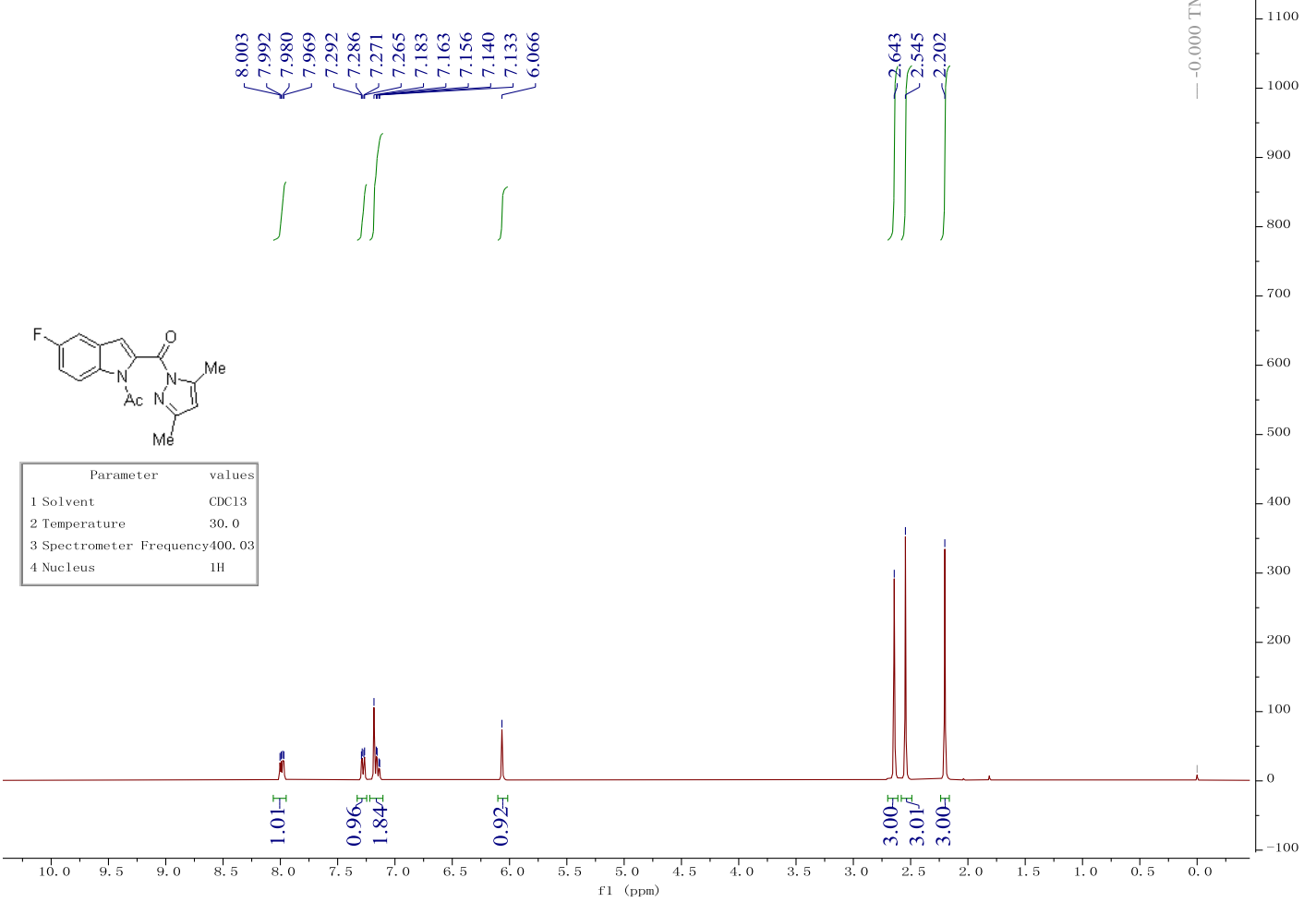

${ }^{13} \mathrm{C}$ NMR Spectrum of $\mathbf{1 f}$

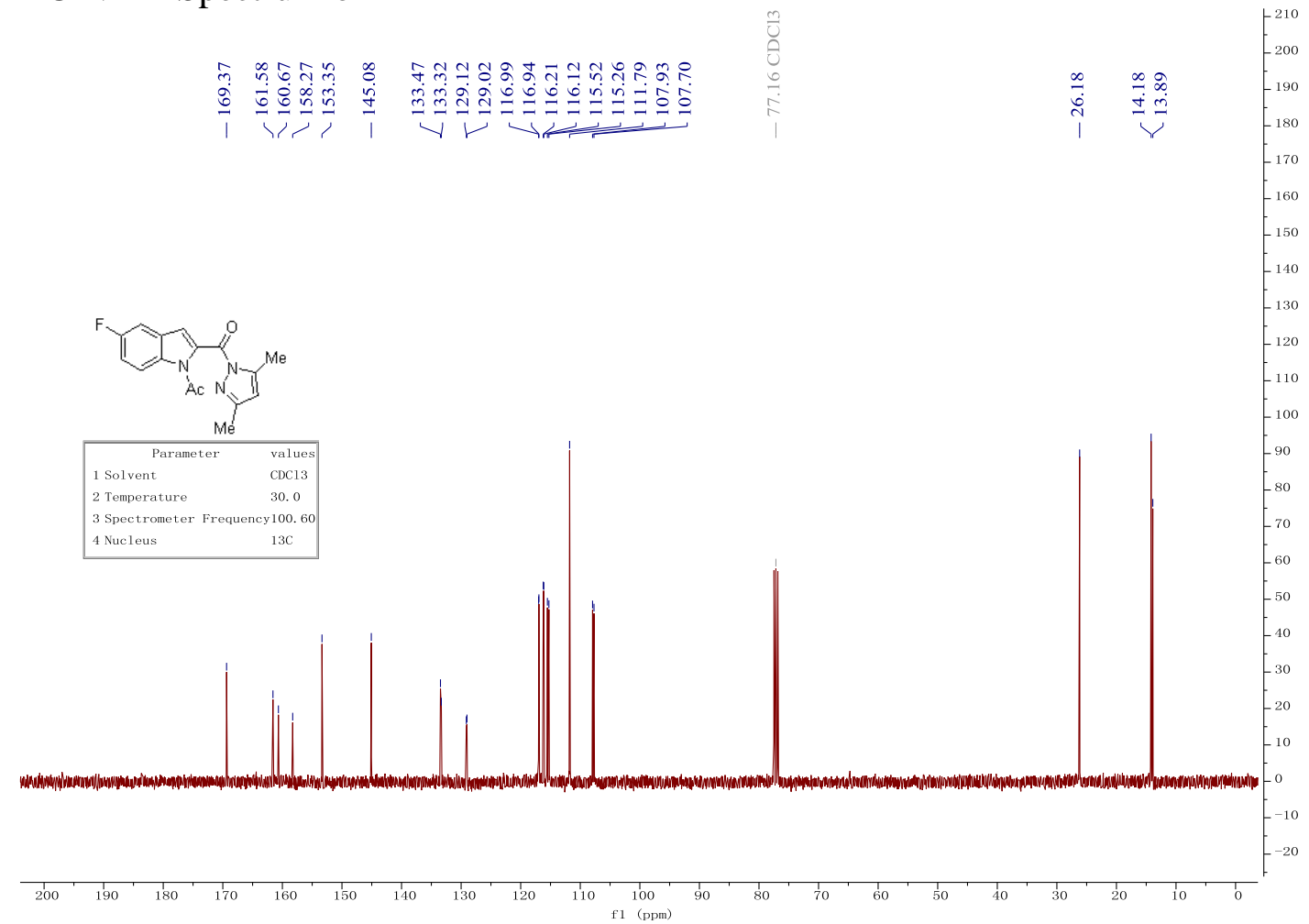


${ }^{19} \mathrm{~F}$ NMR Spectrum of $\mathbf{1 f}$

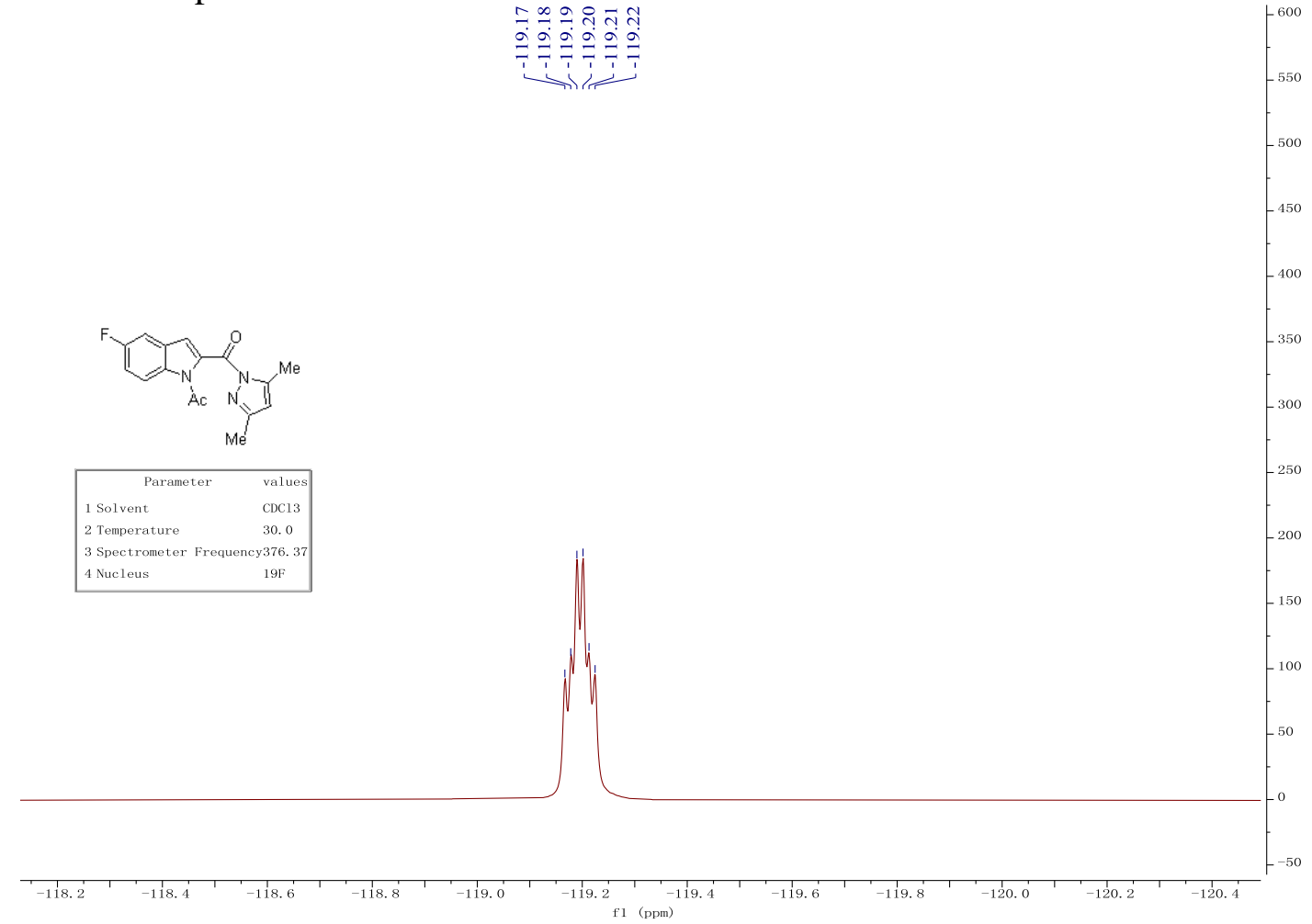

${ }^{1} \mathrm{H}$ NMR Spectrum of $\mathbf{1 g}$

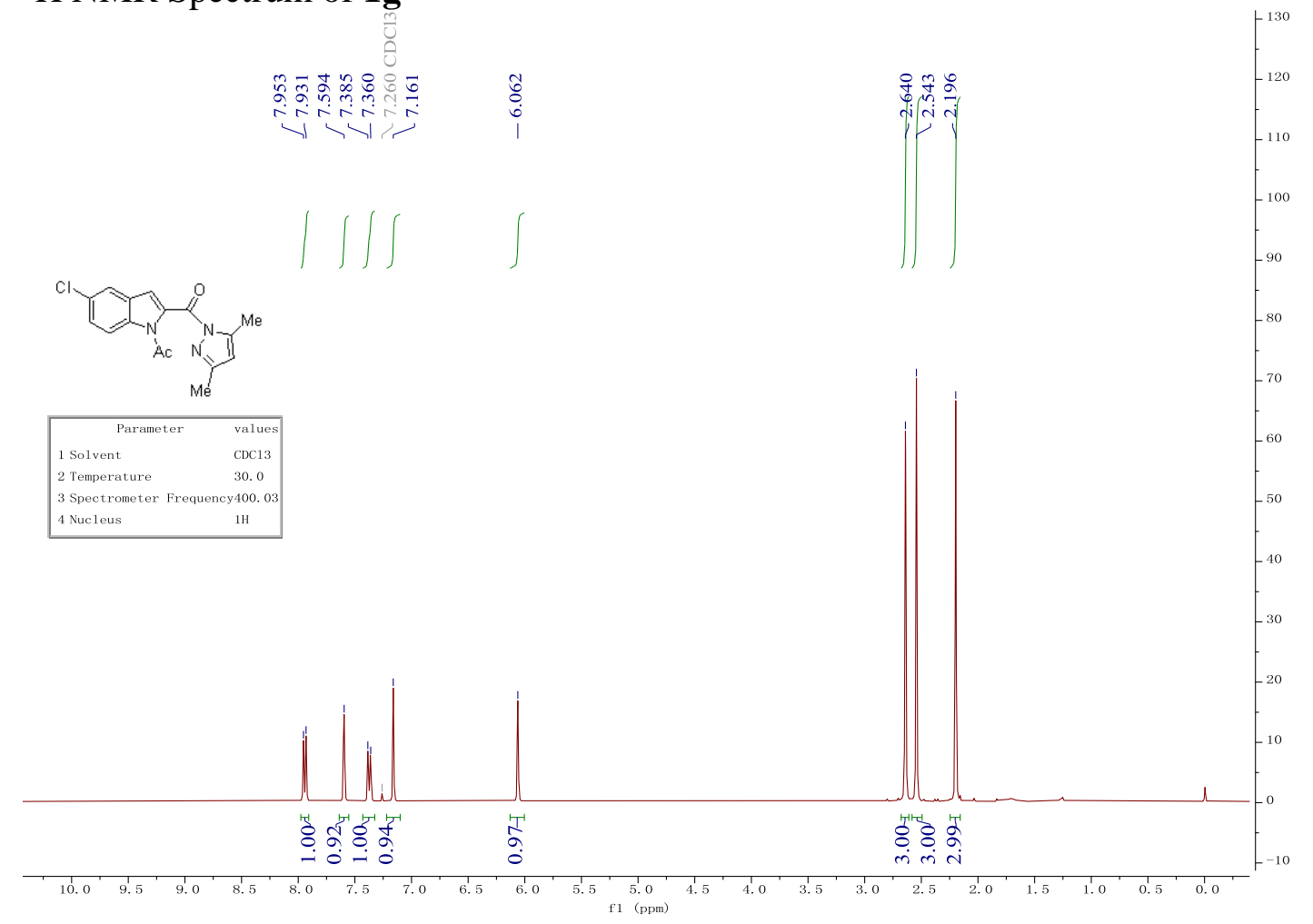


${ }^{13} \mathrm{C}$ NMR Spectrum of $\mathbf{1 g}$

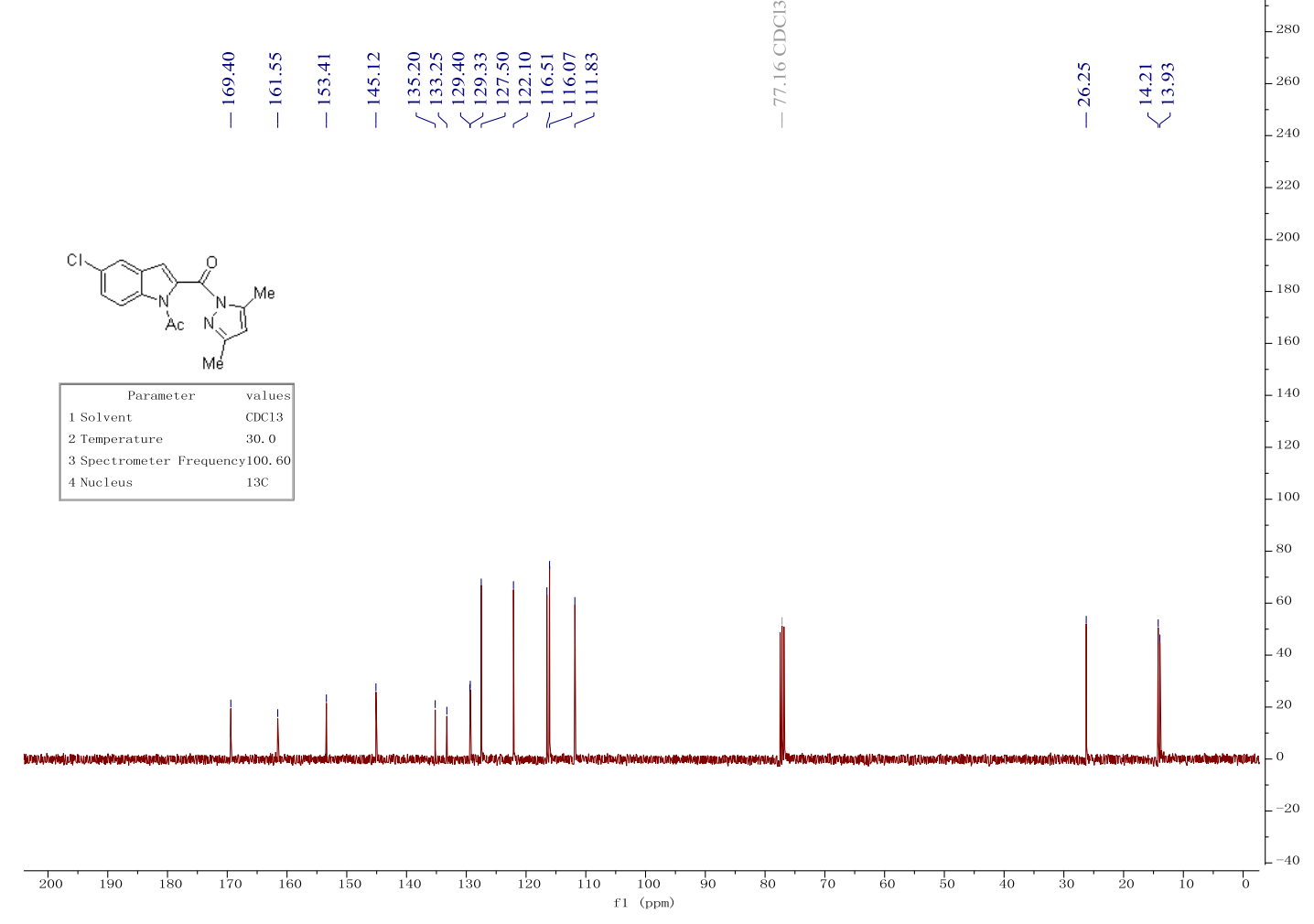

${ }^{1} \mathrm{H}$ NMR Spectrum of $\mathbf{1 h}$

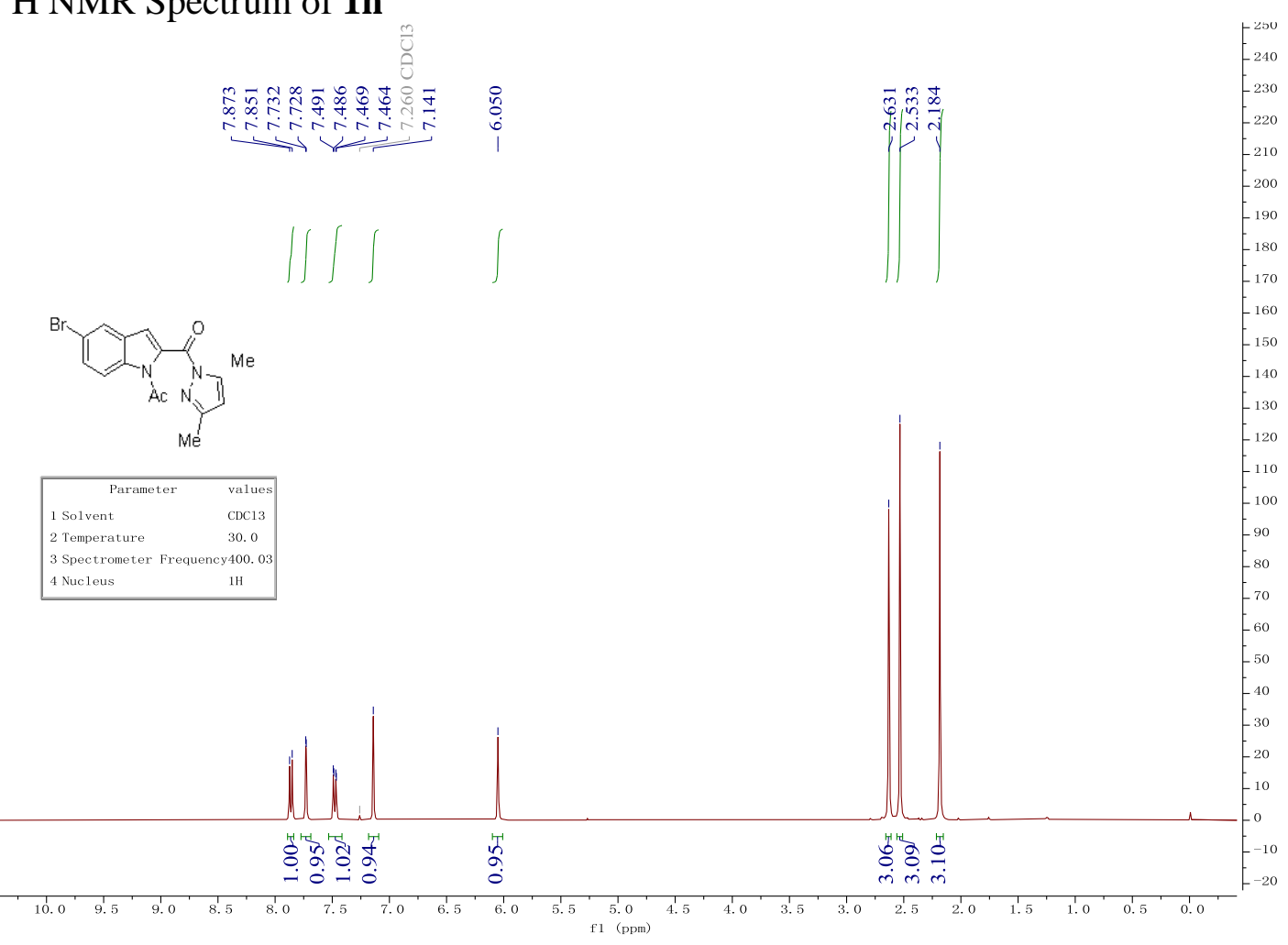


${ }^{13} \mathrm{C}$ NMR Spectrum of $\mathbf{1 h}$
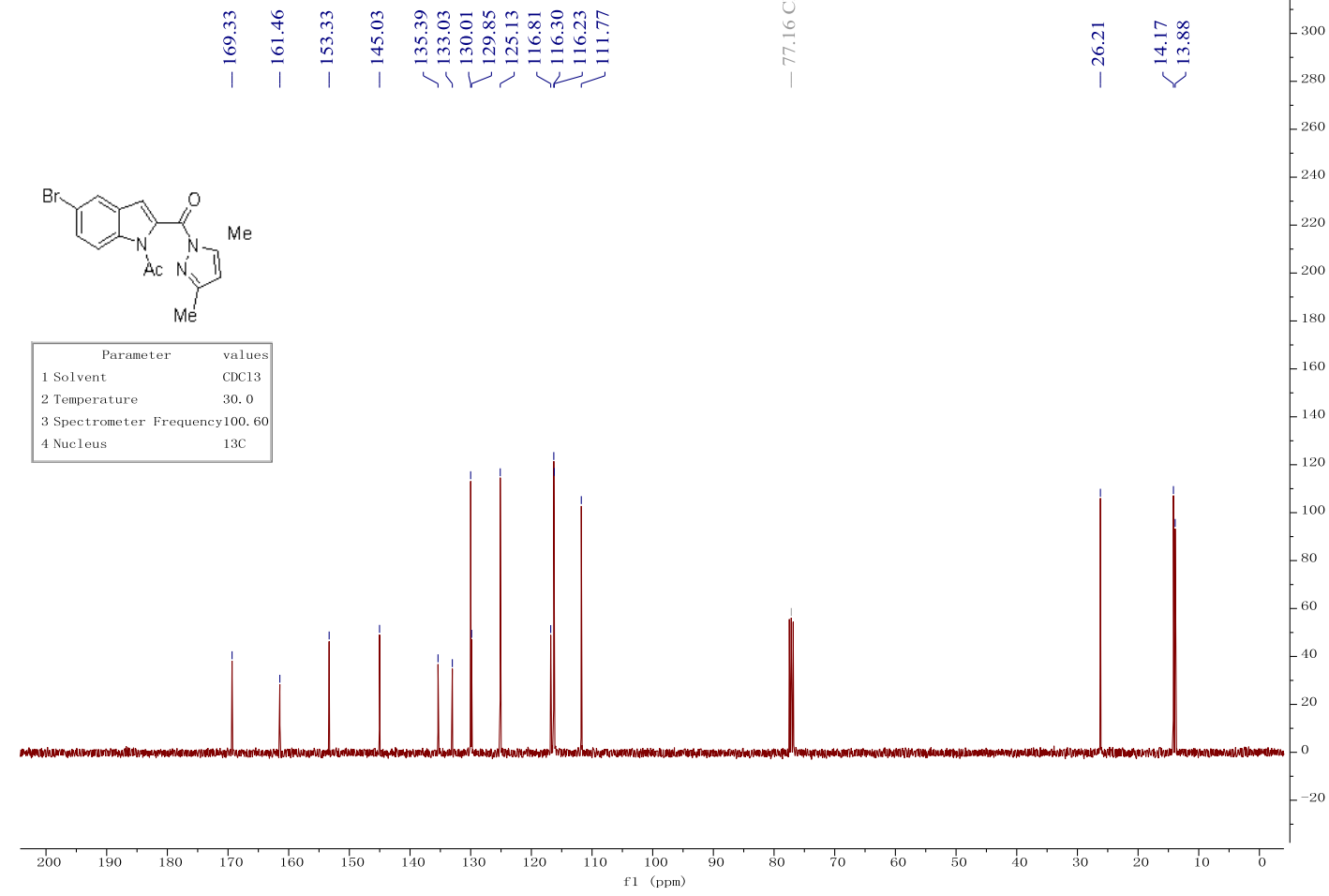

${ }^{1} \mathrm{H}$ NMR Spectrum of $\mathbf{1 i}$

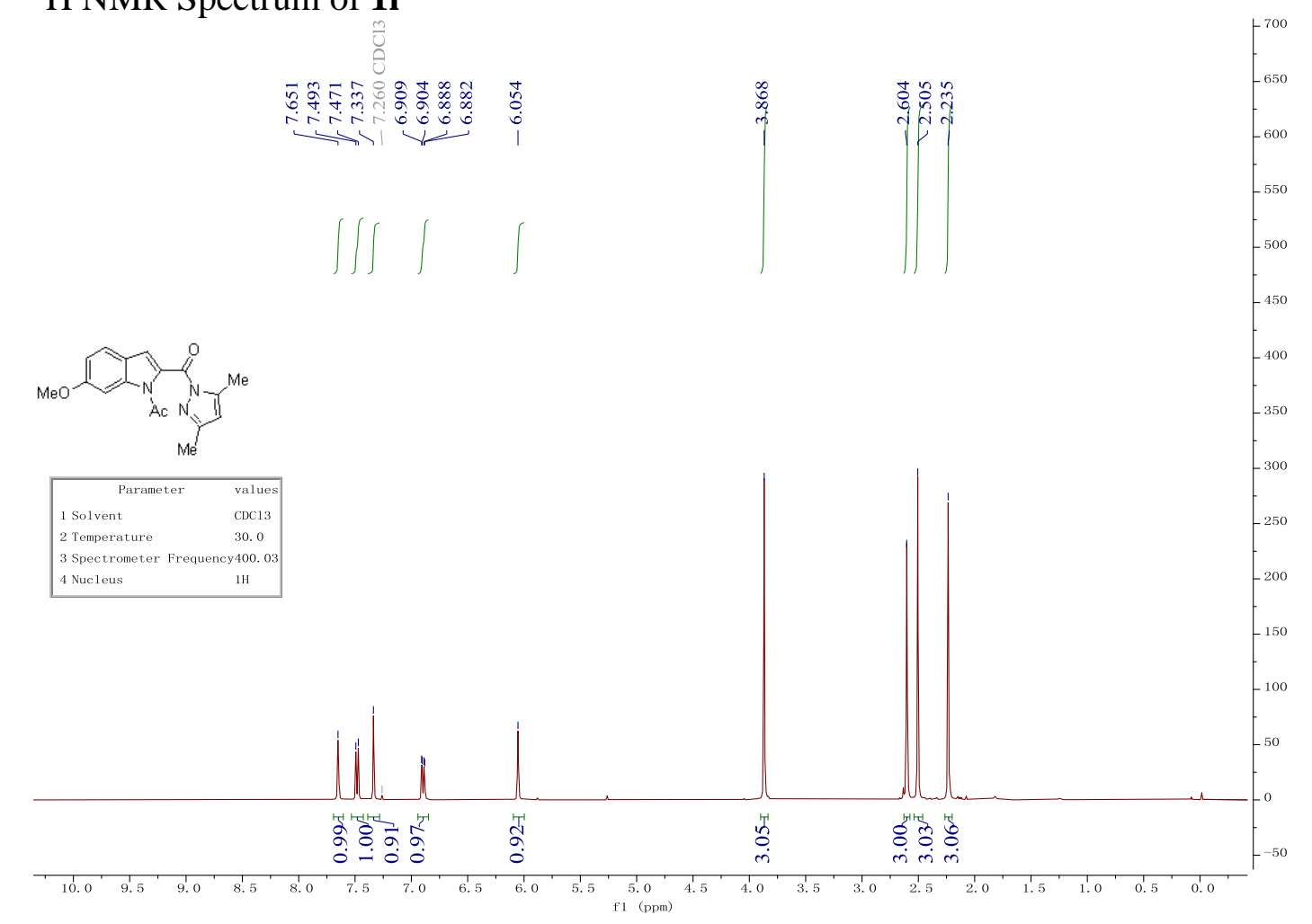


${ }^{13} \mathrm{C}$ NMR Spectrum of $\mathbf{1 i}$

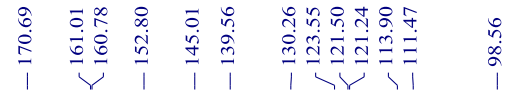

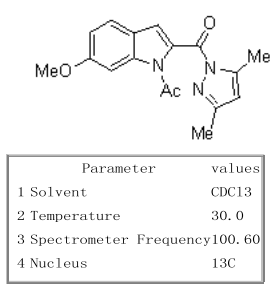

$\begin{array}{ll} & 0 \\ 0 & 8 \\ 0 & 0 \\ 0 & 0 \\ \infty & 0 \\ 0 & 1\end{array}$

$\begin{array}{ll}0 \\ 0 \\ 0 \\ 0 & 0 \\ 0 & n \\ 1 & n\end{array}$
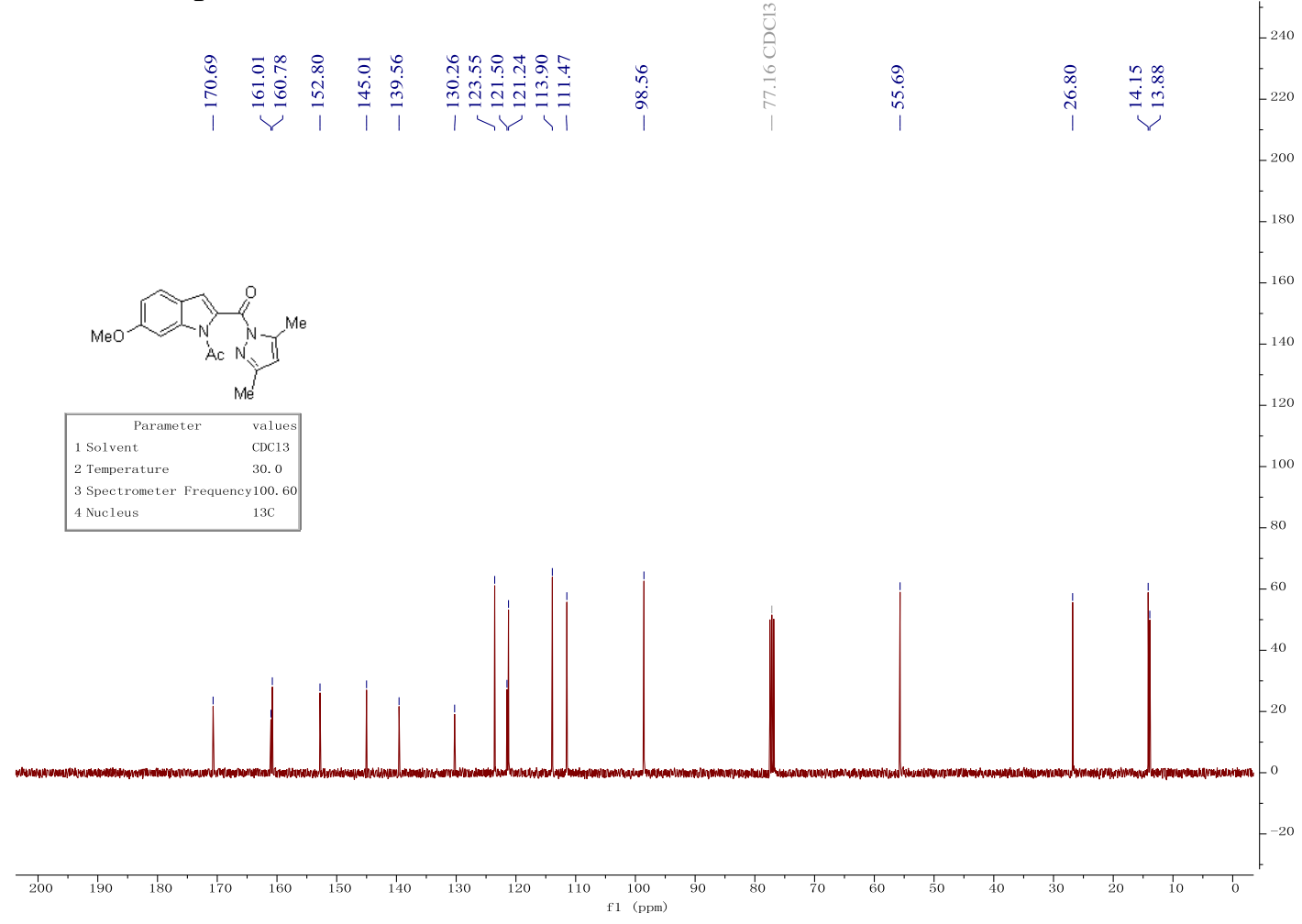

${ }^{1} \mathrm{H}$ NMR Spectrum of $\mathbf{1} \mathbf{j}$

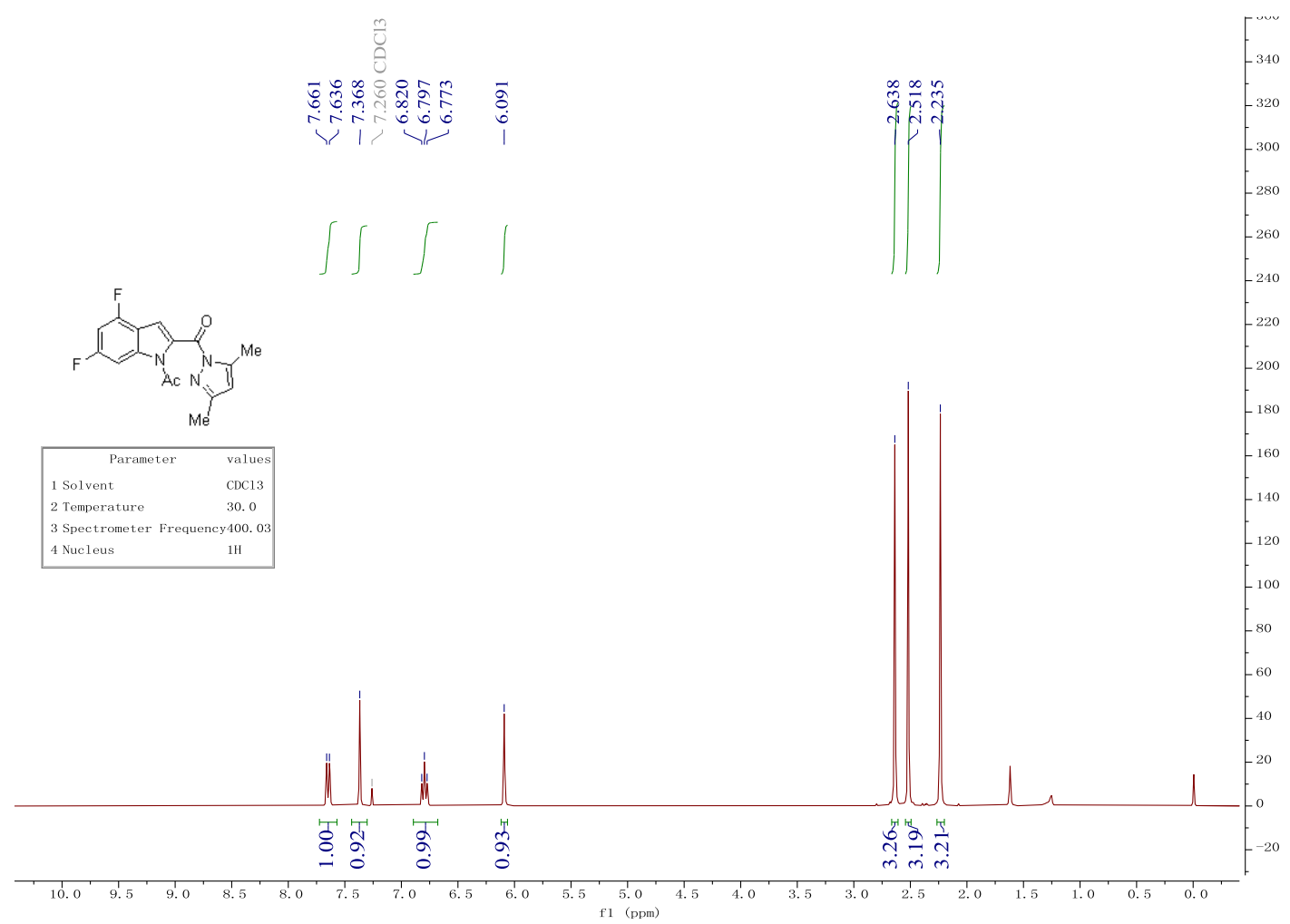




\section{${ }^{13} \mathrm{C}$ NMR Spectrum of $\mathbf{1 j}$}
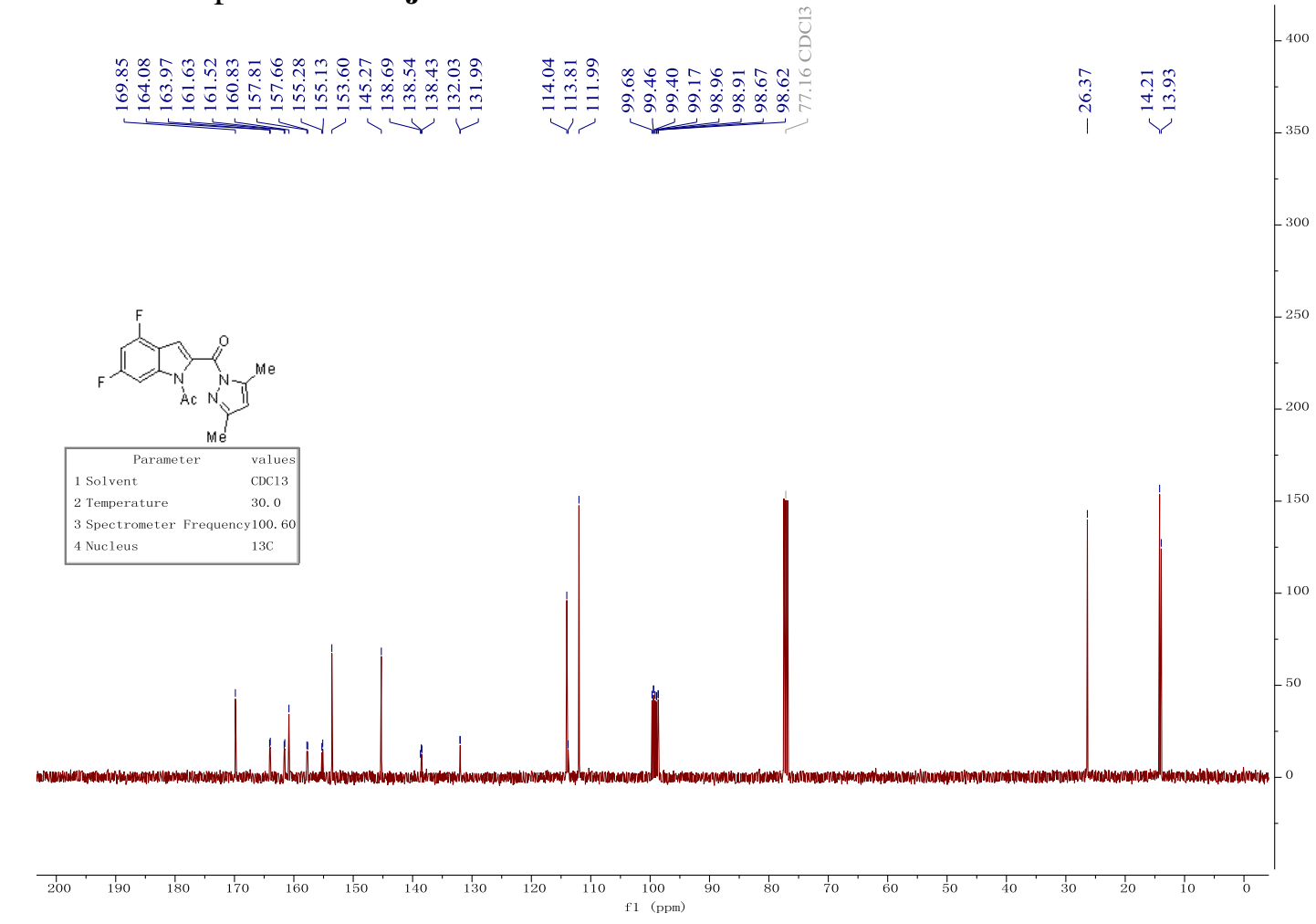

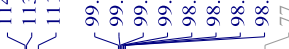

${ }^{19}$ F NMR Spectrum of $\mathbf{1 j}$

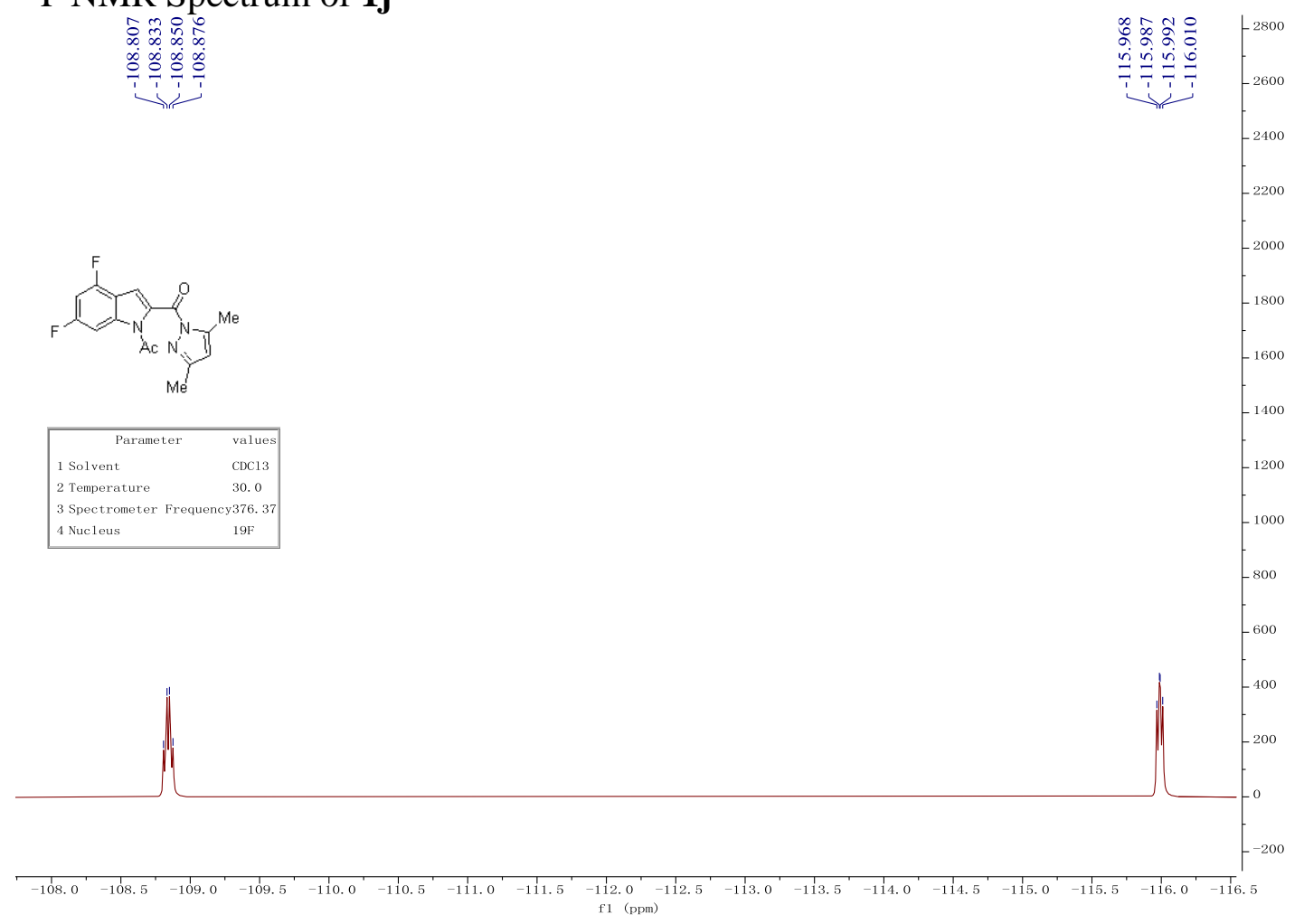


${ }^{1} \mathrm{H}$ NMR Spectrum of $\mathbf{1 k}$

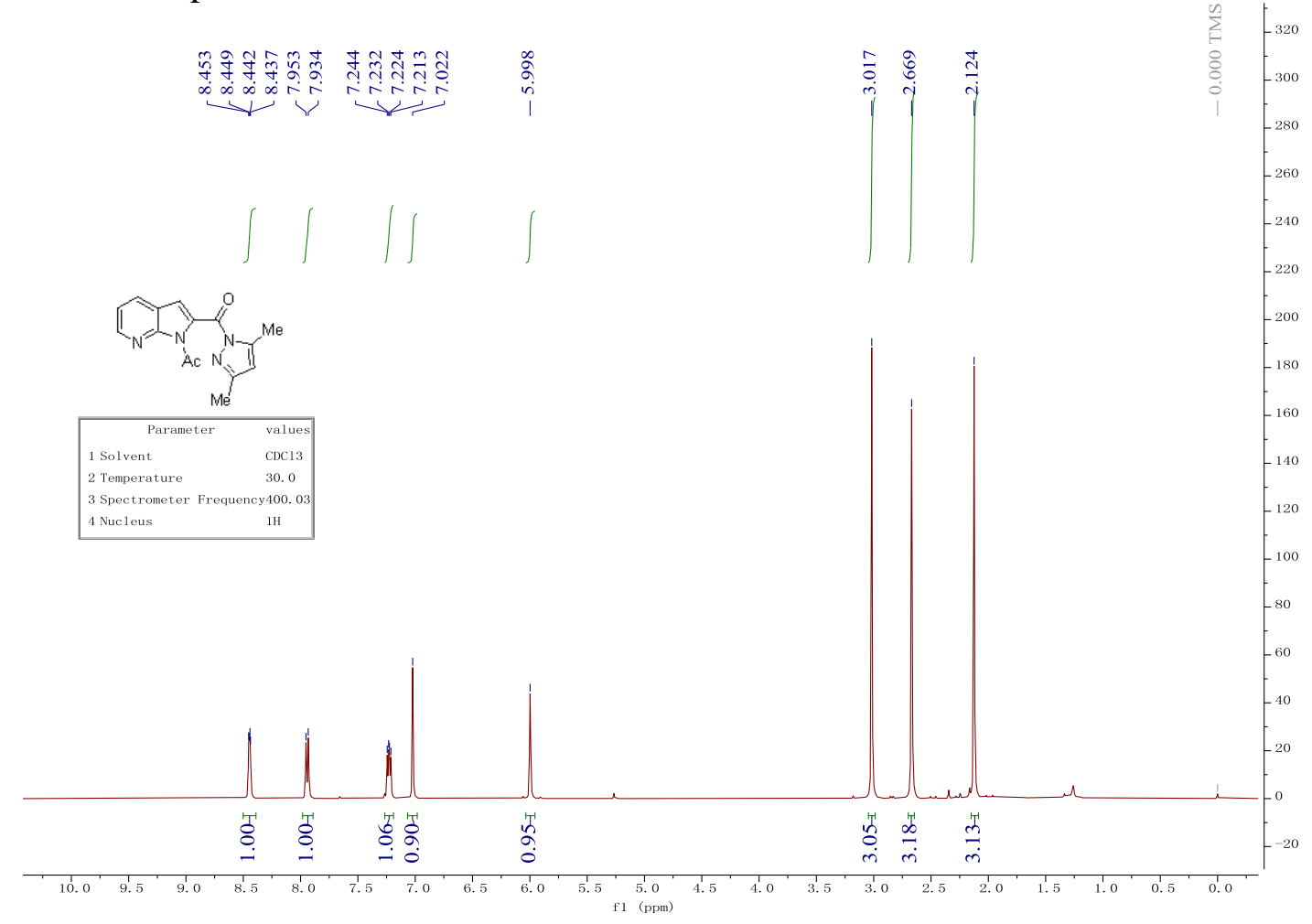

${ }^{13} \mathrm{C}$ NMR Spectrum of $\mathbf{1 k}$

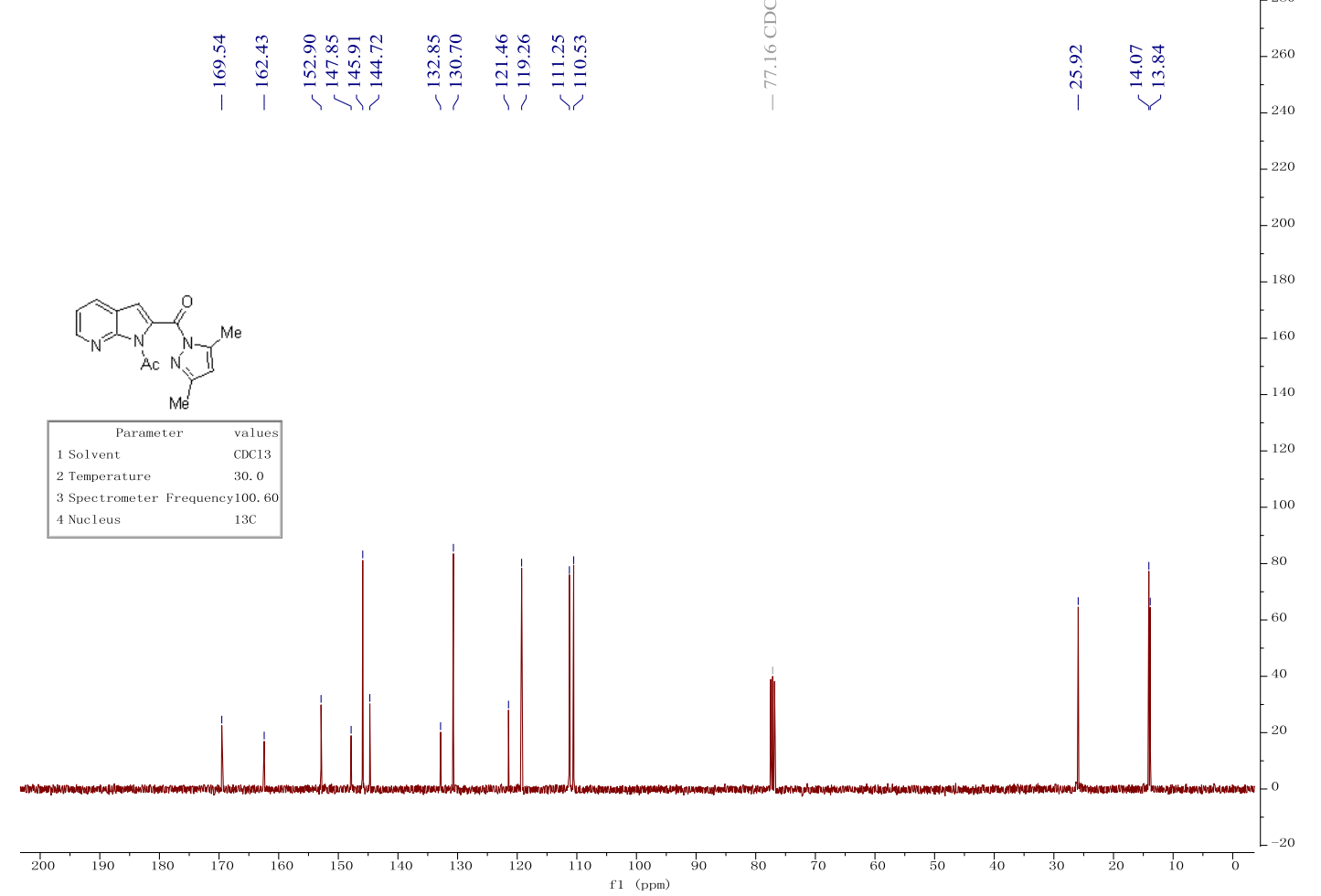


${ }^{1} \mathrm{H}$ NMR Spectrum of $\mathbf{1 l}$

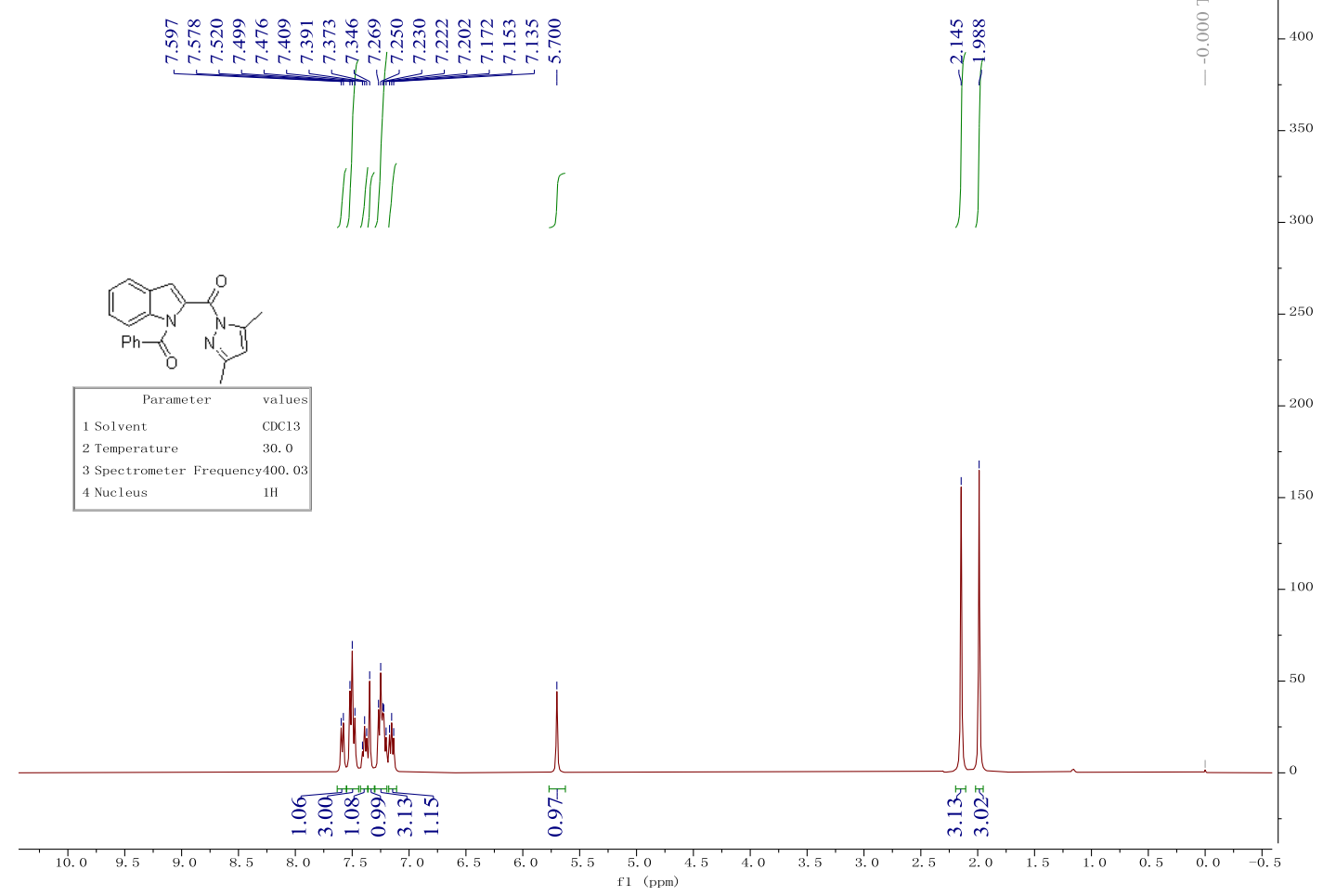

${ }^{13} \mathrm{C}$ NMR Spectrum of $\mathbf{1 l}$

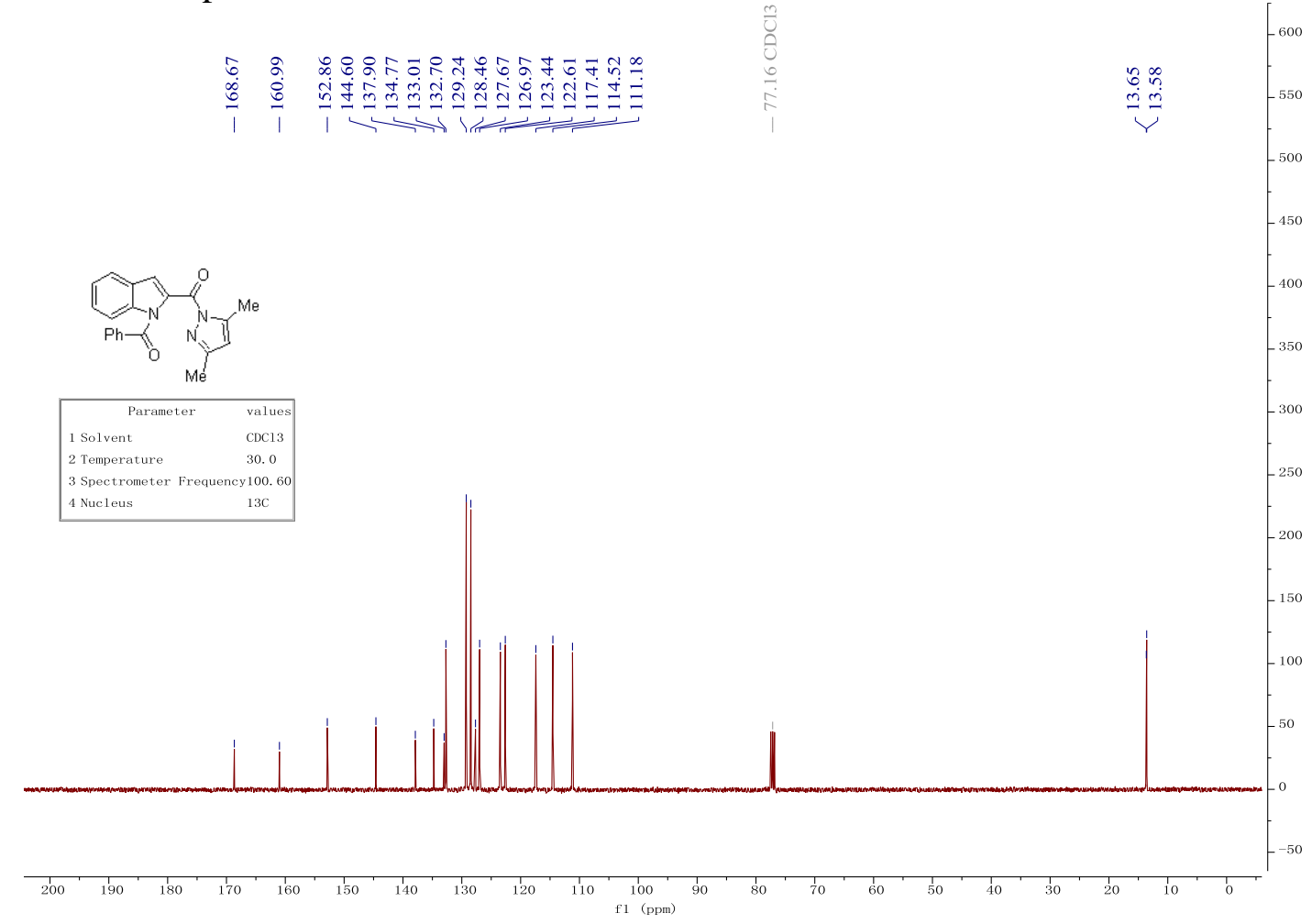




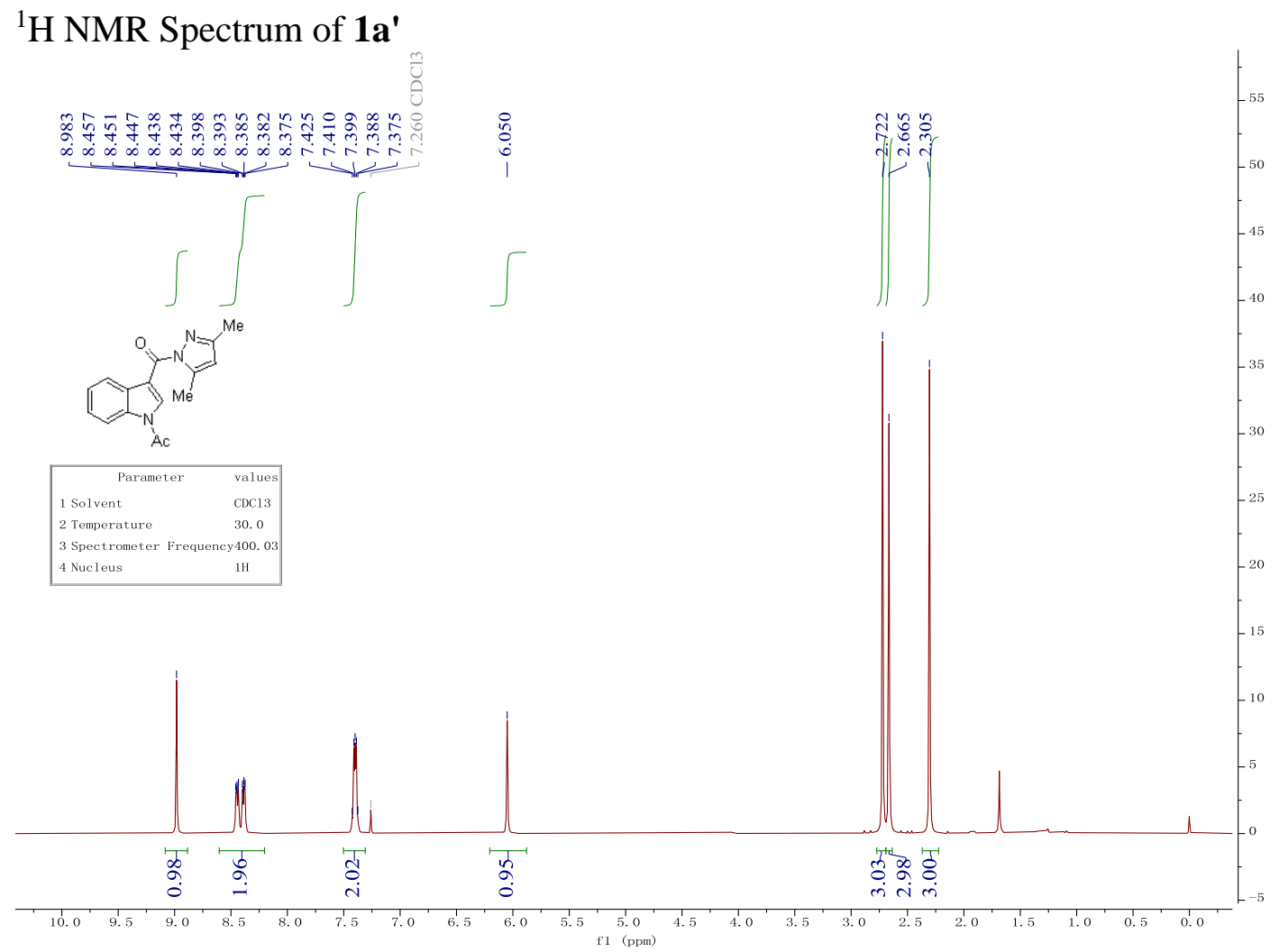

${ }^{13} \mathrm{C}$ NMR Spectrum of $\mathbf{1 a}$

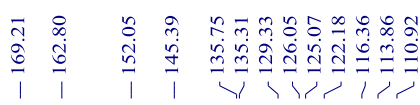

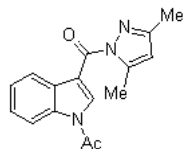

\begin{tabular}{lc}
\multicolumn{1}{c}{ Parameter } & values \\
1 Solvent & CDC13 \\
2 Temperature & 30.0 \\
3 Spectrometer & Frequency 100.60 \\
4 Nucleus & $13 \mathrm{C}$
\end{tabular}

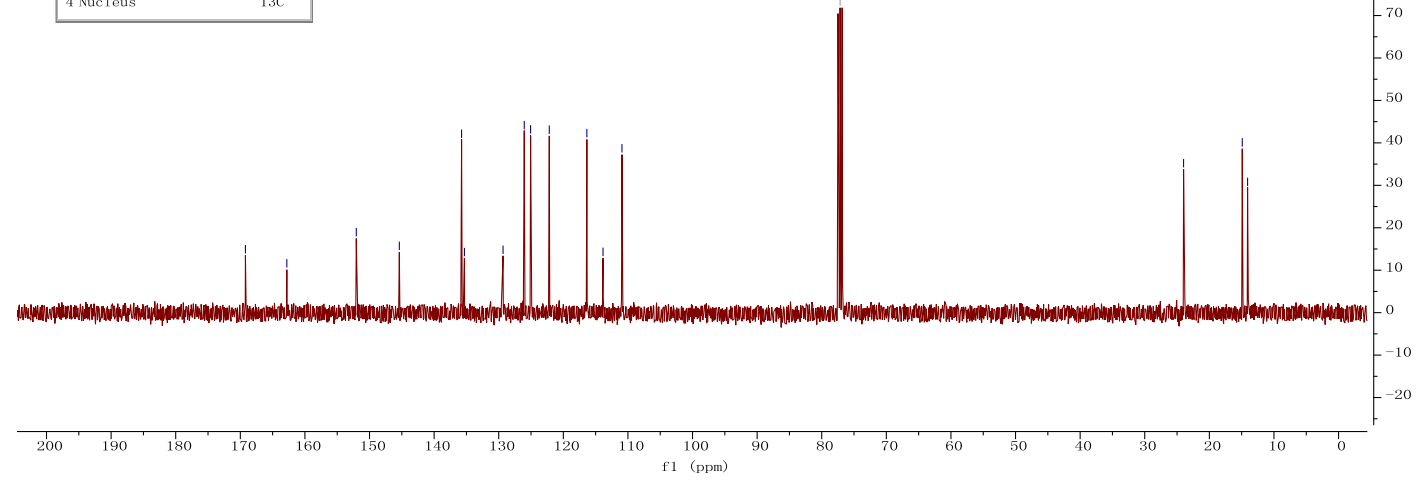


${ }^{1} \mathrm{H}$ NMR Spectrum of $\mathbf{1} \mathbf{b}^{\prime}$
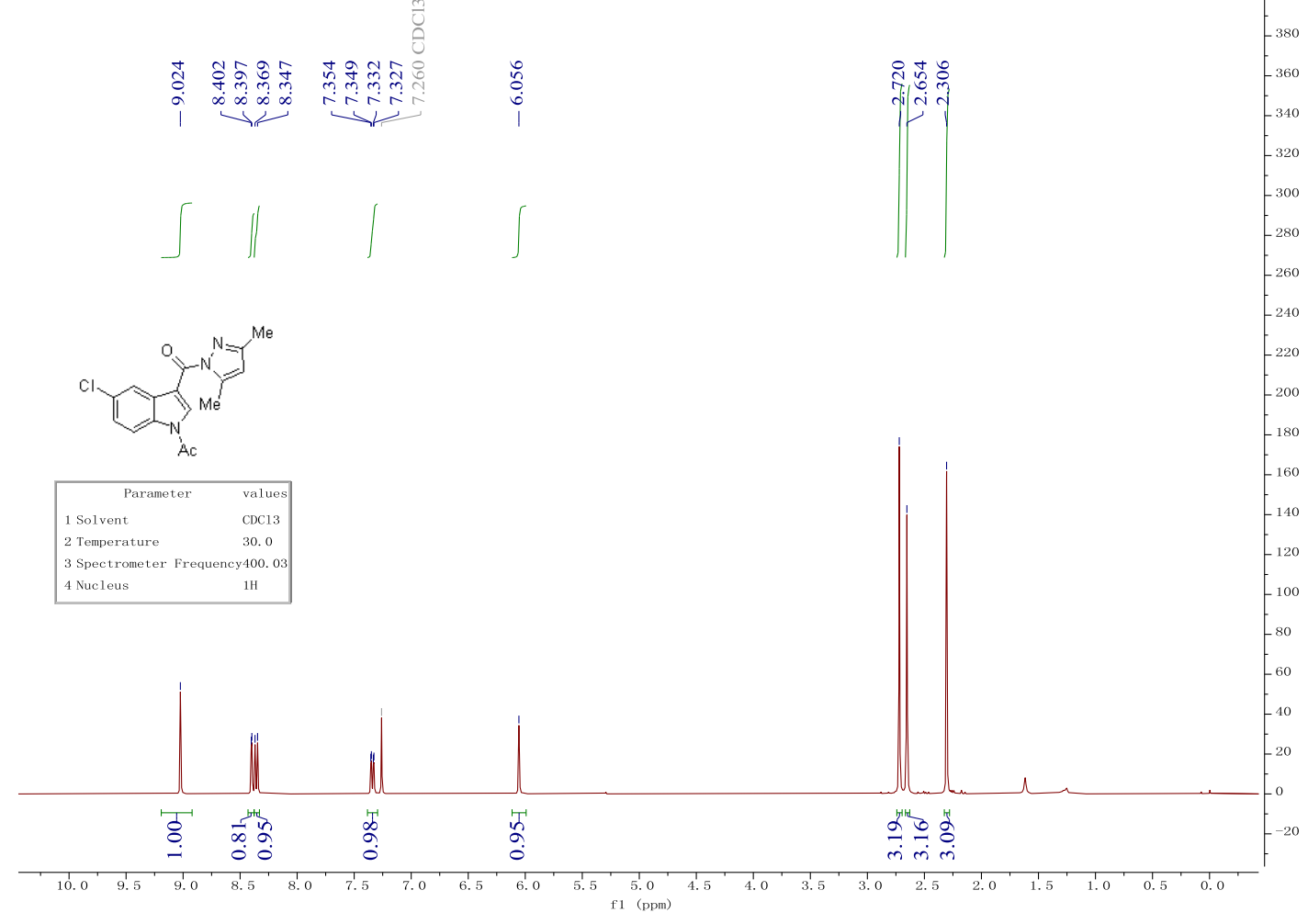

${ }^{13} \mathrm{C}$ NMR Spectrum of $\mathbf{1} \mathbf{b}^{\prime}$
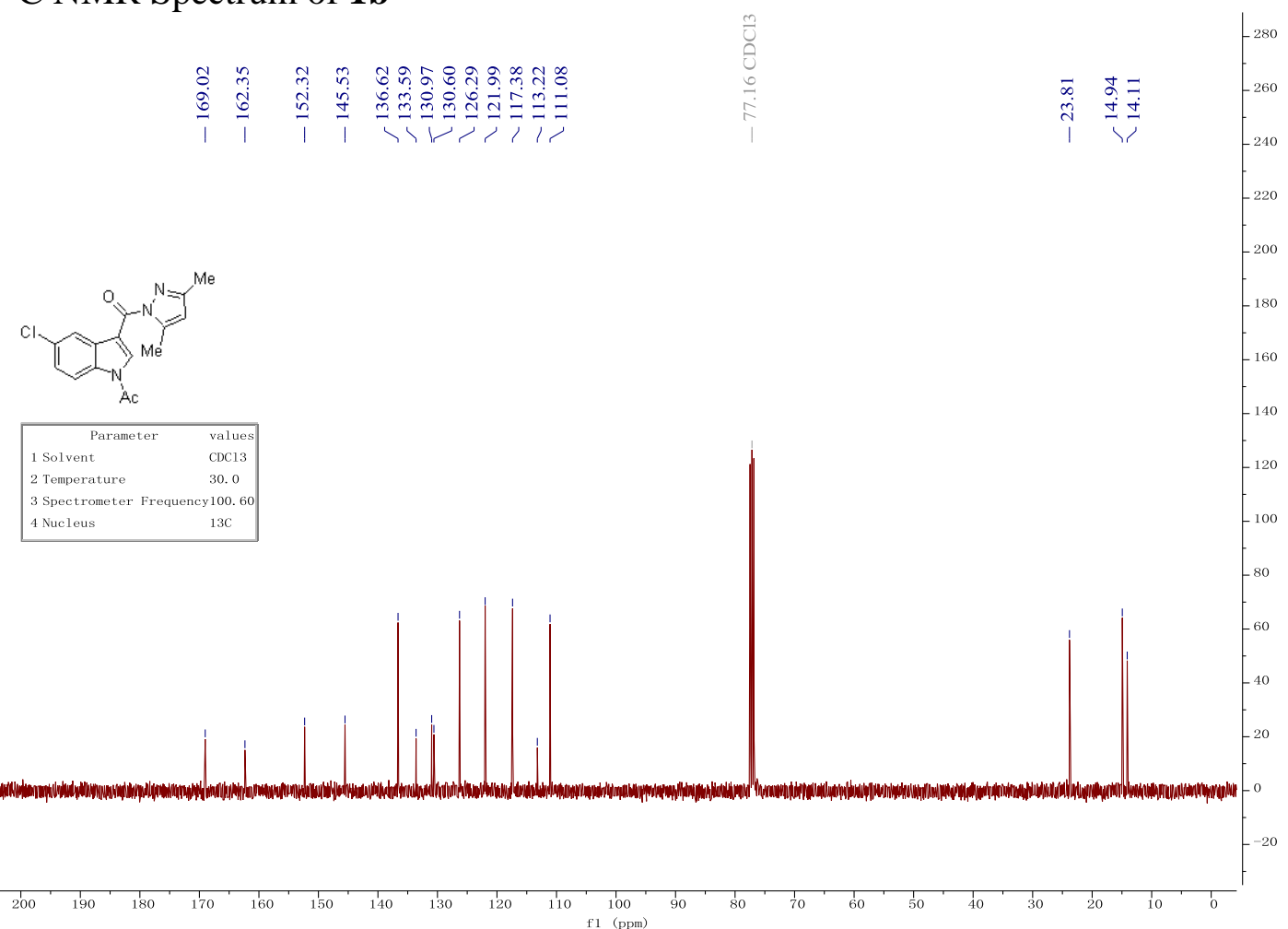


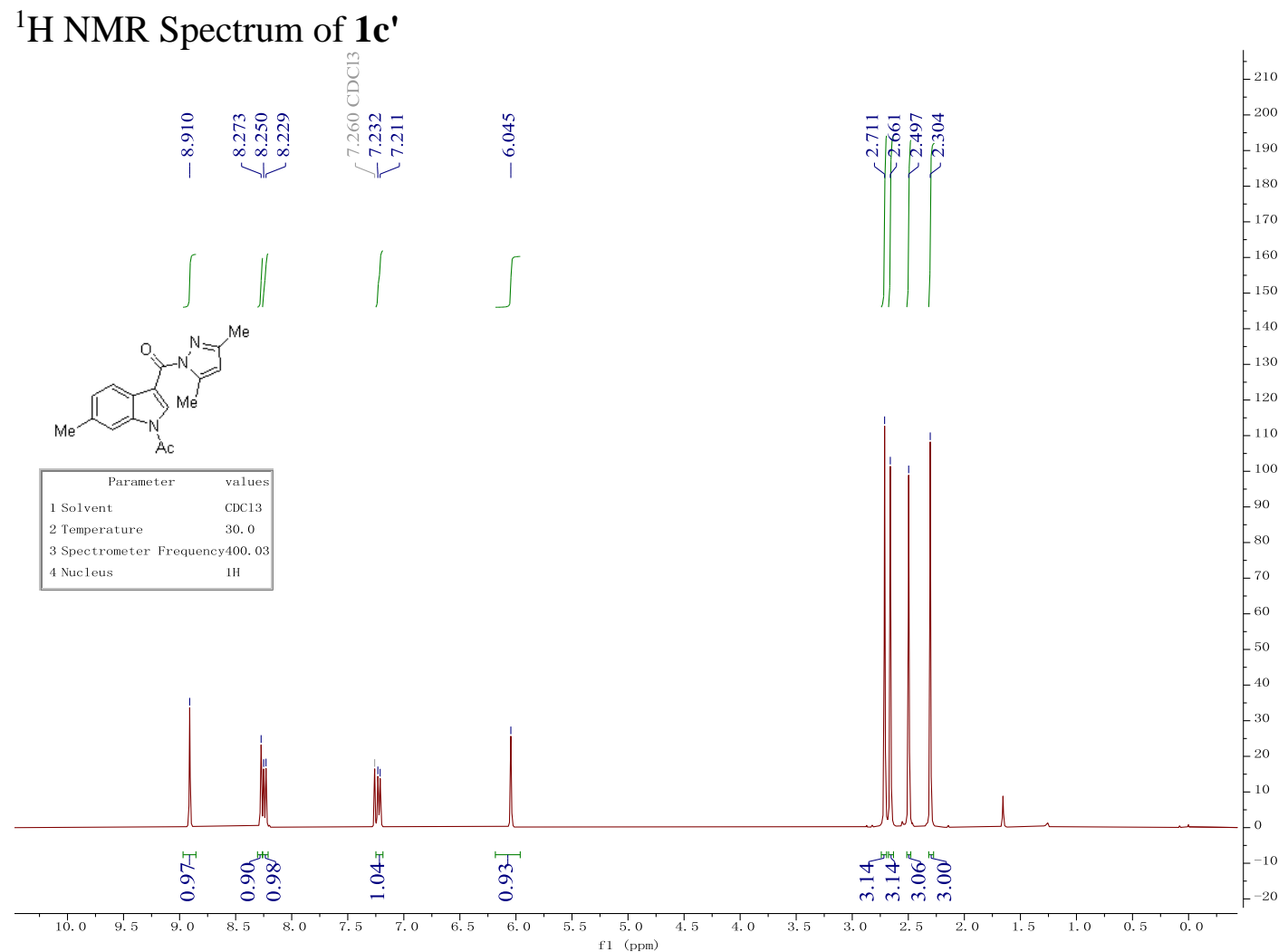

${ }^{13} \mathrm{C}$ NMR Spectrum of $\mathbf{1} \mathbf{c}^{\prime}$

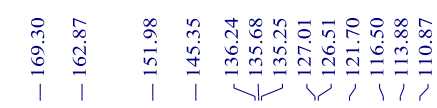

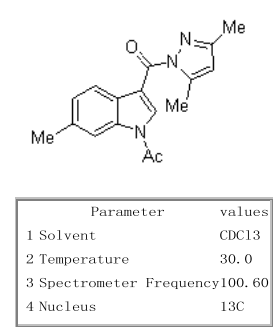


${ }^{1} \mathrm{H}$ NMR Spectrum of $\mathbf{1 d}$

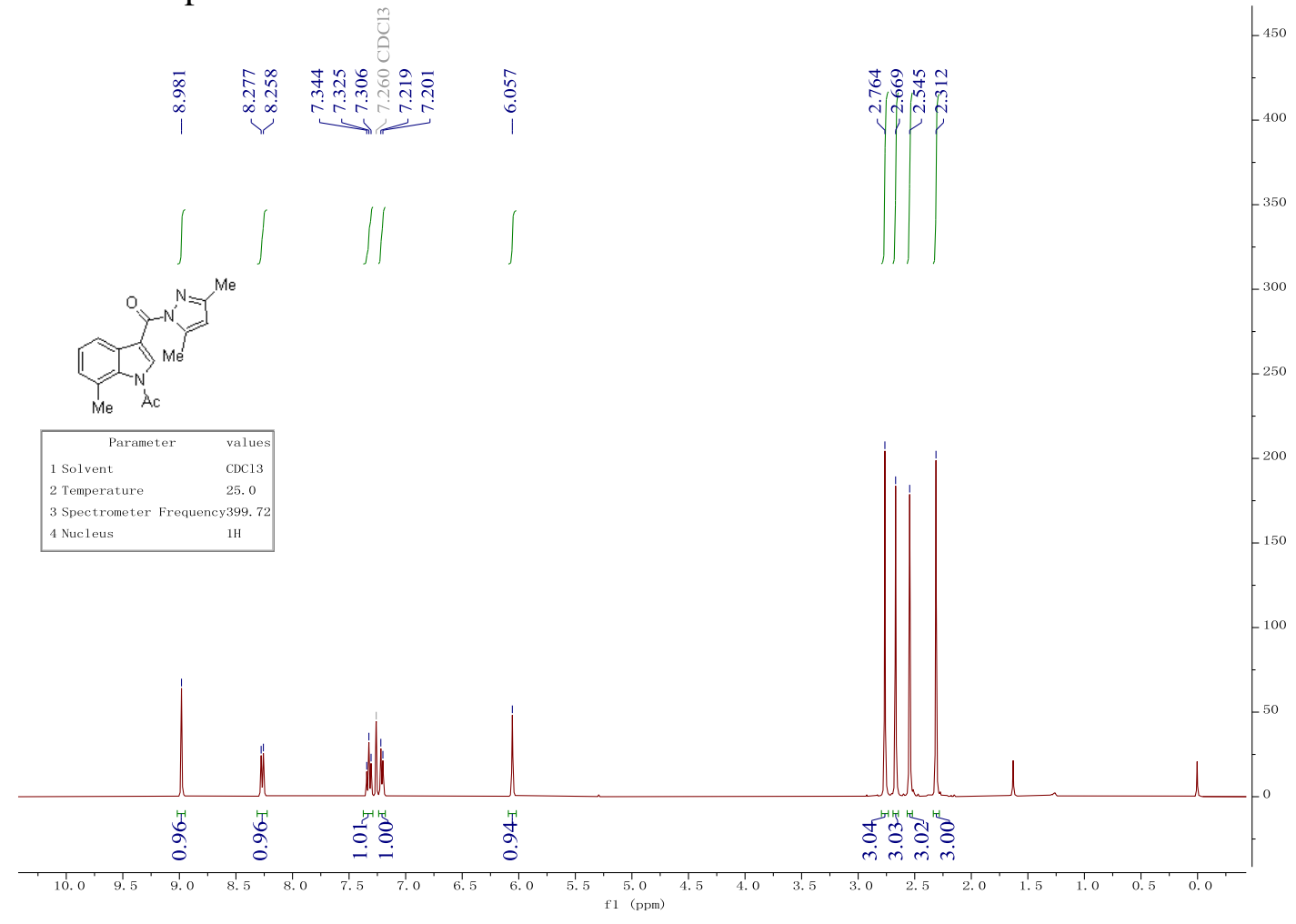

${ }^{13} \mathrm{C}$ NMR Spectrum of $\mathbf{1 d}$

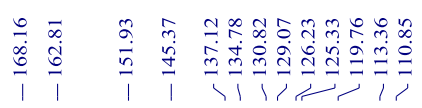
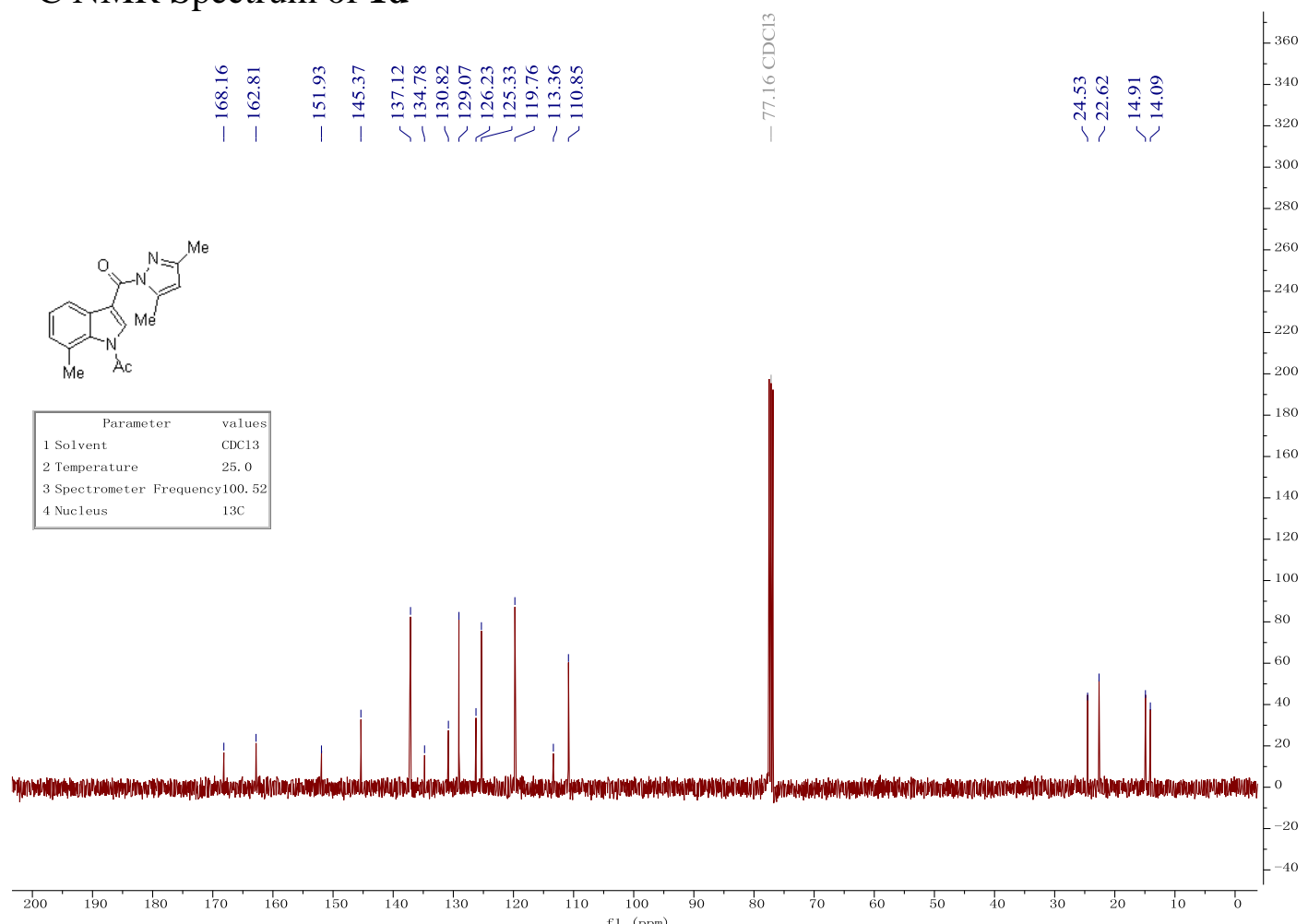
${ }^{1} \mathrm{H}$ NMR Spectrum of $\mathbf{1} \mathbf{e}^{\prime}$

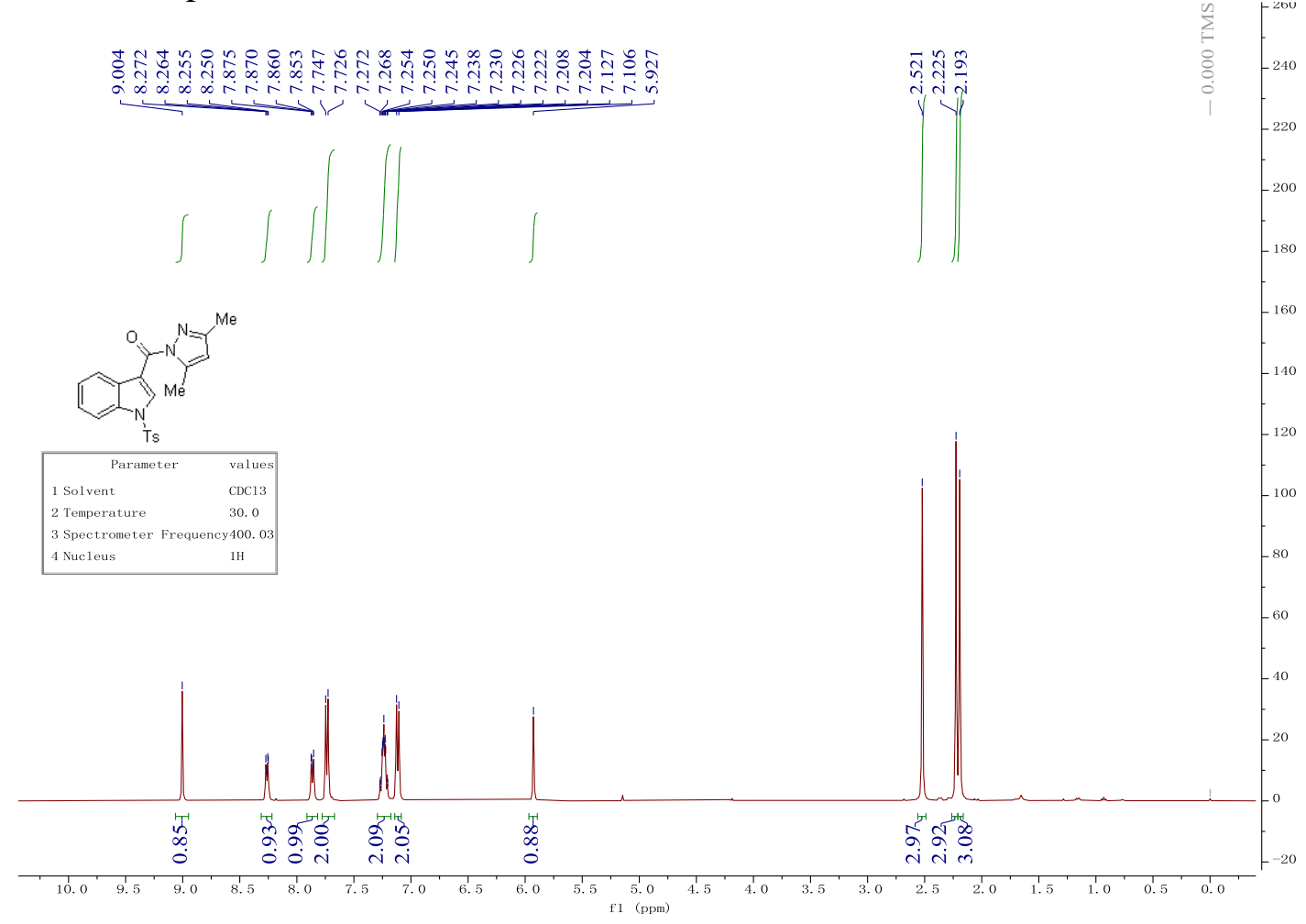

${ }^{13} \mathrm{C}$ NMR Spectrum of $\mathbf{1 e}$

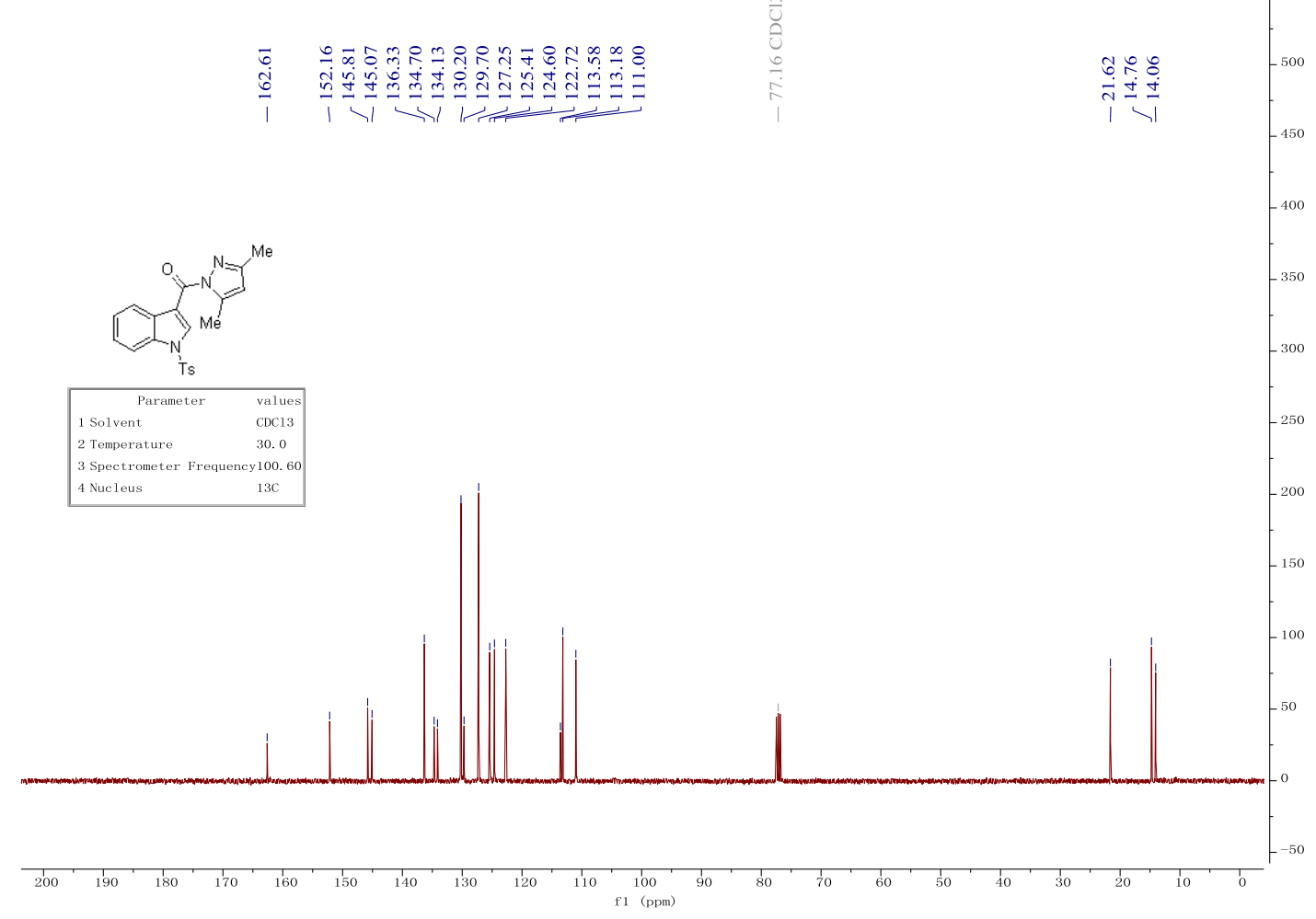


${ }^{1} \mathrm{H}$ NMR Spectrum of $\mathbf{1 f}^{\mathbf{\prime}}$

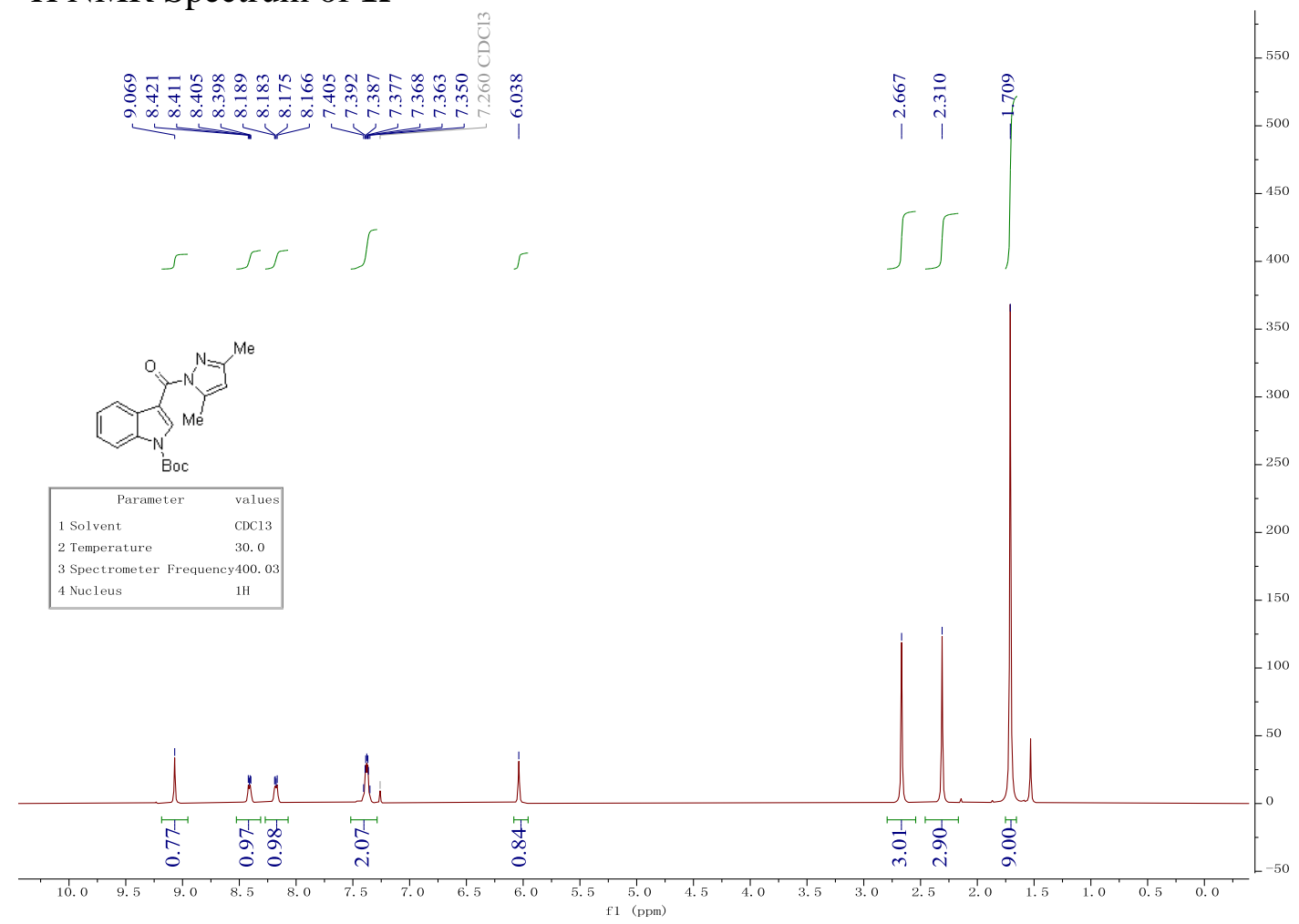

${ }^{13} \mathrm{C}$ NMR Spectrum of $\mathbf{1 f}^{\mathbf{4}}$
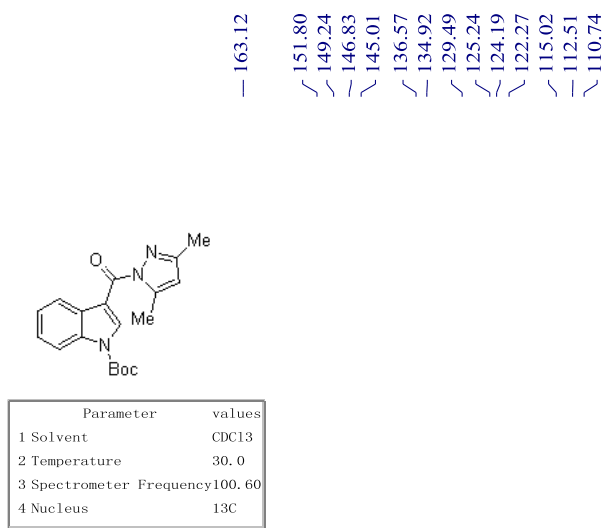

values
CDC13
30.0
100.60
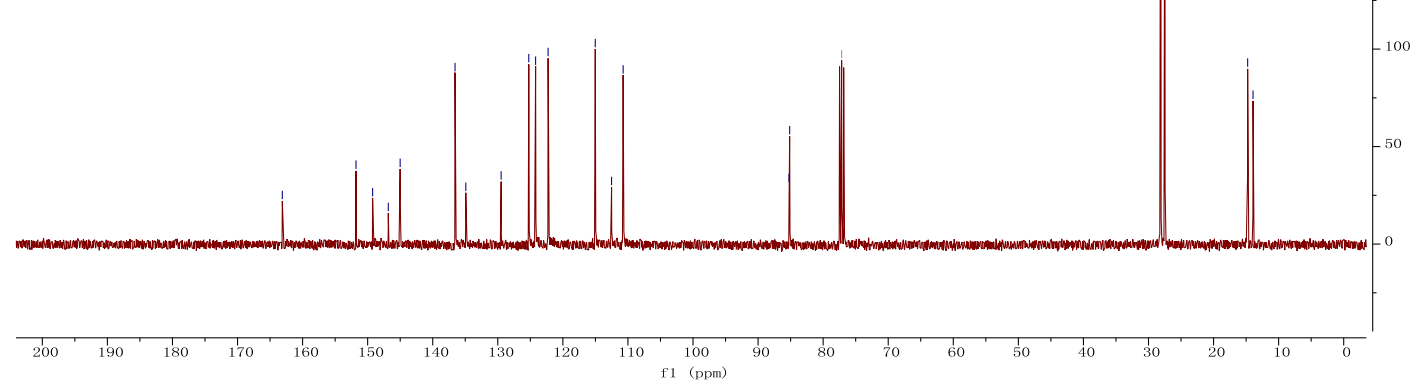
${ }^{1} \mathrm{H}$ NMR Spectrum of $\mathbf{3 a}$

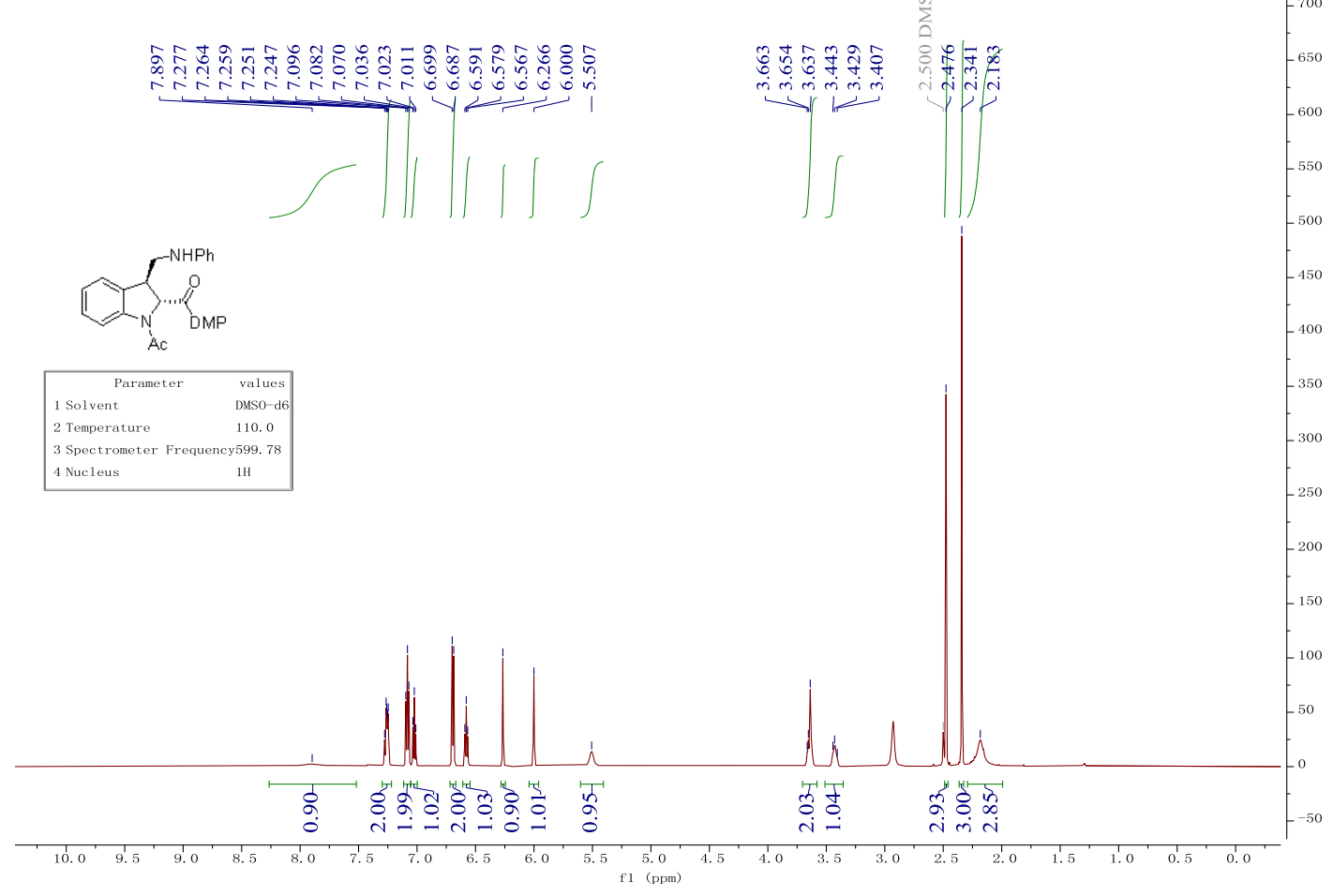

${ }^{13} \mathrm{C}$ NMR Spectrum of $\mathbf{3 a}$

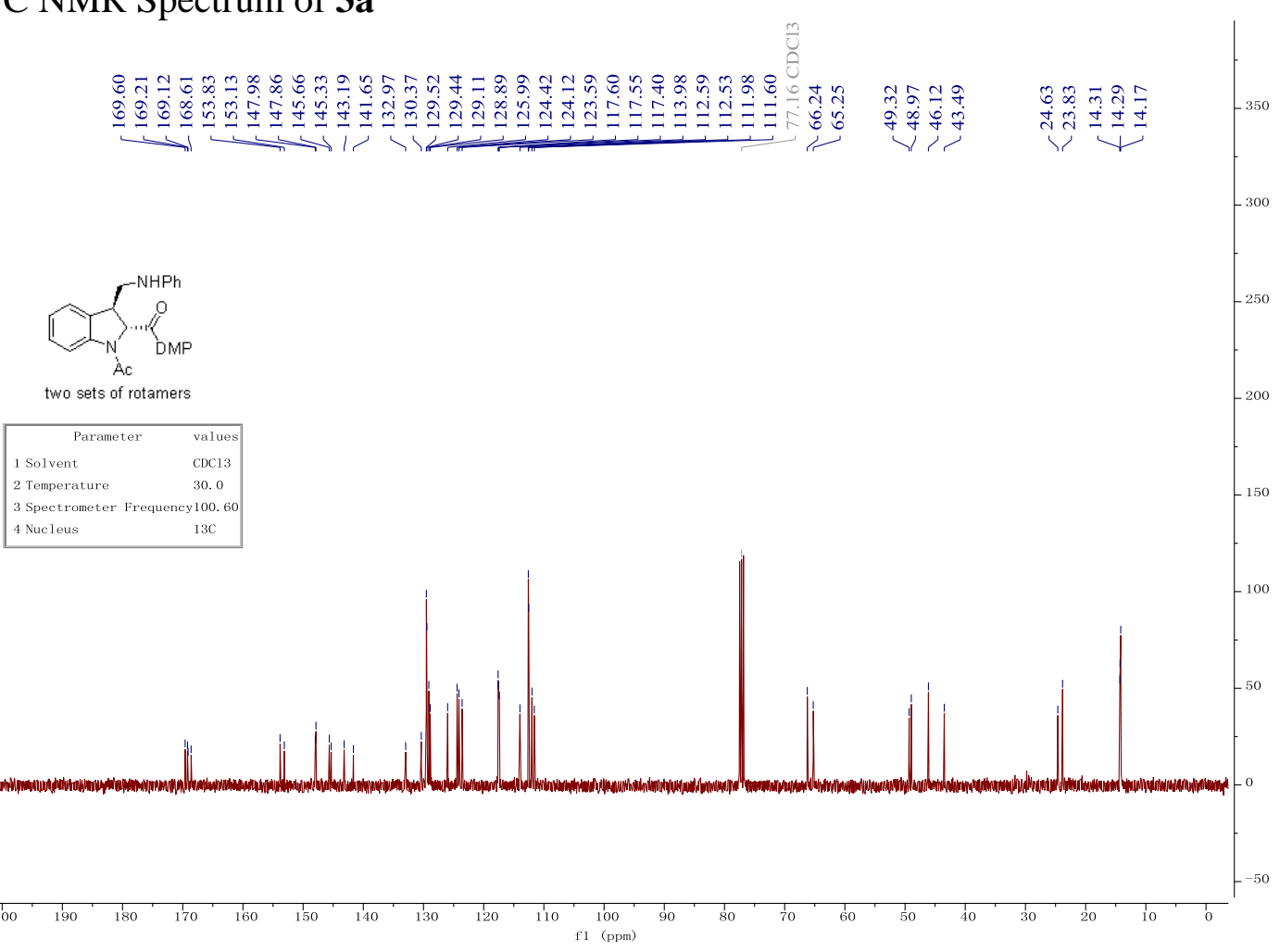


${ }^{1} \mathrm{H}$ NMR Spectrum of $\mathbf{3 b}$

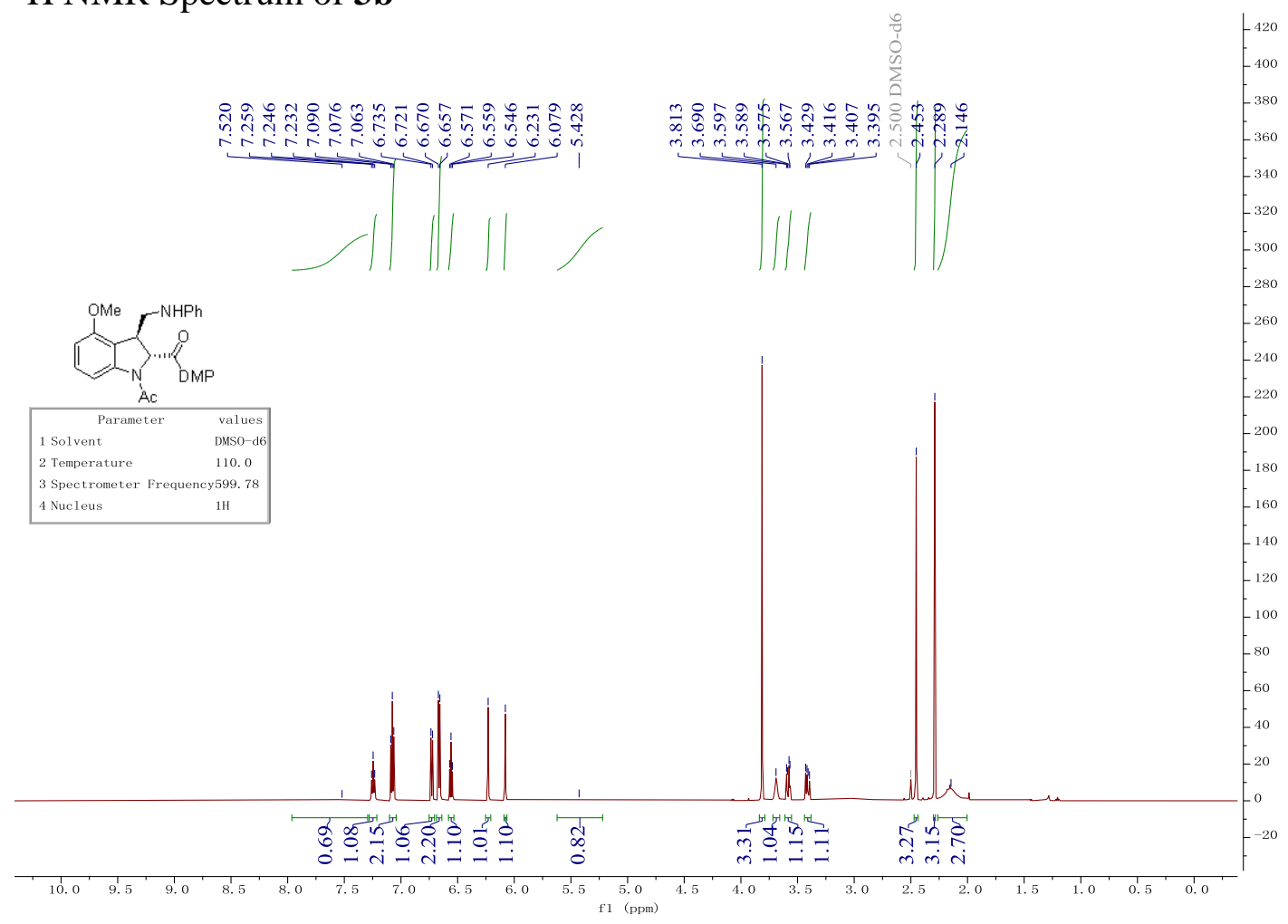

${ }^{13} \mathrm{C}$ NMR Spectrum of $\mathbf{3 b}$

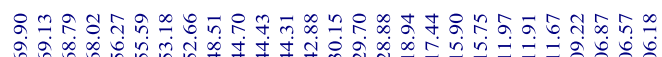

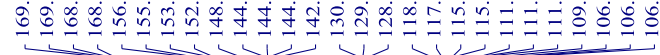

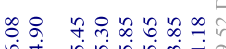

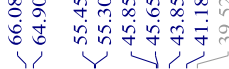

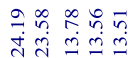
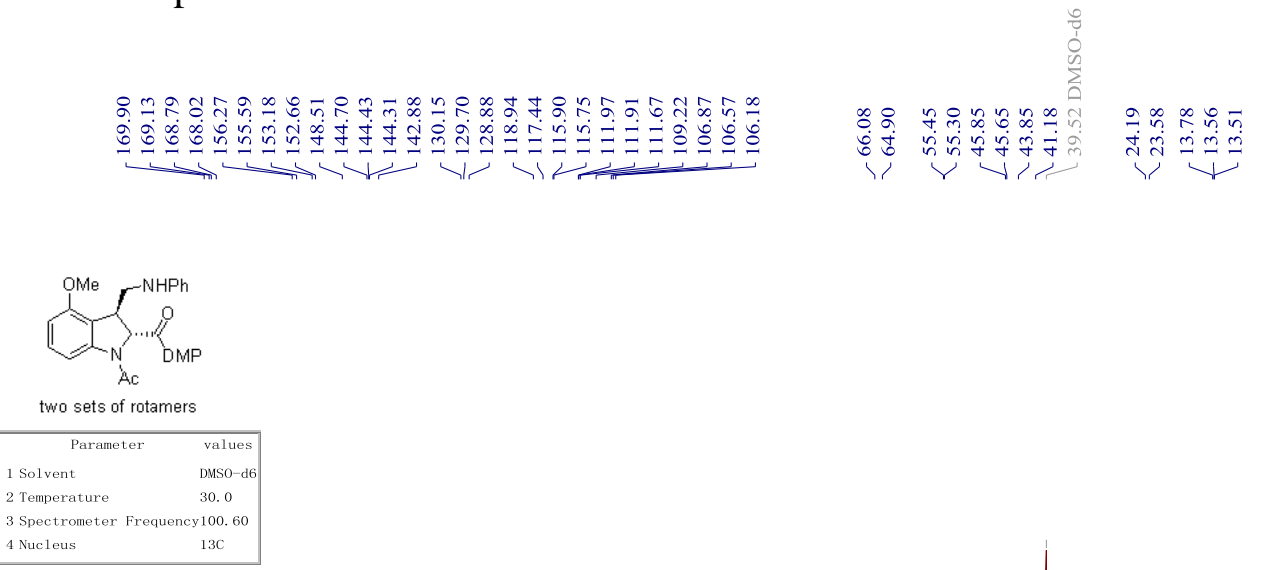

$13 \mathrm{C}$

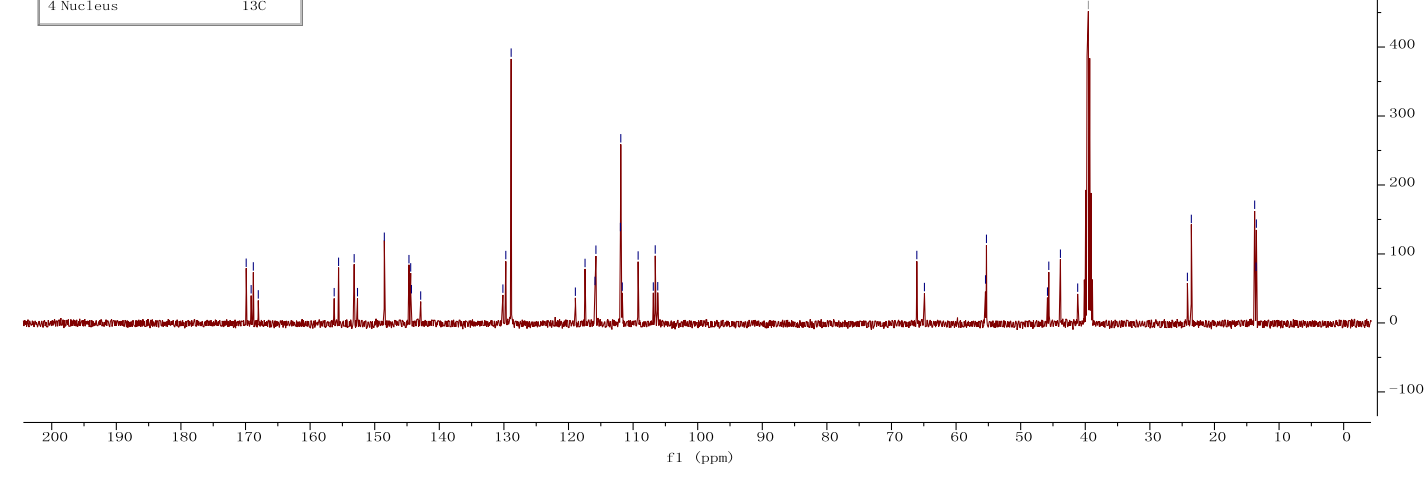


${ }^{1} \mathrm{H}$ NMR Spectrum of $\mathbf{3 c}$

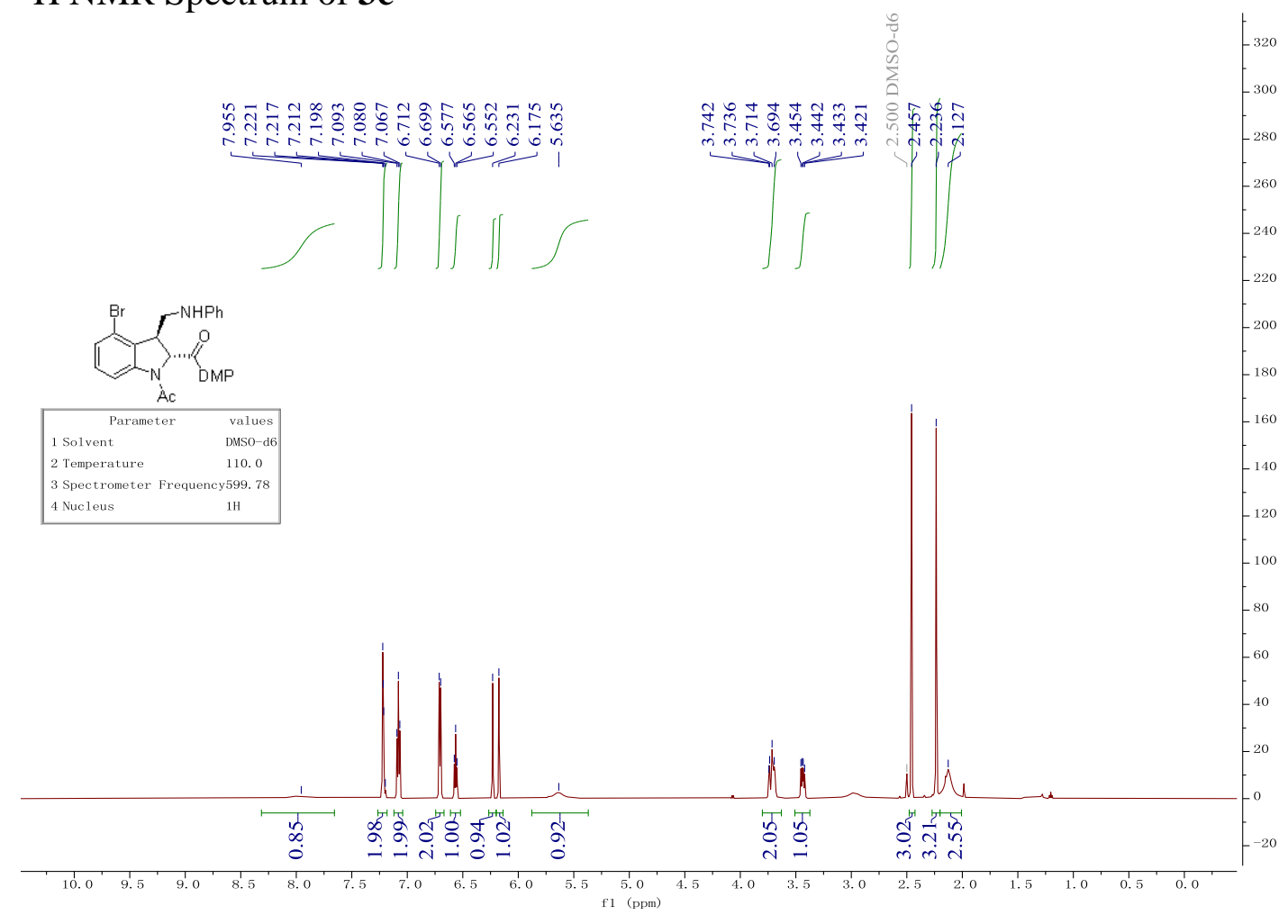

${ }^{13} \mathrm{C}$ NMR Spectrum of $\mathbf{3 c}$

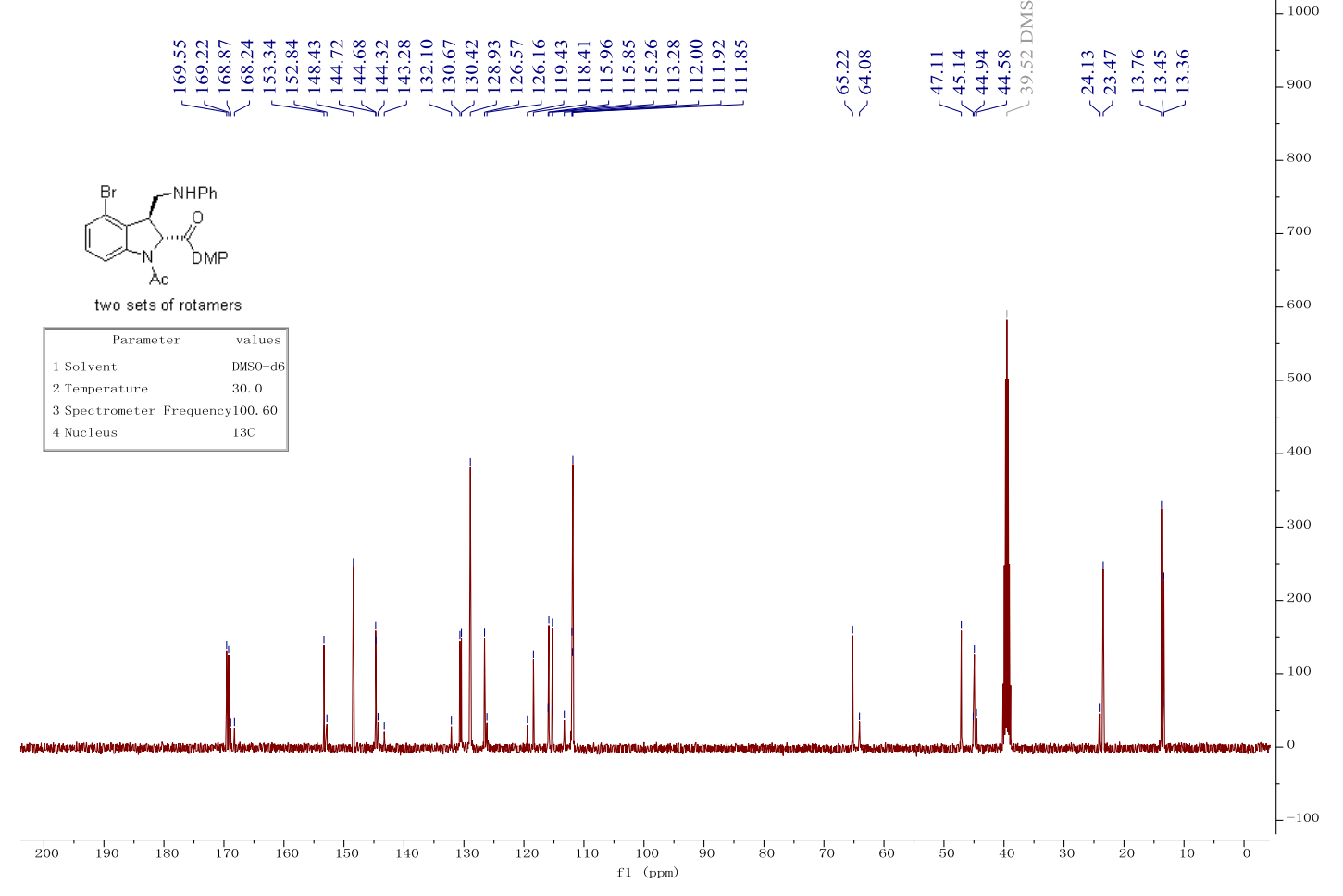


${ }^{1} \mathrm{H}$ NMR Spectrum of $\mathbf{3 d}$

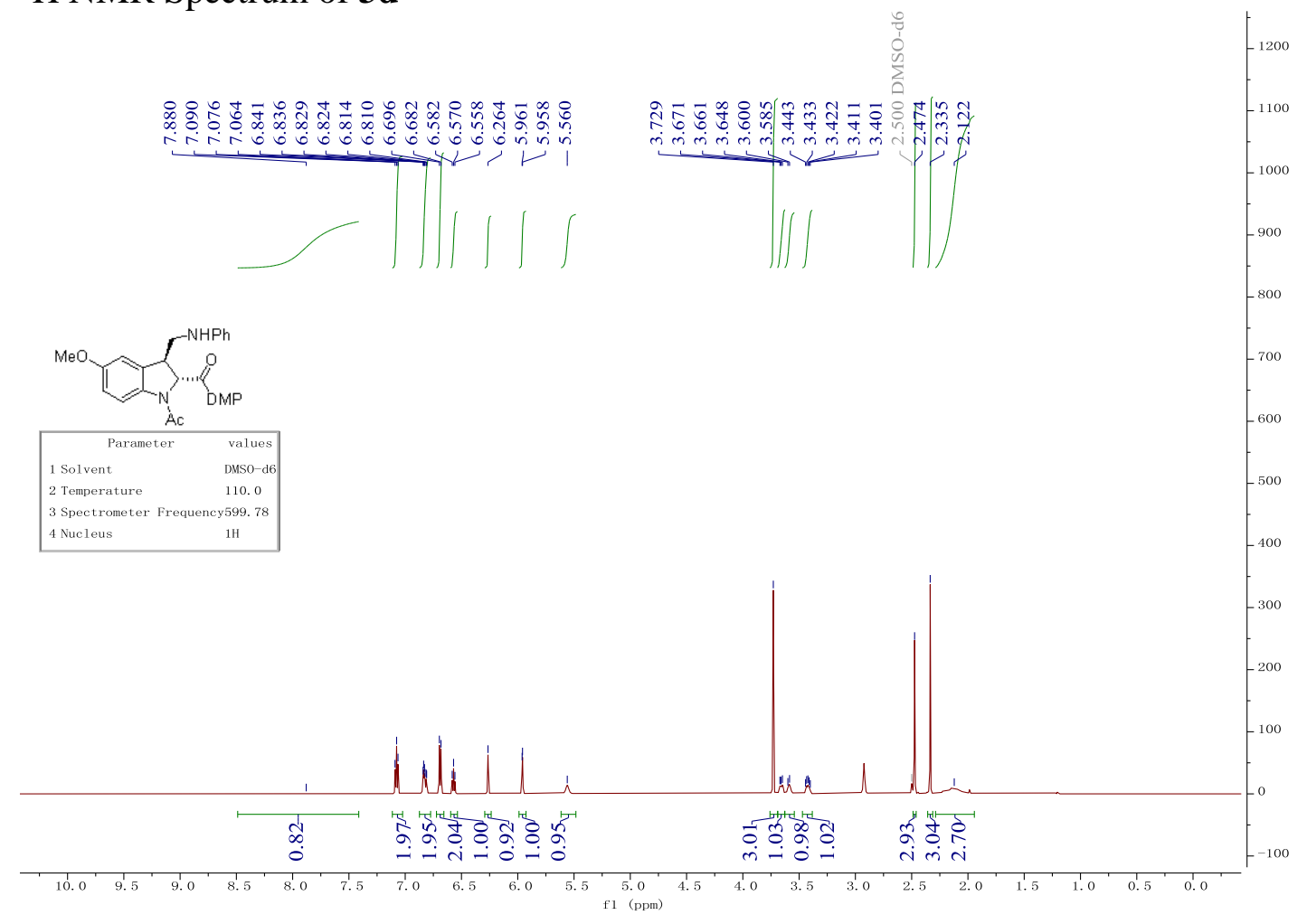

${ }^{13} \mathrm{C}$ NMR Spectrum of $\mathbf{3 d}$

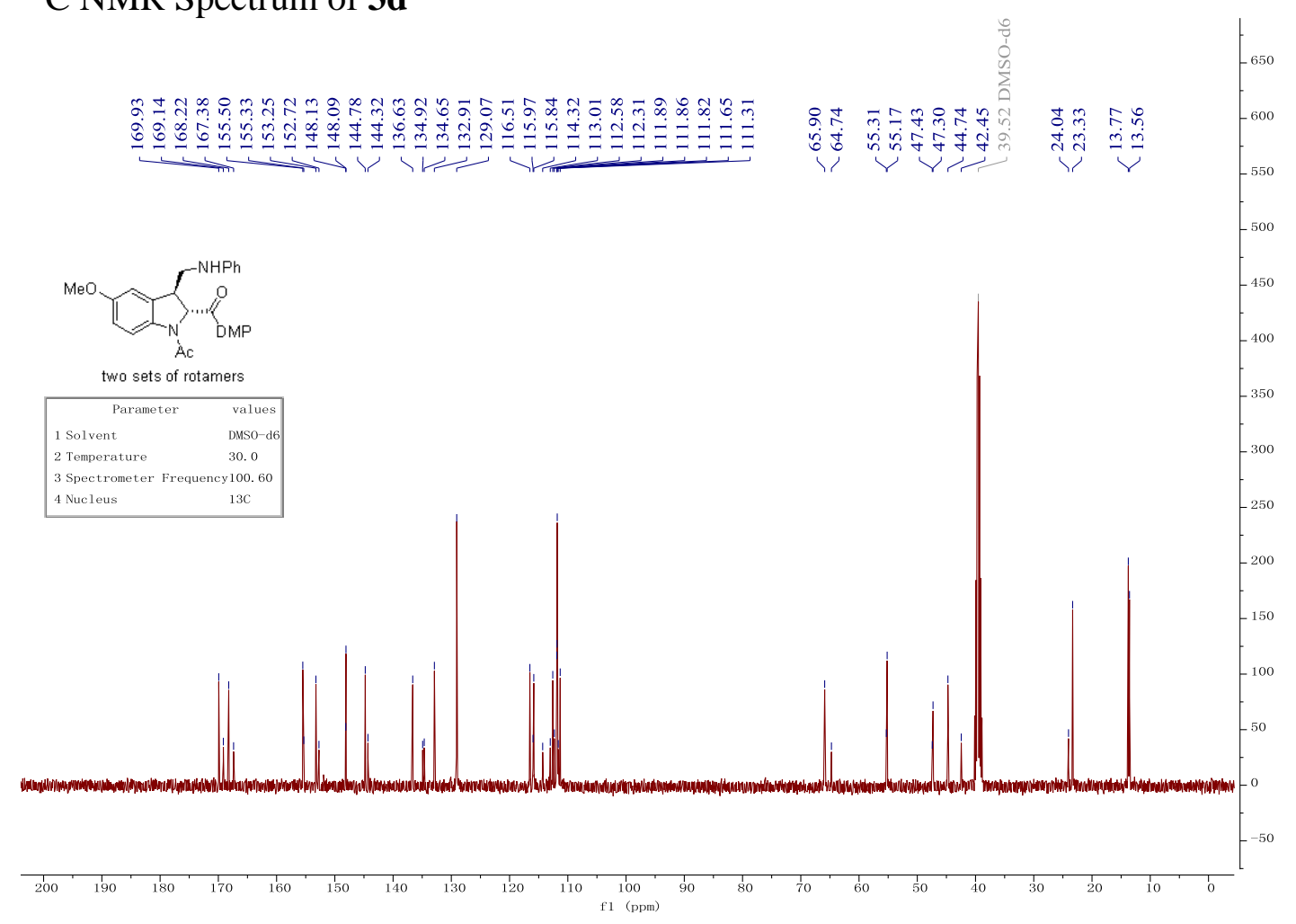


${ }^{1} \mathrm{H}$ NMR Spectrum of $\mathbf{3 e}$

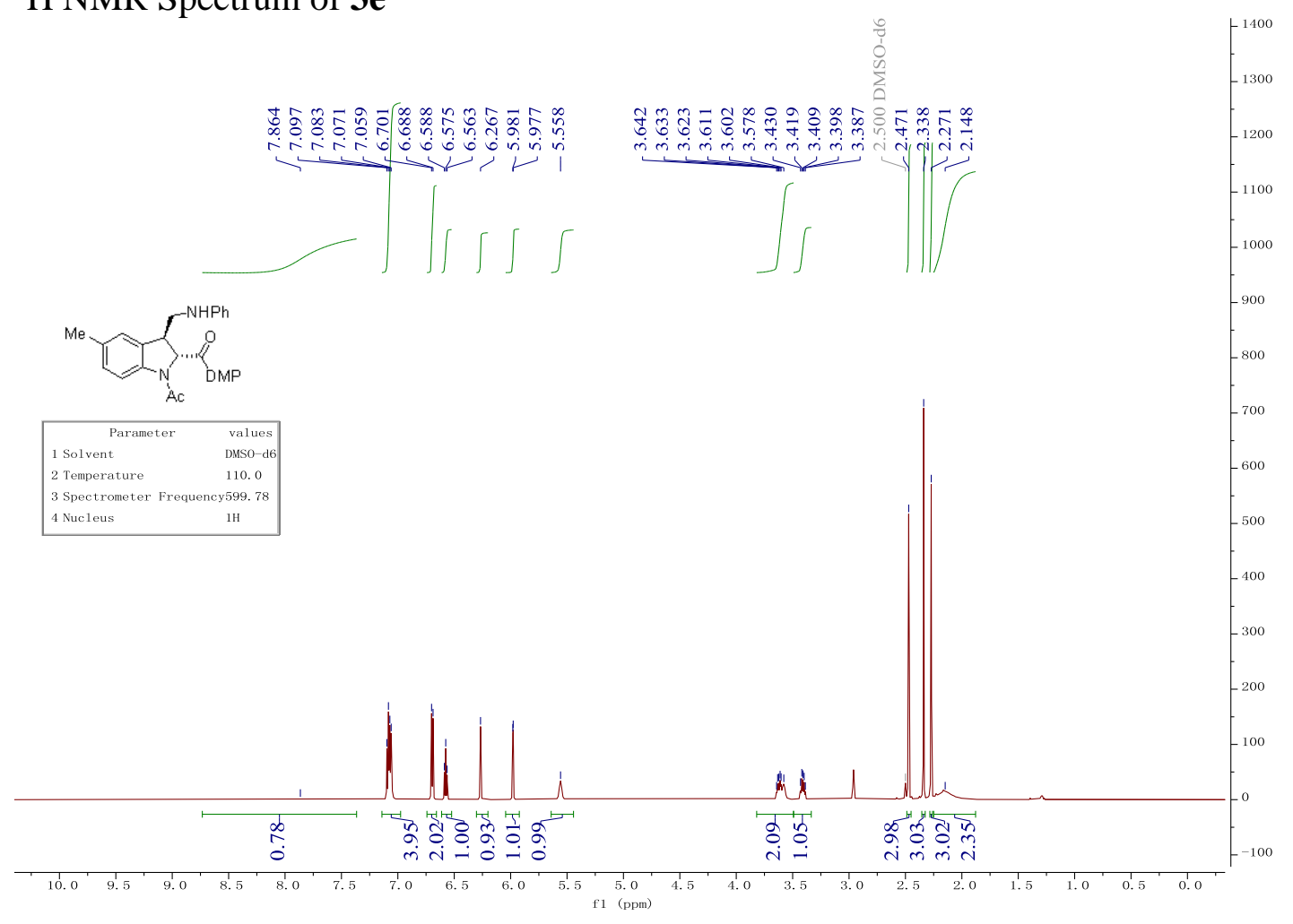

${ }^{13} \mathrm{C}$ NMR Spectrum of $\mathbf{3 e}$

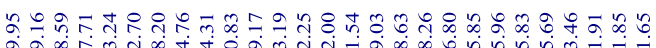

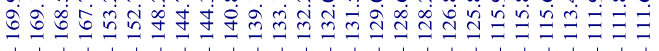

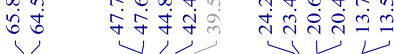

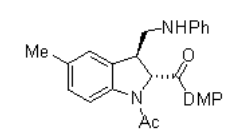

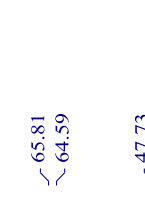

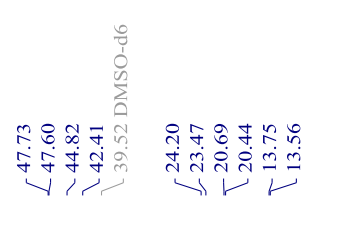

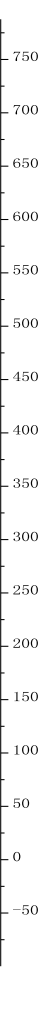


${ }^{1} \mathrm{H}$ NMR Spectrum of $\mathbf{3 f}$
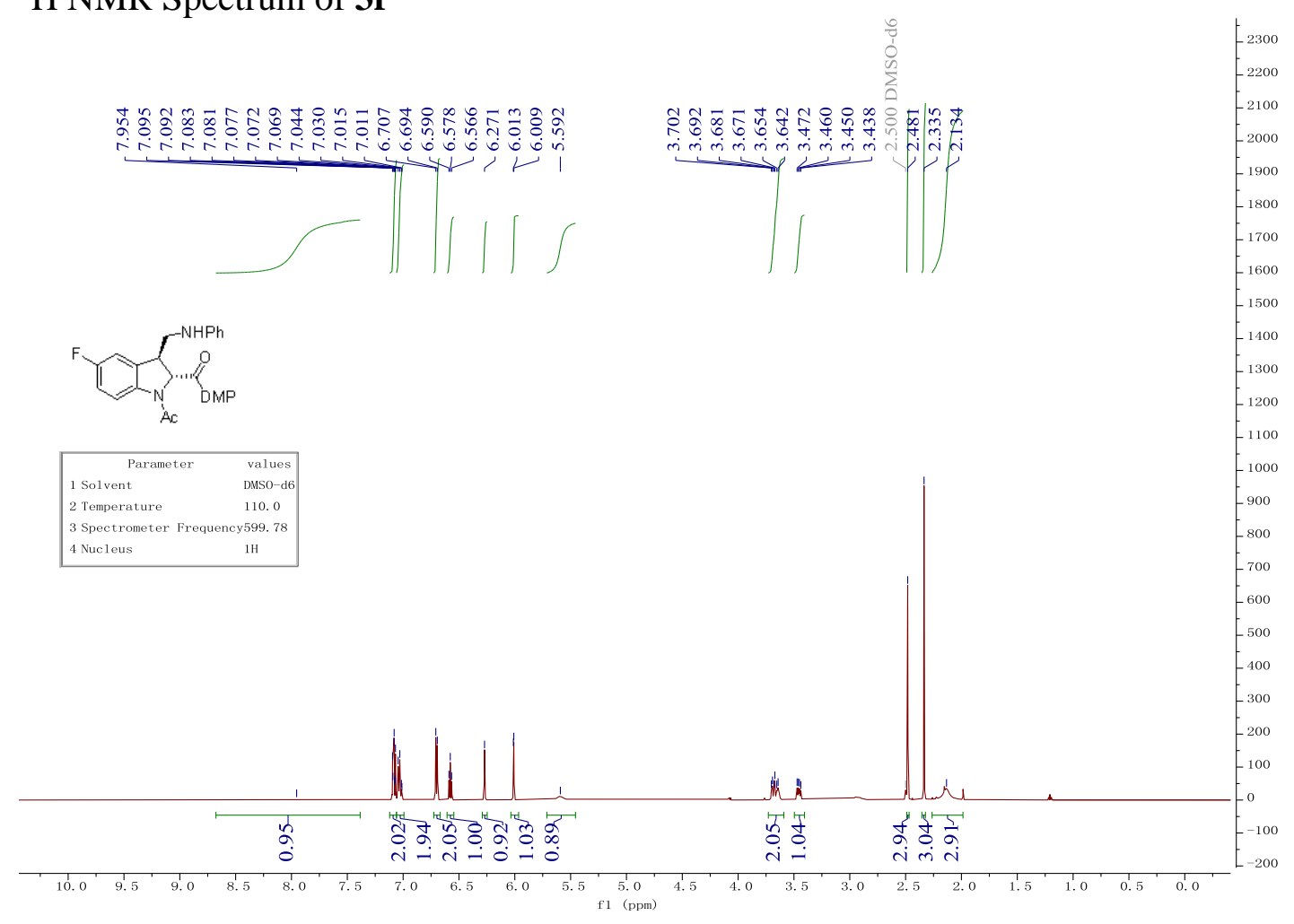

\section{${ }^{13} \mathrm{C}$ NMR Spectrum of $\mathbf{3 f}$}

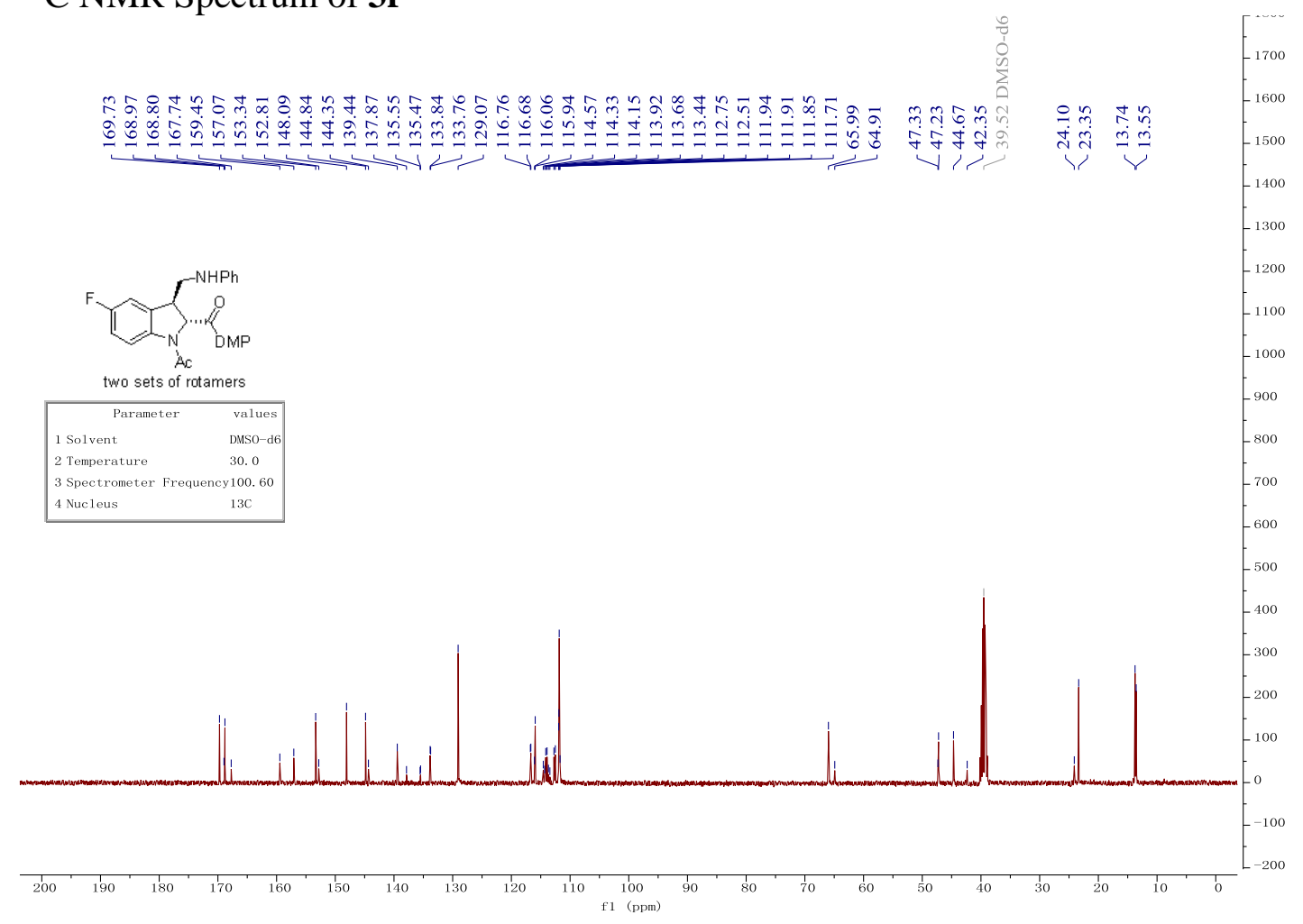




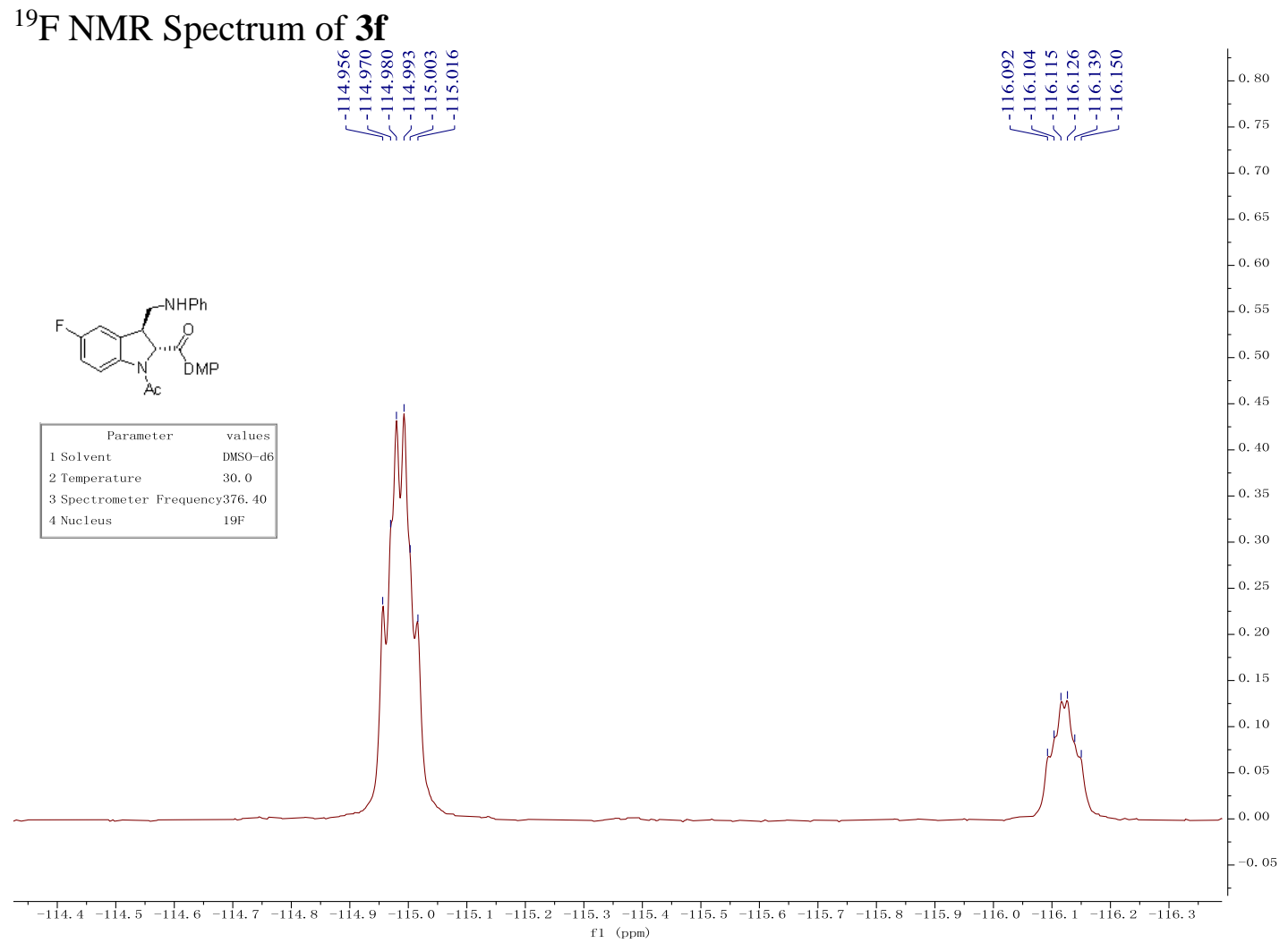

${ }^{1} \mathrm{H}$ NMR Spectrum of $\mathbf{3 g}$

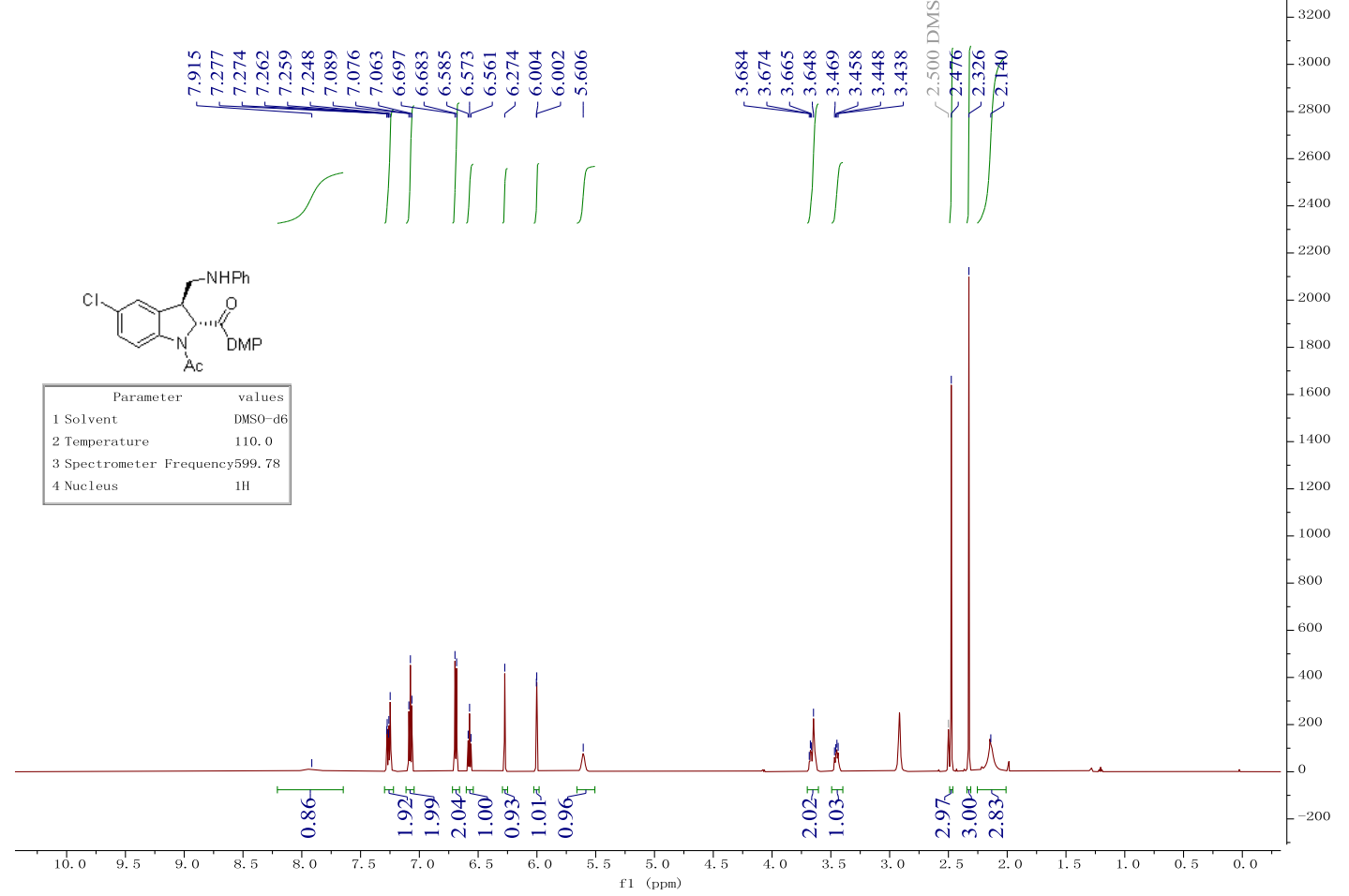


${ }^{13} \mathrm{C}$ NMR Spectrum of $\mathbf{3 g}$

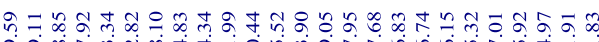

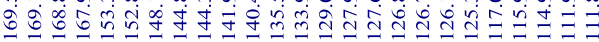

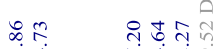

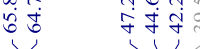

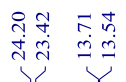

$-100$

(N)

two sets of rotamers

\begin{tabular}{ll}
\multicolumn{1}{r}{ Parameter } & \multicolumn{1}{c}{ values } \\
1 Solvent & DMSO-d6 \\
2 Temperature & 30.0 \\
3 S Specture &
\end{tabular}

3 Spectrometer Frequency 100. 60

$13 \mathrm{C}$

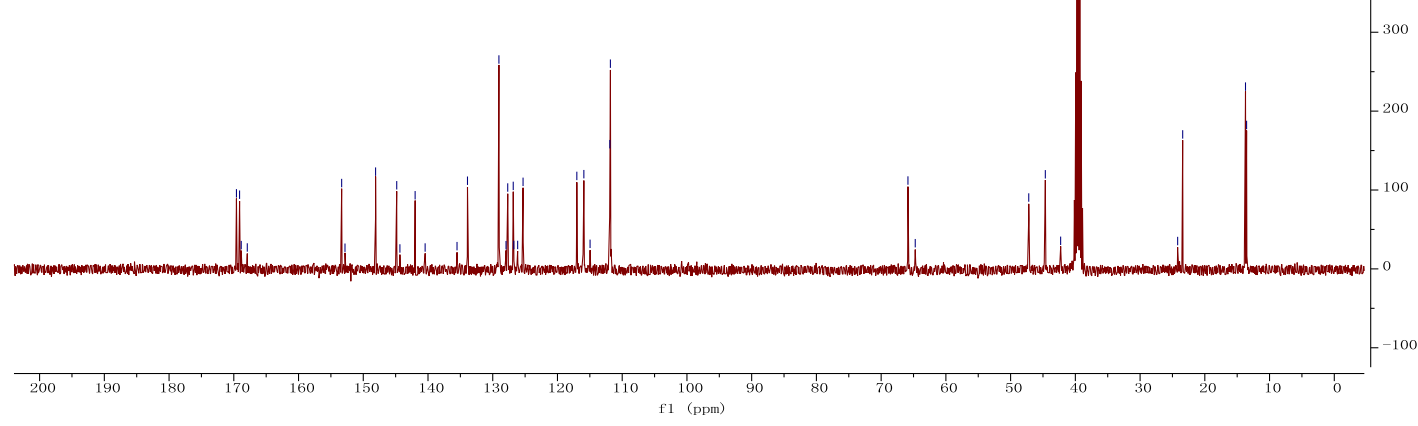

${ }^{1} \mathrm{H}$ NMR Spectrum of $\mathbf{3 h}$
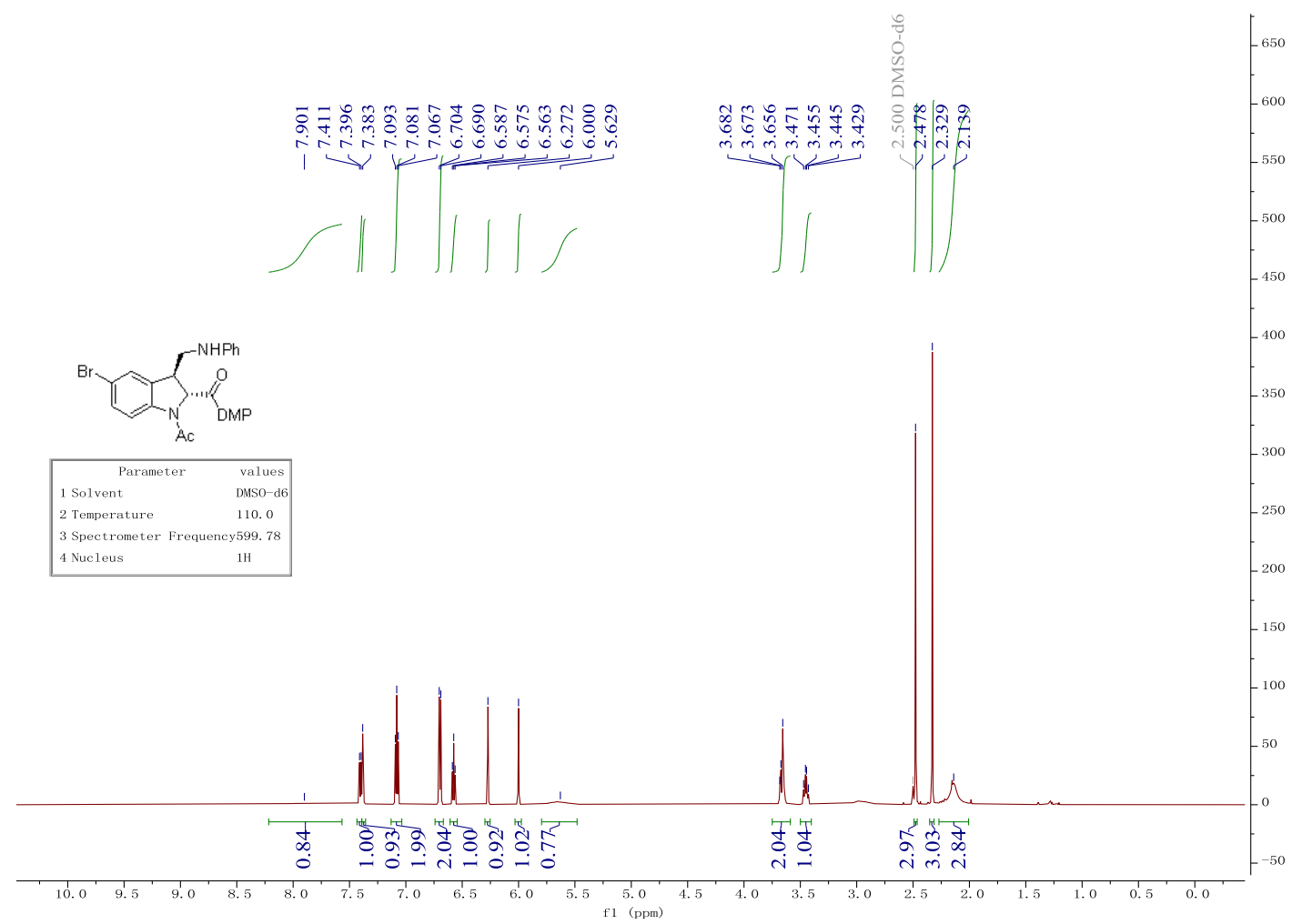
${ }^{13} \mathrm{C}$ NMR Spectrum of $\mathbf{3 h}$

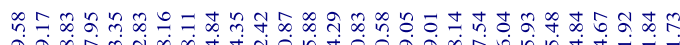

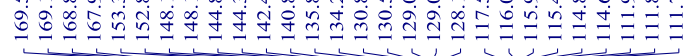

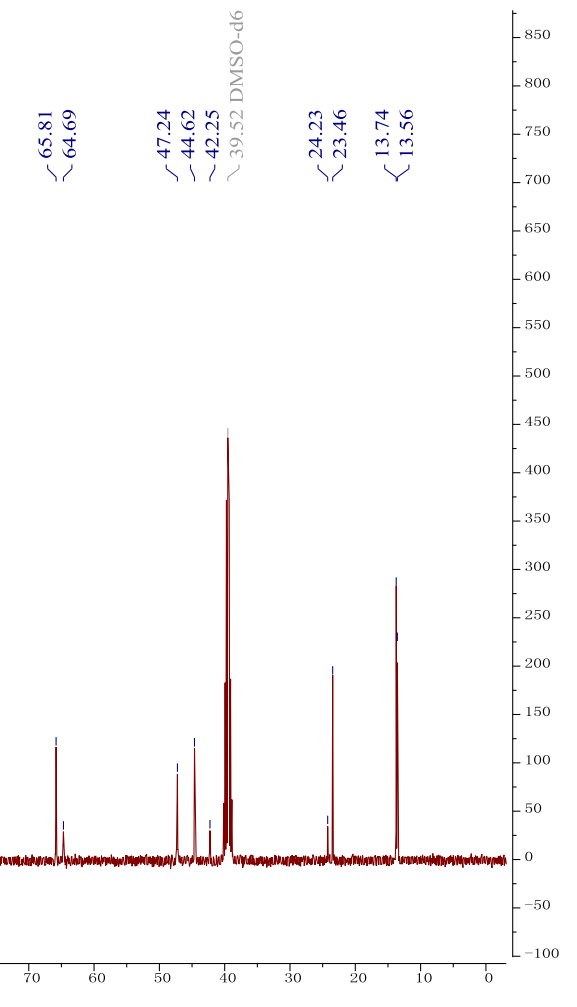

${ }^{1} \mathrm{H}$ NMR Spectrum of $\mathbf{3 i}$

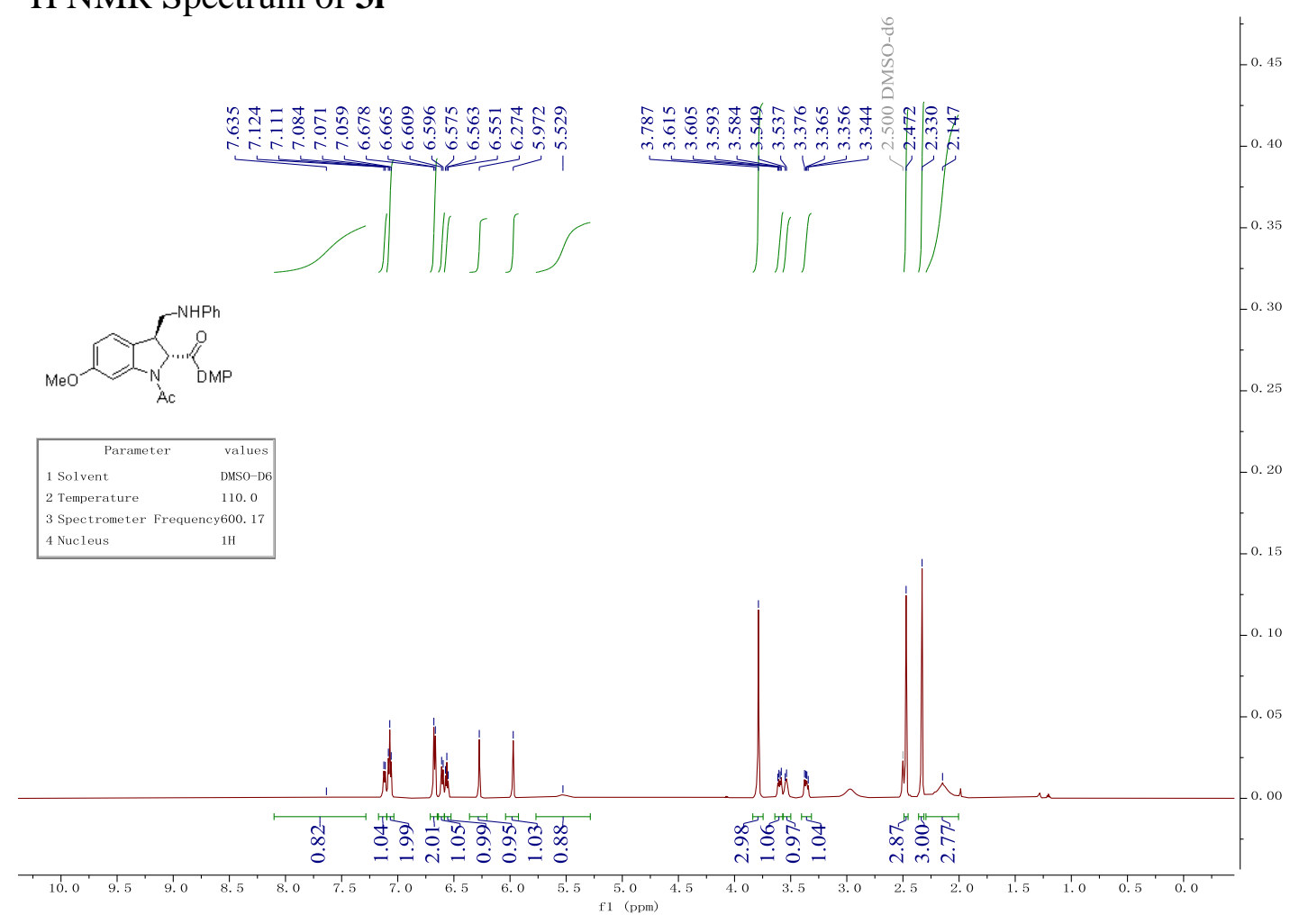


${ }^{13} \mathrm{C}$ NMR Spectrum of $\mathbf{3 i}$

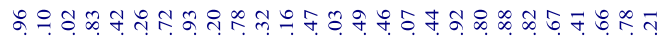

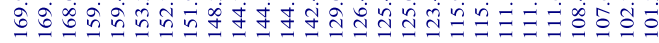

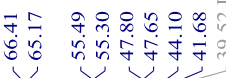

ปูi
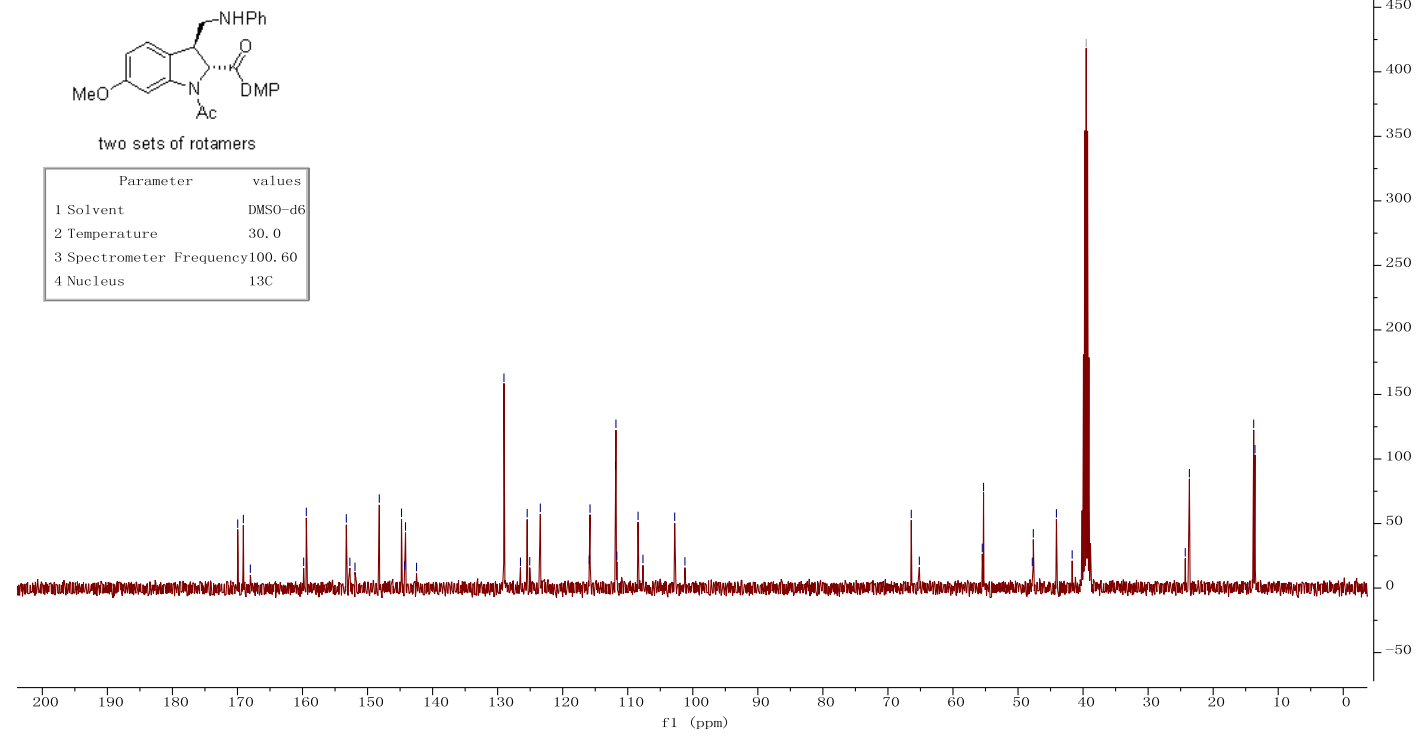

${ }^{1} \mathrm{H}$ NMR Spectrum of $\mathbf{3 j}$
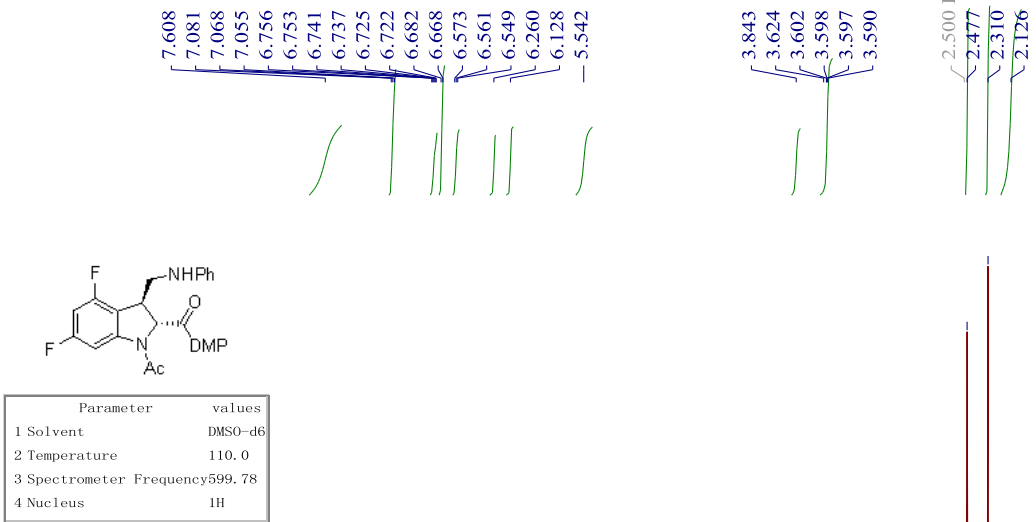

alues
DMSO-d6
10.0
99.78
H

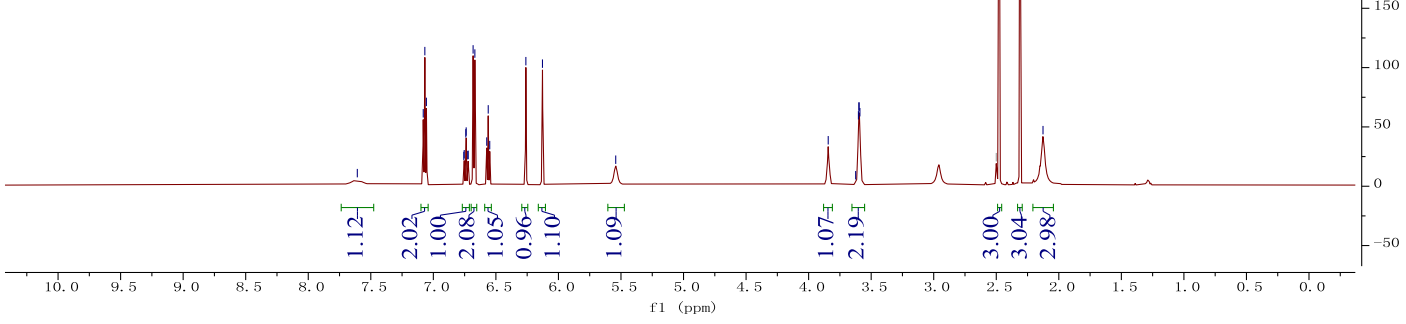


${ }^{13} \mathrm{C}$ NMR Spectrum of $\mathbf{3 j}$

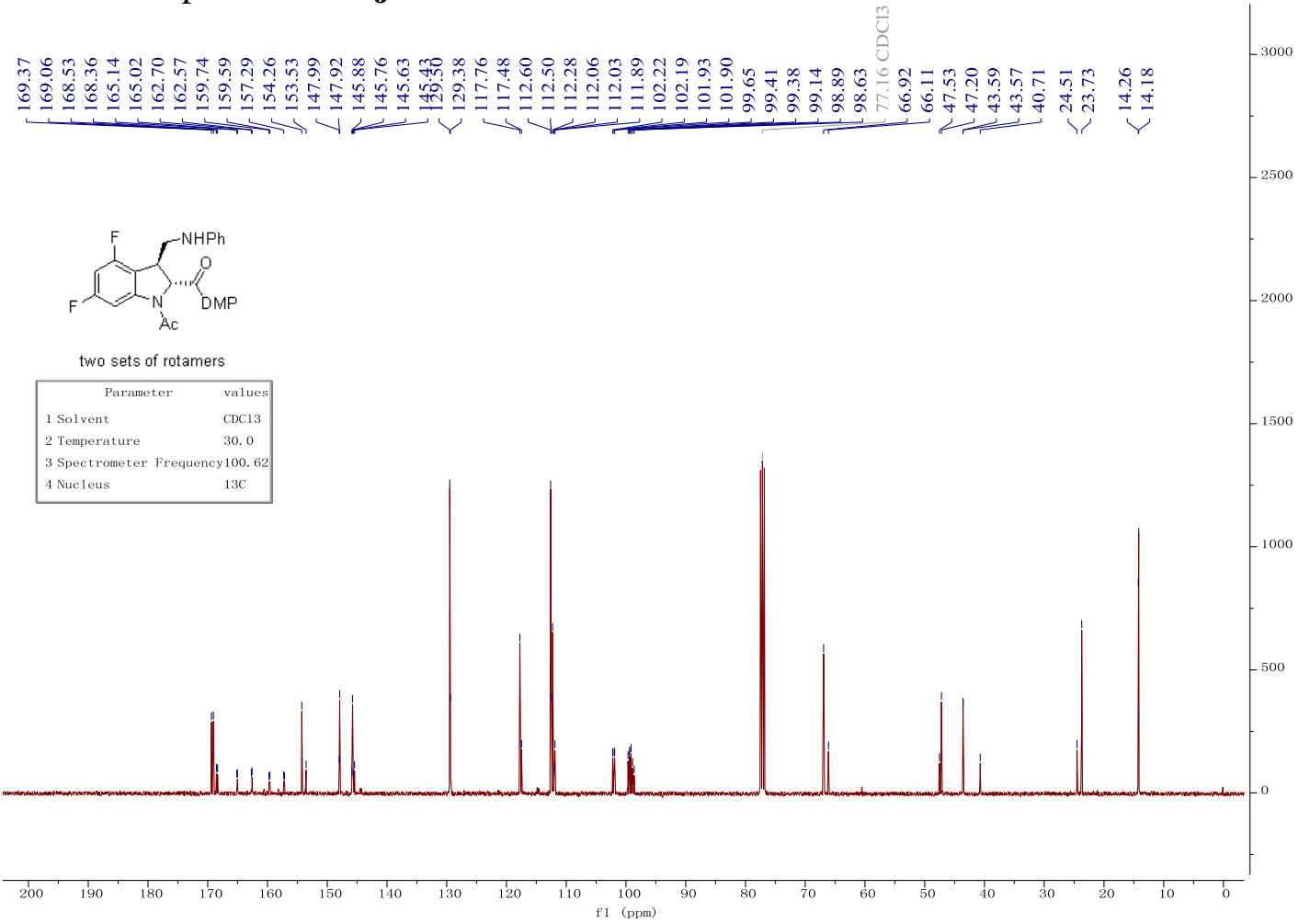

${ }^{19}$ F NMR Spectrum of $\mathbf{3 j}$

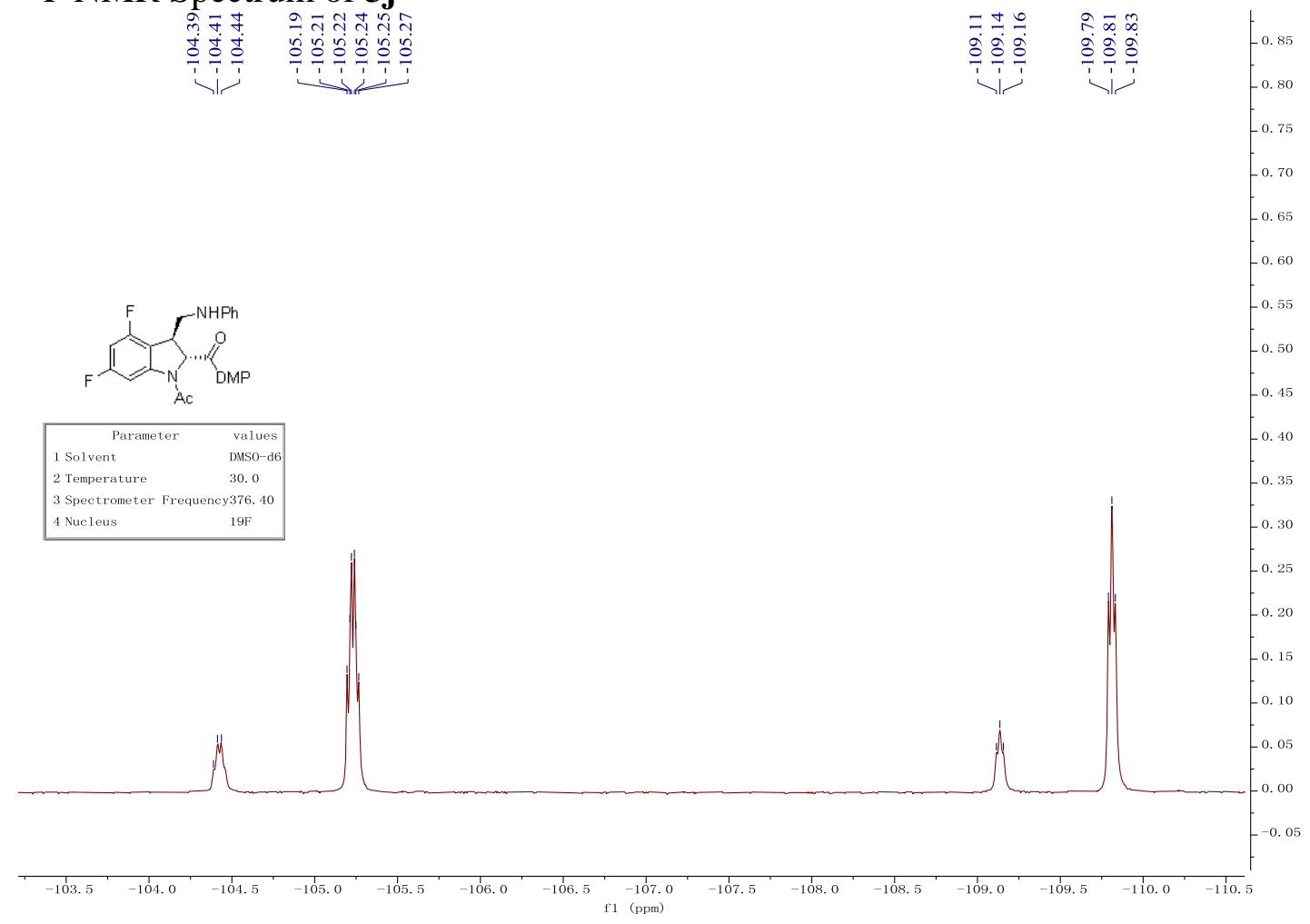


${ }^{1} \mathrm{H}$ NMR Spectrum of $\mathbf{3 k}$

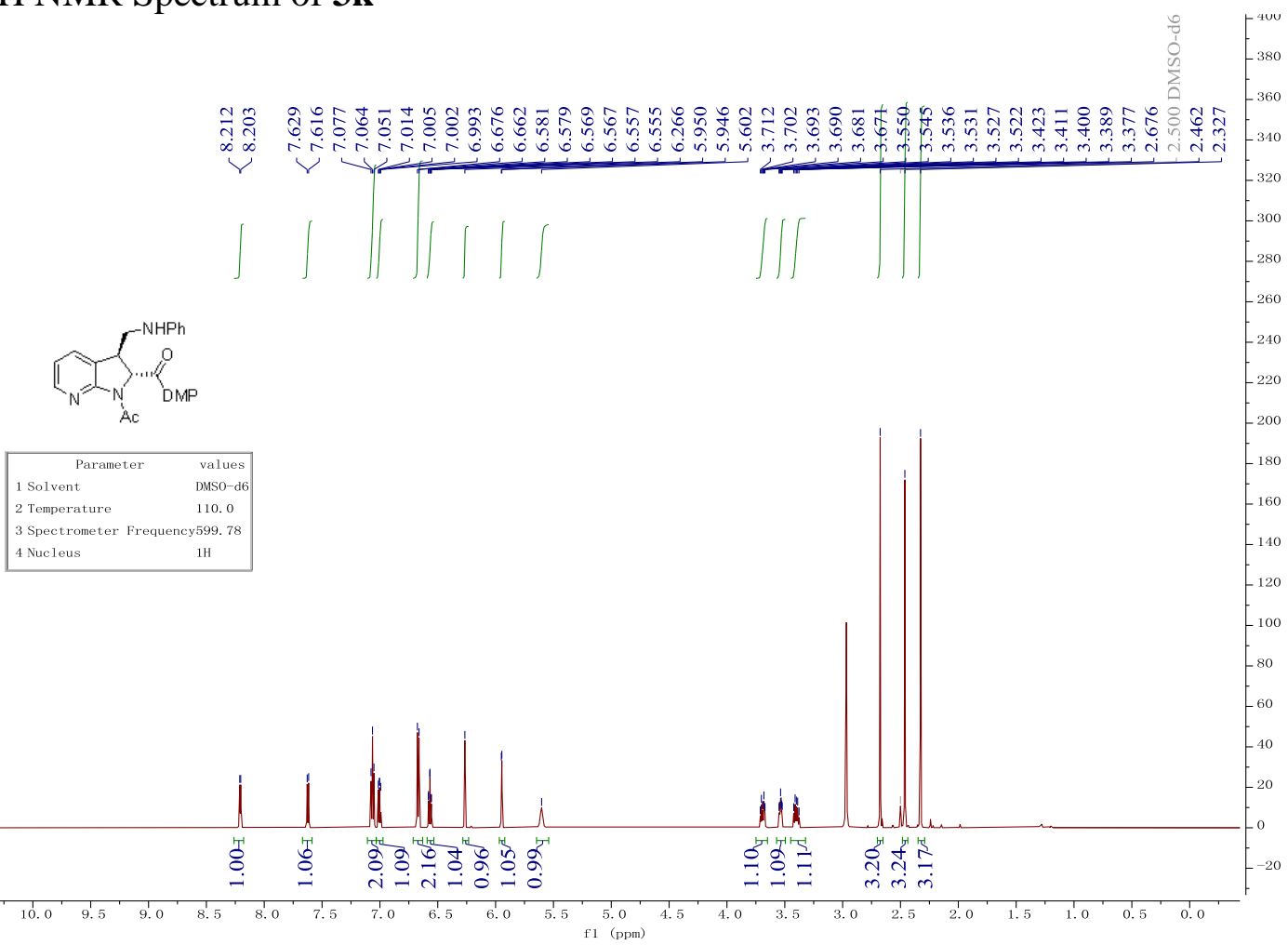

${ }^{13}$ C NMR Spectrum of $\mathbf{3 k}$

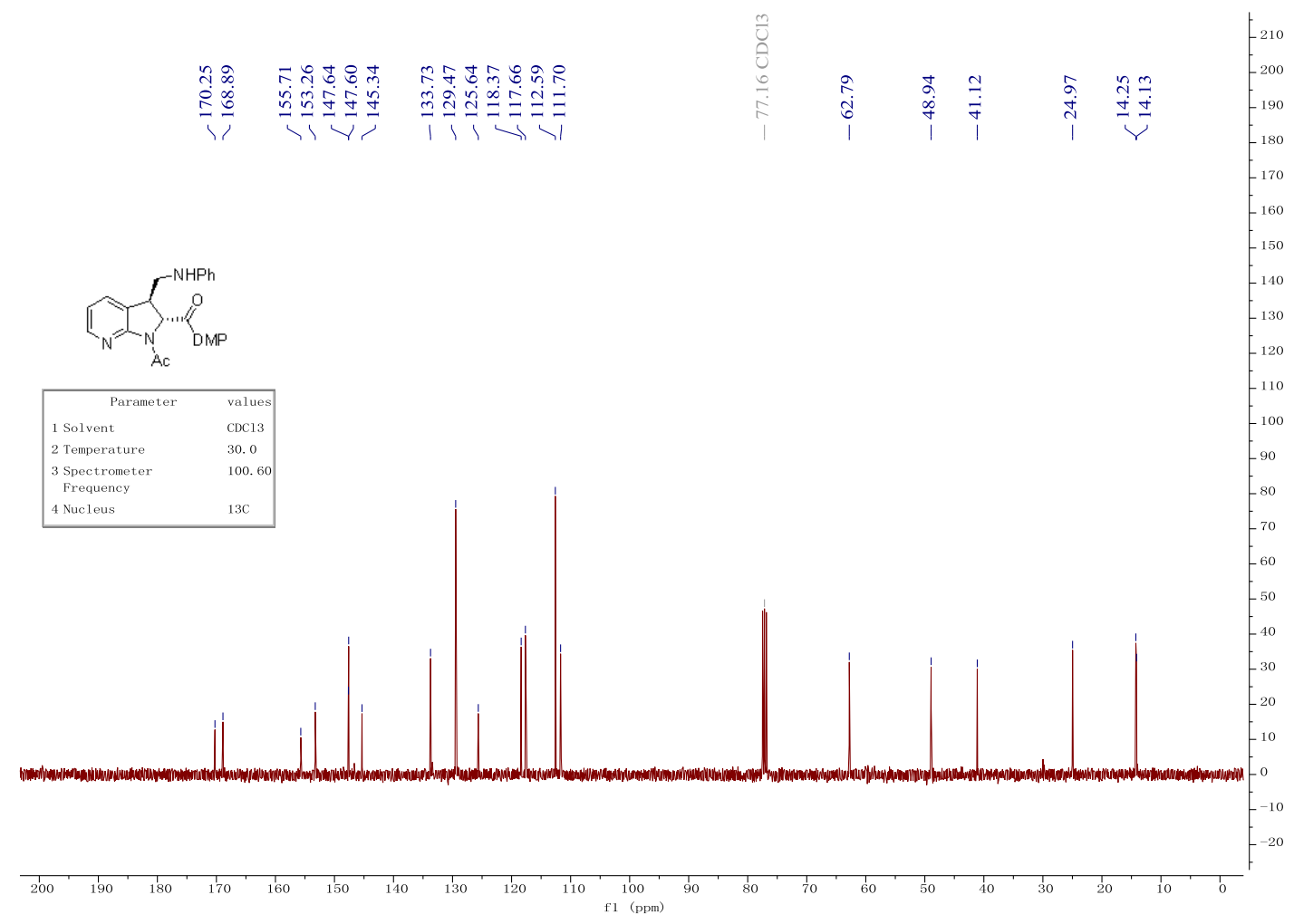




\section{${ }^{1} \mathrm{H}$ NMR Spectrum of $\mathbf{3 l}$}

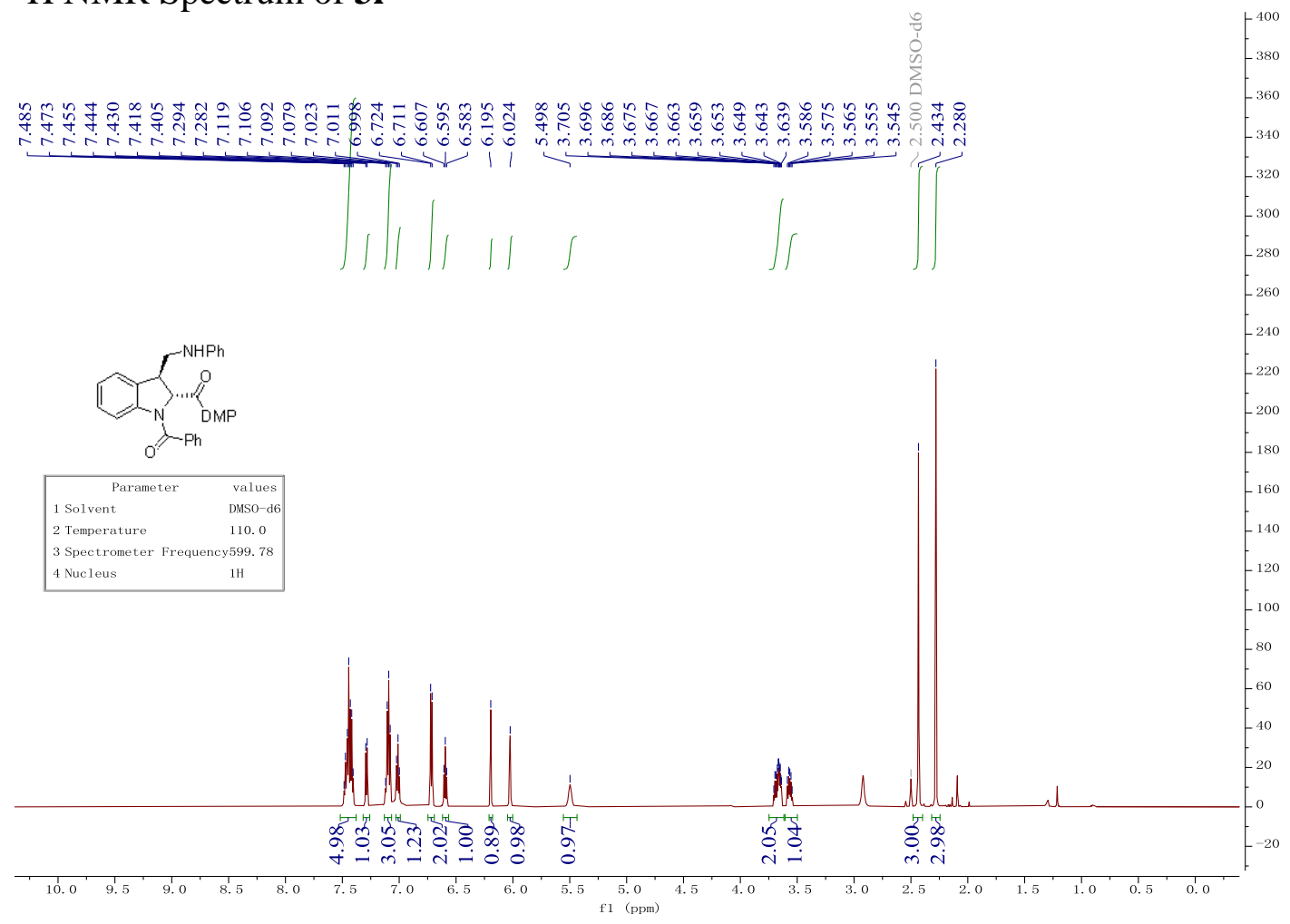

${ }^{13} \mathrm{C}$ NMR Spectrum of $\mathbf{3 l}$

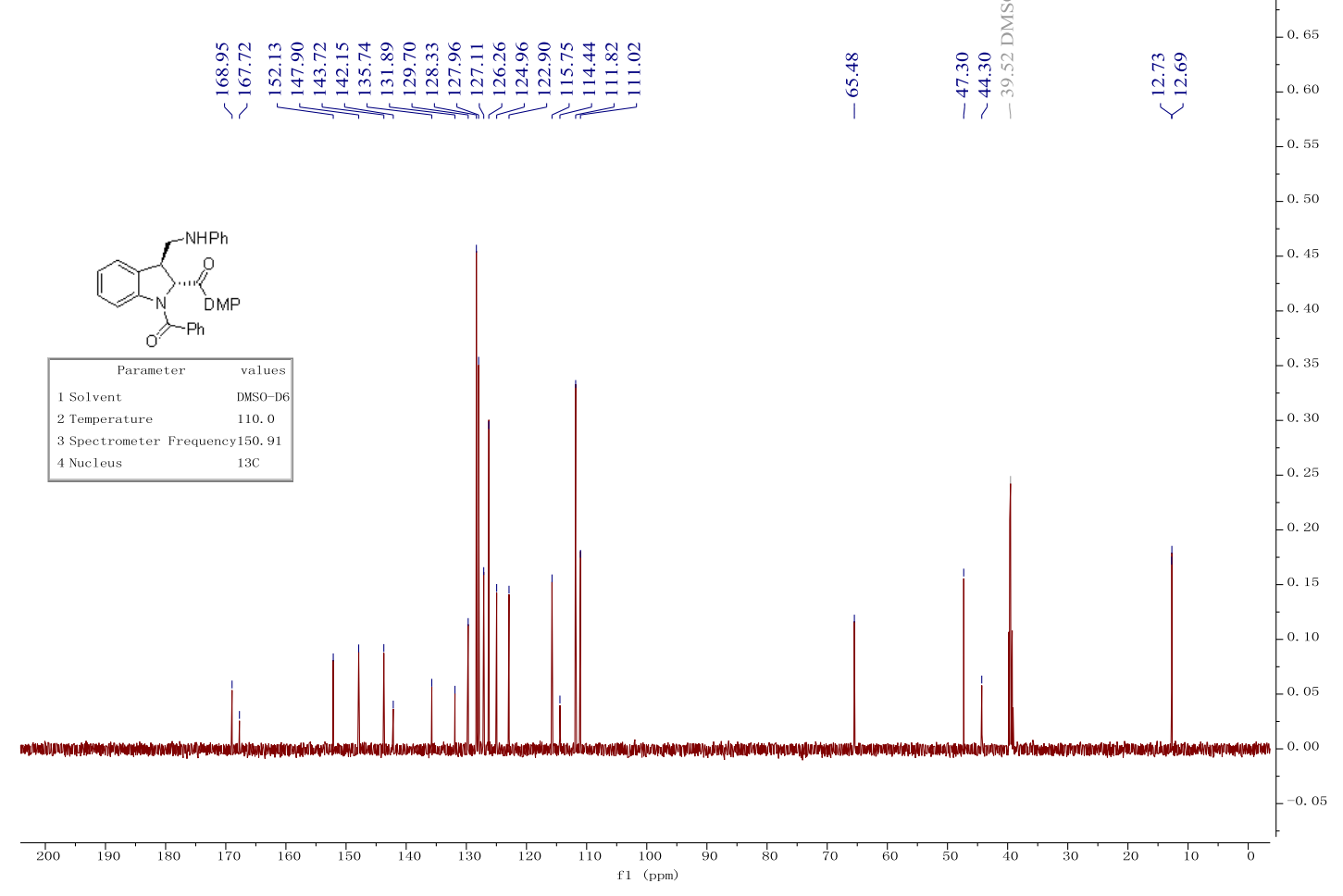


${ }^{1} \mathrm{H}$ NMR Spectrum of $\mathbf{3 m}$

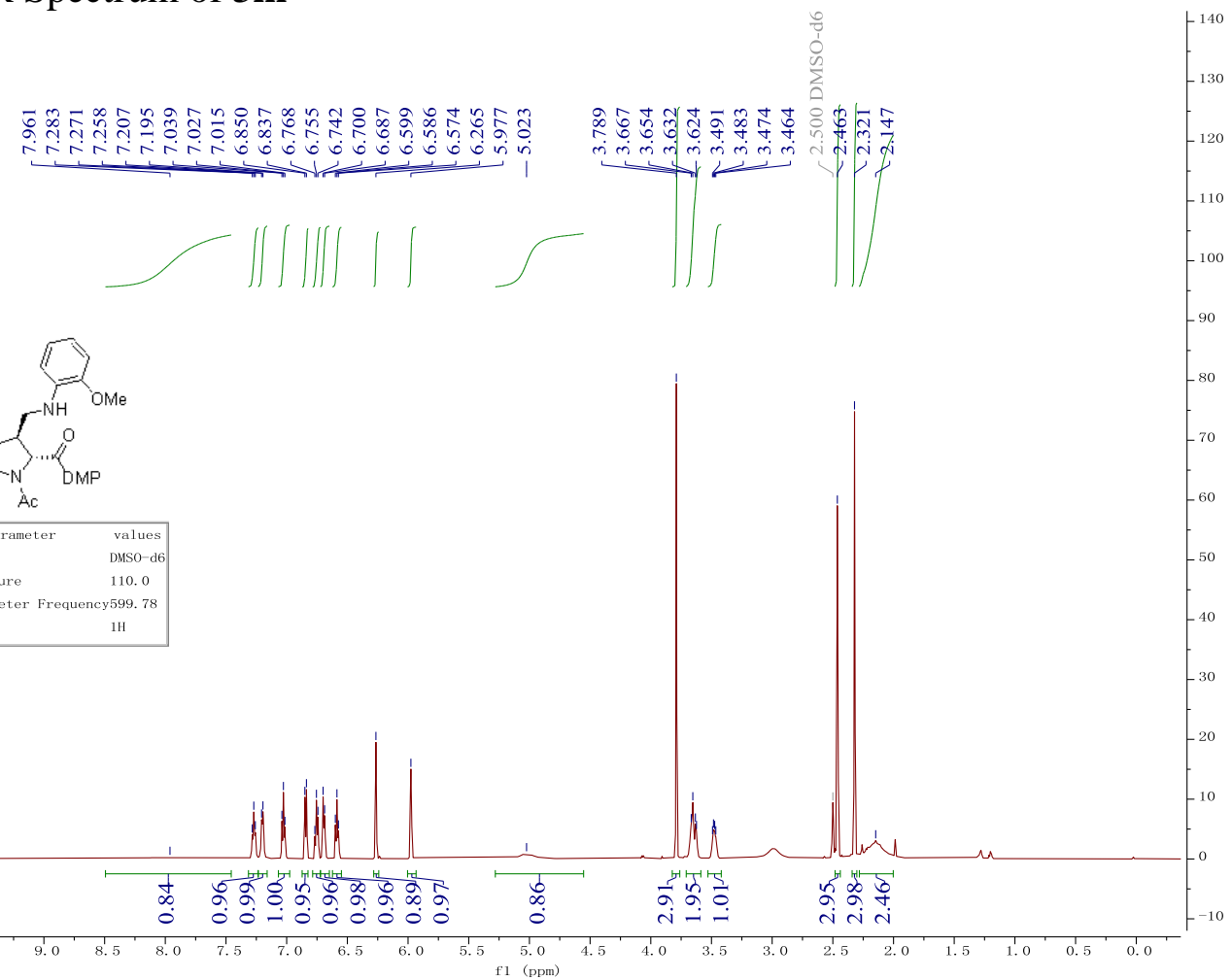

${ }^{13} \mathrm{C}$ NMR Spectrum of $\mathbf{3 m}$

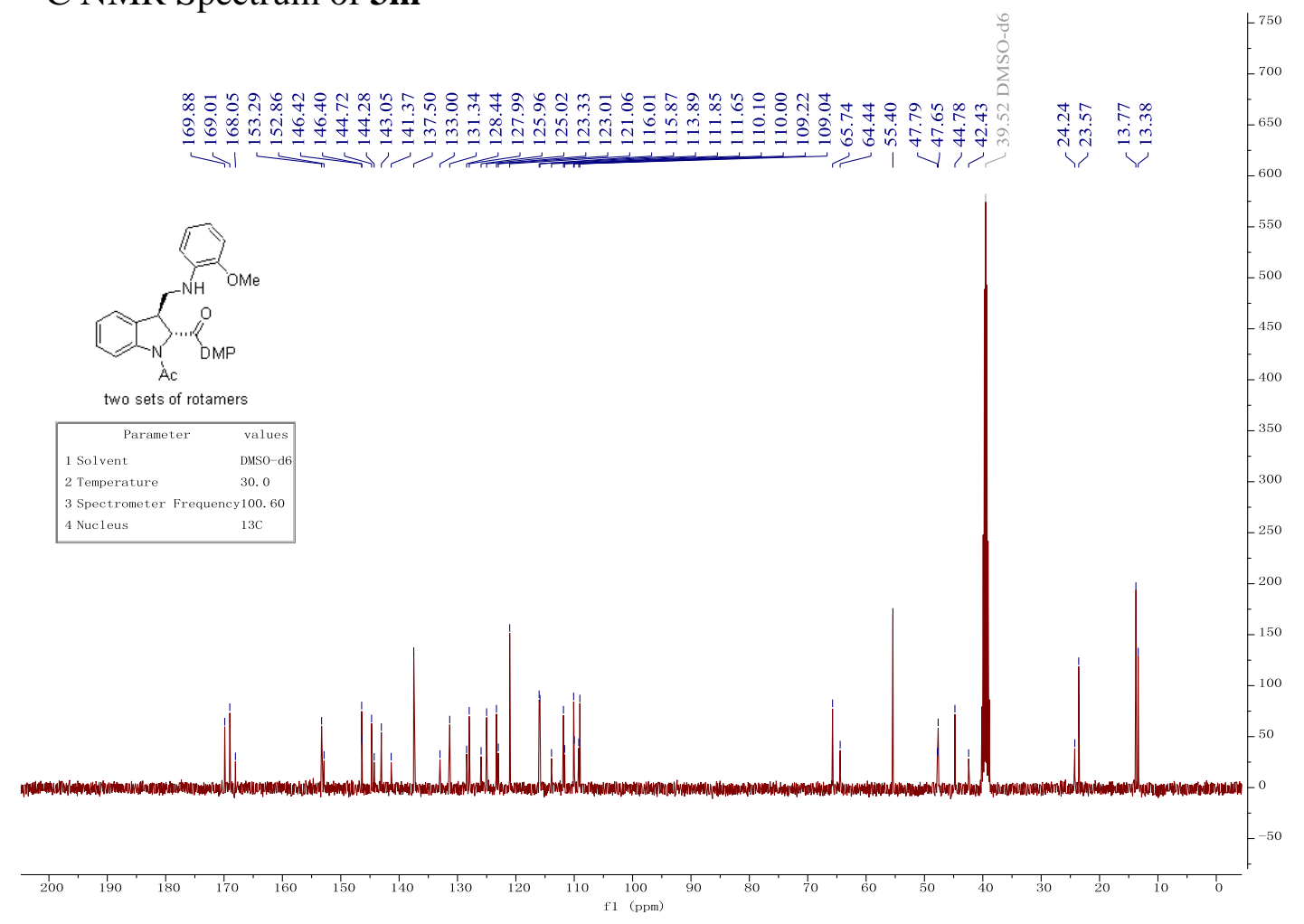


${ }^{1} \mathrm{H}$ NMR Spectrum of $\mathbf{3 n}$

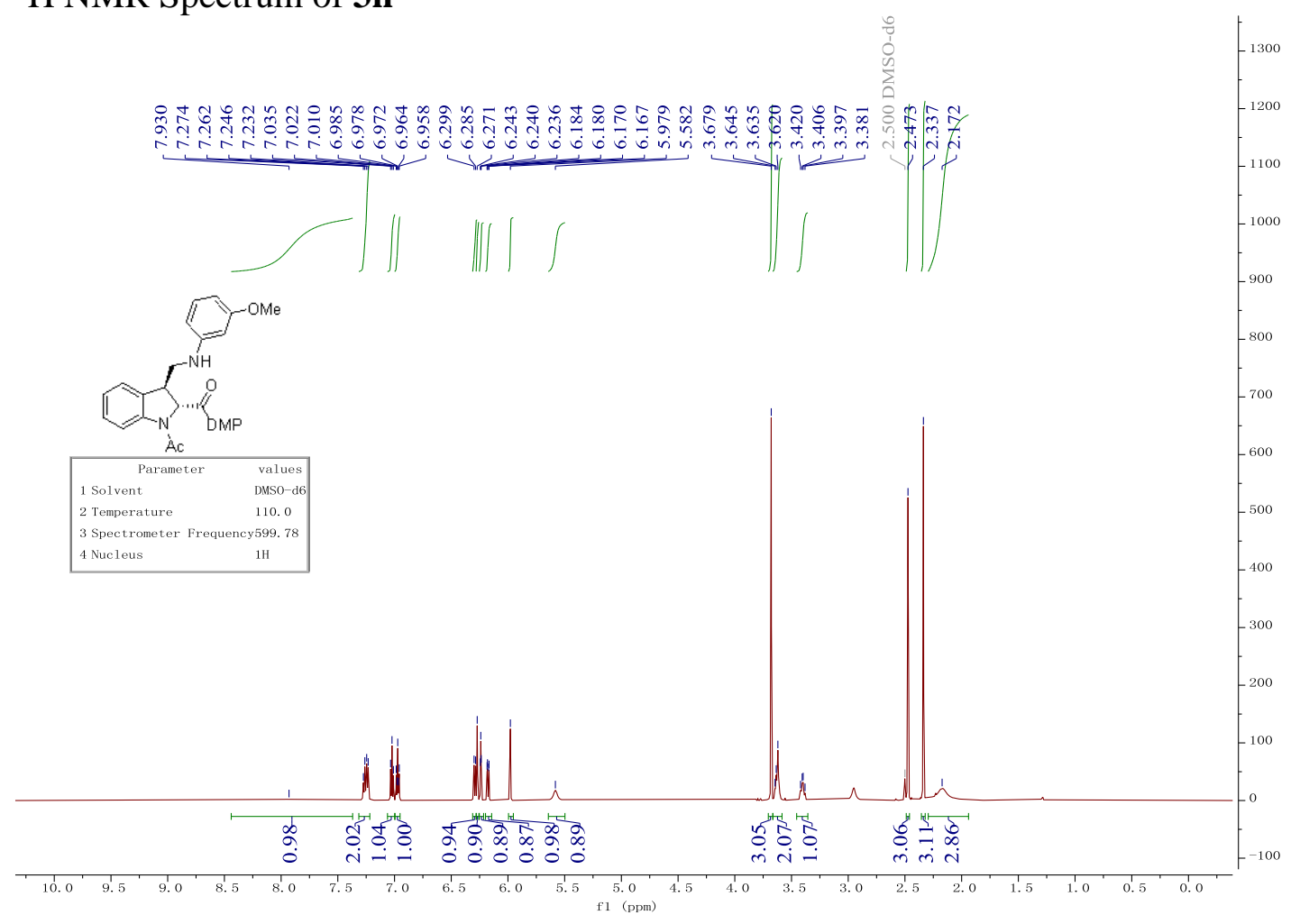

${ }^{13}$ C NMR Spectrum of $\mathbf{3 n}$

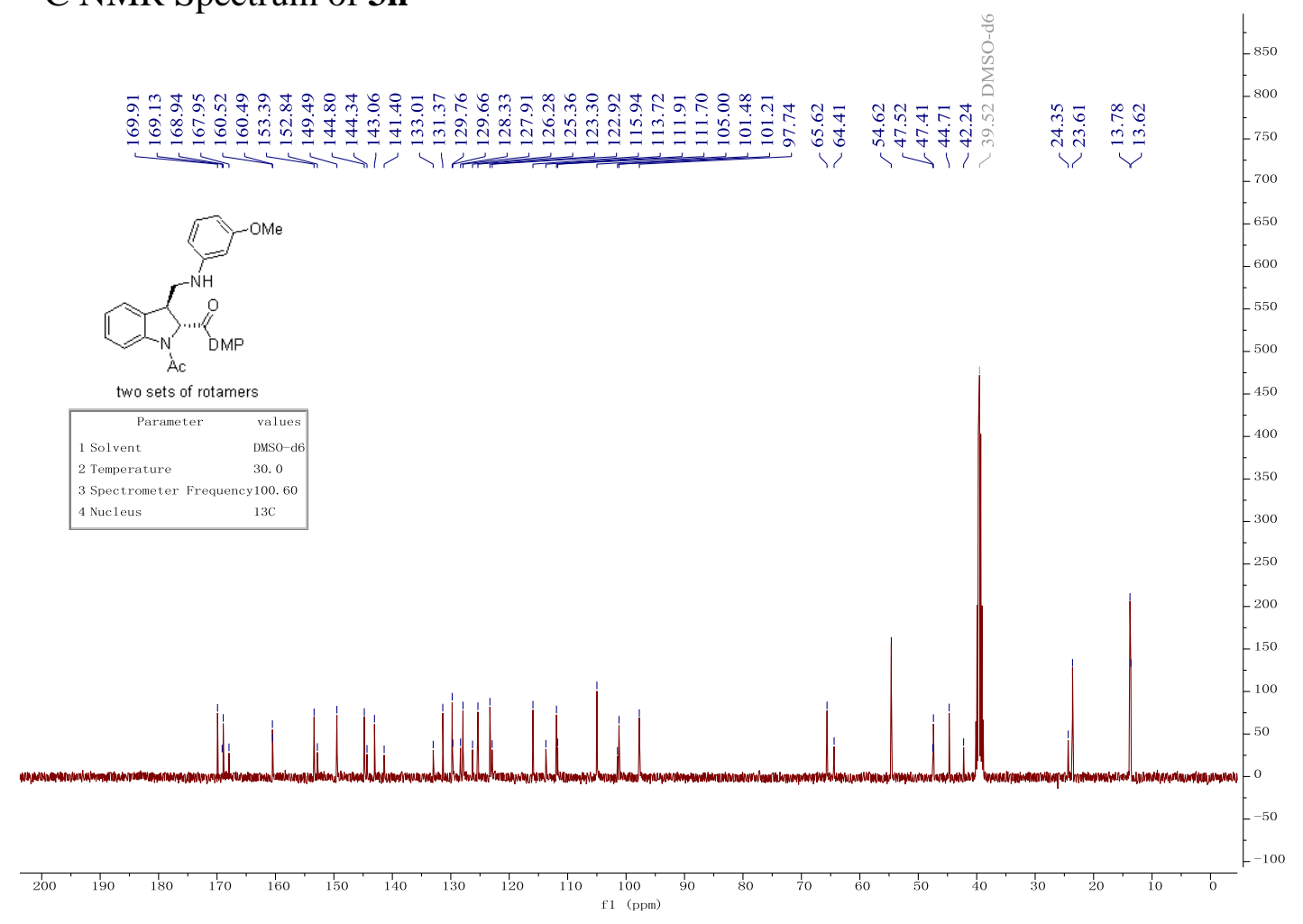


${ }^{1} \mathrm{H}$ NMR Spectrum of $\mathbf{3 o}$

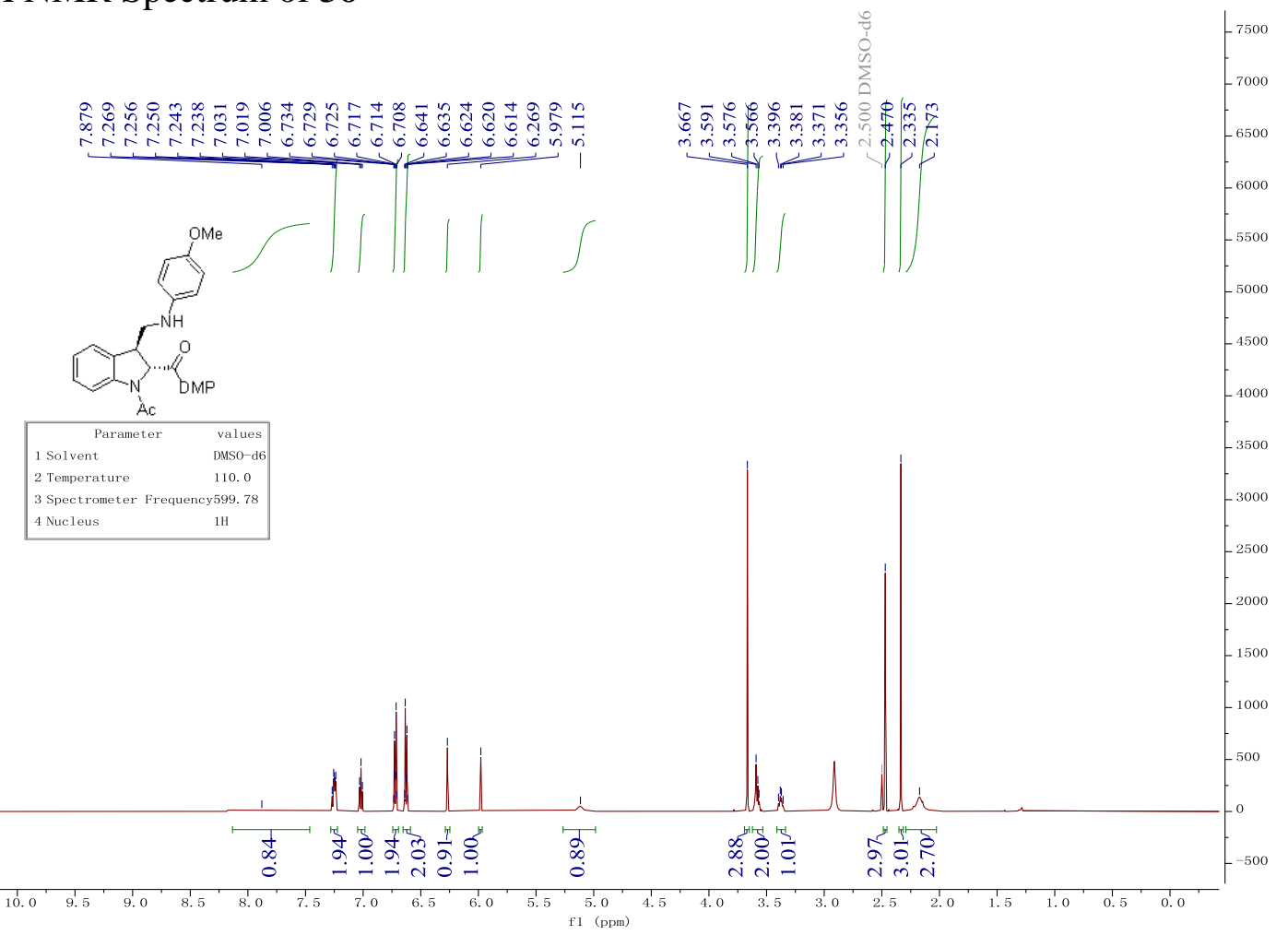

${ }^{13} \mathrm{C}$ NMR Spectrum of $\mathbf{3 o}$

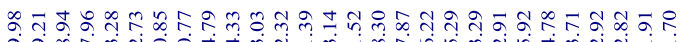

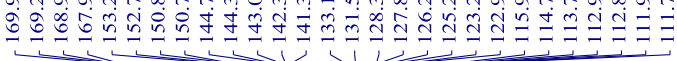

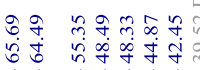

$\sqrt{3}$

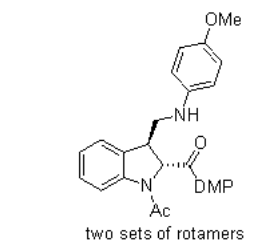

OMe

Paran

1 Solvent

DMSO-d

S Spectrometer Frequency 100.60

4 Nucleus
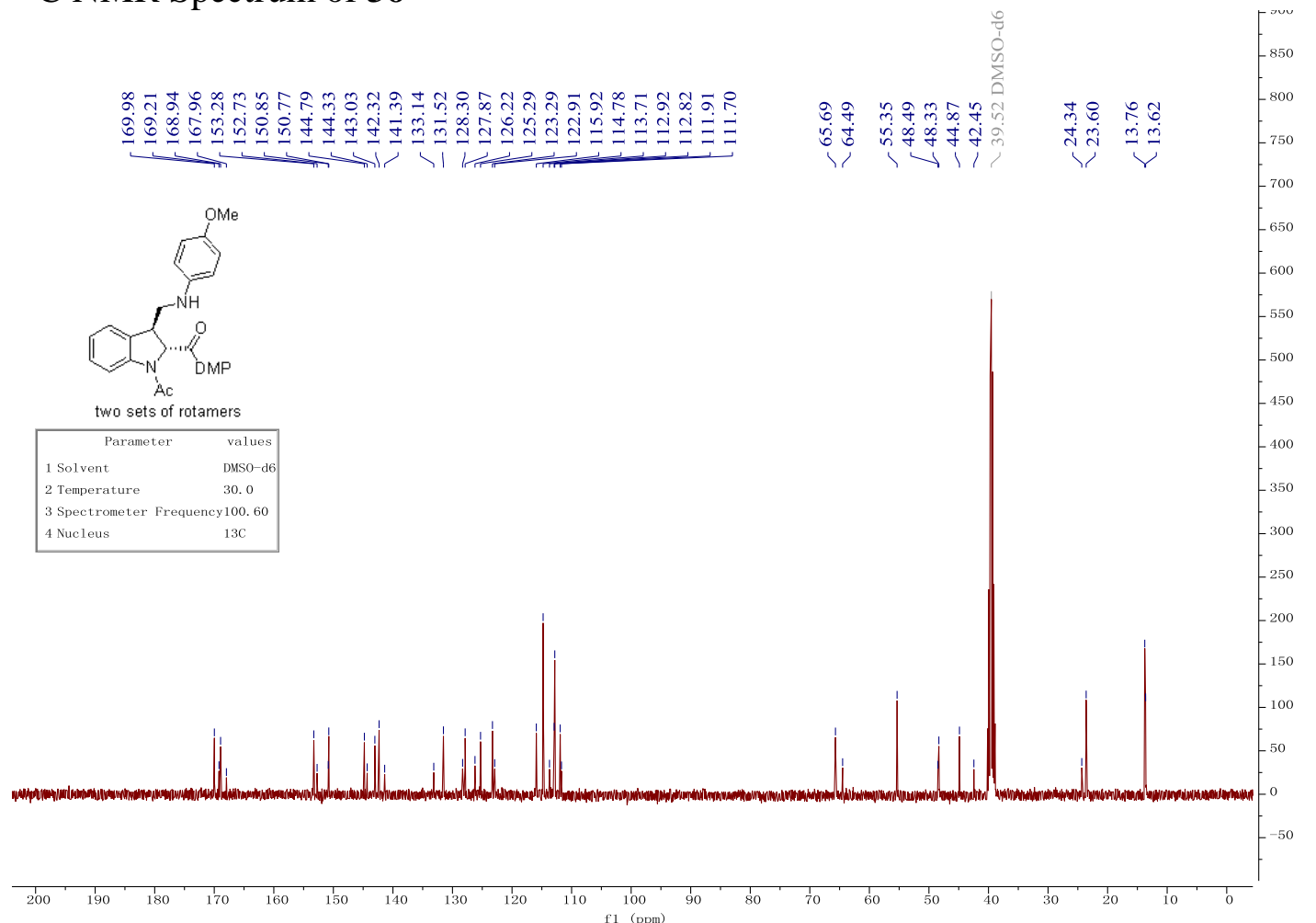
${ }^{1} \mathrm{H}$ NMR Spectrum of $\mathbf{3 p}$

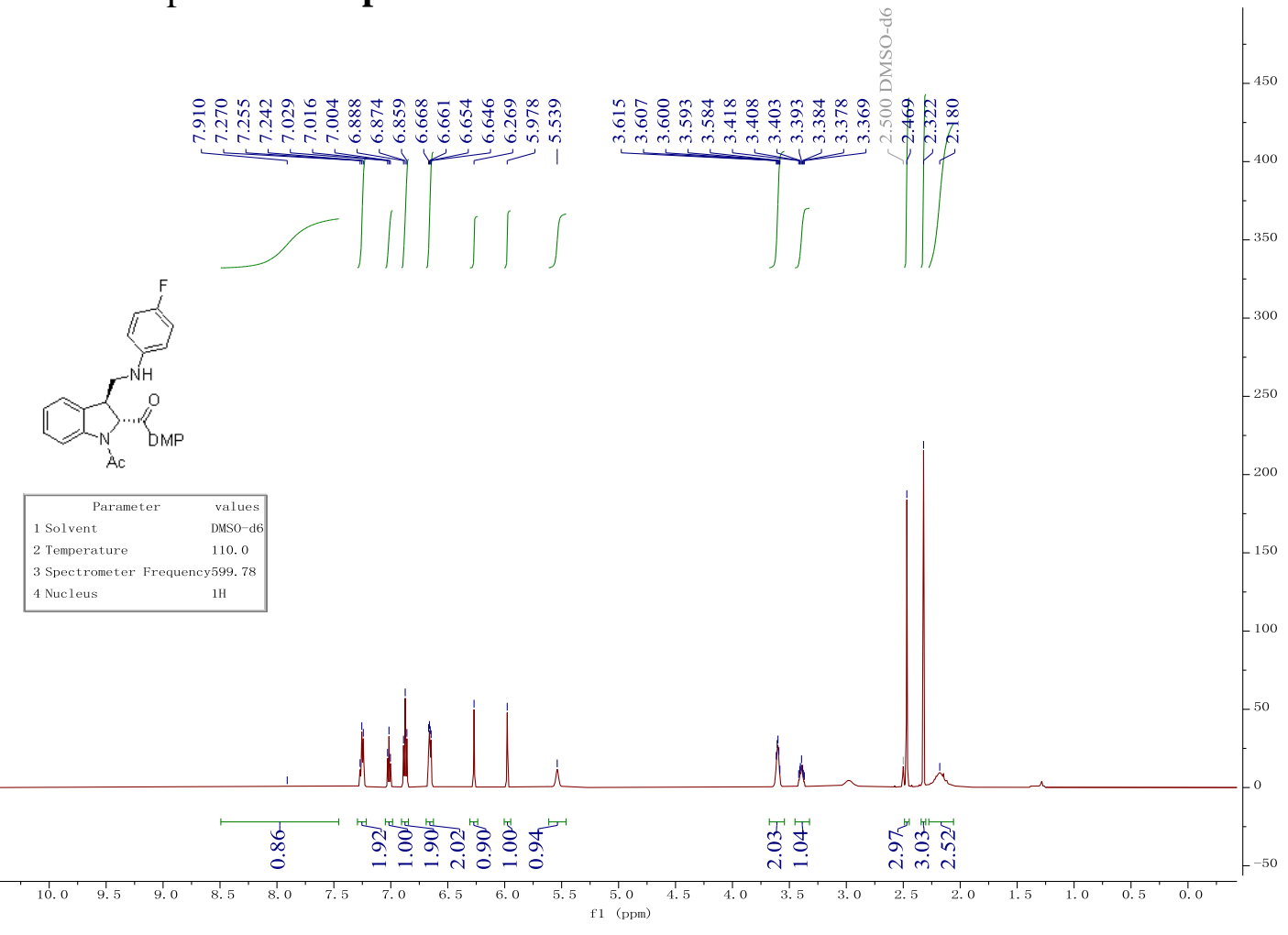

${ }^{13} \mathrm{C}$ NMR Spectrum of $\mathbf{3 p}$

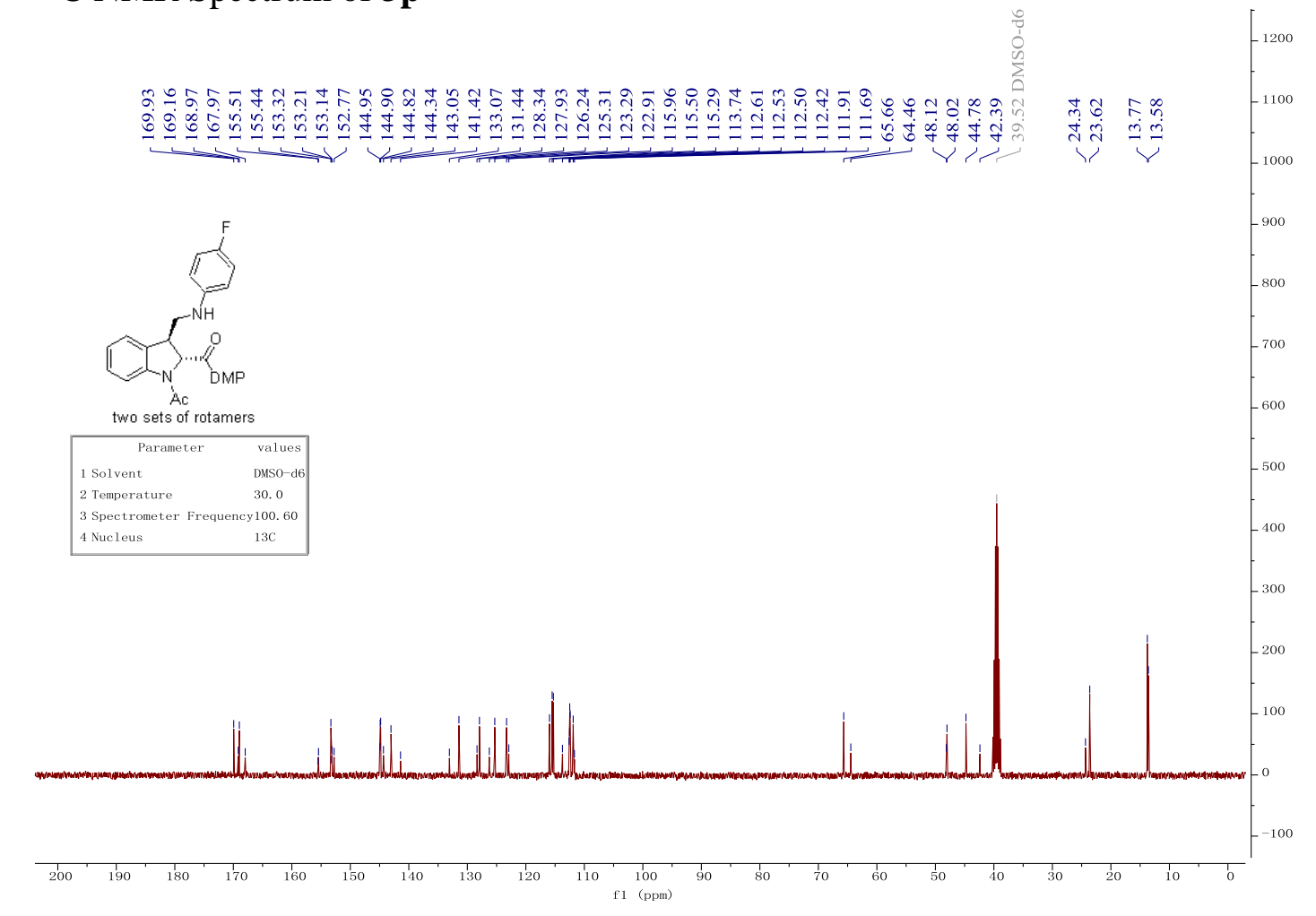


${ }^{19}$ F NMR Spectrum of $\mathbf{3 p}$

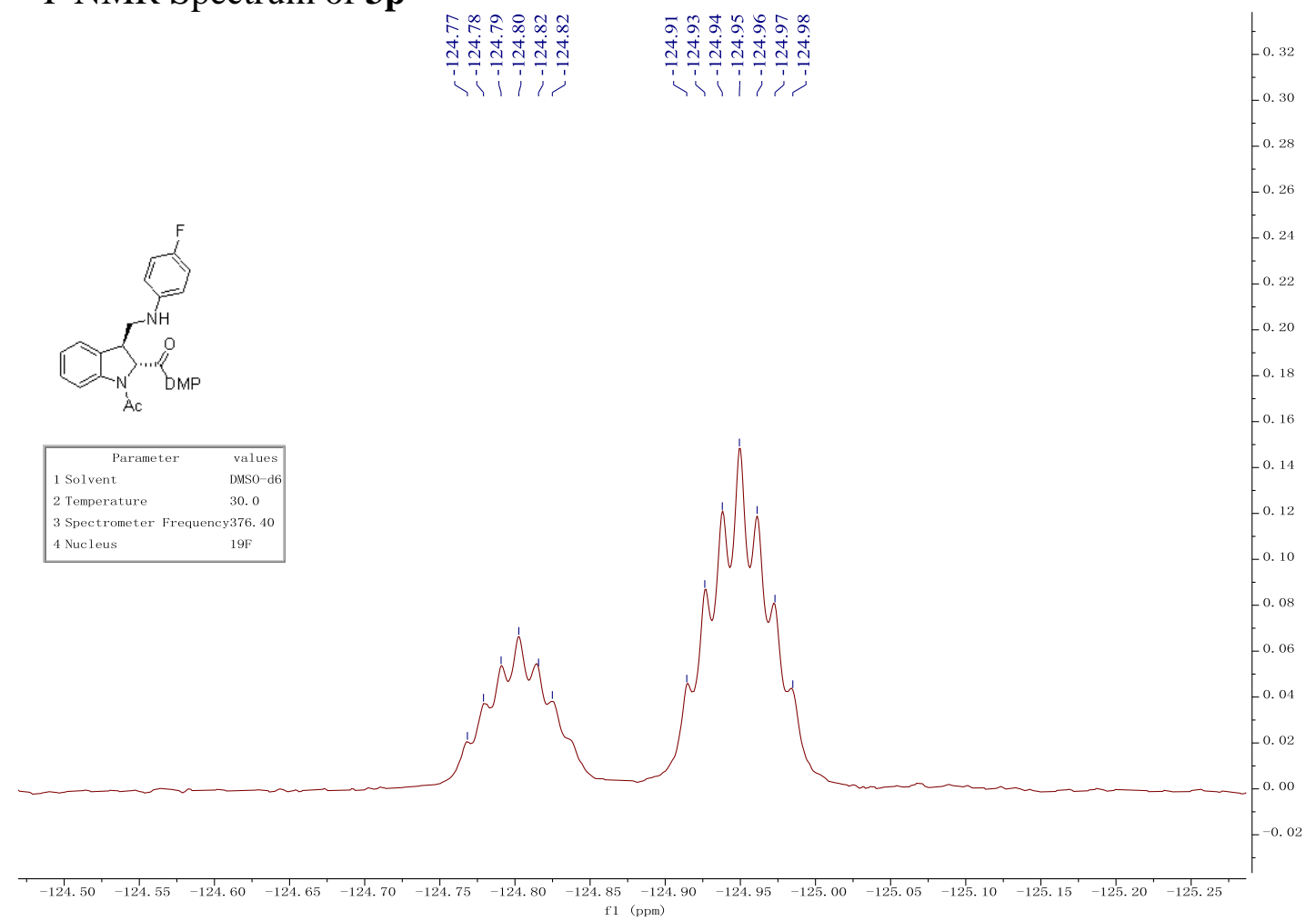

${ }^{1} \mathrm{H}$ NMR Spectrum of $\mathbf{3 q}$

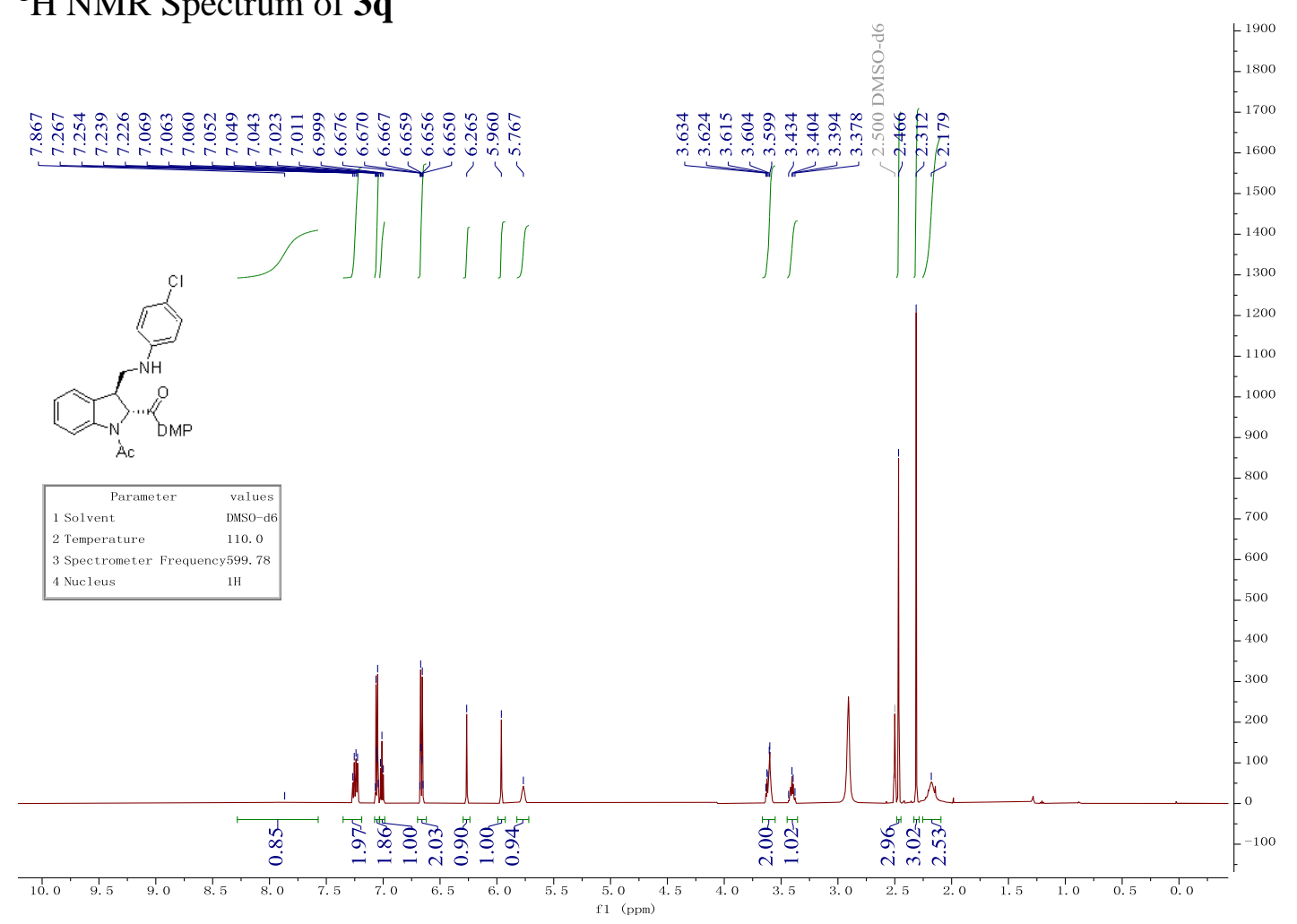


${ }^{13}$ C NMR Spectrum of $\mathbf{3 q}$

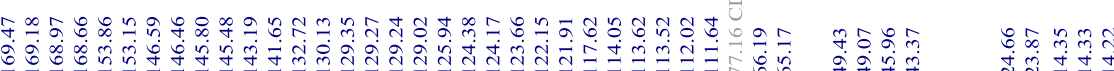

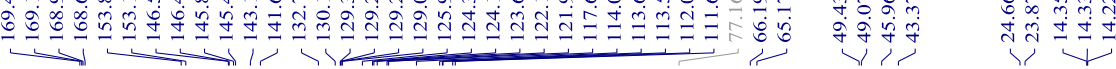
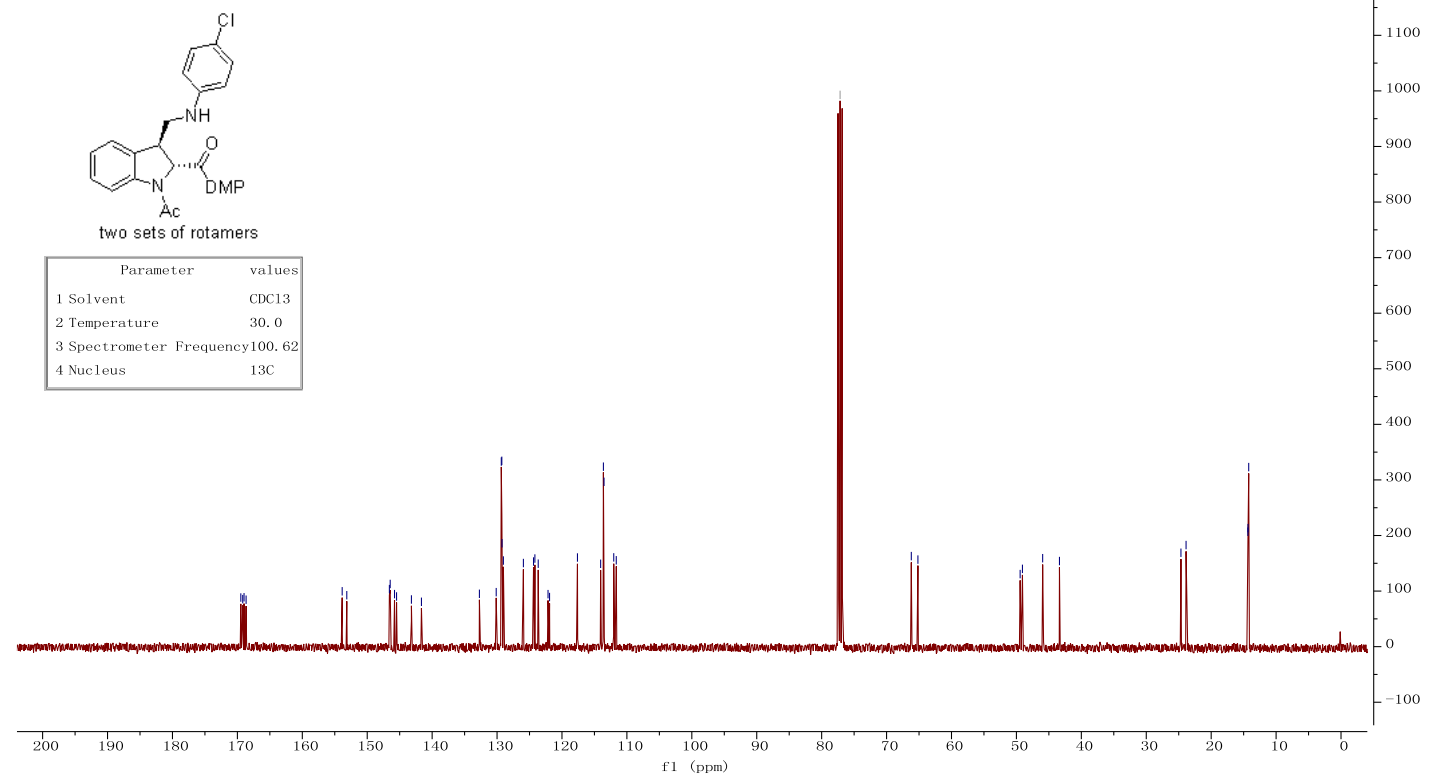

${ }^{1} \mathrm{H}$ NMR Spectrum of $\mathbf{3 r}$

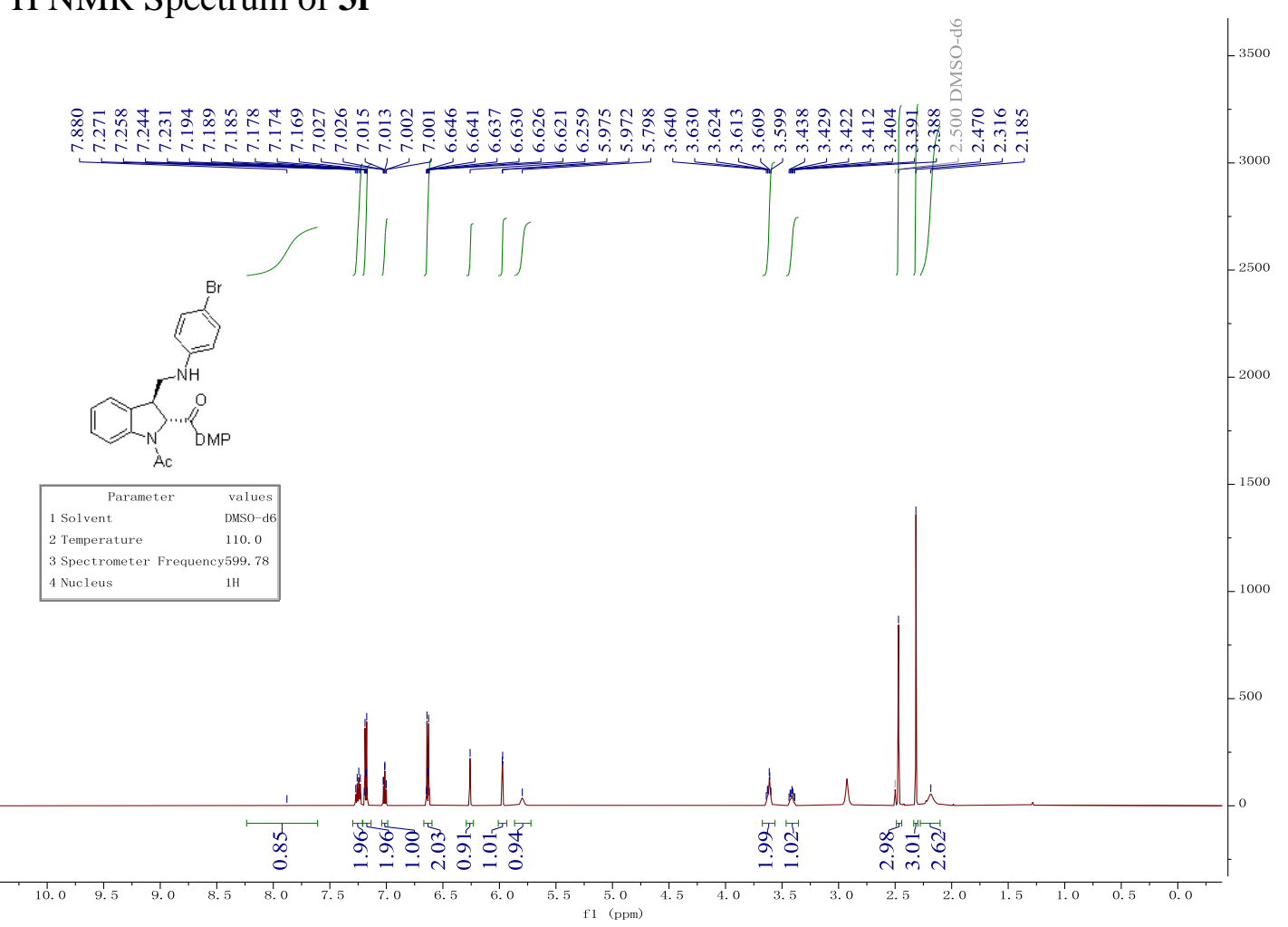


${ }^{13} \mathrm{C}$ NMR Spectrum of $\mathbf{3 r}$

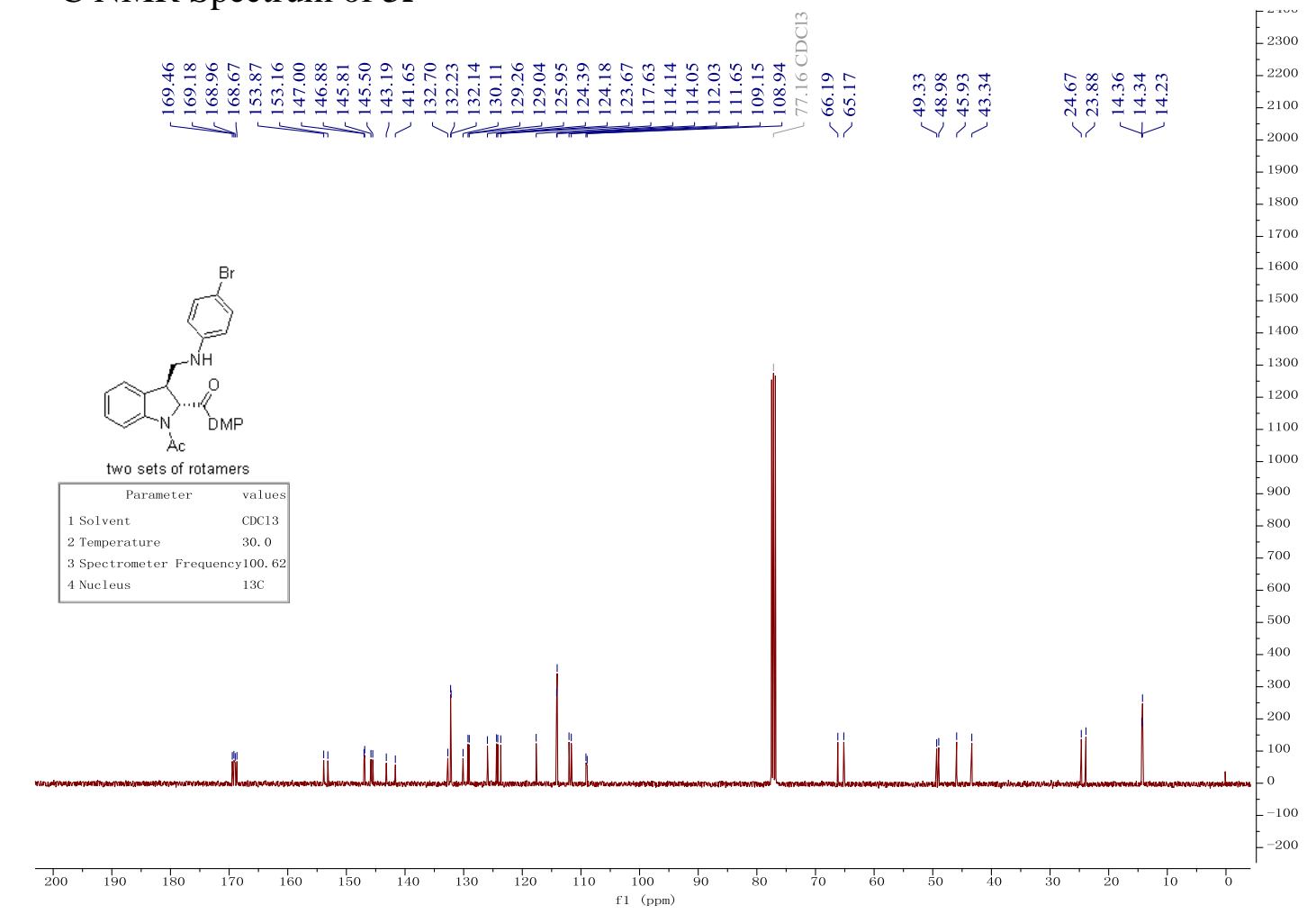

${ }^{1} \mathrm{H}$ NMR Spectrum of $\mathbf{3 s}$

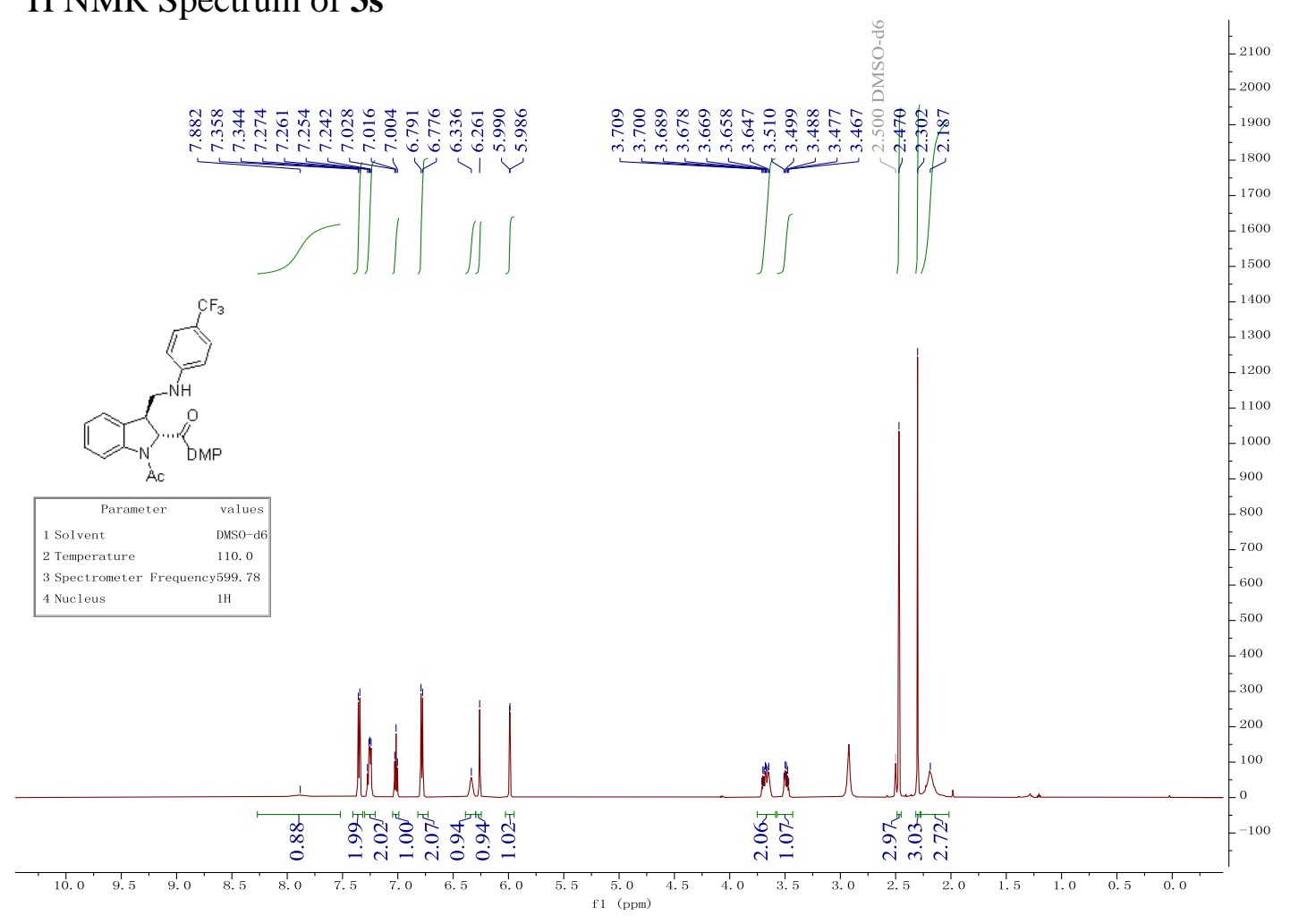


${ }^{13} \mathrm{C}$ NMR Spectrum of $\mathbf{3 s}$

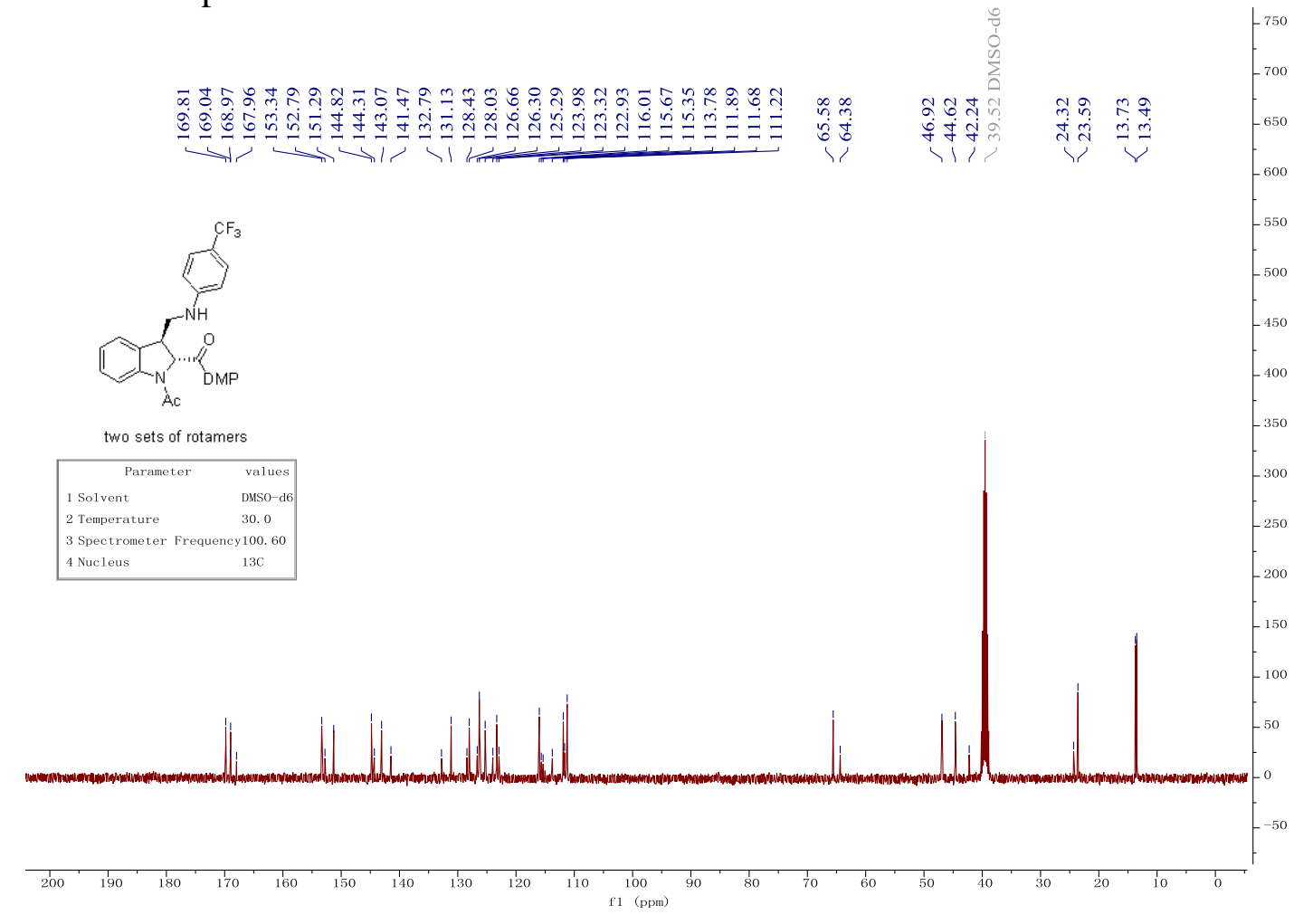

${ }^{19} \mathrm{~F}$ NMR Spectrum of $\mathbf{3 s}$

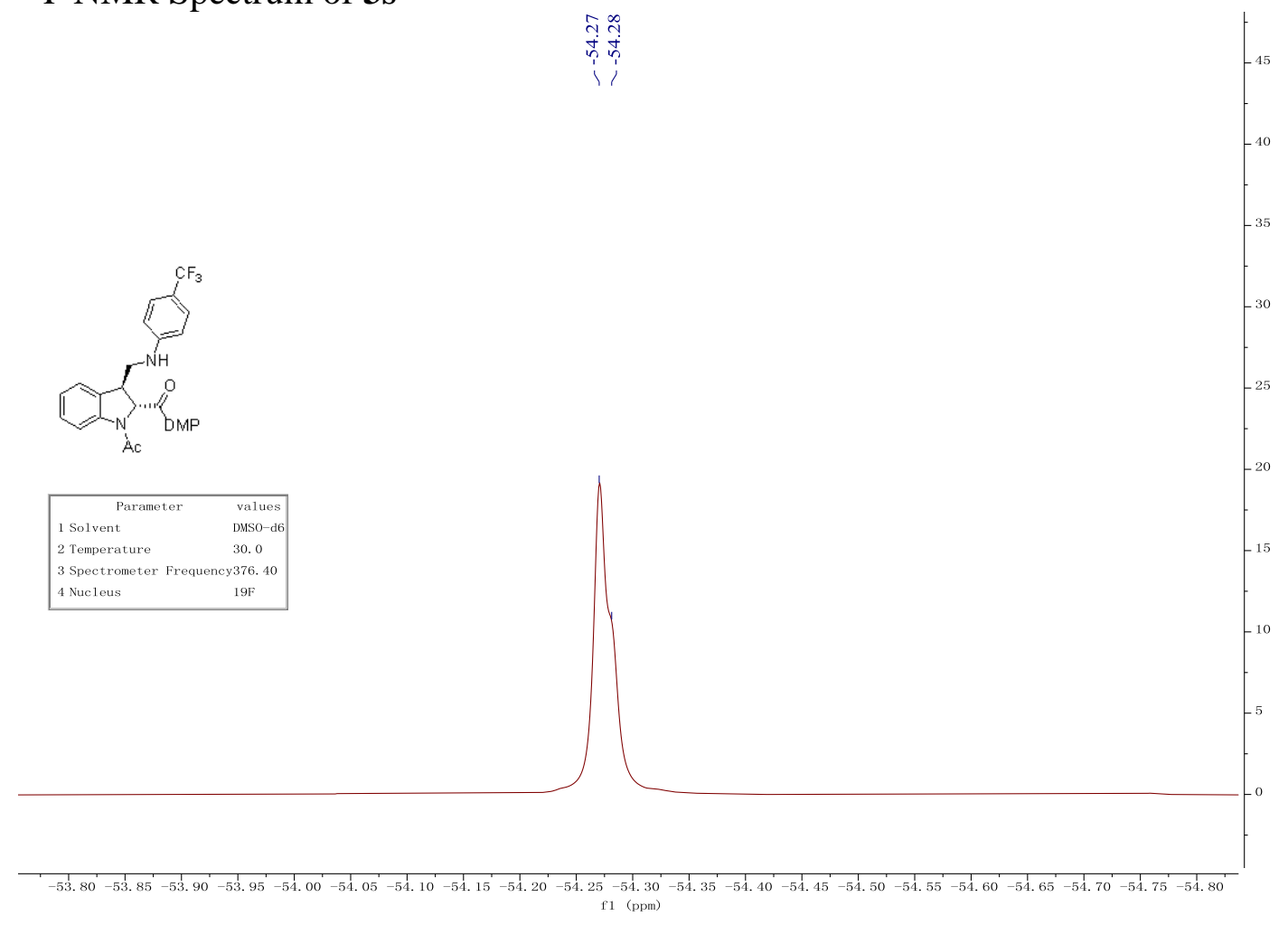


${ }^{1} \mathrm{H}$ NMR Spectrum of $\mathbf{4 a}$

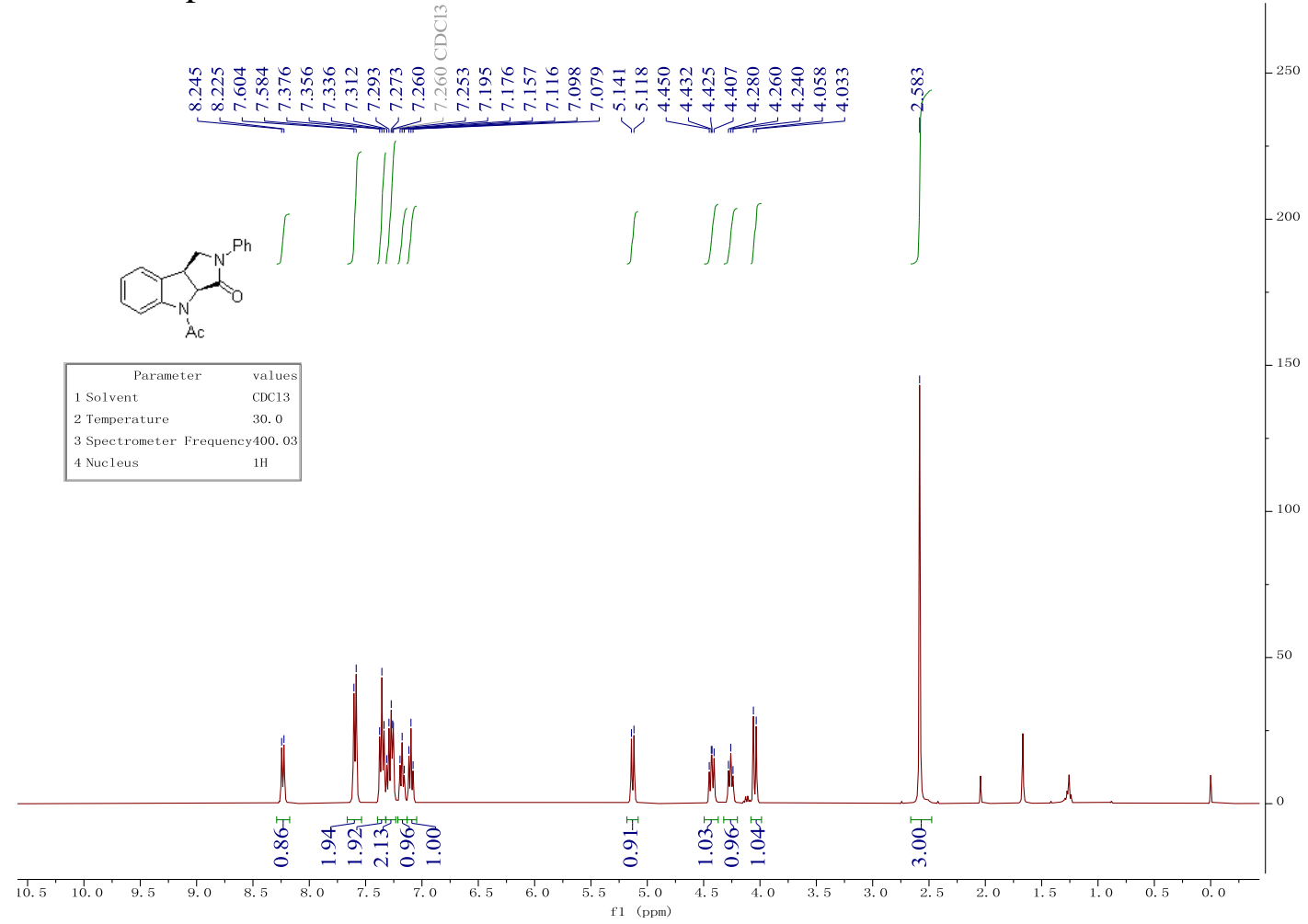

${ }^{13}$ C NMR Spectrum of $\mathbf{4 a}$

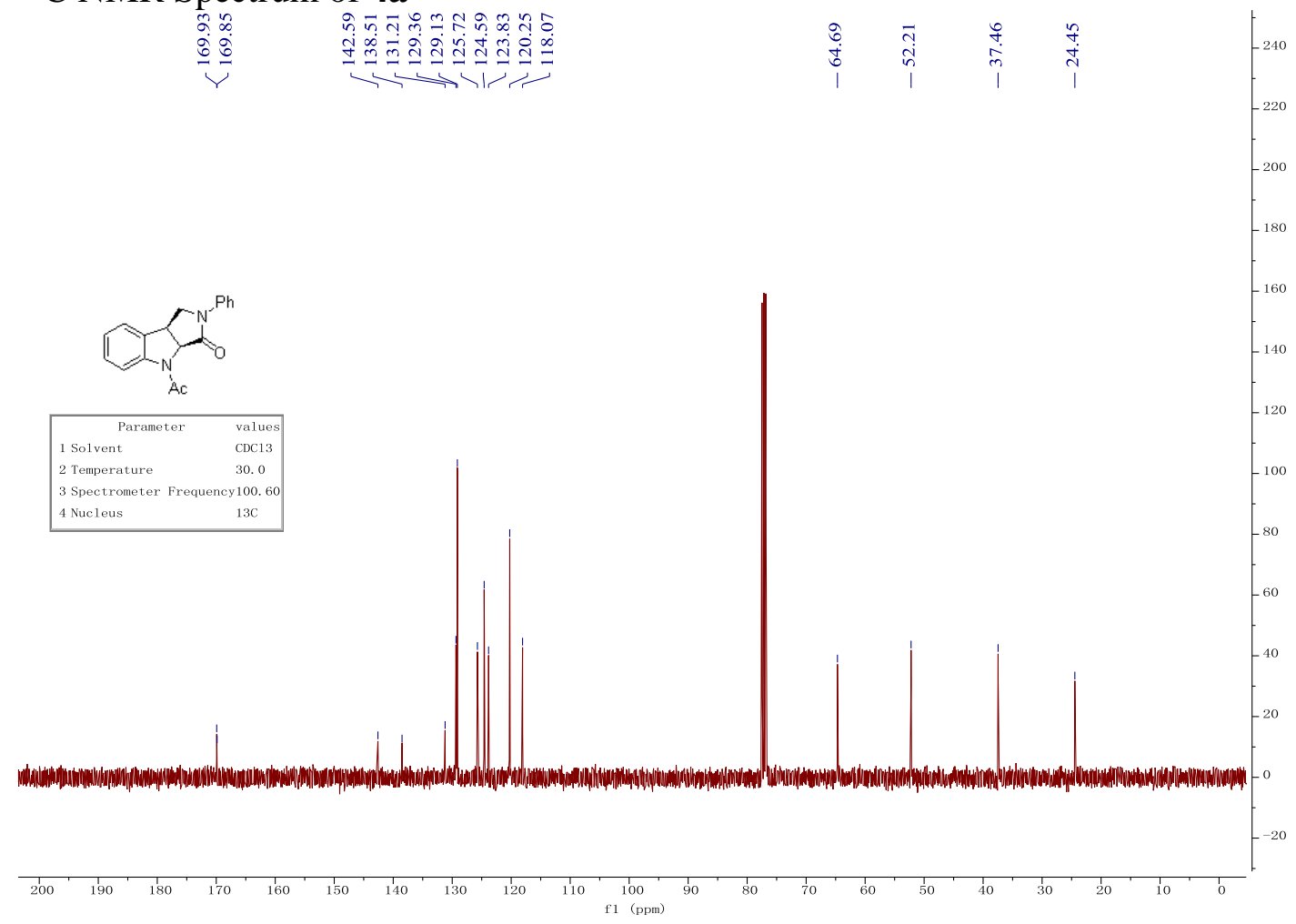


${ }^{1} \mathrm{H}$ NMR Spectrum of $\mathbf{4 b}$
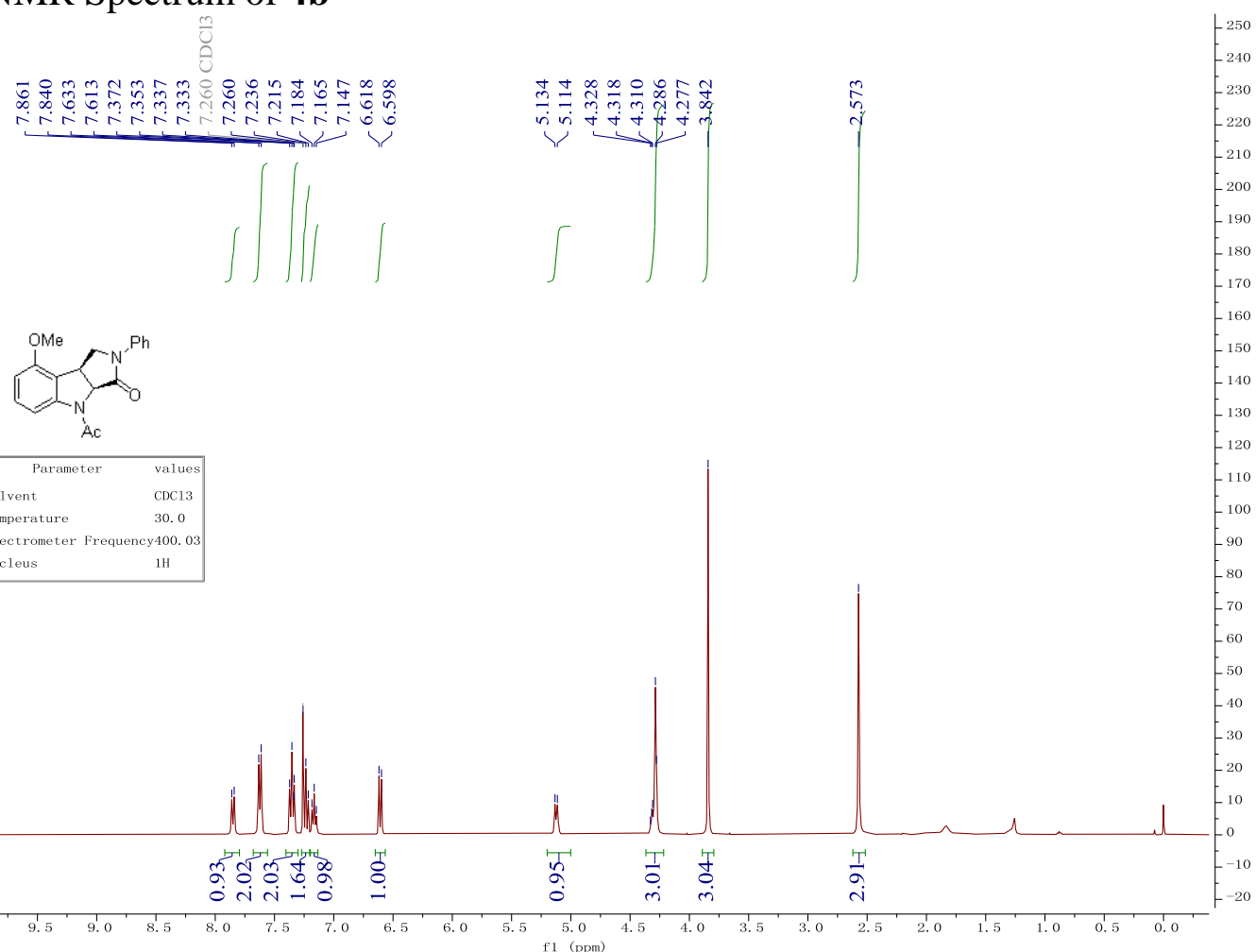

${ }^{13} \mathrm{C}$ NMR Spectrum of $\mathbf{4 b}$

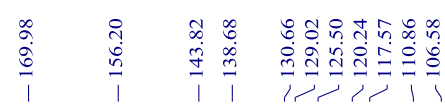
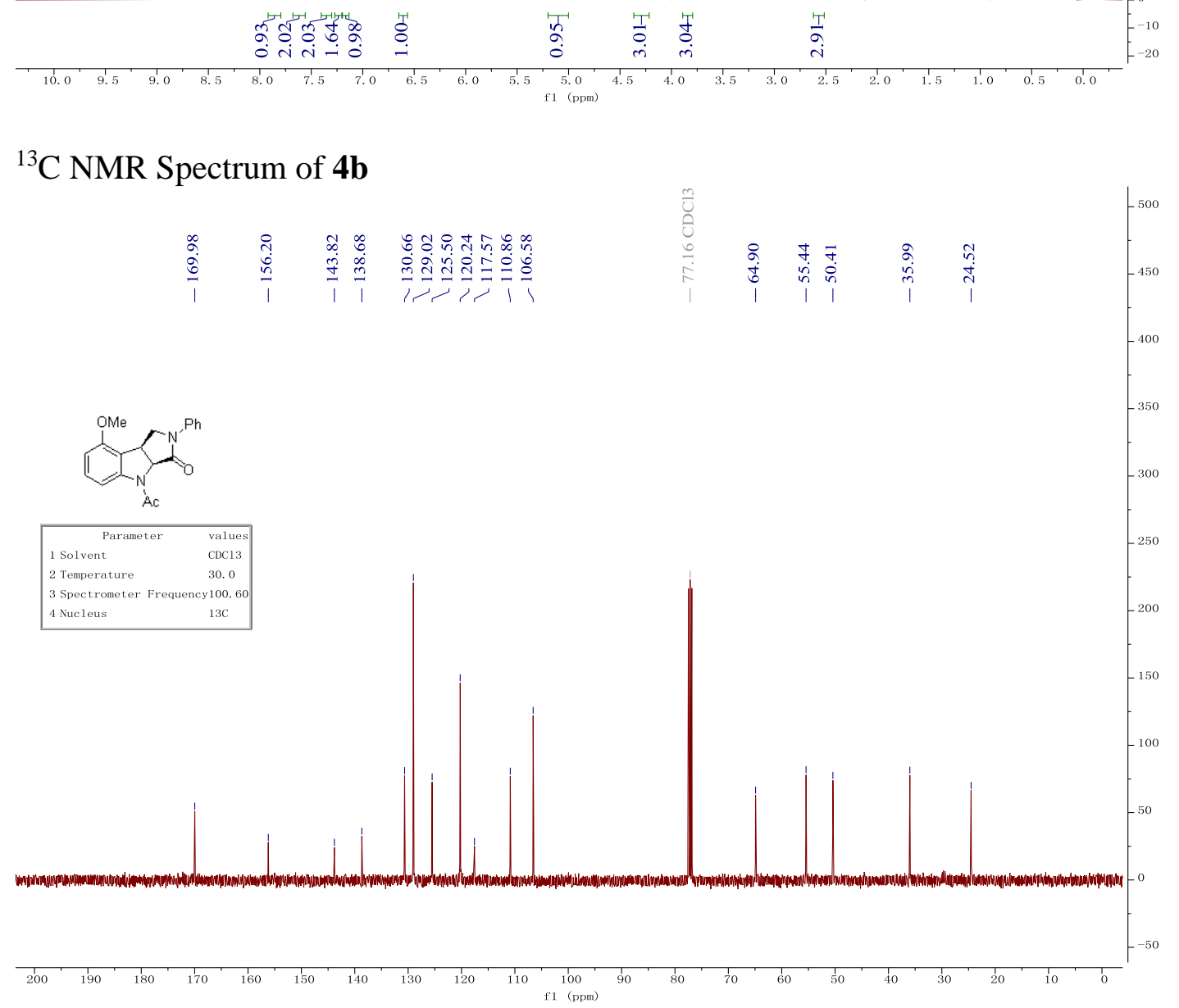
${ }^{1} \mathrm{H}$ NMR Spectrum of $\mathbf{4 c}$

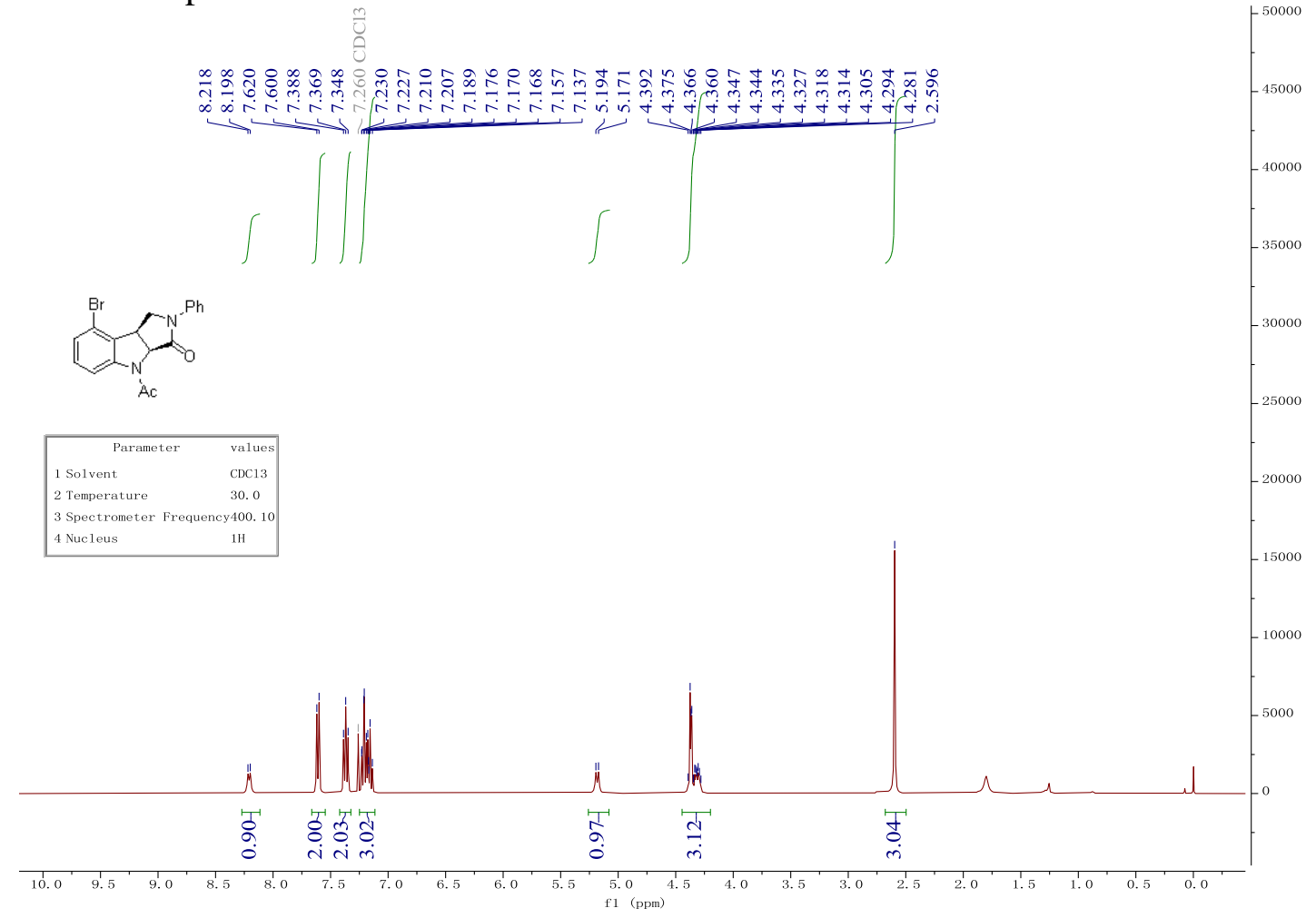

${ }^{13} \mathrm{C}$ NMR Spectrum of $\mathbf{4 c}$

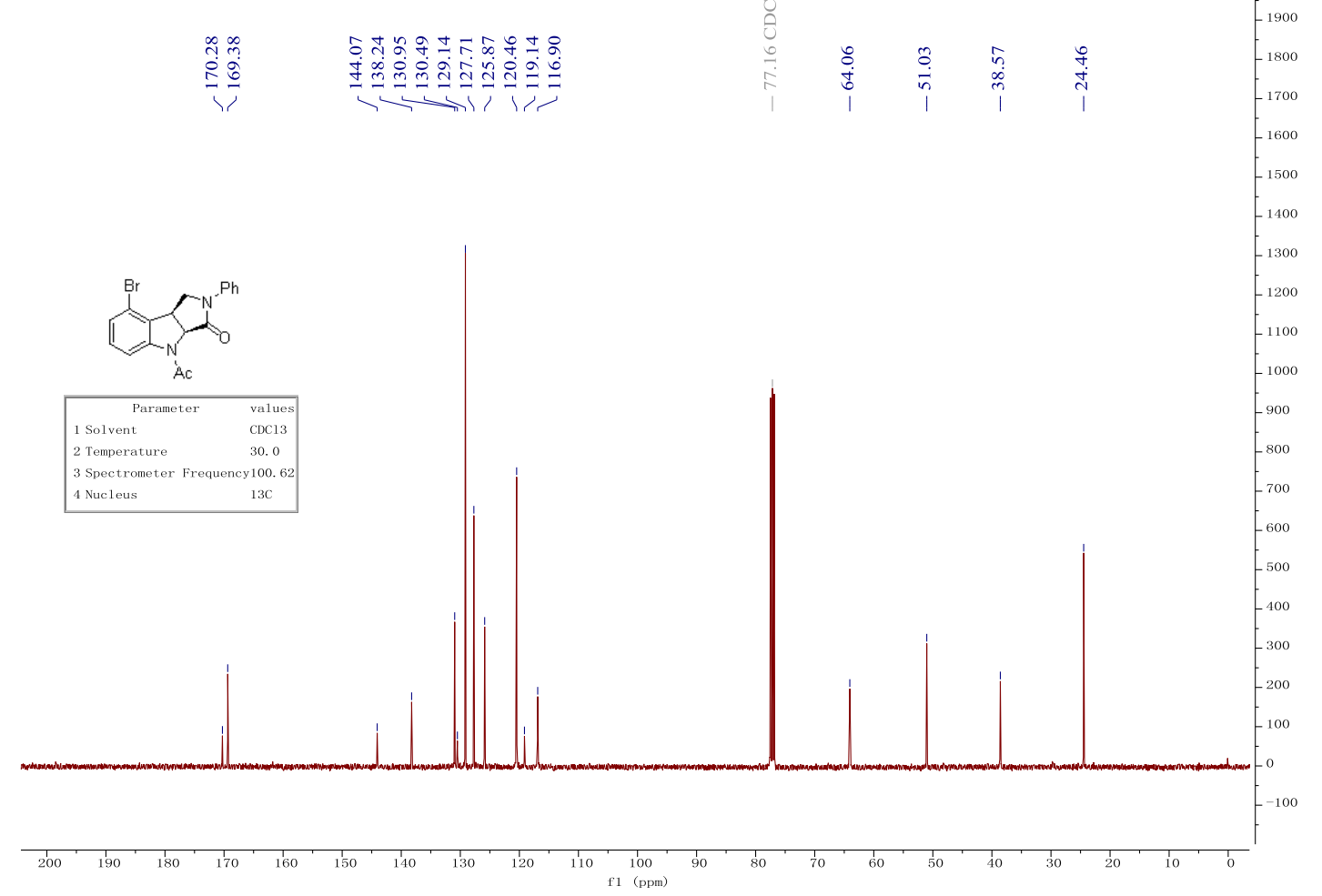


${ }^{1} \mathrm{H}$ NMR Spectrum of $\mathbf{4 d}$

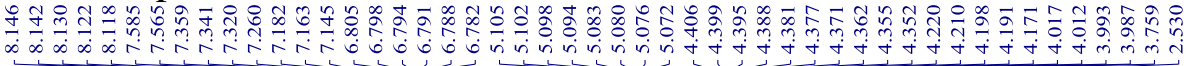
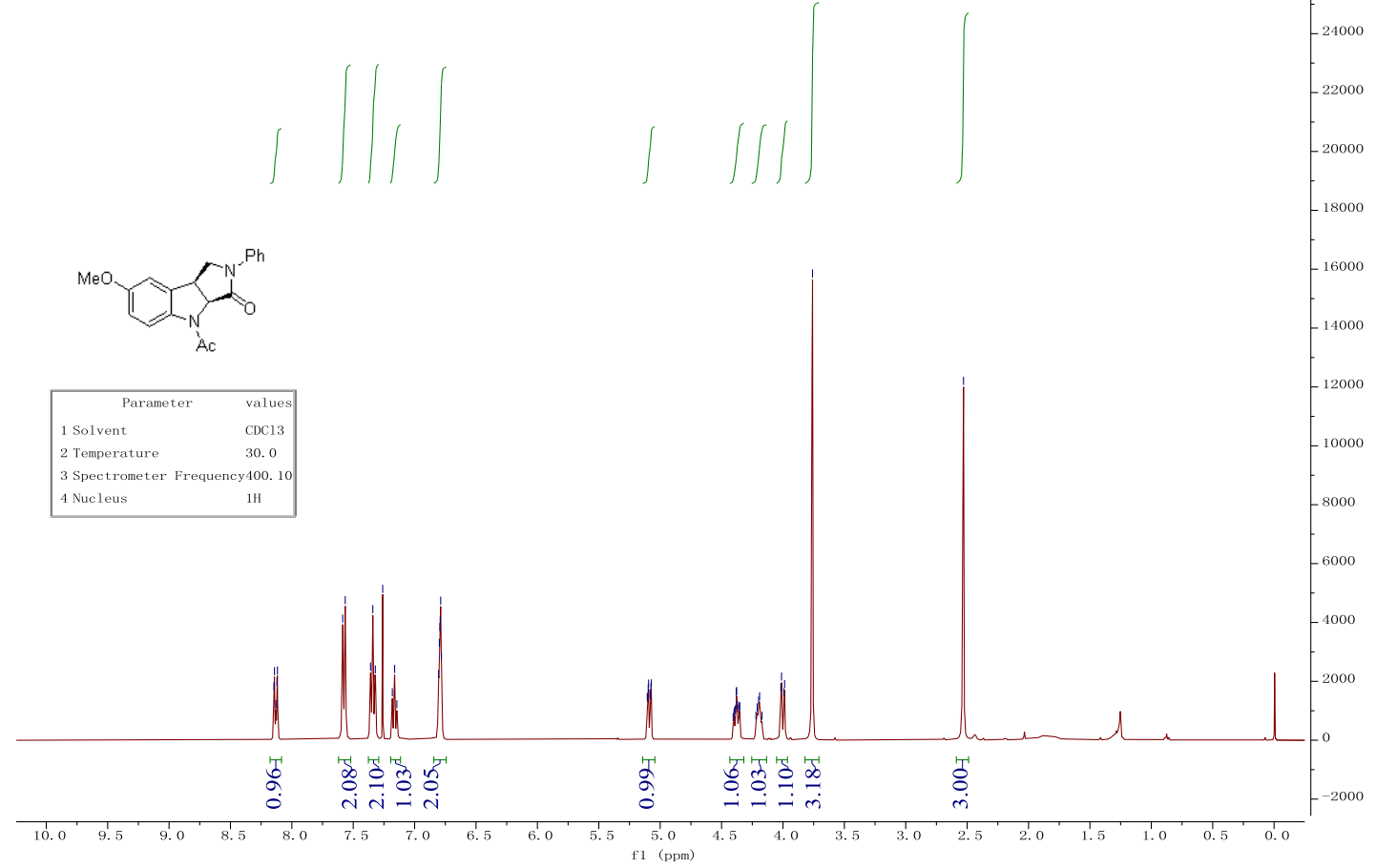

${ }^{13} \mathrm{C}$ NMR Spectrum of $\mathbf{4 d}$

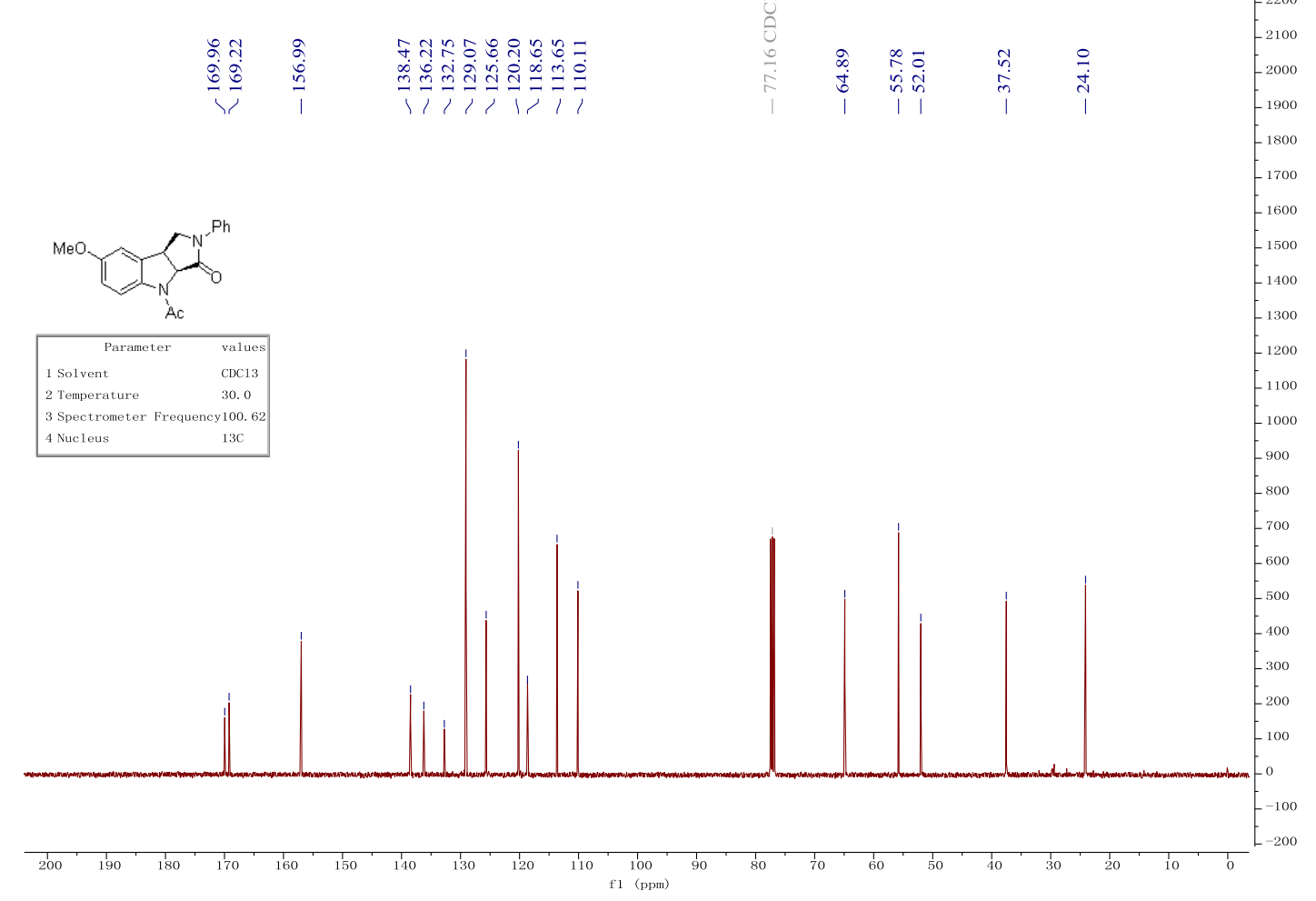


${ }^{1} \mathrm{H}$ NMR Spectrum of $\mathbf{4 e}$

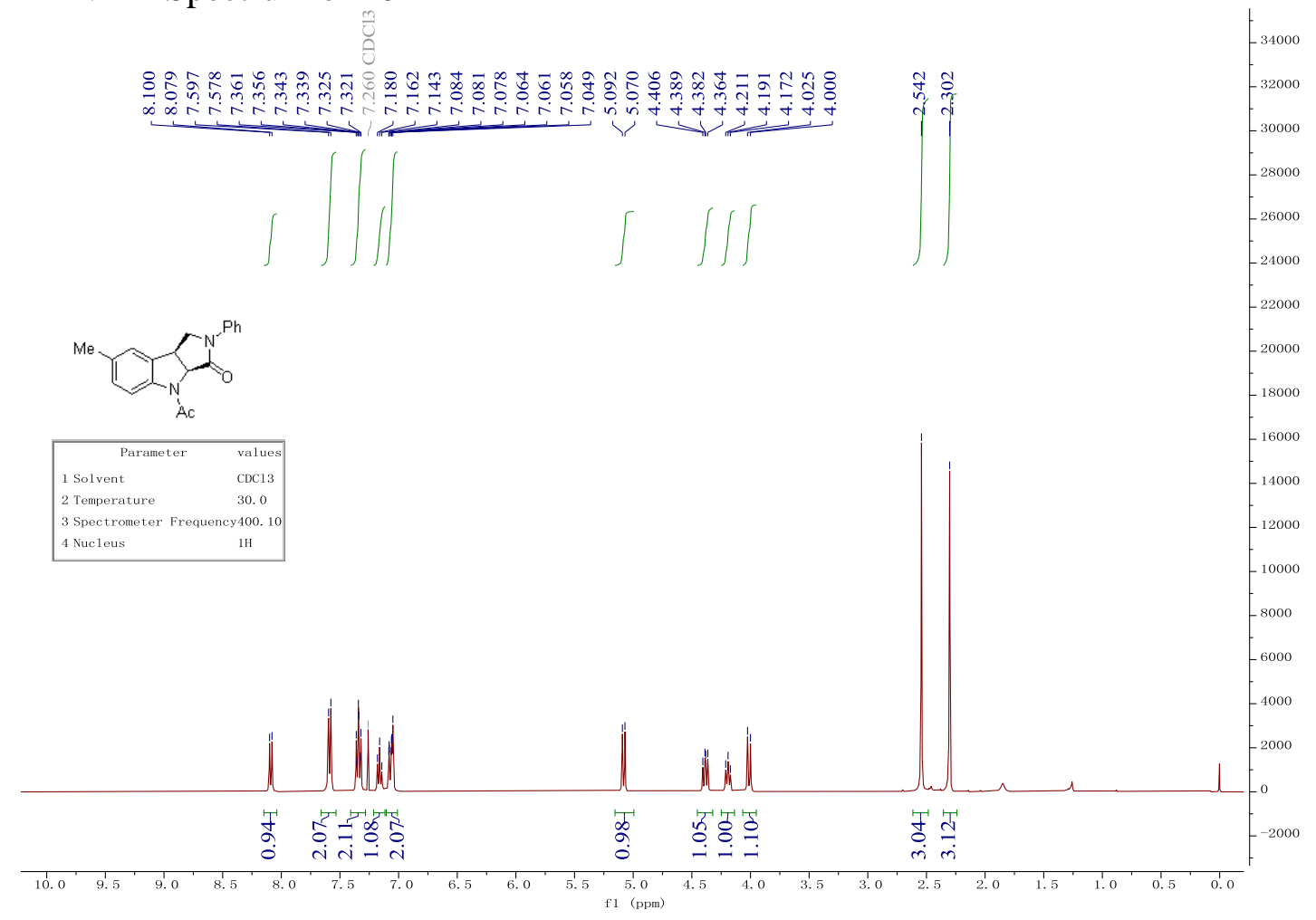

${ }^{13} \mathrm{C}$ NMR Spectrum of $\mathbf{4 e}$

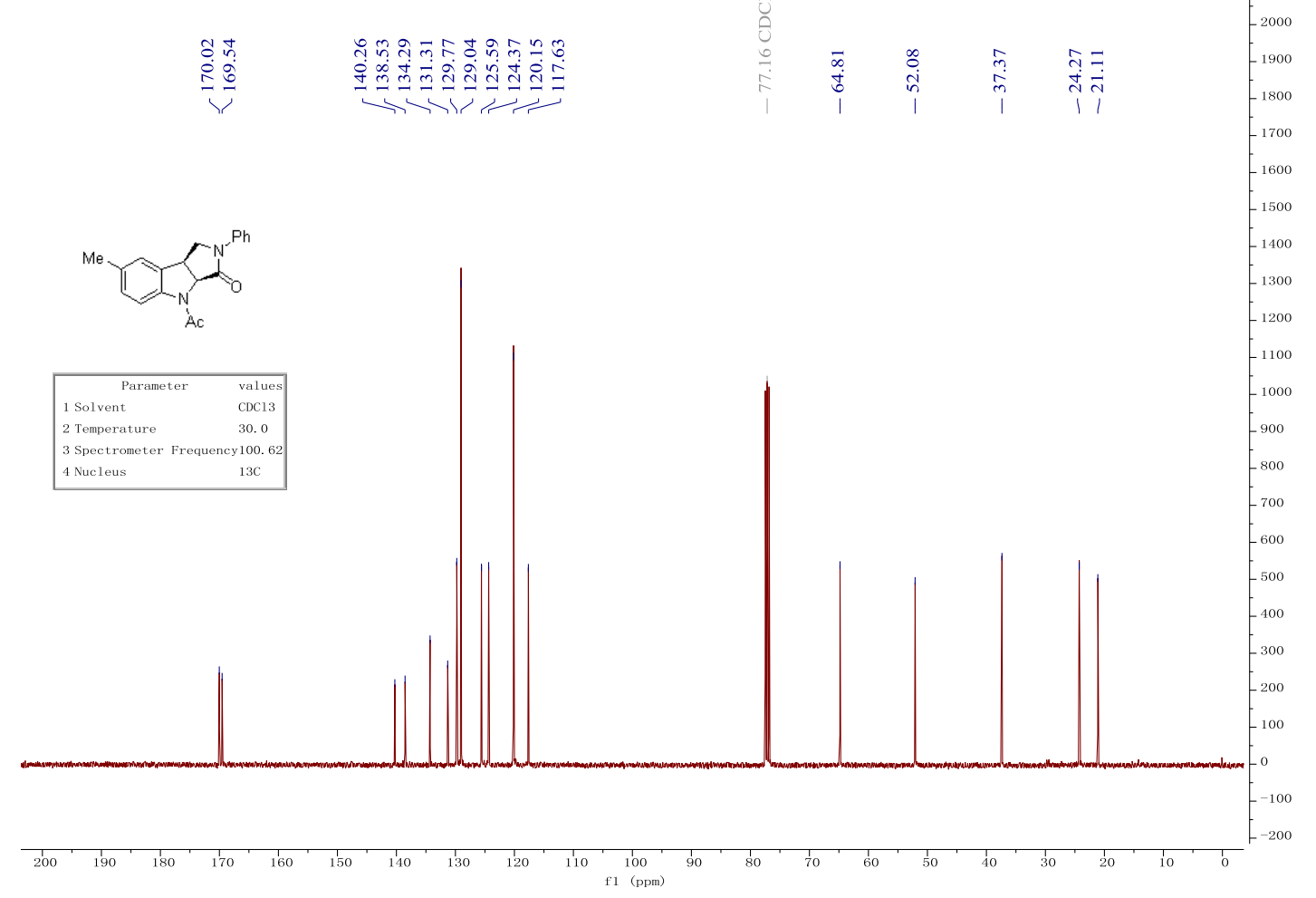


${ }^{1} \mathrm{H}$ NMR Spectrum of $\mathbf{4 f}$

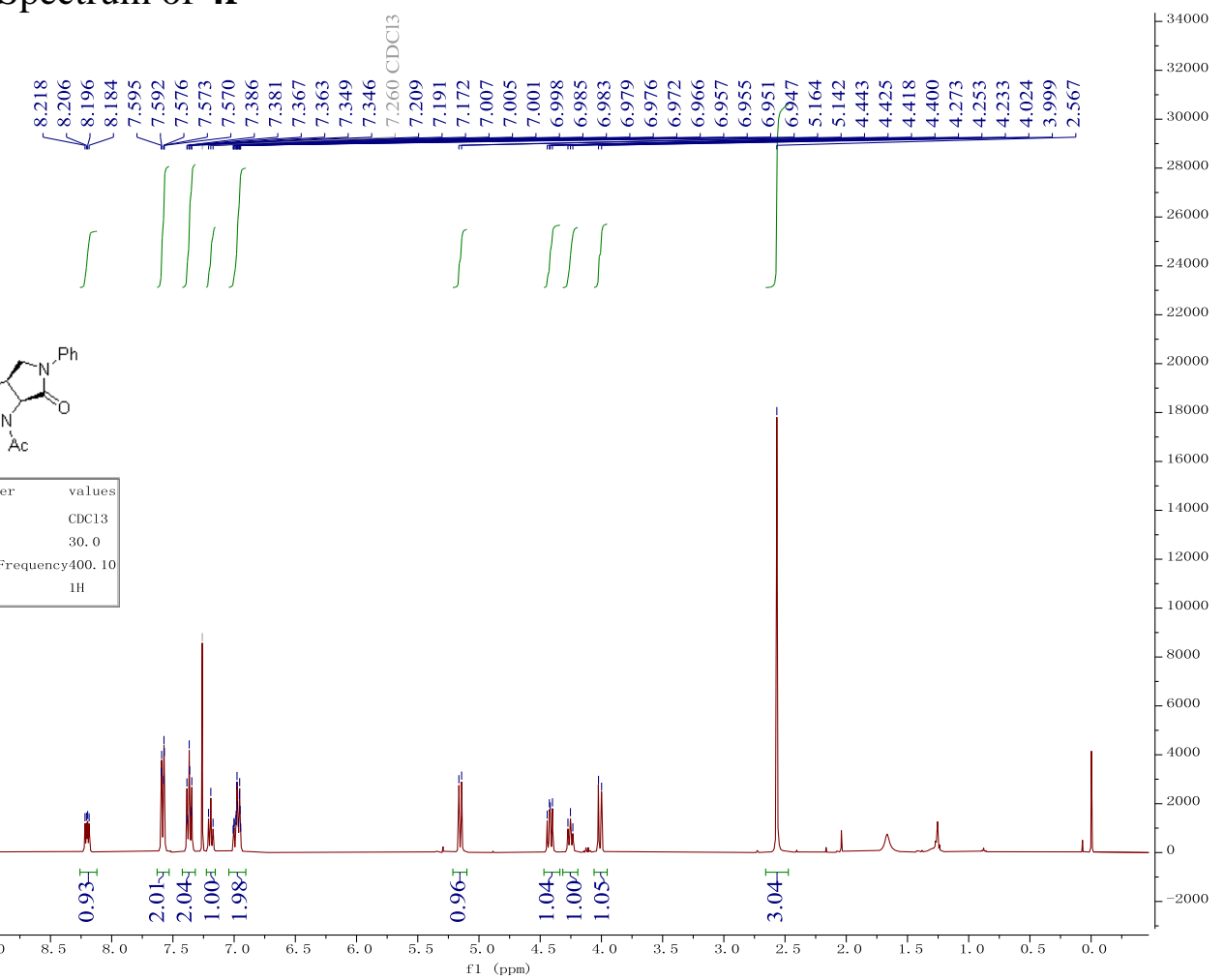

${ }^{13} \mathrm{C}$ NMR Spectrum of $\mathbf{4 f}$

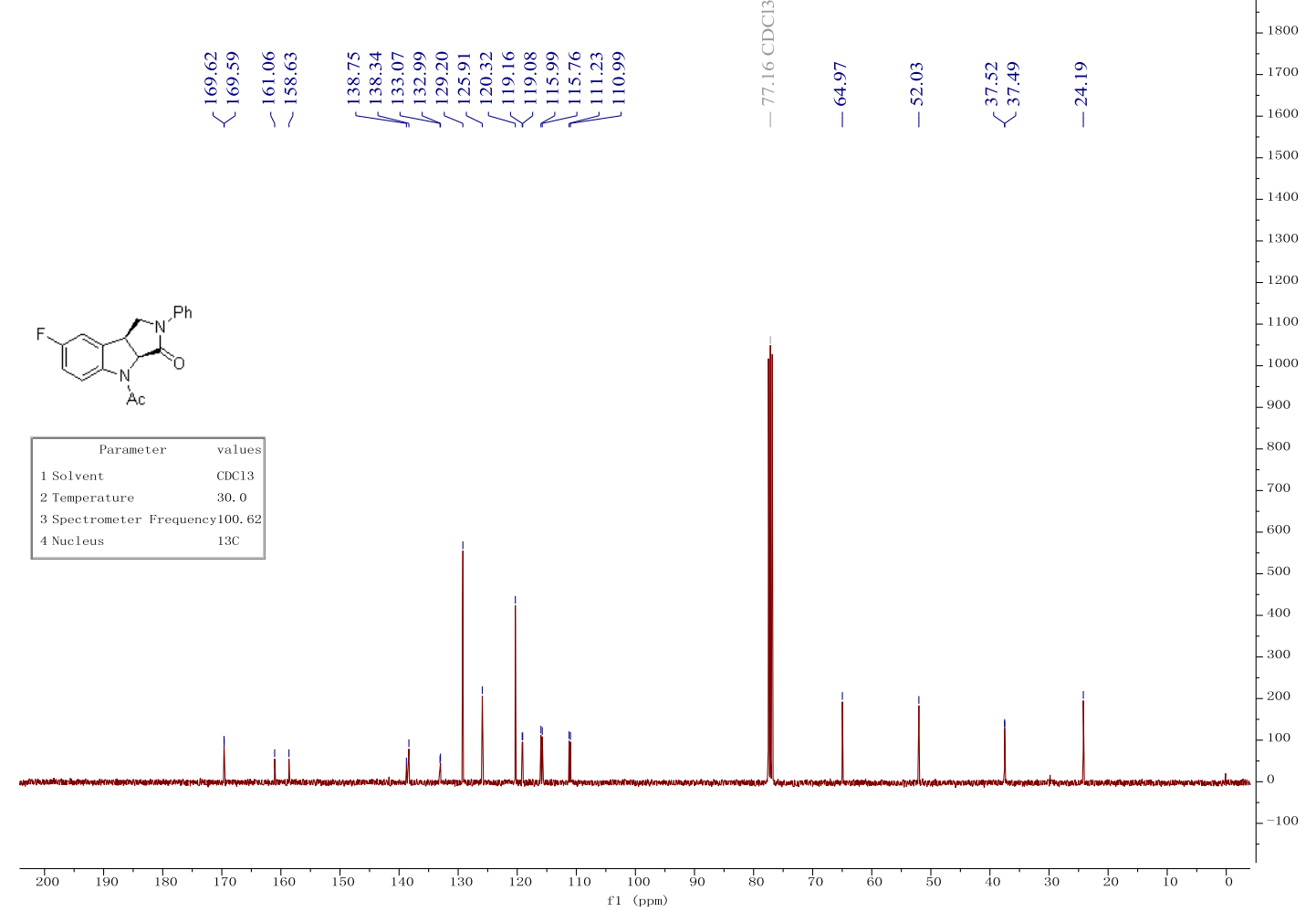


${ }^{19}$ F NMR Spectrum of $\mathbf{4 f}$

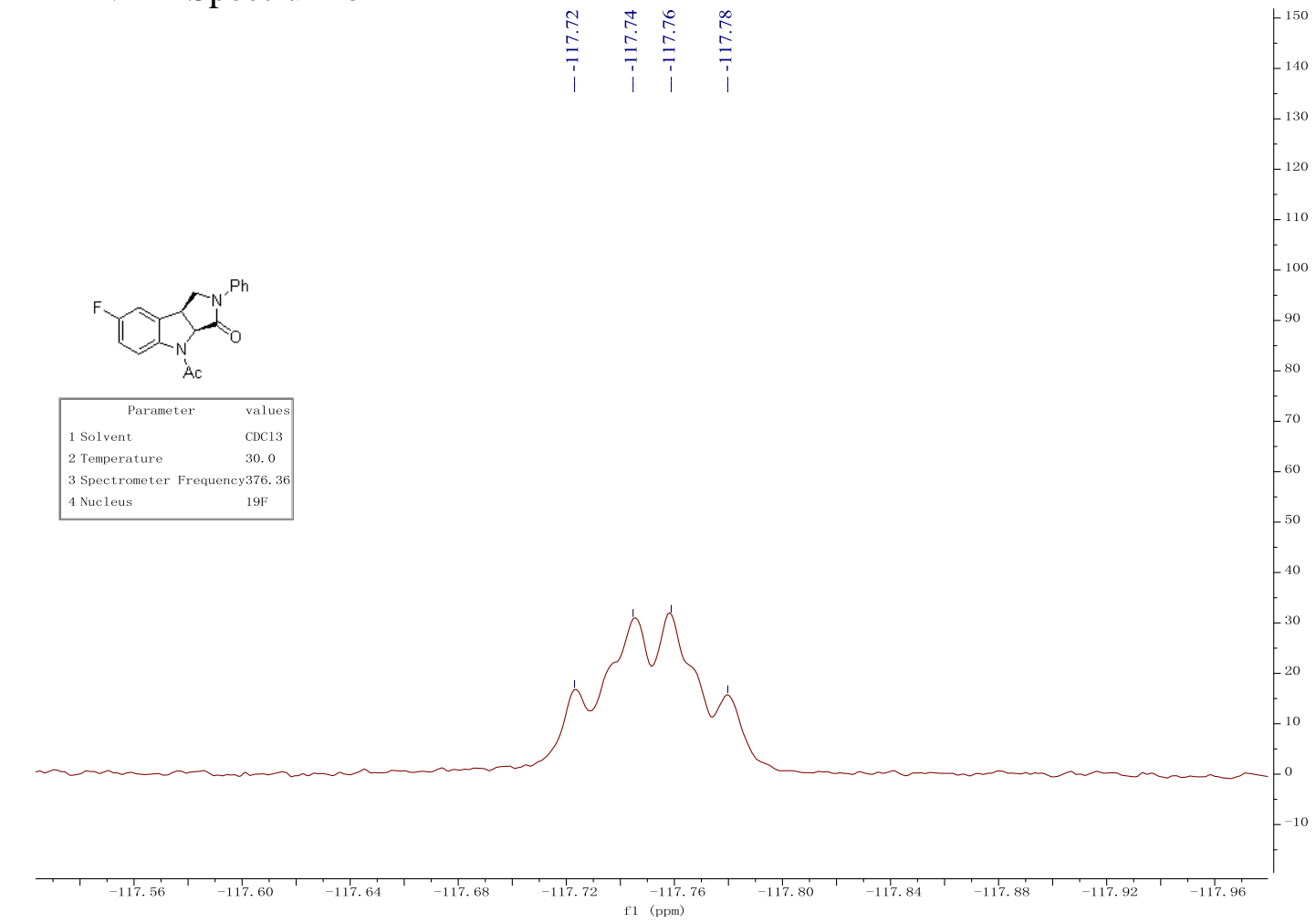

${ }^{1} \mathrm{H}$ NMR Spectrum of $\mathbf{4 g}$
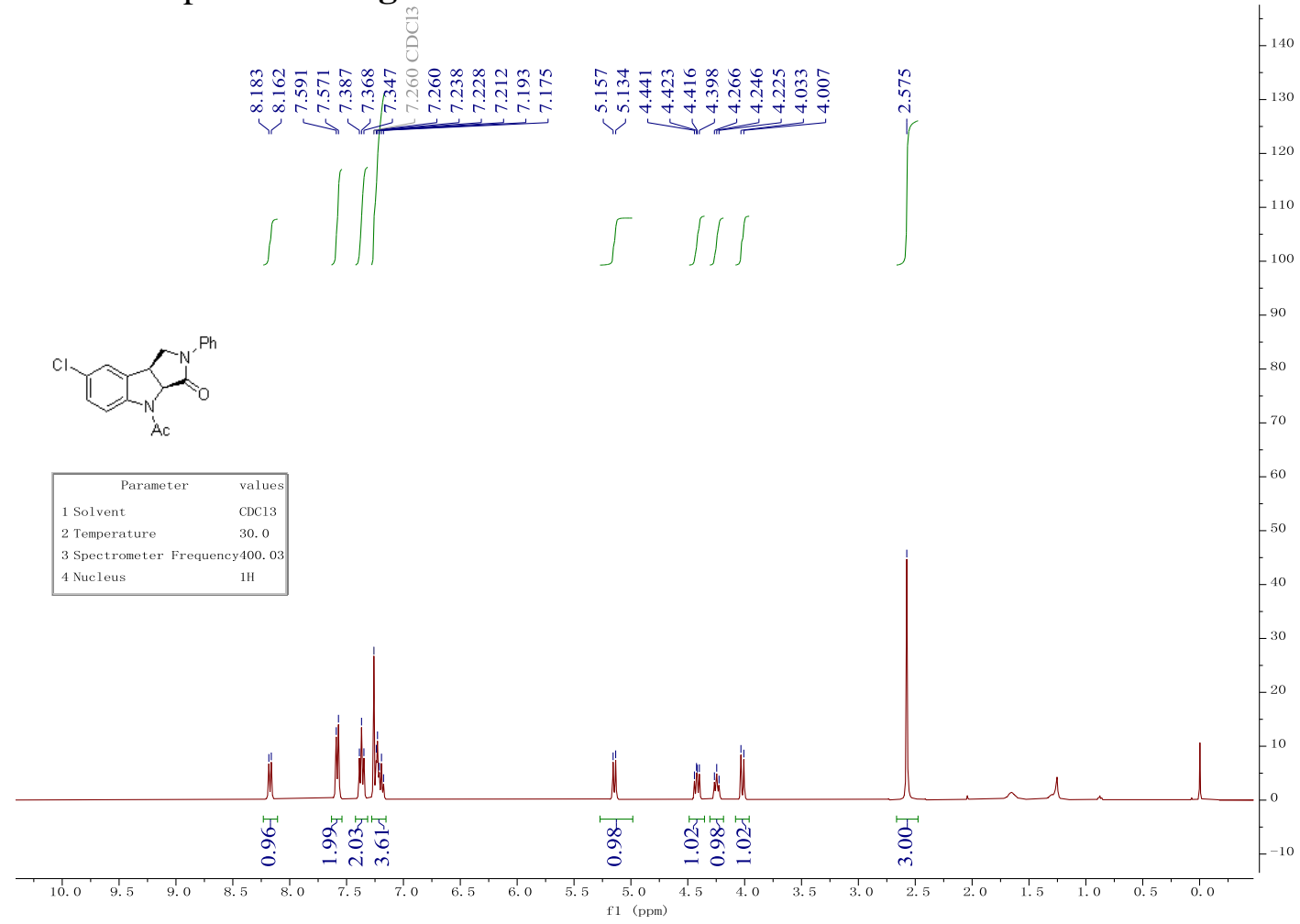
${ }^{13} \mathrm{C}$ NMR Spectrum of $\mathbf{4 g}$

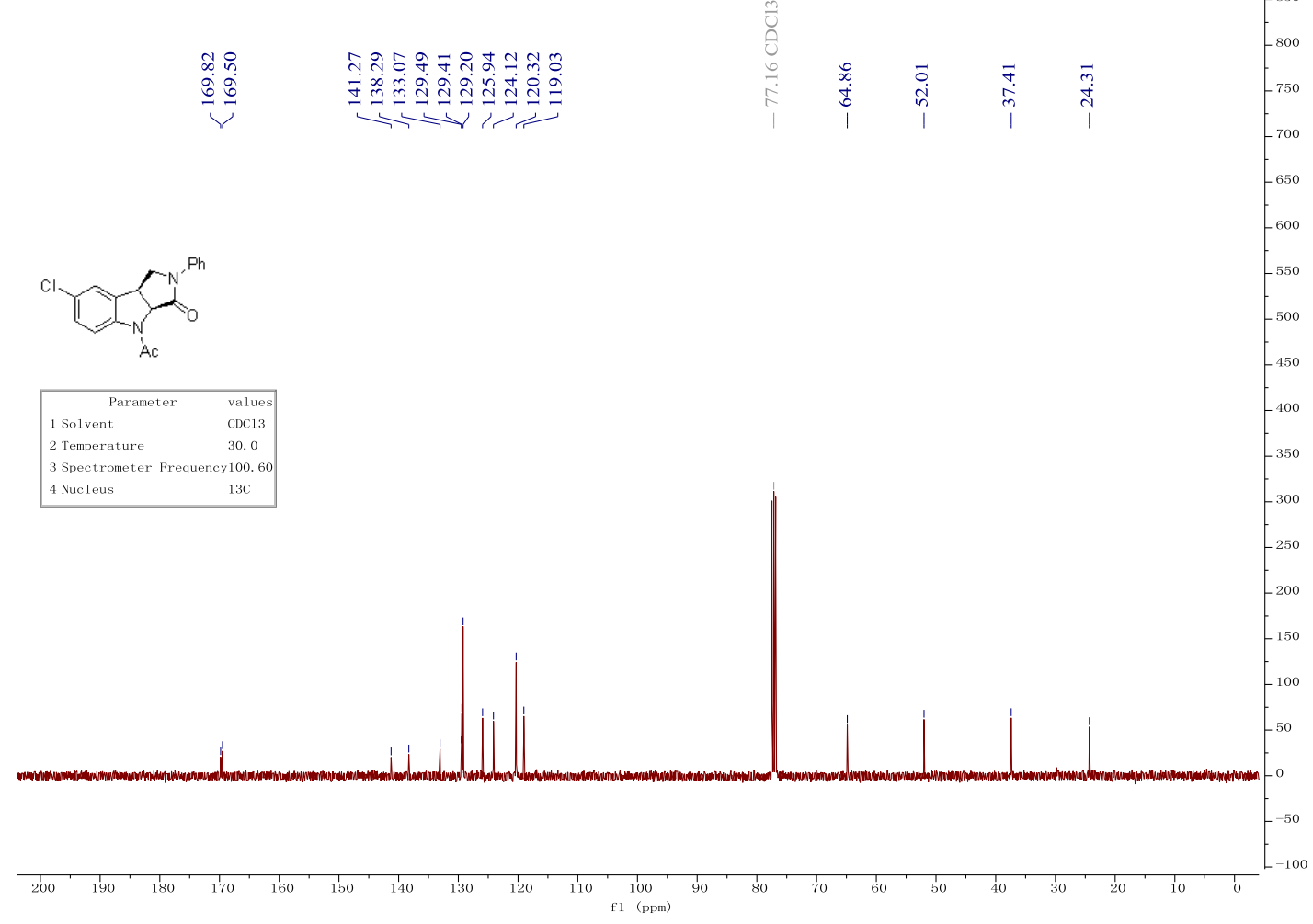

${ }^{1} \mathrm{H}$ NMR Spectrum of $\mathbf{4 h}$

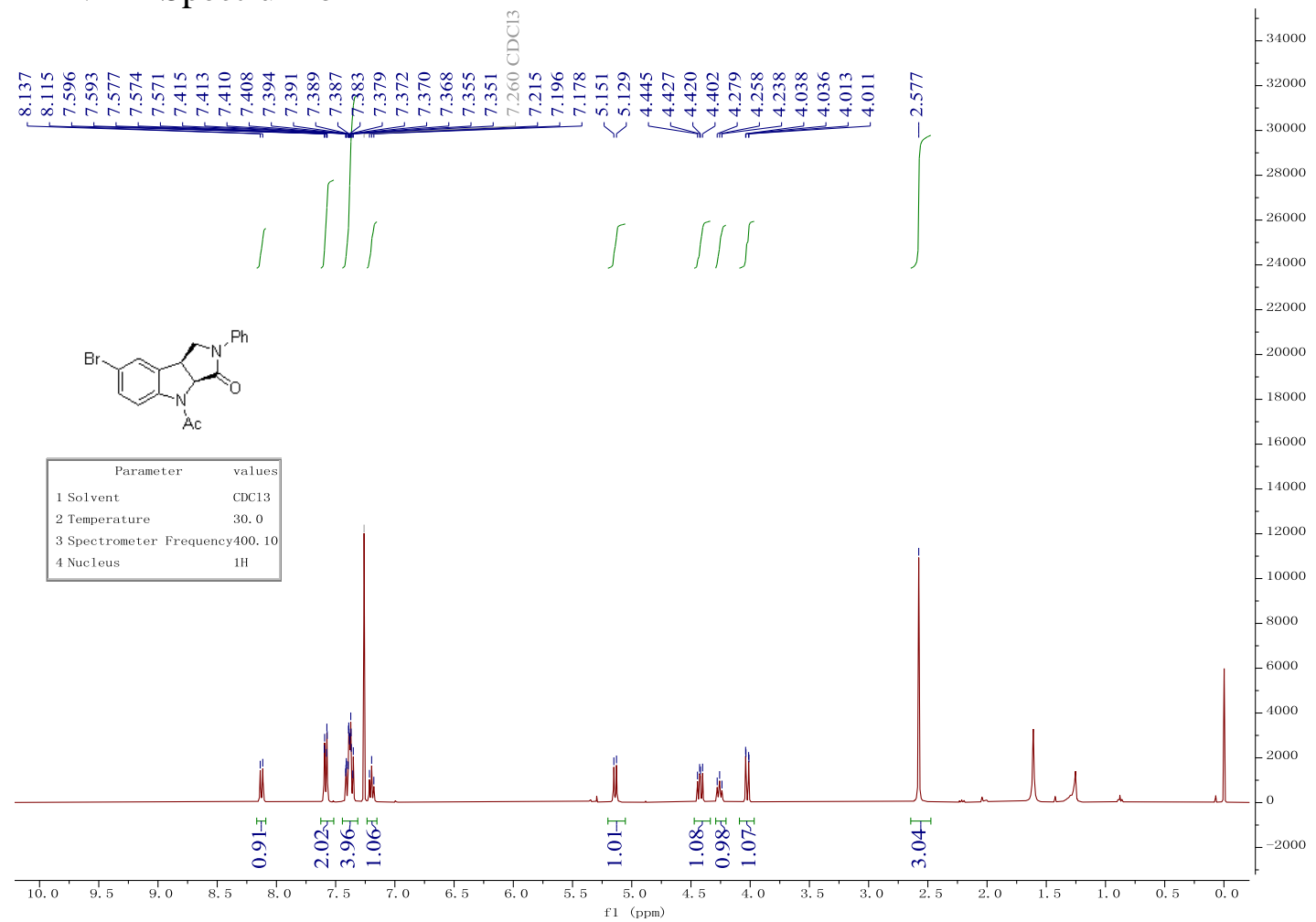


${ }^{13} \mathrm{C}$ NMR Spectrum of $\mathbf{4 h}$

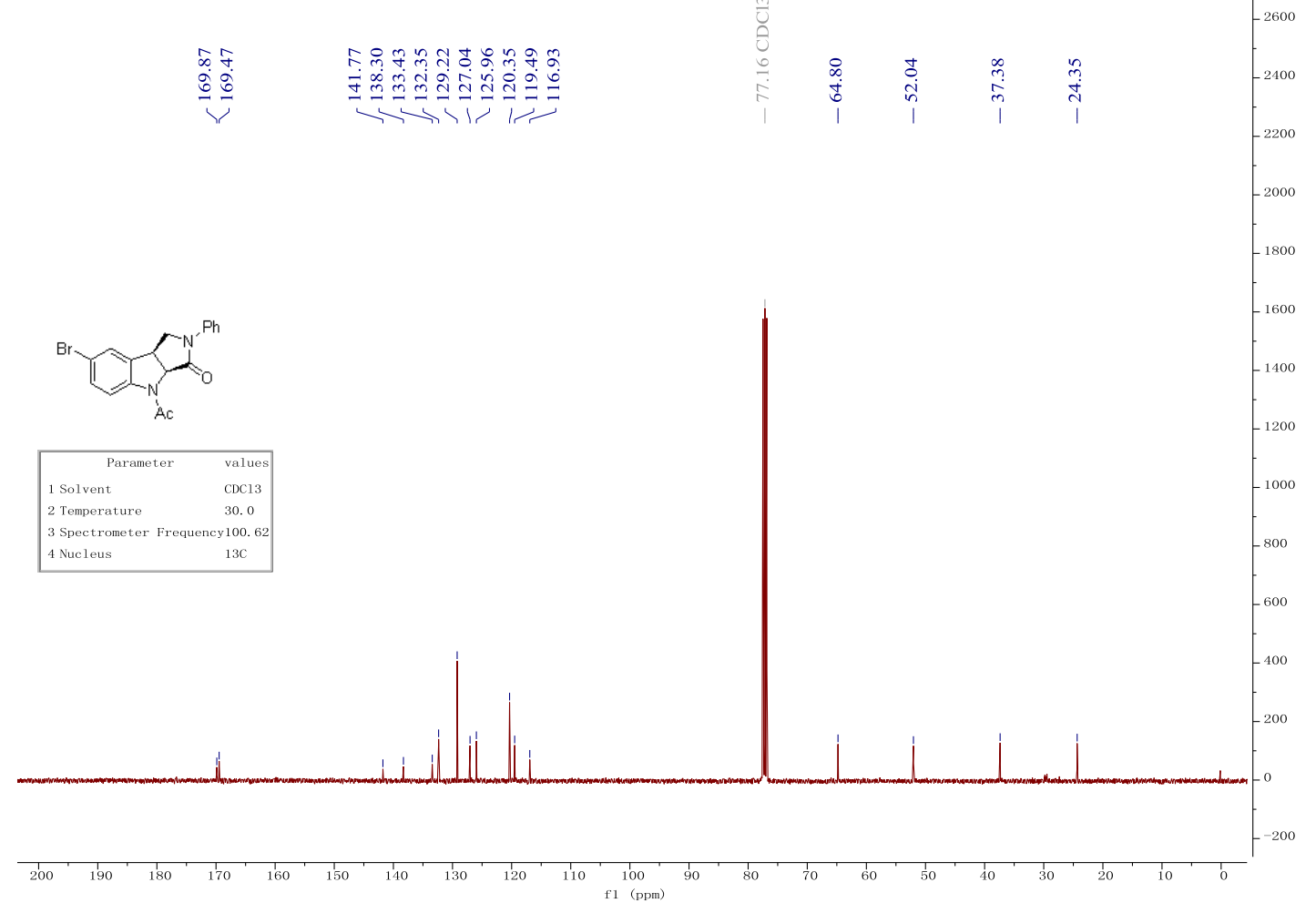

${ }^{1} \mathrm{H}$ NMR Spectrum of $\mathbf{4 i}$

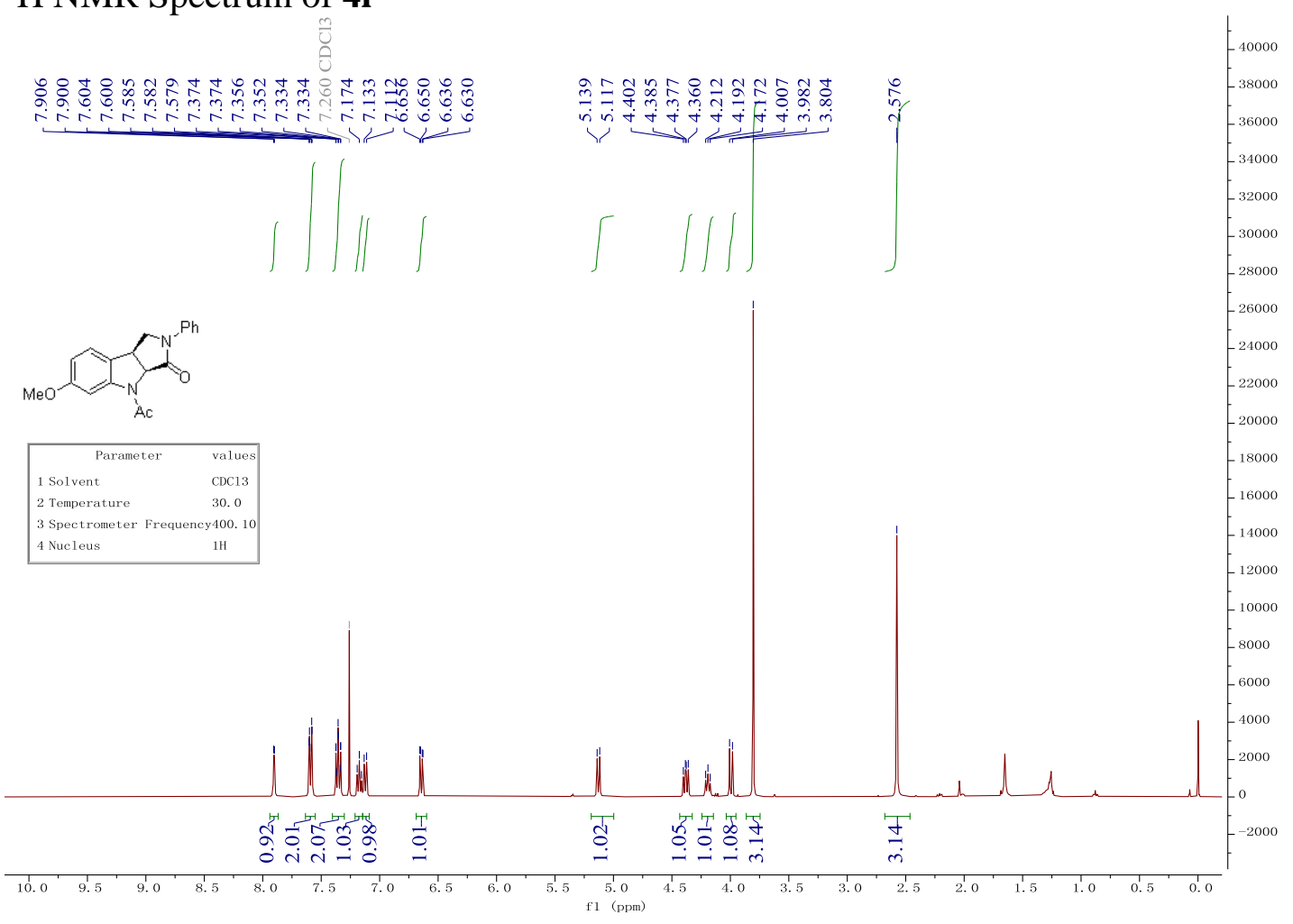


${ }^{13} \mathrm{C}$ NMR Spectrum of $\mathbf{4 i}$

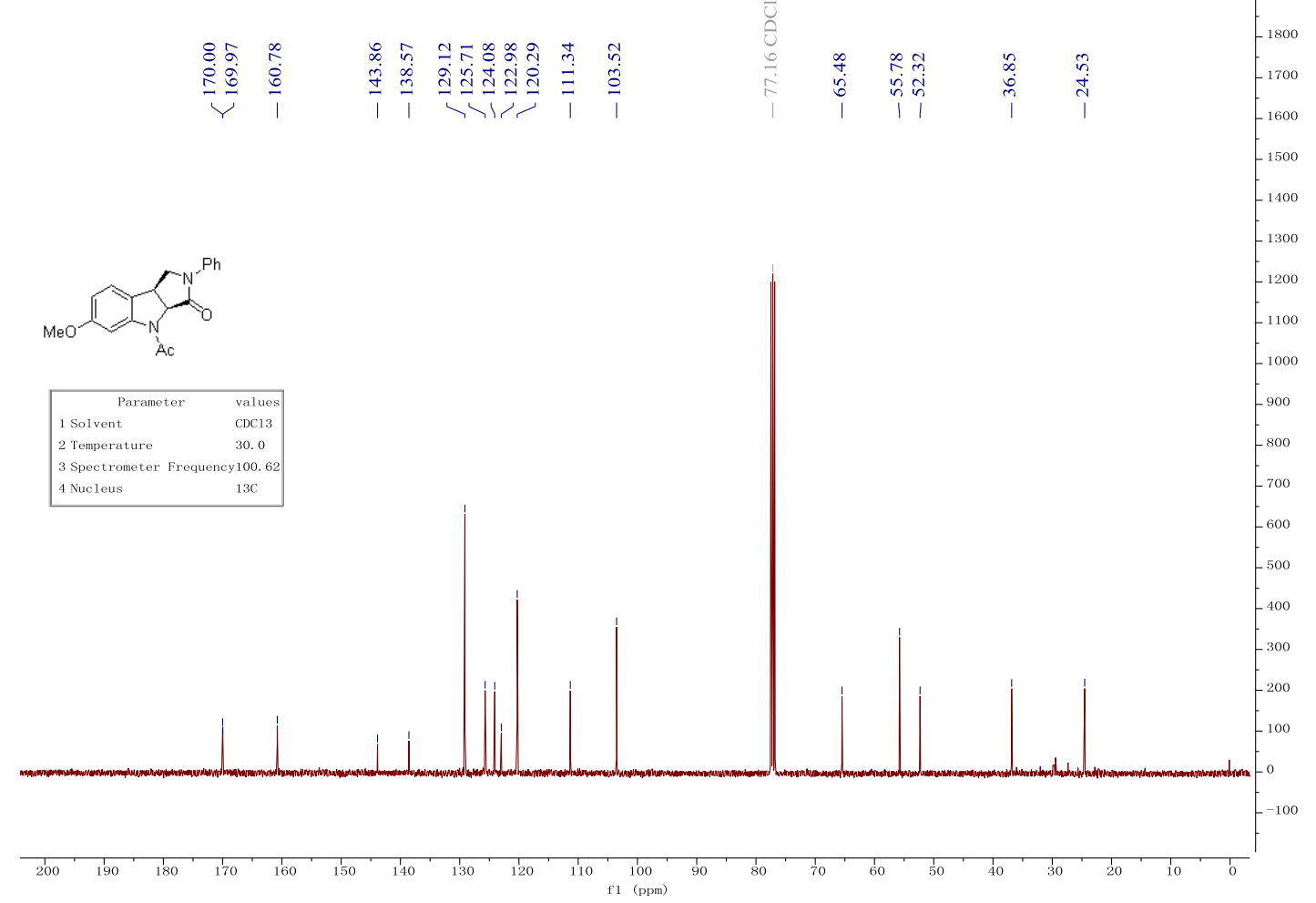

${ }^{1} \mathrm{H}$ NMR Spectrum of $\mathbf{4 j}$
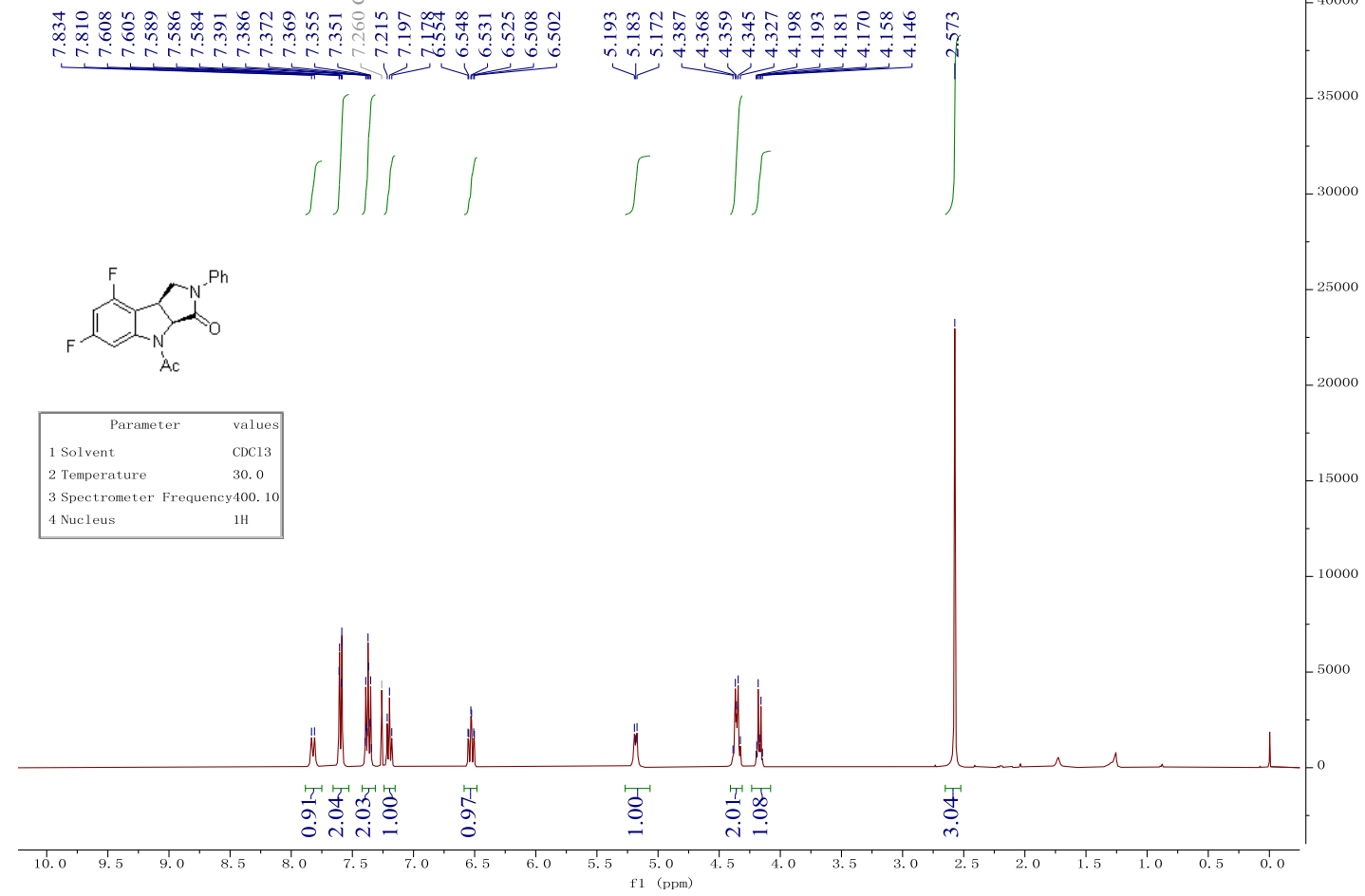
${ }^{13} \mathrm{C}$ NMR Spectrum of $\mathbf{4 j}$

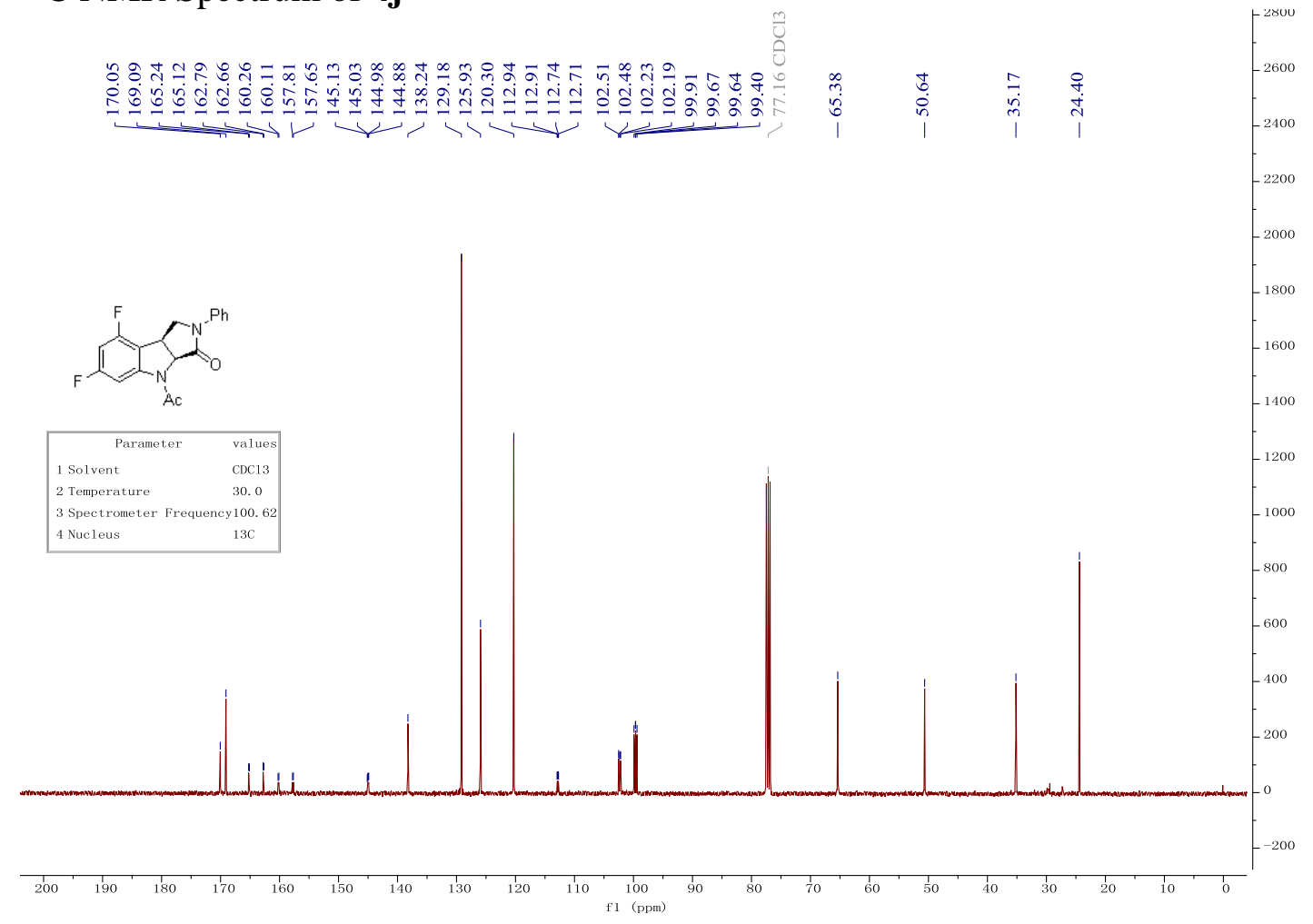

${ }^{19} \mathrm{~F}$ NMR Spectrum of $\mathbf{4 j}$

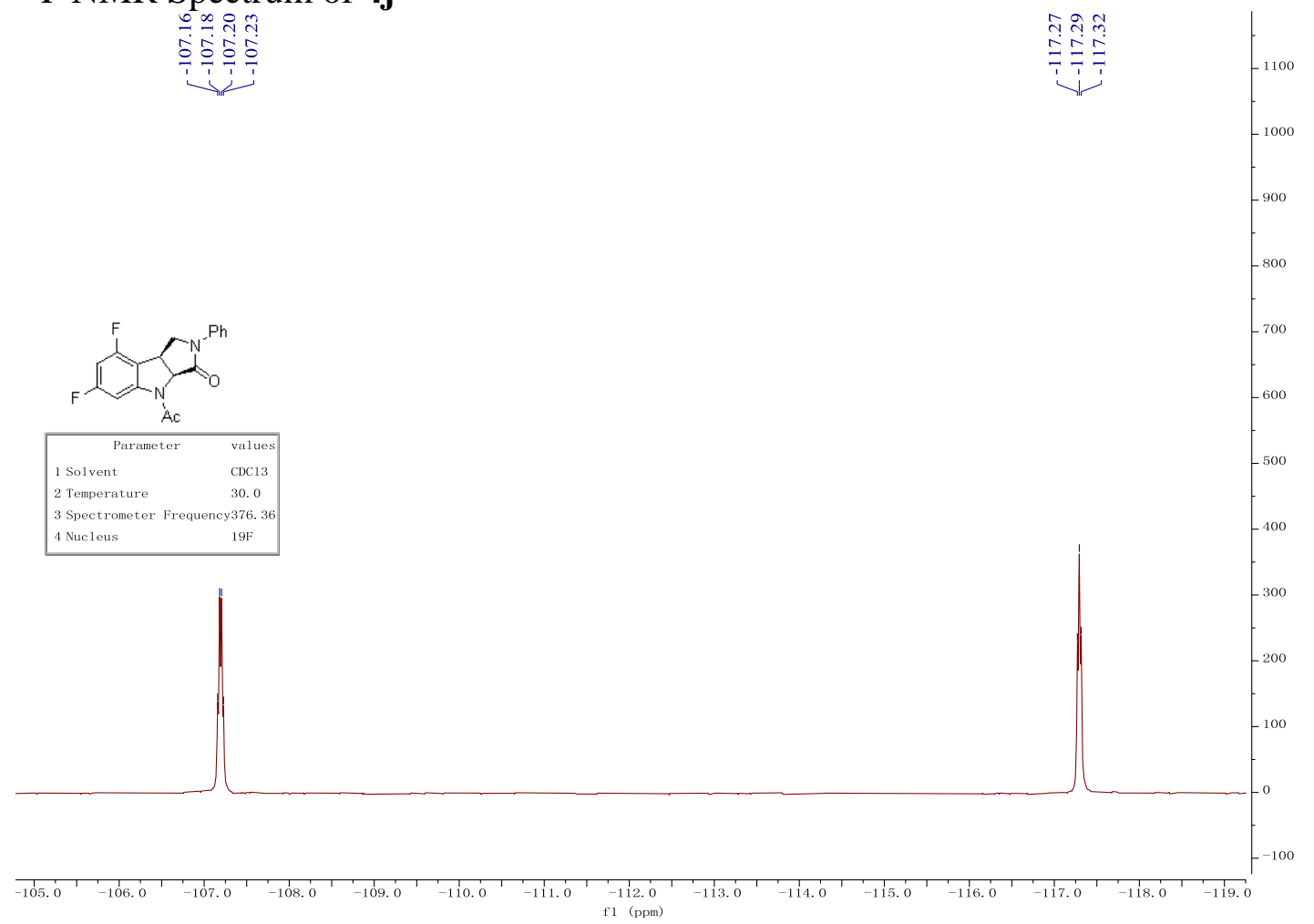

${ }^{1} \mathrm{H}$ NMR Spectrum of $\mathbf{4 k}$ 


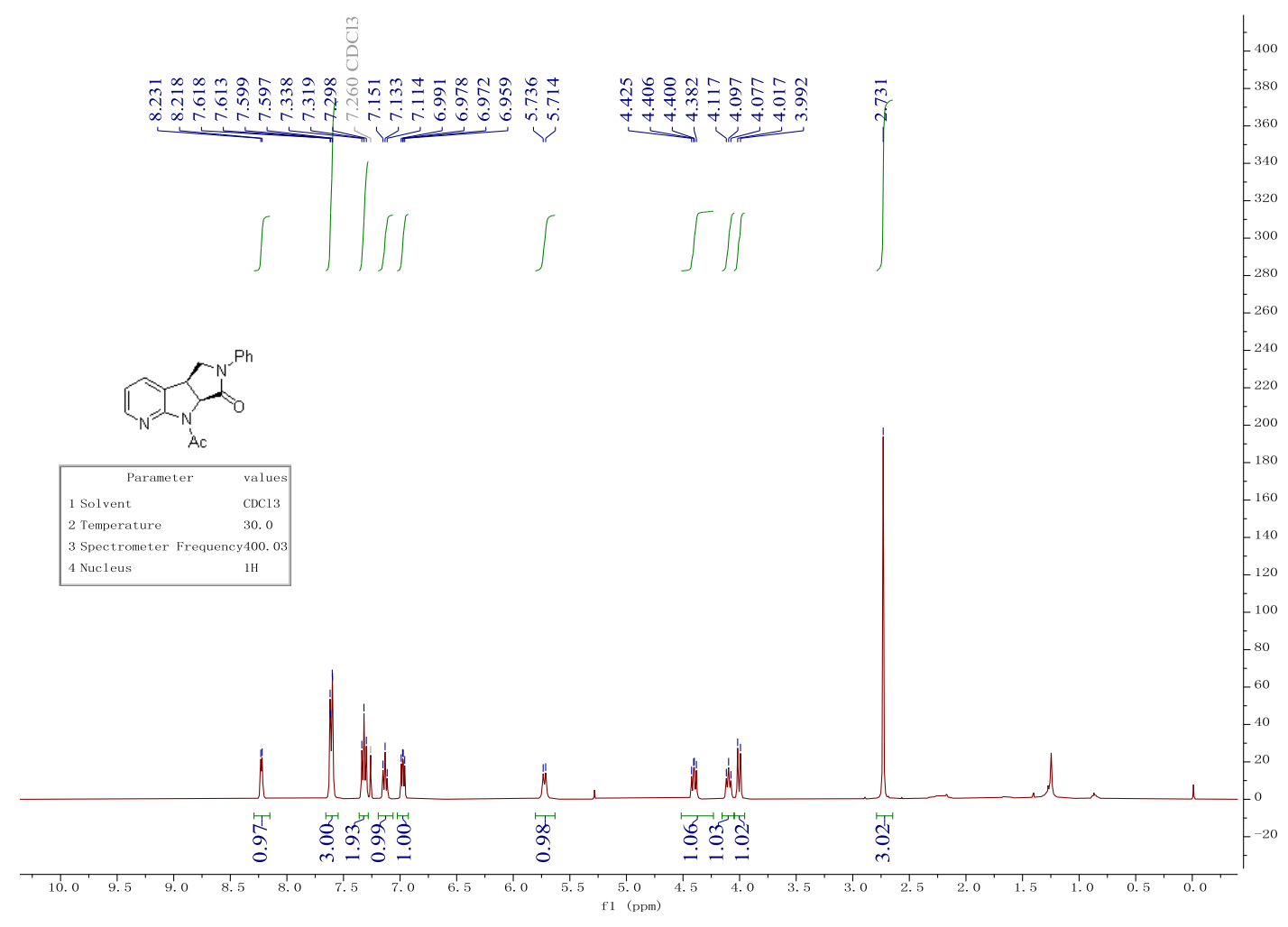

${ }^{13} \mathrm{C}$ NMR Spectrum of $\mathbf{4 k}$

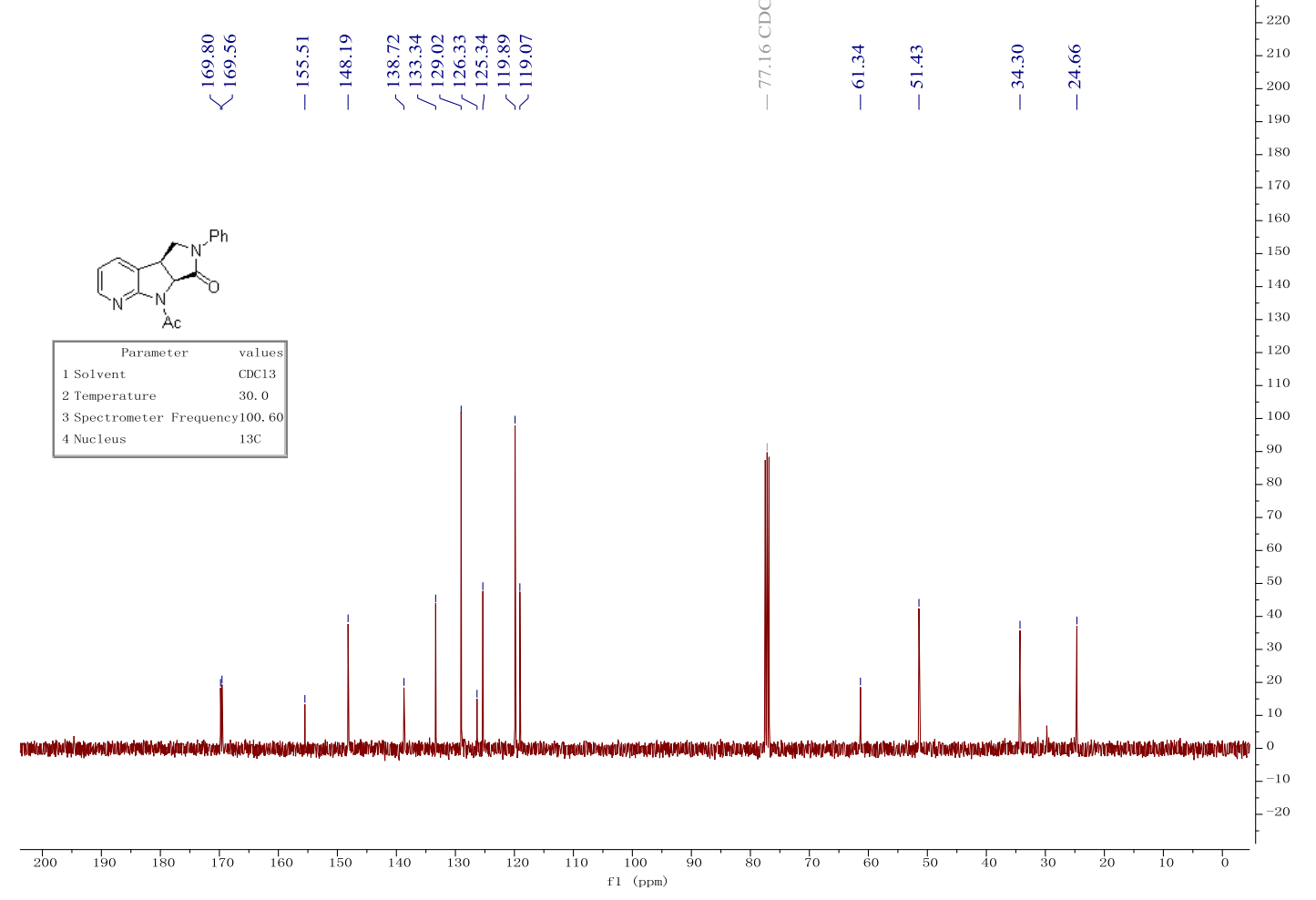


${ }^{1} \mathrm{H}$ NMR Spectrum of $\mathbf{4 l}$

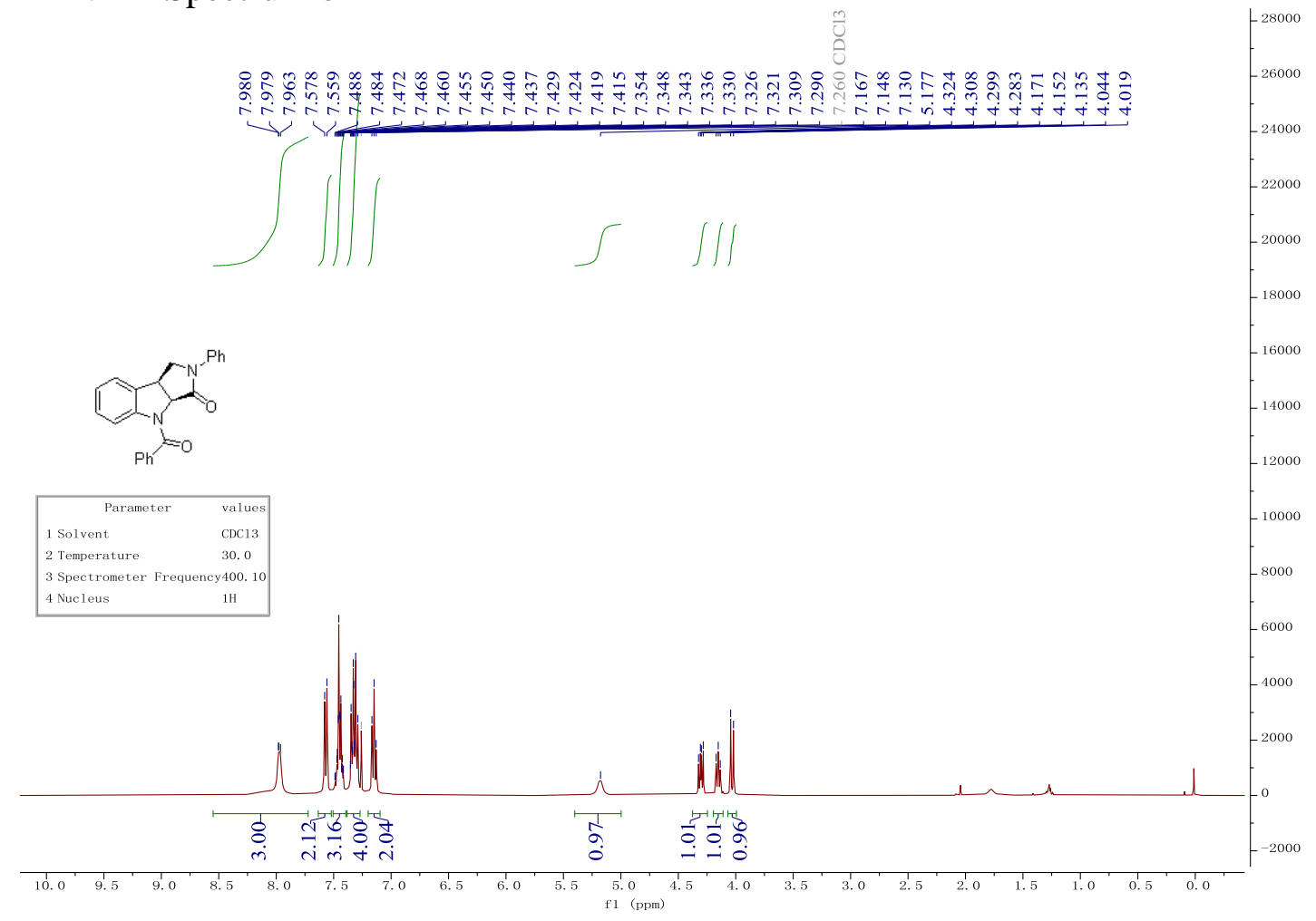

${ }^{13} \mathrm{C}$ NMR Spectrum of $\mathbf{4 I}$

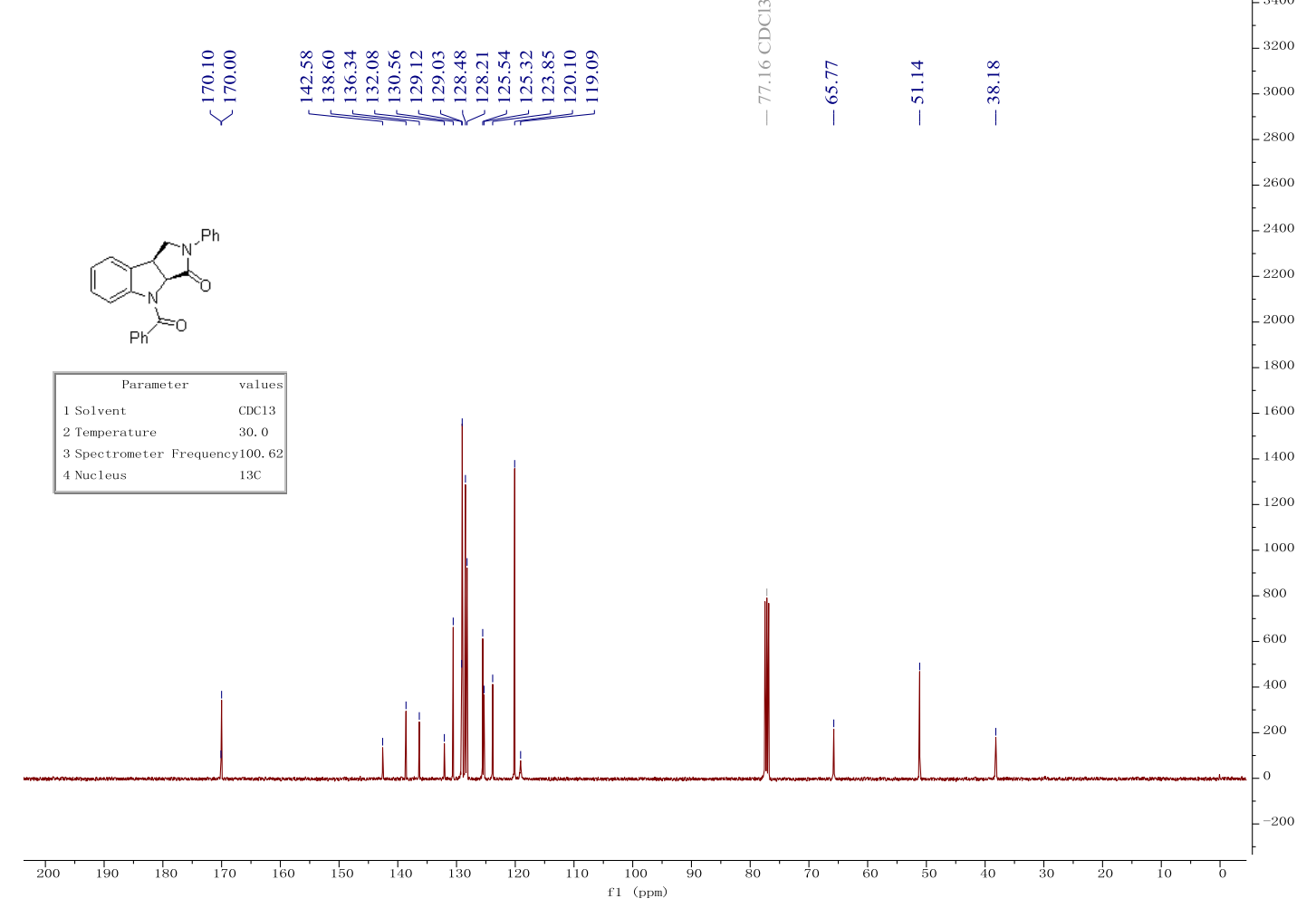


${ }^{1} \mathrm{H}$ NMR Spectrum of $\mathbf{4 m}$

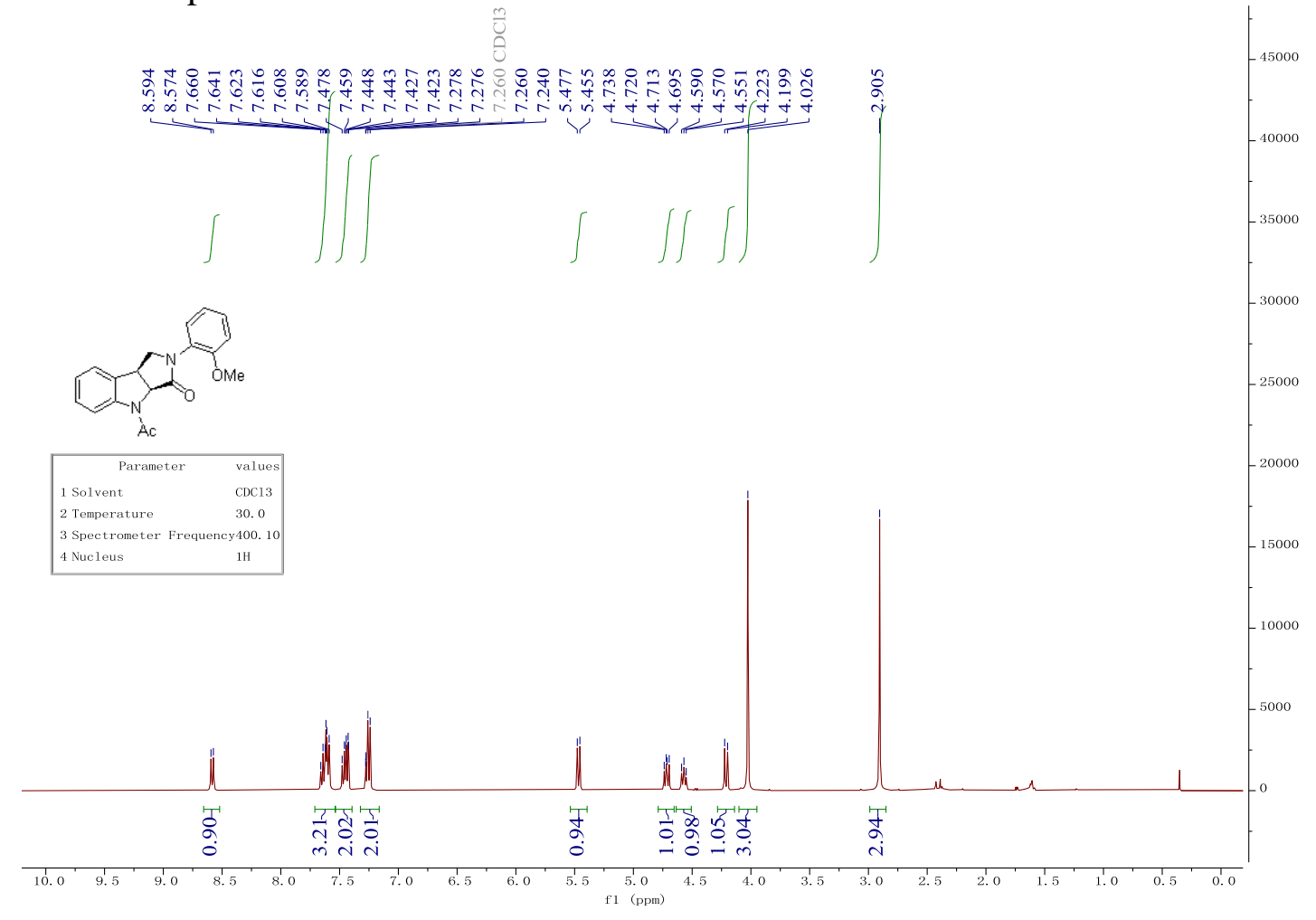

${ }^{13} \mathrm{C}$ NMR Spectrum of $\mathbf{4 m}$

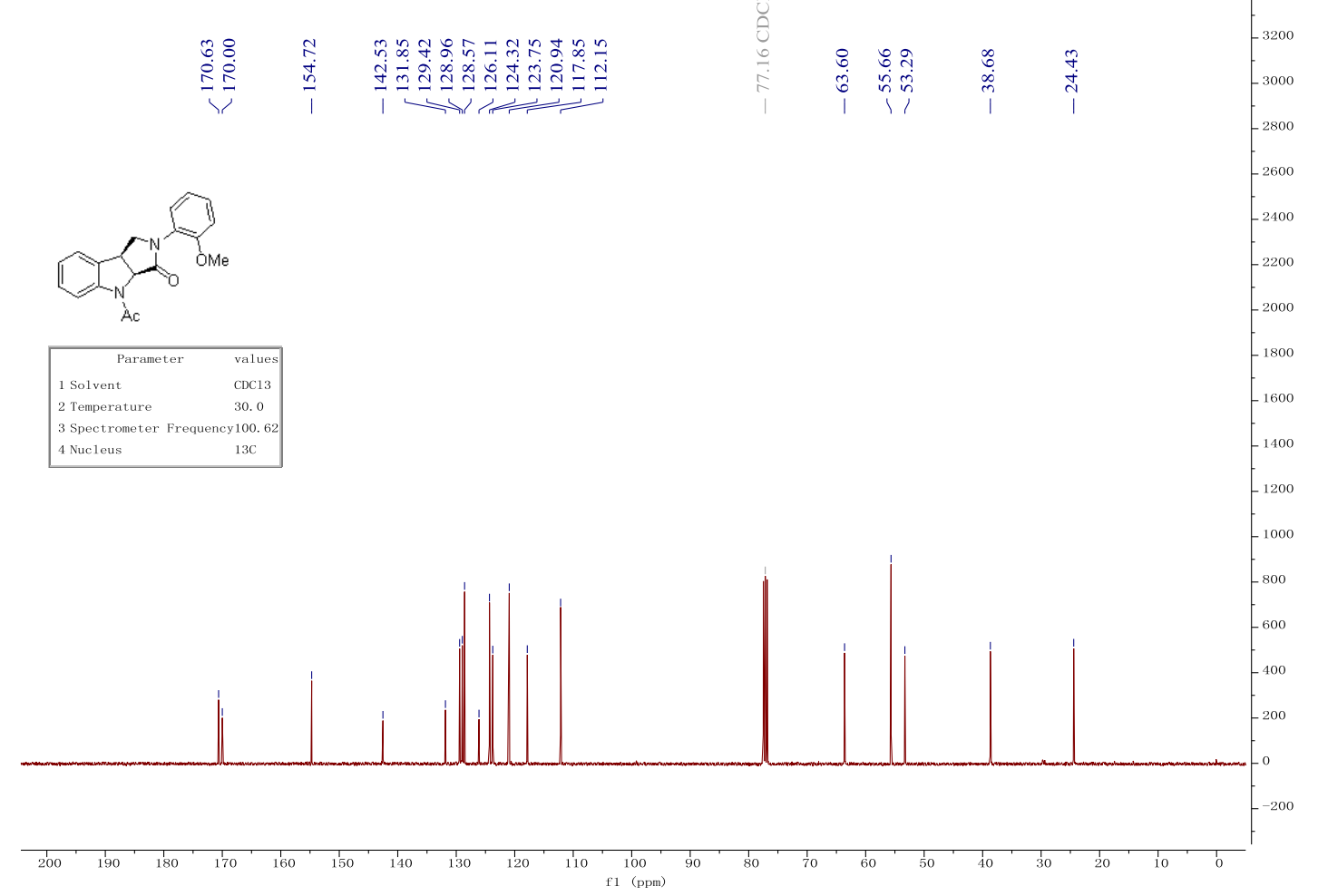


${ }^{1} \mathrm{H}$ NMR Spectrum of $\mathbf{4 n}$

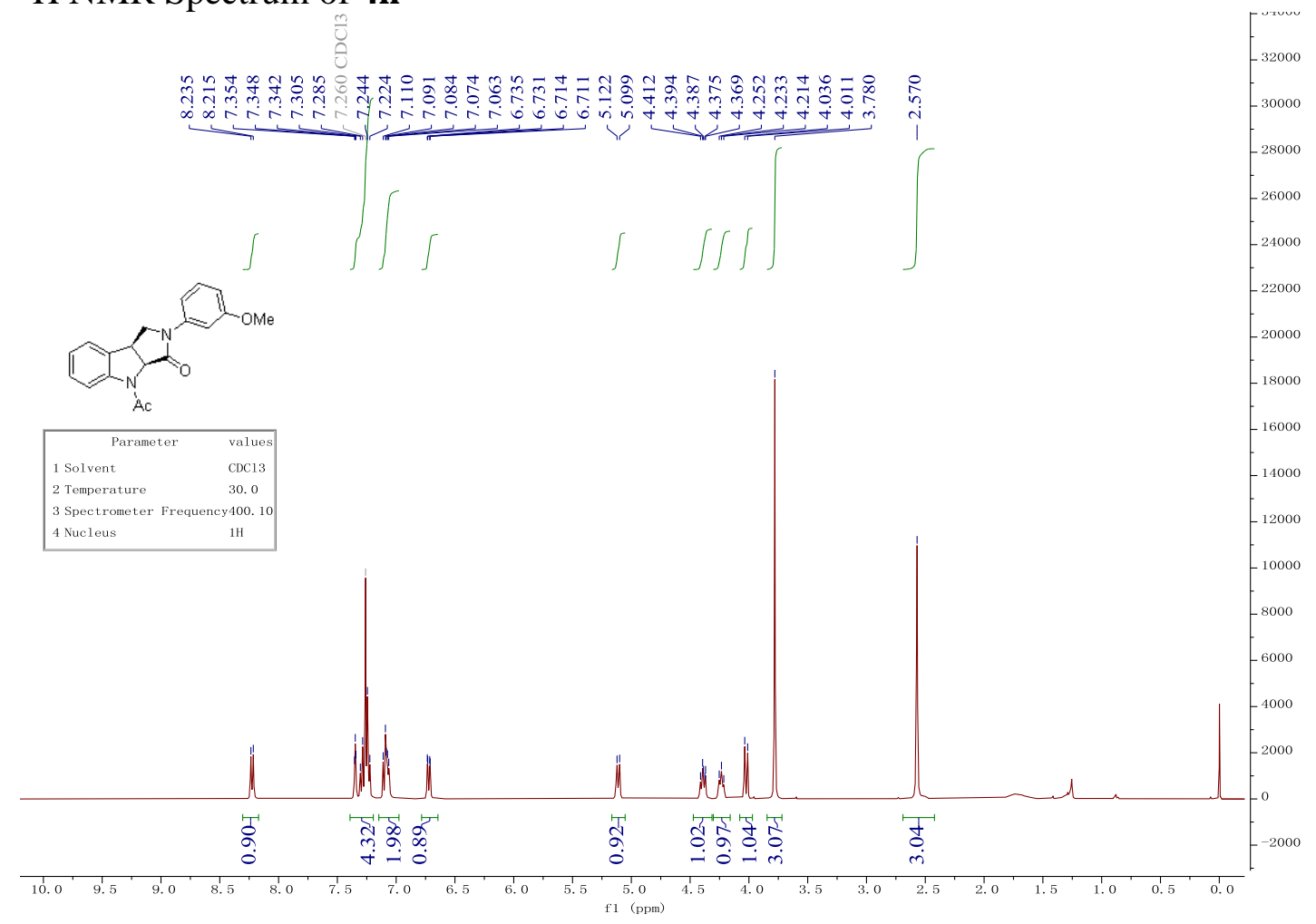

${ }^{13} \mathrm{C}$ NMR Spectrum of $\mathbf{4 n}$

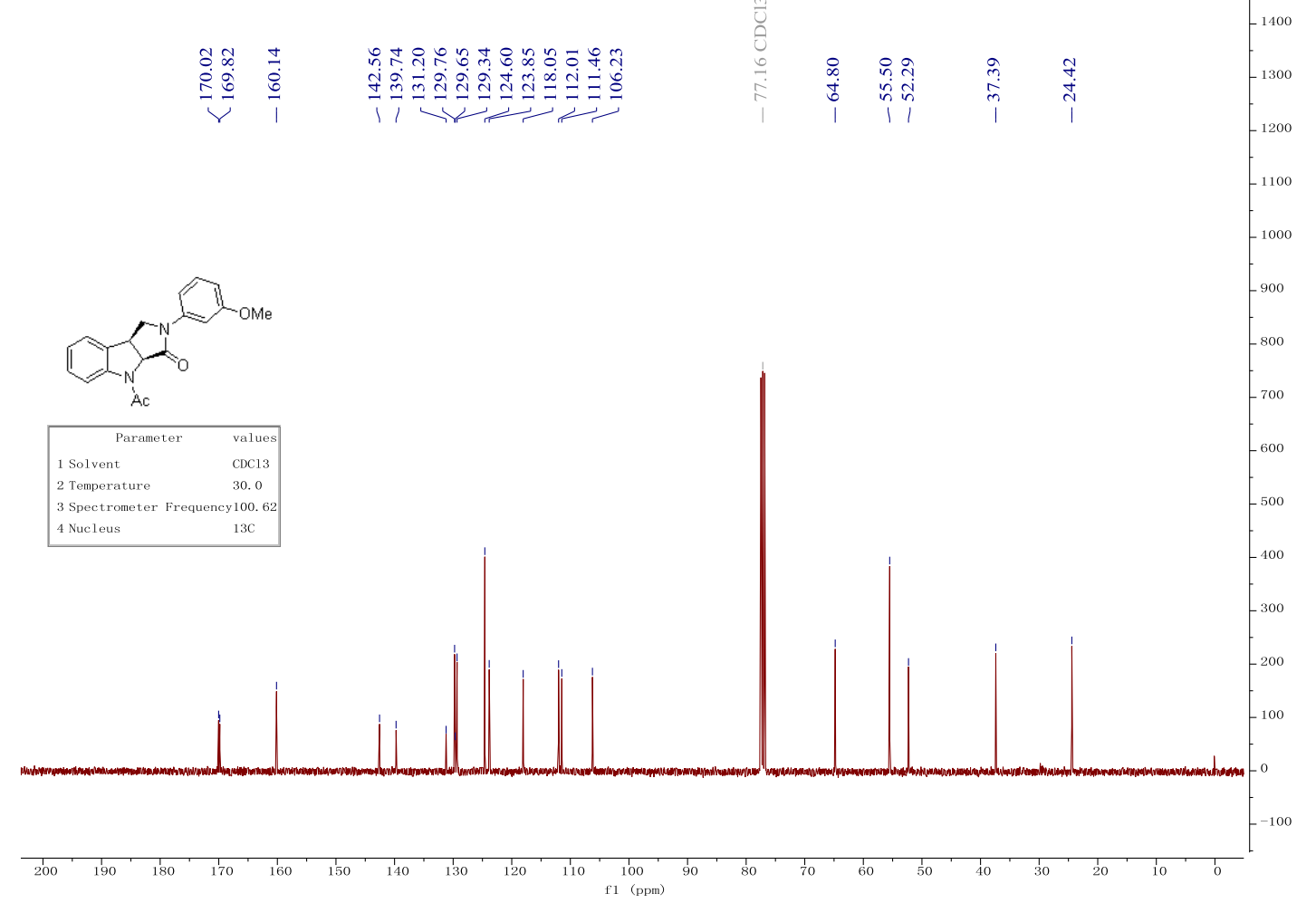


${ }^{1} \mathrm{H}$ NMR Spectrum of 40

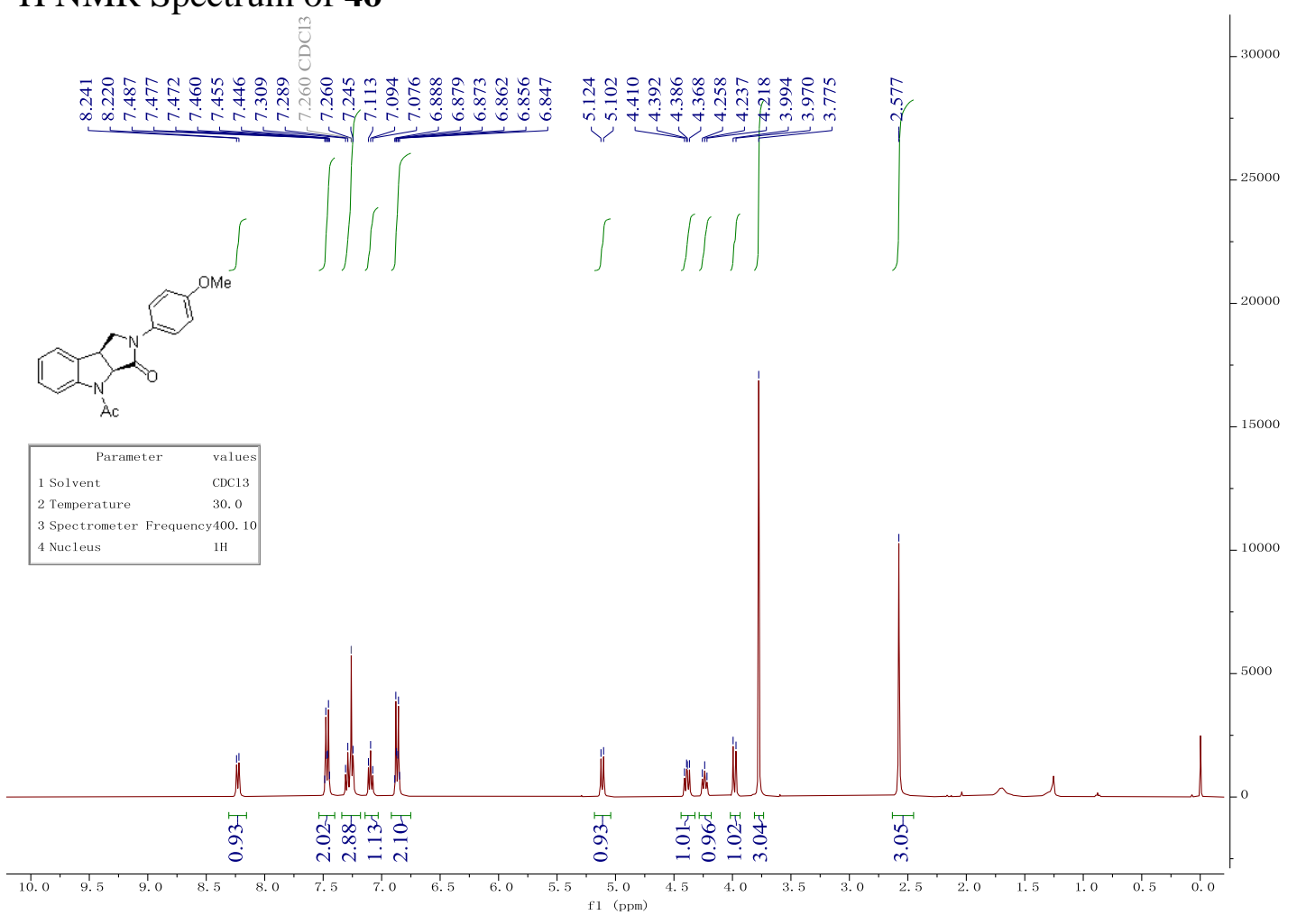

${ }^{13} \mathrm{C}$ NMR Spectrum of 40

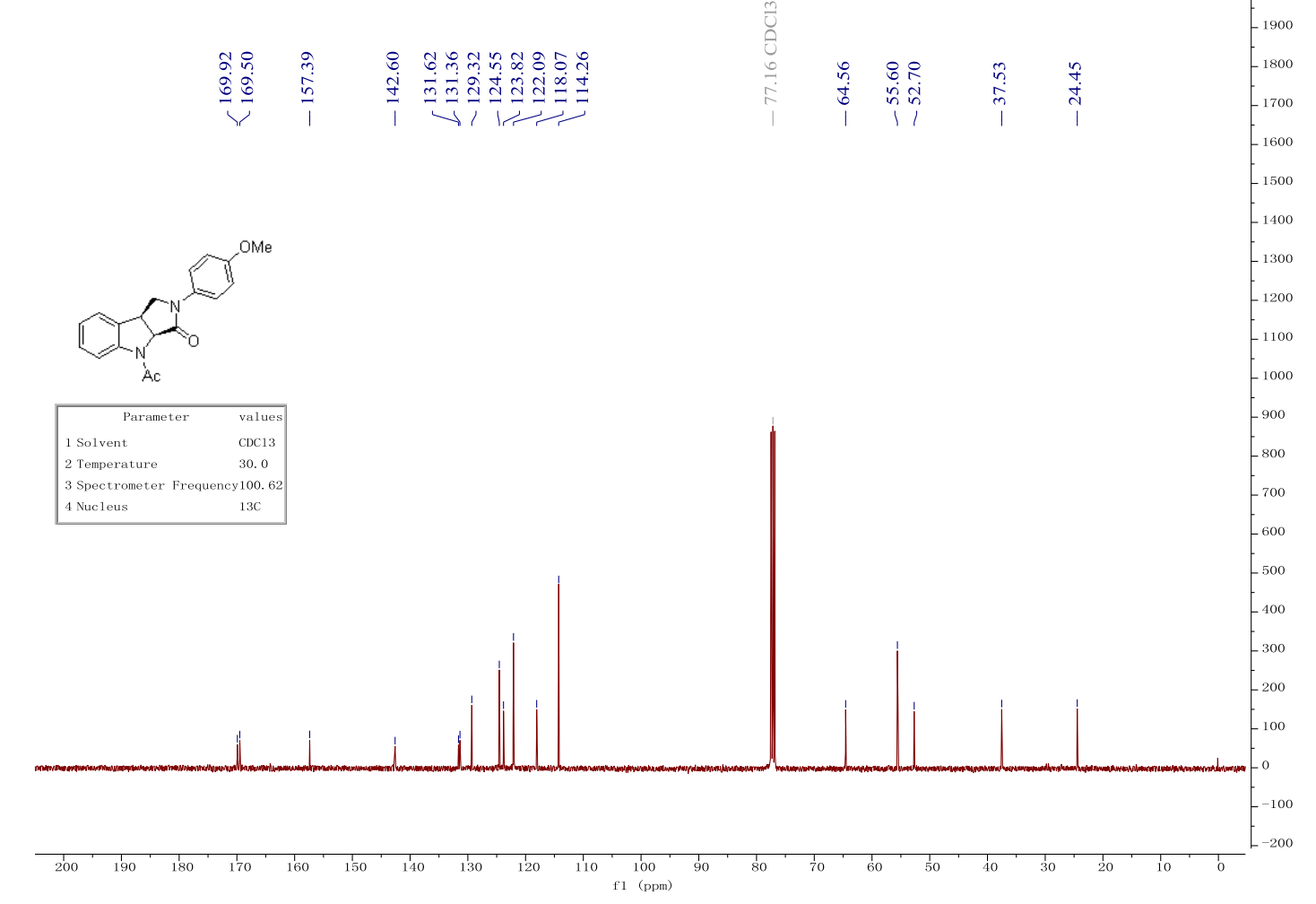




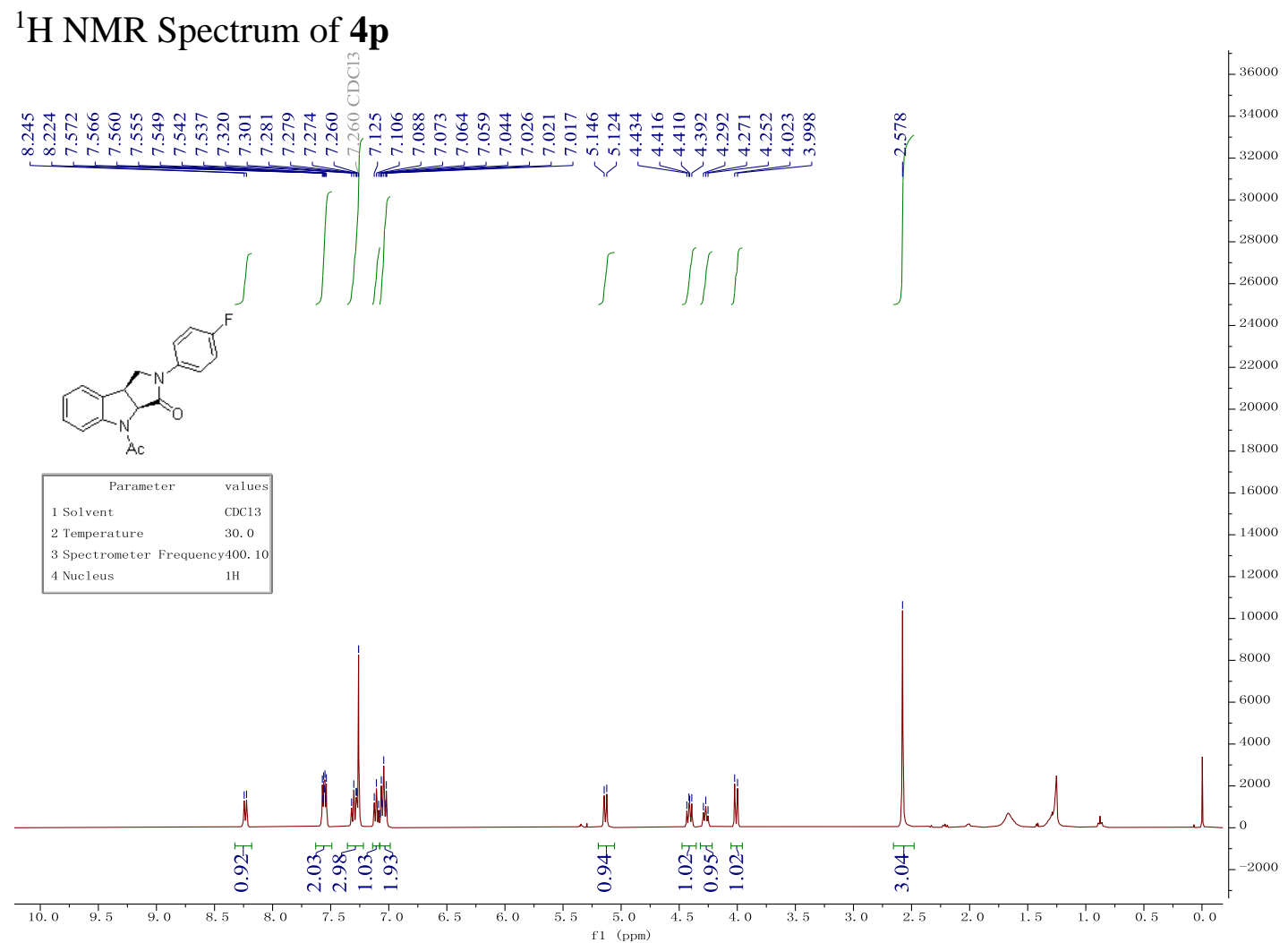

${ }^{13} \mathrm{C}$ NMR Spectrum of $\mathbf{4 p}$

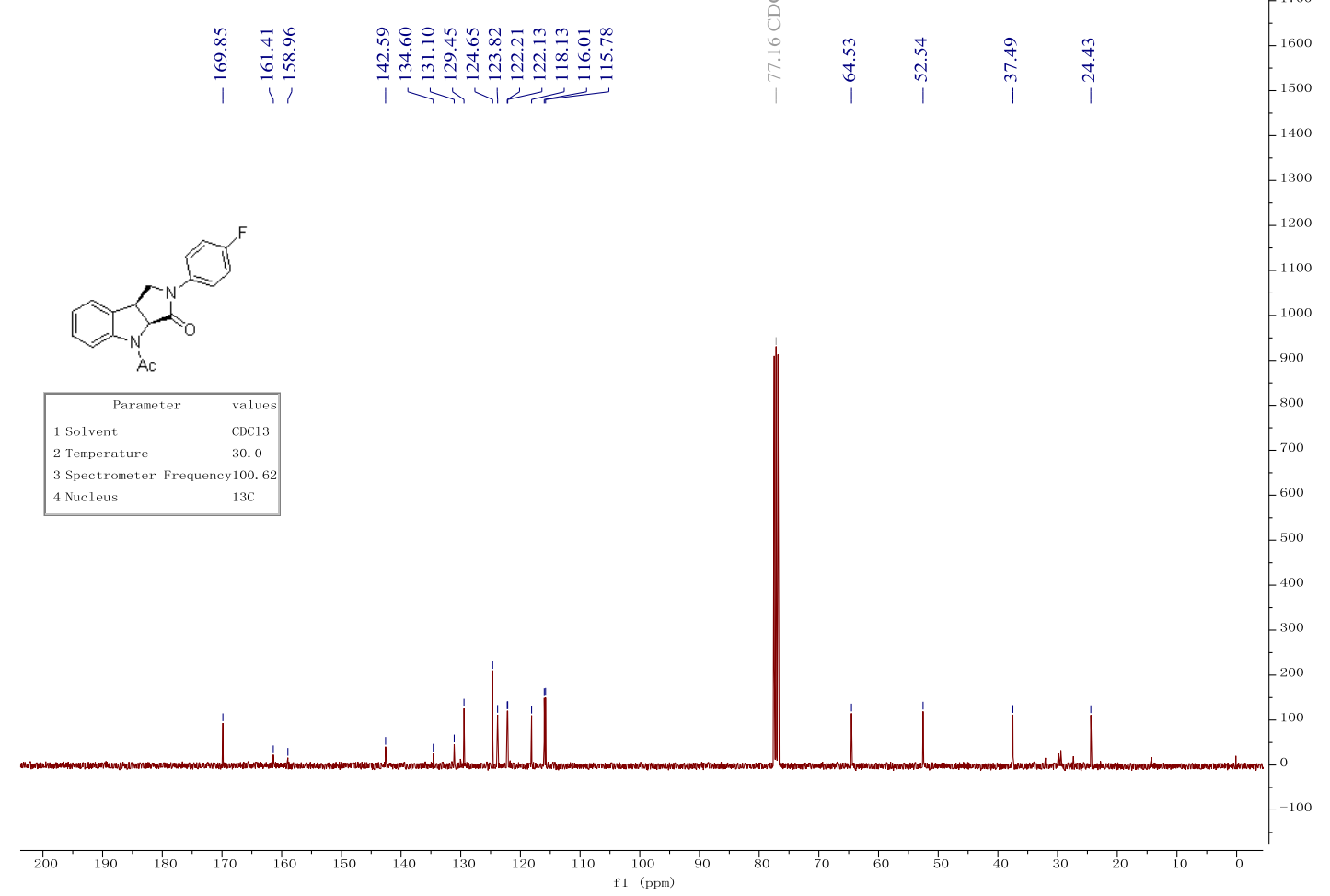


${ }^{19}$ F NMR Spectrum of $\mathbf{4 p}$

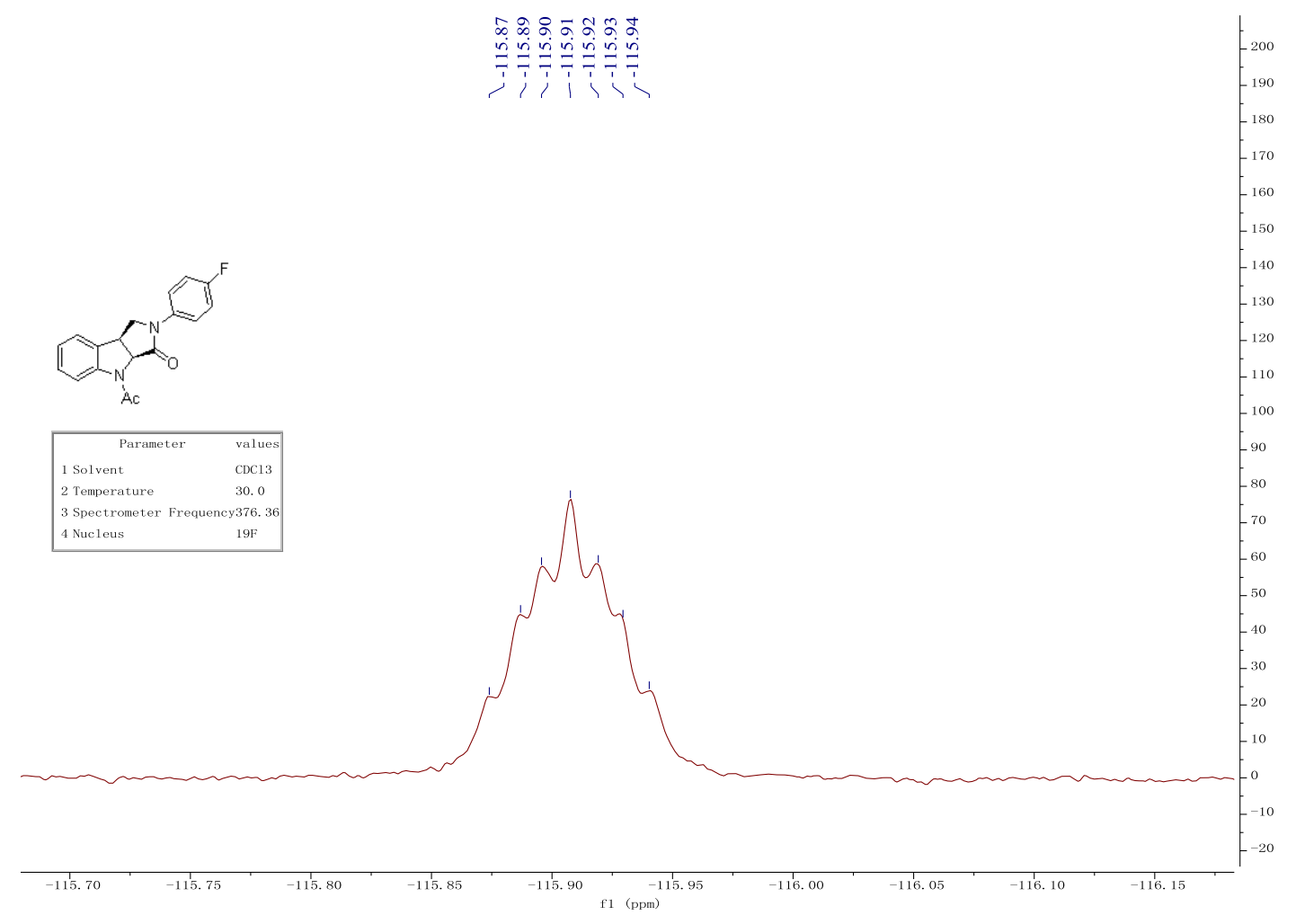

${ }^{1} \mathrm{H}$ NMR Spectrum of $\mathbf{4 q}$

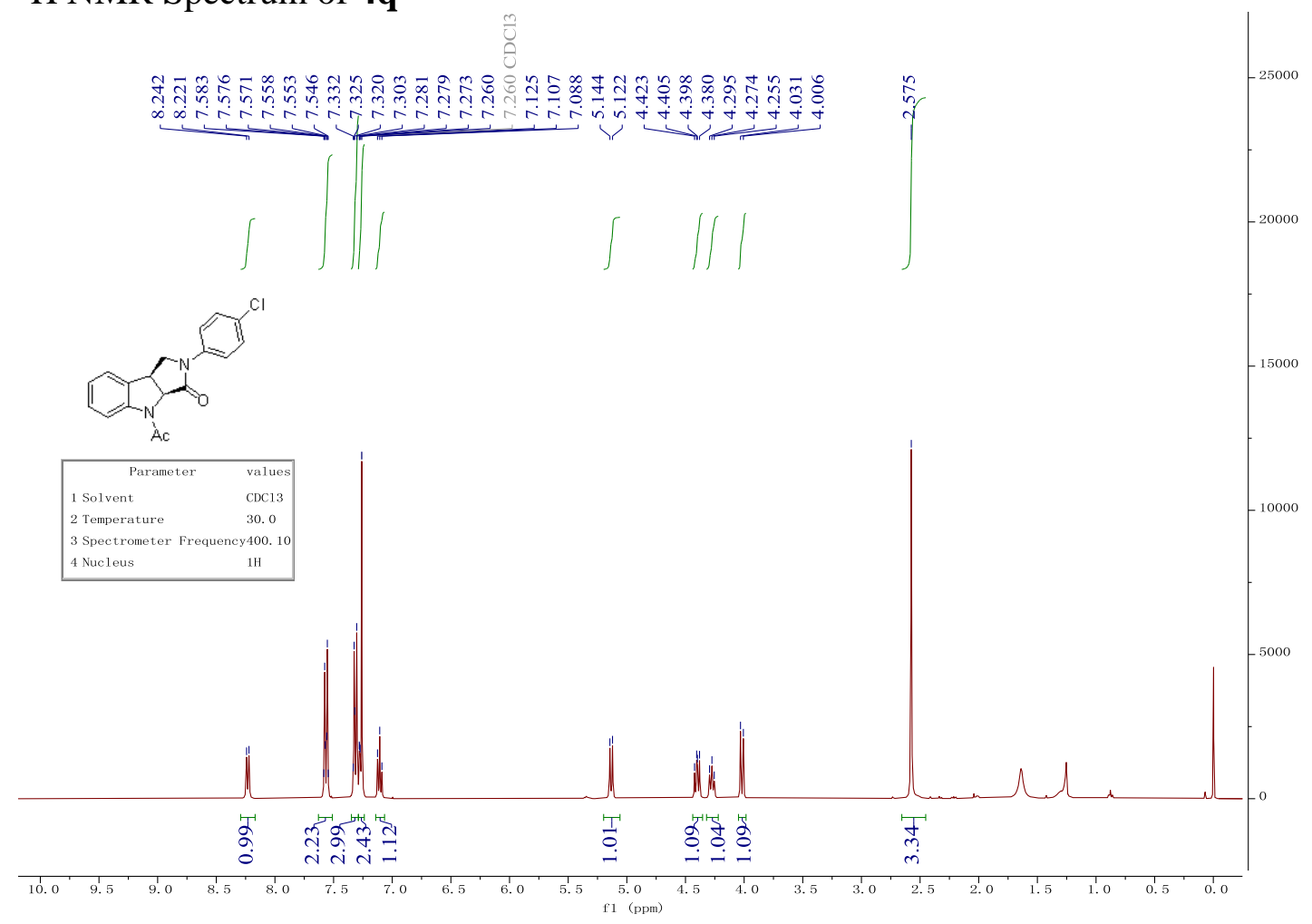


${ }^{13} \mathrm{C}$ NMR Spectrum of $\mathbf{4 q}$

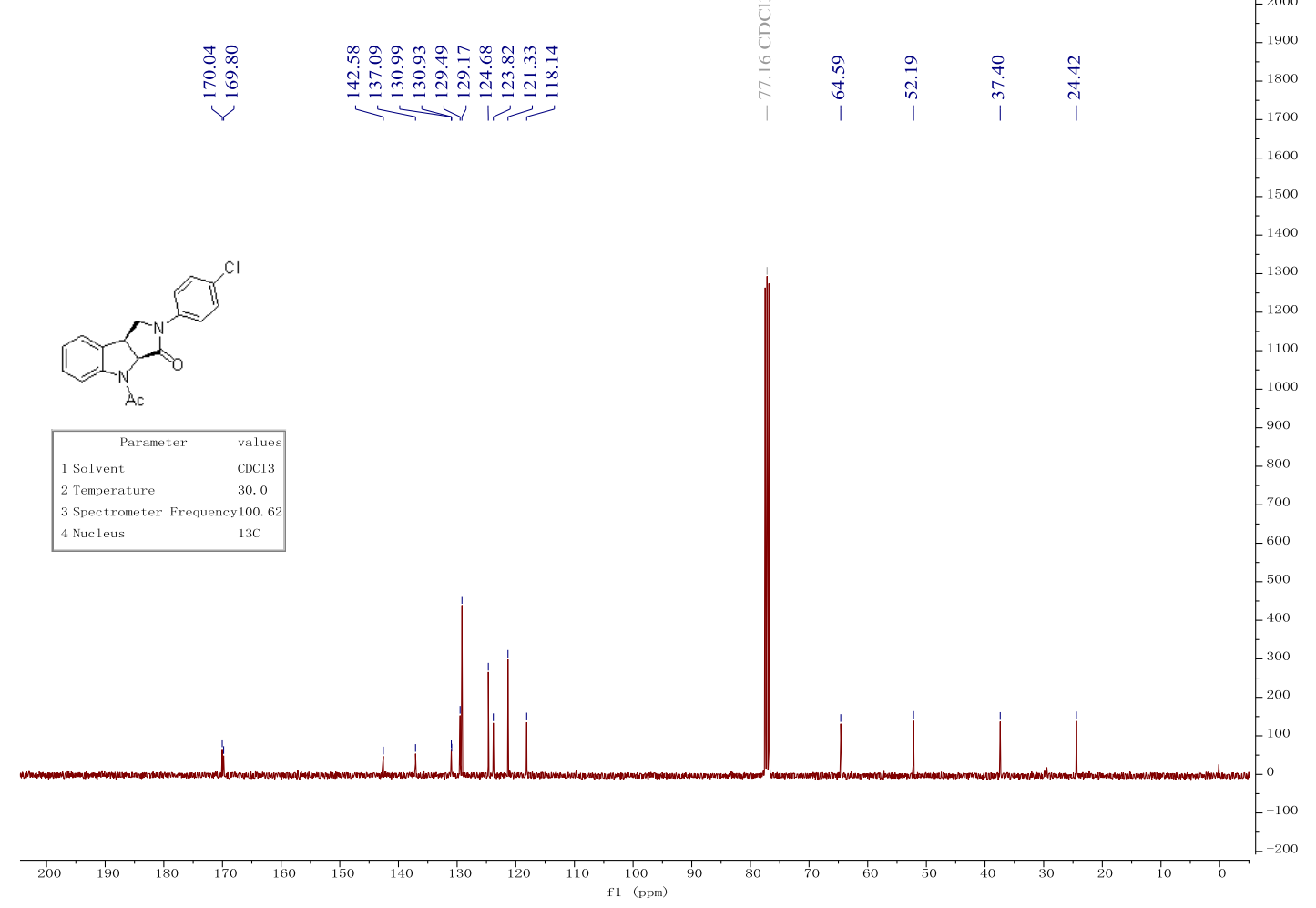

${ }^{1} \mathrm{H}$ NMR Spectrum of $\mathbf{4 r}$

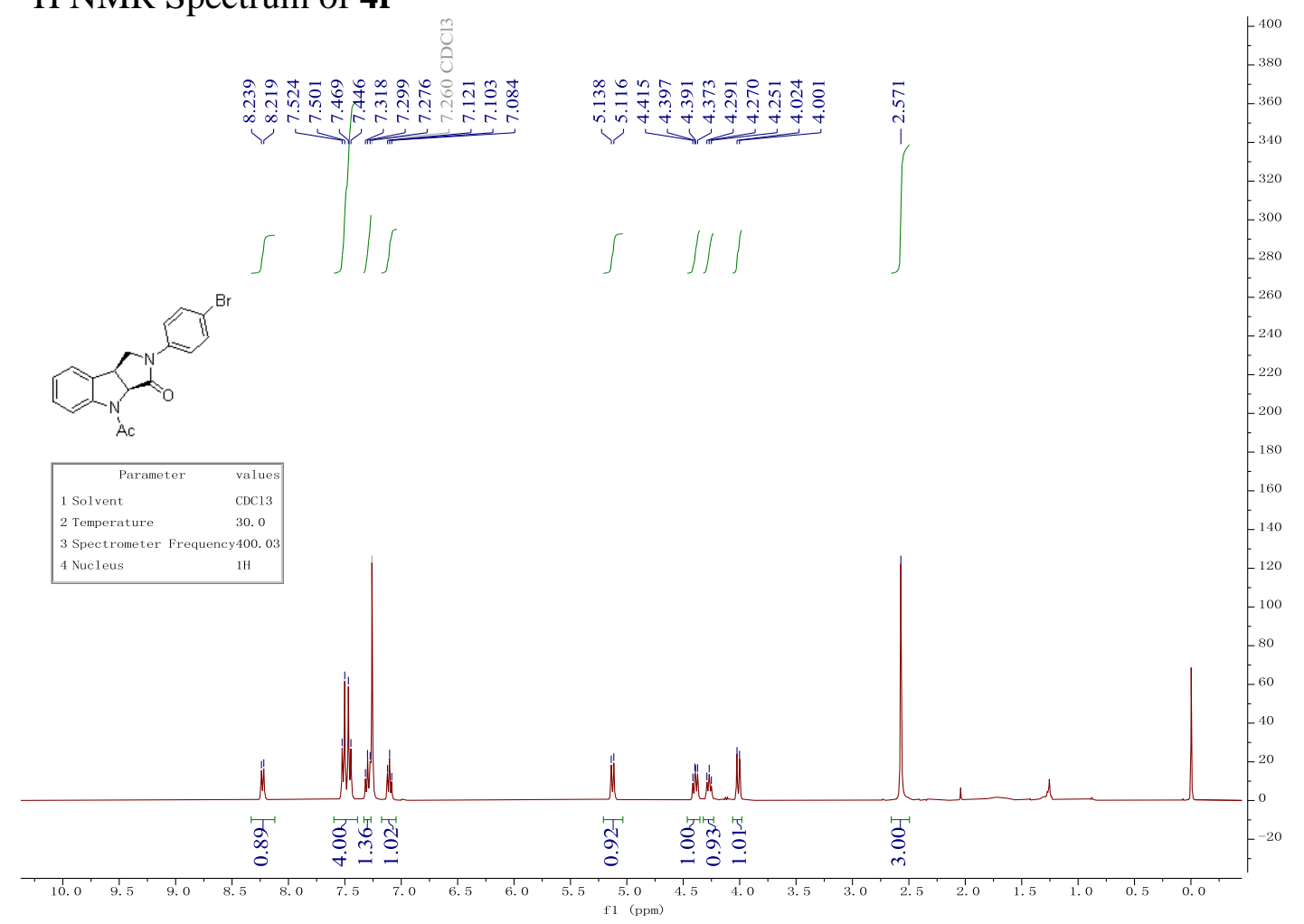


${ }^{13} \mathrm{C}$ NMR Spectrum of $\mathbf{4 r}$
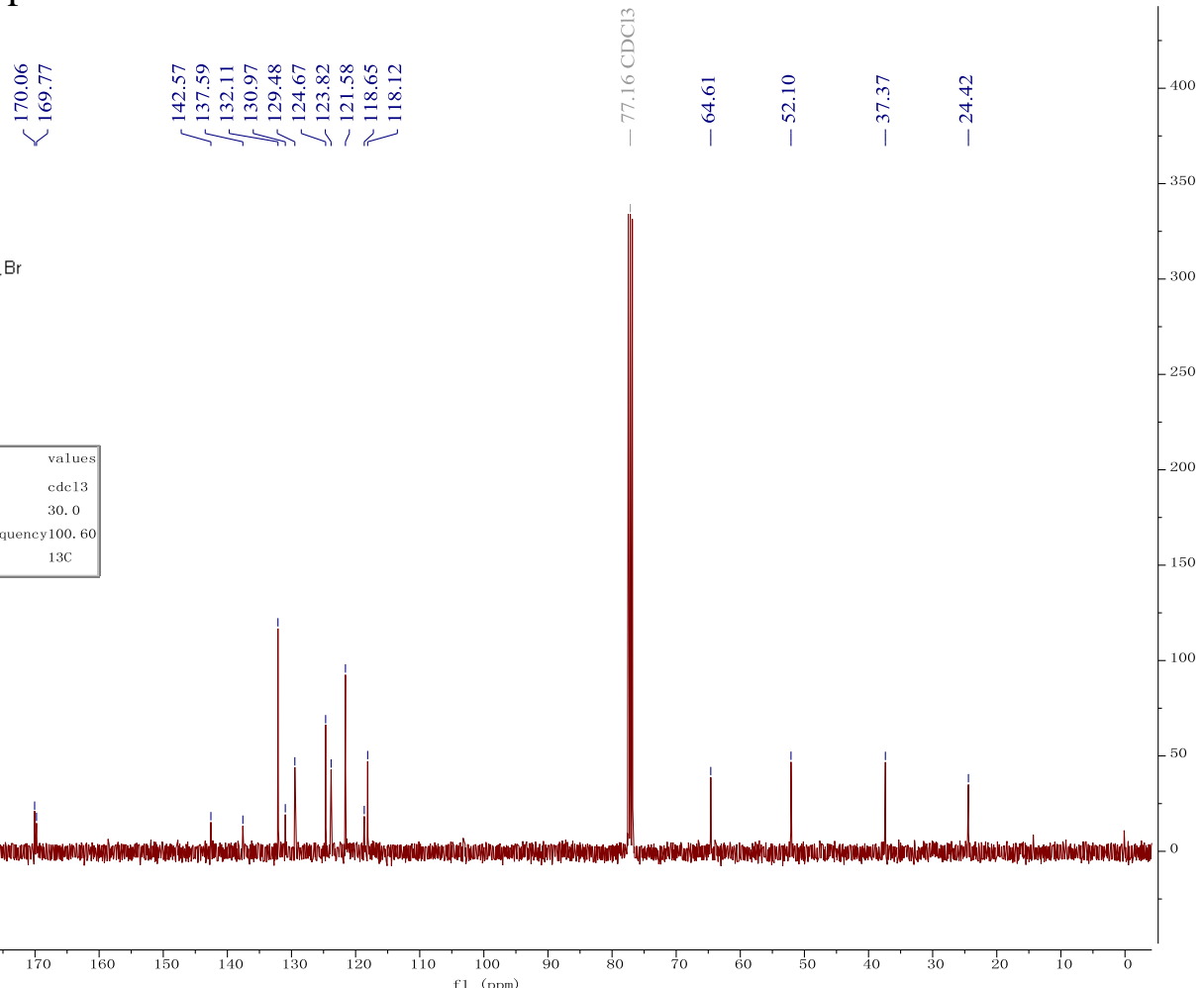

${ }^{1} \mathrm{H}$ NMR Spectrum of $\mathbf{4 s}$

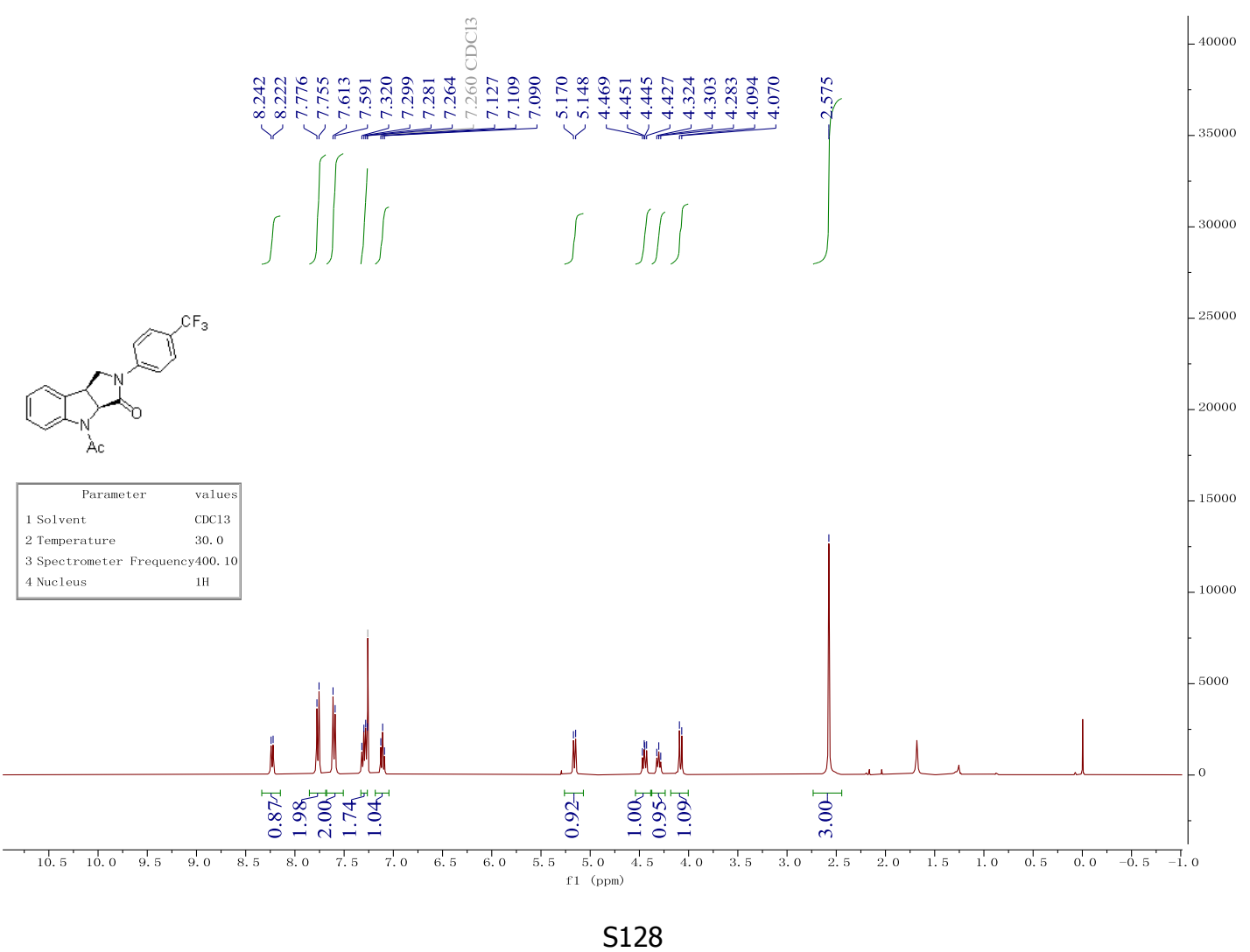


${ }^{13} \mathrm{C}$ NMR Spectrum of $\mathbf{4 s}$

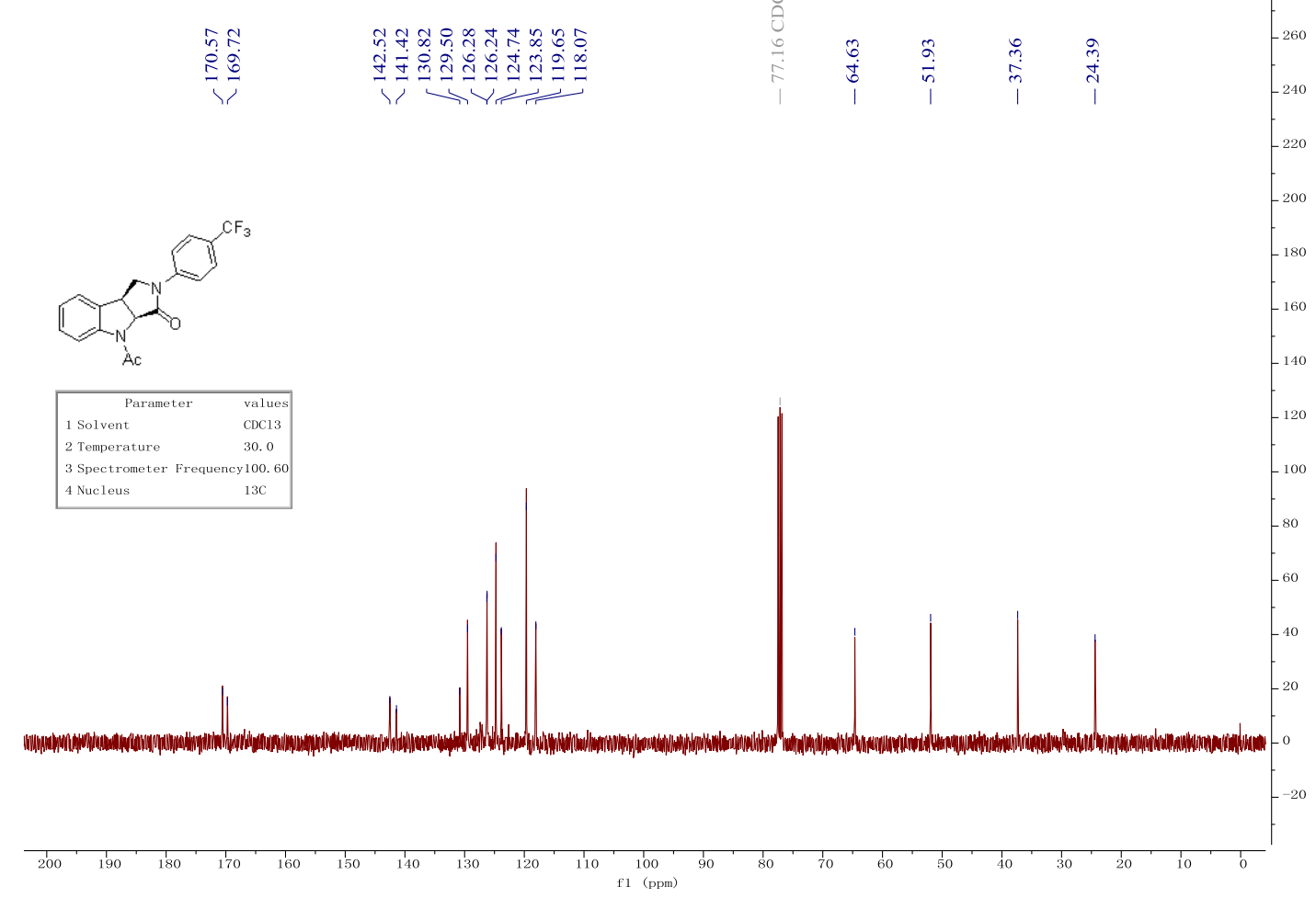

${ }^{19}$ F NMR Spectrum of $\mathbf{4 s}$

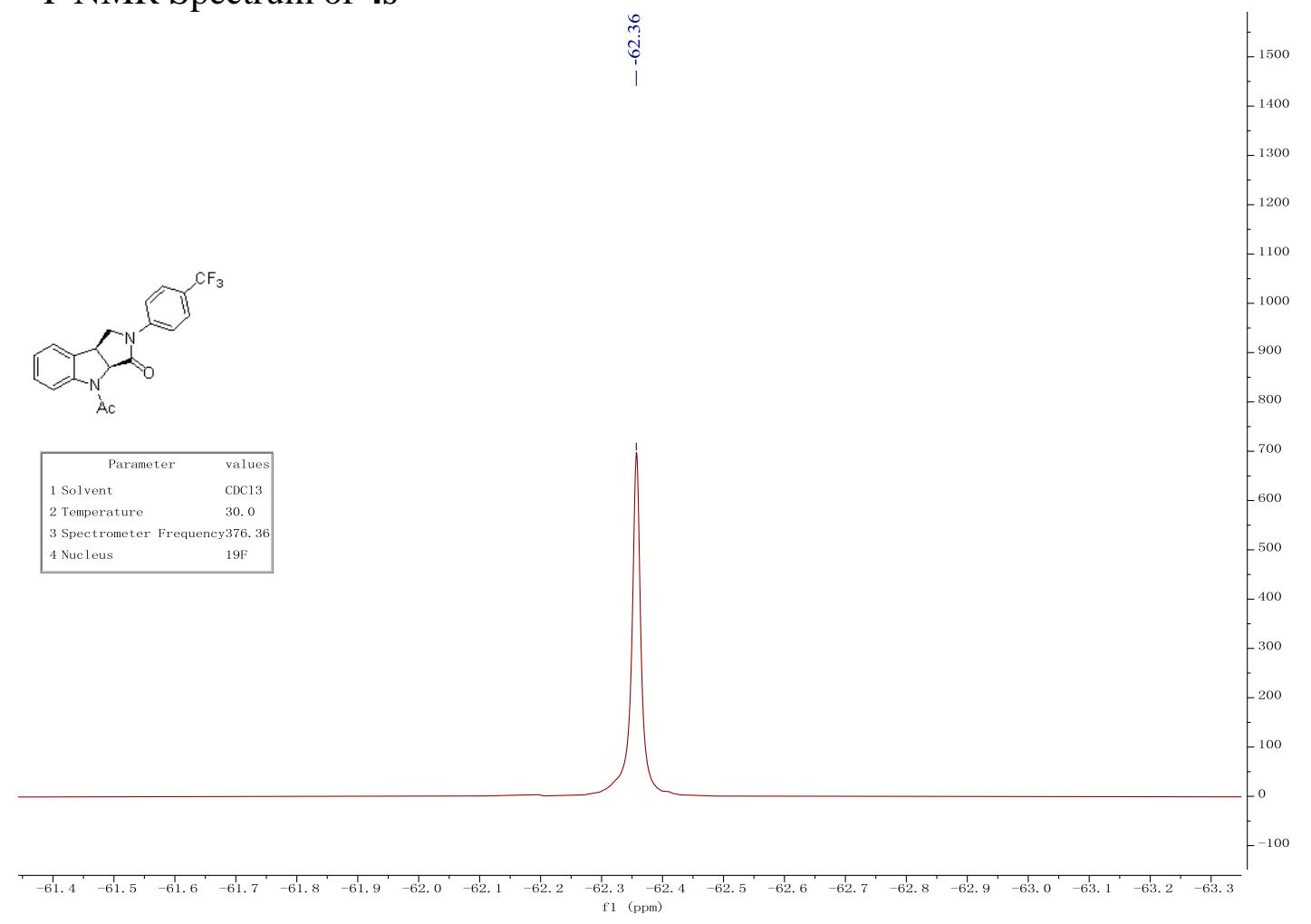




\section{${ }^{1} \mathrm{H}$ NMR Spectrum of $\mathbf{5 a}$}

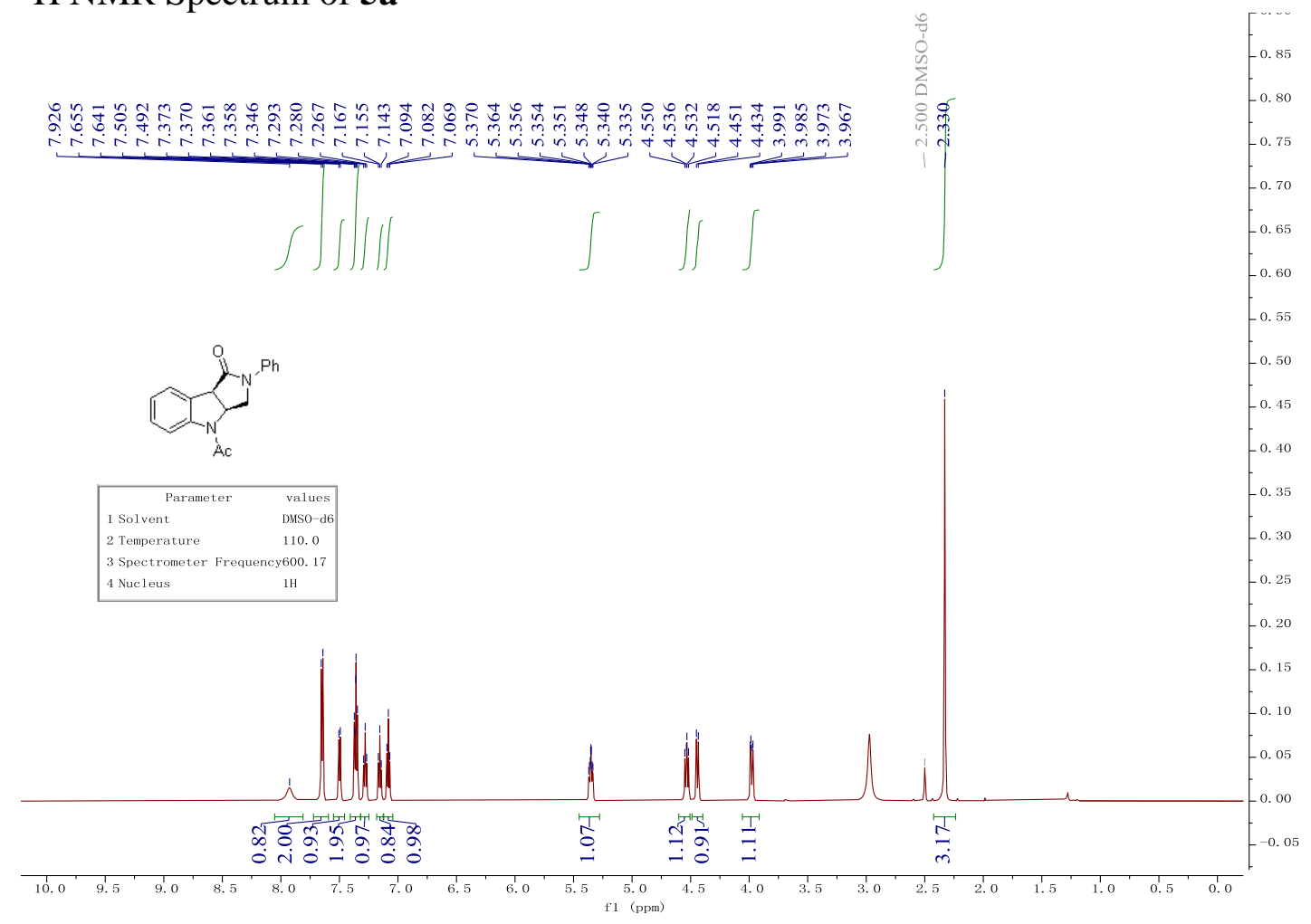

${ }^{13} \mathrm{C}$ NMR Spectrum of $\mathbf{5 a}$

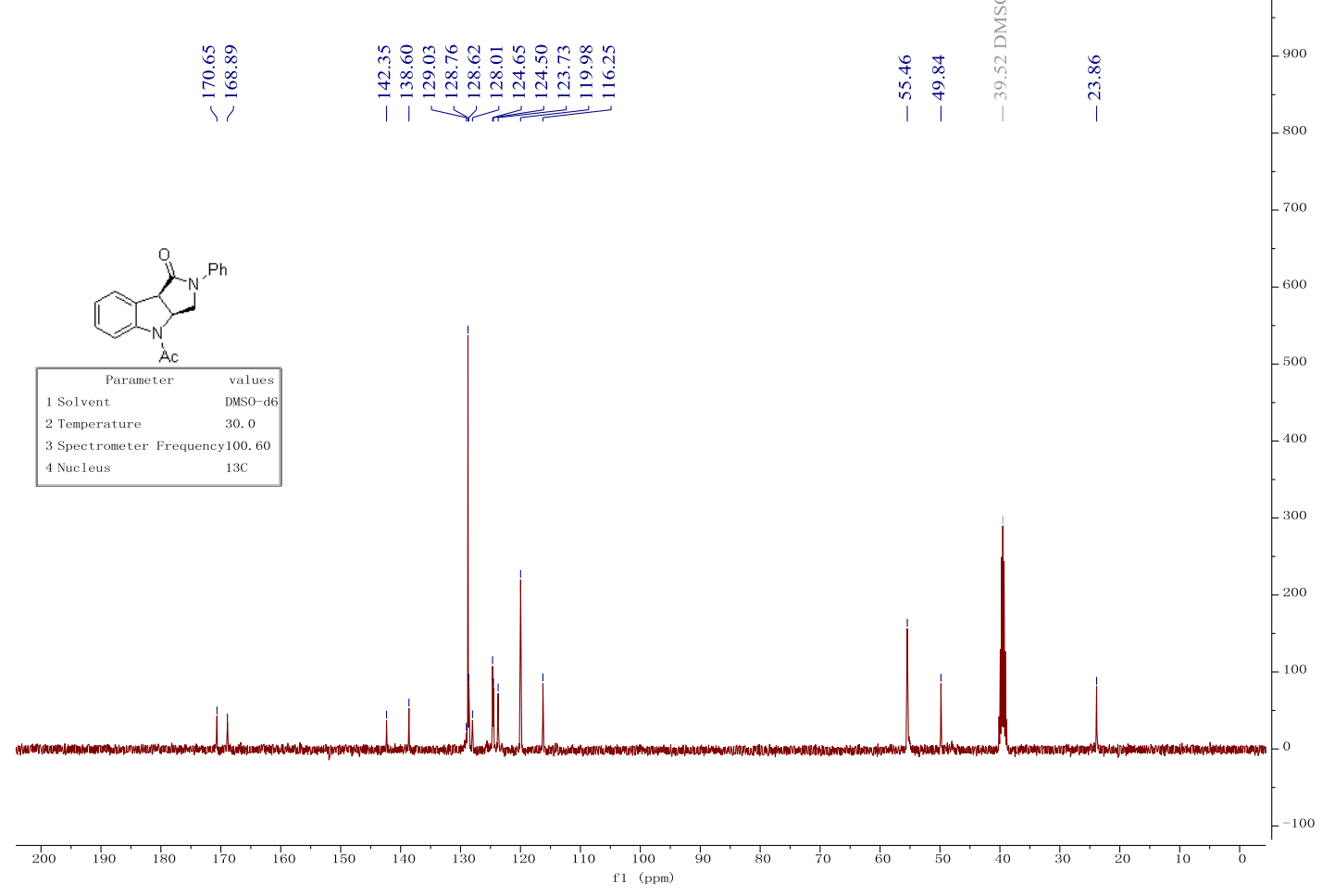


${ }^{1} \mathrm{H}$ NMR Spectrum of $\mathbf{5 b}$
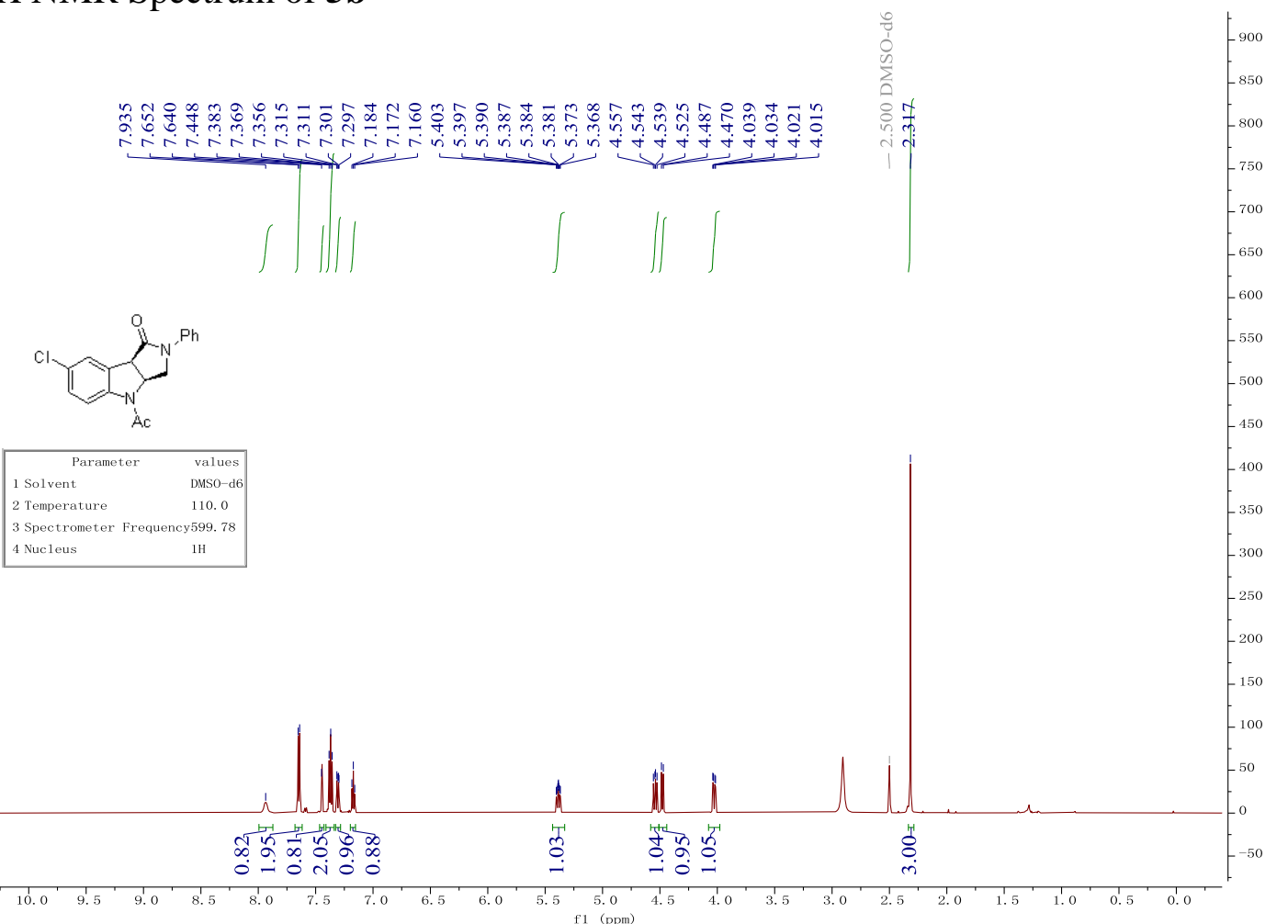

${ }^{13} \mathrm{C}$ NMR Spectrum of $\mathbf{5 b}$

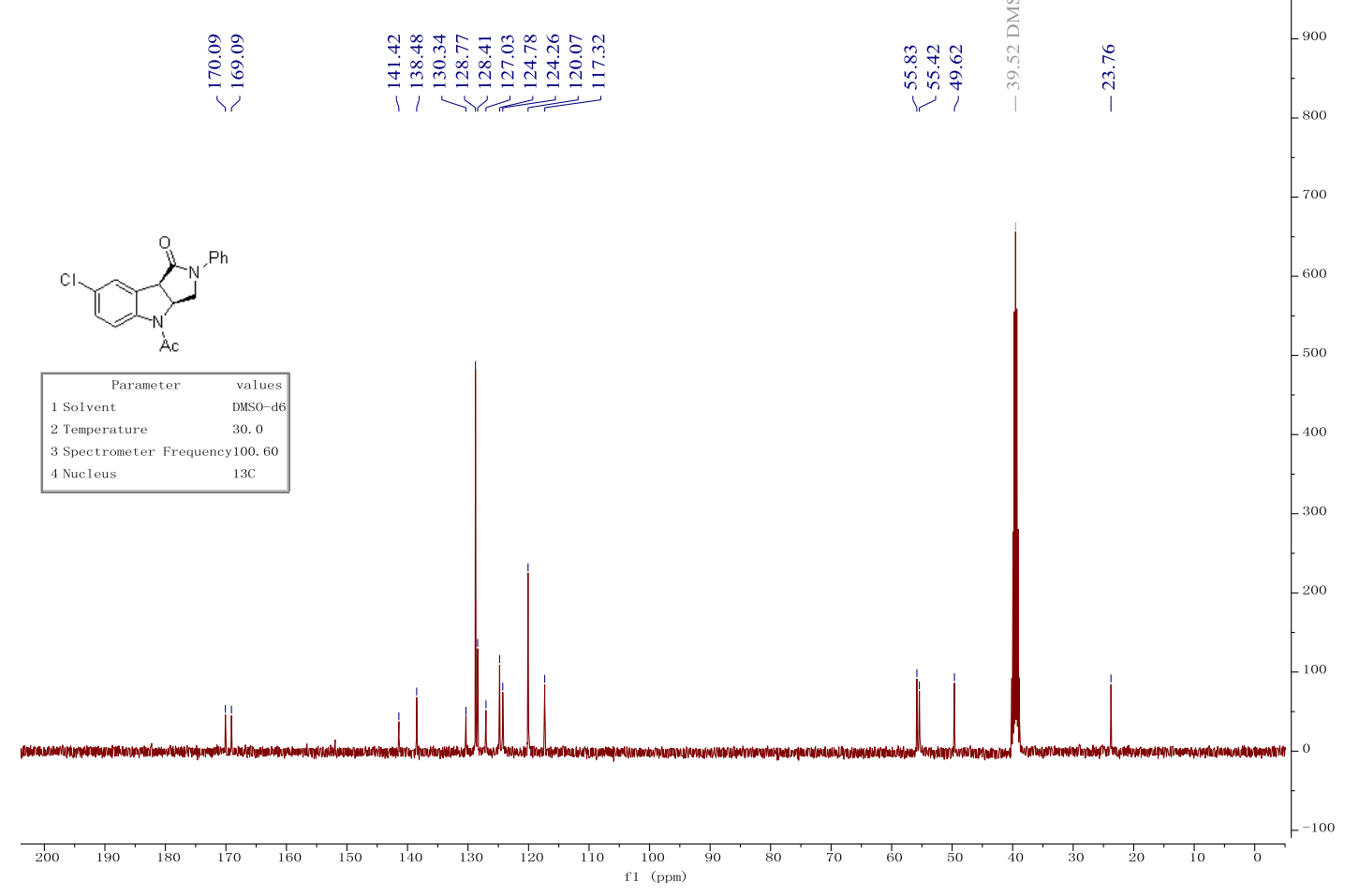


${ }^{1} \mathrm{H}$ NMR Spectrum of $\mathbf{5 c}$

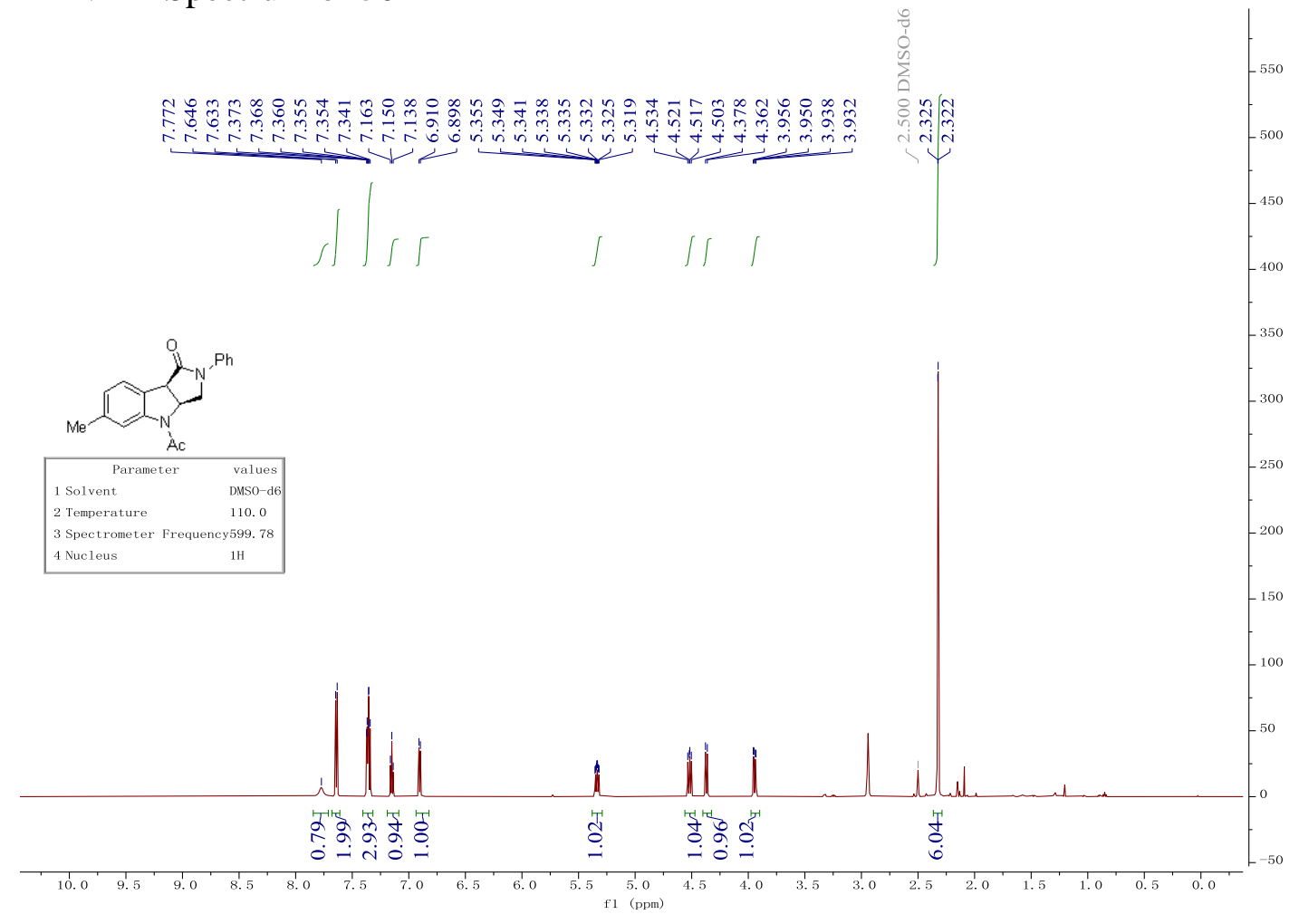

${ }^{13} \mathrm{C}$ NMR Spectrum of $\mathbf{5 c}$

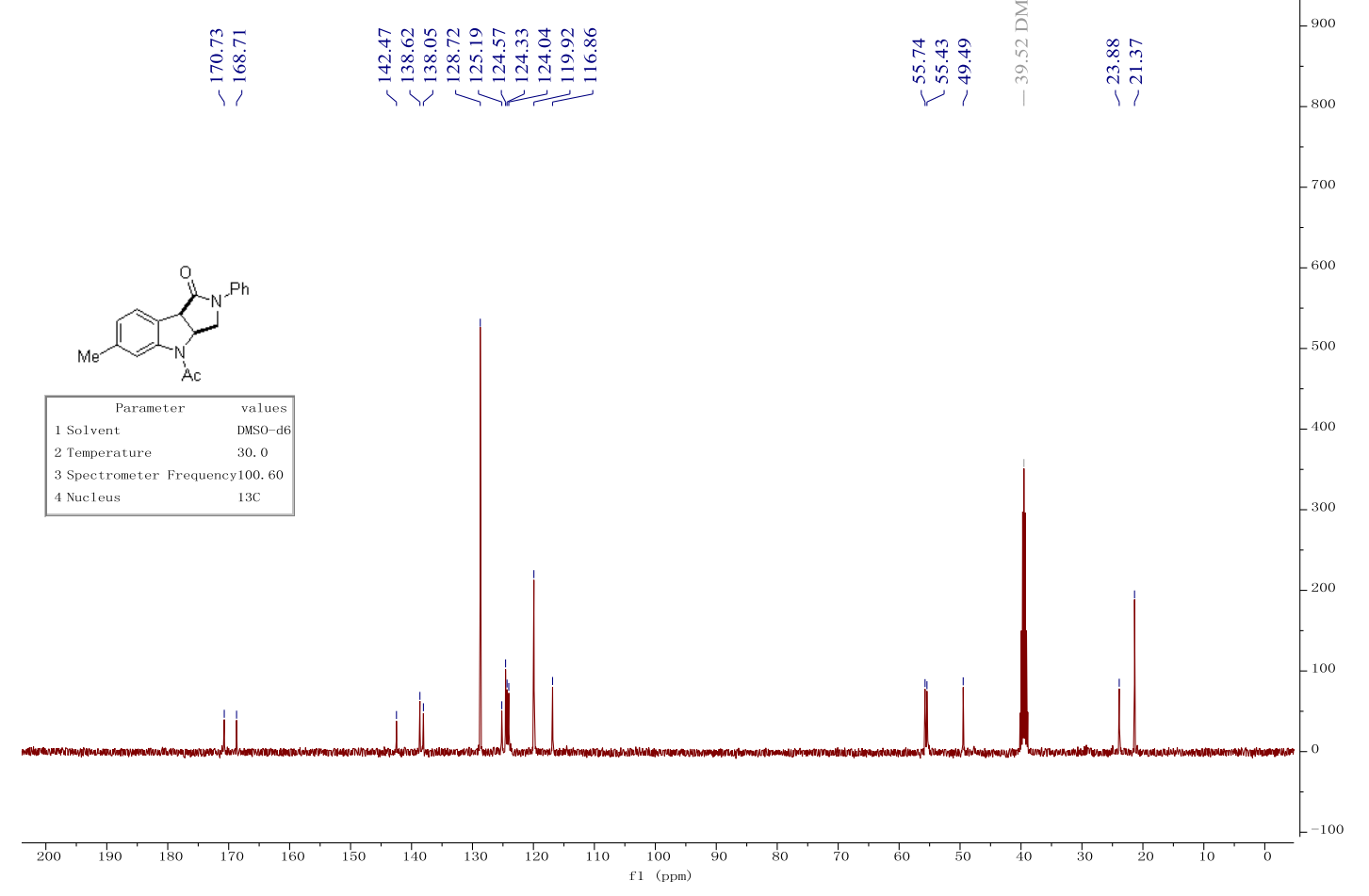


${ }^{1} \mathrm{H}$ NMR Spectrum of $\mathbf{5 d}$

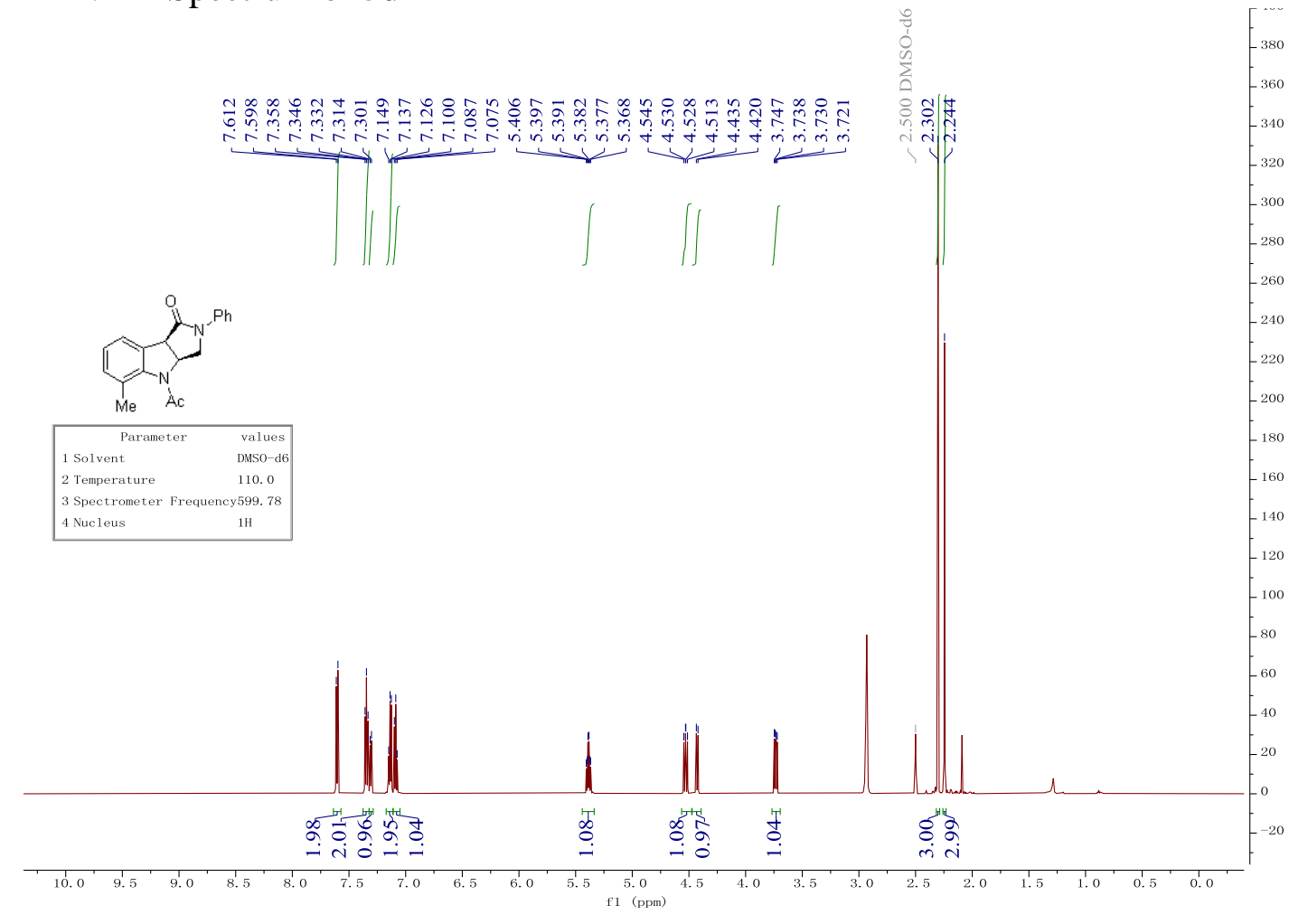

${ }^{13} \mathrm{C}$ NMR Spectrum of $\mathbf{5 d}$

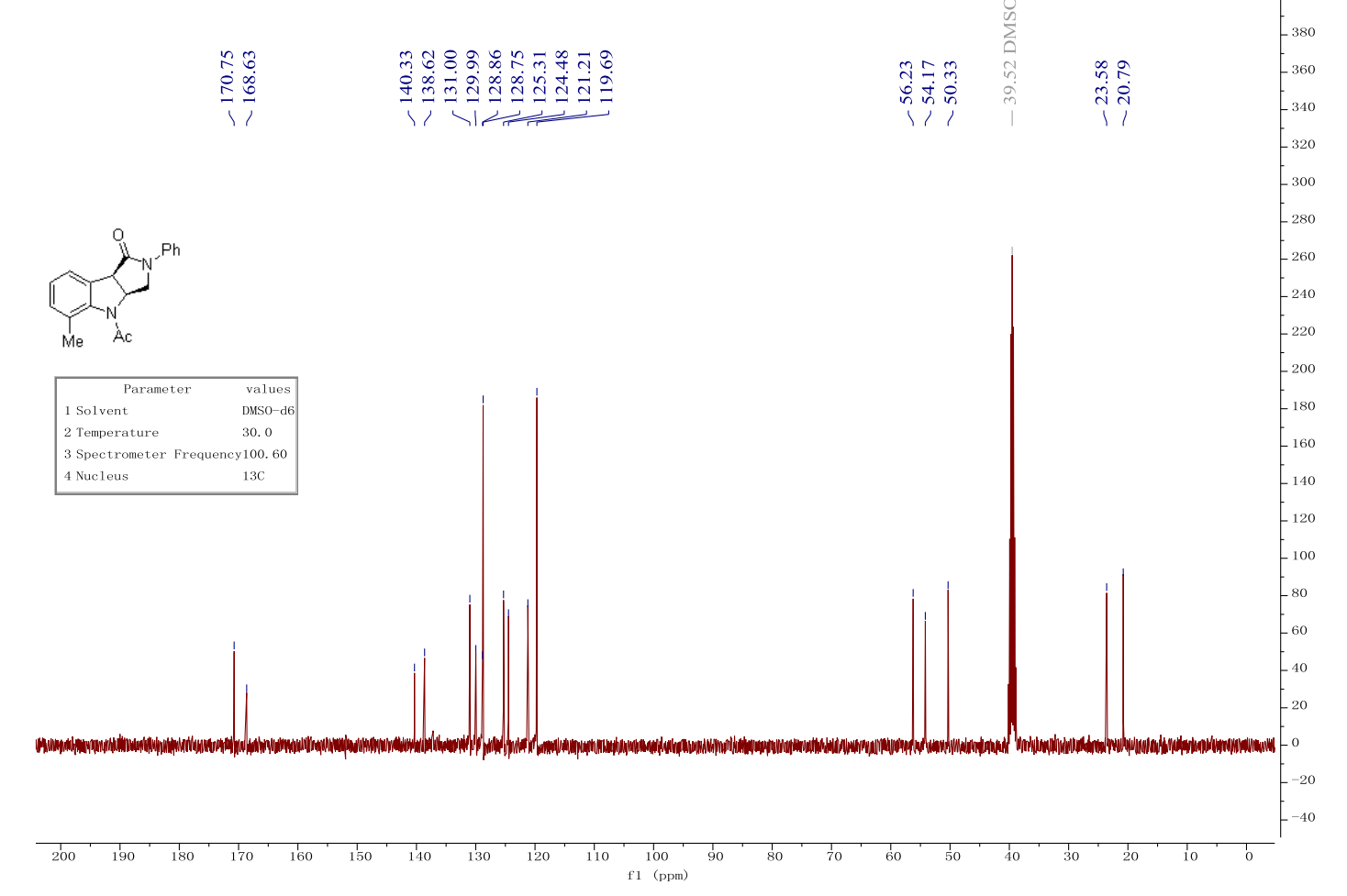




\section{${ }^{1} \mathrm{H}$ NMR Spectrum of $\mathbf{5 e}$}

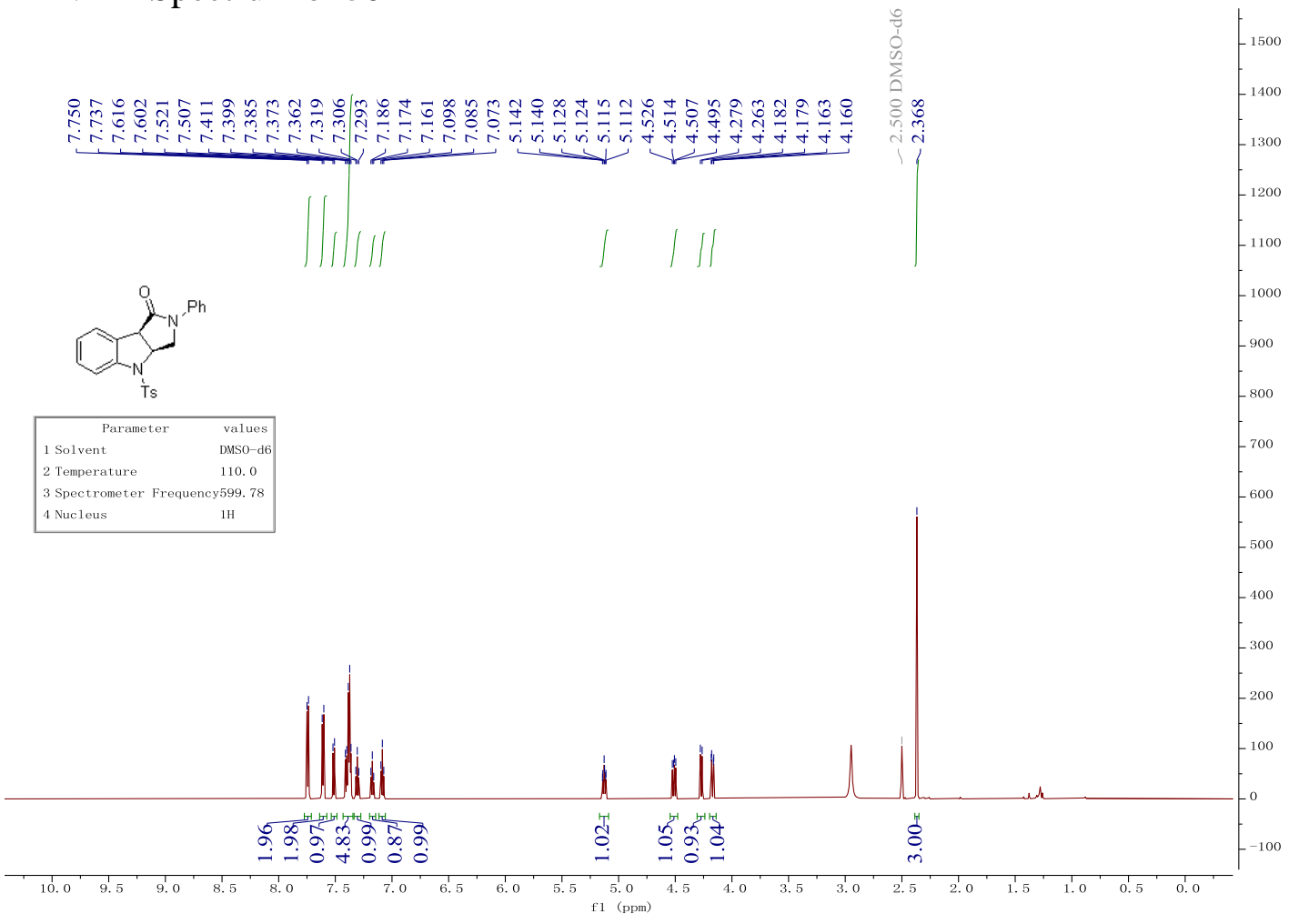

${ }^{13} \mathrm{C}$ NMR Spectrum of $\mathbf{5 e}$

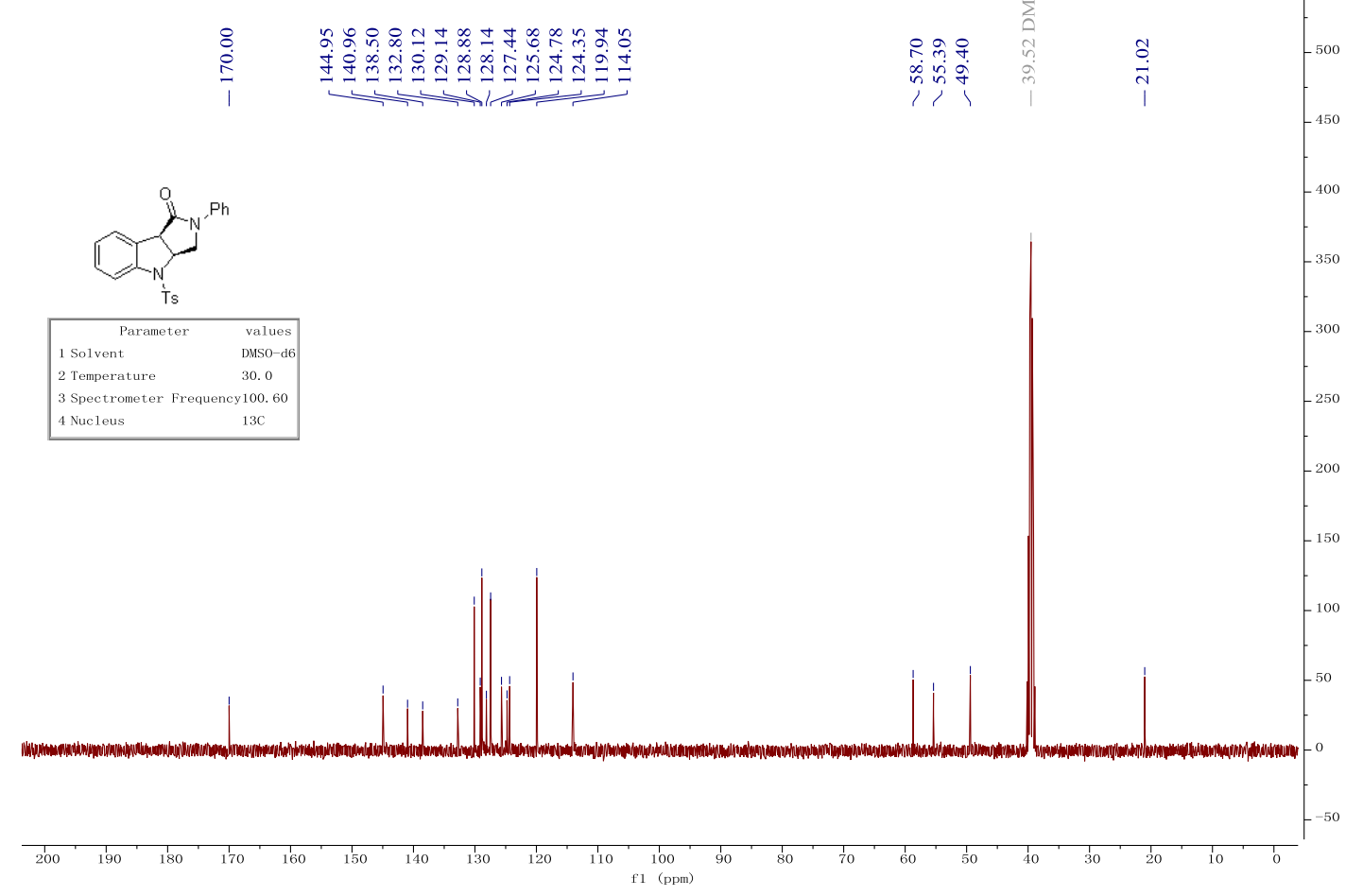


${ }^{1} \mathrm{H}$ NMR Spectrum of $\mathbf{5 f}$

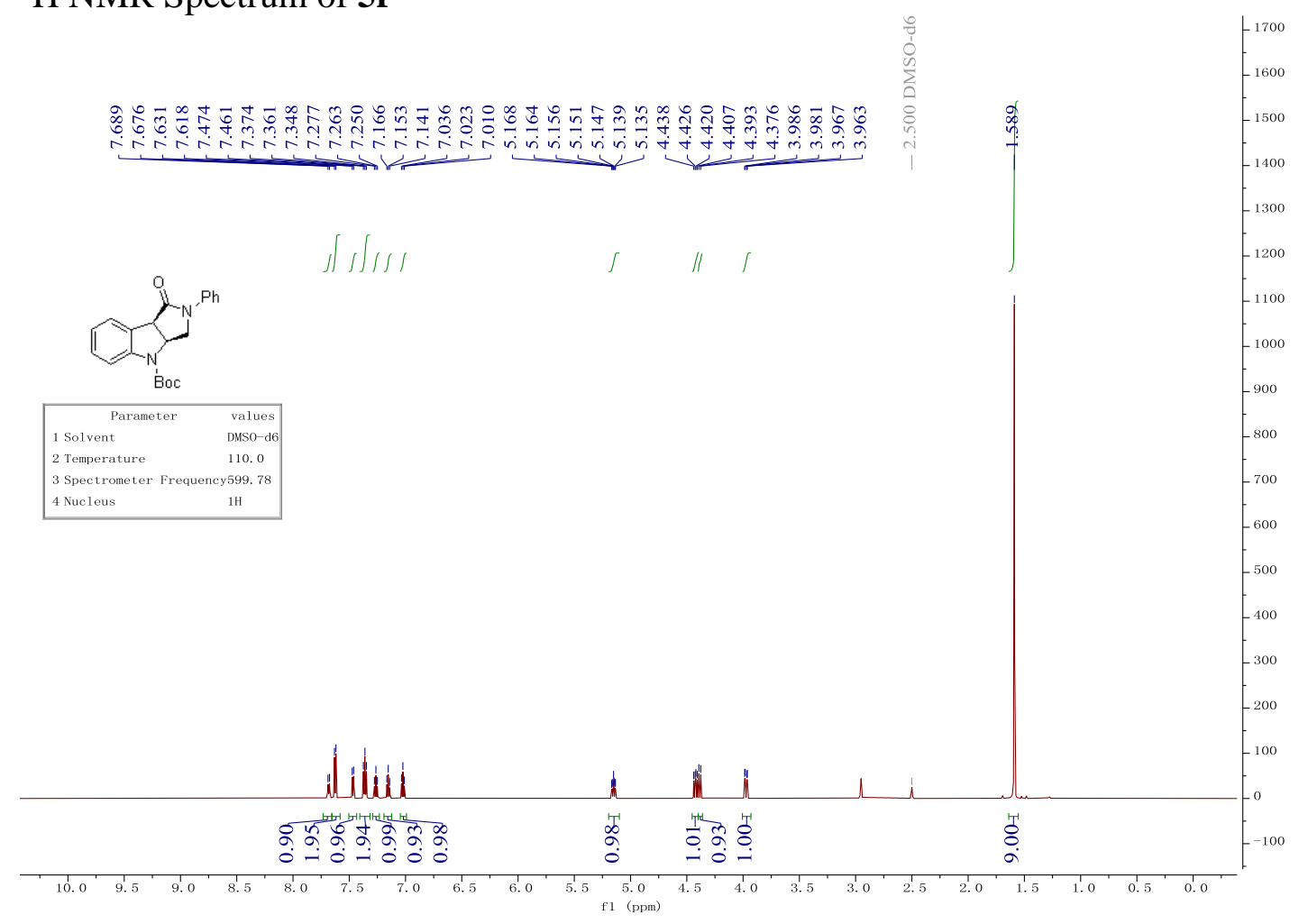

${ }^{13} \mathrm{C}$ NMR Spectrum of $\mathbf{5 f}$

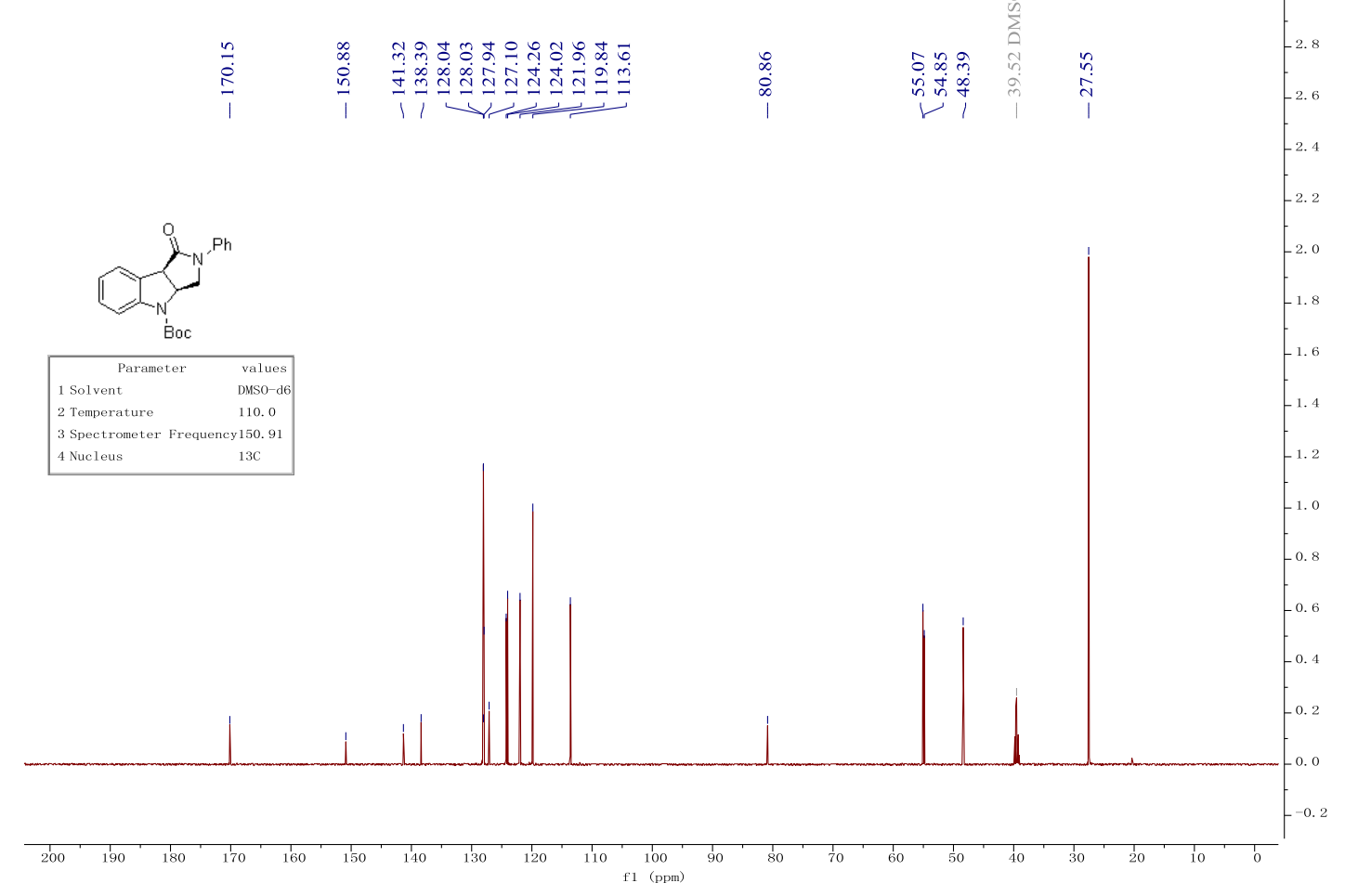


${ }^{1} \mathrm{H}$ NMR Spectrum of $\mathbf{5 g}$

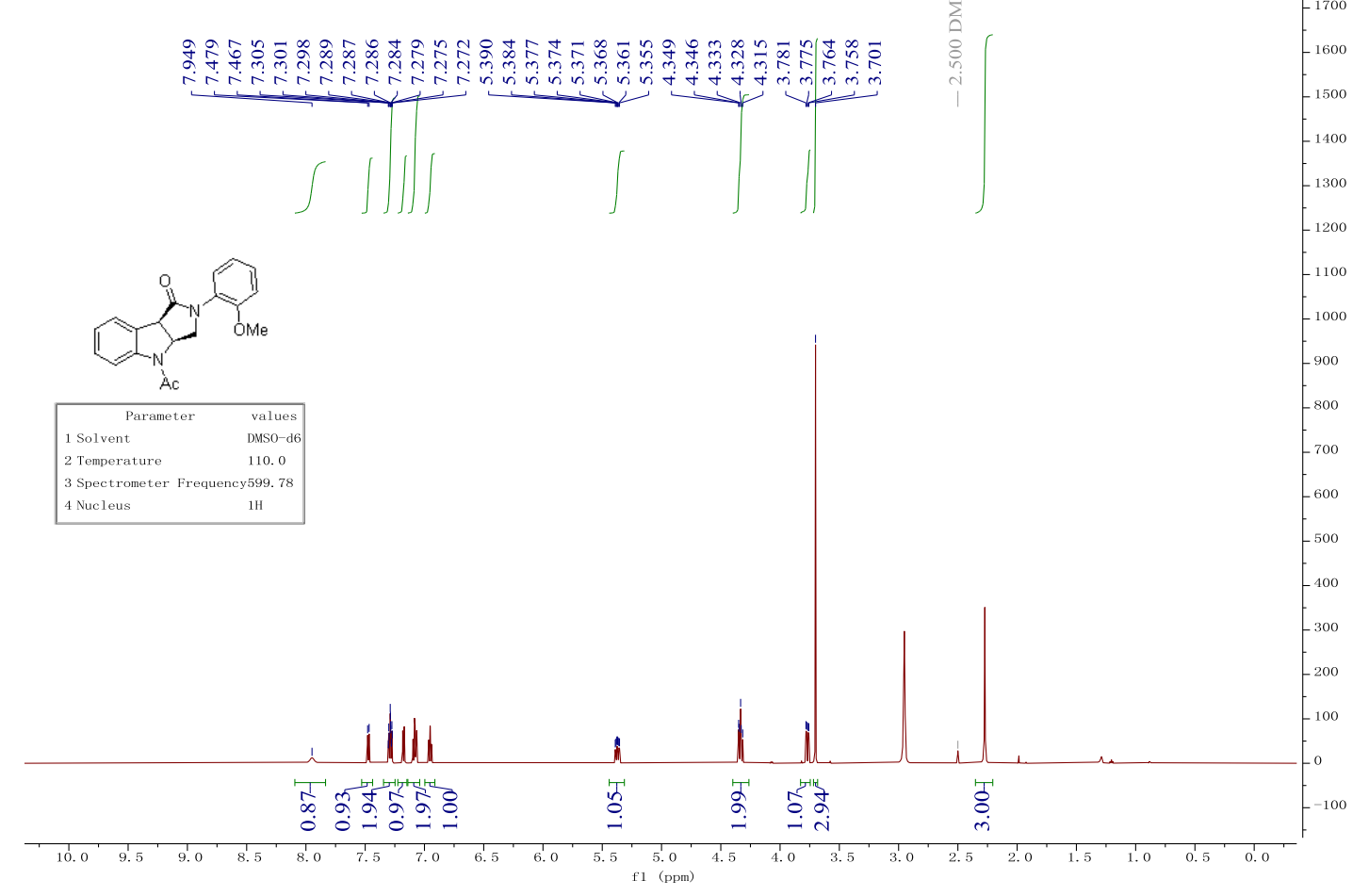

${ }^{13} \mathrm{C}$ NMR Spectrum of $\mathbf{5 g}$

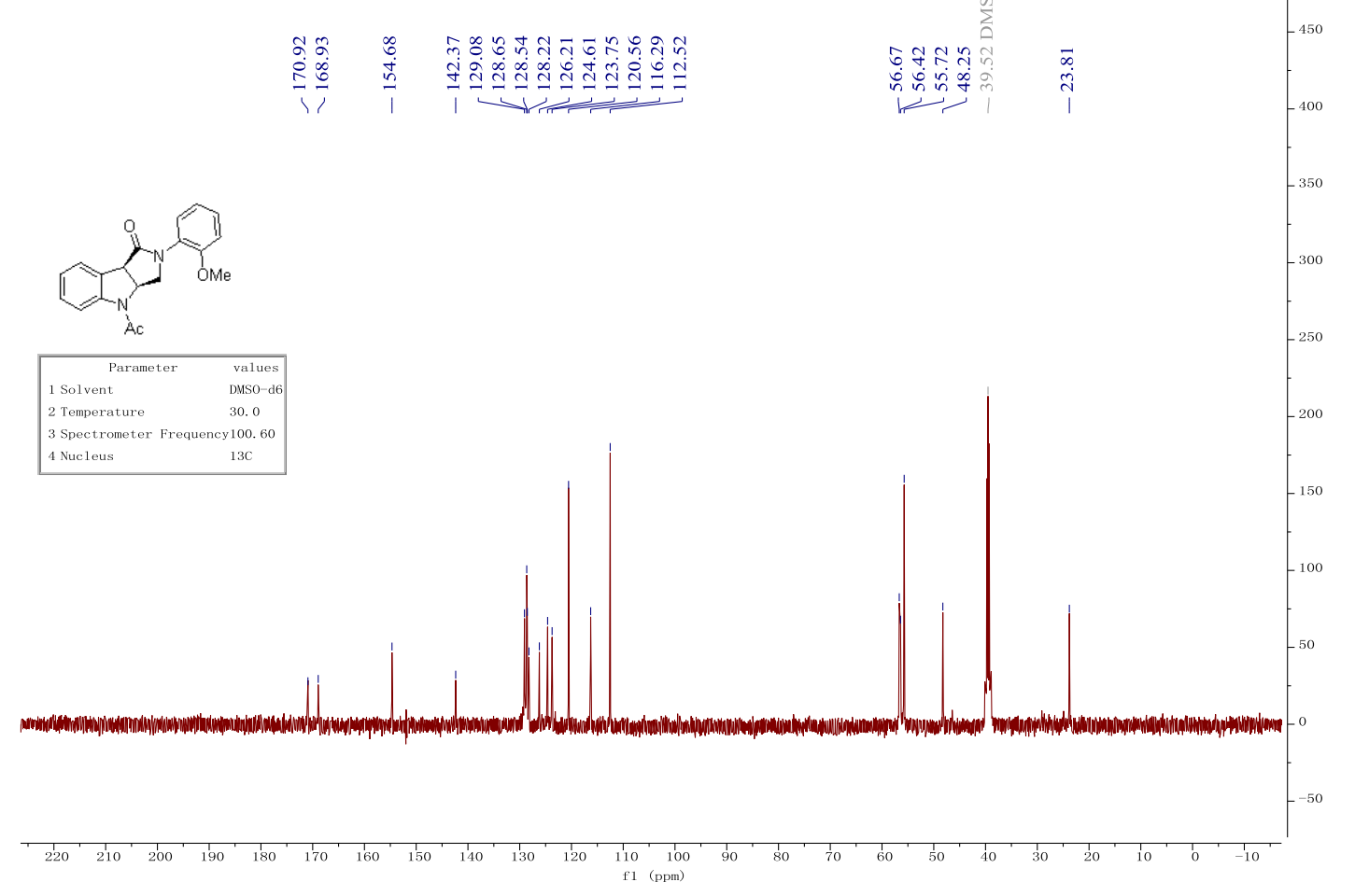


${ }^{1} \mathrm{H}$ NMR Spectrum of $\mathbf{5 h}$

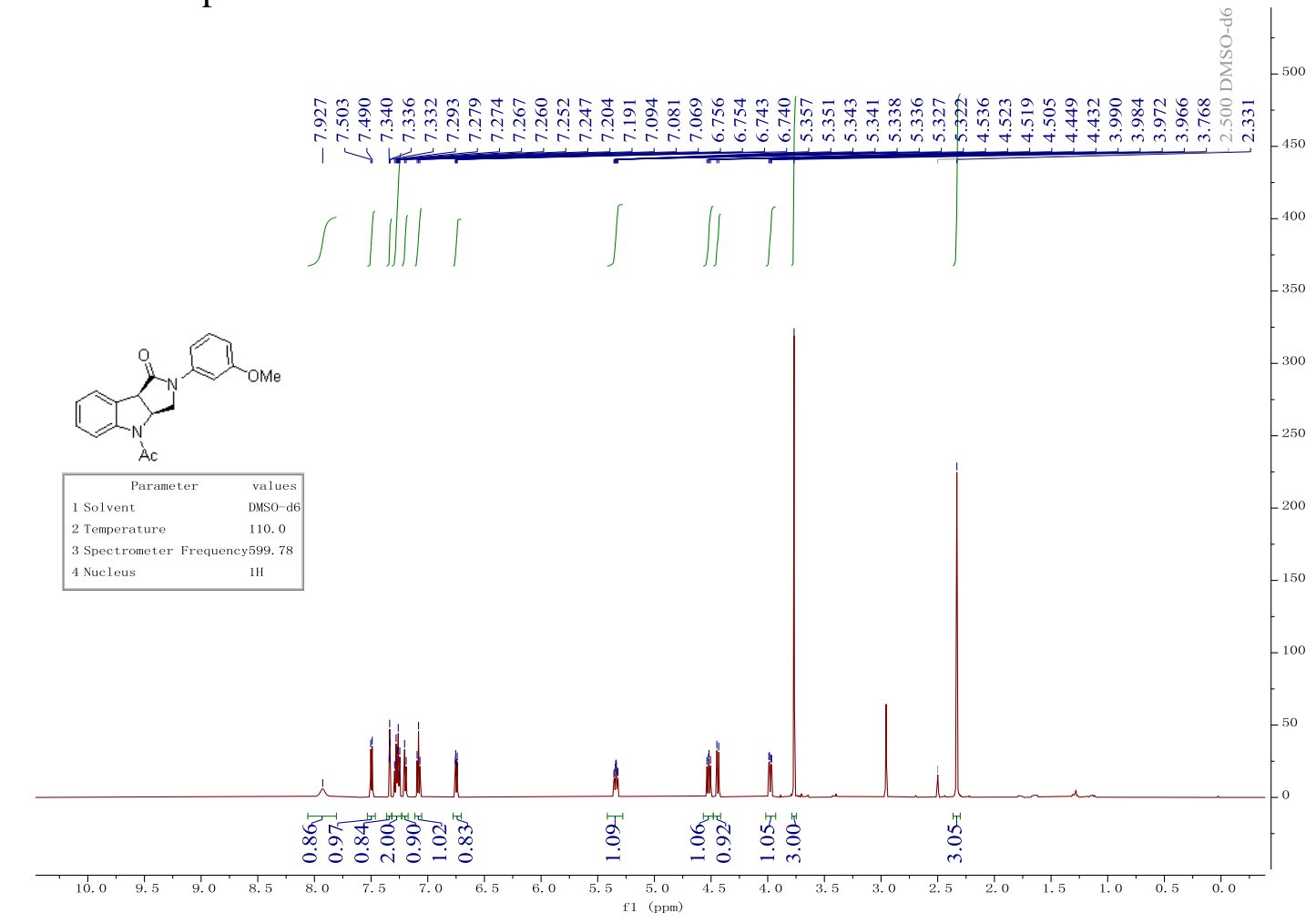

${ }^{13} \mathrm{C}$ NMR Spectrum of $\mathbf{5 h}$

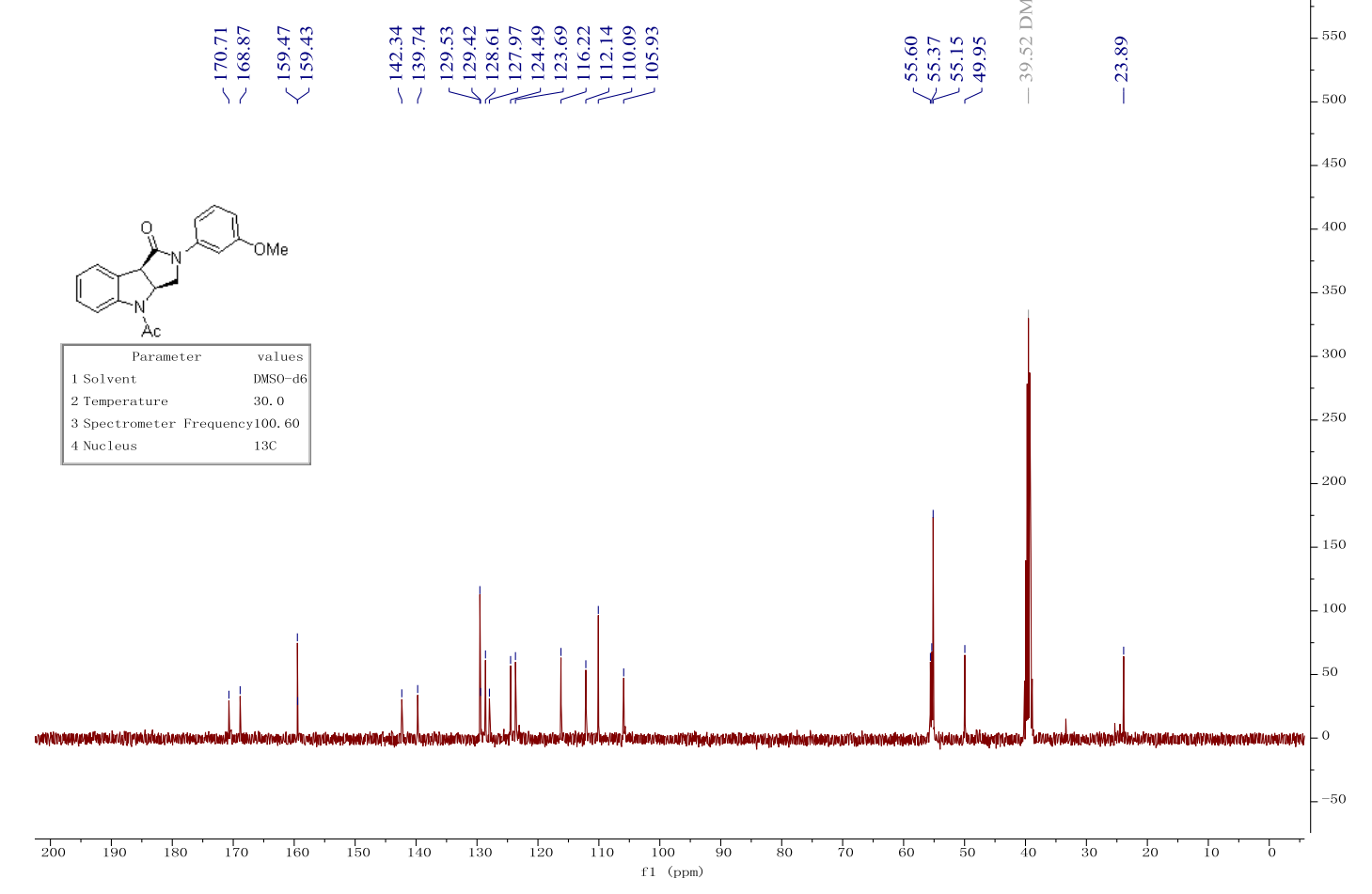


${ }^{1} \mathrm{H}$ NMR Spectrum of $\mathbf{5 i}$

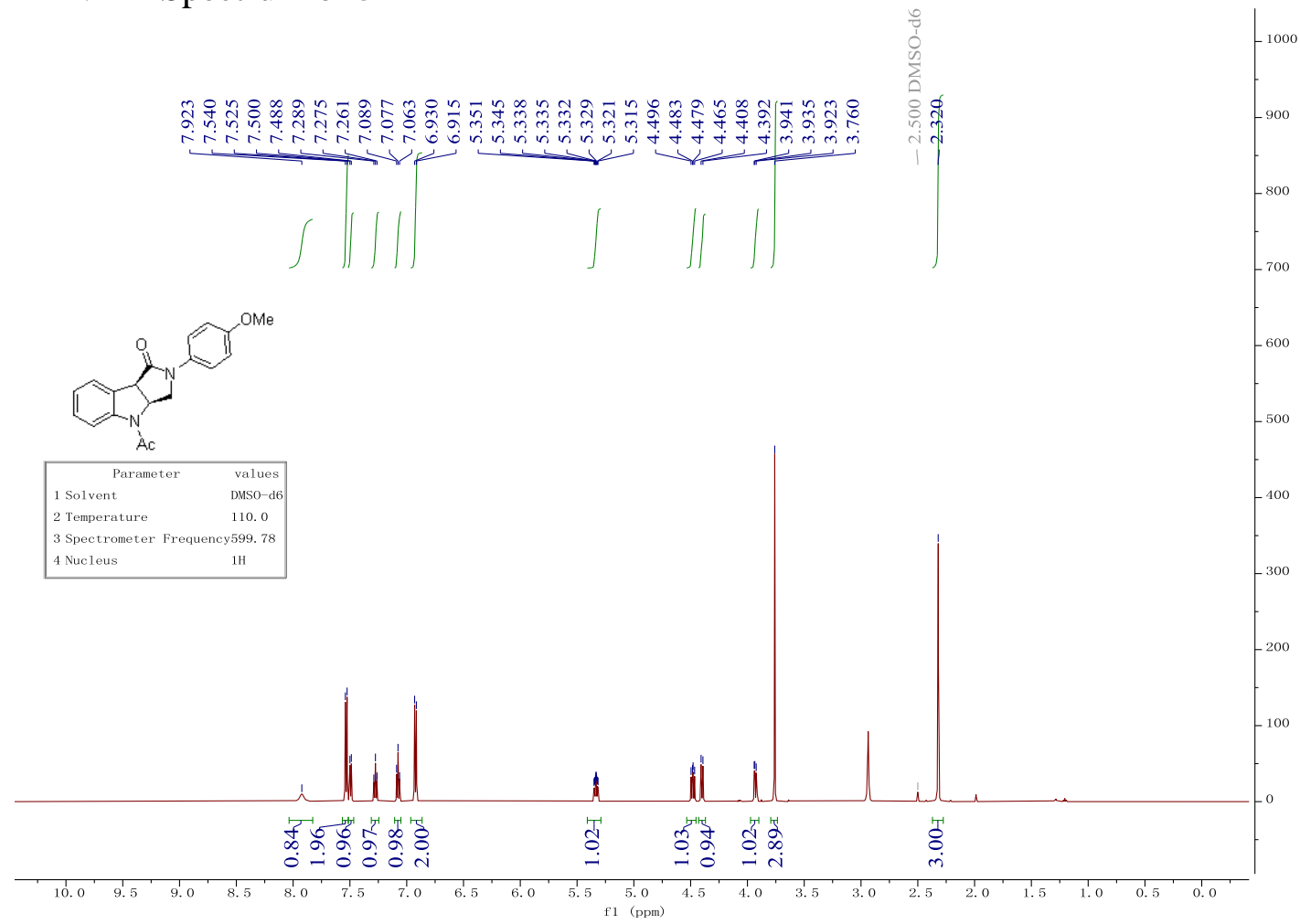

${ }^{13} \mathrm{C}$ NMR Spectrum of $\mathbf{5 i}$

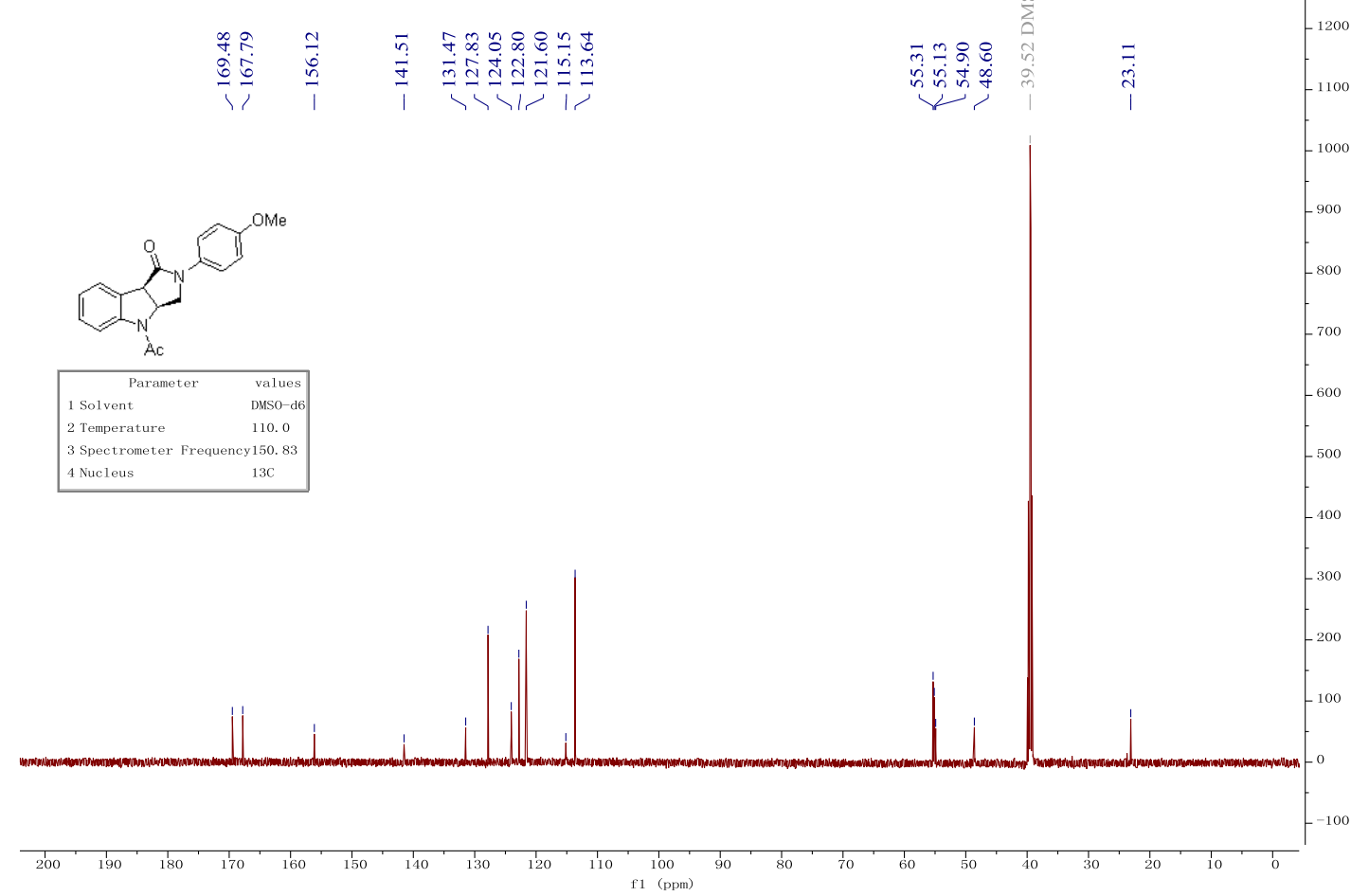


${ }^{1} \mathrm{H}$ NMR Spectrum of $\mathbf{5 j}$

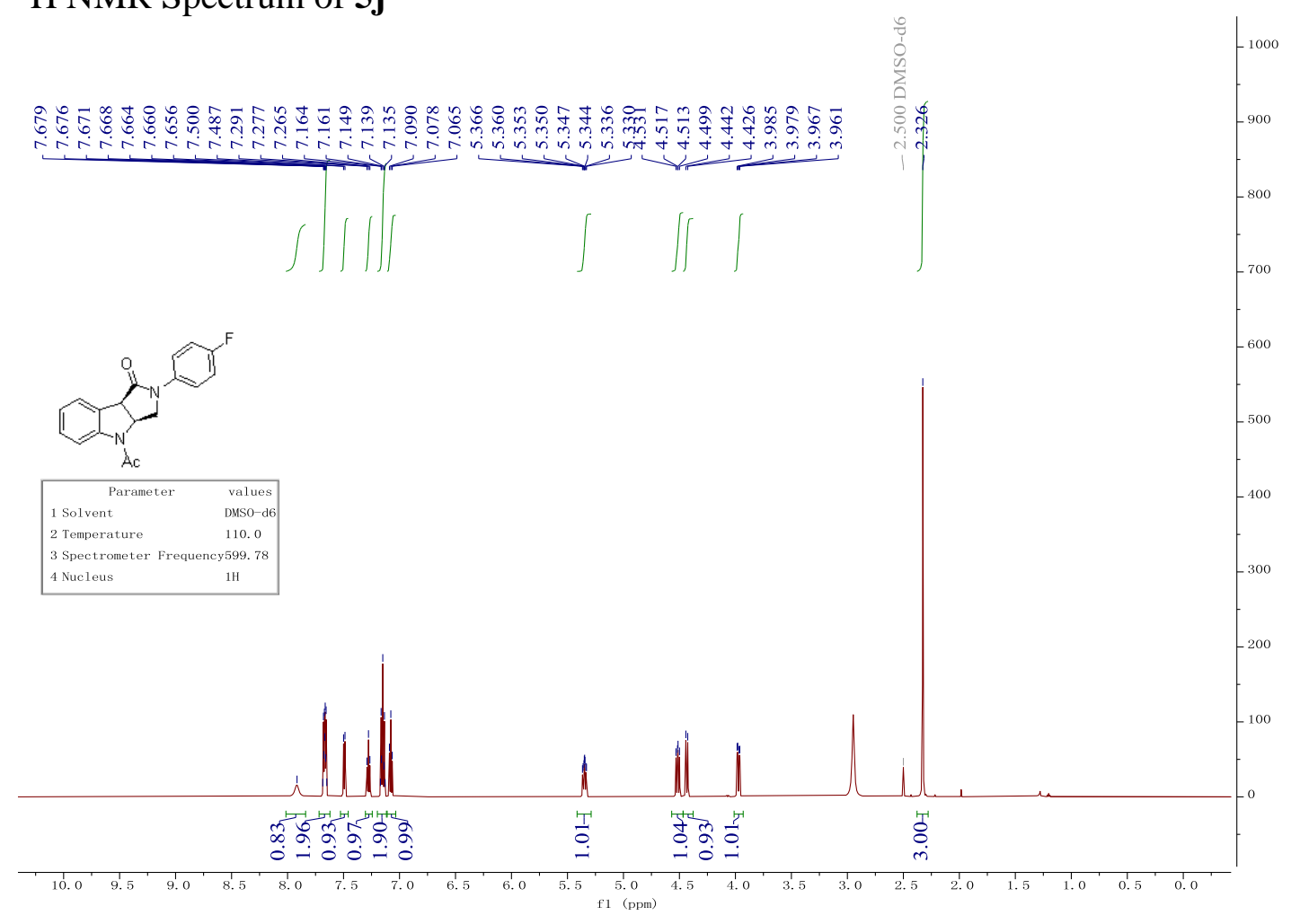

${ }^{13} \mathrm{C}$ NMR Spectrum of $\mathbf{5 j}$

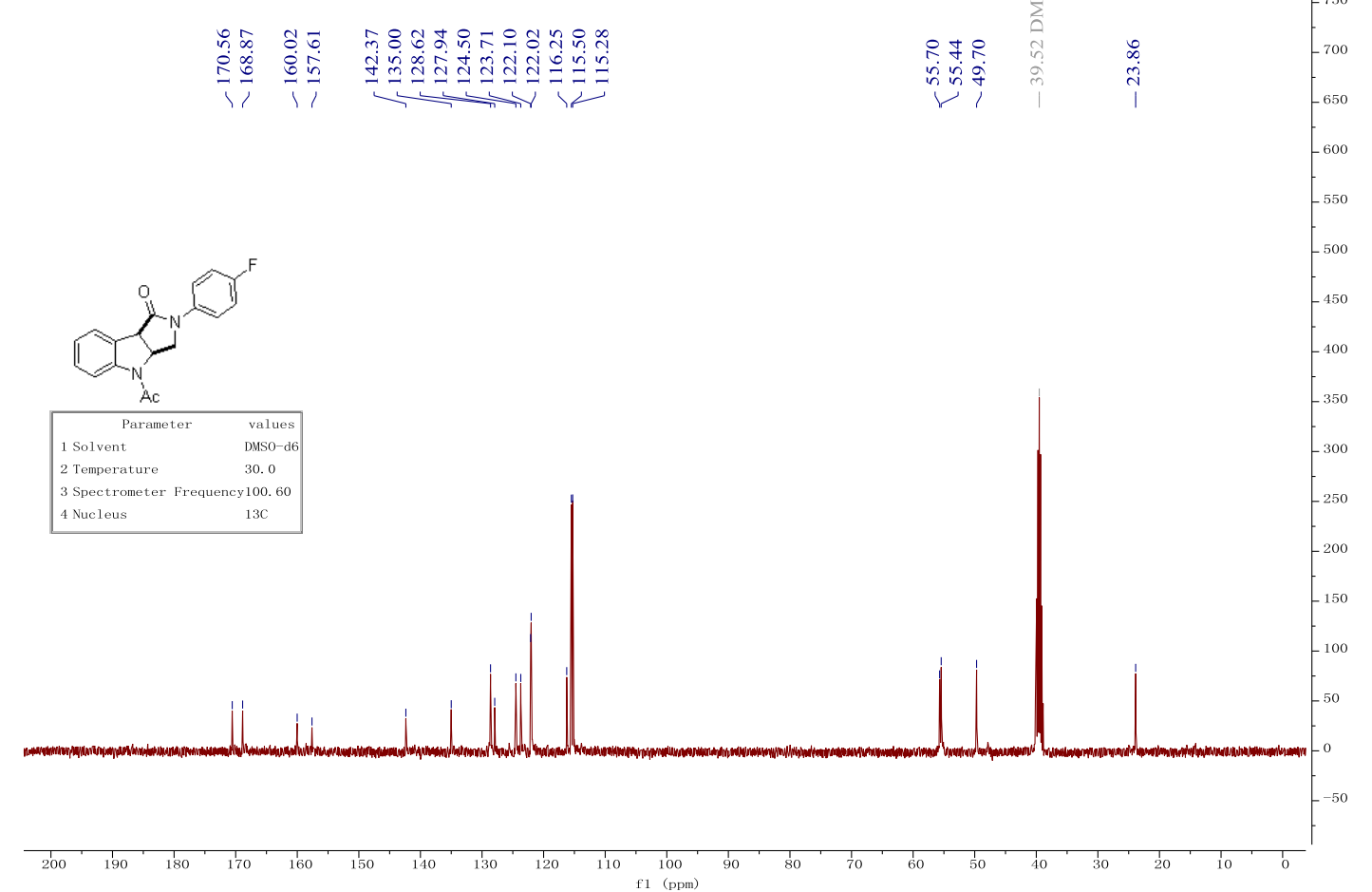

4 
${ }^{19} \mathrm{~F}$ NMR Spectrum of $\mathbf{5 j}$

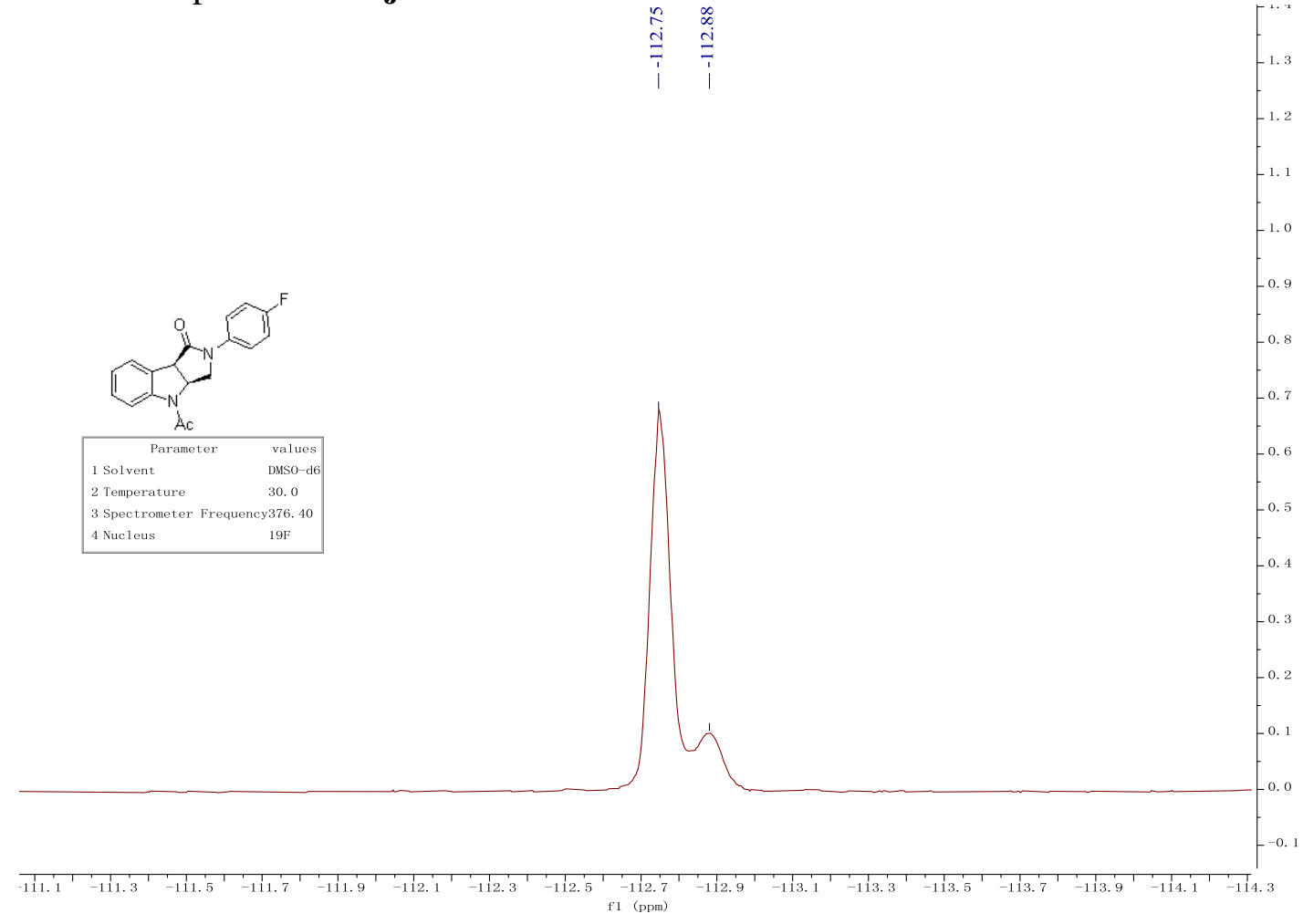

${ }^{1} \mathrm{H}$ NMR Spectrum of $\mathbf{5 k}$

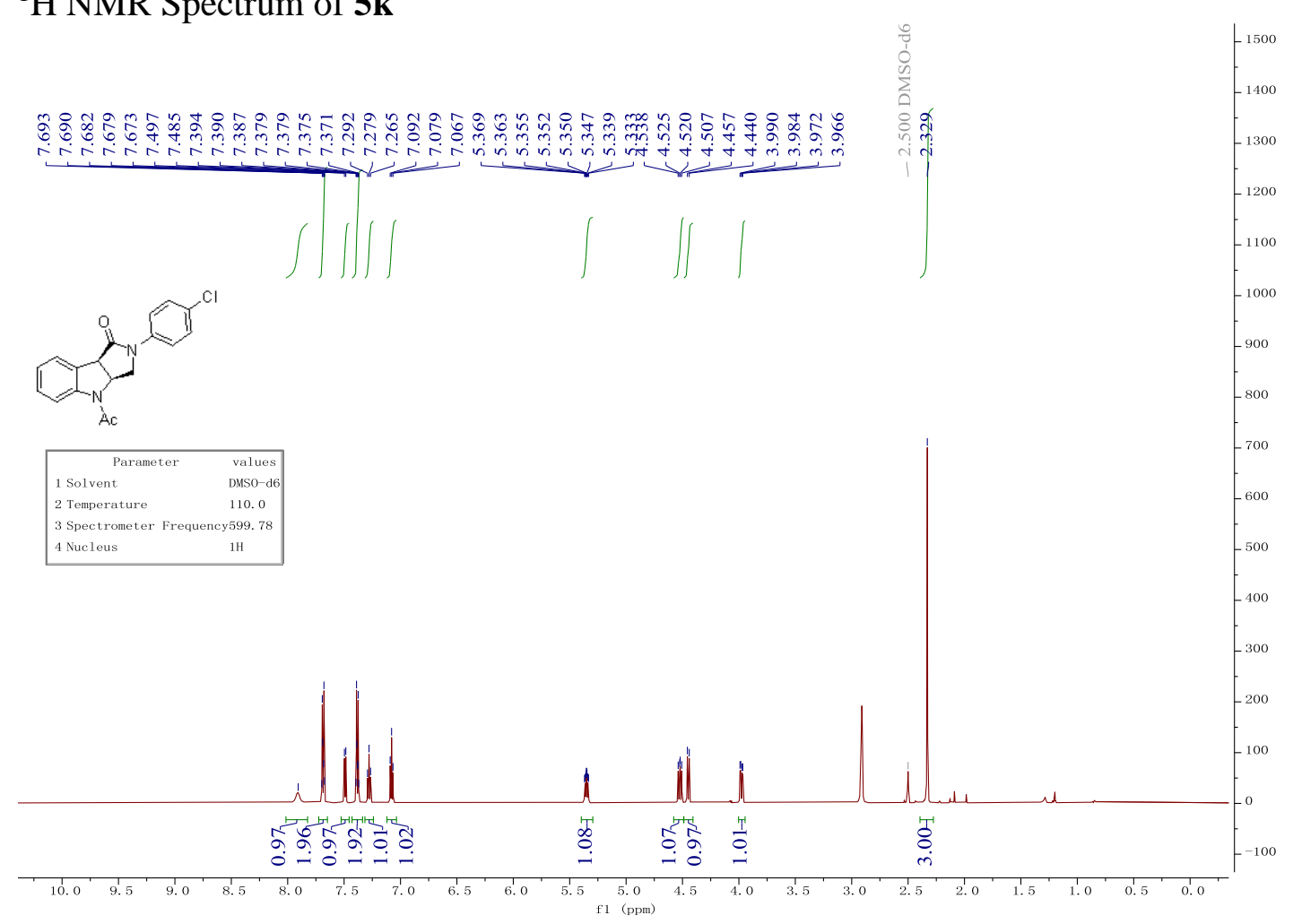


${ }^{13} \mathrm{C}$ NMR Spectrum of $\mathbf{5 k}$

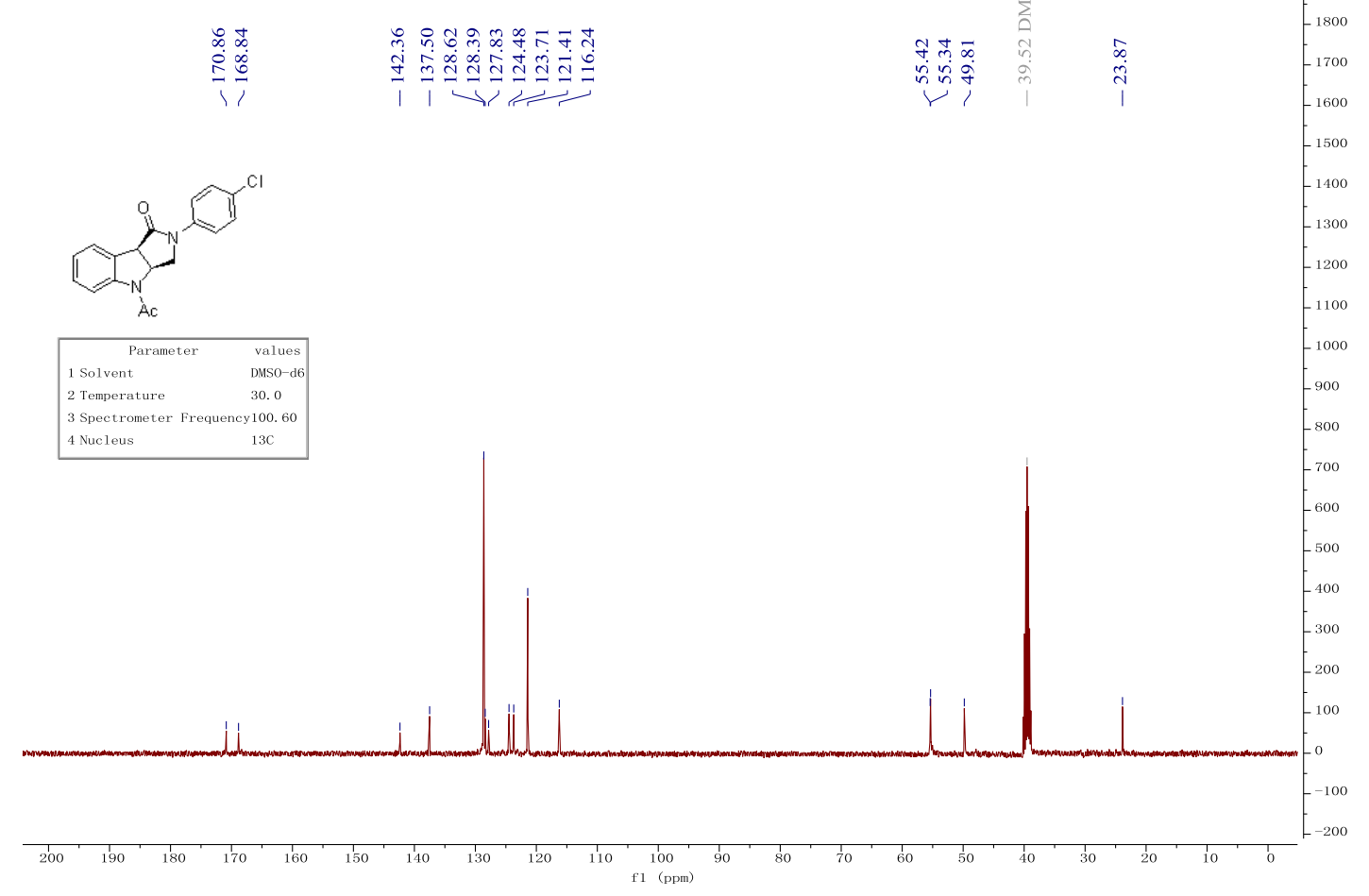

${ }^{1} \mathrm{H}$ NMR Spectrum of $\mathbf{5 l}$

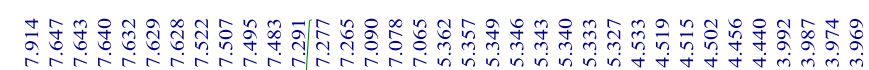
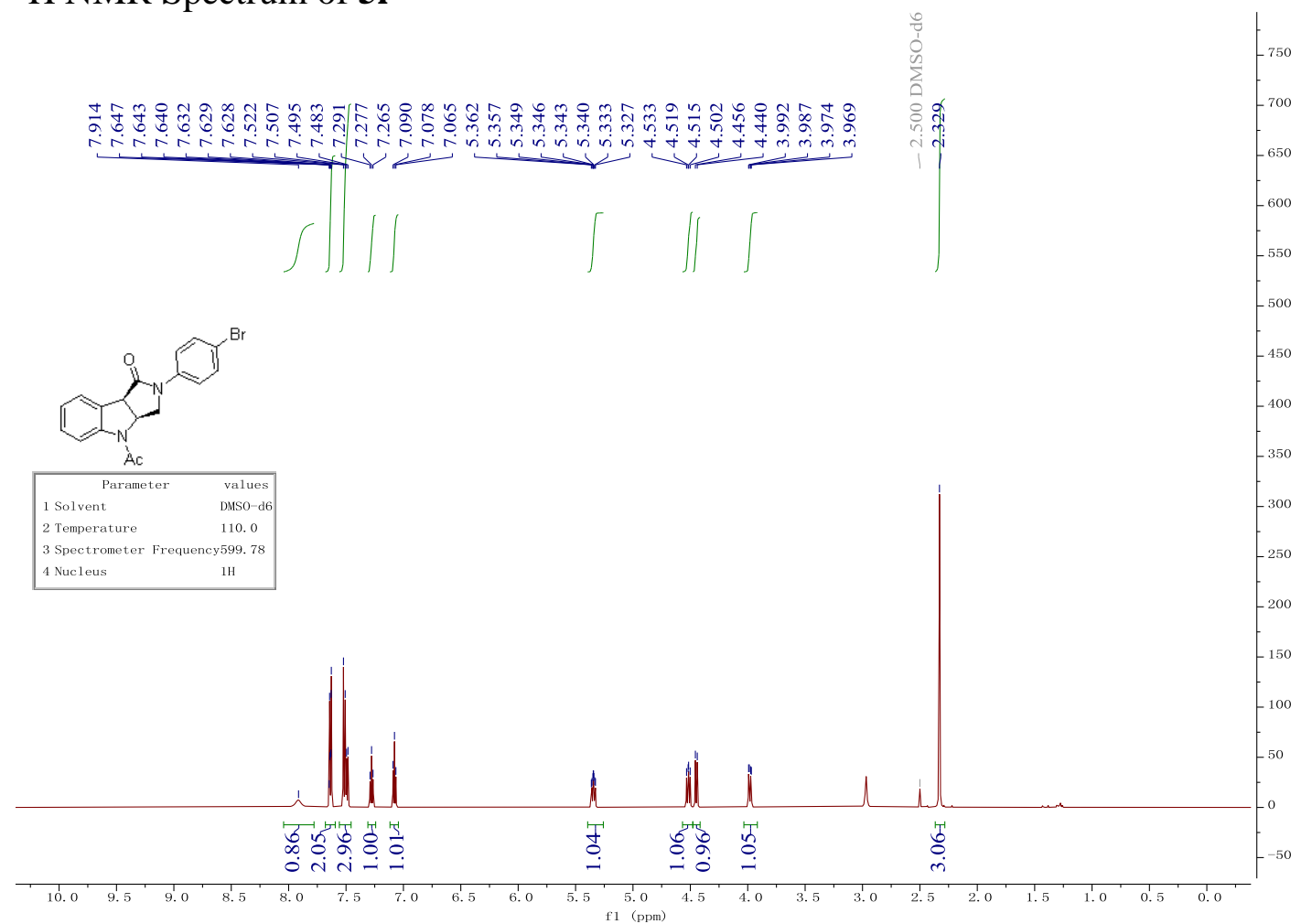
${ }^{13} \mathrm{C}$ NMR Spectrum of $\mathbf{5 l}$

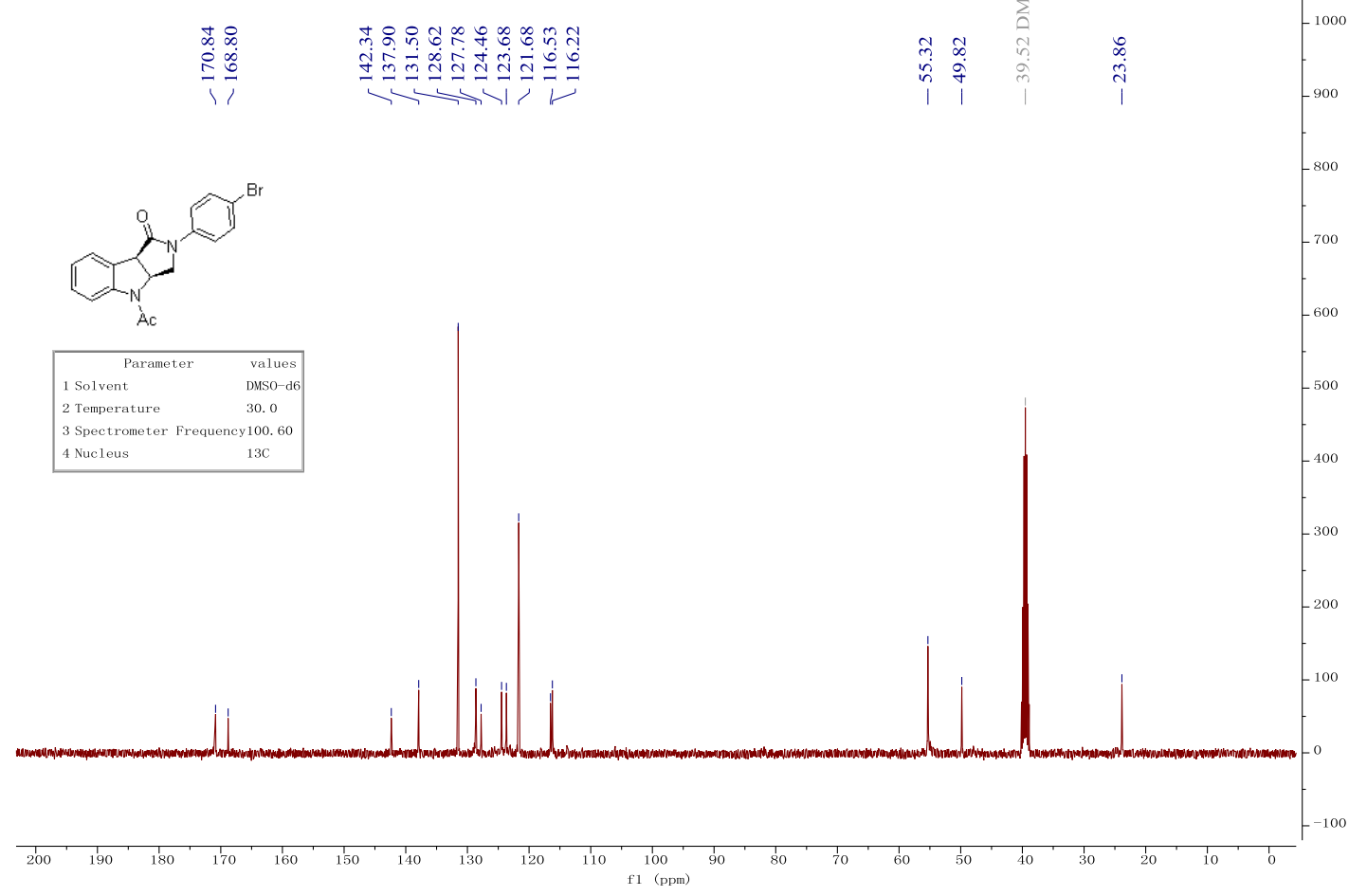

${ }^{1} \mathrm{H}$ NMR Spectrum of $\mathbf{5 m}$

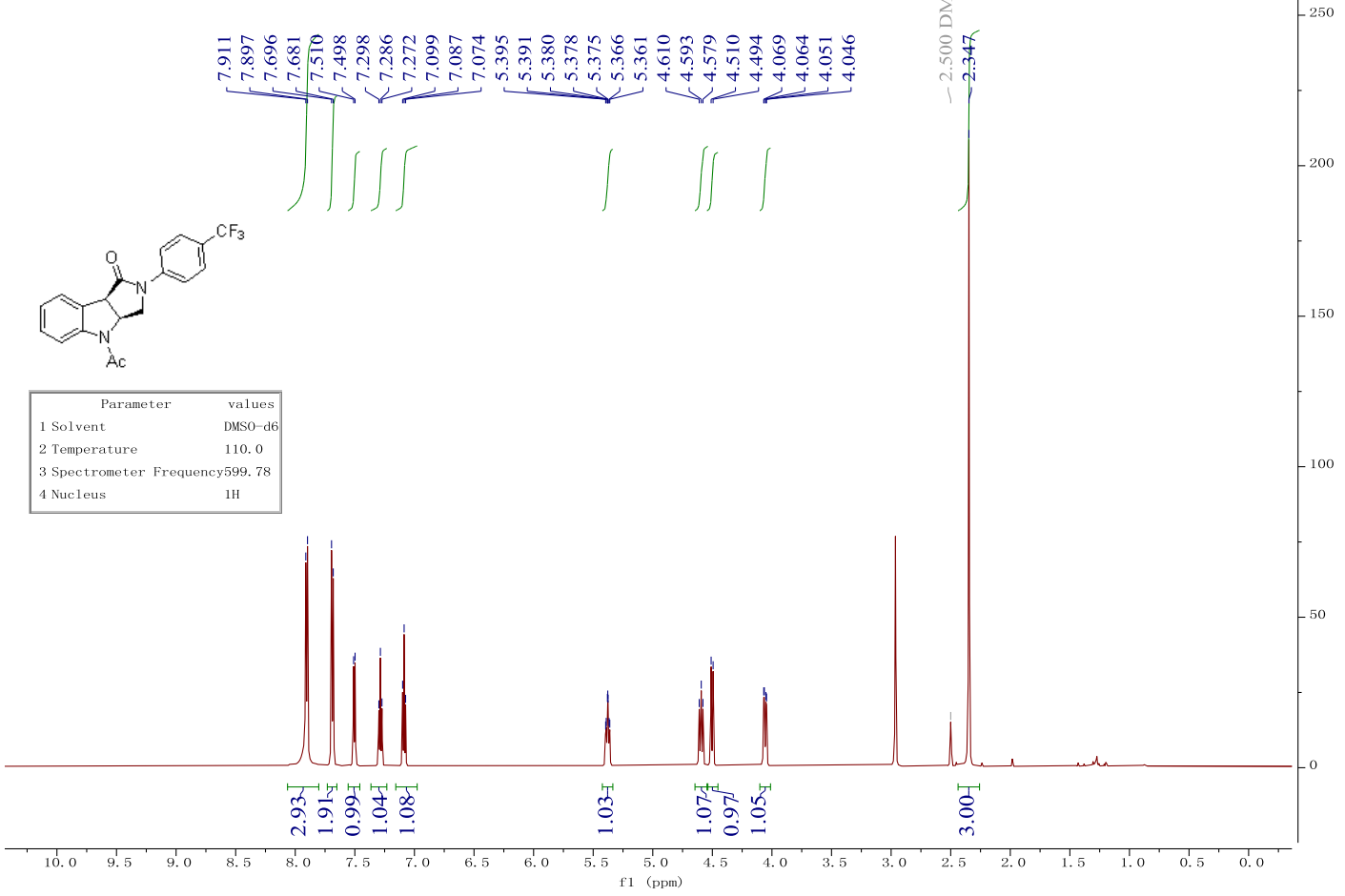


${ }^{13} \mathrm{C}$ NMR Spectrum of $\mathbf{5 m}$

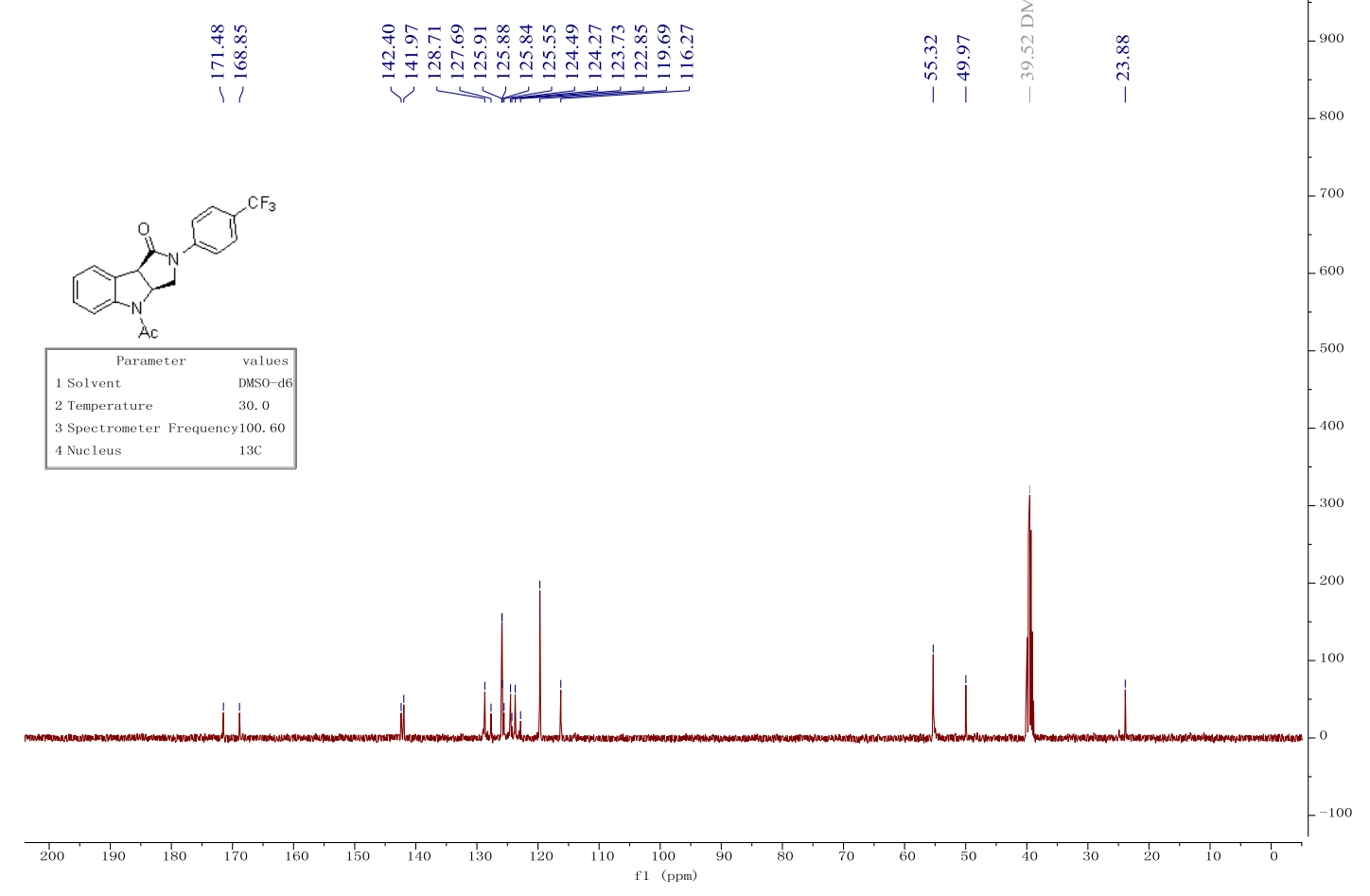

${ }^{19} \mathrm{~F}$ NMR Spectrum of $\mathbf{5 m}$

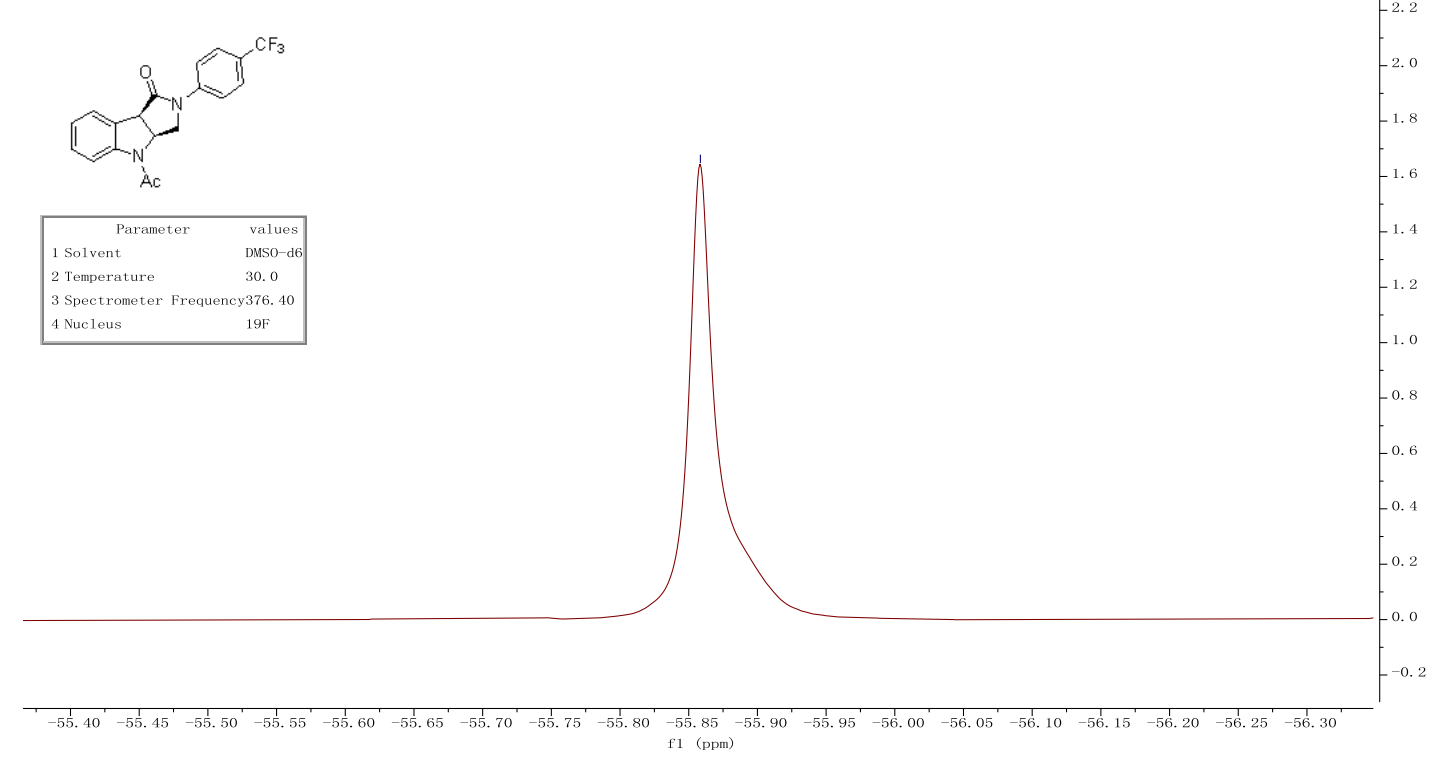


${ }^{1} \mathrm{H}$ NMR Spectrum of 7

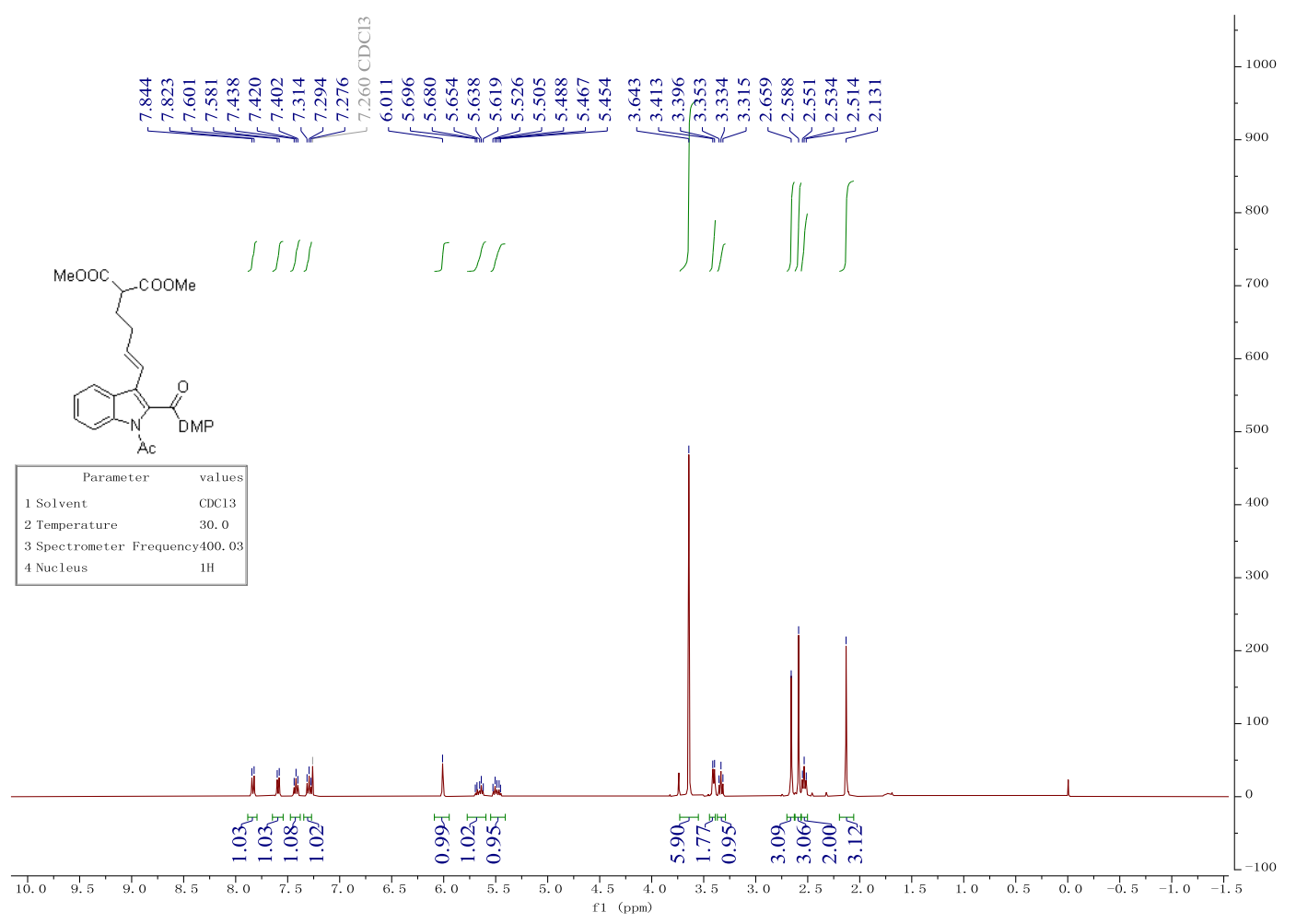

${ }^{13} \mathrm{C}$ NMR Spectrum of 7
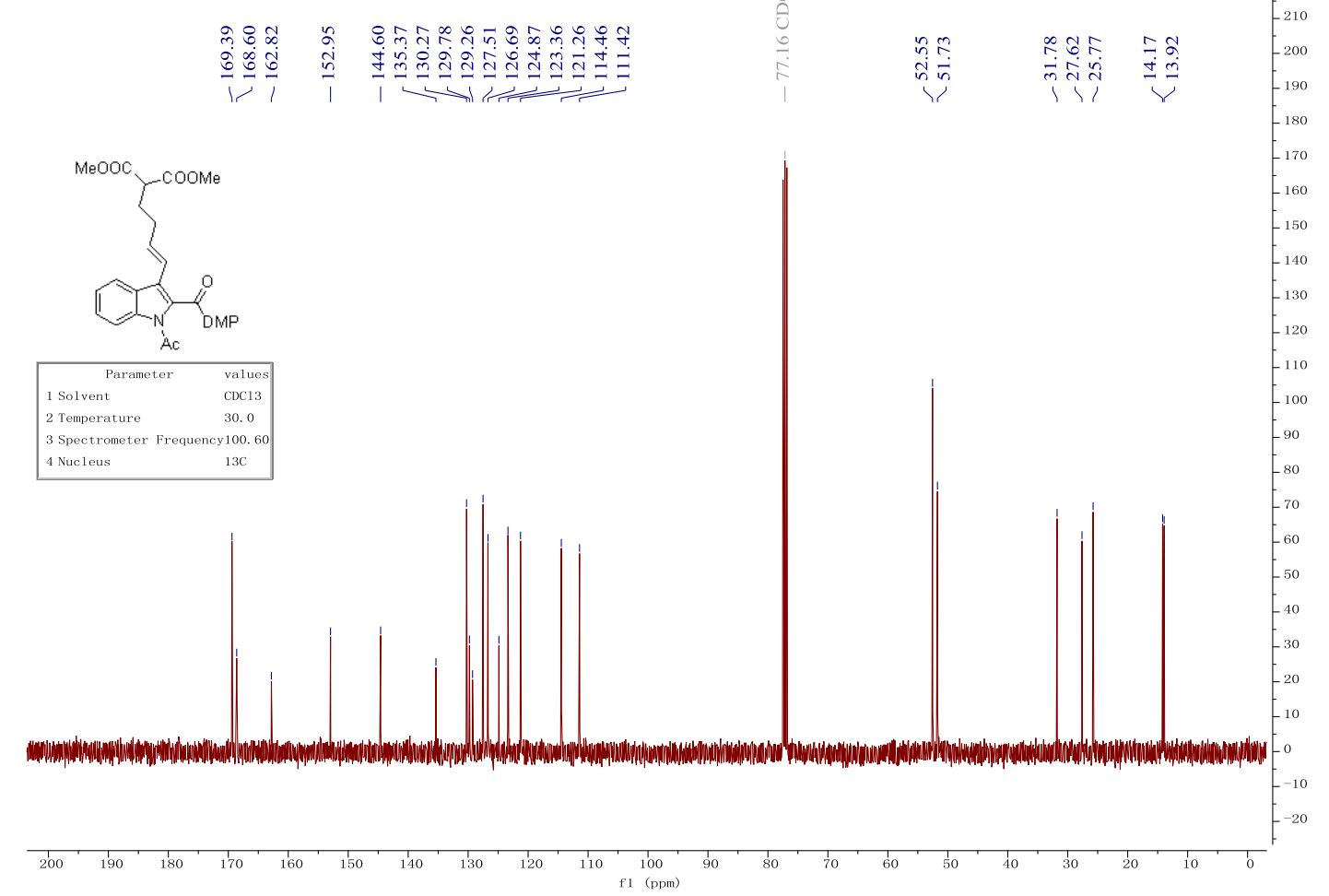